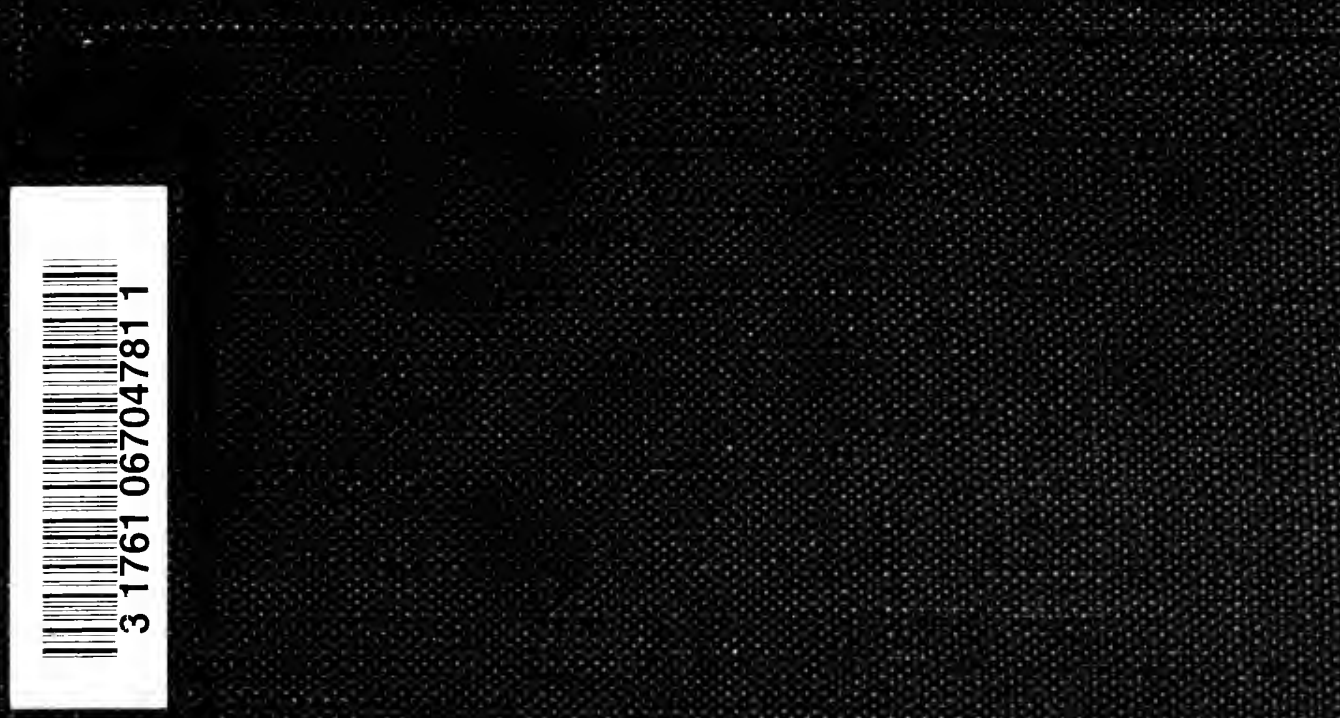





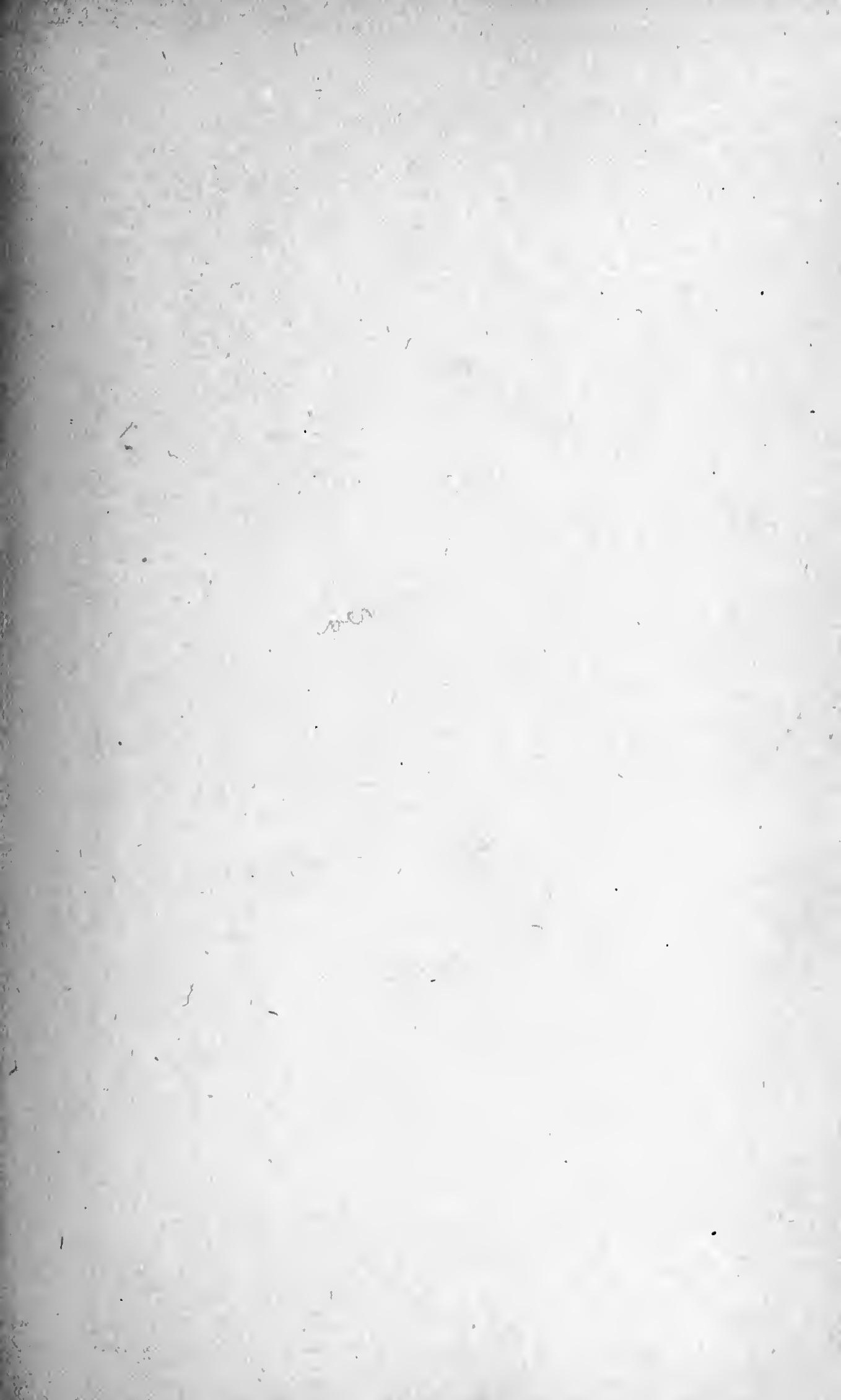


Digitized by the Internet Archive in 2007 with funding from Microsoft Corporation 


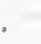




$$
i \quad \ldots
$$




\title{
THE
}

\section{ENDEMIC DISEASES}

\author{
OF THE
}

\section{SOUTHERN STATES}

BY

WILLIAM H. DPEADERICK, M. D.

Member of the Hot Springs Medical Society, the Arkansas Medical Society, and the South ern Medical Association; Fellow of the American Medical Association and A merican

Society of Tropical Medicine; Corresponding Member Societe de Pathologie Exotique (Paris); Member of the Commission for the Study and Prevention of Malaria; Secretary Malaria Section, National Drainage Congress

AND

LOYD THOMPSON, M. D.

Member of the Hot Springs Medical Society, the Arkansas Medical Society, and the Southern Medical Association; Fellow of the American Medical Asscciation; Charter Member of the American Association of Immunologists; First Lieutenant in the Medical Reserve Corps, United States Army; Formerly

Instructor in Clinical Diagnosis and Director of the Clinical Laboratory, University of Arkansas

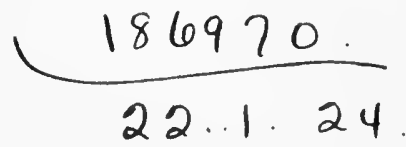

ILLUSTRATED 
Copyright, 1916, by W. B. Saunders Company

PRINTED IN AMERICA

PRESS OF

W. Q. BAUNDERs companY

PMILADELPHIA 


\section{PREFACE}

THE inception of this book was due to the fact that there is no work in existence dealing solely with the endemic diseases of the Southern States.

It must not be inferred that the diseases considered are confined to the states of the South. On the other hand most of them are disseminated throughout the United States and some of them are found in most of the states of the Union. It is true, however, that these diseases are more prevalent in the Southern States.

It has not been deemed advisable to treat of all the diseases that are especially prone to visit our confines at longer or at shorter intervals as the list of such diseases is long and such a work would necessarily be one on tropical diseases in general to which this work makes no pretension. We have concluded that it would be more practical to consider intensively only the endemic diseases of our Southland rather than to devote brief space to each of a large number of tropical diseases the most important of which will soon be of historic interest only.

Filariasis which occurs in an extremely limited area has been omitted.

We are grateful to the publishers for courtesies throughout the publication of the book.

The Authors.

Hot Springs, Arkansas,

March, r9г6. 



\section{CONTENTS}

\section{MALARIA}

WILLIAM H. DEADERICK, M. D.

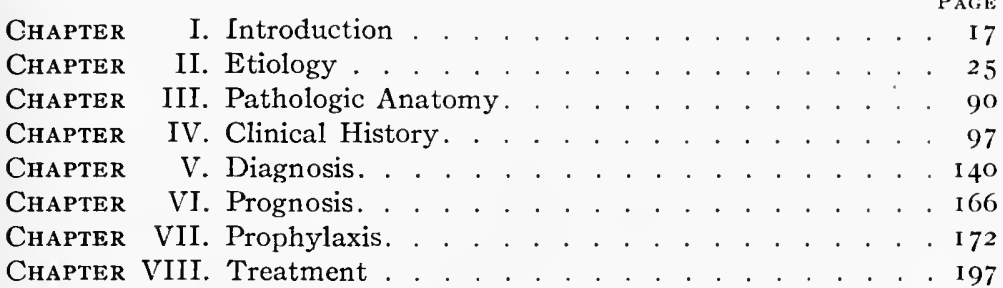

\section{BLACKWATER FEVER}

WILLIAM H. DEADERICK, M. D.

Chapter IX. Introduction . . . . . . . . . . . . . . 2 I9

Chapter X. Etiology . . . . . . . . . . . . 229

Chapter XI. Pathology . . . . . . . . . . . . . . . . . . . 247

Chapter XII. Clinical History. . . . . . . . . . . . . . . . . 250

Chapter XIII. Diagnosis . . . . . . . . . . . . . . . 262

Chapter XIV. Prognosis . . . . . . . . . . . . . . . . 265

Chapter XV. Prophylaxis. . . . . . . . . . . . . 270

Chapter XVI. Treatment . . . . . . . . . . . . . . 272

\section{PELLAGRA}

LOYD THOMPSON, Ph. B., M. D. and

WILLIAM H. DEADERICK, M. D.

Chapter XVII. Introduction ............. 281

Chapter XVIII. Etiology . . . . . . . . . . . . . . . . . . . 287

Chapter XIX. Pathology . . . . . . . . . . . . 3I3

Chapter XX. Clinical History. . . . . . . . . . . . . . 324

Chapter XXI. Diagnosis. . . . . . . . . . . . . . $36+$

Chapter XXII. Prognosis. . . . . . . . . . . . . . . . 379

Chapter XXIII. Prophylaxis. . . . . . . . . . . . 380

Chapter XXIV. Treatment . . . . . . . . . . . 385 


\section{AMEBIC DYSENTERY}

LOYD THOMPSON, Ph. B., M. D. and

WILLIAM H. DEADERICK, M. D.

Chapter XXV. Introduction. . . . . . . . . . . 395

Chapter XXVI. Etiology . . . . . . . . . . . . . . . 401

Chapter XXVII. Pathology. . . . . . . . . . . . . . . 4I3

Cyapter XXVIII. Clinical History . . . . . . . . . . . . . 419

Chapter XXIX. Diagnosis . . . . . . . . . . . . . . 427

Chapter XXX. Prognosis . . . . . . . . . . . . . 434

Chapter XXXI. Prophylaxis . . . . . . . . . . . 436

Chapter XXXil. Treatment . . . . . . . . . . . . . 438

\section{HOOK-WORM DISEASE}

WILLIAM H. DEADERICK, M. D.

Chapter XXXIII. Introduction. . . . . . . . . . . . 445

Chapter XXXIV. Etiology . . . . . . . . . . . 45I

Chapter XXXV. Pathology.............. 460

Chapter XXXVI. Clinical History . . . . . . . . . . . . 462

Chapter XXXVII. Diagnosis ................ . 473

Chapter XXXVIII. Prognosis . . . . . . . . . . . . . 478

Chapter XXXIX. Prophylaxis . . . . . . . . . . . . . 480

Chapter XL. Treatment. . . . . . . . . . . . . . . . . 487

\section{OTHER INTESTINAL PARASITES}

WILLIAM H. DEADERICK, M. D.

Chapter XLI. Other Intestinal Parasites

REFERENCES . . . . . . . . . . . . . . . . 528

INDEX 


\section{ENDEMIC DISEASES OF THE SOUTHERN STATES}

\section{MALARIA}

\section{CHAPTER I}

\section{INTRODUCTION}

History.- The history of malaria may be traced to the age of fable. The story of Hercules and the Hydra is a familiar one. This monster dwelt in the morasses in the neighborhood of the Lake of Lerna, where Hercules was dispatched to destroy him. As each of the nine heads was struck off two new ones appeared. With the aid of his faithful servant, Iolaus, who burned each wound caused by the removed head, the beast was finally conquered. Even before the birth of Christ this myth was construed to typify the reclamation of swamp lands, uninhabitable on account of the presence of malaria. Antipater wrote, "Hercules, the greatest subduer of the foggy atmosphere in times past, was placed among the Gods for having destroyed the Hydra; in other words for having reclaimed the marshy desert." The slaying by Apollo of the Python which arose from the fertile ground after the recession of the flood is similarly interpreted.

More than one thousand years before the birth of Christ malarial disease is mentioned in the Orphic poems, and the tertian and quartan types are alluded to. In the Iliad of Homer, and the Wasps of Aristophanes allusions are made to a fever which was probably malarial. Paludism was probably introduced into Greece from Egypt. According to Groff the word AAT, which is found among the inscriptions of the temple of Denderah, referred to a disease, doubtless malaria, which recurred every year at the same season. 
Hippocrates divided malarial fevers into continuous and intermittent, which he subdivided into quotidian, tertian, and quartan. He recognized the etiologic influence of season, rains, and stagnant water, and the dangers of malignancy, dropsy, and affections of the spleen. Plato describes splenic enlargement, and other early Greek writers undoubtedly refer to malaria.

References by Roman writers to malaria are not numerous, the earliest being that of Plautus, who died r84 B.C. Cato speaks of "black bile and swollen spleen," and Cicero, Varro, Celsus, Livy, and others show unmistakable evidence of a knowledge of the disease.

Passing over the development during the middle ages of the knowledge of malaria, the names of Morton, Lancisi, Sydenham, and Torti appear.

Morton, I697, gave accurate clinic descriptions of the pernicious and intermittent fevers, and attributed them to miasmatic effluvia. He was an ardent advocate of cinchona whose value was at that period being hotly contested.

Sydenham, I723, accurately described the malarial fevers. The intermittent fevers he divided into spring and autumn fevers. He justly concluded that the intermittent and continuous forms of malaria were due to the same cause. Sydenham ably defended cinchona, and after clinic experiments with its use, formulated useful rules for its administration.

Lancisi, I717, stated the etiologic relationship between marshy regions and malaria, and was the first to seek for a microscopic organism as the cause of the disease.

Torti, I 753, wrote an exhaustive treatise upon the various forms of malaria. His classification of the pernicious forms has become classical. Numerous quotations from Torti's treatise are to be found even in recent works upon malaria.

Varro, Ir 8-29 B.C., expressed the opinion that malarial fever was caused by animals so minute that they could not be seen by the naked eye, and which enter the body with the air through the nose and mouth. Similar opinions were held by Columella, Palladius, and Vitruvius. Rasori is quoted as saying, "For many years I have held the opinion that the 
intermittent fevers are produced by parasites which renew the paroxysm by the act of their reproduction which occurs more or less rapidly according to the variety of their species." Le Diberder, r869, maintained that the fever was due to the presence in the blood of animals which preyed upon the blood, and that the paroxysms depended upon reproductive acts between which apyrexia occurred.

Mitchell, I849, claimed to have found in the sputa of malarial subjects fungus spores in great numbers, which he believed to have been inspired with marsh air and to have caused the disease.

Salisbury, r866, announced the discovery in the urine and sweat of malarial patients of a species of alga, palmella, common on the marshy regions along the Ohio and Mississippi Rivers, which he alleged to be the causative element.

Until the true parasite of malaria was discovered, the most widely accepted parasitic theory was that proposed in 1879 by Klebs and Tommasi-Crudeli. These investigators found constantly present in the mud of the Roman marshes a short bacillus. They were able to cultivate it upon fish gelatine, and when injected into rabbits produced a fever similar to malaria. They named it the bacillus malaria.

The malaria parasites were undoubtedly seen and described before Laveran discovered them. In 1847 , Meckel, who first discovered malarial pigment, described bodies containing pigment which correspond to the malarial parasites. Virchow, in 1849 , in a description of the pigment, depicted cells now known to be parasites, as did also Frerichs in 1866 . The pigment was observed also by Dlauhy, Heschl, and Planer. None of these investigators, however, recognized the significance of these bodies, and their parasitic nature was not suspected until 1880 by Laveran, to whom all the more honor is due.

Charles Louis Alphonse Laveran was born at Paris, June I8, 1845. He entered the military service and was assigned to Algeria where his brilliant discovery was made Nov. 6, I880, and announced to the Paris Academy of Medicine, Nov. 23, r880. He was using a one-sixth inch dry lens when examining the blood. 
Laveran's discovery was not accepted by the medical world until several years later; now it has been confirmed the world over. Among Americans who first corroborated Laveran's views may be mentioned Sternberg, Councilman and Abbott, Osler, James, Dock, Thayer and Hewetson, Barker, Woldert and Welch.

The discoveries of Golgi in 1885 were of great importance with reference to the life history of the parasite. He was able to follow tertian and quartan parasites throughout the endogenous cycle of development, and showed that a close relationship existed between certain phases of parasitic growth and certain stages of the paroxysm. Other Italian investigators proved the same for the estivo-autumnal parasites.

A vague suspicion that malaria and mosquitoes were in some definite way connected has been entertained in certain countries for a long period. A definite mosquito theory, however, was born in America. While reference is sometimes made to a paper on the "Mosquital Origin of Malarial Disease," supposed to have been published by Dr. John Crawford in the Baltimore Observer, in 1807 , no such article has been found, and the reference is probably erroneous.

In I 848 , Dr. Josiah Nott, ${ }^{434}$ of Mobile, published a paper upon yellow fever, in which he maintained the dissemination of that disease by insects, and suggested that malaria was spread by the "mosquito of the lowlands."

The most complete theory was proposed by King, ${ }^{435}$ in 1883 . His views are supported by nineteen arguments, most of which are incontestable at the present day.

That mosquitoes are agents in the spread of malaria was advanced by Koch in 1884 , by Laveran in 1884 , by Flugge in $\mathrm{I} 89 \mathrm{I}$, by Manson in 1894 , and by Bignami in 1896 .

Undertaking the work of Manson's suggestion, and after several years $\left(1895^{-1} 898\right)$ of toil and discouragement, Ross proved conclusively that certain species of mosquitoes were concerned in the dissemination of malaria. The debt owed him by mankind was acknowledged by the gift of a Nobel prize.

MacCallum in 1898 demonstrated that the flagella represent male sexual elements analogous to spermatozoa. 
Bass' discovery of the method of cultivating the malarial parasites is the most important landmark in the history of malaria since the discovery of Ross.

Geographic Distribution.-North America.-In the United States it is chiefly the southeastern portion in which malaria is most prevalent. Along the Atlantic Coast, south of New York and especially the lowlands of Maryland and Virginia, and in the Carolinas, Georgia and Florida the disease occurs frequently. Along the Gulf coast and up the Mississippi River and its tributaries, malaria is widely prevalent. The portions of the States lying along the Appalachian Range are almost exempt, but the disease appears as the Mississippi River and the Atlantic Coast upon either side are approached. West of the Mississippi, Arkansas, Louisiana, and 'Texas present the most numerous foci of malaria. In portions of Pennsylvania and New York autochthonous cases are not infrequently observed. In the more southern New England States malaria is still encountered, and in some places is even increasing in frequency. In the neighborhood of the Great Lakes malaria is very rare, excepting possibly that of Lake Erie and of Lake Michigan. In the Central States malaria has almost or quite disappeared, except in certain low river valleys. Along the Pacific Coast the disease is not so frequent as along the Atlantic. In Washington it occurs in the Puget Sound Basin and the Columbia River, Chehalis, and the Yakima valleys. In Oregon malaria is found in the Columbia, Williamette, Rogue, and the Umatilla valleys, and in California in the Sacramento, San Joaquin, Tulare, Kern, and Santa Clara valleys. In certain parts of New Mexico malaria is occasionally met with.

Canada is free from paludism except along the northern shore of Lake Ontario.

In Mexico severe forms of malaria occur, particularly in the low coast regions.

Malaria abounds in Central America along the Atlantic Coast and to a less extent upon the Pacific side.

South America.-The eastern coast of South America is more intensely infested with malaria than is the western coast. Venezuela (in the valleys), Guiana, and the greater portion of 
Brazil are highly malarial. Portions of Paraguay and of Bolivia afford a great many cases, while the disease is much less prevalent in Uruguay and almost absent from the Argentine Republic. On the Pacific border the deep valleys of Peru and of Ecuador are malarial centers.

The entire island of Cuba is malarial to a greater or less extent, as is also Jamaica. Of the Lesser Antilles, St. Vincent and Antigua are highly malarial, and Barbadoes is exempt. Malaria is said to be almost unknown in the Bermudas.

Europe--Great Britain, once infested, is now free from endemic malaria. In Germany the disease occurs infrequently in the Rhine and Danube valleys and near the mouths of rivers along the coast. Malaria is met in Holland, chiefly upon the island of Zeeland and in North and South Holland. The valley of the Danube, in Austria, affords a considerable number of cases. There are a few regions in Hungary in which the disease does not occur, but it is especially along the western half of the southern border that it is prevalent. The marshes along the west coast and the south of France give rise to a number of cases of malaria. In Spain and Portugal malaria occurs in the coast regions and in the larger river valleys. The disease is practically unknown in Norway, but is occasionally reported from Sweden, as well as from certain of the islands of Denmark. In Russia it is in the southern portion, particularly along the coasts and along the valleys of the rivers flowing southward, that malaria is encountered. Cases are occasionally observed in the southwest of Switzerland. The portions of Bulgaria most highly malarial are the Danube valley, the coast region, and the southern part. Almost the whole of Italy is sorely afflicted with malaria, as are also Sicily and Sardinia. Greece is the most severely scourged country of Europe. It is said that in the plains of Thessaly, Phthiotis, Acarnania, Boeotia, Elis, Messenia, Argos, and Laconia hardly a single inhabitant escapes the disease.

Asia.-Asia Minor, Arabia, and Persia present foci of malaria, both in the coast neighborhoods and in the interior lowlands. In the swampy regions of Afghanistan and Beloochistan malaria is common and severe. The foothills of the Himalayas, 
the Duars, and Terai are famous malaria seats. Both the coast regions and the interior highlands of Ceylon are endemic territory. Burmah, Siam, the Malay Peninsula, and French Indo-China are malarial in portions of their extent, and parts of China are intensely infested. Malaria is found in Japan, Formosa, and the Philippines, and portions of the East Indies are among the most highly malarial regions of the world.

Africa.-On the west coast the territory, between the Senegal and the Congo Rivers, is headquarters for malaria of malignant type. Approaching South Africa the disease diminishes in frequency and in severity. On the east the region from Delagoa Bay to Eritrea is malarial. In the interior of Central Africa, excepting the high elevations, malaria is widespread. Malaria abounds in Madagascar, excepting upon the northeast coast and the mountainous interior. Reunion and Mauritius are also malarial. In Egypt it is chiefly the region overflowed by the Nile in which the disease is most prevalent. Malaria abounds about the coasts and marshes of Algeria.

In Australia malaria occurs from Cape York to Brisbane, on the east coast, diminishing toward the south. New Zealand is apparently free from malaria, and the Sandwich Islands and most of the other Pacific islands are markedly exempt.

The relative frequency of the forms of malarial infection varies greatly. It may be stated as a general proposition that the quartan is the rarest form, the tertian is the form prevailing in temperate regions, and the estivo-autumnal in warm and hot climates. There are regions, however, in which the quartan predominates, as in certain portions of Italy and of India; in other localities it is the only form of malaria present, as upon the island of Merite, of the Bismarck Archipelago.

The following table shows the relative frequency of the types of malaria in various regions: 
ENDEMIC DISEASES OF THE SOUTHERN STATES

\begin{tabular}{|c|c|c|c|c|}
\hline Locality & Authority & Tertian & Quartan & $\begin{array}{c}\text { Estivo- } \\
\text { autumnal }\end{array}$ \\
\hline Texas. & Moore ${ }^{436}$ & 23 & $\circ$ & 30 \\
\hline Georgia.. & Curry ${ }^{437}$ & 34 & $\circ$ & 16 \\
\hline Camp Wikoff. & Ewing ${ }^{72}$ & 74 & $\circ$ & 261 \\
\hline New Orleans...... & Charity Hospital Recs. ${ }^{438}$ & 373 & $\mathbf{I}$ & 203 \\
\hline Baltimore. . & Thayer and Hewetson ${ }^{439}$ & 338 & 5 & 188 \\
\hline Panama.... & Kendall $^{440}$ & 22 & $\circ$ & 291 \\
\hline St. Lucia....... & Gray and Low 441 & 12 & 2 & 109 \\
\hline Panama......... & Gorgas $^{42}$ & 4,812 & 8 & 10,815 \\
\hline Italy.......... & Koch $^{43}$ & 32 & 5 & 78 \\
\hline Italy............ & Koch $^{44}$ & 202 & 15 & IgI \\
\hline Greece........... & Cardimatis and Diamessis ${ }^{445}$ & 87 & 3 & 145 \\
\hline Bulgaria.......... & Mollow 46 & 99 & 10 & 67 \\
\hline Italy $\ldots \ldots \ldots \ldots \ldots$ & Italian statistics 447 & 32,392 & 6,846 & 23,520 \\
\hline British Malaya..... & Wright ${ }^{48}$ & 78 & 56 & 117 \\
\hline British Malaya..... & Watson ${ }^{48}$ & 19 & 4 & 28 \\
\hline Philippines....... & Craig ${ }^{449}$ & 98 & 8 & 272 \\
\hline India............ & Hope 450 & 217 & 933 & 547 \\
\hline Cyprus........... & Williamson 451 & 12 & 8 & 4 \\
\hline East Indies........ & $\operatorname{Koch}^{452}$ & 57 & II9 & 123 \\
\hline Philippines........ & Chamberlain 453 & 55 & 3 & 62 \\
\hline India............ & Rogers ${ }^{17}$ & $\mathrm{I}, 372$ & 71 & 1,311 \\
\hline India............. & Buchanan ${ }^{454}$ & 56 & 12 & 118 \\
\hline Assam............ & Bentley 454 & 134 & 46 & 74 \\
\hline Japan............. & Tsuzuki ${ }^{454}$ & 345 & 12 & 107 \\
\hline Togo.............. & Ziemann $^{86}$ & I & 7 & 32 \\
\hline German East Africa. & Meixner ${ }^{33}$ & 5 & I & 102. \\
\hline German East Africa. & Grothusen $^{33}$ & 5 & 7 & 68 \\
\hline Senegal............ & Thiroux and d'Antreville ${ }^{455}$ & 7 & 44 & 266 \\
\hline German East Africa. & Kudicke $^{44}$ & 3 & 2 & 118 \\
\hline German East Africa. & Exner ${ }^{71}$ & II & 4 & 328 \\
\hline German East Africa. & Ollwig71 & 7 & $\circ$ & 134 \\
\hline German East Africa. & Schornich $^{71}$ & I & 2 & 130 \\
\hline
\end{tabular}




\section{CHAPTER II}

\section{ETIOLOGY OF MALARIA}

Malaria is of very complex etiology, depending as it does for its existence upon the life histories of three species of animals. While within the blood of man the parasite is not subject to great variations of environment, no matter what the season or the latitude, nevertheless exposure to cold, wet or heat, dietary or other excesses, will have the effect of awakening latent malaria.

But it is the influence of external factors upon the life history of the mosquito that determines the greatest variations in the prevalence of malaria according to climate, season, temperature, rainfall, altitude, etc.

Climate.-It may be said as a general rule, that the frequency and virulence of malaria increase as we approach the equator. The conditions of warmth and moisture are more propitious for the development of the parasites within the bodies of mosquitoes in tropic than in colder climates; this is especially true of the estivo-autumnal form of the malarial parasite. Exposure to the heat of the tropical sun predisposes to the cerebral forms of pernicious malaria, and undue exposure to the sun's rays is ofttimes sufficient to stimulate sporulation of the parasites of latent malaria.

With respect to latitude Hirsch ${ }^{15}$ reached the following conclusions as to the northern boundary of malaria in the northern hemisphere. The line starts from $55^{\circ} \mathrm{N}$. on the western side of North America, sinks to $45^{\circ}$ on its eastern side, rises to $63^{\circ}$ or $64^{\circ}$ on the western side of the old world (Sweden and Finland), and runs across Northern Asia in about the latitude of $55^{\circ}$.

Long before the discovery of the rôle of the mosquito in malaria it was known that the disease was not endemic unless the summer temperature maintained a certain average. Hirsch maintained that the summer isobar of $59^{\circ}-60.8^{\circ} \mathrm{F}$. marks the 
limit of the occurrence of malarial fever, and that those regions where the mean summer temperature does not reach that height are exempt from the disease. Curiously enough, it has been recently repeatedly demonstrated that this is the lowest temperature at which the parasite will develop in the body of the mosquito.

Season.-While relapses may occur at any season, and in certain tropic regions fresh infections may occur during any period of the year, in all temperate and most tropic regions there are seasons during which the disease is especially prevalent. This is commonly known as the malarial season, and varies according to latitude, temperature, rainfall, soil, etc.

The season of primary attacks depends entirely upon the life-history of the malaria-bearing mosquitoes. This season usually begins a few weeks after the first brood of anophelines appears, which is at the height of summer, and continues, in temperate climates, until after the nights become cool. In each individual locality the beginning of the season is rather definite, the disease recurring at a certain period each year with more or less exactitude. In most of the regions of the Southern States the malarial season begins in the earlier half of July. In the latitude of Baltimore the most notable increase in cases begins during August. The malarial season in California is from August to October.

Where both tertian and estivo-autumnal malaria are endemic, the malarial season is usually ushered in by cases of the former, the estivo-autumnal variety appearing at the height of the season. The pernicious forms of malaria occur with greatest frequency at the height of estivo-autumnal prevalence. In Italy quartan malaria begins late in the summer and continues late in the fall. In America this variety is too infrequent to justify any definite conclusions. Mixed and multiple infections occur more frequently late in the season than early.

Rainfall.-The influence of rainfall upon the extent of malaria is very decided. Breeding places for mosquitoes are essential in the etiology of malaria, and limited pools, such as result from a fall of rain, are well suited to the taste of the malarial mosquitoes. 
Rain has a twofold effect upon the prevalence of malaria. First, exposure to wet is not infrequently followed by a recrudescence of a former infection. This effect is usually immediate. Second, rainfall produces breeding pools for the disseminators of malaria. The effect of fresh breeding places is not shown immediately. Allowing twenty days for the aquatic stages of the mosquito, ten days for the mosquito cycle of the parasite, and a like period for the incubative stage in man, it would be, obviously, several weeks before an increase in malaria could be expected from such a source. This is well exemplified in the tropics, where so much depends upon rainfall. Here the height of the malaria curve is attained toward the end of the rainy season, or shortly after.

A heavy rainfall in the spring and early summer has long had the reputation of being favorable to the spread of malaria.

While rainfall is essential to the development of malaria, if excessive it may have the opposite effect by scouring breeding pools and destroying the contained ova and young of the mosquito. Moderate rains at short intervals are more productive of breeding pools than heavy downpours at long intervals. Hence, the number of rainy days, as well as the actual rainfall in inches, is a factor in the etiology of malaria.

In very low countries rainy years may be healthy years. This is said to be the case in the Netherlands.

Dew and a high atmospheric moisture were formerly accredited with being factors in the cause of malaria. This was doubtless on account of the well-recognized danger of contracting malaria between sunset and sunrise. Other than as an index of ground-moisture it is doubtful whether atmospheric moisture bears any relation to primary infections with malaria.

Soil.-The chemical composition of the soil has an effect upon the reign of malaria only so far as the relation of the soil to the retention of water is concerned. More depends upon the physical conformation than upon the geologic characteristics of the soil. As a rule, clay soils retain moisture better than the sandy, though there are exceptions. Rocky regions are less apt to harbor breeding pools because of good drainage, but pools upon a rock bed are very persistent. The soil must be of such a 
character as to retain surface water sufficiently long for the aquatic stages of mosquito life to be completed.

More depends upon the nature of the subsoil than upon that of the surface soil. Even where the surface soil is very porous, an impervious subsoil favors the accumulation of surface water by preventing further percolation. Thus the height of the ground-water during the malarial season bears a close relation to the volume of the malarial endemic. Proximity to collections of water, by raising the height of the ground-water, favors the development of malaria.

Topography.-In countries designated malarial, regions entirely free from the disease are not uncommon. In a region within a short distance of a severely scourged locality malaria may be entirely absent. The difference in the prevalence of malaria within limited areas is dependent, in great measure, upon the physical characteristics of the surface of the land.

It has been known for centuries that malaria is partial to low marshy places, swamps, lakesides, low coast levels, and river valleys, and especially the deltas of large rivers. The cleaner the banks and the swifter the current of the streams at all stages the less apt they are to be malarious. Streams with sluggish or no currents, and with weedy banks which foster eddies, are breeding places for mosquitoes.

It is generally believed that salt marshes are never malarious, and that anopheles larvæ cannot develop in sea-water. This is, however, not strictly true. DeVogel ${ }^{119}$ has recently shown that anopheles larvæ may develop in sea-water evaporated to half its initial volume, and a number of other observers have found larvæ in salt water. But marshes of pure sea-water are not nearly so noxious as those of brackish water, a mixture of salt and fresh water, which are famous anopheles breeders. It appears that in some instances, where salt water is inimical to the development of the aquatic stages of mosquitoes, they may gradually become accustomed to the environment.

Altitude.-Malaria is essentially a disease of the lowlands, high altitudes being relatively exempt. This is partially accounted for by the better drainage of elevated altitudes and fewer pools in which malarial mosquitoes may breed. The 
lower temperature of high altitudes is also a factor in maintaining a low malarial morbidity in these regions.

It is known that anopheline mosquitoes do not fly to great heights. Hence sleeping in an upper story or in a building situated high above the ground gives a measure of protection from malaria. Laborers employed in highly malarial sections, and who sleep in the surrounding hills, even of moderate altitude, often remain entirely free from infection.

A few hundred feet in altitude may show a more marked difference in the prevalence of malaria than as many miles in latitude.

The general rule that malaria is a disease of low countries has some exceptions. This is especially true in the tropics, where the disease may be encountered at very high altitudes. It may be said that the altitude at which malaria may occur varies in inverse ratio to latitude.

Malaria has been found on Lake Nyssa at an altitude of I,560 meters; at Colico, 2,500 meters; in the Himalaya Mountains, at 2,000 meters; in the Andes, at 2,500 meters; at Blantyre, at 3,000 feet; German East Africa, at I,550 meters; at points in Central Africa, at heights of over 5,000 feet; and in some of the high-lying valleys of Syria, at altitudes of $\mathrm{x}, 200$ meters.

Some of the cases in high altitudes reported as malaria may be mistakes in diagnosis; other cases may be malaria contracted in the lowlands. Thus Tosari, at an elevation of I, I 77 meters, has been cited as a place where malaria prevailed without the presence of mosquitoes, and this was used as an argument against the "mosquito theory." Koch, ${ }^{122}$ investigating the place in I899, examined the blood of eighty-two children; in none was the parasite of malaria detected. The only case of malaria found was in a man who, twelve days before the beginning of his illness, had spent the night in a highly malarial place upon the coast.

However, malaria is endemic in certain places of high altitude. Such are Eritrea, in altitudes of I,750 meters; Upper Tonkin, at I,, 00 meters; parts of Madagascar, at I, I00 meters; parts of Reunion Island, I,200 meters; in Java, at 1,000 meters; and in 
the Philippines it is said that, while certain valleys are almost free from malaria, the hills in the vicinity are notoriously infected. Wright, ${ }^{448}$ in British Malaya, found anopheles larvæ in pools at an elevation of 2,300 feet.

Earthquakes and volcanic eruptions have been followed by a great development of malaria. Examples are cited of Rome in 1703 , in Reggio in 1783 , and Palermo in 1828 . Remarkable instances have occurred in Peru also. The most recent illustration is that of Amboina, in the East Indies, which had until 1835 been remarkably free from malaria. In that year a severe earthquake occurred, and since then the malaria has increased both in extent and intensity.

Such results can be explained only by an increase of stagnant water following these violent disturbances, probably through the interruption of the flow of ground-water.

Inundations.- Since very early times overflows have been recognized as a prolific cause of epidemics of malaria. Tacitus, Suetonious, Livy, Dionysius, Cassio, and Strabo, mention such results from inundations of the Tiber. This stream experienced an overflow in 1695 , which was described by Lancisi. The water covered a broad area of country, filling ditches, sewers and canals. The following June, July and August were extremely hot. An epidemic of malignant malarial fever ensued and, spreading far and wide, occasioned a great mortality.

Epidemics of malaria following overflows of the Nile, Ganges, Indus, Euphrates, Niger, Senegal, Volga, Danube, Saone, Rhone, Loire, Mississippi, and other rivers have been described.

The immediate effect of an inundation is to check the development of malaria. This is a result of a destructive effect of the flood upon the breeding pools of mosquitoes. It is only after the waters have subsided and pools and marshes are left that the epidemic develops.

Trees and Vegetation.-It was formerly believed that, while decaying vegetation was the cause of malaria, living plant life greatly retarded its development. Whole volumes have been devoted to this subject. It was supposed that vegetation filtered the miasm from the air. It was argued that if air vitiated by respiration be confined in a bottle containing a. living plant 
and exposed to the rays of the sun, the carbonic-acid gas will be absorbed and the air restored to its original condition, plant life consuming carbon dioxide and exhaling oxygen. So firm was this belief that in the days of ancient Rome trees were protected by law.

It is needless to say that the protective power of living plants was as much overestimated as the faculty of decaying vegetation to cause malaria. Their power of absorbing moisture from the soil is more than outweighed by the shade they afford the ground.

While the clearing of land of trees and vegetation may be followed by an outbreak of malaria, this may be due to the overturning of the soil, which usually goes hand in hand with opening land, and to the hardships attending such labor. The ultimate effect of clearing trees from the land is to diminish malaria by permitting the sun to dry the soil.

If trees have any protective virtues whatever, it is probably through affording shelter and food for mosquitoes. The culture of eucalyptus trees is now known to have no prophylactic effect upon malaria.

Weeds and other vegetation growing in the water favor the development of mosquito larvæ by protecting the surface of the water from agitation by the wind.

Vegetable decomposition bears no relation to the etiology of malaria other than as an index to heat and moisture.

Wind.-The wind was formerly held responsible for transmitting malaria long distances. It was believed that the malaria of Edinburgh was imported by the winds from Holland, and that Italy became malarious through the agency of the African sirocco. The land breezes, especially if they blew over marshy areas, were regarded as more highly noxious than the sea breezes.

As a matter of fact, the wind has little or no power to transmit malaria for distances of any consequence. While it is theoretically possible for infected mosquitoes to be borne by the wind, in reality these insects, especially the anopheles, being weak fliers, seek shelter while a breeze is blowing. The immunity from mosquito bites afforded by the Indian punkah, or a common fan, is evidence of this. 
Furthermore, the disturbing effect of the wind upon the surface of the water interferes with oviposition of the adults and with respiration of larvæ and pupæ.

Exposure to cold winds may have the effect of arousing latent malaria.

The occurrence of malaria upon shipboard has been cited as an argument that malaria is an air-born disease. Bilge water in the holds of vessels has also been accredited with producing malaria at sea.

Malaria occurring upon ships may be accounted for in several ways. These cases may be manifestations of malaria contracted upon shore. Even cases occurring long after embarking may be explosions of latent malaria. If vessels anchor too close in shore in malarial regions infected mosquitoes may easily gain access to the crew-a half mile from shore is probably a safe distance. It has been proven that mosquitoes may be carried for considerable periods in the holds and sleeping apartments of ships.

There are many places where, notwithstanding apparently favorable topographic and meteorologic conditions, malaria is entirely absent. This is due to the absence of either malariabearing mosquitoes, or malarial parasites, or of both. Among a number of such places may be mentioned the city of Rome and other portions of Italy, Madeira, portions of Cameroon, Chole Islands, portions of India and of Borneo, the French Islands, Ponape, Saipan, Samoa, New Caledonia, Tahiti, Barbadoes, and portions of Brazil and of the Argentine Republic. The majority of such localities are islands and in the southern hemisphere.

Race; Immunity.-Certain protozoan diseases among lower animals confer immunity. In the Texas fever of cattle an attack, if recovered from, is followed by immunity. There are said to be breeds of cattle naturally immune to the disease. In the large game animals of Africa one infection with trypanosoma brucei confers immunity. Koch found that birds that had been infected with proteosoma grassii could not be reinfected.

From analogy it might therefore be expected that immunity 
to malaria might exist with some individuals or races. This is true, however, in only a limited sense.

While the various races of mankind vary somewhat in susceptibility to malaria, none can be said to possess absolute immunity.

Caucasians residing in non-malarial countries are, when exposed, most liable to contract malaria. Negroes bred in

- highly malarial regions are, as long as they remain upon the native soil, least susceptible to paludal infection.

Immunity within the race increases generally as we go toward the equator. Thus the negroes of the Southern States display less immunity than the negroes of the West Indies or of tropic Africa. Likewise it may be said that immunity is much more marked in countries with a high than in those with a low endemic index.

The immunity of the negro race has been variously estimated, some observers maintaining that they are absolutely proof against malarial invasion, while others hold that they are as susceptible as the whites. The truth lies between these two extremes. Adult negroes reared in malarial regions are much less liable to paludism, as long as they remain indigenous, than are the whites. The negro race does not, however, enjoy an absolute but only a relative immunity from malaria.

According to Sternberg, ${ }^{456}$ there were in the department of Texas of the United States Army during the year ending June 30 , r883, among the white soldiers 21.36 per cent., colored, 6.27 per cent. of periodic fevers to all kinds of fevers.

Sternberg ${ }^{456}$ gives the ratio per thousand of mortality from malarial diseases in the United States Army thus:

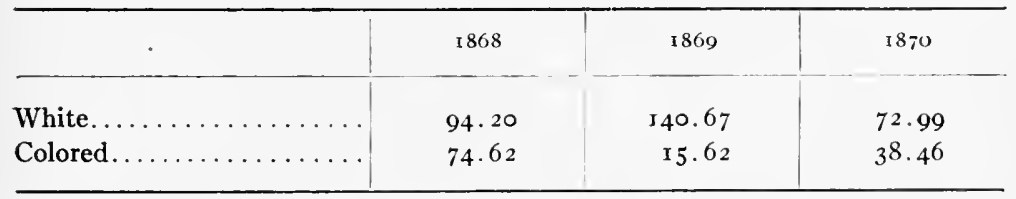

During the Civil War both the morbidity and the mortality from malaria in the negro race were greater than in the white race. However, the negro soldiers are said to have been more 
exposed to malaria than the whites, having been aggregated in malarial localities.

With the better hygienic surroundings and more limited exposure of the whites the negroes would probably be attacked less often than they are. Whether the color, thickness, or other qualities of the skin of this race have anything to do with relative immunity is not known.

At Stephansort, Koch ${ }^{452}$ found various races infected in the following proportions:

\begin{tabular}{|c|c|c|c|}
\hline & $\begin{array}{c}\text { Number of persons } \\
\text { examined }\end{array}$ & $\begin{array}{c}\text { Number infected with } \\
\text { malaria }\end{array}$ & Per cent. \\
\hline Europeans. & $2 \mathrm{I}$ & 12 & 57.1 \\
\hline Chinese. & 240 & 63 & 26.3 \\
\hline Malays. . & 209 & 53 & $25 \cdot 3$ \\
\hline Melanesians. & 264 & 29 & 10.9 \\
\hline Total. & 734 & 157 & 21.4 \\
\hline
\end{tabular}

Immunity from malaria is probably an acquired immunity in the great majority of instances, though the contrary opinion is held by some competent authorities upon the subject. The reasons for believing that this immunity is acquired by repeated infection, especially in childhood, and by prolonged residence in a malarial region, a sort of acclimatization, are, that immunity is much more prevalent in adults than in children; that immunity is often diminished by a change of residence or may be entirely lost by a temporary residence in a nonmalarial climate; and that immunity in an individual may exist toward one form of malaria and not toward others.

That immunity is much more manifest in adults than in children is evident from the consideration of the endemic index of malarial regions, particularly of countries where the latter is high. During the first years of life many individuals examined show evidence of malarial infection, older children in a less proportion, and adults evince a relative immunity. This would hardly be the case if the immunity were racial and congenital. 
The effect of a change of residence upon malarial immunity is a well-known fact. Plehn' ${ }^{2}$ says that the Soudan negroes, relatively immune at home, are of ten afflicted with malaria when going as soldiers to other parts of the continent. Smith ${ }^{457}$ states that, while the native negroes of Sierra Leone are infrequently attacked, and only with mild degrees of malaria, in the West Indies regiment of negroes stationed in Sierra Leone the fever is of a severe and often fatal character.

Individuals once immune to malaria may become susceptible on returning home from a temporary residence in a malariafree country. Plehn ${ }^{2}$ mentions three Cameroon negroes who, shortly after returning from a several years' sojourn in Europe, were attacked with severe remittent fever.

Repeated infection and consequent immunity to one form of malaria does not usually protect the individual from the other forms.

In the South there is little difference between the races as regards susceptibility to the various forms of malarial infection -tertian, quartan, and estivo-autumnal. Clinically, however, pernicious cases, cachexia and hemoglobinuric fever, are rarer in the negro.

Instances of cachexia followed by immunity have been observed, especially by the Italian school. In these cases, after recession of the spleen and liver, and restoration of the blood elements, a stable immunity resulted. Subjects of existing cachexia, even though free from clinic evidences of acute malaria for years, can hardly be regarded as immune.

Rarely are persons encountered in highly malarial localities who have never been attacked with malaria. Such persons are supposed to possess congenital immunity. Celli ${ }^{458}$ obtained precise histories of four persons living in the Pontine Marshes who were absolutely immune, having never had malaria, though they took no prophylactic precautions; their color was good, and their spleens and livers normal. In persons claiming never to have had malaria allowances must be made for the possibility of unrecognized attacks, especially in early childhood, which might give rise to an acquired immunity. 
In conclusion, the resistance of the black race to malaria is due to repeated attacks in early childhood, and not to any great extent to heredity. While in a sense natural selection is a factor, it is largely an individual struggle, the selection of the fittest occurring in infancy, and but little being derived from progenitors.

Sex.-As a general rule, females are less often attacked with malaria than males, though in childhood the proportion is about even.

That women are less frequently infected is not due to a higher degree of resistance, but to the fact that they are less often exposed and are more temperate in their habits. It is probable that if they were equally exposed with males they would be even more often infected than the latter, on account of the greater delicacy of the skin and the manner of dress.

In certain localities women are not less frequently attacked than men. In Panama there is said to be very little, if any, difference between the sexes in this respect. In the Dutch East Indies European women are more susceptible than men. Davidson ${ }^{459}$ says that from $187 \mathrm{I}-75$ the death rate of soldiers' wives in India was 4.20 per thousand, as compared with $2.8 \mathrm{I}$ for the men; and that in Bombay, $1885^{-86}$, the female death rate was I0.14; that of males, 7.56 .

In an institution in Alabama, Sims and Warwick ${ }^{460}$ found among deaf mutes 1.05 per cent. of the males and 6 per cent. of the females infected; among blind, 6 per cent. of the males and 3 per cent. of the females.

Pregnant women are probably less often infected because, on account of their condition, they are less often exposed; when exposed they are very susceptible. The puerperium predisposes to malaria.

Age.-Children are more frequently and more severely afflicted with malaria than adults. This is probably due to their more delicate skin, their manner of dress, sounder and more prolonged sleep, and inability to defend themselves against mosquito bites. The fact that cases of malaria in children more often escape correct diagnosis may account somewhat for the greater frequency, especially of relapses. 
The subjoined figures show the distribution of malaria according to age:

\begin{tabular}{|c|c|c|c|c|c|c|c|c|c|}
\hline & \multicolumn{9}{|c|}{ Age } \\
\hline & $0-10$ & $\mid 10-20$ & $\mid 20-30$ & $30-40$ & $40-50$ & $50-60$ & $60-70$ & $70-80$ & $80-90$ \\
\hline Thayer and Hewetson ${ }^{439} \ldots \ldots$ & 18 & 146 & 204 & 130 & 65 & 36 & I I & 3 & $\mathbf{I}$ \\
\hline $\operatorname{Rogers}^{23} \ldots \ldots \ldots \ldots$ & 2 & I3 & IO & 3 & $\ldots \ldots$ & ... & $\ldots$ & $\ldots$. & $\ldots$ \\
\hline Cardamatis ${ }^{119} \ldots$ & 729 & 499 & 398 & 230 & I 44 & 100 & 55 & 15 & 3 \\
\hline Conti ${ }^{461} \ldots \ldots \ldots \ldots$ & 245 & 146 & $8_{3}$ & $6 \mathrm{I}$ & 63 & & & & \\
\hline Total. & 994 & 804 & 695 & 424 & 272 & I 36 & 66 & I 8 & 4 \\
\hline
\end{tabular}

Endemic Index.-The percentage of children infected in a given locality is the index to the prevalence of malaria in that region. As Ross ${ }^{462}$ expresses it, "There is probably only one really accurate method by which we can determine the degree of malaria in a given locality, and that is by ascertaining the average time in which a newcomer becomes infected. The shorter this period the greater, evidently, the malaria potential of the locality. Native children constitute the class of newcomers most accessible for making the estimate."

The most accurate method of determining the index endemicus of an area is to make a large number of blood examinations of native children at various ages. This requires a great deal of time. It has been repeatedly shown that in regions where malaria prevails extensively a large per cent. of young children harbor the parasites without manifesting any symptoms of the disease, the index decreasing as the age increases. For this reason young natives with latent malaria are the source of the greatest danger to the community.

Endemic indices for the United States have been determined in very few instances.

Sims and Warwick ${ }^{460}$ examined the blood of 610 apparently healthy children and adults in Alabama and found that between 8 and 9 per cent. were infected with malaria.

Surgeon von Ezdorf of the United States Public Health Service examined the blood of a number of persons in several Southern States with the following results: 


\begin{tabular}{|c|c|c|}
\hline & Number examined & Per cent. infected \\
\hline Alabama. & 664 & 3.7 \\
\hline Arkansas.. & 802 & 6.6 \\
\hline North Carolina. & $36 \pm 3$ & 8.5 \\
\hline South Carolina. & 65 & 12.3 \\
\hline
\end{tabular}

In calculating the endemic index a sufficiently large number of persons should be examined in order to eliminate error. It has been estimated that if fifty persons be examined and the blood of twenty-five found to contain parasites, the margin of error being 20 per cent., the index would not be $5 \circ$ per cent. but between 30 and 70 per cent. Furthermore, while a high index indicates widespread malaria, an index of zero must not be construed to indicate an entire absence of the disease, since experience has shown that it may exist where the index, estimated in this manner, is zero.

In comparing the indices of two localities the figures should be taken at corresponding seasons, since the index of a given locality varies according to season.

The prevalence of splenic enlargement has been employed to calculate the extent of paludism, this method requiring much less time than the examination of the blood.

The spleen rate and the endemic index, estimated by a microscopic examination of the blood, do not usually correspond even approximately. Stephens and Christophers ${ }^{463}$ have prepared the following table to illustrate the relation between the spleen rate and the parasite rate:

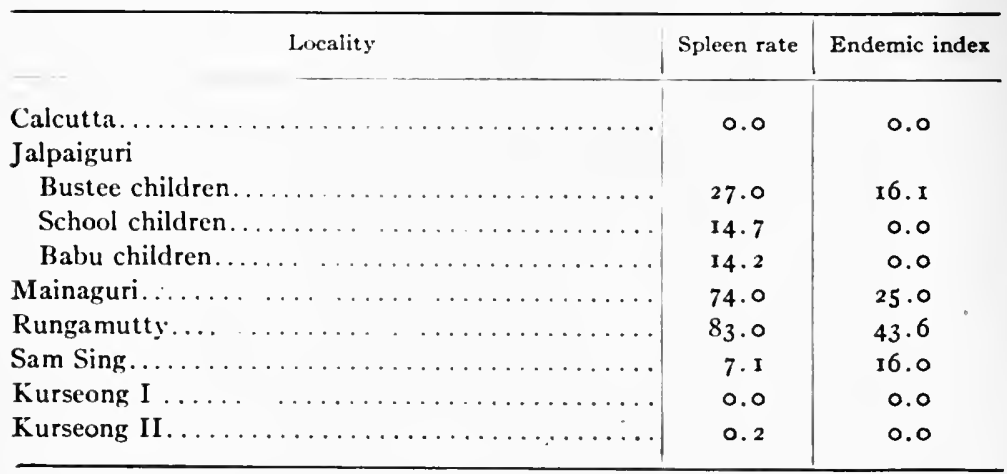


These investigators draw the following conclusions: I. A high endemic index may exist without any appreciable spleen rate (Africa).

2. A high spleen rate may exist in adults without a corresponding parasite infection.

3. In India (Bengal) among children a high spleen rate is a fair indication of the parasite infection.

4. The spleen rate, unlike the parasite rate, increases up to a certain age limit and may be considerable when the parasite rate is nil.

I do not believe that the spleen rate would disclose the true endemic index of regions in the Southern States.

Length of Residence.-In highly malarial regions, especially in the tropics, newcomers are usually infected during the first year.

In the Southern States the period before infection varies greatly according to circumstances. Newcomers who live in hygienic surroundings, and who observe ordinary precautions, may go for years without developing the disease. On the other hand, persons coming South who take no precaution, and who expose themselves carelessly, are liable to be attacked early. Thus it is said that when the Beaumont oil fields were opened up people flocked there from nearly every section of the country, and nearly every newcomer was struck down within a few weeks with malarial fever of some form.

Residence, even prolonged, in a malarial locality does not confer absolute immunity to malaria.

Change of Residence.-The effect of a change of residence upon the immunity in the negro race has been referred to.

It is a common observation that moving to another locality "brings the malaria out of the system." This malaria is usually latent-always so, of course, if the new residence is in a nonmalarial region. It is not uncommon for persons who have never had recognizable paroxysms to suffer an outbreak upon leaving the endemic region.

Occupation.-This is a factor in the etiology of malaria in two respects: first, by reason of certain occupations exposing the person to the bites of mosquitoes; second, by reason of the 
exposure and exertion attending certain occupations awakening latent malaria.

Rural avocations more commonly expose to malaria than urban. Occupations which necessitate residence at highly malarious spots are especially dangerous, as well as those that require being out of doors at night.

Overturning the soil, as in gardening, farming, ditching, railroad, levee, and canal construction, predisposes to malarial infection. Fishermen, soldiers, night-watchmen, engineers, and timber workers are often exposed.

Rice culture, requiring as it does the retention of water from the surface of the ground, is not an unalloyed boon as an innovation into many of our Southern States. The dangers to the community from the growth of rice were recognized many decades ago near Savannah and Charleston.

Social Condition. Civilization.-Formerly malaria attacked all classes. Many noted persons were frequently infected, and James I and Cromwell died of the disease. Moats and lakes near castles and country estates were doubtless to blame.

Now malaria is chiefly a disease of the poor and ignorant classes. The man in the well-constructed and properly screened residence is much less liable to become infected than the one in the loosely built and unprotected hut. The occupations and food of the poorer classes are also factors in the greater prevalence among them.

Persons living in cities and towns are much less apt to be exposed to infection than those living in villages and in the country. Many towns and cities in the heart of malarial areas are relatively free from the disease. Suburbs are more highly malarious than the more densely populated sections, for the reason that the mosquito has more opportunity to breed in the former.

Other Factors.-There are certain factors of the utmost importance in the etiology of malaria, and before the truth was known were looked upon as causing the disease. These are over-work, fatigue, exposure to sun, rain, and cold, excesses in Bacchus and Venus, psychic emotions, loss of sleep, traumatism, surgical intervention, over-eating, hunger, thirst, digestive 
disorders, menstruation, parturition, intercurrent affections, and the administration of certain medicaments.

Watermelons, muscadines, cucumbers, and other articles have yet the reputation in parts of the South of causing chills.

The administration of tuberculin and of potassium iodide is said to be followed not infrequently by outbursts of malaria. It is obvious that the influence of these factors is upon latent malaria, or the parthenogenetic cycle of the parasite's life history.

Insufficient and improper food both lower the resistance to new infections and kindle latent malaria into activity.

While the major portion of many older works on malaria was devoted to the rôle of drinking water in the contagion of malaria, it is now known that it is of minor importance.

In regard to the immunity to malaria enjoyed by opiumeaters, Russell ${ }^{464}$ states that the observations of several surgeons of extensive experience in opium-eating regions confirm the popular belief that the opium-eater in the early stages of the habit, while as yet not constitutionally broken by its long continuance, does, as a matter of fact, enjoy considerable immunity from malarial affections. This writer concludes that this power of opium is partially responsible for its prevalence in some of the eastern countries. Moore ${ }^{436}$ testifies also that opium-smokers are more resistant to malaria.

Epidemics.-Malaria, known as an endemic disease, occasionally prevails so intensely as to acquire the dignity of an epidemic. Becoming more frequent and fatal in its native haunts, it may spread to regions ordinarily immune. and may even assume the extent of a pandemic.

The first pandemic of which we have any knowledge occurred in 1557 to 1558 , and is said to have overspread all of Europe. The next appeared from 1678 to 1682 , and was nearly as extensive as the former. Similar epidemics arose during 17 I $8-1722$, I748-I 750, I 770-I 772 , and $\mathrm{I} 779-\mathrm{I} 783$. During the past century an epidemic occurred from I 806 to $18 \mathrm{I} 2$, and one from I823 to 1827 is said by Hirsch to have been one of the most extensive, severe, and persistent of pandemics, of which reports were received from almost all parts of the world. Between 
1845 and 1849 , and 1855 and 1860 malaria assumed epidemic form, and the great pandemic of 1866 to 1872 marked the invasion of Mauritius and Reunion, where malaria was previously unknown.

What may be called house epidemics or domestic epidemics are common in the experience of many observers.

It is well known that the residents of ceriain houses suffer much from malaria, and that certain houses are seldom free from the disease during the malarial season. For this local conditions are responsible.

I have more than once seen as many as half a dozen cases in one family at the same time, and in many families every member is successively attacked during the season.

Modes of Infection.-The only known modes of transmission of malaria necessary to consider are: (I) congenital; (2) artificial inoculation; and (3) inoculation through the bites of certain species of mosquitoes.

Congenital Malaria.-It was formerly believed that malaria was not infrequently transferred from mother to fetus. Ducheck $^{82}$ published a case in $185^{8}$ of a child whose mother suffered from malarial paroxysms during pregnancy. The child dying three hours after birth, at autopsy the liver and spleen were found to be enlarged, and the spleen and blood of the portal vein contained considerable pigment.

Two cases are reported by Felkin. ${ }^{465}$ In the first case the diagnosis was based upon intrauterine quivering of the fetus, enlarged spleen at birth, and fever later, the date of which is not recorded. In the second case the mother had never had malaria, having never been outside Edinburgh, but the infection is attributed to the father, who had contracted malaria in Africa several years previously and, as Felkin believes, had transmitted the disease to no less than three infants.

Watson ${ }^{466}$ cites the case of a woman who was suffering with tertian ague. On alternate days when she missed the paroxysms she could feel the child shiver with chills. Bark was prescribed and the paroxysms of the fetus were first interrupted, then those of the mother.

However, of numerous cases recorded by a score or more of 
early writers, all are open to two objections: First, the diagnosis was not certainly established; secondly, postnatal infection was not excluded.

Marchiafava and Bignami ${ }^{19}$ mention four cases in which the blood of the fetuses of malarial mothers was entirely negative.

Thayer ${ }^{467}$ records a case of a negress who had had malaria at least five months and whose blood contained three groups of the quartan parasites when she gave birth, during a paroxysm, to a child whose blood, upon repeated examination, was found free from parasites and pigments. While both parasites and pigment were found upon the maternal side of the placenta, none were found upon the fetal side.

Sereni, ${ }^{23}$ who examined the blood of four infants born of malarial mothers, was unable to find evidences of malaria in any case.

Ziemann, ${ }^{86}$ likewise, in four cases of new-born children of malarial infected mothers, had uniformly negative results.

I have upon several occasions obtained blood from infants, immediately after birth, whose mothers harbored malarial parasites, and in no case have parasites been detected. Similar results have been obtained by Bastianelli, ${ }^{19}$ Caccini, ${ }^{19}$ Borne,${ }^{41}$ Schoo, ${ }^{41}$ F. Plehn, ${ }^{2}$ Terburgh, ${ }^{86}$ A. Plehn, ${ }^{36}$ Wellman, ${ }^{119}$ and others.

Pezopoulos and Cardamatis, ${ }^{468}$ based the following conclusions upon six cases, five full-termed labors and one abortion, which they studied.

I. In the blood of the six mothers there were parasites, more or less abundant.

2. In the blood of the new-born and of the aborted fetus, examined a few hours after expulsion, there were no parasites.

3. In the blood of the liver and spleen, as well as in sections of these organs of the two fetuses which were examined postmortem, no parasites were found.

4. In the blood taken from the maternal surface of the placentæ of the five new-born children there were parasites in abundance, while in the blood taken from the fetal surface there were no parasites, or at most a very few. 
5. In blood taken from the umbilical cord no parasites were found.

6. In the blood of the placenta of the aborted fetus no parasites could be detected.

Bein and Kohlstock ${ }^{29}$ are said to have found malarial parasites in the blood of a four-months-old child born some time after the arrival of the mother in a region free from malaria.

Winslow ${ }^{469}$ records a case which he believes to be congenital, though the parasites were not detected until the child was eleven weeks old.

A case of malarial fever in infancy thought to be maternal in origin is reported by Peters. ${ }^{470}$ The examination of the blood on the second and third days after birth was negative, though parasites were found on the fifty-first day.

Moffatt ${ }^{471}$ observed a case supposed to be congenital malaria, though the parasites were not detected before the seventh week.

Bouzian, ${ }^{472}$ in Algeria, detected parasites in the blood of an infant only twelve days old.

Hitte $^{473}$ collected from the literature twenty-one cases of malaria considered congenital. In thirteen of these the blood was not examined; in one parasites were detected four months after birth, and in five parasites were found from several weeks to two months after birth. The remaining two cases were observed by Hitte personally, who claims to have found parasites in the blood obtained from the umbilical cords. The mothers of both children were suffering with malaria.

Parasites were found by Simms and Warwick ${ }^{460}$ in the blood of three babies between three and seven days old; the mothers had previously had malarial paroxysms.

Holt ${ }^{474}$ mentions a case in which he states there seems little doubt that the disease was contracted in utero. The mother had been suffering with tertian intermittent. Eighteen hours after birth the child showed evidences of a malarial paroxysm. The next day malaria organisms were found in the blood.

Ecocomous $^{475}$ reports six cases with almost conclusive evidence of congenital origin. In each of these cases the blood, examined from eight to forty-eight hours after birth, contained 
malarial parasites. The mothers had, previous to delivery, suffered with malaria.

$\mathrm{BeI}^{476}$ mentions a female patient who died of pernicious malaria. The parasite was found in the blood, pericardium, meninges, and spleen, as well as in a seventh-month fetus.

As may be inferred, properly proven cases of congenital malaria are rare. This reluctance of the parasites to pass through the placenta is in keeping with their aversion to leave the blood-vessels. It has been pointed out that no parasites are found in the hemorrhages and perivascular exudates in cases of pernicious malaria, though they may exist in hordes within the vessels. Congenital malaria is probably to be explained in the majority of cases through placental lesions permitting direct mingling of maternal and fetal blood during parturition.

Inoculation.-Even before the parasite of malaria was discovered Gerhardt ${ }^{439}$ succeeded, employing the blood of malarial subjects, in inoculating healthy persons with malaria.

Since then many similar experiments have been performed. Tertian malaria has been transmitted by inoculation by Bein, Bacelli, and Celli and Santori; estivo-autumnal by Gualdi and Antolisei, Di Mattei, Celli and Santori, Bastianelli and Bignami, and Elting.

The injection of blood containing only crescents gave negative results in the experiments of Thayer, Bastianelli, Bignami, and Elting. Di Mattei and Calandruccio, however, observed an irregular form of fever to follow such an injection. This can be explained only by parthenogenesis.

The injection of blood containing certain species of parasites is followed by fever characteristic of that species, and these parasites are to be found in the blood of the person inoculated.

There are those who cannot be successfully inoculated with one species of parasite but can with another. It has been shown also that one species of parasite often disappears from the blood upon inoculation with a different species.

The degree of development of the parasites apparently has no effect upon the result, since the disease develops as readily after the injection of blood containing adult organisms as after that containing young parasites. It is immaterial also whether the 
blood be injected intravenously or subcutaneously. A very small amount of blood, even less than one drop, is often sufficient for inoculation.

The injection of defibrinated blood, of centrifugalized corpuscles, and of blood diluted with an equal quantity of distilled water and inoculated immediately trave given positive results. The injection of dissolved dried blood, and blood diluted with an equal quantity of distilled water and allowed to stand an hour, have proven negative.

Jeffries $^{477}$ reports the case of a New York surgeon who had never had malaria, supposed to have contracted the disease by pricking his finger several times during an operation upon a woman infected with malaria. Sixteen days after the operation the surgeon had the first chill and had several subsequently. The blood contained many estivo-autumnal parasites.

Efforts to inoculate the lower animals with human malaria have been fruitless. Such attempts have been made upon horses, mules, dogs, monkeys, rabbits, mice, guinea-pigs, hedgehogs, bats, wolves, cats, pigeons, doves, magpies, screechowls, turtles, frogs, and lizards.

\section{DISSEMINATION OF MALARIA BY MOSQUITOES}

The discovery by Ross of the rôle of the mosquito in the dissemination of malaria is the most startling achievement of modern medical science.

Mosquitoes do not cause malaria; they carry it from infected to healthy persons. The parasites, sucked with blood from a malarial individual, undergo a cycle of development within the body of the mosquito, and are then inoculated into healthy persons. Man is merely the intermediate host of the parasite, the mosquito is the definitive host, and it has been said that man gives malaria to the mosquito, and not the mosquito to man.

Not all species of mosquitoes can serve as hosts for the malaria parasite. It is only certain members of the subfamily Anophelinæ that have been found to act in this capacity. Of this subfamily the following have been determined, with more or less certainty, to be malaria carriers: 
Anopheles annulipes.

Anopheles bifurcatus.

Anopheles cohaesa.

Anopheles crucians.

Anopheles farauti.

Anopheles formosaensis.

Anopheles maculipennis.

Anopheles martini.

Anopheles pseudopunctipennis.

Anopheles pursati.

Anopheles quadrimaculatus.

Anopheles tarsimaculata.

Anopheles vincenti.

Cellia albimanus.

Cellia argyrotarsus.

Cellia pharoensis.

Cycloleppteron grabhamii.

Myzomyia Christophersi.

Myzomia culicifacies.

Myzomyia funesta.

Myzomyia Hispaniola.

Myzomyia Ludlowii.
Myzomyia Lutzii.

Myzomyia picta.

Myzomyia Rosii.

Myzomyia Turkhudi.

Myzorhynchus barbirostris.

Myzorhynchus Coustani.

Myzorhynchus fuliginosus.

Myzorhynchus paludis.

Myzorhynchus sinensis.

Myzorhynchus umbrosus.

Myzorhynchus Ziemanni.

Nyssorhynchus annulipes.

Nyssorhynchus Jamesii.

Nyssorhynchus maculatus.

Nyssorhynchus maculipalpis.

Nyssorhynchus Stephensii.

Nyssorhynchus Theobaldi.

Nyssorhynchus Willmori.

Pyretophorus Chaudoyei.

Pyretophorus costalis.

Pyretophorus jeyporensis.

Pyretophorus superpictus.

Not all of these mosquitoes serve equally well as hosts of the malaria parasites. Myzomyia Rosii is a very poor carrier of malaria, while the Christophersi is a very efficient carrier.

As yet very little is known of the relation between the species of mosquitoes and species of malarial parasites. Pyretophorus costalis is known to transmit tertian, quartan, and estivo-autumnal malaria, while Myzorhynchus sinesis carries tertian and quartan, but not estivo-autumnal malaria.

It is possible that some mosquitoes acquire a sort of immunity to malaria which may account for their incompetence as malaria disseminators. There are certain regions where, in spite of members of a malaria-bearing species of mosquito and the immigration of infected persons, malaria does not spread, though temperature and other conditions are apparently favorable.

The food of mosquitoes has much to do with their susceptibility to infection. Experiments have shown that Anopheles maculipennis fed upon fruits and sweets are not readily infected from feeding upon malarial blood, but if allowed only water for several days before and after feeding on malarial blood they are almost always infected. 
One feeding upon blood containing parasites does not always suffice to infect the mosquito. Daniels, ${ }^{478}$ investigating this subject, examined 57 mosquitoes which had fed once or oftener at intervals of two days.

\footnotetext{
Per cent.

Nineteen fed only once, and five had zygotes......... 26.0

Thirteen fed twice, and six had zygotes............. 46.0

Sixteen fed three times, and ten had zygotes......... 62.0

Nine fed four times, and six had zygotes.............6.6.6

Of these 57 anopheles 27 , or 47.5 per cent., were infected.
}

The effect of fertilization upon the power of mosquitoes to transmit malaria is not definitely settled, but it is thought by some that fertilized females are the most desirable, if not indeed the sole, hosts of the parasite.

In order that anopheline mosquitoes may be infected from malarial blood it is necessary that the sexual forms of the parasite be present in sufficient numbers, of proper maturity, and suitable proportion of sexes.

How is the existence of the malaria parasite perpetuated? Why does not the disease become extinct over winter when there are apparently no mosquitoes to further the life history of the organism?

The subject of latent or chronic malaria furnishes the solution. The parasites here lie dormant or undergo parthenogenesis at longer or shorter intervals, and are ready the following season for the sexual cycle in the body of the definitive host, the mosquito.

It is possible that in a few instances the parasites persist in the bodies of hibernating mosquitoes. While some investigations have led to a different conclusion, Martirano has found in the neighborhood of Rome as late as the middle of March that from $\mathrm{I}$ to 5 per cent. of anophelines were infected, and Stephens and Christophers observed at Freetown, during the dry season, that from 5 to 20 per cent. were infected. ${ }^{41}$

From analogy with the transference of Texas fever hematozoa by the tick to its progeny, it has been sought to establish such an inheritance of malaria parasites by mosquitoes, but it must be considered as yet unproven that infected mosquitoes can communicate the infection to their offspring. 
The relation of the mosquito to malaria explains the prevalence of the latter with reference to season, temperature, and rainfall. It explains malaria as a disease chiefly of low altitudes and marshy regions; a disease of the country rather than of the city. House epidemics of malaria are thus rendered clear and the relation of ship malaria and proximity to the shore become obvious. The bearing of age, sex, and occupation upon the endemic is in thorough harmony with the theory. That malaria is more easily contracted at night is understood from the feeding habits of the malarial mosquitoes. That all measures directed toward the prevention of mosquito bites are followed by a commensurate reduction of the prevalence of malaria is one of the strongest arguments. The analogy with filiariasis, Texas fever, hematozoan infection of birds, and similar diseases strengthens the theory. Numerous and accurate experiments have absolutely proven the dissemination of malaria by certain mosquitoes.

The sexual cycle of the parasite within the mosquito has been followed many times.

An objection that has been frequently urged against the "mosquito theory" is that there are numerous localities in which mosquitoes abound and from which malaria is entirely absent; indeed, mosquitoes are said to be well nigh intolerable in portions of the arctic regions. It must be remembered, however, that only a certain subfamily of mosquitoes can serve as hosts for the parasite. Furthermore, the surrounding temperature must be suitable for the sexual development of the parasite within the definitive host.

It was formerly maintained that there were highly malarial regions in which there were no mosquitoes, and a number of such places have been reported. But in each case where investigated by a competent observer anopheline mosquitoes are such that they may be easily overlooked except by an expert. Retiring to dark recesses during the day, biting only at night, and not singing a great deal, their presence may not be felt, especially by persons in whom the bites do not cause much irritation.

.It may therefore be stated confidently that there is no endemic malaria without mosquitoes. 
The misproportion between the number of infected anophelines and the number of cases of malaria has been cited to overthrow the mosquito doctrine. In Algiers Sergent ${ }^{41}$ found 4 per cent. of the anopheles and roo per cent. of the children infected. A. Plehn ${ }^{86}$ found in one of the most malarial localities, Cameroon, among 860 anopheles, only 2.2 per cent. infected. Stephens and Christophers ${ }^{47}$ believe that about 5 per cent. of all the anopheles of tropic Africa are infected. At Aro they found the sporozoit rate in anopheles caught in native huts to be 50 per cent.

It should be borne in mind that one infected anopheline mosquito is capable of infecting a number of persons. Also the sporozoit rate varies according to season and according to the kind of mosquito, since it has been shown that some species are better malaria carriers than others.

Finally, it has been maintained that persons who have never had malaria have contracted it in uninhabited wildernesses, where, obviously, only uninfected anophelines would be found, since man is the only intermediate host of the parasite.

To this may be answered that no such instance has been so accurately reported as to prove conclusively that infection has ever occurred under these circumstances.

\section{THE MALARIA-BEARING MOSQUITOES}

The genus Anopheles was established in 1818 by Johann Meigen. The bestowal of the name appears prescient, since anopheles signifies troublesome or hurtful.

Of the fifty or more species and subspecies of anophelines now known eight occur in the United States: $A$. maculipennis, A. punctipennis, A. crucians, A. franciscanus, $A$. pseudopunctipennis, $A$. barberi, C. argyrotarsus, and C. albipes.

Breeding Places. - The different species of anophelines vary a great deal in the choice of a breeding place. Furthermore, with each species there may be said to be places of choice and places of necessity.

Contrary to the usual custom of culex, the anopheles usually selects water more or less pure in which to deposit her ova. 
Ground-water appearing at the surface is especially suitable. Pools of at least some degree of permanence are preferred to those which might dry before the aquatic stage of the insect is completed. Natural accumulations of water more often contain anopheles larvæ than do artificial collections. Pools, ponds, swamps, inlets of lakes, and of small, slowly flowing streams, ditches along roadsides, canals, borrow pits along railroads and levees, and rice fields are common breeding places. Water contained in the tracks of animals may harbor larvæ.

When water is scarce, as during the dry season, anopheline larvæ may be found in tubs, barrels, buckets, bottles, cisterns, mollusc and cocoanut shells, in water retained by the leaves and stalks of tropic plants, or even within vases in dwellings, though these locations are to be regarded as places of necessity and not of choice.

In regard to salt water as a medium for anopheline larvæ many opinions are held. It seems that the species indigenous to the United States do not breed in salt water, and this was the experience of Celli and other Italian investigators. However, Centanni and Orta ${ }^{480}$ found anopheles larvæ in water containing 8.77 per $\mathrm{I}, 000$ of sodium chloride. Ficalbi and others ${ }^{480}$ found them in water containing 40 to 50 grams of sodium chloride per liter. In Algiers and the Dutch Indies anophelines are found breeding in concentrated sea-water, and $B^{2} k^{481}$ found $M$. Ludlowii breeding in sea-water in the Philippines. Bancroft in Queensland found a species of anopheles breeding in sea-water, and at Accra, on the west coast of Africa, Stephens and Christophers $^{482}$ obtained numerous anopheline larvæ from water containing 6 per cent. of salt. De Vogel ${ }^{119}$ at Semarang found certain kinds of anopheles breeding not only in sea-water, but in that which had been condensed to half its volume.

Ova of Anophelines.-These mosquitoes do not deposit their eggs in a raft-like mass, as do tle culex. They are laid in irregular piles, but soon become scattered, lie horizontally, and may form attractive patterns upon the surface of the water. In captivity the eggs may be laid upon some floating object.

The ova are from 0.7 to $1.0 \mathrm{~mm}$. in length by about $0.16 \mathrm{~mm}$. in breadth. They are fusiform in shape and somewhat broader 
at one end than at the other. The lower surface is convex, the upper nearly flat. From the middle third of each side protrudes a transversely corrugated membrane which acts as a floal, some-

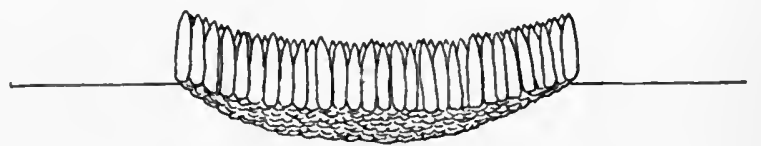

Fig. I.-A raft of culex ova.

what after the manner of the air chambers of a lifeboat. Around the margin of the upper surface of the ovum is a frill, usually transversely corrugated. When first laid the eggs are whitish,

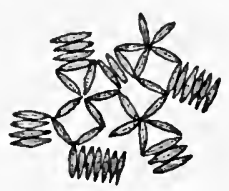

Fig. 2.-Patterns assumed by anopheles ova.

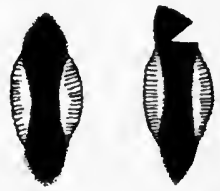

Fig. 3.-Anopheles ova.

but soon become almost black. The head of the larva lies in the broad end of the egg and escapes by loosening a circular cap from this end. It is said that if an ovum is partially removed from the water the broad end always hangs downward in order

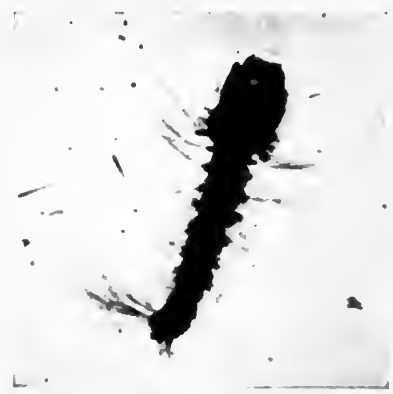

Fig. 4. A young anopheles larva. Magnified.

that the larva may be born into the water instead of into the air.

The duration of the egg stage varies with the temperature, but is generally from two to four days.

Stephens and Christophers ${ }^{120}$ did not succeed in hatching the 


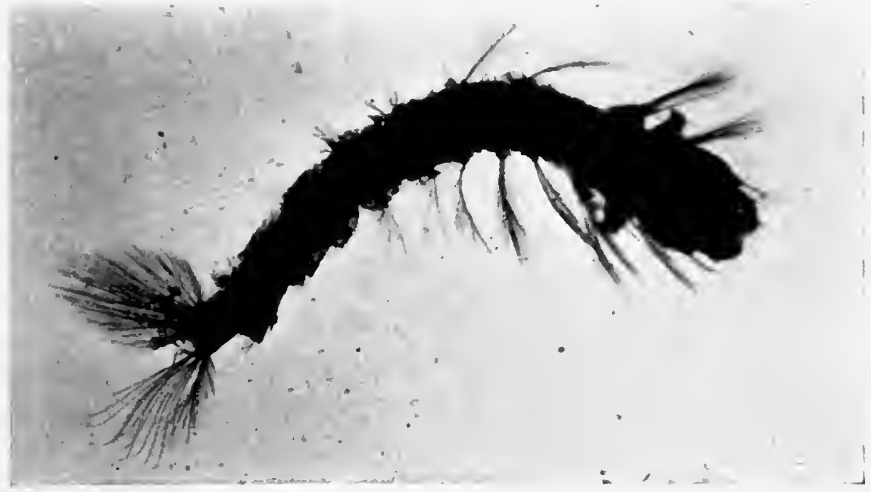

Fig. 5.-Half-grown anopheles larva. Magnified.

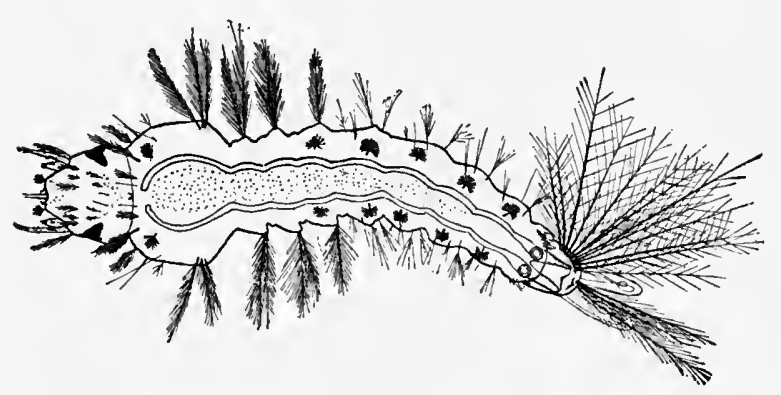

Fig. 6.-Full-grown anopheles larva.

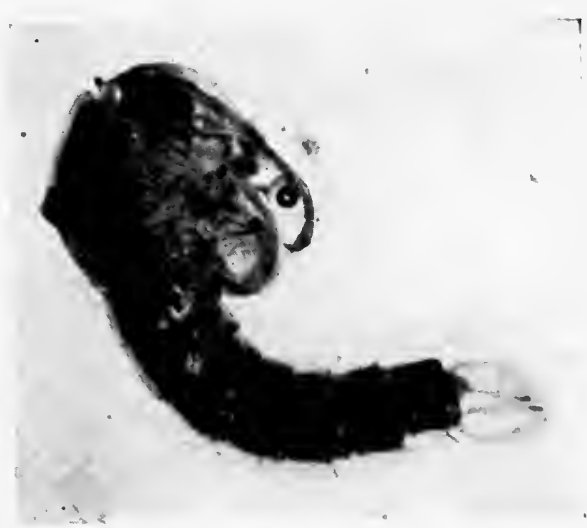

Fig. 7.-Anopheles pupa. Magnified. 
ova after desiccation for two or three days, but Grassi ${ }^{480}$ is said to have hatched them after keeping them dry for twelve days.

The Larva.-The head of the anopheles larva is more or less globular; the eyes are situated laterally at the broadest part of the head. The antennæ are rod-shaped and unjointed; at the end are two leaflets, between which arises a branched hair. The mouth parts consist of the feeding brushes or whorl-organs,

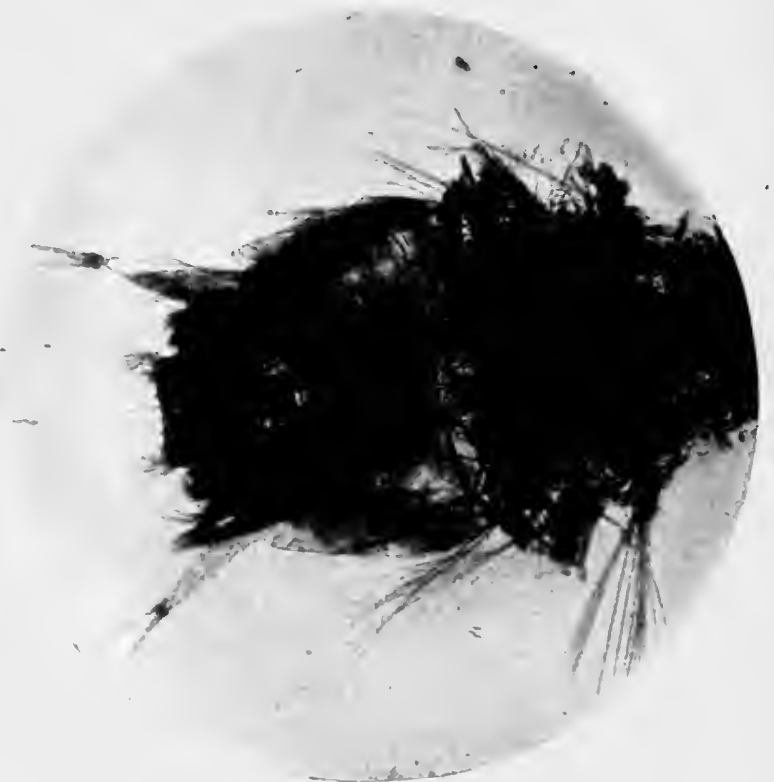

Fig. 8.-Head of anopheles. Magnified.

the maxillary palps, the mandibles, the under lip, and the clypeus.

The neck is very narrow in the full-grown larva.

In the young larva the thorax is little, if any, wider than the head, but in older specimens it is much wider.

There are nine post-thoracic segments. The first three segments bear branched lateral hairs. The third to the seventh segments have upon the dorsum a pair of fan-shaped structures, known as the palmate hairs.

The eighth segment contains the two openings of the respiratory system, which ends abruptly at the dorsum of this segment 
without the prolonged breathing tube of the other subfamilies.

The ninth or caudal segment bears four flaps containing respiratory branchiæ. This segment is armed with two large tufts of hair

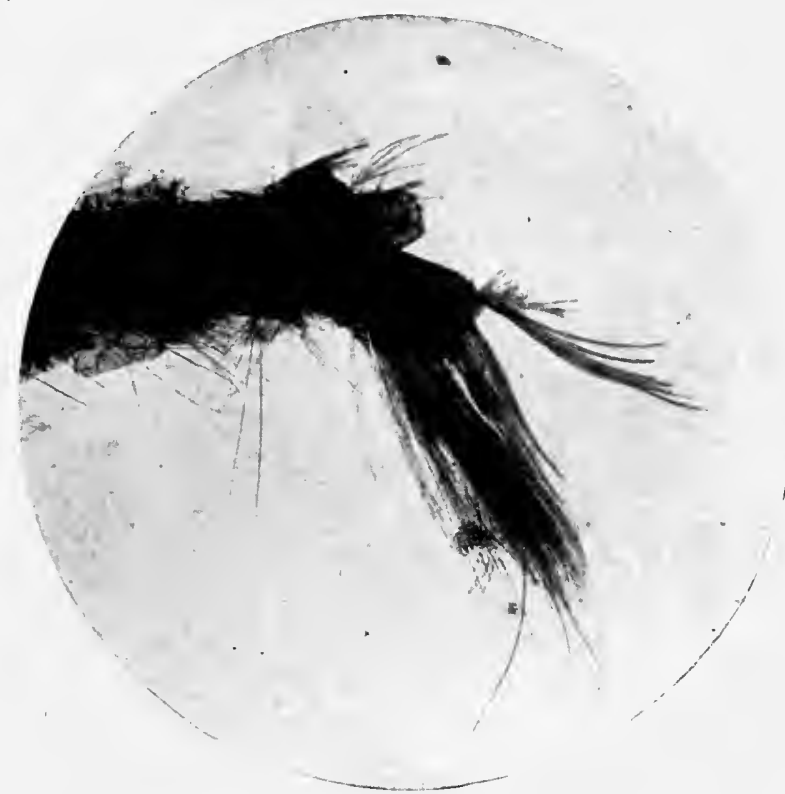

Fig. 9.-Tail of anopheles larva. Magnified.

The color of the larva varies greatly, according to food and environment, and may be grayish, green, almost black, reddish, or mottled with black or white.

The full-grown larva is about $8 \mathrm{~mm}$. in length.

Anopheline larvæ are omnivorous. Their diet consists of the spores of algæ, diatoms, animalcules, bacilli, other larvæ, moulted skins, mosquitoes, and other small insects. In captivity they eat dry rice greedily.

The customary location of these larvæ is at the surface of the water near the edge of the pool where they may remain almost mo-

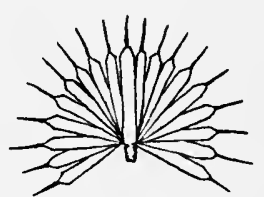

Fig. 10.-A palmate hair tionless for long periods. The characteristic position is parallel with the surface of the water and so close to it that a portion of the dorsum appears to rise above the surface, which, 
however, is not the case. When feeding, the constant motion of the mouth parts creates a flow of water toward the mouth, bringing in small particles of food. The head is often rotated suddenly, so that it turns through an arc of I 80 degrees the lower

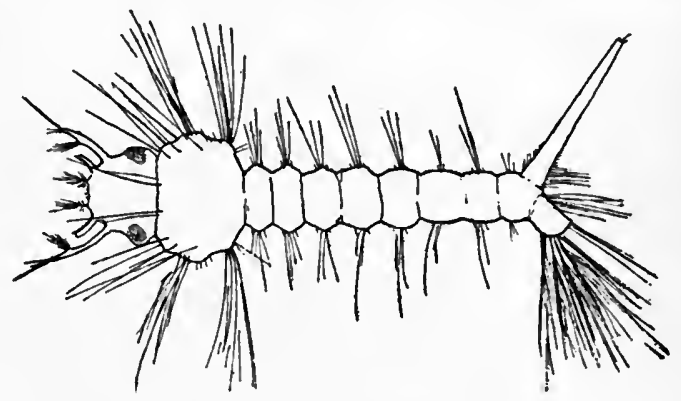

Fig. Ir.-Adult culex larva.

surface looking uppermost. On taking hold of something too large to swallow the larva will of ten shake the head vigorously and may bend the body to steady the particles against the last segments of the body. In captivity they of ten rest with the tail against the sides of the container and the head toward the center, when numerous forming a fringe around the circumference. Locomotion is very jerky and irregular. When disturbed they

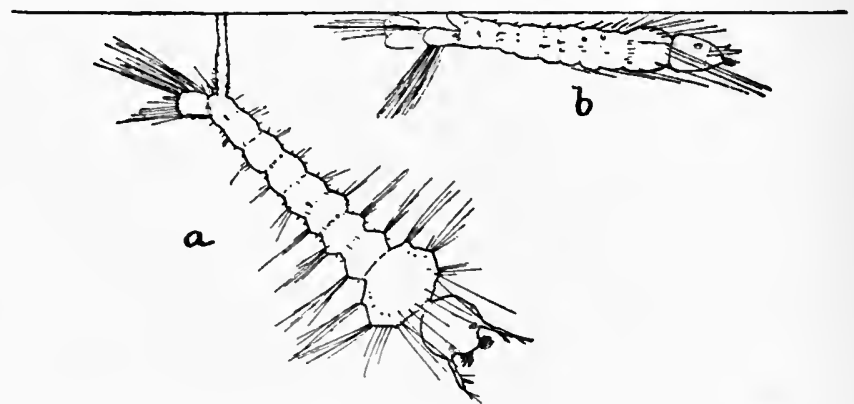

Fig. 12.-Resting positions of larvæ: $a$, Culex; $b$, anopheles.

not infrequently feign death. From the behavior of the anopheline larvæ it does not appear that the sense of sight is very acute.

Culex larvæ have been thawed out of ice in which they were imbedded and have proceeded to develop into insects, but 
so far as I am aware this has not been done with anopheles. The latter have, however, been found in water under a frozen

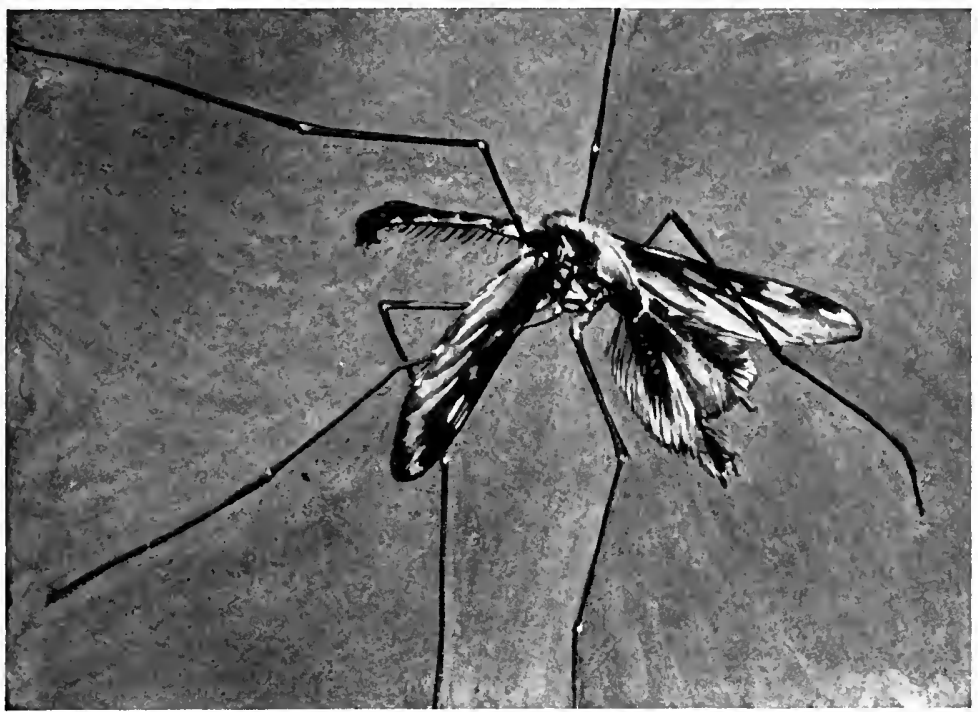

Fig. I3.-Male anopheles.

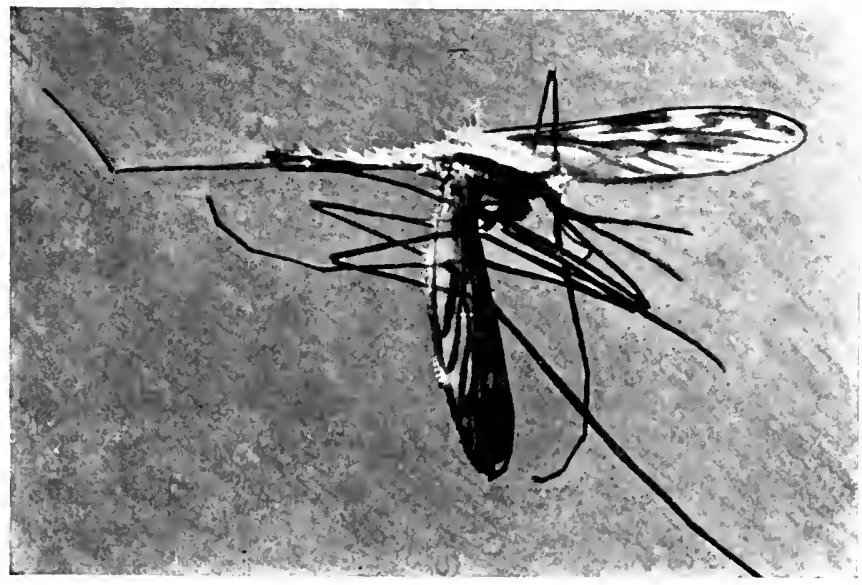

Fig. 14.-Female anopheles.

surface. They may exist for a few hours to a few days upon moist mud.

The duration of the larval stage varies according to tem- 
perature, food, and possibly other conditions. The limits may be placed at from ten to twenty-six days. In warm climates when suitable food is abundant the duration is ordinarily twelve or fourteen days. In cooler climates and seasons the duration is longer.

Anopheles and culex larvæ may be differentiated by the following gross characteristics:

Anopheles

Habitually at the surface of the water.

Position parallel with the surface.

No respiratory tube.

In full-grown larvæ the head is smaller than the thorax.

\section{Culex}

At the surface to breathe only.

Hangs at an angle of 50 to 60 degrees to the surface.

Large respiratory tube.

The Pupa.-While the larva bears some resemblance to the imago, the pupa resembles neither. It has been compared in shape to a hypertrophied comma. The anopheles resembles culex more closely in this stage than in any other. The head and thorax are enclosed together in a semitransparent shell, through which portions of the mouth parts, wings, and legs may be detected. Respiration is no longer transacted through the eighth abdominal segment, as in the larva, but through the trumpet-shaped spiracles or syphons of the thorax. This necessitates a change in position, the abdomen hanging or rather curved around the cephalo-thoracic segment. The eighth abdominal segment bears a pair of broad paddles for locomotion.

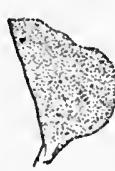

a.

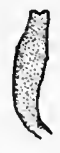

b
Fig. $x_{5} .-$ Breathing syphons of $(a)$ anopheles and $(b)$ culex pupa.

The young pupa is rather light in color, but rapidly becomes darker.

The pupæ are more easily alarmed than the larvæ, and when disturbed dart wildly downward with rapid jerks. Being of lower specific gravity than the water, they rise quickly without effort. They do not eat.

Italian investigators ${ }^{480}$ observed that the nymphæ of some mosquitoes resisted freezing and desiccation to a remarkable degree. Enclosed for several hours in ice they were yet able to develop, and kept in dry soil for two or three days they developed when placed in water. 
The duration of the pupal stage is ordinarily from two to five days.

The following points may serve to distinguish anopheles and culex pupæ:

Anopheles

Position in water more horizontal.

Syphons short, square truncated ends, attached to middle of thorax.

Longer anteroposteriorly, narrower laterally.

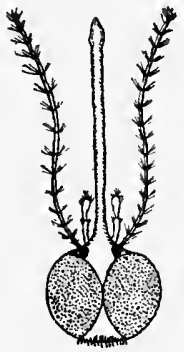

a

\section{Culex}

Position more vertical.

Syphons long and narrow, slit-like opening, attached to posterior part of thorax.

Shorter and broader.

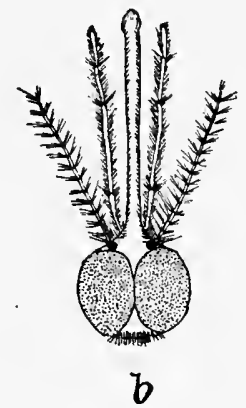

Fig. I6.-Heads of $(a)$ culex and $(b)$ anopheles females.

When approaching the emergence of the imago the pupa becomes motionless at the surface of the water; the abdomen is extended parallel with the surface; minute air bubbles are seen under the membrane, which then splits along the dorsal line of the thorax. The imago emerges head first, then the thorax and wings, then the legs. At this stage the insect is very liable to be drowned by a breeze or by a ripple in the water.

The Imago.-Anopheles is distinguished from the culex especially by the palpi, which in the former are in both sexes almost as long as the proboscis, in the latter the palpi of the female being very short. Anopheles is more slender, the head is smaller, and the legs more delicate. The palpi of the female, being thickly scaled and lying close to the proboscis, give the impression of a thick beak, which contrasts strongly with the short palpi and slender proboscis of the culex. The wings of the anopheles are speckled, which is not the case with any of the common species of culex.

The sitting of anopheles is characteristic. The body of 
the insect is at an angle with the surface upon which it rests, the proboscis pointing toward the surface, sometimes even touching it. This angle varies in different anopheline species, in some being almost a right angle, when the insect appears almost like standing upon its head. The proboscis, head, thorax and abdomen are in the same line. The mosquito at rest has been compared to a brad-awl stuck into the wall. It often rests upon the first two pairs of legs, waving the last pair in the air. Culex at rest is angular and humpbacked. Stegomyia may be recognized by its smooth, velvety coat of jet black and

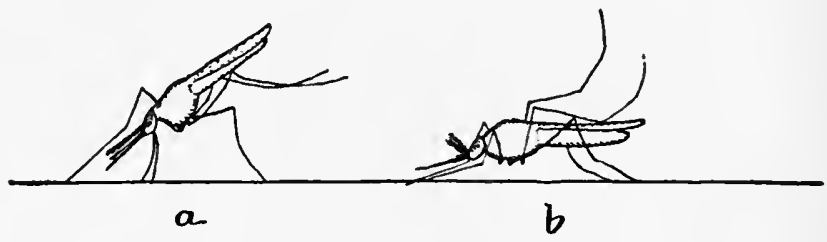

Fig. 17.-Resting positions of $(a)$ anopheles and (b) culex.

silvery white, the banded legs and abdomen, and the lyreshaped ornamentation of the thorax.

The principal malaria carriers of the United States are thus described by Giles:484

Anopheles Maculipennis (Meigen).-"Wings with four tufted spots on the wing-field, the costa being uniformly dark except at the apex, where its color fades to form a fairly distinct spot; tarsi unbanded, but with an apical yellowish spot to the first joints. Thorax with four broad ferruginous stripes formed of golden hairs, between which the darker ground color is left bare, with a tuft of large golden scales on the anterior border. Abdominal segments brown with yellowish basal markings; anterior femora not thickened at the base.

"Female.-Head with two patches of creamy scales divided by a central line, the rest of the head with black scales, a small tuft of white hairs in front; borders of the eyes white; eyes deep purplish black; antennæ dark brown with pale bands and with ferruginous basal joint, pale pubescence, and brown hairs; proboscis brown; palpi yellowish brown with dense, dark scales at the base, which is swollen, shorter than the proboscis. Legs 
with pale coxæ; femora and tibiæ yellowish brown below, covered with dark brown scales above; knee spot yellow, apex of the tibiæ paler; tarsi slightly darker than the rest of the leg.

"Male.-Antennæ banded, plume hairs brown, last joint darker; proboscis black to dark brown; palpi dark brown; the last two joints, which are clubbed, have a number of short golden hairs internally and are yellow in color, clothed with thick black scales, through which the yellow underground shows; the last joint is truncated. Length 4 to $7.5 \mathrm{~mm}$., male, to 8 or Io $\mathrm{mm}$., female. This species varies greatly in size, the wings shown me in Italy by Professor Grassi being quite small, while some Canadian specimens in the British Museum are huge gnats, and to illustrate this I have superposed on the plate the drawing of one of Grassi's specimens on the outline of a Canadian specimen."

Anopheles Crucians (Wied)._-"Wings with white spots here and there on the brown veins, uniform along the costa; tarsi unbanded, dusky brown; abdomen uniformly brown with gray hairs. Thorax red brown with linear markings."

Description from Wied, A. Z. I., page r2: "Tawney; the thorax with three deeper-tinted lines; the abdomen covered with gray hairs; the wings with dusky spots and costa. Length $21 / 2$ lines (German)."

"Coquillett, in his recent synoptic table of North American Culicidæ, states that 'the scales of the last veins are white, marked with three black spots; palpi with white at the bases of the last four joints,' and without any spot on the costa, as seen in $A$. punctipennis.

"Professor Nuttall sent Mr. Theobald two females from America in spirit, which, although much damaged, show the two features mentioned by Coquillett very clearly, readily distinguishing the species from the C. punctipennis of Say."

The hatching of the first brood of anophelines bears an intimate relation to the seasonal occurrences of malaria. The seasonal variations of different species are probably dependent upon the presence or absence of breeding pools suitable to particular species. Temperature also exerts an influence, the hibernating females of some species leaving winter quarters 
earlier than others, and hibernating larvæ mature at different temperatures.

While the anophelines are mosquitoes of low altitudes, they may be found at considerable elevations. Thus in the Alps they are found at an altitude of $\mathrm{I}, \mathrm{I} 45$ meters; in the Apennines at 1,283 meters; in Java at $\mathrm{r}, 000$ meters; at Harrar at 2,000 meters; in Africa at 1,900 meters, and in the high plateaus of Mexico at 2,000 meters. ${ }^{480}$

It is the rule among mosquitoes that only the females are blood suckers, hence it is this sex alone that is concerned in the
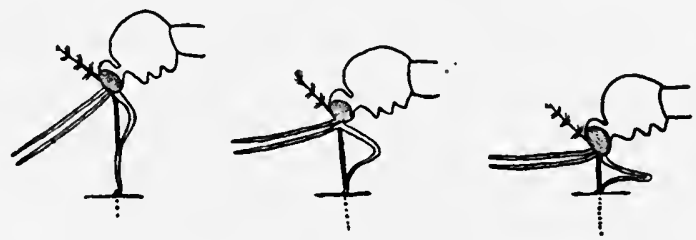

Fig. 18.-A female mosquito in the act of biting.

propagation of malaria. The female insect sucks not only the blood of man, but of other mammals, birds, occasionally of coldblooded animals, and even other insects.

There are a few exceptions to the rule that males do not bite. While males do not infrequently light upon the skin and probe around with the proboscis, they usually fly away without partaking of any blood. The habitual diet of male mosquitoes is vegetarian. They are very fond of fruits, as bananas, dates, pears, apples, melons, and of the nectar of flowers, wine and beer.

Anopheline mosquitoes rarely suck blood except during the night. After feeding they usually retire to remote and dark corners or to breeding places to oviposit. During the day their reserved habits make them difficult of detection.

A point of great practical interest is the length of flight of the mosquito and the extent to which it may be borne by the wind. It is a general rule that mosquitoes do not migrate far from their native pools or from dwellings where nourishment may be obtained.

It is very unusual for anophelines to fly farther than a few 
hundred yards, and half a mile may be regarded as the maximum limit of flight. They are poorer flyers than most other species. For this reason they are less often borne by the wind, since they seek shelter when a breeze arises. While the wind is not so generally a vehicle for the dissemination of mosquitoes as commonly believed, certain species, especially of salt-water breeders, are borne by the wind for several miles.

The preference of anophelines for certain colors has been

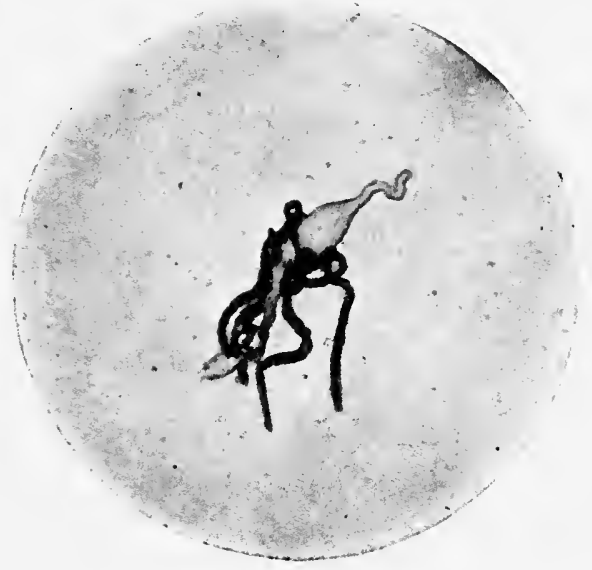

Fig. 19.-Midgut and Malpighian tubules of anopheles.

demonstrated by Galli-Valerio and De Jongh ${ }^{480}$ who counted I 9 anophelines resting upon dark colors and 33 upon bright colors, and 349 culex upon dark colors and I 20 upon bright.

Mosquitoes are fond of the odor of leather and are usually plentiful upon harness hanging in stables. They are said to prefer the odor of the negro to that of the white man.

Anophelines, like other malefactors, prefer darkness rather than light, and seek the sequestered nooks during the day.

A meal of blood is a prerequisite to fertilization. Females confined with males, then isolated and fed, do not deposit fertile eggs, but must be fed first. A single fertilization is sufficient for several batches of eggs. These are usually deposited between dusk and dawn. Still water is necessary, since the female may be drowned if the surface is agitated. The female sits upon 
the water or upon the edge of floating leaves or débris. The ova of anophelines are deposited upon the water in clumps, but soon separate and lie horizontally. A batch of ova usually numbers from 100 to 150 . Pressat ${ }^{485}$ has calculated, on a basis of 150 ova for each female, hatching 50 per cent. females, that a single female in one season produces about $5,000,000,000$ mosquitoes.

It is impossible to determine the length of life of mosquitoes in nature, though even in captivity they have been kept for weeks. Anophelines have been kept alive five days without food or water, and for about two months fed upon bananas. The males are not so long lived as the females.

Stephens and Christophers ${ }^{120}$ say there is evidence that the ova can survive for some months in moist earth and exposed to frost. Eysell ${ }^{486}$ and Galli-Valerio and De Jongh ${ }^{480}$ state that the ova of most species of mosquitoes of the northern temperate and frigid zones may hibernate.

Mosquitoes hibernate in the larval stage also. Smith ${ }^{480}$ found, in New Jersey, larvæ of culex pungens in ice contained in pitcher plants, and believes that larval hibernation must be extremely common. Mitchell ${ }^{488}$ found anopheles larvæ in tanks and barrels in the Botanical Gardens of Washington, D. C., during winter, and Woldert ${ }^{487}$ found these larvæ in December at Tyler, Texas.

Mitchell ${ }^{488}$ believes it probable that mosquitoes do not hibernate in the pupal stage, though Galli-Valerio and De Jongh ${ }^{480}$ maintain the opposite opinion.

It is chiefly in the winged stage that mosquitoes hibernate. In the late fall the males die, the fecundated females seeking shelter in dwellings, cellars, stables, barns, cisterns, hollow trees, or under bridges.

\section{THE PARASITES OF MALARIA}

Zoological Relations. - The parasites of malaria belong to the animal kingdom, to the division of protozoa, to the class of sporozoa, and to the order of hemosporidia. The hemocytozoa are not peculiar to man, but are found in other classes of vertebrates, and are distributed by Manson ${ }^{29}$ into three genera, as follows: 


\section{HEMOCYTOZOA}

I. Genus Hamamoba

Names

H. subtertiana.

H. quartan.

H. tertian.

H. relicta (Proteosoma).

H. Danielewski (Halteridium).

H. Kochi.

H. melaniphera.

H. Metchnikovi.

\section{Hosts}

The malaria parasites of man, the sexual phase being evolved in mosquitoes of the genus Anopheles.

Birds: sexual phase in mosquitoes of the genus Culex.

Birds.

Several species of monkeys.

Bat (Miniopterus Shredeibersii).

Trionyx indicus.

2. Genus Piroplasma

P. bigeminum.

P. canis.

P. ovis.

P. equi.

P. hominis.

Bovines: transmitted by the cattle tick (Boophilus bovis).

Dogs.

Sheep.

Horse.

Man.

H. ranarum (Drepanidium).

3. Genus Hemogregarina

H. splendens.

H. Magna.

H. lacertarum.

Frog (Rana esculenta).

Frog (Rana esculenta).

Frog (Rana esculenta).

Lizard (Lacerta muralis).

About twenty additional but less readily procured species.

There are three sharply defined species of parasites of malaria, the parasite of tertian malaria, the parasite of quartan malaria, and the parasite of estivo-autumnal malaria. The latter is divided by most observers into three, or at least two, varieties, the tertian and the quotidian, of which latter variety a pigmented form and an unpigmented form are described. $\mathrm{My}$ opinion is that there are two varieties of the estivo-autumnal parasite, the tertian and the quotidian, and that the pigmented and the unpigmented quotidians are merely forms of one variety.

Stephens ${ }^{489}$ described a form of the parasite which he believed was a new species and named it plasmodium tenue. It was characterized by extreme ameboid movement, irregularity of form, abundance and irregularity of chromatin and an absence of pigment.

I have recently obtained similar specimens from a case of 
comatose malaria and believe that this organism is an atypical form of estivo-autumnal parasite.

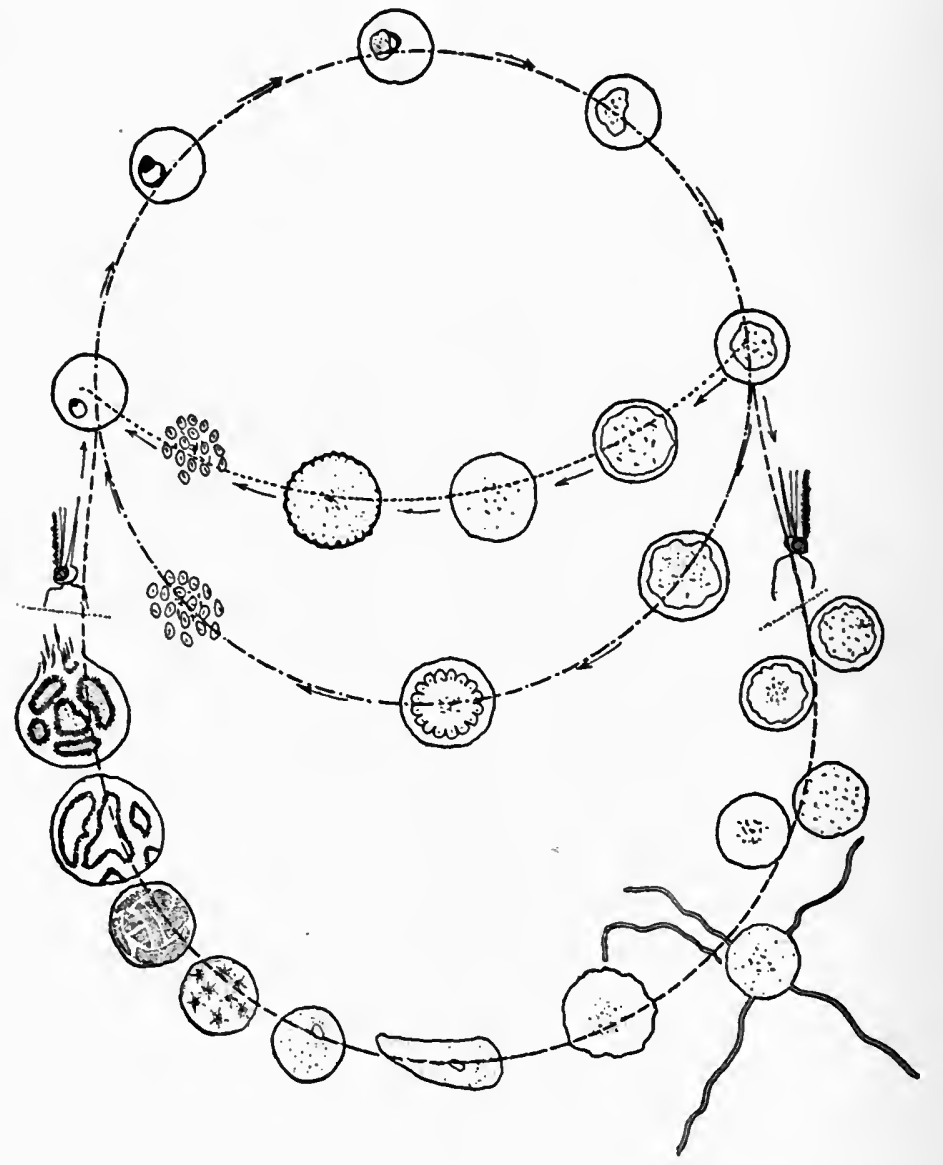

Fig. 20.-Diagram illustrating the cycles of the parasite.

-.-...-. Schizogonic cycle.

- - - - Sporogonic cycle.

.......... Parthenogenetic cycle.

Biology.-The life history of the parasites of malaria is somewhat complicated, inasmuch as man, the mosquito, and the parasite are involved, and as there are three species of parasites and each species has three biologic cycles. These three cycles are: 
I. The schizogonic, or human cycle, also called the asexual cycle, monogonic cycle, endogenous cycle, cycle of Golgi, or trophic cycle.
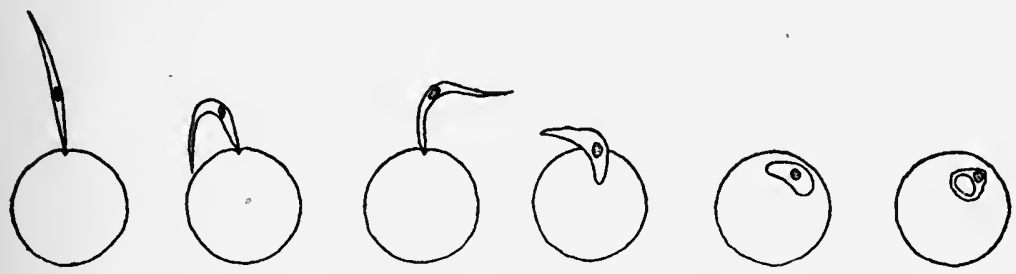

Fig. 21.-The entrance of the sporozoit into the red cell.

2. The sporogonic or mosquito cycle, also called the sexual cycle, amphigonic cycle, exogenous cycle, or cycle of Ross.

3. The parthenogenetic cycle, or reproduction by unfertilized macrogametes; the cycle of chronic malaria, of latency and relapses, an immaculate conception yielding saviors to the species necessary for its salvation at a time of crisis, a vicarious atonement of macrogametes that the human cycle may be saved.

The first cycle is that of active malaria; the last two are destined for the perpetuation of the species, and without them the interruption of the schizogonic cycle would result in the extermination of the species.

The Schizogonic Cycle.-In the act of biting, the mosquito injects into the blood sporozoits, elongated or needle-shaped organisms. The sporozoits have the power of bending, contraction, and of locomotion, and each immediately penetrates into a red blood-cell. Here it loses its slender form and appears as a mere dot of protoplasm, whose index of refraction varies but little from that of the red blood-cell. The size of the young parasite varies in different species, but is about I or 2 microns in diameter. Ameboid motion is more or less active, pseudopodia being protruded and retracted, the parasite even changing its position within the cell, and has no constant form. There is usually only one parasite in each infected cell, but there may be several. As the parasite grows it acquires pigment, a few grains at first, gradually increasing in amount with the growth of the parasite. This pigment is from the hemoglobin of the 
infected cell, and occurs in the form of grains, rods, or clumps. The adult parasite occupies a relatively large portion of the cell, and ameboid motion is less active, though the pigment may be in violent motion. The organism is composed of cell protoplasm, nucleus, and nucleolus, but appears structureless in fresh, unstained blood. Prior to sporulation the pigment becomes concentrated and fused, and fission occurs, dividing the parasite more or less symmetrically into spores, constituting the

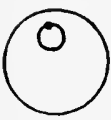

a

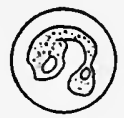

b

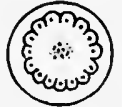

e

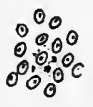

d

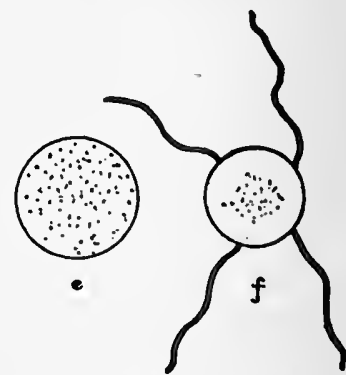

Fig. 22.-Diagram representing the development of the malarial parasite: $a$, Young form; $b$, half-grown parasite; $c$, sporulating body; $d$, free spores; $e_{\text {, }}$ macrogamete; $f$, microgametocyte.

so-called rosette or marguerite forms, each spore containing a fragment of nucleus. The cell ruptures and the spores, or merozoites, escape into the blood current, where they rapidly enter the blood-cells to repeat the cycle. The corpuscular remnants and the pigment are rapidly taken up by the phagocytes.

Rowley-Lawson ${ }^{490}$ has attempted to prove that the malaria parasite is extracellular throughout its life cycle, migrating from cell to cell, destroying each before it is abandoned, and that in the brief intervals between, the parasite is free in the blood serum. These observations have not been confirmed; on the other hand the experiments of Bass and Johns ${ }^{350}$ seems to overthrow them.

Instead of proceeding to sporulation some of the parasites develop into sexual forms, or gametes, large parasites of round, ovoid, spindle, or crescentic shape. It is these bodies which are taken up by the mosquito, undergo a sexual cycle in its midgut, develop into sporozoits, which are injected into man, 
where they pass through the schizogonic cycle above outlined.

The Tertian Parasite (Hamamaba vivax, H. tertiance, Plasmodium vivax).-The duration of the asexual cycle of the simple tertian parasite is forty-eight hours. The young parasites are about one-fifth the size of the red blood corpuscles and unpigmented. They are difficult to distinguish from the young parasites of the other species. They are actively ameboid, protruding and retracting short pseudopodia with rapidity; $\mathrm{Y}$-shapes, T-shapes, and crosses are common forms. The index of refraction of the parasites is low, so that their contours are not easily distinguished from the substance of the red blood-cells. As the parasite increases in size pigment gradually appears. The pigment of the tertian parasite is fine, rod-shaped, rather light in color, and in active motion. This motion of the pigment has been compared to the bubbling of boiling water and to the swarming of insects. The infected red cell becomes enlarged, swollen and pale. The half-grown parasite fills about half or two-thirds of the red blood corpuscle. At this stage the parasite assumes fantastic and bizarre shapes. The adult parasite is more or less spherical, as large as or larger than a normal red cell, and occupies three-fourths or four-fifths of the swollen infected cell, the margin of which may be difficult to see on account of its pale color. The pigment tends to become more abundant about the periphery. When sporulation is imminent the parasite and its pigment become less active or motionless, the pigment gathers in clumps at the center, and radial striations appear from the periphery toward the center. Usually the sporulating tertian parasite is not so symmetrical as the corresponding stage of the quartan, resembling, rather, a bunch of grapes or a mulberry. Less often there are two concentric rows of spores. The spores are small and vary in number from twelve or fourteen to twenty-six, oftenest sixteen, and more often an even than an uneven number. Sporulation takes place, especially in the circulation of certain viscera, but sporulating tertian parasites are much more frequently encountered in the peripheral circulation than in the case of estivo-autumnal infections.

The parasites develop with remarkable uniformity, nearly all 


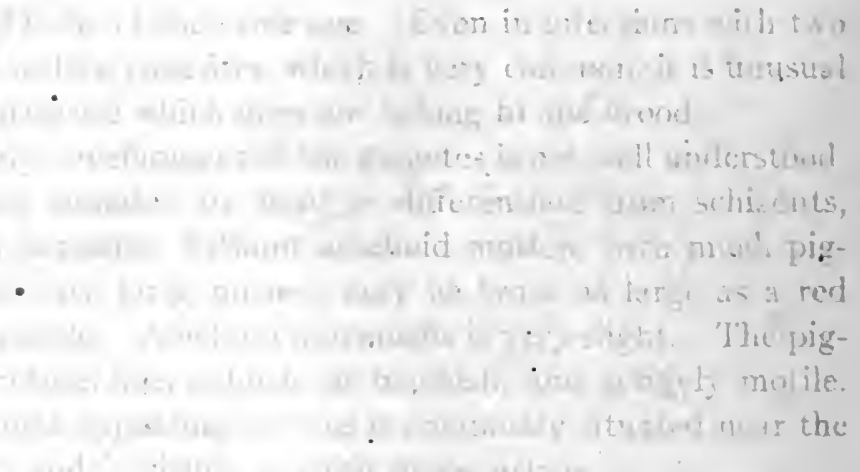

DESCRIPTION OF PLATES I AND II

Various forms of malarial parasites: Figs. I to ro inclusive, tertian parasites; Figs. II to 19 inclusive, quartan parasites; Figs. 20 to 26 inclusive, estivoautumnal parasites.

1.--Normal red blood cell. 2.- Young tertian ring. 3.-Large tertian ring. 4.- Half-grown tertian parasite. 5--Infected cell showing Schüffner's dots. 6.-Adult tertian parasite. 7.-Beginning sporulation. 8.-Sporulation completed. 9.-Tertian microgametocyte. Io.-Tertian macrogamete. II.-Young quartan ring. 12.-Older quartan ring. 13.-Quartan band. 14.-Older quartan band. 15.-Full-grown quartan parasite. 16.- Mature parasite with divided chromatin. 17.- Sporulation completed. 18.-Quartan microgametocyte. 19.-Quartan macrocyte. 20.- Young estivo-autumnal ring. 21.-Large estivo-autumnal ring. 22.-Mature parasite. 23.-Sporulation completed. 24.-Estivo-autumnal microgametocyte. 25.-Estivo-autumnal macrogamete. 26.-Estivo-autumnal ovoid. 
PLATE I

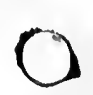

2

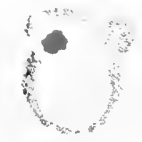

3

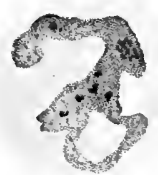

4.

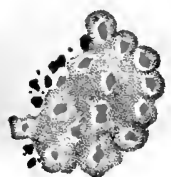

7

0

11

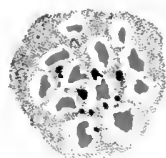

6

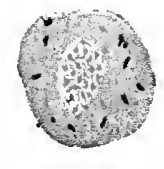

9

$c$

12 

PLATE II

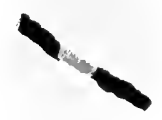

13

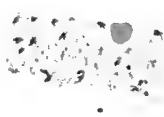

14

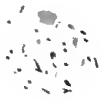

15

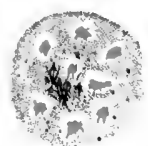

16
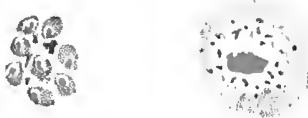

18

19

0

0

21

22

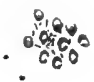

23

24

25

26

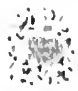

\% 

Microgametocyles

Plasma hyaline.

Pigment abundant in thick rods, brownish yellow.

Not larger than a red blood-cell.

Chromatin profuse.

Little ameboid motion.

Nucleus toward center.

\section{Macrogametes}

Plasma granular.

Pigment in fine rods and granules, brownish black.

Larger than red cell.

Chromatin less abundant.

More or less ameboid motion.

Nucleus toward periphery.

In stained films the early stage of the tertian parasite is seen as a ring. Often the ring is not of the same thickness throughout its circumference, but is composed of a thin segment, and a thicker segment, the chromatin being upon the thin segment. Usually the chromatin dot is immediately within the ring, but it may lie outside, and is surrounded by a pale zone. The achromatic zone may be regarded as the nucleus and the chromatin as the nucleolus. As the parasite develops one arc becomes much thickened, giving the appearance of the signet ring. The forms of the half-grown parasites are varied and peculiar. Pigment appears first and most abundantly in the peripheral region, and does not invade the clear zone. The red cell is enlarged and does not stain deeply. A peculiar stippling of the infected red cells is shown in films containing tertian parasites and stained with the Romanowsky stain or one of its modifications. When the parasite fills one-third of the cell a number of fine, red-stained points, Schüffner's dots, appear, which increase in size but not in number as the parasite grows. In the process of sporulation the chromatin becomes subdivided and surrounded by a clear zone, and encircled by the blue cytoplasm, constituting the spore.

The Quartan Parasite (Hamamaba malarice, H. quartane, Plasmodium malaria, Laverania malaria).- The duration of the schizogonic cycle of the quartan parasite is seventy-two hours. The young forms of the parasite appear as small, hyaline, unpigmented bits of protoplasm. They are highly refractive and the contour is much more sharply defined than the tertian parasite. Ameboid motion is sluggish and the organism may be watched sometime until motion is detected. Pigment appears within twenty-four hours. It is in larger quantities than in the tertian parasite, in coarser grains or rods, and dark brown or 
biack in color. The pigment is accumulated around the margin, and its motion is very slow. In the half-grown parasites the peculiar forms observed in the tertian organisms are not seen, and ameboid movements become more sluggish or cease altogether. The red blood-cell infested with the quartan parasite does not enlarge and decolorize as in tertian infections, but, if there is any deviation from normal, becomes smaller and darker, perhaps greenish and brassy. The adult parasites are almost as large as the red cells. Prior to sporulation the pigment collects toward the center, often in a radial arrangement. Sporulation proceeds after the manner of the tertian parasite, but is slower. The sporula ting forms are beautifully symmetrical and are typical rosettes. The spores are round or oval, relatively large and six to twelve in number, of tenest eight. Sporulating quartan parasites are much more commonly observed in the peripheral blood than are the corresponding forms of the other species.

Quartan gametes are but rarely encountered. The macrogametes are spherical in shape, and as long as they remain intracorpuscular are smaller than tertian gametes, but are equally as large when they become extracorpuscular. Exflagellated microgametocytes have been observed; they are somewhat smaller than the tertian forms, but no less active.

The staining reactions of the quartan parasites are similar to those of the tertian. The young form is a ring and so closely resembles the tertian that it cannot be determined with certainty. After twelve to twenty-four hours the parasite becomes disc- or band-shaped. The latter forms are characteristic. The parasite extends across the center of the infested cell as a more or less broad band, often rather quadrilateral, the pigment being arranged more profusely along the margin of the band. The chromatin body of the quartan species stains less intensely and splits earlier than the tertian. The adult usually fills the corpuscle, which may be no longer apparent. The sexes of the gametes are differentiated by the same characters as in the tertian.

The Estivo-autumnal Parasite (Hamamœba pracox, Plasmodium pracox, Hamameba immaculata, Laverania pracox, 
Hamomenas pracox, Plasmodium immacuiatum, Hamamaba parva, Hamatozoon falciform, Plasmodium falciparum).-The young forms of the estivo-autumnal parasites are similar to those of the other species, but are smaller, being from one-fifth to one-sixth the size of the infested corpuscle. Ameboid motion is rather active, stars, crosses, and irregular shapes occurring in succession. At rest the parasites appear annular or discoid. More than one parasite in a single cell is relatively more common than in tertians and quartan infections. Advanced stages of development are rarely seen in peripheral blood. The infested red cells often become shriveled, crenated, darker, and of a brassy hue. The adult parasites do not attain the size of the red blood corpuscles. Sporulation proceeds in a manner similar to that of the simple tertian parasite. The spores number from five to twenty-five or even thirty. Sporulation is not so uniform as in other infections; sporulating forms may be associated with young or half-grown parasites.

Estivo-autumnal gametes occur in the form of crescents, and of fusiform, ovoid, and spherical bodies. The crescents are characteristic, being found in this form of malaria alone. They are cylindrical, tapering slightly at each extremity, and slightly curved upon themselves. They are longer than the diameter of the red cell and about a third as broad as long. The changes from crescent to ovoid and round bodies may be easily observed under the microscope. The gametes appear only after the infection has persisted for about a week. The crescent may lie within the cell or may have the appearance of the red cell being attached to the concave side; in some instances there is no evidence left of the infested blood-cell. The cell may be stretched across the concavity of the crescent, and is usually almost decolorized. The crescents have given evidence of a double outline. They possess no ameboid movement, and the pigment in the form of rods or granules is motionless. Before exflagellation the crescent assumes the spheric form, smaller than the tertian, and similar to the quartan.

The staining reactions of the estivo-autumnal schizonts are similar to those of the tertian and quartan. The young parasites are unpigmented rings, resembling the simple tertian rings, 
but are smaller and more delicate. Typical signet rings and rings without nodes are seen. The ring may be distorted or become broken and extended like a delicate thread or a narrow band. The largest rings are about one-half the diameter of the red cell. The pigment is sparse. Both the sporulating body and the individual spores are small. The central region of the crescent is almost achromatic, the extremities staining more deeply. Chromatin is not always visible in the crescents, but is usually seen, as is the pigment, in the achromatic area.

Mixed infections with quotidian and tertian estivo-autumnal parasites are very common.

The differences between these parasites are thus tabulated by Craig: ${ }^{: 91}$

The Hyaline Body

\begin{tabular}{|c|c|c|}
\hline $\begin{array}{c}\text { Stage of } \\
\text { development }\end{array}$ & Quotidian & Malignant tertian \\
\hline Size.. & Minute, one-sixth of corpuscle. & $\begin{array}{l}\text { Larger, one-third to one-quarter } \\
\text { of corpuscle. }\end{array}$ \\
\hline Shape & Ring or perfectly round. & Signet-ring shape. \\
\hline Outline & Indistinct. & Clear cut and refractive. \\
\hline Motion... & Very active. & Sluggish. \\
\hline Corpuscle. & $\begin{array}{l}\text { Very dark green, wrinkled. } \\
\text { Crenated. }\end{array}$ & Light green, less wrinkled. \\
\hline Number... & $\begin{array}{l}\text { More than one parasite in a cor- } \\
\text { puscle, common. }\end{array}$ & $\begin{array}{l}\text { Very seldom more than one par- } \\
\text { asite in a corpuscle. }\end{array}$ \\
\hline
\end{tabular}

The Pigmented Body

\begin{tabular}{|c|c|c|}
\hline Size. & One-quarter size of corpuscle. & One-half size of corpuscle. \\
\hline Shap & $\begin{array}{l}\text { Round. Loses ring form beiore } \\
\text { pigmentation. }\end{array}$ & $\begin{array}{l}\text { Ring form becomes pigmented; } \\
\text { afterward the parasite is round. }\end{array}$ \\
\hline Motion & Ameboid motion is lost. & $\begin{array}{l}\text { Ameboid motion continues. Is } \\
\text { sluggish. }\end{array}$ \\
\hline Outline. & More sharply defined. & $\begin{array}{l}\text { Very sharply defined and refrac- } \\
\text { tive. The protoplasm firmly } \\
\text { granular. }\end{array}$ \\
\hline Pigment. & $\begin{array}{l}\text { One or two coarse granules per- } \\
\text { fectly motionless. }\end{array}$ & $\begin{array}{l}\text { Several minute grains having a } \\
\text { rapid vibratory motion. }\end{array}$ \\
\hline Number. & $\begin{array}{l}\text { May be more than one in a cor- } \\
\text { puscle. }\end{array}$ & $\begin{array}{l}\text { Never more than one in a cor- } \\
\text { puscle. }\end{array}$ \\
\hline Corpuscle. & $\begin{array}{l}\text { Very green in color, often cre- } \\
\text { nated. }\end{array}$ & Lighter in color, seldom crenated. \\
\hline
\end{tabular}


The Segmented Body

\begin{tabular}{c|l|l}
\hline $\begin{array}{c}\text { Place of seg- } \\
\text { mentation. }\end{array}$ & $\begin{array}{l}\text { Within the red blood corpuscles, } \\
\text { as a rule. }\end{array}$ & $\begin{array}{l}\text { Outside the red blood corpuscles } \\
\text { as a rule. } \\
\text { Ten to fifteen or more. }\end{array}$ \\
$\begin{array}{l}\text { segments. } \\
\text { The crescent } \\
\text { phase. }\end{array}$ & $\begin{array}{l}\text { Six to eight. } \\
\text { Crescents small and plump, con- } \\
\text { taining small a mount of pig- } \\
\text { ment. Always present double } \\
\text { outline. }\end{array}$ & $\begin{array}{l}\text { Crescents long, narrow, deeply } \\
\text { pigmented. Double outline less } \\
\text { common. }\end{array}$ \\
$\begin{aligned} \text { Cycle of de- } \\
\text { velopment. }\end{aligned}$ & Twenty-four hours. & Forty-eight hours. \\
\hline
\end{tabular}

The following table will serve to distinguish the sexes of the gametes:

Microgametocytes

Protoplasm stains very slightly.

Pigment distributed throughout the parasite.

Reniform short and broad.

Chromatin in several scattered masses.

\section{Macrogametes}

Protoplasm stains more intensely.

Pigment collected near center often in a circle.

Crescentic, longer and narrower.

Chromatin in one or two large masses near the center.

The chief characteristics of the species of malaria parasites may be tabulated as follows:

\begin{tabular}{|c|c|c|c|}
\hline & Tertian & Quartan & Estivo-autumnal \\
\hline $\begin{array}{l}\text { Length of asex- } \\
\text { ual cycle. }\end{array}$ & Forty-eight hours. & Seventy-two hours. & $\begin{array}{l}\text { Twenty-four hours, } \\
\text { forty-eight hours, } \\
\text { or irregular. }\end{array}$ \\
\hline $\begin{array}{l}\text { Site of sporula- } \\
\text { tion. }\end{array}$ & $\begin{array}{l}\text { May sporulate in } \\
\text { peripheral blood, } \\
\text { chiefly in visceral } \\
\text { circulation. }\end{array}$ & $\begin{array}{l}\text { Equally in peripheral } \\
\text { and visceral blood. }\end{array}$ & $\begin{array}{l}\text { Visceral circula- } \\
\text { tion. }\end{array}$ \\
\hline Movements. & Active. & Sluggish. & Active. \\
\hline Pigment. & $\begin{array}{l}\text { Fine, yellowish or } \\
\text { dark brown, scat- } \\
\text { tered, actively } \\
\text { motile. }\end{array}$ & $\begin{array}{l}\text { Coarse, dark brown or } \\
\text { black (at periphery), } \\
\text { slightly motile. }\end{array}$ & Scanty, fine. \\
\hline $\begin{array}{l}\text { Effect on red } \\
\text { cell. }\end{array}$ & $\begin{array}{l}\text { Enlarged, decolor- } \\
\text { ized, Schüffner's } \\
\text { dots in stained } \\
\text { films. }\end{array}$ & $\begin{array}{l}\text { Normal size or smaller, } \\
\text { often dark and } \\
\text { brassy. }\end{array}$ & $\begin{array}{l}\text { Often shrunken, } \\
\text { may be dark and } \\
\text { brassy. }\end{array}$ \\
\hline Size of adult. & $\begin{array}{l}\text { As large as normal } \\
\text { red corpuscles. }\end{array}$ & $\begin{array}{l}\text { Smaller than normal } \\
\text { corpuscles. }\end{array}$ & $\begin{array}{l}\text { Much smaller than } \\
\text { normal corpuscles. }\end{array}$ \\
\hline Sporocyte. & Mulberry shape. & $\begin{array}{l}\text { Symmetrical daisy } \\
\text { shape. }\end{array}$ & Irregular. \\
\hline $\begin{array}{l}\text { Spores. } \\
\text { Gametes. }\end{array}$ & $\begin{array}{l}\text { I } 2-26 \text {, of tenest I } 6 . \\
\text { Spheric. }\end{array}$ & $\begin{array}{l}6-12, \text { of tenest } 8 . \\
\text { Spheric. }\end{array}$ & $\begin{array}{l}5-30 . \\
\text { Crescentic. }\end{array}$ \\
\hline
\end{tabular}


It is not infrequently difficult to differentiate young tertian from young estivo-autumnal rings, though the following table adapted from Ewing ${ }^{492}$ will enable a comparison of the main feature:

Tertian

Nucleus achromatic to methylene-blue.

Ring usually coarse and irregular.

One or two grains of pigment almost invariably present.

Ring usually pigmented before chromatin subdivides.

Infected cell swollen.

\section{Estivo-autumnal}

Nucleus stains intensely with methylene-blue.

Ring geometrically circular, delicate, usually a typical signet-like swelling.

Pigment almost constantly absent.

Chromatin always subdivides before pigment appears.

Infected cell shrunken.

The Sporogonic Cycle.-If the anopheline mosquito obtains blood containing only schizonts, the latter soon perish in the digestive canal of the insect. However, if the blood contains mature gametes of both sexes these undergo the exogenous cycle, to be prepared to reinfect man bitten by the infected mosquito. The portion of the mosquito in which this transformation takes place is the stomach or midgut. Shortly after the infested blood has been sucked into the stomach the microgametocytes exflagellate, the microgametes become free, and the macrogametes emit small protuberances to receive the flagella or spermatozoa. The latter forces an entrance into the macrogamete at the site of the protuberance and the resulting fertilized body is known as the zygote. All this has taken place within the first few hours. In its early stages the zygote resembles the ovoid body, but is larger. It is pigmented, it enlarges, becomes pyriform, and has the power of locomotion. The zygote burrows through the epithelial coat of the midgut to the tunica elasticomuscularis, becomes spheric and encysted, and is known as the oocyst. It enlarges so that it projects like a spheric excrescence into the body cavity or blood sinus, where it is nourished. The stomach of a badly infected mosquito may be studded with these outgrowths. The oocyst attains a size of 40 to 70 microns in diameter. Its nuclear chromatin divides and subdivides, each portion surrounded by protoplasm, polygonal or irregular in shape from pressure, being known as the sporoblast. Each 
sporoblast splits into a large number of sporozoits, each enclosing a bit of chromatin. The sporozoits remain attached by one end to the residual body of the sporoblast until the oocyst bursts, when the sporozoits escape into the body cavity. Finally through the lacunar circulation, they arrive at the salivary glands, where they congregate in hordes. The sporozoits number from a few hundreds to ten thousand or more. Each measures about I4 microns in length, about eight times as long as broad, being very slender, tapering at both ends, and endowed with serpentine movements. From the salivary glands the sporozoits are injected by the mosquito, in the act of preying upon its victim, where each sporozoit soon enters a red cell and goes through the schizogonic cycle. The duration of the mosquito cycle varies from eight to sixteen or more days, depending mainly upon the temperature, but possibly also upon other factors.

The three species of parasites are closely similar in their stages of exogenous development. The differences between the tertian and the estivo-autumnal organisms are that in the former the zygote is round or oval instead of pyriform or ovoid, the protoplasm is less refractive, the characteristic pigment maintains, the sporoblasts are larger and less numerous, the sporozoits are less dense and more regularly arranged, often radially within the sporoblast, and black spores have not been found.

The quartan parasite is the most difficult to develop within the mosquito.

The Parthenogenetic Cycle.-Parthenogenesis, or virgin birth, is reproduction by unfertilized females.

This phenomenon, known also as the "alternation of generations," has been most carefully studied in plant lice, the Aphida. The eggs, which are laid in the fall and have hibernated, hatch in the spring into females, having the power of giving birth, without fertilization, to viviparous young, which inherit the faculty of parthenogenesis, and procreate in this manner until the advent of cold weather or the failure of nourishment when males and oviparous females are brought forth. From these latter, after copulation, ova are produced, and the cycle recommences. 
Parthenogenetic reproduction is known to occur in a number of species, as hemoproteus, certain rotifera, jellyfish, worms, entomostracea, acarina, and certain insects, the silk-moth, mosquitoes, gall-flies, ants, bees, wasps, chironomus, etc.

This life cycle of the parasite of malaria is the most recently recognized and least known of its cycles. Since the discovery of the parasite the gametes have been regarded as closely allied with the chronic malaria and relapses. Golgi plainly stated as his belief that the crescent was the parasite of fevers recurring at long intervals.

Canalis, ${ }^{493}$ in 1889 , described and pictured spheric bodies derived from crescents in the act of sporulation. In I890, Antolisei and Angelini ${ }^{492}$ confirmed the observation of Canalis. Lewkowicz ${ }^{492}$ reported, in 1897 , that he had seen sporulating crescents some of which contained as many as thirty spores.

Grassi ${ }^{494}$ expressed the opinion in I90I that the parasites of malaria underwent a parthenogenetic cycle of development whereby the species was perpetuated after the death of the schizonts.

He cited a number of arguments in support of the theory, and referred to a similar process in other protozoa, Adelea, Trichospherium, and Volvox.

It was Schaudinn ${ }^{494}$ who, in I902, first observed and correctly interpreted parthenogenesis of tertian macrogametes, and who thus describes the process of parthenogenesis as he observed it in tertian malaria. The chromatin of the parthenogametes collects in coarse fragments and cords toward one end of the bean-shaped nucleus, and stains intensely, while the other somewhat larger half contains fewer and smaller chromatin particles and stains faintly. The nucleus then divides into two, one containing the coarse, deeply staining chromatin, and the other the fine, diffusely staining chromatin, the former resembling the nucleus of a schizont before nuclear proliferation. A constriction may be perceptible about the parasite almost separating a deeply staining, highly pigmented portion containing the pale staining nucleus from a lightly stained and less pigmented portion in which lies the deeper stained nucleus. This nucleus now subdivides and the portion of the plasma in which 
it lies proceeds to sporulation in a manner similar to schizogonic sporulation, the spores becoming typical schizonts.

Maurer, ${ }^{495}$ in 1902 , observed sporulation of estivo-autumnal gametes, and construed it as parthenogenesis.

Ziemann ${ }^{86}$ believes that he has seen parthenogenetic reproduction of quartan gametes.

Blüml and Metz ${ }^{496}$ observed sporulating parthenogametes in six preparations taken from five patients with tertian malaria.

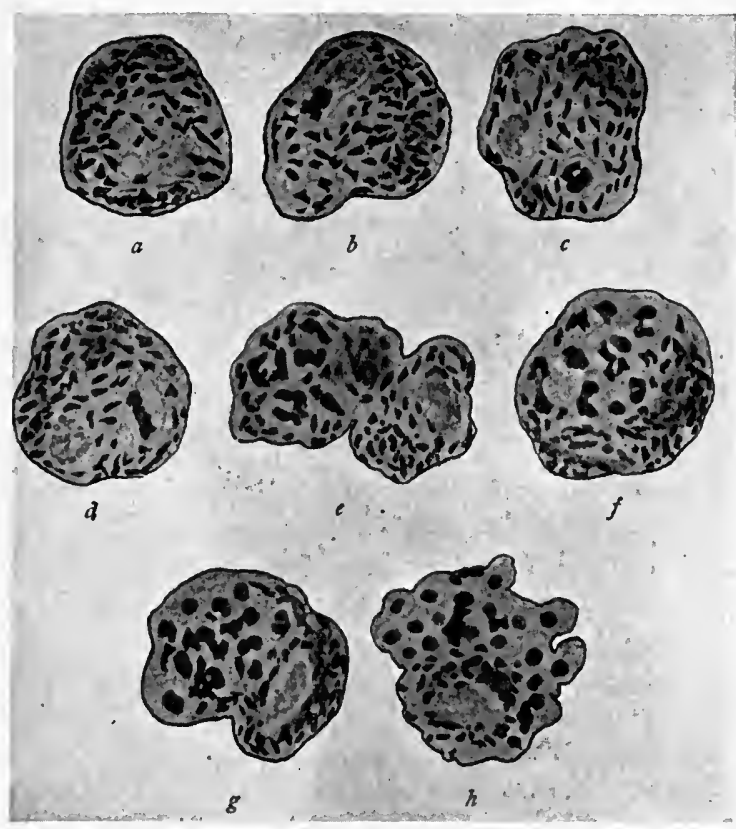

Fig. 23.-Parthenogenesis of the tertian parasite (after Schaudinn).

The process was identical with that described by Schaudinn. Young and sporulating schizonts and young gametes were present in these same preparations.

Bass and Johns ${ }^{350}$ observed a process resembling parthenogenesis in some of their cultivation experiments.

Are the parthenogametes identical with true macrogametes which do not proceed to sporulation? Such a doubt is hardly justifiable from a review of the scientific studies of the learned Schaudinn and of the other observers cited above. Craig, ${ }^{497}$ 
however, has recently adduced evidence that latency and relapses are dependent upon resting bodies, the products of intracorpuscular conjugation of young schizonts. He does not, however, follow these forms further than the completion of conjugation, and while it is possible that this is the origin of the parthenogametes, there is as yet no positive evidence that the latter are not true macrogametes.

Cultivation Experiments.-Coronado ${ }^{491}$ claimed to have been successful in cultivating the malarial parasites from water which he believed infected. He stated that the entire cycle could be followed. Miller ${ }^{23}$ also believed that he had cultivated the organisms. These experiments have been repeated, but the results could not be confirmed, hence were probably incorrect.

Sakharov, Rosenbach, Blumer, Hamburger and Mitchell ${ }^{467}$ succeeded in maintaining the organisms alive for several days in the bodies of leeches which had sucked the blood from malarial patients.

In I9I I, Bass ${ }^{349}$ announced the successful cultivation of the three varieties of malarial parasites. The technic as developed by Bass and Johns $\mathrm{s}^{350}$ may be summarized as follows:

The blood is collected from the patient's vein at the bend of the elbow, unnecessary exposure to air and agitation being avoided. One-tenth of a cubic centimeter of a 50 per cent. solution of dextrose for each ro c.c. of blood to be taken is placed in the defibrinating tube before the blood is drawn. The blood is defibrinated by gently stirring or whipping with a glass rod, care being taken not to whip in air or cause bubbles.

The column of blood must be 2.5 to $5 \mathrm{~cm}$. deep in the tube, giving a column of serum $\mathrm{x} .25$ to $2.5 \mathrm{~cm}$. deep above the cells after the latter have settled. Development of the parasites takes place at the top of the column of precipitated cells, all parasites below the top layer dying in from two to twenty hours. The parasites grow best at an incubator temperature of about $40^{\circ}$.

When more than one generation of parasites is to be grown it is necessary to remove the leucocytes at the time the culture is made in order to avoid destruction of the organisms by them at 
the time of segmentation. This is done by centrifuging the blood sufficiently to force the leucocytes to the surface of the cells, drawing off the supernatant serum and carefully pipetting the cells and plasmodia from about the middle of the centrifuged mass. The latter are then placed in the decanted serum and incubated.

Three successive generations sometimes grow in such a culture

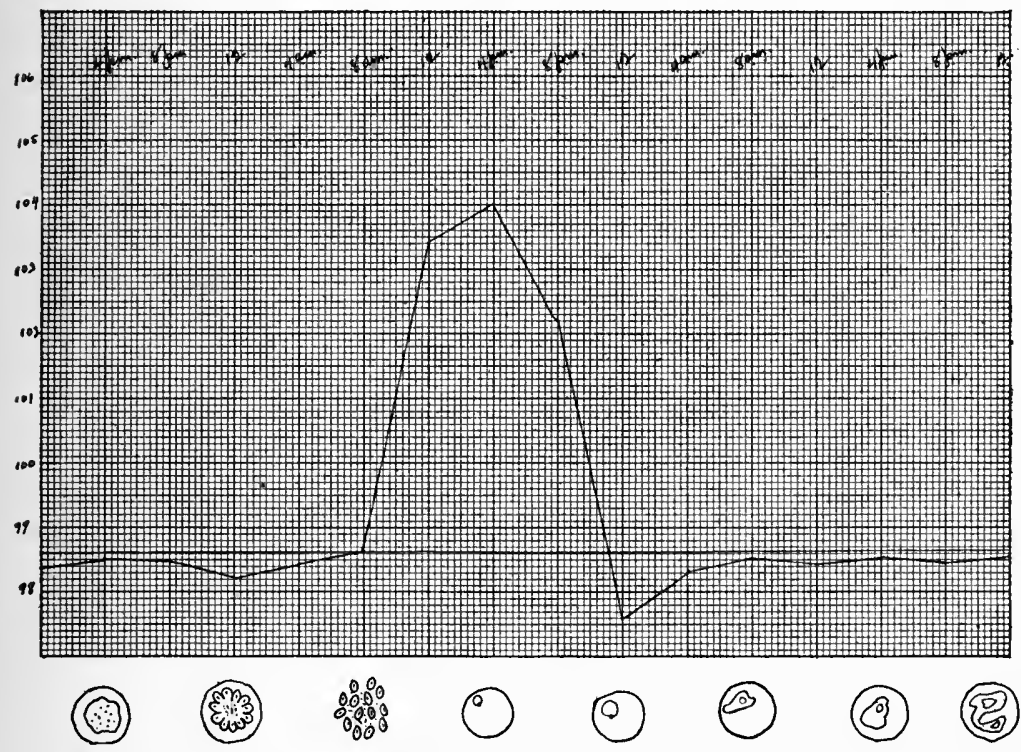

Fig. 24. - The relation between the stages of the parasite and the paroxysm.

but it is usually necessary, after each segmentation, to transfer a portion of the cells and parasites to a tube containing recently prepared cells and serum.

Only asexual forms have been cultivated but organisms suggesting parthenogenesis have been observed.

Leucocytes do not attack intracorpuscular plasmodia but engulf them immediately upon becoming free after segmentation.

The young parasites are soon destroyed by the serum and enter fresh red cells only when these lie in contact with the cell in which sporulation takes place and then only at the point of juxtaposition. 
Culture of parasites by this method has been confirmed by Lavinder, ${ }^{351}$ Ziemann, ${ }^{352}$ Thompson and Thompson ${ }^{353}$ and others.

\section{PATHOGENESIS}

Much fanciful speculation had been indulged in as to the cause of the paroxysms until Golgi and others of the Italian school showed that it was closely connected with the life history of the malarial parasites.

The paroxysm is not immediately associated with the gradual vegetative growth of the parasite within the corpuscle, but, occurring more or less abruptly as it does, is simultaneous with sporulation and the sudden discharge into the blood stream of a new generation of parasites. In what manner does this process produce so peculiar a phenomenon as the malarial paroxysm? Golgi ${ }^{19}$ was of the opinion that it was dependent upon the entrance into fresh red cells of the young generation of parasites. This, however, is shown to be erroneous by the fact that a properly timed and adequate dose of quinine given before the chill is expected does not prevent the access though it does destroy the young parasites, preventing their invasion of the cells.

The true explanation of the origin of the paroxysm is through the agency of a toxin liberated by the sporulative act. The existence of a toxin, the product of the malarial parasite, is almost universally assumed by students of malaria. The grounds for this assumption may be recounted as follows:

I. An analogy with other infectious diseases.

2. Immunity: this immunity is not absolute, but that a relative immunity to malaria exists there is no room for doubt.

3. The formation of an antitoxin, Ford's ${ }^{498}$ experiments being conclusive as to the existence of such.

4. Degenerative changes in the kidneys, liver, spleen, and other organs not otherwise explainable.

5. Blood changes, as anemia out of proportion to the number of parasites, and brassy degeneration, stippling, and polychromatophilia of the red cells.

6. Increased toxicity of the urine and sweat. 
7. The existence of coma in malaria without parasites or pigment in the brain.

8. The fever and its relation to parasitic sporulation.

9. Experimental proof. The negative results of Gualdi, ${ }^{86}$ Montesano, ${ }^{86}$ Mannaberg, ${ }^{82}$ and Celli ${ }^{458}$ are devoid of weight against the convincing experiments of Rosenau, Parker, Francis and Beyer, ${ }^{499}$ who demonstrated the existence in malarial blood of a poison capable of reproducing the symptoms of the disease when injected into the veins of other men.

The experiments of Brown ${ }^{500}$ would seem to indicate that the phenomena in human beings infected with malaria are, at least in part, directly referable to the toxic action of the malarial pigment.

The parasites of tertian and quartan infections develop uniformly, one generation at a time; hence typic paroxysms are the rule. Sometimes, it is true, sporulating forms are met with between the accesses, but a certain dose of toxin is necessary to excite a fit. The estivo-autumnal parasites, on the other hand, do not sporulate so uniformly; hence the poison is liberated in broken doses and typic paroxysms are more frequently lacking and the fever more continuous or irregular. If sporulation occurred more nearly simultaneously, as in the tertian and quartan forms of malaria, it is probable that the sudden discharge of the more highly poisonous estivo-autumnai toxin would be more often attended with serious consequences. The uniform sporulation of the tertian and quartan parasites may be likened to a body of soldiers firing by volleys, while that of the estivo-autumnal parasites is similar to soldiers firing at will.

The change of type of malarial attacks has been used as an argument for the unity of the malarial parasites. It is well known, however, that such occurrences are best explained by a number of different species. Quotidian malarial paroxysms due to two generations of tertian organisms may become tertian in character by the destruction of one generation. Quotidian paroxysms due to a triple quartan infection may become quartan or double quartan by the death of two generations or of a single generation of parasites. On the contrary, tertian 
and quartan accesses may become quotidian by the development into activity of one or two additional generations.

It is remarkable in multiple infections by different generations of the same species of parasite that they almost always sporulate on different days and very often about the same time each day. Thus it is very rare in double tertian infections that two paroxysms should occur within one day followed by a fever-free day. This is probably best explained by the mode of infection. It is known that the malarial mosquitoes feed almost solely at night and usually only once during the night. If a subject is inoculated by the mosquito on two successive nights it is obvious that the parasite would become mature with an interval of about twenty-four hours between the generations. If inoculation should occur upon three or more successive nights it is probable that the third and succeeding generations would sporulate, after incubation, simultaneously with the first and second. The interval between multiple quartan infections may be explained similarly.

Latency and relapses were formerly explained upon the theory that so long as the parasites remained below a certain level of asexual reproduction the disease was latent, and when the parasites exceeded in number this level a relapse occurred. Sims has estimated the greatest number of adult parasites which the body can endure without symptoms as about two billions. It is probable that brief periods of latency may be thus explained, especially in persons possessing a relative immunity, but it is evident that this is not a common mode, particularly of relapses at long intervals, since the asexual cycle is known to wear out spontaneously after certain periods. These relapses at long intervals can be explained by parthenogenesis alone. After the schizonts have perished, while the microgametocytes do not persist long, the macrogametes remain for indefinite periods. They may sporulate more or less regularly, causing paroxysms at intervals of about a week, or multiples thereof, or may lie dormant until aroused into reproductive activity by exposure or dietary or other excesses. It is highly probable that the parthenogenetic cycle of reproduction is conducted almost altogether in the visceral circulation, particularly in the spleen. 
As evidence of this may be cited the outbreaks of malaria following cold douching, electrical stimulation, and trauma of the splenic region.

The anemia of malaria depends upon three factors: (I) The mechanical destruction of cells by the parasites; (2) the effect of toxins; and (3) the activity of the blood-making organs. Liberated hemoglobin is transformed by the liver into bile pigment. When the hemoglobin is liberated too fast for the liver to utilize, hemoglobinemia results, and hemosiderin is precipitated from the blood. The increased activity of the liver results in polycholia and icterus. It is probable that jaundice is due also, when the liver capacity is overtaxed, to hemoglobinemia. When hemoglobinemia exceeds a certain limit the hemoglobin is excreted by the kidneys, resulting in hemoglobinuria.

Splenic enlargement is effected through hyperemia, deposition of detritus of destroyed erythrocytes, accumulation of parasites, and hyperplasia of the pulp.

Spontaneous cure is probably due to the natural weakening of the reproductive powers of the parasite, a phenomenon occurring in strains throughout the vegetable and animal kingdoms, and possibly also to the influence of an antitoxin. Phagocytosis plays, in my opinion, a much less prominent rôle than is usually attributed to it. It is probable that this function ' is exercised mainly after the parasites have lost vitality from other causes:

\section{ETIOLOGY OF PERNICIOUS MALARIA}

Pernicious malaria is almost as varied in pathogenesis as it is in manifestations. Not only are its several forms associated with unlike conditions, but for the explanation of some the presence of several different factors is necessary. Thus comatose malaria may be associated with at least two different forms of the parasite; the peripheral blood may show very great numbers of these parasites or they may be scanty; in the brain they may be found in hordes, even to the occlusion of small vessels, or they may be entirely absent. 
As may be inferred, no one etiologic element can account for all cases, even of the same type. Probably the only essential common factor is the presence of the malarial parasite, the manifestations of which run the gamut from the mildest intermittent to the profoundest cachexia, from the most artfully masked to the deadliest pernicious.

Until comparatively recently it was believed that infections with the so-called benign organisms never gave rise to pernicious symptoms. I have, however, collected ${ }^{146}$ a number of cases in which these organisms were responsible for pernicious attacks.

Pernicious symptoms occur more rarely in connection with the quartan infections than with simple tertian. The reasons for this are probably the relative rarity of quartan fever and the more even distribution of parasites throughout the circulation, there being slight tendency to form accumulatio $s$.

It is not yet known with certainty which variety of the estivo-autumnal parasite gives rise most frequently to perniciousness. That the crescentic form of the parasite has an intimate relation to the production of the pernicious fevers is improbable, for the following reasons: First, crescents alone may be found in the peripheral blood, and intense localization of active forms be present in the brain or other viscera. The number of parasites in the superficial circulation is not a reliable guide to the severity of the attack. Of Ewing's 64 cerebral cases no parasites were identified in I I, and in many of his 33 cases in which crescents alone were found the search was successful only after one and two hours. Second, crescents are rarely, if ever, present in the parasitic localizations and thrombi are frequently observed in pernicious cases.

Of the pathogenic factors which excite perniciousness the following are to be regarded as the most important and approximately of relatively equal importance:

I. An excessive number of parasites.

2. Localizations of parasites.

3. Toxins.

4. Individual predisposition and external etiologic influences.

Number of Parasites.-Golgi's law, that the number of parasites determines the severity of the attack, has been generally 
accepted. Cases in which the parasites are in very great numbers in the peripheral blood are usually accompanied by coma. That the parasites are abundant, either absolutely in the body as a whole or relatively in certain areas, probably holds good in a great majority of the cases, though we cannot always attribute perniciousness to the large number of parasitic forms. I have observed one case of severe comatose malaria in a boy, aged twelve, in whose peripheral blood the parasites were scanty.

On the other hand, the superficial circulation may be teeming with parasites, while the patient experiences only a mild attack. Thus A. Plehn ${ }^{36}$ gives the histories of two cases in which the symptoms were slight though the peripheral blood showed as many as thirty-five and forty-six tropic parasites, respectively, to each field of the microscope.

It is highly probable that an enormous number of parasites, equally distributed, depend for their power to elicit pernicious symptoms upon the increased quantity of toxin elaborated.

Localizations of Parasites.-Accumulations of parasites in the brain were first described by Planer (1845) and by Frerichs (I86I); those in the liver by Guarnieri (I867). Localizations in the brain have been found associated with a wide variety of cerebral symptoms: in the mucosa of the alimentary tract, with gastro-intestinal symptoms and typical algid attacks; in the heart, with cardiac symptoms; in the medulla, with bulbar paralysis; in the retina, with amblyopia; in the pancreas, with hemorrhagic pancreatitis, etc. In proportion to the amount of damage sustained by the kidneys in malaria there is less tendency for parasites in pernicious attacks to accumulate in these organs than in any other of the body.

These localizations consist, in the main, of parasite-infected red blood-cells. There may be, however, pigmented leucocytes and free parasites and pigment. The parasites in each particular case may be of the same or of different stages of development. The pigmented and sporulating forms are probably oftenest seen, but the earlier phases are frequently observed. It would seem reasonable that the crescents, on account of their size, would frequently form an important element in these 
accumulations of parasites, but such does not appear to be the case.

The symptoms present in cases in which, on post-mortem examination, localization of parasites are demonstrated are not always referable to these aggregations alone, since changes are frequently observed which are secondary to parasitic thrombosis, and may outweigh the latter in pathogenic importance.

The most conspicuous of these changes are perivascular exudation, hemorrhage, and necrosis. The hemorrhages are usually punctate.

This propensity of the parasites in pernicious fever to congregate undoubtedly explains the course of many cases, but by no means all. Fatal cases of comatose malaria have been observed with no parasites at all in the brain. It is not known whether parasitic thrombi may exist without producing symptoms.

Toxins.-The evidence of the existence of a toxin in malaria has been detailed above.

Individual Predisposition and External Etiologic Influences. -These influences, in many instances; doubtless not only induce the attack but determine its type. Organs or systems enfeebled by antecedent ailments are apt to play the title rôle in the pernicious tragedy. Thus algid and choleraic attacks may be associated with a history of intestinal catarrh; the comatose and delirious cases, with a history of abuse of alcohol; the convulsive, with epilepsy, etc. It is not improbable that some cases of dysenteric, cardialgic, syncopal, tetanic, cataleptic, paralytic, pneumonic, pleuritic, gastralgic, and other forms described by the older writers may be similarly explained.

Malarial subjects who are much exposed to the heat of the sun are liable to be stricken with pernicious fever, especially of the cerebral type. This danger is enhanced if to the solar heat are added fatigue, deficient or improper food, or other hardships. Certain psychic states have causative significance.

In addition to the four principal factors enumerated, congestion of viscera and parasitic obstruction of the hepatic capillaries have been regarded as important. It is probable that they have little influence. 
A feeble phagocytic activity was considered by Golgi as predisposing to pernicious attacks. In the present state of our knowledge it is impossible to define the relation of this function to perniciousness.

A consideration of the relative frequency with which the several factors are concerned in the pathogenesis of the various forms of pernicious malaria will necessarily be brief. In the comatose variety any of the four chief agents may take part; idiosyncrasy and external influences may unite with any of the other factors; an extraordinary number of parasites in the general circulation, without accumulations in the brain, is productive of coma probably because of the toxin. 


\section{PATHOLOGIC ANATOMY OF MALARIA}

\section{ACUTE MALARIA}

Intravascular melanin, which is a product of hemoglobin converted through the biologic agency of the malarial parasites, is the pathognomonic anatomic feature of malaria. Melanin occurs in the tissues also, but here there is some doubt as to its origin. It is brownish black in color, occurs in fine grains, coarse particles, or in lumps; does not yield the reaction for iron, and is insoluble in acids, but is readily dissolved by ammonium sulphide. This should not be confused with hemosiderin, which is a chemic derivation of the hemoglobin of broken-down red blood-cells; is yellowish in color; responds to the reaction for iron; is insoluble in acids, alkalies, alcohol and water; and exists especially extravascularly. It is regarded as a result of prolonged hemoglobinemia following severe or chronic infections.

The general plan of distribution of melanin may be thus stated: In the blood current it may exist free or, more commonly, is contained within the phagocytes and the red cells infected with pigmented parasites, and is more abundant in the capillaries than in the larger vessels. In the viscera it is oftenest seen in the spleen, bone-marrow, brain, and liver, especially in the endothelial cells, but in the spleen and bone-marrow it exists also outside the vessels and either between or within the cells proper to these tissues.

The distribution of the parasites varies according to the type of the attack; it has been shown that the latter depends largely upon the localizations of the parasites. They are usually abundant in the splenic blood irrespective of the form assumed by the attack. It occasionally happens that death supervenes, notwithstanding a progressive diminution of the parasites, so that the latter may be scanty or even absent. In the spleen 
are found not only schizonts, but also numerous sexual forms, which are likewise usually found in the bone-marrow and even in the liver, but in the brain gametes are conspicuously few. Parasitic development is checked almost immediately upon the death of the host.

The spleen is always more or less enlarged, though perhaps slightly so in acute cases following recent infection. The edges are often rounded, the organ tending to lose its characteristic contour and to assume a spheric shape. The color varies from reddish brown to almost black, being darker in old malarials. In consistence it is usually softer than normal, often semifluid, sometimes resembling a bag of pulp. The capsule is thinned, occasionally adherent to the adjacent organs, and is very liable to rupture. The cut surface is dark in proportion to the age of the infection. The pigmentation is occasionally uniform and the tissue hardly distinguishable, though, as a rule, the Malpighian bodies stand out distinctly. The venous sinuses are often dilated. Microscopically there is enormous cellular hyperplasia with distention of Mall's pulp cords. The spleen cells are everywhere intercalated with red blood corpuscles, a large per cent. of which are infected. The parasites may be in the same or in different stages of development. The pigment is contained in the large mononucleated leucocytes, endothelial cells, and giant cells. The latter contain also red cells, parasites, and even small phagocytes, and are most abundant in the splenic vein. They sometimes show evidences of necrosis. The Malpighian bodies and the fibrous trabeculæ are usually unpigmented. Mitotic cells may be found in the pulp and in the Malpighian bodies. The circulation may be so obstructed that edema, interstitial hemorrhage, and cellular necrosis may occur.

The liver is generally enlarged, but in a less proportion as to frequéncy and size than the spleen. The color is usually a dirty brown, the surface is sleek, and the form is preserved. The consistence may be normal or somewhat diminished. The parenchyma presents a reddish-brown color after recent infection and the cut surface drips blood. The gall-bladder is often distended with a quantity of dark, inspissated bile. 
Microscopically parasites are not so abundant as in the spleen. Pigment is found in the vessels, especially in the blood capillaries. Here are found also altered parasites, melaniferous leucocytes, and large endothelial cells containing coarse grains of pigment. The macrophages are sometimes of an enormous size. The pigmented endothelial cells are swollen and the capillaries are not infrequently entirely obstructed with pigmented cellular elements. The hepatic cells do not contain melanin, but are frequently charged with hemosiderin, and may show evidences of cloudy swelling, atrophy, or necrosis. Karyokinesis is occasionally noted. Areas of focal necrosis have been described.

The kidneys on gross inspection show few changes; they may be slightly enlarged and hyperemic. Microscopic examination shows a marked pigmentation of the Malpighian corpuscles, together with degenerated tubular epithelium. While the epithelium of the tubules may be healthy, it often shows cloudy swelling and necrosis. In the straight tubules there may be casts of various sorts. Melanin is found in the glomeruli, less often in the tubules. The cells may contain hemosiderin granules. Parasites are rare in the glomerular vessels, but may be found in the intertubular capillaries. Ewing's ${ }^{501}$ case with massing of the parasites in the renal capillaries has been mentioned. A true glomerulitis has been found in cases of the algid type.

In cerebral cases the only variation from the normal condition of the stomach and bowels may be a slight pigmentation. In fatal cases of the algid and choleraic forms the gastrointestinal tract may contain a bloody fluid and the mucous membrane may be swollen, hyperemic, pigmented, necrotic or ulcerated. The follicles and Peyer's patches may be hypertrophied and prominent. Microscopically there is vivid injection, parasitic and pigmentary thrombosis of the capillaries, hemorrhagic points, and necrosis. The peritoneum is usually normal.

Macroscopically the lungs may show nothing abnormal save, probably, slight results of hypostasis, which in some cases may be cadaveric lesions. Occasionally there are hemorrhagic areas. Microscopically neither pigment nor parasites are so 
evident as in certain of the other organs. The capillaries are congested, sometimes thrombosed, and contain infected erythrocytes, phagocytes, which often show signs of degeneration, and macrophages. The capillary epithelium may be swollen, but is only occasionally pigmented. The pleuræ show nothing abnormal.

The heart muscle is ordinarily pale and flabby, but the muscular fibers do not usually afford degenerative signs. The capillaries may contain parasites in greater or less number, and the endothelium may be swollen. Cases in which the parasites are very numerous in the cardiac capillaries, such as that of Ewing, ${ }^{124}$ are very rare.

In cerebral cases the meninges of the brain are deeply hyperemic, and excess of serum is found in the meshes of the pia, in the ventricles, and at the base of the brain. The cerebral substance is commonly darkly pigmented and congested, and may show hemorrhages, usually punctiform, occasionally larger. The hemorrhages occur oftener in the cerebrum, but may be present in the cerebellum. In the abdominal form the brain may show but few pathologic changes. Microscopically in the cerebral cases the capillaries are seen to be filled, even to occlusion, with pigment, parasites, and phagocytes, the later in the same or in different stages of schizogony; gametes are seldom found. In some instances nearly every red cell contains one or more parasites. Localization of parasites are found not only in the cerebrum, but also in the cerebellum and medulla. The capillary endothelium may be swollen, pigmented, and undergoing fatty degeneration. Secondary changes, such as perivascular exudation, hemorrhages, and necrosis, are not uncommon results of thrombosis. Degenerative changes in the ganglion cells have been detected.

The bone-marrow is of a dark color approaching that of the spleen, and sometimes diffluent. Microscopic examination reveals hyperemia, the capillaries being engorged with pigmented parasites and giant cells clinging to the vessel walls. The parasites exist as free spores, schizonts, which are frequently sporulating, and gametes in large numbers. Extravascular parasites and free pigment are also found. 


\section{CHRONIC MALARIA}

The spleen is always enlarged. The form is usually preserved. Its average weight is from 700 to 800 grams, though it may attain four or five times this weight. In consistence it is usually firmer than normal. The capsule is thickened, especially at the convexity. Upon the surface are scattered indurated whitish plaques of fibrous, occasionally of calcareous consistence, evidences of perisplenitis. Adhesions to the diaphragm or other parts are not infrequent. Subcapsular infarcts are occasionally encountered. In section the parenchyma is usually found firm; only rarely is it of diminished consistence. The color varies from that of muscular tissue to slate color. The thickened trabeculæ, like white bands, are very evident. The Malpighian follicles are sometimes conspicuous, sometimes indistinct. In old cases there is an overgrowth of connective tissue, particularly near the capsule. Histologically the chief changes found are trabecular hyperplasia and venous dilatation. The process sometimes resembles a hypertrophic cirrhosis. The fibrous trabeculx are hypertrophied and there is formation of new connective tissue. The venules are notably dilated, the walls thickened, and the blood rich in pigmented leucocytes and macrophages. The deposition of pigment is in general similar to that in acute malaria. There is at times little change in the lymphoid tissue forming the arterial sheaths and Malpighian bodies, but this may be hyperplastic. Necrosis of the spleen pulp is observed, surrounded by evidences of regeneration. These regenerative processes consist chiefly of increased vascularization, formation of connective-tissue network enclosing giant cells, and hyperplasia of lymphoid tissue beginning in the Malpighian bodies.

The liver is not so constantly enlarged as is the spleen and never attains so excessive a degree of hypertrophy. It may weigh from 2 to $4 \mathrm{~kg}$. In rare instances it is atrophic. The consistence is firm, occasionally somewhat doughy. The capsule is tense and may be thickened. There may be present whitish bands or patches, the results of perihepatitis. . The color varies from reddish to almost black. The cut surface 
is usually found to be congested and may drip with blood. The color is more or less dark red. There may sometimes be detected on gross inspection an increase of connective tissue. Microscopically the hepatic cells are seen to be hypertrophied and hyperplastic, showing evidences of cloudy swelling and necrosis, or atrophied as a consequence of vascular dilatation. In certain areas there may be a complete disappearance of hepatic cells which are replaced by connective tissue, Kupffer's cells, or beginning formation of new hepatic cells. The nuclei are frequently multiple, and when single may be much larger than normal and contain one to two nucleoli. The hepatic cells may be charged with hemosiderin. Pigment is contained in the endothelial and Kupffer's cells, especially in congested areas and in the periphery of the lobule. There is sometimes diffuse overgrowth of connective tissue. The blood capillaries are usually dilated and congested with blood rich in pigmented leucocytes; the circulation is commonly sluggish. The bile capillaries are ordinarily unaltered. The perivascular lymph channels may be dilated. Amyloid degeneration, beginning apparently at the periphery of the lobules, is not rare.

The kidneys are usually increased in volume and in weight. The contracted kidney has been described in connection with malaria, but there is some doubt as to the etiologic relationship. The surface of the kidney is smooth, the color is dark red, and the consistence is slightly increased. Upon section the cortical substance is reddish gray. The pyramids are markedly hyperemic, the red tint being most decided at the border of the pyramidal substance. Upon microscopic examination the convoluted tubules and ascending limb of Henle's loop are found dilated. The epithelium is swollen, charged with hemosiderin, and may be undergoing degeneration. In the collecting tubules the epithelium is, as a rule, only slightly altered. These tubules rarely contain granular or hyaline casts or desquamated epithelium. Bowman's capsule presents changes similar to those of the convoluted tubules. The renal arterioles are congested and the capillaries are dilated and gorged with blood rich in leucocytes, more marked in the pyramidal than in the cortical substance. Malanemia is not 
so decided in the kidney even when profuse in the spleen and liver. There is generally little change in the connective tissue. Here and there is a slight thickening of the intertubular connective tissue. The blood-vessels, the glomeruli, and the walls of the renal tubules may undergo amyloid degeneration. This is more diffuse in the kidneys in chronic malaria than in the other organs.

The alimentary tract may show evidence of amyloid degeneration in the stomach or bowel and dysenteric lesions in the colon.

In the lungs may be pigmentation and anemia, and in the pleural cavity an effusion.

The heart is relaxed and often dilated and sometimes shows evidence of degeneration of the musculature.

The bone-marrow is of firmer consistence and more deeply colored than normal, especially toward the ends of the long bones. There is usually a decrease of fat and a proliferation of marrow cells, together with large cells, some undergoing karyokinesis, lymphoid cells, and nucleated red cells. The vessel walls are thickened. In some instances there is atrophy of the bone-marrow.

The elimination of the pigment probably consumes three or four months after the cessation of infection, though this varies with the activity of the eliminative process. 


\section{CHAPTER IV}

\section{CLINICAL HISTORY OF MALARIA}

\section{ACUTE MALARIA}

Incubation.-The period of incubation varies within very wide limits. It may be stated as a general proposition that the incubation period is longest in quartan infections and shortest in the estivo-autumnal. The average period is, for quartan, - twelve to eighteen days; tertian, six to fourteen days, and estivoautumnal, two to ten days. Much longer periods running into several months, have been reliably recorded. These must be regarded as cases of chronic malaria where the latent stage precedes the active, and are analogous to those cases of syphilis in which the secondary manifestations occur without recognized primary lesion, and are to be explained satisfactorily only by parthenogenesis.

General Description of a Malarial Paroxysm.-The forms of acute malaria have so many points in common that it is convenient to describe first the typic malarial paroxysm.

Prodromata may be perceived by the patient. They may correspond to the last few parasitic sporulations preceding that which causes the paroxysms, or may occur only a few hours before the access. They are ill-defined, but usually consist of languor, anorexia, headache, aching of the loins and hips, thirst, epigastric distress, a disposition to stretch and yawn, and chilliness along the course of the spine. These symptoms may be so slight as to escape attention. The typic malarial paroxysm comprises three well-marked stages: the cold stage, the hot stage, and the sweating stage.

The cold stage presents itself with the rapid intensification of the prodromata described. The sensation of coldness spreads to every part of the body. The skin becomes pale, especially the lips and the ears, as well as the nails, and the papillæ of the skin stand out, forming the so-called "goose-skin." The patient shivers, sometimes so violently that he shakes the bed; 
he covers up, his teeth chatter, and he looks and feels cold. The slightest motion of the body or of the bedclothing increases the vehemence of these phenomena. Notwithstanding these evidences of coldness, the thermometer shows an elevation of internal temperature. The fever may even precede the cold stage. The patient complains of a tight headache, a backache, precordial oppression, and dyspnea. He often complains of general soreness, as severe as if having been beaten. He may suffer with nausea and vomiting of bile. There is apt to be frequent micturition of small quantities of limpid urine. The respiration is rapid and tremulous. The pulse is accelerated, diminished in volume, and increased in tension. The cold stage may last from a few minutes to two or three hours.

With the onset of the hot stage hot flashes alternate with cold until the sense of heat becoming general, the patient presents a very different picture from that of the first stage. He begins to uncover, the skin is flushed and hot, the pulse full and bounding, the respiration deeper, and the urine is scanty and high colored. There may be constipation or diarrhea. The tongue is coated, bulky, and usually shows indentations produced by the teeth. Herpes appears upon the lips or nose. The spleen is enlarged and the upper half of the abdomen is tender on pressure. The headache, soreness, nausea, and vomiting continue, there is often great thirst and epigastric pain, and the temperature continues to rise.

When the temperature is at its height the sweating stage is ushered in by crisis. Beads of perspiration begin to appear upon the face, then a universal sweat breaks out, and the skin, which was first cold and rough, then hot and dry, now becomes moist and natural. The temperature falls to normal, often a little below; the pulse and respiration resume their normal features. The soreness disappears, the thirst ceases, and the patient often feels so comfortable that he takes a short nap.

Such is the typic procession of one of the most remarkable events in the category of disease. The conspicuous changes, the swift succession of stages, and the punctual periodicity of paroxysms are unparalleled in pathology. 
In some paroxysms, however, one or two stages may be missing. The temperature may rise unaccompanied by a cold stage or may fall to normal unattended by sweats. This constitutes the so-called dumb chill. The cold stage is the least constant, the hot stage the most so. The cold stage is most constant in quartan fever, least so in estivo-autumnal infections.

Simple Tertian Infection.-Infection with a single brood of simple tertian parasites causes a paroxysm every other day.

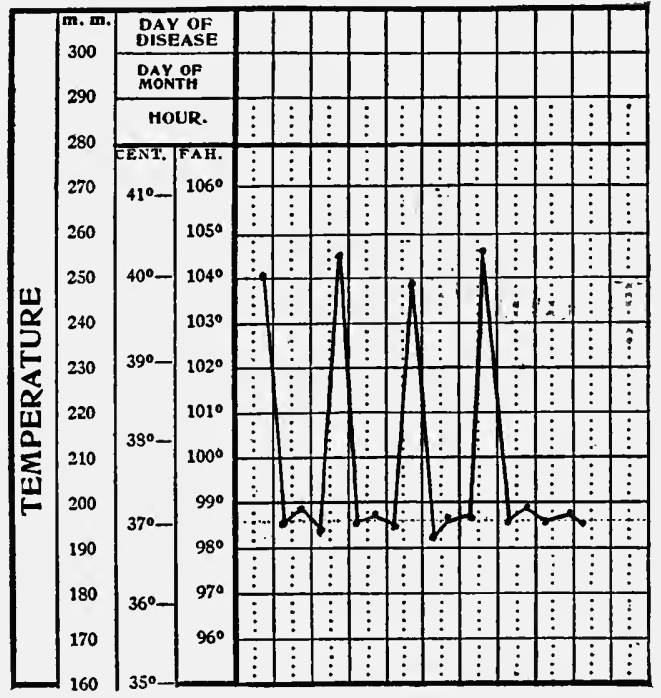

Fig. 25.-Single tertian infection.

That the parasites are in the same stage of development, causes great regularity in the course. From the beginning of one paroxysm to the beginning of another is almost precisely fortyeight hours. When the interval is not quite so long, as sometimes happens, the paroxysms are said to anticipate; when longer, as is more rarely the case, they are said to postpone or to retard. Postponing is usually regarded as evidence of abating activity.

In more than half the simple tertian cases the infection is double; that is, there are two distinct generations of parasites. These generally mature on alternate days, two paroxysms on 
one day with an intervening day of apyrexia being extremely rare. The paroxysms may occur at the same time every day and be similar in every respect. Usually, however, there is a perceptible difference between the paroxysms of successive days, a difference consisting of time of onset, severity, and relative length of the stages of the paroxysms. It very rarely happens that the paroxysms are so lengthened, and one so anticipates that its onset occurs during the latter stage of the preceding paroxysm. They are styled subintrant attacks.

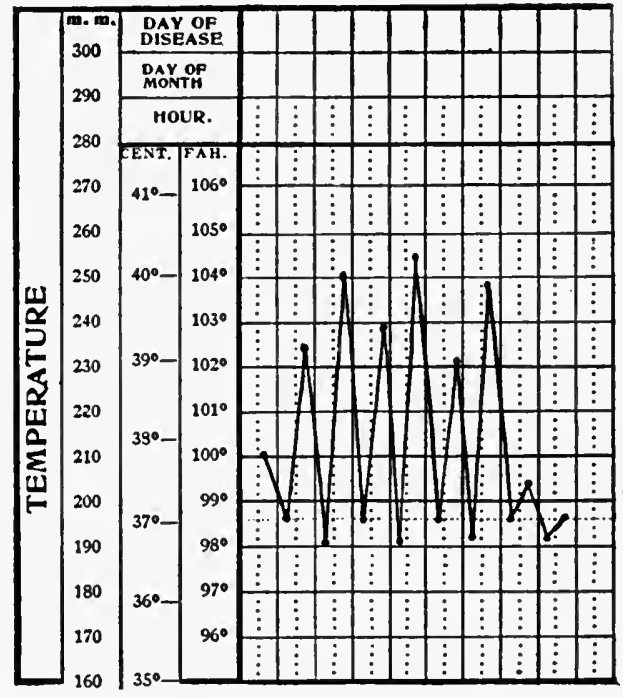

Fig. 26.-Double tertian infection.

A change of type from quotidian to tertian paroxysms, or vice versa, is commonly observed. The change from quotidian to tertian may be spontaneous or the result of incomplete medication or improvement in hygienic conditions, one group of parasites perishing. A change from tertian to quotidian may occur without apparent cause or following indiscretions of various sorts.

The onset is probably more common during the morning, but this is neither constant nor of diagnostic dignity. The invasion is almost always with a chill. The temperature rises suddenly and falls likewise. Commonly there are no grave 
symptoms, but a mild delirium is not rare. The temperature usually goes as high as $103^{\circ}$ to $105^{\circ} \mathrm{F}$. The average duration of the paroxysm is from eight to twelve hours.

During apyrexia the patient may feel perfectly well except slight weakness, headache or vertigo. $\mathrm{He}$ is usually able to attend to his duties. The tendency to spontaneous cure is greater than in either of the other forms of malaria, the attack not infrequently subsiding after a number of paroxysms, without any medication or with only a purgative.

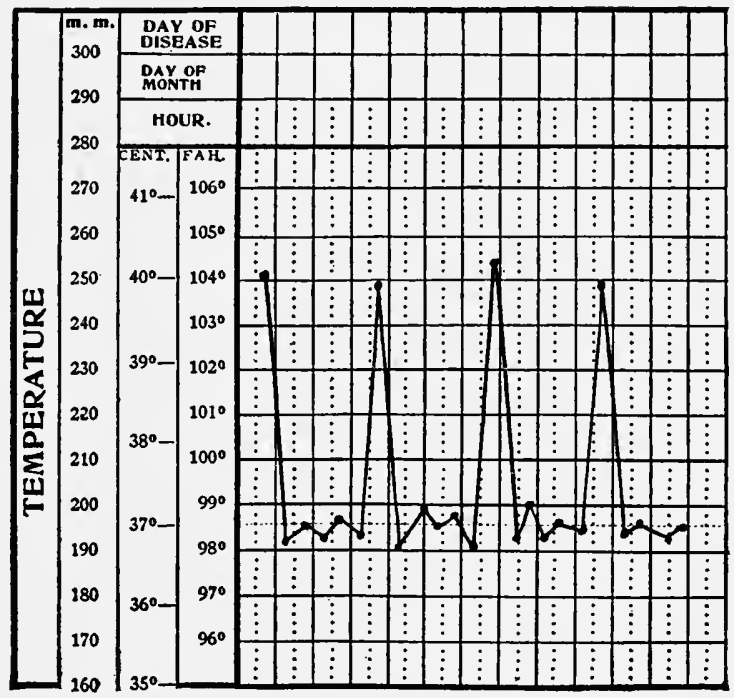

Fig. 27.-Single quartan infection.

Quartan Infection.-The quartan parasite accomplishes its endogenous cycle in seventy-two hours. Hence infection with a single generation of quartan parasites produces a paroxysm followed by two days of apyrexia and a second paroxysm on the fourth day. Such attacks are popularly known in the South as "third-day chills." A double quartan infection reverses the course, causing two paroxysms on successive days, followed by a day of apyrexia. Triple quartan infections, the parasites maturing on successive days, give rise to quotidian fever. As in simple tertian intermittents, quartan accesses sometimes anticipate or retard. Subintrance in triple infections is rarely 
scen, due probably to the shorter duration of the paroxysms. Changes of type between single, double, and triple quartan are sometimes observed.

The onset, especially in single infections, occurs probably more often during the afternoon hours. The symptoms are those of the typic paroxysm and are well marked. The cold stage is not only more constant than in the other forms, but is more intense and usually of longer duration. The three stages are sharply contrasted. Pernicious symptoms are very rarely

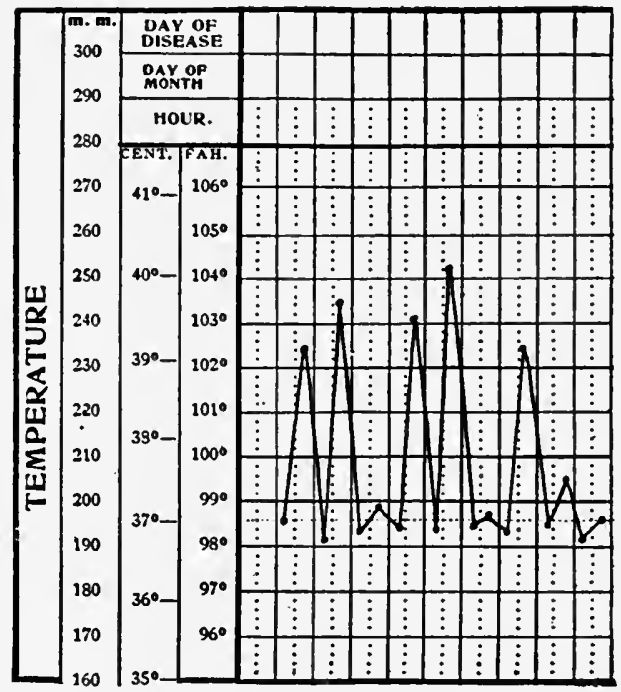

Fig. 28.-Double quartan infection.

observed in connection with quartan infections. This maybe accounted for by the more equal distribution through the circulation of the parasites which show no tendency to congregate, and by the longer apyrexial periods between the paroxysms. The average duration of the paroxysm is eight or ten hours.

Estivo-autumnal Infection.- The chief feature of infection with estivo-autumnal parasites is the irregularity of the course as contrasted with that of tertian and quartan infections. A classification is difficult and, while that into estivo-autumnal or malignant tertian and quotidian is perhaps best, these may be clinically indistinguishable. 
Malignant Tertian.-This form of infection is due to parasites which tend to mature in forty-eight hours. It is characterized

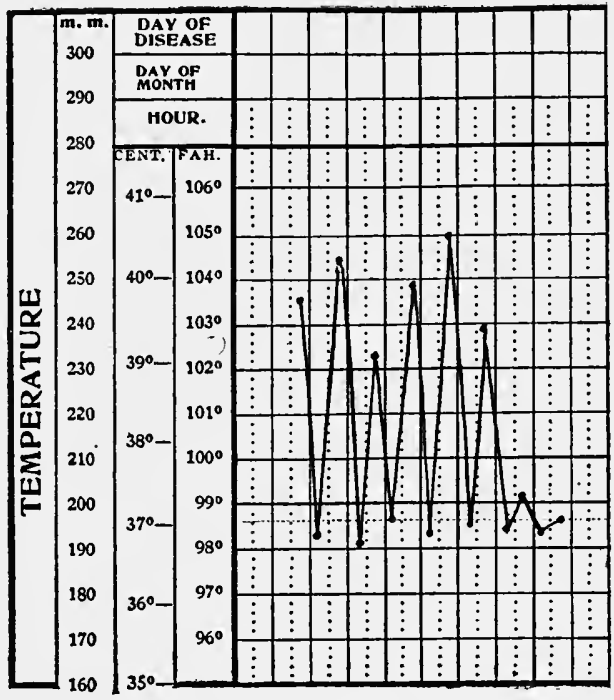

Fig. z9.-Triple quartan infection.

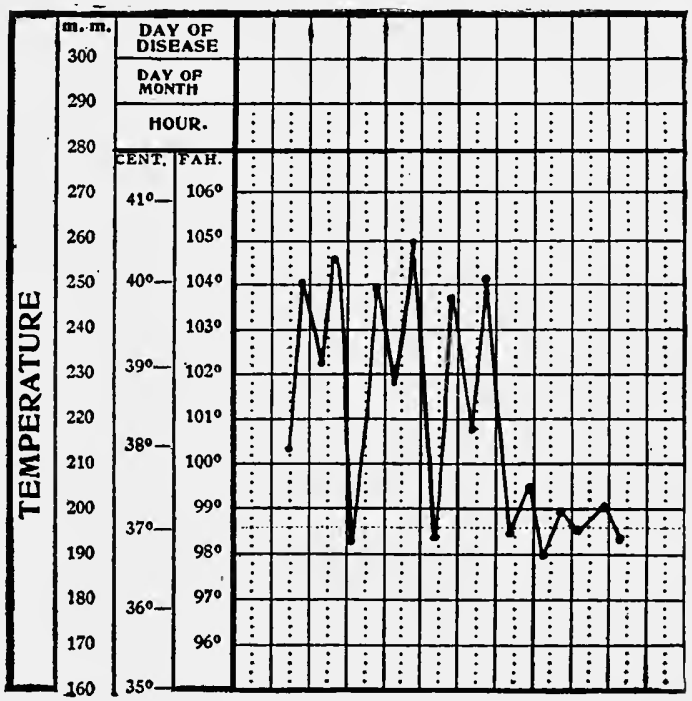

Fig. 30.-Tertian estivo-autumnal malaria.

by a long paroxysm and a short apyrexia. The duration of 
the access is from twenty-four to forty-eight hours or more. Anticipation and subintrance are oftener observed here than in any other form of malaria.

Prodromal symptoms are usually pronounced. The cold stage is often not manifest; the sweating stage is less commonly missing. The typic temperature is characteristic. It rises abruptly, often as high as $104^{\circ} \mathrm{F}$. On reaching its height it remits with slight oscillations for a few hours. It then makes a marked remission and again rises suddenly, usually higher than

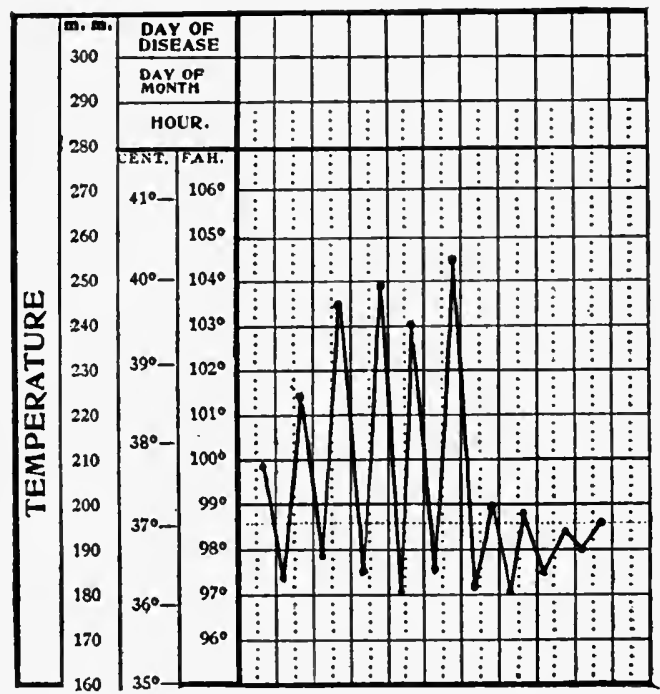

Frg. 3r.-Quotidian estivo-autumnal malaria.

before. The final fall is by crisis. It is customary, following Marchiafava and Bignami, to divide this course into five stages: (I) the rise of invasion; (2) the fastigium; (3) the pseudocrisis; (4) the precritical rise; $(5)$ the crisis. This curve is simulated only by simple tertian fever with subintrant attacks, which is uncommon. Unfortunately this typic sequence is far from constant; the modifications are very numerous and are too irregular to analyze.

The symptoms of the hot stage are more pronounced than in the infections previously described. The headache and backache are worse, the general depression is more profound, stupor 
and delirium may appear and pernicious symptoms may arise. During the short interval the patient does not regain his ease as in the simple intermittent fevers, but the aching and prostration continue, and he may be unaware that the fever has left.

Quotidian.-The quotidian estivo-autumnal fever is more regular in its course than the tertian, especially at first, though there is nothing characteristic in the temperature curve, which may closely resemble a double tertian or a triple quartan. Later it is apt to lose some of its regularity by anticipation or by lengthening of the paroxysms, whose average duration is from six to ten hours. The chill is rather more constant than in the tertian; otherwise the symptoms are identical, the patient not regaining strength from one paroxysm to another. In the interval the temperature is prone to sink, even as low as to $95^{\circ} \mathrm{F}$.

Mixed Infections.-Infections with two or more species of the malaria parasite are known as mixed or combined infections. The most frequent combination is of simple tertian and estivoautumnal. As a rule, one parasitic form predominates and produces its usual picture; this may, however, be considerably modified by the other group of parasites. The most frequent modification in the temperature chart is a tendency to continuity. The paroxysms are not usually as typic as in simple infections and are not so regular in their occurrence.

Analysis of Symptoms.-Temperature.-The main characteristics of malarial temperature have been given when treating of the several forms. It remains only to consider a few general traits.

The feature of the temperature in uncomplicated and untreated malaria is periodicity. The temperature is, in a great majority of instances, intermittent.

In tertian and quartan infections the temperature usually rises rapidly after the onset, reaching the acme during the second stage of the paroxysm and declining during the third to normal or a little below normal. This is also the usual course in quotidian estivo-autumnal, the temperature chart showing symmetric ascent and descent, producing an arrow-head appearance. In this infection the temperature descends rather 
lower during the fever-free interval than in the others. The typic run of tertian estivo-autumnal has been given as follows: (I) the stage of initial ascent; (2) the fastigium, during which the temperature may show fluctuations of a half degree to a degree; (3) the pseudocrisis; (4) the precritical or final ascent; and $(5)$ the crisis.

Continued temperature in malaria is not as common as usually regarded; remittent fever was formally thought to be the rule in the summer-autumn malarial fevers. The causes of this error are three: first, in tertian estivo-autumnal infections the apyretic interval is short; second, this interval often occurs during the night or early morning hours; thirdly, the patient, guided by the discomfort which continues during the interval, usually denies that he has been free from fever. Nevertheless, a continued temperature is occasionally noted in malaria, especially in tertian estivo-autumnal infections.

The duration of untreated acute malaria is too indefinite to permit of exact statements. While simple tertian may terminate after a few paroxysms, an estivo-autumnal fever may continue three or four weeks if it does not in the meantime become pernicious.

A post-malarial secondary fever, or spodogenous fever, is occasionally observed after the infection, particularly estivoautumnal, has lasted for some time. It persists for days or weeks uninfluenced by quinine. The blood examination is negative for parasites.

Circulatory System.-The Blood.-The volume of the blood as a whole is somewhat diminished. The specific gravity is only slightly lowered, and usually only nearly compensated by the excretion of fluids. The density, at first lowered, approaches normal as the infection persists. The experiments that have been performed with reference to the tonicity of the blood in malaria have uniformly shown that this is increased.

The tertian parasite, more abundant in the deep circulation, may be observed in the peripheral circulation throughout the course of the asexual cycle, excepting the sporulating forms, which are only exceptionally seen. The gametes are not infrequently detected. 
The quartan parasite is most evenly distributed, being about equally common in the visceral and superficial blood. Furthermore, all stages of the asexual development, including the sporulating forms, may be followed in blood obtained from the peripheral circulation. Quartan gametes are rarely seen.

The estivo-autumnal parasites are seen only in the earliest phases. In some localities the gametes are very commonly observed after the infection has persisted a week or more; in others, even where severe infections of long standing are encountered, they are more rarely noted.

Pigment is most frequently contained within the large mononuclear, less often the polymorphonuclear, leucocytes, but may exist free in the blood current. It is of a dark reddish-brown or black color, and occurs as granules, rodlets, or irregular clumps.

One of the best-known facts in the study of malaria is the rapid and widespread destruction of the red blood-cells. A certain number of erythrocytes perish with each parasitic sporulation like soldiers after a volley from the enemy. It is not uncommon for a fourth to a half million red cells per cubic millimeter to be destroyed during each of the first two or three paroxysms, and this may progress until the count is considerably less than one million per cubic millimeter. The anemia is commonly in proportion to the severity and duration of the attack. Restitution of the red cells is more rapid and certain with tertian and quartan than with estivo-autumnal infections. Race, age, and constitution are also factors in the rapidity of reconstruction.

The cells containing the simple tertian parasites are swollen and somewhat decolorized. Those containing the quartan parasites are shrunken and somewhat darker in color. The cells harboring estivo-autumnal organisms have the appearance of old gold or of brass, and become somewhat smaller. A curious appearance of some infected cells is what has been termed stippling. This may be seen in both simple tertian and estivo-autumnal infections, but presents features more or less characteristic in each. In simple tertian the dots are fine and abundant. In estivo-autumnal they are coarse, irregular, 
may be cleft-like, and few in number-from two to six. The fine stippling of tertian infection is also known as "Schüffner's dots." Stippling is brought out by staining.

Changes occur also in non-infected cells. The commonest of these are, in my experience, in the order named: the occurrence of macrocytes and microcytes, polychromatophiles, and poikilocytes. Nucleated reds are occasionally observed. Basophile granulation is sometimes noted.

Retraction of hemoglobin and vacuolization are common findings in malarial blood.

The hemoglobin generally falls decidedly. Its curve is apt to run parallel with and a little below that of the red cells, and is slower in returning to normal. The hemoglobin content is no guide as to the severity of the disease.

The leucocytes are, in benign malaria, usually slightly diminished. A leucocytosis is found only in pernicious malaria or in association with complications. The differential formula is the most noteworthy feature. Its peculiarity is the large mononuclear increase. Eosinophilia in my experience denotes complications, ordinarily intestinal helminthiasis.

The leucocytes occasionally undergo degenerative changes, among which are fatty degeneration and vacuolization of the protoplasm and fragmentation and chromatolysis of the nucleus.

The blood platelets are somewhat increased in malaria, especially during the interval following a severe attack.

The Wassermann reaction is occasionally positive in malaria. Of $\mathrm{r}, 957$ cases of diseases other than syphilis examined by Craig and Nichols, ${ }^{302}$ four of the positive cases were in patients with malaria. In forty-six cases of malaria, Boehm ${ }^{503}$ found the Wassermann positive in sixteen cases or 34.8 per cent.

Sutherland and Mitra ${ }^{432}$ examined the blood of fifty patients in whom parasites were present and nine gave positive Wassermanns; at least three were probably syphilitic.

At the height of the fever the pulse may reach I 30 or more. During the interval it usually becomes almost quite normal in tertian and quartan infections. In estivo-autumnal fever it depends upon the severity of the attack and the resistance of 
the patient. An anemic murmur may be heard over the heart. A sense of precordial oppression or acute pain are common complaints.

Respiratory Organs.-Respiration is usually accelerated in proportion to the temperature. Cough is a frequent symptom. In children a frequently repeated superficial hacking cough is often an indication of nausea. Bronchial catarrh is not infrequently observed, accompanied by sibilant râles on auscultation. Epistaxis may occur and is occasionally alarmingly profuse.

Gastro-intestinal Organs.-While the paroxysm is on, the appetite is usually completely lost. In tertian and quartan malaria this may be regained during the interval, but in estivoautumnal anorexia generally persists throughout apyrexia. The patient ordinarily complains of a bitter taste in the mouth and fulness, discomfort or pain in the epigastric region. The tongue is large, flabby, thickly coated, usually anemic, and showing the prints of the teeth along the edges. Nausea is nearly a constant symptom, and retching and vomiting are distressing. The vomitus consists of matters ingested, bile or slimy mucus. The bowels are constipated, regular, or loose, in the order of frequency named; choleraic or dysenteric discharges occasionally appear. More or less enlargement of the spleen is a usual occurrence, together with pain and tenderness in the left hypochondrium. In primary acute infections the enlargement may not be prominent; in later infections the spleen is often palpable beyond the costal margin. The spleen is rarely much enlarged in the negro. Enlargement of the liver is much less contant and less marked than splenic hypertrophy. There usually exists tenderness in the epigastric and right hypochondriac regions.

Genito-urinary Organs.--Urine.-As a general rule, the urine emitted during a cold stage is pale in color and that of the stage of fever highly colored, but individual circumstances may produce numerous exceptions to this rule. In certain cases of estivo-autumnal fever the urine may be very highly colored and contain a heavy deposit. In these cases the urine contains biliary coloring matters and an excess of urobilin. The diazo 
reaction sometimes obtains. The indican is frequently increased. In tertian and quartan cases the quantity of the urine is somewhat augmented, in estivo-autumnal diminished. The reaction is acid, varying directly with the concentration of the urine. The specific gravity does not always bear a definite relation to the amount of the urine, as might be inferred, but may be relatively high when the urine is abundant. or low with scanty urine.

The output of urea is increased. The increase begins several hours before the attack, attains its maximum toward the end of the cold stage, declining to or below normal at the end of the paroxysm.

The uric acid content of the urine is only slightly, if at all, modified. The amount of chlorides runs parallel with the quantity of urine. The phosphates are eliminated in quantities less than normal during the fever, and in greater quantities during apyrexia. The entire twenty-four-hour urine commonly shows an increase. The variations in the excretion of the sulphates are similar to those in regard to urea.

The elimination of the sodium and potassium bases is very inconstant, both as to quantity and as to the stage of the disease during which elimination takes place. Malarial urine contains an excess of iron, especially after the paroxysm. It is dependent upon and proportionate to the destruction of erythrocytes.

The occurrence of albumin in the urine is relatively infrequent in the mild attacks of simple intermittent which terminates after one or two paroxysms. In severe estivo-autumnal infections, however, it is exceedingly frequent. Its frequency varies not only with the type and severity of the attack, but also with locality and other circumstances. The following reports are tabulated to show the extent of these differences, the denominator indicating the number of cases of malaria, the numerator the number in which albumin was found:

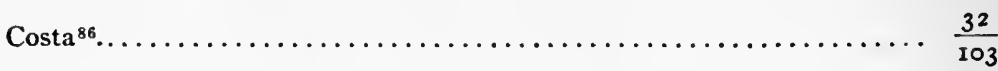

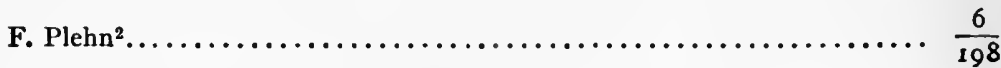




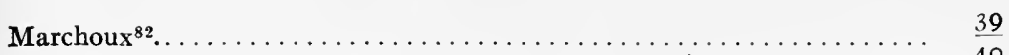

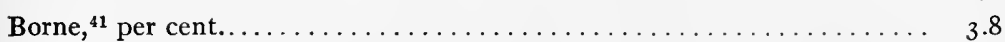

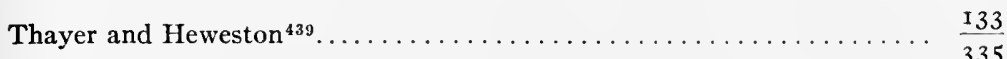

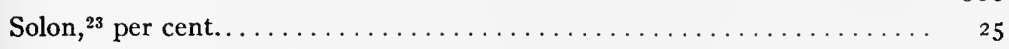

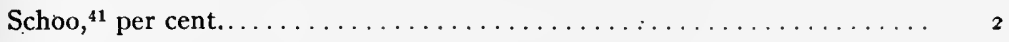

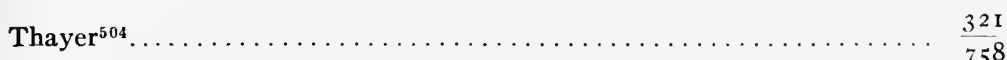

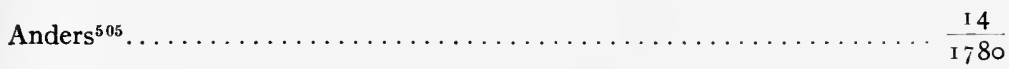

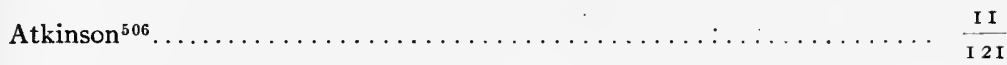

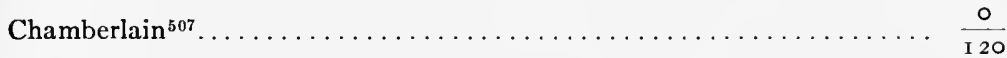

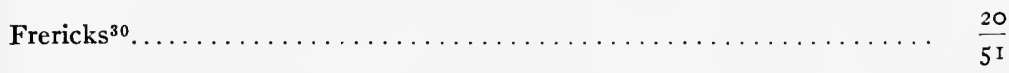

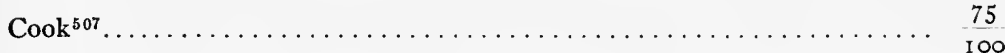

An increased toxicity of the urine has been found in a large per cent. of cases of malaria, greatest during apyrexia and usually intensifying with each successive paroxysm.

Nervous System.-Headache is one of the most invariable symptoms of malaria. Backache and somatic soreness are severe. Sometimes hyperesthesia is seen. Vertigo is the rule, especially when the patient is upright. Neuralgia, facial or intercostal, is a not infrequent symptom. Stupor and delirium are present in grave cases, particularly in children.

Skin.-During the first stage of the paroxysm the skin is blanched and cold; during the second stage hot, dry, and perhaps turgid; during the third bathed with sweat, becoming natural toward apyrexia. Icterus is not a pronounced symptom in acute cases except in certain pernicious forms. With the possible exception of pneumonia, herpes is seen more frequently in malaria than in any other disease. Its commonest sites are the lips and nose, but it may appear elsewhere. It is not nearly so common in the negro as in the white. Urticaria and erythema are sometimes observed.

\section{PERNICIOUS MALARIA}

Pernicious malaria is that form of malaria, extremely acute, which, independently of complications, endangers life in a few 
hours or a few days. This gravity may be due to the intensification of ordinary malarial symptoms or to the advent of unusual ones. It should be clearly understood that pernicious fever is not a pathologic entity, but is a form of malaria from the simple modes of which it sometimes differs only in degree. Its pathogenesis is intimately associated with the life history of the malaria parasite, much more so than is hemoglobinuric fever. Intermediate forms may be encountered which may be difficult to place, as cases with slight somnolence, abundant sweats, or cold surface.

More than thirty so-called varieties of pernicious malaria have been described. A partial list of these includes the apoplectic, ataxic, comatose, sudoral or diaphoretic, delirious, eclamptic or convulsive, tetanic, typhoid, amaurotic, aphasic, ardent, exanthematous, hemiplegic, hydrophobic, neuralgic, cerebromeningeal, cardialgic, dyspneic or asthmatic, pneumonic, pleuritic, syncopal, hemoptoic, algid, choleraic, dysenteric, gastric or gastralgic, hemorrhagic, bilious or hepatic, lymphatic, rheumatic and nephritic forms.

Any classification is not absolutely essential, and all are more or less arbitrary. Nevertheless, for convenience, all forms of true pernicious malaria may be easily and logically arranged into (I) cerebrospinal, (2) thoracic, and (3) abdominal forms.

Cerebrospinal Forms.-The representative type of cerebrospinal pernicious malaria is the comatose variety, which is, as well, the most frequent of all varieties.

Comatose malaria may make its appearance as the first manifestation of malaria or, more commonly, after the lapse of one or more paroxysms, typic or irregular. Violent headache, stupid countenance, and somnolence interrupted by frequent sighing, with a mild grade of mental aberration and defective articulation and vision, are not uncommon prodromata. These may, however, be so slight as to escape notice. The onset of cerebral symptoms may be with violent abruptness (the apoplectic form of some writers) or, as is most common, begin within a few hours after the commencement of the paroxysm with somnclence, which gradually deepens into stupor and coma. It has occasionally happened that malarial coma has 
come on during natural sleep, the condition of the patient being discovered by accident. Convulsions may precede the coma, especially in children, or there may be extreme restlessness, gritting the teeth, and jactitation. The cerebral symptoms may vary from the marked drowsiness to profoundest coma. The eyes may be closed or open (coma-vigil). The pupils are usually equal and dilated or contracted, but may be unequal and may or may not react to light. Strabismus is an occasional symptom. The face is congested in individuals recently attacked or pallid in older sufferers. The skin is at first hot and dry, perhaps slightly jaundiced; later it may be bathed with sweat. Petechiæ are occasionally seen. Trismus may be present, but the extremities are usually completely relaxed, though sensation and motion are often not entirely abolished, as sometimes evidenced by resistance to hypodermic medication. Cases manifesting muscular rigidity and tonic contractures have been reported. Hyperesthesia and muscular tremors are not infrequently present. The reflexes may be increased or diminished.

There may be twitching of the muscles of the face, usually confined to one side. Loud calls may not elicit response, and shaking only groans and unintelligible utterances. The coma may be intermittent, running parallel with the temperature. The fever in most cases varies from $10 \mathrm{r}^{\circ}$ to $103^{\circ} \mathrm{F}$., but may be subnormal or hyperpyrexial. The pulse is at first full and bounding, later small, rapid, and feeble. Dilatation of the right side of the heart may exist and an anemic murmur may sometimes be heard. The respiration may be quiet, slow or rapid, or blowing and stertorous, with Cheyne-Stokes characteristics late in the course. Edema of the lungs is an occasional late occurrence. Nausea and vomiting are seen early in the attack, if they are present at all. The mouth and tongue are dry, the latter deeply coated. Herpes and sordes are sometimes noted. Hiccough is an occasional symptom. The tongue when protruded may be drawn to one side. In cases of recent infection the spleen may be only slightly or not at all enlarged; in other cases it may be greatly enlarged, constituting a valuable diagnostic sign. 
The liver may be tender, but is usually not much enlarged. The evacuations of bowels and bladder may be involuntary or there may be retention of urine. The bowels are often constipated.

In favorable cases the coma gradually fades, consciousness slowly dawns, the temperature drops to or below normal, the pulse regains its normal characteristics, and, save the physical weakness and a degree of mental hebetude, all is well with the patient.

In unfavorable cases the coma becomes absolute, the pulse becomes rapid, thready, and irregular, the breathing is stertorous and of Cheyne-Stokes type, tracheal rattling appears, the face becomes cyanotic, and death ensues from convulsions or from collapse.

The duration of an attack is from a few hours to a few days. It usually holds true that the successive paroxysms increase in severity and danger to the patient. In the interval the patient may be apathetic or may complain of headache. The relapse may appear in the form of a different type of pernicious paroxysm, as algid or choleraic, but such cases are very rare.

As intimated, the apoplectic form of pernicious malaria is merely a fulminant variety of comatose malaria. In these rare cases the onset is equally as sudden as in cerebral hemorrhage, whence the name.

Symptoms originating from the cerebellum are present in rare instances. Such are slow, monotonous speech, drowsiness, severe depression and incoördination of voluntary movements.

Cases in which hemiplegia occurs have sometimes been described as the hemiplegic form of pernicious malaria; cases with aphasia, as the aphasic form. These two are not infrequently associated. Paraplegia is a very rare development in pernicious malaria.

A mild delirium is frequently present in the cerebrospinal forms of pernicious malaria. When it is conspicuous it forms the so-called delirious type. In this probably more than in any other form do predispositions have a causal part, especially alcoholism, nervous predisposition, mental fatigue, and exposure to solar heat. Delirium in this condition may vary 
from quiet to maniacal. Cases resembling rabies have been designated intermittens hydrophobica.

Convulsive or eclamptic pernicious malaria is a variety of the comatose type in which convulsions are a prominent feature. It is especially common in children. The convulsions may be confined to certain muscle groups or may be general. In one of my cases the little patient had twelve convulsions in an hour. Epileptiform convulsions have been described, but it is probable that most of these cases are complicated with true epilepsy.

The most prominent symptoms are usually trismus and opisthotonos; emprosthotonos and pleurosthotonos are but rarely observed.

Occasionally amaurosis arises in the course of a comatose attack. It may be transient or, in rare instances, permanent. In the only case occurring under my observation vision began to improve at the end of the attack, but was not fully restored until after several weeks.

A rare form of pernicious malaria, the ataxic, has been described. The principal symptoms are scanning speech, dysarthria, weakness of lower limbs, vertigo, unsteady gait with a disposition to fall forward, muscular tremors, and exaggerated reflexes.

Manson $^{29}$ thus describes the so-called ardent fever: "In the course of what seems to be an ordinary malarial attack the body temperature, instead of stopping at $104^{\circ}$ or $105^{\circ} \mathrm{F}$, may continue to rise and, passing $107^{\circ} \mathrm{F}$., rapidly mount to I1 $0^{\circ}$ or even to $112^{\circ} \mathrm{F}$. The patient, after a brief stage of wild, maniacal, or perhaps muttering delirium, becomes rapidly unconscious, then comatose, and dies within a few hours or perhaps within an hour after the onset of the pernicious symptoms."

In typhoid pernicious the clinic picture is almost identical with that presented in typhoid fever. The temperature is periodically intermittent or, as is more common, remittent, and usually ranges from $\operatorname{ror}^{\circ}$ to $103^{\circ} \mathrm{F}$., but may reach $106^{\circ} \mathrm{F}$. There are headache, backache, rapid pulse, torpid digestive tract, sordes, splenomegaly, apathy, and stupor. There may 
be diarrhea or constipation, and bilious vomiting occurs in some cases. The abdomen is usually tympanitic and there may exist tenderness and gurgling in the right iliac fossa. Epistaxis is frequent. Incoherent speech, delirium, and incontinence of urine and feces are symptoms of severe cases.

Thoracic Forms.-The immunity of the organs of the chest to localizations of the malarial parasites and to the effects of their toxins is remarkable. Indeed, the thoracic forms are much rarer than the records would import, for the older writers especially were prone to attribute any complication that might present itself to the effect of the mysterious malarial poison.

Formerly cases of pneumonic pernicious malaria were more frequently reported than at present. Since more exact methods of observation have come into use it is certain that many of these cases were complicating lobar pneumonias. That the malarial parasite is unable to cause true inflammation of lung tissue is now widely recognized. Nevertheless grave symptoms referable to the lung, and more or less resembling pneumonia, may arise in malarial infections. Cases presenting profuse hemorrhages from the lungs and nose have been recorded but rarely.

Abdominal Forms.-The type of abdominal pernicious malaria is the algid. The picture presented is that of abdominal shock; it is peritonism minus the peritonitis.

The algid symptoms may appear insidiously, but much more frequently supervene after the course of one or more simple paroxysms. Usually the first symptoms that attract the attention to the condition of the patient are the bad pulse and cold surface. Soon the Hippocratic facies is assumed. The eyes are deeply sunken and surrounded by dark circles, the nose appears sharp, the alæ nasi dilate with respiration, the tip of the nose and the ears are icy cold. The temples and cheeks are hollowed, the cheek bones project, the pupils are dilated, the conjunctivæ are bluish white, the eyes have a peculiar anxious expression, and the breath is cool. The skin is pale, having the appearance of absolute bloodlessness rather than that of cyanosis. The surface of the body is bathed with a clammy sweat, is cold, and gives the sensation to the hand of handling a catfish. The fingers and toes often have the shrunken appear- 
ance of the washerwoman's hand. The prostration is extreme and the voice is weak, low, and cracked. The patient complains of burning heat within and begs piteously for cold drinks, which are, as a rule, immediately rejected by the stomach. The intelligence remains clear and occasionally "the patient, overcome by sad apprehensions, considers himself lost, bewails his situation, but is not delirious," though usually he is indifferent to his peril. The temperature may be subnormal or slightly elevated, seldom reaching $104^{\circ} \mathrm{F}$. The pulse is rapid, filiform, of low tension, and often intermittent. Later it usually becomes imperceptible at the radial. The heart sounds are extremely feeble. The respiration is very rapid, superficial, and frequently interrupted with deep sighs. The tongue is tremulous, cold, and usually moist and smooth. Vomiting is a common symptom. The bowels are sometimes constipated, but often loose. The abdomen may be slightly tympanitic, or scaphoid and tender, especially in the upper half. The urine is scanty, highly colored, and of high specific gravity. The duration of the attack is short, rarely longer than twelve hours after the onset of algidity. In fatal cases the symptoms progress rapidly and the patient dies as if in peaceful sleep. In favorable cases the character of the circulation and respiration improves, the body warmth is gradually restored, the patient ceases to complain, and convalescence is impeded only by the extreme weakness.

When, in addition to the symptoms of algidity already detailed, there exist symptoms simulating true cholera, there is the variety of algid malaria usually spoken of as choleraic pernicious. The onset is with profuse diarrhea and vomiting. The stools are thin and watery and often rice-water-like. There may likewise be shown the muscular cramps of the lower limbs frequent in cholera. The temperature is usually elevated, and pains in the abdomen and precordia and singultus may be experienced. The urine is usually scanty and may become suppressed.

The condition of algor with which drenching diaphoresis occurs constitutes the so-called sudoral or diaphoretic form of pernicious fever. These sweats, which are so profuse that not 
only the clothing of the patient, but also the bedclothes are saturated, usually supervene toward the close of a paroxysm. The celebrated Torti, who was himself the victim of such an attack, says that he was just congratulating himself upon escaping the fever when the abundant sweats occurred to convince him that his condition was critical.

In the course of an algid access syncope occasionally occurs when any exertion, even the slightest, is attempted or when the patient's head is lifted from the pillow. This dangerous symptom usually comes quite unexpectedly, and if the patient survives the first onset a subsequent one may rapidly prove fatal.

The gastralgic or cardialgic type is characterized by excruciating pain in the abdomen, especially the epigastric region, or in the precordia. The pain is often so intense that the patient doubles up and rolls in agony upon the bed. The abdomen is tender and vomiting is a common symptom. There may be hematemesis, sometimes profuse. Diarrhea and singultus are occasionally symptoms.

The frequent occurrence of severe dysenteric symptoms, due solely to malarial infection, has been definitely demonstrated. The attack may follow other forms of abdominal pernicious or may come on suddenly. There are frequent actions of bloody mucus, violent tenesmus, colicky pains in the abdomen, elevation of temperature, and sometimes emaciation. Algid symptoms are not common. Occasionally abundant hemorrhages from the bowels occur. They may prove rapidly fatal, especially if the patient is already markedly anemic.

Icterus and bilious vomiting are not rare in malaria. As a rule, these are not grave symptoms, but there are cases in which their persistence and intensity form a complex of symptoms described as bilious pernicious malaria. The fever is usually high, nausea constant, icterus marked, and vomiting of bile distressing. Bile is present in the urine, often in quantities, and sometimes albumin. Epistaxis and hematemesis have been noted. The epigastrium is often painful and singultus may add to the discomfort of the patient. Toward the end of the severe cases there are apathy and carphology and the scene usually closes with delirium and coma. 
Cases are not rarely observed in which the symptoms closely simulate peritonitis, and cases have even been operated upon for appendicitis.

The urine is usually highly colored. The amount varies inversely with the quantity of sweat, bowel movement, and vomited matter; the specific gravity varies inversely with the amount. Early in the attack albumin may be absent, though later it is often present in large quantities, together with numerous tube casts.

The blood in various forms of pernicious malaria shows, besides parasitic findings previously mentioned, a pronounced reduction of red cells, averaging a half to one million per paroxysm. Polychromatophilia of red cells may be observed. Contrary to the case of simple malaria, there is usually a pronounced leucocytosis. There may be as many as 35,000 per cubic millimeter.

\section{CHRONIC MALARIA}

There is a great deal of confusion as to what is comprehended by chronic malaria. Much of this chaos is due to including the manifestations of malarial cachexia with those of chronic malaria, between which, however, there are essential differences. Chronic malaria implies a supply of vital resistance equal to the demand; malarial cachexia denotes an exhaustion of this supply. Chronic malaria is an antagonistic equilibrium between parasite and host; cachexia, a rupture of equilibrium. Chronic malaria is a conflict, cachexia a conquest. The relation between chronic malaria and cachexia has been fitly compared to that existing between a compensated heart lesion and broken compensation. Chronic malaria is an active form of malaria; cachexia is a sequel. Câchexia being a sequel, usually of chronic malaria, it may be difficult to say where the influence of the latter ends and the former begins. On the other hand, it is frequently difficult or impossible to differentiate between a relapse in chronic malaria and a reinfection.

For convenience of study, chronic malaria may be divided into a latent or passive stage and an active stage or stage of relapse. 
It is more frequently observed in children. It has already been shown that the frequency with which children native to the soil are infected constitutes the true endemic index of a locality.

Chronic malaria may be due to one infection, but, occurring chiefly in regions where repeated reinfection is possible, it is highly probable that reinfection is an important factor.

An analysis by me of a large number of cases shows the following results: Quartan and tertian infections are more prone to relapse than estivo-autumnal. The percentage of relapses to total number of cases of quartan, tertian, and estivo-autumnal is, respectively, 65,55 , and 45 . The pertinacity of quartan may be regarded as a conservative effort of nature to perpetuate the species. It is, indeed, remarkable how this form of the parasite is conserved in certain places where it is so very rare. However, the greatest tendency to relapse shown by the benign infections is more than counterbalanced by the severity of the symptoms of the estivo-autumnal relapses. Hence it is undoubtedly true that the estivo-autumnal parasites are the most important factors in chronic malaria.

It is the parthenogenetic cycle of the parasite that is chiefly concerned in the pathogenesis of chronic malaria, though the asexual forms also have a rôle. The parthenogametes are the parasites of the latent stage, the schizonts of the active stage. Parthenogenesis is the bridge across the gap caused by interruption of the schizogonic cycle.

The most frequent course is for chronic malaria to follow one or more acute attacks. In some instances, however, the latent stage may precede the active. Thus it is not extremely rare to meet cases with evidences of chronic malaria which have no history of active manifestations.

The latent stage of chronic malaria resembles in some respects a period of incubation; in fact, the cases reported with unduly long stages of incubation are doubtless nothing but latent stages of the chronic disease. During the latent stage parasites may or may not be found in the peripheral blood.

Symptoms during the latent stage may be altogether absent, 
in which case latency is absolute, or there may be present certain symptoms, subjectively insignificant, constituting relative latency. These symptoms are ordinarily similar to the prodromata of acute malaria: malaise, loss of appetite, aching of the back and legs, digestive disorders, etc., together with anemia and enlarged spleen. Latent malaria is the source of very numerous infections, and is of the utmost importance from the viewpoint of prophylaxis.

The duration of latency is exceedingly variable. Relapses occur at shorter or at longer intervals.

Relapses at short intervals have been recognized since the time of Hippocrates. Later the septenary periods were noted for a tendency to show relapses, and this idea is still largely prevalent among the laity. This shorter interval of latency corresponds more or less closely to the sexual cycle of the parasite and to the period of incubation. It is also in harmony with the law of Treille ${ }^{127}$ and with the studies of Cohen ${ }^{508}$ upon the period of freedom from paroxysms following a single injection of quinine. The duration of this period is from five to twenty-one days, oftenest from five to ten. Relapses at shorter intervals occasionally exhibit a striking periodicity.

Relapses at longer intervals occur at from one to twelve months, exceptionally longer. Very long periods of freedom have been recorded, even up to sixty years. ${ }^{509}$ Undoubtedly many of these are errors, due either to mistaken diagnosis or to the occurrence in the interval of unrecognized or masked paroxysms. However, periods as long as three years have been reliably recorded. ${ }^{86}$

It being clinically impossible to distinguish between a relapse and a reinfection, I have adopted Celli's ${ }^{458}$ rule, it being equally adapted to the seasonal prevalence of malaria in this country. This authority regards as a relapse every case of fever which repeats itself in the same individual during the epidemic year of malaria, from July of one year to the end of the following June. It is true that this may include some cases of reinfection, but it is unquestionably the most practical guide and eliminates a maximum of error.

It is ordinarily the relapse that brings the chronic malarial 
to the physician. The relapse may consist of one or more typic malarial paroxysms or they may be atypic. Very often the first stage of the paroxysm is wanting. They may exhibit quotidian, tertian, or quartan periodicity, or may be altogether irregular. The patient usually has an anemic tint and may, in advanced cases, be jaundiced. Occasionally the complexion may be earthy, at other times bronzed. The skin is dry and often scaly. The eyes may be deep set; they often bear a haggard, restless expression. The patient appears aged beyond his years. The condition of nourishment is poor, and there may be edema of the face and feet. There are weakness of arms and legs and an indisposition to physical exertion. The pulse is accelerated, weak and sometimes irregular. Percussion may reveal an increase in the cardiac dulness, and auscultation an anemic murmur. Dyspnea on slight exertion, a feeling of weight or pain in the precordia, and palpitation are not infrequent symptoms.

The extent of the blood destruction depends upon the severity and proximity of the paroxysms and the activity of the bloodmaking organs. The number of red cells frequently falls to one million per cubic millimeter or even less. In other cases the destroyed cells are nearly replaced within a short time after the relapse. The hemoglobin percentage is sometimes disproportionately lower than the red cell count, though occasionally it may be normal or above. The leucocyte formula is similar to that of acute infections. Parasites of the asexual cycle are usually found in the peripheral blood. Tertian gametes are frequently seen, while quartan are rare. The frequency of crescents and ovoids is very variable. In my experience they are not often seen in blood obtained from the superficial circulation. They are also infrequent in the experience of some other observers. The majority of writers, however, have seen estivo-autumnal gametes in a considerable proportion of their cases.

Other blood changes, as nucleated red cells, microcytes, macrocytes, and poikilocytes, are more common in chronic than in acute malaria.

The respiration is usually quickened, especially after exercise. 
Chronic bronchial catarrh, usually of a mild degree, is not a rare condition in chronic malaria, and epistaxis is sometimes profuse.

Digestive disorders are very common and marked meteorism may exist. The condition of the bowels is not constant, diarrhea sometimes alternating with constipation. Dysenteric manifestations are frequent.

The spleen may be of normal proportions in mild cases, but is usually enlarged, sometimes enormously so, passing the median line of the abdomen and the iliac crest. It may or may not be tender or painful; in the former case the pain is usually of a pulling nature and referred to the left shoulder. If perisplenitis with adhesions does not occur, the spleen may be movable or floating. Often the spleen enlarges during the active stage to recede slowly during latency. The liver is of ten slightly enlarged and tender.

Headache, nervousness, restlessness, vertigo, insomnia, and, in severe cases, impaired memory, are observed. The urine is often albuminous.

Masked Malaria.-Masked or larvate malaria, like pernicious malaria, needs complete overhauling. Nearly every disease in the category has been confounded with malaria and classed as larvate. This heterogeneous group has been expanded to embrace diseases unrelated in any way to malaria, diseases complicating malaria, and symptoms and sequelæ of malaria. The frequency of masked malaria varies inversely with the care employed in diagnosis. Masked malaria is merely atypic malaria. . The symptoms being of little value in diagnosis, this must be made by the anamnesis, the microscopic examination of the blood, and by the therapeutic test. Nervous, gastrointestinal, and cutaneous disorders are those most frequently recorded as masked. Most of these are to be considered under Complications and Sequelæ.

\section{COMPLICATIONS AND SEQUEL}

Circulatory System.-Malaria is very frequently complicated by heart disorders. In the negro population of the South, in whom syphilis, abuse of alcohol and tobacco, pneumonia, 
and other etiologic factors are very prevalent, lesions of the circulatory system, particularly valvular lesions of the heart, often add to the gravity of severe malarial infections. These are to be regarded almost invariably as complications and not as sequelæ. Circulatory lesions, the direct result of malarial invasion, are remarkably rare. Many such cases were reported before the discovery of the parasite, but are for this reason practically valueless.

Probably the commonest cardiac sequel of malaria is myocarditis. Slight evidences of degeneration of the heart muscle are sometimes found after death in cases which presented no symptoms during life.

Angina pectoris is occasionally observed in connection with malaria. It may exist as a complication or as the cardialgic type of pernicious malaria.

Much was formerly written about malarial endocarditis, aortitis, and endarteritis. These occur but rarely and only as complications. Pericarditis and aortic aneurism are usually complications. Phlebitis and thrombosis have been seen, more often in cases of cachexia.

There is absolutely no evidence that malaria is a causative factor in either lymphangitis or lymphadenitis, the so-called malarial bubo, these conditions occurring only as complications. A suppurating bubo, like other septic processes, may be accompanied by an intermittent temperature.

Respiratory System.-Coryza may occur as a complication to malaria, especially during unseasonable weather. Bronchitis is a common complication, during a portion of the malarial season occurring with marked frequency. It is observed oftener in the negro than in the white race. Subacute or chronic bronchitis is usual in chronic malaria and cachexia.

A peculiar condition of the pulmonary apices has been described. It consists of a rapid and transient congestion of the apex of one or both lungs, arising and disappearing with the paroxysm. The cough is dry and painful, the expectoration is scanty, occasionally bloody, and there may be bronchial breathing and increased vocal fremitus. I have had no experience with this complication. 
Pneumonia was long considered a manifestation or a sequel of malaria, but it is now known that they are entirely distinct diseases.

Gastro-intestinal Tract.-Stomatitis is sometimes observed in malaria. Parotitis is an infrequent complication. Dyspeptic symptoms denoting chronic gastric catarrh are not uncommon in cases of chronic malaria and cachexia. Gastric ulcer in association with amyloid changes in the mucosa is rarely noted. Hematemesis occasionally assumes alarming proportions.

Enteritis is a much more frequent sequela of malaria than ordinarily regarded. The inflammation may advance to ulceration. The process has been frequently demonstrated by autopsies to be due to accumulations of parasites in the intestinal mucosa. Diarrhea is common, especially in persons improperly fed. Profuse hemorrhage occasionally occurs, in which case the microscopic examination of the blood is of the greatest value in differentiating the disease from typhoid fever.

Dysenteric symptoms arising in the course of malaria and the dysenteric form of pernicious malaria have been considered. Well-marked dysentery may be present either as a complication or as a sequela. Often the dysenteric symptoms predominate, thus constituting one of the commonest forms of masked malaria.

Besides the ameba, other intestinal parasites may complicate paludism. Of these by far the most common is the Ascaris lumbricoides. Uncinariasis is not an infrequent complication in some sections. I have observed an infection with Hymenlepis nana associated with malaria. It is not improbable that intestinal helminthiasis aggravates the anemia and the gastrointestinal and nervous symptoms. In examining the blood for the malaria parasite the presence of eosinophilia calls for an examination of the feces.

Cirrhosis of the liver the direct result of malarial infection, described as relatively frequent in certain portions of the tropics, appears to be rare in this section. Hypertrophic hepatitis may result from prolonged infection. Ascites occasionally develops, particularly in chronic malaria and cachexia.

The Blood and Spleen.-Leukemia follows malaria only 
rarely, probably never as a true sequela, though many cases of leukemia give a history of past malaria.

The relation of splenic anemia to malaria is not clear. Splenic anemia may come into consideration in differential diagnosis.

Malarial Cachexia.-In this condition the parasites have obtained undisputed possession of the host. The defensive forces have been completely conquered, the blood-making organs can no longer meet the demand made upon them, and toxins, unopposed, work changes, often irreparable, in important organs. Cachexia has been classified as dry or humid, according to the absence or presence of anasarca, and as acute or chronic. Acute cachexia is characterized by a rapid onset and development of symptoms and usually follows acute malaria, occasionally after only one or two attacks. These cases are infrequent. Chronic cachexia, the usual form, is a sequela of chronic malaria.

Malarial cachexia is found where the severe forms of malaria are endemic. It may be stated as a general rule that the frequency of cachexia among the white race is an index to the prevalence of grave infections. It is much more common in the white race than in the negro. While negro children are not infrequently the subjects of malarial cachexia, it is much rarer in the adult negro. Of adults, males are more commonly cachectic than females; among children the proportion is about even. The condition rarely develops in persons of the better class, but is seen in those living under improper hygienic conditions and who neglect the treatment of acute malaria.

Cases of cachexia developing without outbreaks of malaria have been reported, but are subject to question. Infections with the estivo-autumnal parasites are followed by cachexia much more frequently than tertian and quartan infections.

The cachectic usually presents a singular appearance. The emaciated limbs are in marked contrast to the big belly, and the features are aged beyond the years. The most pronounced phenomena are the anemia and the enlarged spleen. The red blood-cells may be reduced to seven or eight hundred thousand per cubic millimeter. The leucocytes are generally normal in 
MALARIA
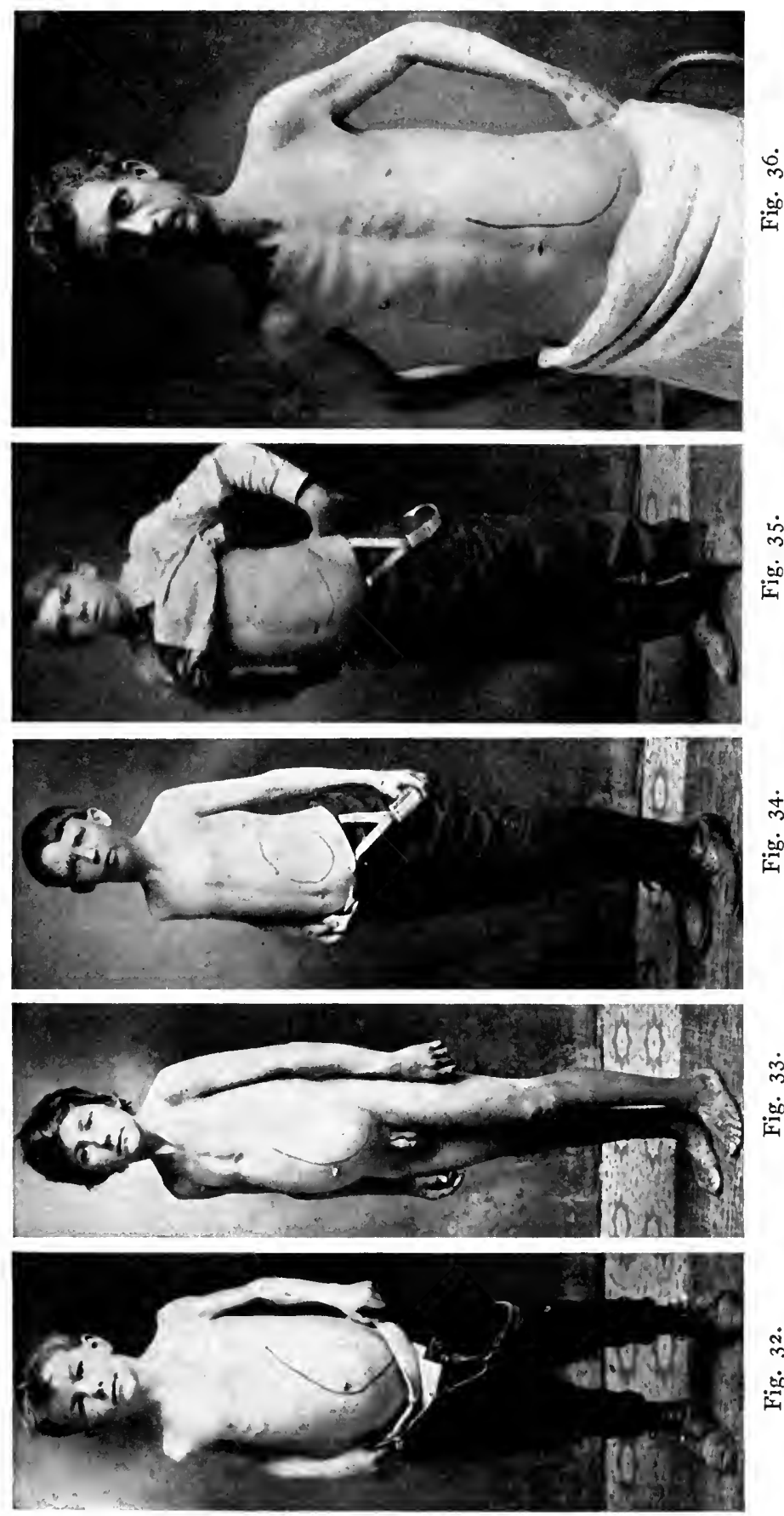

I 27

:

垔

i்

si

步

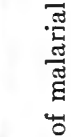

要

$\dot{m}$

:

i்

我 
number or a little below. Numerous differential counts have shown a relative increase of the large mononuclear elements. The red cells may show basophile degeneration, polychromatophilia, poikilocytosis and nuclei, but none of these changes are by any means constant. According to my experience parasites are rarely found in the peripheral blood. The spleen often extends to the umbilicus and to the crest of the ilium, sometimes beyond. It is usually hard and the anterior border presents a sharp edge. Pain and tenderness on pressure are not always felt. Occasionally a bruit is to be detected over the splenic area.

The pulse is small, compressible, and may be irregular. Palpitation of the heart and hemorrhages, especially epistaxis, may occur. An ancmic murmur over the precordia is often heard. Myocarditis and dilatation are not infrequent. The breath is short, sometimes amounting to actual dyspnea. A cough is common and signs of bronchitis may be elicited. Pulmonary edema is a late symptom.

The temperature may be normal or subnormal for long periods, though evening rises are often observed. Typic paroxysms are not frequent. Fever often follows imprudences. Whether the fever of cachexia is due directly to parasitic activity or to organic changes is not definitely known. The appetite is generally poor and the digestion tardy. Epigastric pain, nausea, and vomiting may be complained of. The tongue and oral mucous membrane are pale. Diarrhea and dysentery frequently occur. Meteorism is common. The liver is usually somewhat enlarged at first; later it may become atrophic. Ascites is not a rare manifestation. When fever exists the urine is ordinarily scanty and highly colored. Delayed development of the genitals is common in the young, and diminished sexual power is not rare in the adult. Indifference, intellectual torpor, somnolence, headache, and vertigo are observed in cachectics. Resistance to cold is lessened and rheumatic pains are experienced. The skin is pallid, dry, and rough, and may exhibit sores or purpuric spots. Anasarca may supervene.

Pneumonia, dysentery, hemoglobinuric fever, and nephritis are common complications of cachexia, and amyloid degeneration, especially of the kidneys, an occasional sequela. Perisplenitis occasionally occurs and may be the cause of severe 
pain, especially if adhesions take place. A heavy spleen may cause relaxation of its supports and become "floating" or "wandering." This condition is seen more often in multiparous females. By pressure on neighboring organs a wandering spleen may cause pain, digestive disorders, or even intestinal occlusion. The pedicle may become twisted.

Rupture of the spleen is an infrequent complication of malarial cachexia. It is very rarely associated with acute malaria. I observed a case in a subject of cachexia who had a large, hard spleen. Recovery followed, notwithstanding extensive hemorrhage. The symptoms of rupture of the spleen consist of violent pain in the splenic region referred to the left shoulder, together.with evidences of shock and hemorrhage. The mortality of cases not treated with splenectomy is exceedingly high. Death may occur in from a few hours to five days.

Abscess of the spleen is a rarer complication of malaria even than rupture. I am able to collect from the literature mention of not more than fifty cases. The common pyogenic cocci and the bacillus coli communis are the bacteria usually present in splenic abscesses. The symptoms are sometimes as vague as those of abscess of the liver. There may be pain in the left hypochondrium, especially if the abscess is superficial and the peritoneum is involved. The pain is often referred to the left shoulder. Pain may, however, be entirely wanting. The temperature is usually elevated, though in rare instances it may be normal or subnormal. It is usually intermittent and associated with chills and sweats. Emaciation may be extreme, notwithstanding the appetite is sometimes voracious. Abscess of the spleen'may be confused with uncomplicated malaria and renal, gastric, or pancreatic disease. The presence of leucocytosis and the therapeutic tests are valuable diagnostic points. Signorelli's spleen point may be a useful guide. This is a painful area corresponding to the fifth intercostal space near the left nipple. Without operation the prognosis is very grave; with timely incision and drainage a considerable per cent. recover.

Infarcts and gangrene of the spleen are noted among the rare complications of the spleen in malarial cachexia. 
Genito-urinary Organs. Nephritis.-The frequency of nephritis recorded as a sequela of malaria varies greatly with locality, type of fever, and with the idea of what constitutes nephritis. The real frequency of nephritis in malaria is in about $1 / 2$ to 2 per cent. of the cases of tertian and quartan infections and 2 to 5 per cent. in estivo-autumnal. The negro is apparently more susceptible to the renal lesions of malaria than is the white. More cases are seen in the months during which the estivo-autumnal fevers prevail. The middle aged and the old are less often attacked than the young. Nephritis is much more prone to result from chronic than from acute malaria. The nephritis is most often acute, but it is highly probable that malaria is an important factor in the etiology of chronic nephritis. Chronic nephritis may follow the acute form or may exist as such from the beginning. True hemorrhagic nephritis is rare. There is ordinarily nothing characteristic either in the symptoms or pathologic histology of the nephritis of malaria. Amyloid degeneration is a renal sequela of malaria.

Occasionally intense lumbar pain, closely simulating renal colic, is experienced as a complication of malaria. This colic usually responds promptly to quinine, though the pathogenesis is not clear.

Forms of orchitis and epididymitis have for a long time been attributed to malaria. Either may complicate malaria. I have seen several cases of epididymitis associated with malaria, there being, however, in each case a history of venereal disease. There is at the present time absolutely no evidence that either orchitis or epididymitis is ever a true sequela of malarial disease. The same may be said of hydrocele, which some observers have ascribed to malaria.

It is doubtful whether genuine atrophy of the testicles ever occurs as the result of malaria. It is more probable that these cases are due to improper development, the result of cachexia, climate, or other factors. Metrorrhagia and, more often, menorrhagia and amenorrhea are not infrequently seen with malaria. Sterility has been charged to paludism.

It was formerly believed that pregnancy conferred a degree of 
immunity against malaria. This is now known not to be true. If the pregnant woman is attacked less often with malaria it is because she is less often exposed to infection, and not on account of an immunity which pregnancy confers upon her.

A list of cases is appended to give an idea of the frequency with which abortion and premature labor occur as the result of malaria complicating pregnancy. The first column of figures records the number of cases in which the complication appeared; the second column shows the number of abortions and premature labors which occurred:

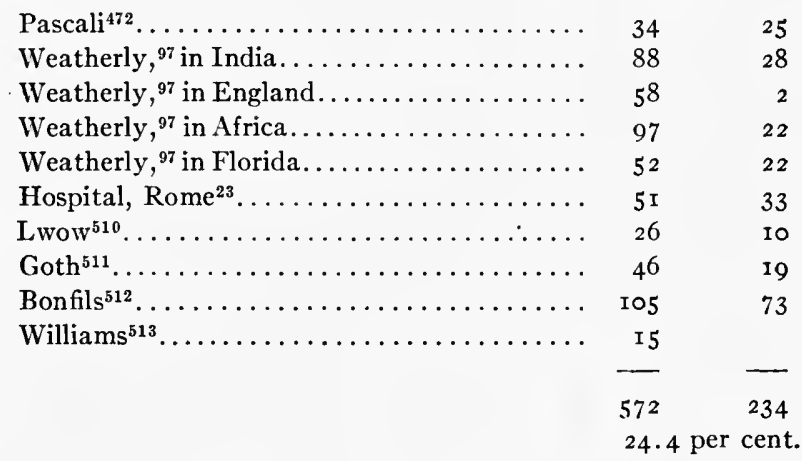

This percentage is at least twice as large as that resulting from my experience.

I am inclined to believe that the negro is much less liable to suffer abortion or premature delivery than is the white. This is in keeping with the well-known relative immunity of the negro to some of the effects of malaria.

Cases accompanied by continued high temperature, retching, and vomiting, and which are more resistant to treatment, are those in which abortion most frequently occurs. Hence it follows that abortion is more often due to estivo-autumnal fever than to tertian and quartan, and to multiple infections than to single.

The danger of abortion and premature delivery is greater in proportion as the pregnancy is advanced.

The factors in the interruption of pregnancy are probably fever, retching, vomiting, anemia, and toxins. It is not improbable that in some cases parasitic localizations in the uterine 
vessels excite pains or cause placental separation, though for this theory there is as yet no pathologic proof.

If the malarial infection does not terminate the pregnancy the labor at full term is apt to be slow, especially the first stage.

Children born at full term of malarial mothers are apt to be smaller and lighter than normal, and the mortality is higher.

Labor of ten rekindles latent malaria, which, in the puerperium, is not infrequently atypic, the first or third stages of the paroxysm or complete intermission of the temperature sometimes lacking. Subinvolution, postpartum hemorrhage, and suppression of milk may occur with puerperal malaria.

Nervous System.-It is often impossible to determine whether nervous manifestations in malaria are complications or sequelæ. It is certain that many cases reported as due to malaria are purely complications. This is the case with multiple neuritis, of which numerous cases have been ascribed to malaria. In by far the majority of cases the existence of malaria was not established by blood examination; in others it is not certain that the neuritis was due to malaria.

Neuritis occurring during and after malarial disease has often been described. Paraplegia and symptoms resembling multiple sclerosis are also occasionally observed.

Hemiplegia, paraplegia, and various monoplegias, either with or without aphasia or sensory disturbances, are not infrequently associated with malaria.

Cerebellar syndromes are rarely observed in malaria. They consist of general weakness, rigidity, and pain in the back of the neck, intense headache, ataxic gait with a tendency to fall backward and to the left, tremors, incoördination of movement, dysarthria, nystagmus, and vomiting. The tertian parasite is usually present in these cases.

Bulbar symptoms are occasionally encountered. Such are hypoglossal and facial paralysis, ataxia of arm, dysarthria or anarthria, and staggering gait. These symptoms are usually obstinate.

Various psychoses occur in connection with malaria, either during or following acute or chronic malaria. The commonest 
of these disorders are weakened memory, melancholia, mania and delusional insanity. Suicidal and erotic tendencies may be observed.

Hysteria is not a rare phenomenon during paludism. It is probably the result of anemia in predisposed persons. As it may assume any of a multitude of forms, its chief significance is from the viewpoint of diagnosis.

A mild neurasthenia is probably due directly to malaria in some instances, and preëxisting neurasthenia is often aggravated by malarial infection. The usual symptoms are restlessness, nervousness, insomnia, and annoying distinctness of the heart beat on retiring.

It was formerly believed that intercurrent malaria exercised a beneficial influence upon epilepsy. So far from this being the case, however, epilepsy is frequently aggravated by paludal infection.

Violent choreic symptoms are among the rare nervous phenomena.

The Eye.-Injection of the conjunctiva is not infrequently associated with neuralgia of the fifth nerve. True intermittent conjunctivitis occurs but rarely if at all. Both interstitial and dendritic keratitis are occasionally observed with malaria, though it is doubtful whether either form can be attributed to malaria. The same probably holds true for vesicular keratitis or the so-called corneal herpes. In pernicious seizures with coma-vigil the eyes are more or less exposed to damage.

Iritis exists as a complication of malaria in rare instances. Choroiditis occasionally occurs in connection with retinitis. Optic neuritis is observed chiefly in cachectics. In the majority of cases it proceeds to atrophy.

Retinal hemorrhages are oftenest minute and located far forward, hence they may be easily overlooked. Occasionally, however, they are peripapillary or macular and of large size; in the latter case the prognosis is more serious. Persistent or periodic amaurosis without evident retinal changes is sometimes seen. I have seen one case of hemianopia following pernicious malaria of the comatose form which terminated in complete restoration of vision. Rarer optic manifestations oc- 
curring in conjunction with malaria are hemorrhage and infiltration into the vitreous humor.

The Ear.-Otalgia, labyrinthine vertigo, otitis media, and lesions of the internal ear and auditory nerve have been described as occurring with malaria, but in no case has the blood been examined.

The senses of taste and smell are said to be diminished or abolished in rare cases of malaria.

The Skin.-The frequency with which herpes occurs in malaria has already been mentioned. Next to herpes, urticaria is the most frequent cutaneous lesion associated with malaria. The possibility of the eruption being caused by quinine should be remembered. Erythema is not an uncommon eruption with malaria, and may simulate the eruption of scarlatina. Pruritus may be present. Erythema nodosum has occasionally been observed. Petechiæ and large purpuric spots are not rare in subjects of chronic malaria and of cachexia. In these patients ulcers and furunculosis may exist as complications.

The occurrence of herpes zoster in malaria is very variable. In 616 cases of malaria studied by Thayer and Hewetson ${ }^{439}$ herpes zoster occurred only once, and this complication existed but once in $\mathrm{I}, 780$ cases of malaria reviewed by Anders. ${ }^{505}$ On the other hand, Winfield ${ }^{514}$ found malarial parasites in the blood of 14 out of 25 cases of herpes zoster. I have recently observed 9 cases of herpes zoster. In 3 the blood examined revealed estivo-autumnal parasties; in 2 there was a history of recent malaria and the examination of the blood was negative.

As previously mentioned, purpura simplex is not an uncommon occurrence in malaria. Purpuric eruptions may also, but rarely, be noted in hemoglobinuric fever. But true purpura hemorrhagica is very rarely seen in malaria. About seven cases are recorded, one of which I observed in the South.

Malaria undoubtedly predisposes to the development of gangrene, especially when it has become chronic or has advanced to cachexia. More than this, however, cannot be said of the part played by malaria in the etiology of gangrene. Gangrene of almost every part of the surface of the body has been observed in malarial subjects. The gangrene is more commonly 
of the dry variety. Local asphyxia not followed by gangrene occurs also. Raynaud's disease, or symmetric gangrene, has been thought to be due to malaria in many instances, but reports of cases in which the malarial parasite was present in the blood are still rare.

Other Conditions and Diseases.-At various times malaria has been supposed to predispose to certain diseases. Such were typhoid fever and diabetes. It has also been thought to exert an antagonistic influence toward other diseases, as tuberculosis, cancer, and influenza. It is probable that any predisposing power on the part of malaria to other diseases is only indirect. It is a priori improbable that a disease conferring only relative immunity toward itself should immunize against or antagonize other diseases, and such is the result of experience.

Typhoid Fever.-The complication of typhoid fever with malaria is not very rare. A search of the literature reveals records of 215 cases in which the presence of malaria parasites and the typhoid bacilli or the Widal reaction conclusively proved the association.

Typhoid fever is more frequently complicated with tertian than with estivo-autumnal malaria. Craig has reported the only case of simultaneous typhoid fever and quartan malaria of which I have any knowledge.

Usually the malarial symptoms arise and the parasites are detected during convalescence from the typhoid fever, though they may be present during the course of the latter. When the onset of the malaria precedes that of the typhoid fever, the malarial parasites often disappear from the peripheral circulation upon the advent of the typhoid fever, sometimes reappearing and producing symptoms during the convalescence from typhoid. This is analogous to the result of inoculating a given variety of malarial parasites into a malarial patient harboring a different form, the older infection usually surrendering to the fresh. Malaria occurring at the height of typhoid fever may or may not modify the course of the latter. The mortality of the complication of these two fevers is higher than that of uncomplicated typhoid.

The term "typhomalarial fever," if used at all, should be 
restricted to those cases in which exact methods of diagnosis prove it applicable. However, the combination of typhoid and malarial fevers is no more entitled, either by virtue of intimacy or frequency, to a hyphenated appellation than is tuberculosis or gonorrhea in association with malaria. It was formerly believed that a mysterious fusion of both diseases produced a hybrid pathologic entity. It is now definitely known that this is not the case. Such a diagnosis is ordinarily a compromise based on a lack of frankness to acknowledge inability to diagnose certain cases of fever in the earliest stages, and is not only loose and unscientific, but is, in many instances, actually harmful, as it often leads to the abuse of purgatives and quinine. There is no question but that nearly all of the so-called "typhomalaria" is pure typhoid fever.

Diabetes.-This is undoubtedly a rare complication. I recall the case of a diabetic, passing more than 5 per cent. of sugar, who was attacked with estivo-autumnal malaria. The latter ran an uneventful course and seemed to have no effect on the sugar excretion. The patient died several months later of bronchitis.

Polyuria in malarial subjects has already been mentioned.

Tuberculosis.-In the South, where tuberculosis is very prevalent in the negro race, the negro death rate from tuberculosis ranging from 100 to 150 per cent. higher than in the white race, tuberculosis and malaria not infrequently concur, especially in the colored race. Malarial cachexia predisposes to tuberculosis only in a slight measure, if at all. The negro, who is less of ten the subject of cachexia than the white, more often shows the combination of malaria and tuberculosis. The malaria may prove rapidly fatal, both diseases may be unmodified in their progress, or the tuberculosis may assume a more rapid course. The old idea that the two diseases are antagonistic is disproved by their not uncommon occurrence in the same individual, as I have frequently observed.

Influenza.-Anders ${ }^{515}$ believes that there exists a decided antagonism between malaria and influenza. Simms and Warwick, ${ }^{460}$ however, mention simultaneous epidemics of malaria and influenza in Alabama, when, "of those infected with malaria, 
60 per cent. were brought down with this disease, and it was much more severe than in those who were not infected."

Cancer.-Based on the supposition that cancer is not so frequent in tropic latitudes, and on the report of Krzowitz, in 1776 , of a case of the breast healing after an attack of double tertian malaria, Loeffler ${ }^{516}$ assumed an antagonsim between the two diseases, and proposed as a therapeutic measure, the inoculation with malaria of cancerous patients. A few experiments and numerous reports of cancer among tropic people and malarial subjects have shown the absolute uselessness of such a procedure. On the other hand, it is believed that malaria of long standing predisposes to cancer of the liver.

Smallpox is an infrequent complication of malaria. Laveran ${ }^{23}$ observed several such cases in Constantine. The malaria parasites usually disappear from the blood with the onset of the smallpox where the onset of the latter. succeeded that of the former. The mortality of these cases was unusually high. Pyemic foci and hemorrhages were observed.

Syphilis is a common complication of paludism. Under these circumstances syphilis is more rapid in its course and is rebellious to treatment in proportion to the chronicity of the malarial infection. In malarial cachectics antisyphilitic treatment is sometimes all but impotent. Syphilitic buboes are more apt to suppurate and become ugly indolent ulcers. Malarial invasion may arouse latent syphilis.

Malaria in Children.-In older children there is nothing unusual in the malarial attacks. In infants and young children there are several points which deserve a brief consideration.

The type of fever is more often quotidian, sometimes tertian or double quotidian, rarely quartan. The paroxysm occurs more often during the night than in the case with the adult, the fever being often detected for the first time in the morning.

The first stage is rarely typic, the rigor being replaced by coldness of the extremities, pallor, slight cyanosis, especially of the lips, and nails, vomiting, drowsiness, and sometimes convulsions. During the second stage the fever is ordinarily higher than in the adult. Gastro-intestinal symptoms, particularly vomiting and diarrhea, are common. Thirst is usually intense. 
The most common complaints are pain in the head and epigastric region. Enlargement of the spleen is more constant than in the adult. Torticollis and erythema may be noted. Atypical forms and dangerous symptoms, especially on the part of the nervous and gastro-intestinal systems, are frequent. Edema, ascites, and purpura are not uncommon.

Malaria in the Negro.-The relative immunity of the negro race to the severe manifestations of malaria and to hemoglobinuric fever has been mentioned. It remains only to cite a few clinic features of malaria in this race.

Estivo-autumnal malaria is much more common in the colored race than are tertian and quartan. The paroxysms usually occur during the day, but night paroxysms are more common than in the white race. A well-defined and severe cold stage, while not at all rare in the negro, is more frequently lacking than in the accesses in the white. Herpes is relatively rarer in the black. Uncontrollable vomiting is not nearly so frequent in the colored race as in the white. Marked splenic enlargement is much less common in the negro, palpable spleens in the adult negro being infrequent. The extremely low hemoglobin percentages, which are not rare in chronic malaria and cachexia of white persons, are far less frequently observed in the negro. As previously stated, cachexia is decidedly more prevalent in the white race. Malaria parasites are altogether absent from the peripheral blood of negroes in a larger per cent. of cases than they are wanting in white patients. When present they are more frequently scanty. On the other hand, the negro may harbor large numbers of parasites without manifesting any symptoms. Pulmonary complications, bronchitis, pneumonia, and tuberculosis are more frequent in the negro. Nephritis is another complication of which this is true. Hysteria and other neuroses are probably more common in the colored female. The abuse of snuff, which is undermining the nervous stability of the majority of adult negro females in the South, may help to account for this. There is less tendency to abort during pregnancy complicated with malaria in the colored females than in the white. Spontaneous cure after only one or two paroxysms is a common termination of malaria in the negro. Every 
physician practising among this race is familiar with the frequency with which their attacks of malaria end after a "round" of purgative and a potion of "tea" of some sort. The grave forms of malaria occurring less often, the mortality is consequently lower in the negro race.

The Surgical Aspect of Malaria.-Trauma may aggravate active malaria or arouse it from latency. On the other hand, malarial infection reacts upon wounds. Slight wounds, such as that caused by the extraction of a tooth, may in cachectics give rise to excessive hemorrhage. Fractures heal more slowly in malarial subjects. I have more than once observed suppuration, ulceration, and sloughing in the wounds, aseptically treated, of malarial persons, especially sawmill employees and timbermen. If surgical measures are contemplated in patients with a history of recent malaria the blood should be examined carefully for evidences of malaria, which, if present, might figure in the result. 


\section{CHAPTER V}

\section{DIAGNOSIS OF MALARIA}

There are three sources from which information may be drawn to make a diagnosis of malaria: first, from the symptoms; second, from the examination of the blood; and third, from the effect of quinine upon the symptoms.

I. Of the clinic history the most important feature to be considered is periodicity. Tertian and quartan periodicity are pathognomonic of malaria. Sometimes the statements of patients cannot be relied on with respect to the course of their ailments, and tertian and quartan periodicity must be absolutely determined to be of diagnostic value. By this is not meant that the disease must be observed by the physician untreated until such periodicity is established, but that value of this symptom is in proportion to the reliability of the source from which the history is derived. Unfortunately, this periodicity is of little value in estivo-autumnal infections, in which the importance and difficulty of diagnosis are greater.

Quotidian periodicity is not only worthless, but actually misleading in the diagnosis of malaria. It is especially in septic conditions that mistakes are oftenest made, where not infrequently is the rhythmic quotidian succession of chill, fever, and sweat mistaken for the metric march of malaria. A noted clinician has said that he has rarely seen a case of abscess of the liver that had not been drenched with quinine, and his experience is not unique in this respect. Malaria is by no means the only condition accompanied with cold, hot and sweating stages, and one or two of these stages are sometimes wanting in malaria. Abscess of the liver, gall-stone disease, tuberculosis, and numerous other diseases may exhibit temperature charts closely resembling that of malaria.

It should be borne in mind, however, that quotidian fever in malaria may show tertian or quartan periodicity. Thus in 
double tertian the paroxysms of the first and third days may occur at a certain hour in the morning, and those of the second and fourth days at a certain hour in the afternoon. Tertian periodicity in quotidian fever is valuable from a diagnostic view in proportion as the paroxysms on successive days are separated from a given hour, or, in other words, as the alternate paroxysms approach a forty-eight-hour interval, while the accesses on successive days are distant, by more or less, from a twenty-fourhour interval. Quartan periodicity in quotidian fever rarely comes into consideration in diagnosis on account of the relative rarity of the triple quartan infections, the promptness with which the microscope decides the matter, and the more frequent tendency of one or two of the three groups of parasites to sporulate approximately twenty-four hours after the last preceding. The course of a double quartan infection, two successive fever days followed by a fever-free day, is pathognomonic.

It may be stated, as a general rule, that tertian and quartan periodicity are of importance in diagnosis in proportion to the length of the series of paroxysms, since it is not impossible that fever on only two days separated by one or two days of apyrexia might occur adventitiously from causes other than malaria. It is the repetition of this succession that indicates malaria; hence the periodicity must be perfectly established.

The characteristic curve of tertian estivo-autumnal fever is probably pathognomonic, but can be obtained in only a small proportion of cases in private practice. The clinical course of estivo-autumnal infections is of much less value in diagnosis than that of tertian and quartan.

The value of enlargement of the spleen in the diagnosis of malaria has certainly been over-rated. In regions where there is little malaria, the endemic index being low, it is probably a point of some worth. On the other hand, in malarial regions of high index endemicus it is worth much less. It is almost valueless in malaria occurring in negroes, as it is infrequently sufficiently enlarged to be palpable, and unless palpable is of no diagnostic value. Physicians in malarial regions are all 
familiar with the frequency with which the enlarged spleen of the subject of chronic malaria or cachexia complicates other diseases. If the physician is sufficiently familiar with the patient to know that the splenic enlargement is acute, it becomes a matter of some importance, but the statement of the patient as to the former condition of the organ, even when the latter is immense, is not always to be relied upon.

Herpes when present is an aid to diagnosis. The only disease in which it occurs with anything like the frequency it does in malaria is pneumonia.

2. The microscopic examination of the blood for the diagnosis of malaria determines the presence or absence of parasites, pigment, and leucocytosis, and the numeric relation of the leucocytes.

Before attempting the diagnosis of malaria by the microscopic examination of the blood the beginner must become thoroughly familiar with the appearance of normal blood and with the technic of examination, and he should not rely too much upon the result of an examination until he has had considerable experience with malarial blood.

While Laveran made his discovery with a one-sixth-inch lens, only a one-twelfth-inch oil immersion lens, with appropriate condenser and diaphragm, should be employed, and the mechanic stage greatly facilitates the work. Thin slides and coverglasses should be used.

While stained films of the blood have a wider field of usefulness to the general practitioner than preparations of the unstained blood, he should become familiar with the technic of each.

When about to obtain blood to be examined, fresh and unstained, several slides and cover-glasses, having been washed thoroughly with soap and water, then with alcohol, should be rubbed thoroughly with an old, clean handkerchief and gently warmed. While the blood may be obtained elsewhere, the lobe of the ear has advantages over other locations: it is less sensitive, it being possible to obtain blood from sleeping children without awakening them; the instrument and the blood may be kept from the view of the patient, an advantage when dealing 


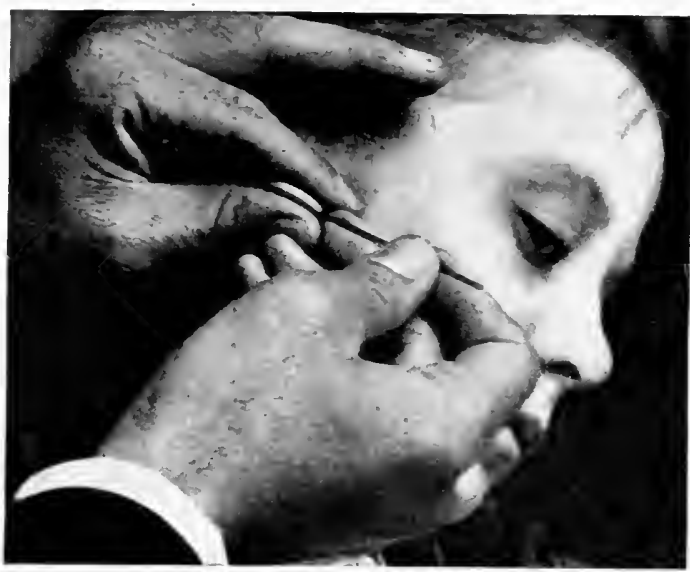

Fig. 37.-Making the puncture.

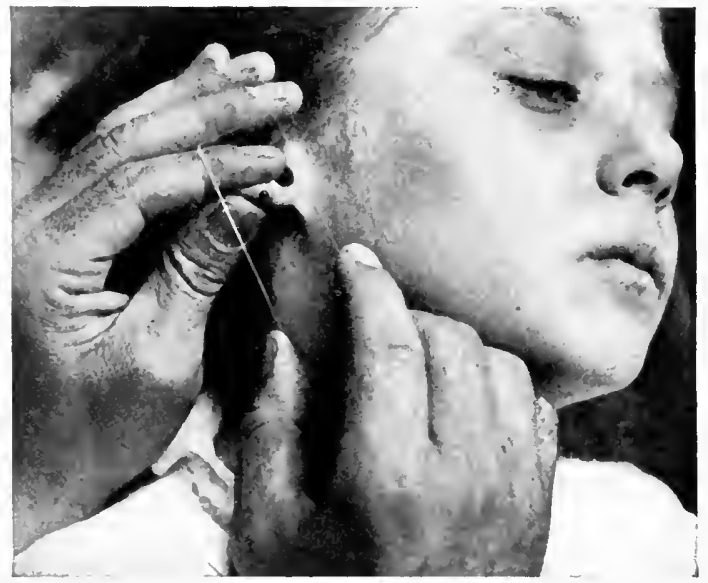

Fig. 38.-Obtaining the blood.

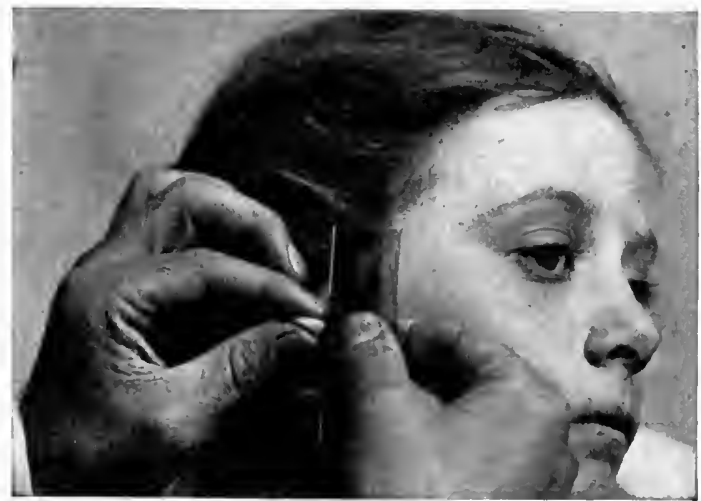

Fig. 39.-Obtaining the blood between slide and cover-glass. 
with children and nervous persons. More blood is easily obtained if desired to make a hemoglobin estimation, blood count, or Widal test. The lobe of the ear should be cleaned with soap and water, then with alcohol, and should be dried thoroughly. It is then grasped between the thumb and forefinger, the latter uppermost. The puncture is made preferably with a large straight Hagedorn needle and should be made quickly to the depth of about one-eighth inch. The first one or two drops should be wiped away and one chosen which is not too large.

The cover-glass, held by diagonal corners between the thumb and forefinger or, better, by means of forceps, is applied to the summit of the blood-drop and laid face down upon the slide. Care must be taken to touch only the top of the drop and not the skin, otherwise the blood smeared upon the cover-glass will have begun to coagulate around the margin and will not spread freely. It is a common mistake to take too large a drop of blood, and if the blood extends to the edges of the cover-glass and the center of the film has a ground-glass appearance it should be discarded. If the blood does not spread freely and evenly it is better not to use pressure, but the cover-glass may be gently pushed by the needle applied to its edge. Several preparations should be made to insure a good one, and each time the ear should be wiped free of blood and a fresh drop taken. A rim of vaseline around the edges of the cover-glass will preserve the specimen longer.

As simple as this seems, it requires considerable practice to obtain films in which the red cells lie side by side and not in rouleaux.

Hayem's method gives better results in the hands of the amateur. A square cover-glass is placed upon a slide in such a manner that one edge of the cover-glass coincides exactly with the edge of the slide near its middle. Held rather firmly in this position by the thumb and forefinger, the coapted edges are applied to the blood-drop, when the blood spreads evenly between the slide and cover-glass. When the blood has almost reached the opposite edge of the cover-glass, enough blood has been obtained. Two cover-glasses may be used instead of a 


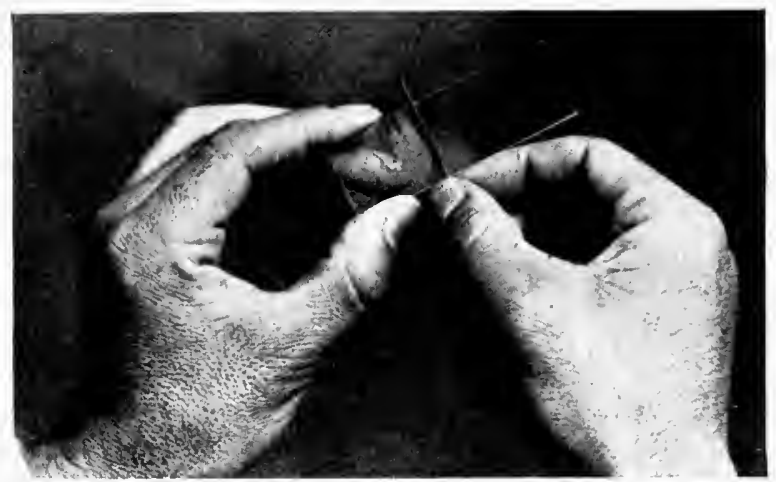

Fig. 40.-Making the spread.

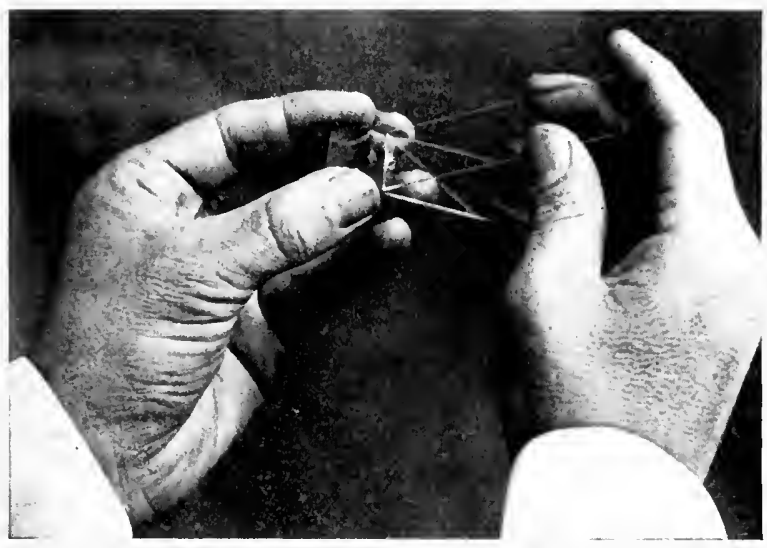

Fig. 4r.-Using another slide to spread the blood.

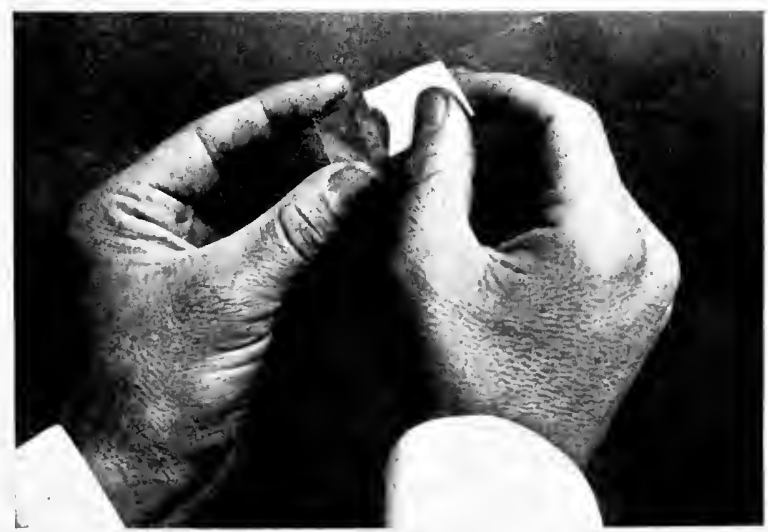

Fig. 42.-The cigarette-paper method. 
slide and cover-glass, and when ready to be examined one of the cover-glasses cemented at the corner or edge to a slide by means of Canada balsam.

The advantages of dried films over fresh preparations of blood are several. Cleanliness of the part from which the blood is taken and the size of the drop are not so important. This advantage is appreciated by the practitioner who often has to make the preparation in the remote corner of an ill-lighted cabin. The slides may be laid aside and examined at leisure, weeks or even months later. The differential leucocyte count can be made on the same slide.

It is not necessary that the region from which the blood is taken should be perfectly clean, but if prespiration is present this should be wiped off. The ear is held and the puncture made as described for wet films. The slide held in the right hand, is rested against the thumb and forefinger holding the lobe of the ear, and gradually lowered until it receives the drop of blood near one end.

The smear may be made by either one of three useful methods. The simplest is to hold the slide in the left hand and with the right lay the shaft of the needle across the drop of blood. After waiting a few moments for the blood to spread out between the needle and the slide, the needle is evenly and gradually drawn to the opposite end of the slide. Drying the film by rapidly waving it in the air preserves the form of the red cells.

Instead of the needle the end of another slide may be applied to the drop of blood so that the two slides meet at an angle of about 45 degrees; after waiting for the blood to spread along the edge of the slide, the upper slide is then drawn to the opposite end of the lower, and the film dried by waving.

Cigarette paper may be used as follows: Strips about threefourths of an inch wide are cut perpendicularly to the ribs of the paper; the end of one of these strips, the original machinecut edge, is applied to the blood-drop near the end of the slide, and after a few moments drawn to the opposite end of the slide. Other paper may be employed if cigarette paper is not available, but does not answer so well. 
If flies gain access to unstained films, they will rapidly devour the blood.

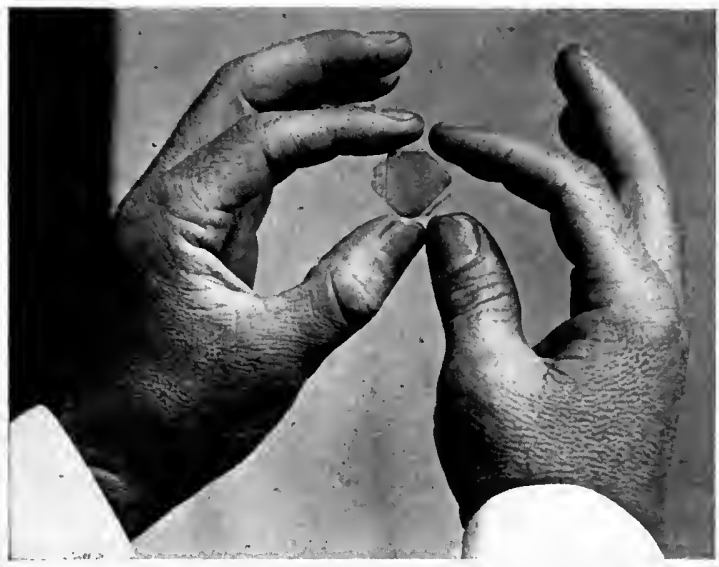

Fig. 43.-Making films upon cover-glasses.

Many staining methods have been proposed to demonstrate the malarial parasite in the blood. A common mistake for the

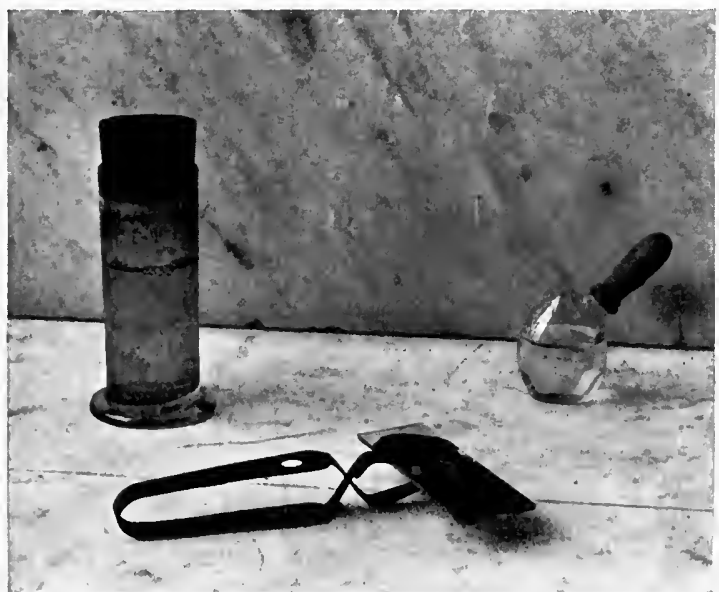

Fig. 44.- If the forceps are applied to the center of the slide the stain will not run off.

student to make is to attribute bad results to the stain, and to discard a method before he has become familiar with it. In 
no branch of pathology is attention to minute details of technic of greater influence upon results, and a method should be thoroughly mastered before passing judgment upon it.

Malaria parasites take basic stains, of which methylene blue is most frequently employed.

Wright's stain gives beautiful results but does not keep well after being mixed. It has the advantage also of being one of the quickest stains.

I am now using the following method. The films are fixed in absolute methyl alcohol for about half a minute. After drying, this stain is freshly mixed and poured on; allow it to remain ten or fifteen minutes:

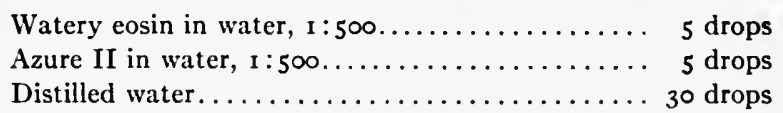

Wash in distilled water, dry with filter paper, place cedar oil directly on the film and examine.

The examination should be protracted for thirty minutes before being pronounced negative. While parasites, if present, are usually found within five or ten minutes, it is not uncommon to detect the first organisms after a search of twenty to thirty minutes.

Cedar oil may be removed from the film by wiping gently with a soft cloth moistened with xylol.

The "thick film process" is occasionally useful where the parasites are very scanty. The blood is smeared upon the slide in a much thicker layer than for other methods. After drying, a little distilled water is added and allowed to remain fifteen minutes, which causes the dissolution of the hemoglobin. After drying again the film is stained by one of the usual methods. While the outline of the red cells are still visible, the cells are transparent and parasites may be detected, though lying under several cells. The advantage of this method is that a much larger volume of blood may be examined in a shorter space of time than is the case with the thin film. The method described by Henson ${ }^{517}$ is valuable also.

Flagella are much more easily demonstrated in the gametes of 
the estivo-autumnal than of the tertian and quartan parasites. The crescent becomes oval and then spheric before exflagellation is observed. To encourage this process a number of rather thick drops of blood are placed upon a series of slides. The slides are then inverted, with the hanging drops over holes cut in blotting paper, moistened with water, and spread on a pane of glass. A series of moist chambers is thus made. A slide is removed at intervals of five minutes, the blood spread in the usual manner and stained. Exflagellation is also observed in preparations of fresh blood. The warm stage, breathing upon the specimen, and the addition of a little water are recommended to hasten the process.

Sources of Error.-In the examination of blood for malarial parasites there are several objects which may mislead. Pitfalls are probably more common in fresh blood than in stained films. Vacuoles and retractions of hemoglobin in red cells of fresh preparations are delusive and not infrequently mistaken for the young hyaline forms of the parasite. They are most common in the center of the cell, while parasites are found in any portion. Vacuoles are highly refractive, having well-defined, clear-cut edges; the margins of the parasites are dim and fade gradually into the substance of the red cells. The vacuoles may show slight changes of form, but do not possess true ameboid motion nor pigment. While the vacuoles are perfectly clear, the parasites show a slight opalescence. In stained specimens areas which do not take the stain may deceive. These areas may be of circular form in the center of the cell, or of ring form surrounding the center, or may be oval, horseshoe-shaped, crucial or irregular. When present they are apt to be abundant in some portions of the film and entirely absent elsewhere.

Crenation of red cells may present a hyaline appearance somewhat resembling an ameboid parasite. Their nature may be determined by changing the focus.

Bent or buckled corpuscles occasionally resemble crescents. The absence of pigment and the size of the corpuscle should, however, enable a distinction. Overlapping of the corpuscles produce a ring or crescent appearance which deceives the beginner. 
The object in stained spreads which proves most deceiving to the inexperienced is probably the blood platelet. These corpuscles may lie upon or within the red cells, in the center, near the periphery, or only partially enclosed by them. They are from one-seventh to one-half the size of a red blood-cell, and are round, oval, or elongated in shape. They are often of mulberry shape and reticular structure, and, with the Romanowsky class of stains, approach more nearly purple or lilac than the characteristic blue of the parasites. The margin is surrounded by a pale or unstained area resembling a halo. There is, of course, an absence of pigment and chromatine. Occurring in groups, as it frequently does, it has not rarely been mistaken for a sporulating body and isolated for a free spore. Bodies resembling free spores should, however, be disregarded for diagnostic purposes.

The nuclei of nucleated red corpuscles may be mistaken for parasites, but this should rarely occur if the morphology and staining reactions of both bodies are borne in mind.

Pigmented leucocytes have been mistaken for parasites, but the ameboid motion of the former in fresh specimens and the staining reactions in dried films should prevent confusion.

Hemokonia, or blood-dust, may be confused with free spores. They are small, highly refractive, micrococcus-like bodies averaging one-half micron in diameter and possessed of very animated motion. As stated, free spores should not be sought for diagnosis, and bodies resembling them should be ignored.

Extraneous dirt, leucocyte granulations, and stain precipitates must be carefully distinguished from pigment.

The amateur in examinations of malarial blood is apt to become decidedly discouraged, even when he has satisfactorily mastered the technic in the laboratory. Most students gain the impression that all that is necessary to find the parasites is to locate a malarial subject with any form of the disease and obtain the necessary blood at any stage of parasitic development, to stain it properly, and to inspect it under a high-power lens. Usually this is what he has been taught by text- 
books and by teachers, and when he fails to detect the characteristic organisms in undoubted cases of malaria he is disgusted. The results of such teaching throw discredit upon a discovery whose practical importance is unsurpassed in modern medicine.

To estimate the value of a report on the result of microscopic examination of the blood for malarial parasites it is always desirable to know something of the experience of the examiner. In addition to competence and proper technic there are several factors which influence the result of the examination for parasites. The most important of these are: (a) the previous administration of quinine; $(b)$ the stage of development of the organisms; $(c)$ the stage of the disease; $(d)$ the type of infection; $(e)$ race; $(f)$ locality; and $(g)$ individual circumstances.

(a) The previous administration of quinine, even in small quantities, renders it almost useless to examine the blood with the expectation of finding parasites. Even where the quantity of the drug is insufficient to have any effect on the symptoms, it will ordinarily cause a disappearance of the parasites from the peripheral circulation. The half-poisoned parasites which persist in some instances are frequently unrecognizable with reference to type.

(b) The quartan parasite is nearly evenly distributed in all its phases, from the youngest form to the sporulating body, throughout the superficial and deep circulation. Hence, when dealing with this type it makes little difference at what period the blood is examined. But with the estivo-autumnal organism it is only the early stages, the small rings, that are observed with any degree of frequency in the peripheral blood, and if the examination is made when the parasite has reached a later stage of development it will probably be missed. Instead of resembling the quartan parasite in habit of distribution it seems to imitate its more distant relative, the Leishman-Donovan parasite. Later phases of the simple tertian hematozoon are less commonly found in examinations of the peripheral blood than those of the quartan, but are much more frequently observed than those of the estivo-autumnal. Sporulating bodies of the quartan type are not uncommon in the cutaneous blood, 
while those of the tertian are much less common and those of the estivo-autumnal extremely uncommon.

The frequency with which crescents are detected varies within the broadest confines. In the experience of some they are rare, while other observers note them frequently in estivo-autumnal infections. Tertian gametes are not rarely observed in the blood of the superficial circulation, while quartan gametes are scarcely found.

(c) In acute untreated malaria the parasite can be detected at some stage of its growth in almost roo per cent. of cases. If not found at the first examination, as frequently occurs, subsequent searches are usually successful. On the contrary, in chronic malaria the parasites are far from constant during the stage of latency, and prolonged search may fail to reveal them during the relapse. Parasites are often absent from the peripheral blood of malarial cachectics. In the paramalarial syndrome, hemoglobinuric fever, the parasites, if present before onset, afterward disappear in the majority of cases.

$(d)$ The behavior of the different kinds of parasites in their various stages has been referred to. As a rule, the quartan parasite is most certainly found on first examination, the estivoautumnal least so, on account of its habit of resorting to the deep circulation when approaching maturity. It is very unfortunate for rapid diagnosis that the estivo-autumnal parasites are less readily detected than those of the benign infections, but, fortunately, are usually found easily in pernicious cases of estivo-autumnal infection.

(e) That malaria parasites are found less frequently and in smaller numbers in the superficial circulation of negroes with malaria $\mathrm{I}$ am convinced, though the difference is slight.

$(f)$ Along the northern borders of malarial distribution the parasites are probably more readily detected. This may be accounted for partially by the greater relative frequency of simple tertian infections. Whether the more northern negro shows the same scanty distribution of parasites in the peripheral blood as manifested by his southern brother I have no means of determining. It is surprising with what frequency crescents are found in higher latitudes in the blood of patients moving 
from highly malarial localities where crescents are not so frequently observed. Whether this is a conservative measure related to the relative rarity of anopheline mosquitoes cannot be stated positively, but it is known that the life histories of animals are, in some instances, peculiarly interdependent, especially in the case of parasite and host.

(g) Why it is that in certain unquestionable cases of malaria which have received no quinine and in which every condition seems favorable to finding the parasites prolonged and repeated examination shows none is not known, but such cases are sometimes encountered.

As before said, where the specific can be withheld and repeated examinations made by a competent microscopist if not found at the first examination, the parasite may be found in almost roo per cent. of cases of malaria. The question, which is of the utmost practical importance to the physician, arises: In what proportion of cases is the parasite to be found at a single examination? On this depends in great measure the practical value of Laveran's discovery, for in not a few cases in general practice for reasons of convenience the examination cannot be repeated; in others in which the diagnosis seems more or less clear urgent symptoms are demanding the specific. The two factors which, more than the others, influence the result are whether or not the patient has received quinine and the phase of parasitic development attained when the blood is withdrawn for the examination. Neither of these factors is always within the control of the physician who desires to make a diagnosis upon examination of the blood taken. when the patient first comes under his observation. Since a very large proportion of the malaria of the land is treated by country doctors, the practical value of a diagnostic test is largely in proportion as it is applicable by them.

With reference to the number of cases in which the parasite can be found at the first examination I will state my experience. From a record kept of the number of malarial cases which had taken quinine in some form before coming under observation it was learned that this reached something over 50 per cent. of the total number of cases treated. The diagnosis in these 
cases was obviously based upon the clinical history and the therapeutic test, since the search for parasites in the blood of persons having received quinine is so discouraging that this has not been done in routine work, but only in special cases. Allowing for errors in diagnosis might reduce this number to 50 per cent. A specimen of blood was always taken from malarial patients who had not recently received quinine when they came under observation for the first time, irrespective of the stage of the access. The blood from frank cases only has been included, no cases of atypic or latent malaria or of cachexia figuring in the result. Parasites were found in approximately two-thirds of the cases and the examination was negative in about one-third. No difference as to clinical course, severity, or the efficacy of quinine could be detected between the cases in which parasites were found and those in which none were observed. From this experience it may be inferred that in localities in which half of the malarial subjects take quinine in some form before consulting a physician the parasite can be detected at a single examination of the peripheral blood, taken at random with respect to the stage of parasitic growth, in approximately onethird of the cases only. The prevalence of self-medication with quinine products depends largely upon local custom and upon the energy of the patent-medicine industry.

My experience being somewhat at variance with the conventional text-book teaching, I feel it incumbent to cite the experience of others in this matter of the most vital interest.

Craig ${ }^{491}$ says, "Often if the blood be examined but once none at all will be found."

Fornario ${ }^{518}$ observes that the parasites are missed with extreme frequency, and Soliani, ${ }^{520}$ in an analysis of $6 \mathrm{I}_{2} 2$ cases under his care, says that in many cases the first examination was negative.

McElroy ${ }^{519}$ says, "I have been struck with the frequency with which I have been unable to find parasites in cases where I am strongly impressed with the malarial nature from the clinical history."

$\mathrm{Plehn}^{521}$ states that the parasites are frequently lacking in the malaria of natives, or at least they are not found in the peripheral 
blood, where the temperature curve is typic and pigmented leucocytes indicated malaria.

The experience of Ewing ${ }^{72}$ at Camp Wikoff is interesting. "In the 605 cases of malaria the plasmodia were found in the blood of 335 cases, while in 270 cases the diagnosis was based upon the clinical history and the discovery in the blood of evidences of malarial infection. The evidences of malarial infection in the blood consisted ( $\mathrm{I}$ ) usually in the presence of intracellular bodies so much affected by quinine that their exact type could not be positively determined; or (2) in the presence of typic pigmented leucocytes; or (3) in chronic cases of distinct clinical character in the presence of marked anemia."

Leonard Rogers, ${ }^{23}$ than whom there is no more competent observer, says: "As long ago as 1896 I showed from an examination of roo cases of consecutive malarial fever before the administration of quinine that in only one-third of them could the malarial parasite be found by means of a prolonged search of a single blood film."

Delaney' ${ }^{522}$ experience is even more disheartening. He concludes: "I think that I shall be supported by most competent observers in India that this ( 17 per cent.) about represents the percentage of success in finding malarial parasites in the malarial fevers of India at a single examination, and on this point both text-books and writers on the subject are, I consider, very misleading."

Such quotations from practical workers and deep observers could be multiplied, but could add no further weight to the authority of those cited.

The above statements are not meant to cast the slightest doubt upon the etiologic rôle of the parasite of malaria, or its presence in every case of acute untreated malaria, or its great diagnostic value under certain circumstances, but are intended to demonstrate that the detection of the parasite is subject to several conditions. In probably no other disease, associated with a pathognomonic sign which can be elicited in almost 100 per cent. of cases, is its detection so dependent upon conditions beyond the control of the physician.

What is the value of a positive result of examination of the 
blood for malarial organisms? This parasite is thoroughly established as the sole cause of malaria, and its pathogenic reputation has never been marred by rumors of etiologic association with other diseases. But is the parasite, when present, responsible for the symptoms which instigate the blood examination?

In localities where a considerable per cent. of the inhabitants carry malaria germs in their blood without showing malarial symptoms it is manifestly possible that parasites might be found in the blood of such inhabitants during the course of other ailments. And such is actually the case in certain regions with a very high endemic index, to such an extent, indeed, that the widely experienced Albert Plehn, ${ }^{36}$ in Cameroon, declared that the presence or absence of malarial parasites in the blood of the West African coast negro is of no diagnostic value.

In cases of coma in which malarial parasites are detected and which give a history of exposure to violent heat or of the abuse of alcohol, it is not infrequently difficult to determine the part played by the parasite. In cases of coma accompanied by malarial parasites in the blood and albumin and casts in the urine the diagnosis may be obscure. Fever occurring during the puerperium in subjects of former malaria will make the thoughtful physician uneasy for a short while at least, even if parasites are found on blood examination.

These are mainly problems, however, which are involved in other fields of diagnosis and serve to impress the fact that complications must be excluded or, if found, weighed. While these contingencies should not be lost sight of, in the immense majority of cases in this country active forms of the malarial parasite detected in the blood are responsible for the symptoms which bring the patient under the care of the physiçian or which prompt the physician to make the examination.

It will be noted that the word active is emphasized. What, then, is the value to be attached to the discovery of gametes alone?

Formerly it was believed that the sole function of these peculiar bodies was the perpetuation of the species through the mosquito cycle. Under this limited view the detection of gam- 
etes alone was on a diagnostic par with anemia and splenomegaly, sequelæ of malaria, and not necessarily proof of existing malaria, even latent. Since it has become known however, that under certain not well understood conditions the macrogametes can immediately, by the process of parthenogenesis, give rise to pyrogenic parasites without undergoing the mosquito cycle, our views must be modified, and these forms must be regarded clinically as the parasites of latent malaria. Relative to active malaria, they may be looked upon as evidences of past and potential, but not necessarily of present, active malaria.

What is the diagnostic value of a negative result?

I can by no means agree with those who maintain that such a result positively excludes a diagnosis of malaria. The failure to find parasites in the blood of a single film taken without reference to the period of the paroxysm, while of some value, is not conclusive, and if the patient has recently received quinine is absolutely worthless. On the other hand, if the blood of a patient who has not recently taken quinine be examined repeatedly by a competent person with the result that no parasites are found, it is very strong evidence against malaria. The diagnostic value, then, of a negative finding depends upon the presence or absence of the conditions which have been enumerated, the chief of which is the administration of quinine.

When the examination of the peripheral blood is negative, puncture of the spleen has been advised, as the parasites in all stages are easily detected in the blood of this organ. This procedure, however, is attended with some degree of danger, especially of hemorrhage, and should be resorted to only in cases where an immediate diagnosis is imperative. It has been estimated that the mortality of aspiration of the spleen is I $1 / 2$ per cent. ${ }^{41}$

Upon failure to discover parasites in the blood there are two other blood signs which must be considered. These are the presence of pigment and a relative increase in the large mononuclear leucocytes. These signs are termed subsidiary evidences of malaria, because, being secondary in diagnostic importance to the parasites, they are generally called upon only in the absence of the latter. 
Melanin is pathognomonic of malaria, and its presence is not contingent upon the stage of development of the parasites or upon the previous administration of quinine. Theoretically, therefore, it should be of the greatest significance in the diagnosis of malaria. There are, however, certain circumstances which detract from its practical value. Free pigment, or that lying upon red blood-cells, should be ignored in the diagnosis, as it cannot be distinguished from adventitious detritus. Within the large mononuclear leucocytes, the leucocytes in which it is most frequently found, it must be carefully distinguished from the minute pigment-like granulations which may occur normally in these cells to the number of one, two, or three to each cell. This requires a considerable degree of experience. Coarse granules of pigment are much more readily recognized, especially in fresh blood. In stained films precipitates may prove very confusing. Pigment may persist for two or three days after the last paroxysm in tertian and quartan infections, and for a much longer period in estivo-autumnal. It is more valuable as a diagnostic sign of chronic malaria than of acute.

The second subsidiary sign of malaria, a relative increase of the large moncnuclear leucocytes, is under some conditions a valuable aid to a diagnosis. The proportion of large mononuclear elements in the differential count is modified by certain factors which detract somewhat from its value.

In early childhood there is normally an increase of mononuclear leucocytes; hence this sign need not be sought for in the malaria of young children.

As with other diagnostic evidences of malaria, this sign is unfortunately more constant and more marked in tertian and quartan infections than in estivo-autumnal.

The increase of the large mononuclear leucocytes in malaria is generally in inverse proportion to the height of the temperature, being most decided in the interval, and may be absent during pyrexia. An increase may be wanting also early in first attacks.

When there is evidence of leucocytosis the differential count alone must not be relied upon, since an absolute increase may exist under these circumstances when the differential count 
will show only a small per cent. Here the absolute count must be made also.

In differentiating malaria from typhoid fever the differential count is of value only in the first two weeks of a fever, since after that time the relative proportions of the leucocytes are similar in the two diseases.

Notwithstanding its difficulties, the differential leucocyte count, made by an experienced examiner, may render important aid in the diagnosis of malaria where the parasite cannot be detected, and a mononuclear leucocytosis reaching i 5 per cent. must be regarded as strong evidence of malaria.

Besides the presence of pigment and a large mononuclear increase there is another point ascertained by microscopic examination of the blood; this is the presence or absence of leucocytosis. Between malaria and typhoid fever this point has no differential value, and it will be remembered that a leucocytosis is frequent in pernicious malaria. It is, however, in septic conditions which sometimes so closely resemble malaria in which a marked leucocytosis may serve to exclude malaria.

The Therapeutic Test.-Here diagnosis and treatment meet very closely, the former encroaching somewhat upon the field of the latter, the diagnostic test often becoming a therapeutic and life-saving measure. The therapeutic test is of especial value in cases which have already had insufficient quinine, thereby causing only the disappearance of the parasites from the superficial circulation and distortion of the fever curve. In these cases it has at least as much standing in clinical medicine as antisyphilitics in obscure cases thought to be syphilis or antitoxin in cases of suspicious angina in which a bacteriologic examination is impossible. When properly applied it can hardly be productive of harm.

A fever which resists quinine is not a malarial fever. In order to test the resistance of fever to quinine the drug must be continued for a sufficient length of time and in proper doses at suitable intervals, and, what is most important, it must be absorbed.

The maximum period of resistance of malaria to quinine is ordinarily stated as four days. As far as my observations go, 
they tend to show that in many cases the fever is broken by the end of thirty-six hours, in at least half of the cases in fortyeight hours, and in three-fourths the cases in sixty hours. It is highly probable that in cases of malaria persisting longer than four days the specific is not being absorbed. A case is recalled in which the fever continued notwithstanding the administration in capsules of 24 grains of a soluble salt of quinine during the twenty-four hours for nearly six days. Parasites having been found before the quinine was begun, the drug was then given in solution, when the fever responded during the seventh day. There had been no evidences of cinchonism until the solution was employed. Cinchonism, however, is not a guide in the employment of the therapeutic test; the specific is directed toward the parasites and not toward the patient, and patients manifest various degrees of sensitiveness toward quinine.

Owing to the conditions under which the therapeutic test is usually employed it is better to use moderate doses at regular intervals during both day and night. Three or four grains every three hours are sufficient. Pills and tablets of quinine should never be relied upon; the result may be not only misleading but dangerous. Capsules, if fresh, are usually satisfactory; a few pin punctures in each end aids solution. Where the fever persists and there is reason to believe that the medicine is not being absorbed it should be given in solution or even intramuscularly.

The period of apyrexia following a single dose of quinine corresponds closely to the parthenogenetic cycle. The conquest of the schizonts seems to be a signal for the macrogametes to lay aside the conventionality of slow sexual reproduction and to conscript recruits rapidly by parthenogenesis.

Not every fever which discontinues after the administration of quinine can be considered malarial, since such an occurrence is occasionally coincidental. Furthermore, it is well known that quinine has no little antipyretic influence upon certain conditions, particularly septic.

It is probably superfluous to say that the diagnosis of malaria does not always consist alone in the mere mechanic application 
of a single test, but that in some cases the keenest clinic judgment is required. Of the several diagnostic signs which we possess each is valuable and each has its limitations.

It should be a routine practice to take a specimen of blood from each fever patient.

In dealing with a disease in which the blood examination affords pathognomonic evidence and for which we possess a specific the dilemma is often faced, where the examination of the first specimen of blood is negative, of having to decide whether it is best to wait a few hours for an absolutely certain diagnosis or to take advantage of every hour and begin the treatment immediately. If quinine has already been taken the chances are that further examinations would also be negative, and the better course would be to proceed with the specific. If quinine has not already been taken and the symptoms are not urgent the case may be treated symptomatically for a little while, during which time the blood is examined at appropriate intervals.

In hospital practice the practical value of the blood examination for malaria parasites is inestimable; in general practice, especially in the rural districts, its value is more limited. In general practice, especially in the country, the therapeutic test is of great value; in hospital practice it is less often justifiable.

\section{DIFFERENTIAL DIAGNOSIS}

Abscess of the Liver.-Septic conditions are very often diagnosed as malaria; this is especially true of hepatic abscess. There are two classes of cases of abscess of the liver that may be difficult at first examination to distinguish from malaria: first, where the local symptoms are absent or not well defined; second, where there is enlargement of both liver and spleen and a history of both dysentery and malaria. The fact that these patients have usually been drenched unsystematically with quinine may complicate the diagnosis. In typic cases of hepatic abscess there is usually a history of dysentery, and amebæ may be present in the feces. There is usually a dragging pain in the liver, sometimes referred to the right shoulder, increase 
of liver dulness, and tenderness on pressure. The spleen is not necessarily enlarged. The temperature does not often rise high and there is apt to be profuse perspiration, especially during sleep. On microscopic examination of the blood there is usually a leucocytosis to be found, though this is wanting in a few cases, and its absence should not be taken to exclude abscess. There are neither parasites, pigment, nor a relative increase of the large mononuclear leucocytes. Exploratory aspiration is valuable in some cases. Jaundice is a very variable symptom and may be misleading.

Infective endocarditis may present periodic paroxysms of chill, fever, and sweat. The physical examination of the heart and the microscopic examination of the blood should establish the diagnosis.

Puerperal Septicemia.-Women who have had malaria during pregnancy are prone to suffer relapses during the puerperium. In this condition malaria is not infrequently atypic; the first or third stages of the paroxysm are sometimes lacking and complete intermission of temperature is often wanting. The following may serve to differentiate typic cases of malaria and puerperal sepsis:

\section{Malaria}

Onset from a few hours to twenty-one days after labor.

Often a history of malaria.

Temperature curve more or less typic.

Symptoms decline with temperature.

No local symptoms.

Blood examination positive.

Therapeutic test positive.

\section{Puerperal Seplicemia}

Rare after the fifth day.

Malarial history usually absent.

Irregular.

No relation between symptoms and temperature.

Local symptoms present.

Negative.

Negative.

The so-called urethral fever may be accompanied by paroxysms somewhat resembling those of malaria. I have recently seen a case in which the introduction of a steel sound every other day was accompanied for a short time by corresponding paroxysms not due to malaria. The differentiation from malaria should present no difficulties.

Perinephric abscess, pyelitis, cholecystitis, and other septic processes may be associated with fever which bears a more or 
less close resemblance to that of malaria. Local symptoms, the blood examination, and the therapeutic test rarely leave the diagnosis in doubt but a short while.

In proportion to the reliance placed upon symptomatology in the differentiation of typhoid and malarial fevers so frequently will mistakes occur. Chills, continued fever, bronchitis, enlarged spleen, slight tenderness and gurgling in the right iliac fossa, tympanites, diarrhea, the Diazo reaction, delirium, and the typhoid state may occur with either disease. Herpes is strongly indicative of malaria and rose spots of typhoid fever, but these spots are more frequently absent than present in the typhoid fever of warm countries.

A correct diagnosis must rest upon the results of the examination of the blood and the therapeutic test.

Tuberculosis is sometimes similar in its course to malaria. It is especially so in the early stage. when the local signs and symptoms are ill defined or absent and the bacillus cannot be detected, and in the stage of secondary infection when septic symptoms supervene. Miliary tuberculosis has not infrequently been mistaken for malaria. For the diagnosis between tuberculosis and malaria the microscopic examination of the blood and sputum, the physical examination and therapeutic test are usually ample.

Influenza has sometimes been confused with malaria. If the epidemic occurrence, different seasonal prevalence, catarrhal and other symptoms are insufficient upon which to make a diagnosis, the absence of characteristic blood findings is generally conclusive.

Yellow fever in some cases so closely resembles the so-called bilious remittent fever that in regions where both diseases occur the differential diagnosis by clinical history alone is impossible. In such instances the microscope becomes an instrument of the greatest good not only to the individual, but to the community.

The frequency with which dysentery is associated with malaria as a complication and as a sequel renders the microscopic examination of the blood very important in these cases.

Patients with syphilis manifesting quotidian fever not infre- 
quently receive quinine instead of antisyphilitics. The microscope, the therapeutic test, the Wassermann reaction, and the history should form the basis of the diagnosis.

Before the geographic distribution of the hook-worm and its importance in the production of anemia became recognized, uncinariasis was not distinguished from chronic malaria and cachexia. The detection of the ova in the feces, the presence of eosinophilia, the absence of parasites and the subsidiary evidences of malaria in the blood render such a mistake at the present time inexcusable.

Leukemia must occasionally be taken into consideration in the differential diagnosis of malaria, in which case the microscopic examination of the blood is absolutely essential.

The differentiation of Banti's disease from chronic malaria and cachexia may be extremely difficult. We will not solve the mysteries of splenomegaly until we learn a safe method of obtaining blood from the spleen.

\section{THE DIAGNOSIS OF PERNICIOUS MALARIA}

In the immense majority of cases examination of the peripheral blood will reveal the presence of the organisms. The value of this is inestimable and is paralleled only by the importance of making blood examinations in all cases. It is not extremely uncommon in our cities for subjects of pernicious attacks found in coma to be taken to the police station instead of the hospital and the true condition not suspected until the patients fail to "sober up" in due time, when it is usually too late for treatment to avail.

In cases showing the presence of parasites, complications must be rigidly excluded. In some cases this is attended with difficulties.

In comatose malaria, besides the evidence obtained by an examination of the blood, a history of exposure to or attacks of malaria, the general appearance and age of the patient, the absence of atheroma, the early elevation of temperature, and perhaps the enlargement of the spleen and slight jaundice should exclude cerebral hemorrhage. The differentiation of 
malarial coma from sunstroke is often hard; in fact, the two not infrequently coexist, in which case it may be impossible to apportion the etiologic share of each in the clinical picture. Cardamatis $^{523}$ states that in this type of pernicious malaria coma is the dominating symptom, while in sunstroke are observed coma, convulsions, delirium, and hyperpyrexia. Uremic coma may simulate that due to malaria. Unfortunately, the urinalysis throws no light on the diagnosis, as in both conditions we may find albumin and casts. The blood examination, the temperature, and the anamnesis serve to make the diagnosis. For the differentiation of alcoholic from malarial coma the blood examination is essential. The history may be of value, but the odor of the breath may be misleading. To discriminate between malarial coma and diabetic coma the presence of the parasites, on one hand, and of glycosuria, on the other, are sufficient. In differentiating between the various comas with reference to malaria two points should be remembered: First, that comatose malaria may occur in persons with the odor of alcohol on the breath, and, secondly, that coma from causes other than malaria may attack malarial cachectics. To distinguish epilepsy, opium poisoning, tetanus, and meningitis from pernicious malaria should rarely present difficulties if the blood is examined.

Algid attacks sometimes resemble perforation of typhoid, or gastric ulcers, or rupture of the spleen. The microscope and the local symptoms should render the diagnosis certain. The cases resembling appendicitis and peritonitis have been mentioned; here, again, the microscopic examination of the blood may save lives. In countries in which cholera is endemic, the diagnosis between this disease and the choleraic type of pernicious malaria was formerly difficult or impossible. Laveran's discovery has removed this difficulty and rendered possible a diagnosis of the utmost importance. The finding of the hematozoa differentiates the hemorrhagic, bilious, and typhoid types from typhoid and yellow fevers. 


\section{CHAPTER VI}

\section{PROGNOSIS OF MALARIA}

Spontaneous Recovery.-While physicians in malarial regions often see patients whose paroxysms, typic and with characteristic periodicity, have ceased without medication or after nothing but a purgative dose, it is doubtful whether this cessation may with propriety be termed a cure. In the majority of instances relapses follow at shorter or longer intervals. It is better, therefore, for practical purposes to consider this but a transition from active malaria to latency. The greater frequency with which gametes are found after the so-called spontaneous recovery justifies this assumption.

Spontaneous cure occurs more frequently in tertian and quartan infections. This statement applies merely to the temporary cessation of paroxysms and not to the tendency to relapse.

It is more frequently observed in the negro than in the white race, permanent cures occurring not rarely in the former race in the absence of all medication.

Sex may exert a slight influence upon the tendency to spontaneous recovery, the female, on account of less severe exposure to deleterious influences, probably manifesting a greater disposition.

The discontinuance of paroxysms may be sudden or more often gradual, the accesses becoming less severe or the interval longer, or in infections with more than one generation of parasites one may be suddenly destroyed, the others later.

Prognosis.-This is influenced to some extent by locality. It is manifest that in regions where only the tertian and quartan infections are prevalent the mortality is less than where severe estivo-autumnal fevers are widespread. There is, furthermore, quite a difference in the mortality rate in countries where the estivo-autumnal infections are equally distributed.

Race as a factor in the mortality of malaria has already been dealt with. 
A majority of deaths from malaria occur in children. There is no doubt but that many children die of malaria which has not been diagnosed in time. In the young, pernicious symptoms, especially cerebral, are prone to supervene, or the attack may be followed by extreme anemia and dropsies. Malaria is likewise much more serious in advanced age than in the intermediate ages.

Occupation and social conditions play a part in prognosis. Excessive toil and exposure may render pernicious attacks otherwise benign, and timely treatment, usually resorted to by the better classes, enhances the chance of recovery.

The outlook is probably more favorable in attacks occurring without the malarial season than within.

Manifestly the condition of the patient with reference to the results of previous disease is of importance. Anemia, alcoholism, dysentery, and other conditions not fully recovered from, contribute gravity to the prospect.

The type of malarial infection is of the greatest importance. In the tertian and quartan types it is only very rarely that serious symptoms result. It is not yet certainly known in which variety of estivo-autumnal infection the prognosis is most grave.

Postponement and anticipation of the paroxysms were formerly regarded as favorable and unfavorable, respectively. However, owing to the irregularity of the estivo-autumnal fevers, these can be said strictly to be properties of tertian and quartan infections only, and are consequently of little prognostic import. Violent headache, somnolence, sighing respiration, slight mental aberration, defective articulation and vision, cold surface, and rapid, feeble pulse are some of the symptoms which forebode evil.

The prognostic value of the microscopic examination of the blood is limited. While, as a general rule, the severity of the attack is in proportion to the number of parasites, these are sometimes scanty in the peripheral circulation even in grave cases. Sporulating and advanced stages of estivo-autumnal parasites are rarely seen in the superficial blood except in extremely severe cases.

While in tertian and quartan infections a paroxysm may be 
predicted approximately from the results of blood examination, such an attempt with estivo-autumnal malaria may prove misleading. An impending paroxysm dependent on mature parasites in the visceral circulation cannot be foretold.

Intercurrent diseases complicating malaria aggravate the prognosis. This is especially the case in chronic malaria and cachexia, with which pneumonia, dysentery, and other diseases form frequently fatal associations.

The gravity of pregnancy as a complication of malaria has been considered.

In nephritis of malarial origin the prospect is, as a rule, good. If, however, the patient is repeatedly subjected to malaria or other harmful influences the prognosis is not propitious.

The prognosis of the nervous sequelæ is ordinarily favorable. The various paralyses and mental symptoms are generally transitory, but may occasionally become persistent. Bulbar symptoms are usually slow to disappear.

The course of chronic cachexia may be extended for years; acute cachexia runs a more rapid course. In mild cases a change of climate and tonic treatment do a great deal for the patient; advanced cases rarely recover. Death may occur from exhaustion, but is more commonly due to pernicious malaria and to complications, of which the most frequent are pneumonia and nephritis. Hence the danger to the cachectic is not confined to the malarial season, but he is in danger throughout the entire year.

Mortality.-The true mortality of malaria is difficult to estimate. While statistics are not lacking, the different conditions under which they are complied must be considered, some being from charity hospitals, some from private practice, some from military practice, from various localities, etc. It is, furthermore, undoubtedly true that a considerable proportion of malarial cases does not come to the notice of physicians. The variety of forms which malaria assumes is another obstacle. It is probable that many cases ascribed to complications, fancied or real, are due to malaria.

Bearing these points in mind, the following figures are presented, showing a mortality of 2.89 per cent.: 


\begin{tabular}{|c|c|c|c|}
\hline Author & Locality & Cases & Deaths \\
\hline Laveram $^{23}$. & Turko-Russian War & I 40,000 & 1,092 \\
\hline Laveran ${ }^{23}$. & Constantine ....... & $\mathbf{I}, 3$ Io & o \\
\hline Laveran ${ }^{23}$ & Italian Army . . . . . . . & 4,856 & 13 \\
\hline Schellong ${ }^{160}$. & New Guinea.......... & I,954 & 22 \\
\hline $\operatorname{Ross}^{524}$ & Greece............. & 960,048 & 5,9 I 6 \\
\hline $\operatorname{Ross}^{524}$.. & Hong Kong........... & $7,35^{2}$ & 984 \\
\hline Ewing ${ }^{72}$. & Camp Wikoff.......... & 605 & 39 \\
\hline $\operatorname{Smart}^{526}$ & Civil War........... & $\mathbf{I}, 373,355$ & I 5,423 \\
\hline Travers ${ }^{48} \ldots$ & Malay States.......... & 3,397 & 348 \\
\hline Terburgh ${ }^{461}$. & Dutch Indies......... & 2,308, I 28 & I I 4,490 \\
\hline Cardamatis $^{119}$. & 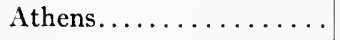 & 22,6 I 8 & I 5 \\
\hline $\mathrm{Koch}^{444} \ldots$ & Grosseto........ & $28 I$ & $\circ$ \\
\hline $\mathrm{Koch}^{79} \ldots$ & East Africa....... & 63 & 2 \\
\hline Hagen $^{527} \ldots . . \ldots \ldots$ & Papua......... & 301 & 23 \\
\hline British Colonial Reports ${ }^{528}$.. & British Colonies .. & I 2,6 I 7 & 618 \\
\hline Wright ${ }^{448}$ & British Malaya..... & I 7,648 & 680 \\
\hline $\mathrm{Haw}^{529} \ldots$ & Baberton.......... & 449 & 14 \\
\hline Hope $^{450}$. & North Bengal. & $\mathbf{I}, 784$ & 0 \\
\hline Laveran ${ }^{530}$ & Algiers.......... & 98,774 & 7,432 \\
\hline Gorgas $^{170} \ldots$ & Panama.......... & 1,055 & 5 \\
\hline Erni $^{531} \ldots \ldots \ldots \ldots$ & Dutch Indies....... & I 16,879 & $73 I$ \\
\hline $\begin{array}{l}\text { United States Marine Hos- } \\
\text { pital }^{532} \ldots \ldots \ldots \ldots \ldots \ldots \ldots\end{array}$ & General... & $6,6 \mathrm{I} 8$ & 20 \\
\hline Various Hospital Reports... & Southern States... & I,294 & 30 \\
\hline $\begin{array}{c}\text { German Protectorate Re- } \\
\text { ports }^{533} \ldots \ldots \ldots \ldots \ldots \ldots \ldots\end{array}$ & German Protectorates... & 5,003 & 32 \\
\hline \multirow[t]{2}{*}{ Malaria Society.47. } & \multirow[t]{2}{*}{ Italy $\ldots \ldots \ldots \ldots \ldots$} & 22,792 & I 20 \\
\hline & & 5, IO9, О०I & I 48,055 \\
\hline
\end{tabular}

Prognosis of Pernicious Malaria.-The prognosis of pernicious malaria is extremely grave. It depends upon the physical condition and age of the patient, the type and severity of the attack, and the promptness and efficiency of the treatment. Anemia from previous attacks of malaria or other cases, alcoholism, or organic disease of important viscera, add to the gravity of the case. The cerebral types are less serious in the young and vigorous, very fatal in the aged. As a rule, patients seen early and treated skilfully and energetically have a better chance for life, but many cases end fatally in spite of the best and most timely treatment.

The number of parasites in the peripheral circulation is not 
always a reliable guide as to the severity of progress of the attack. With apparent amelioration of the symptoms the physician should be circumspect in his prognosis and bear in mind the possibility of further paroxysms.

In my opinion the algid type is the most lethal, the typhoid and the dysenteric least so.

The following list of 27,039 cases of pernicious malaria, compiled from the literature, shows a mortality of 26.6 per cent. The first column of figures shows the number of cases, the second the number of fatalities:

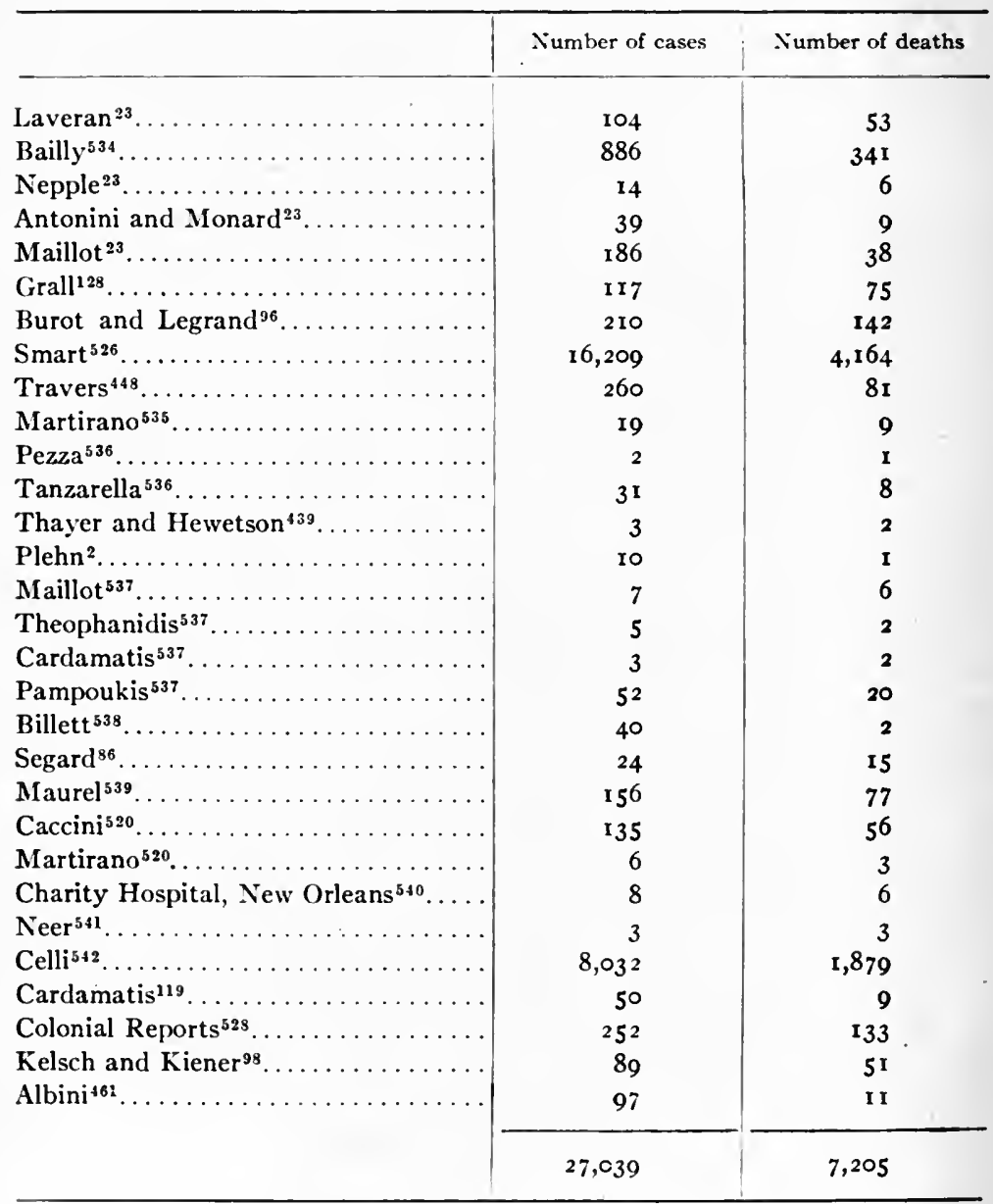


Six hundred and eighty-nine cases of specified type give the following respective mortalities:

\begin{tabular}{|c|c|c|c|c|c|}
\hline & Comatose & Delirious & Algid & Typhoid & Ataxic \\
\hline $\begin{array}{l}\text { Maillot }^{23} \ldots \ldots \ldots \\
\text { Schellong }^{160} \ldots \ldots \ldots \\
\text { Plehn }^{2} \ldots \ldots \ldots \ldots \\
\text { Maillot }^{537} \ldots \ldots \ldots \\
\text { Theophanidis }^{537} \ldots \\
\text { Cardamatis }^{537} \ldots \ldots \\
\text { Pampoukis }^{523} \ldots \ldots \\
\text { Billet }^{538} \ldots \ldots \ldots \ldots \\
\text { Maurel }^{539} \ldots \ldots \ldots \ldots \\
\text { Neer }^{541} \ldots \ldots \ldots \ldots\end{array}$ & $\begin{array}{c}77-14 \\
7-6 \\
10-I \\
\ldots \ldots \ldots \\
\ldots \ldots \ldots \ldots \\
\ldots \ldots \ldots \ldots \\
52-20 \\
\ldots \ldots \ldots \\
297-103 \\
3-3\end{array}$ & 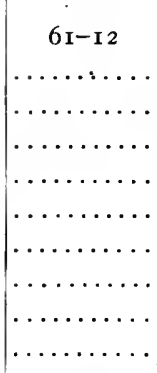 & $\begin{array}{c}48-12 \\
\ldots \ldots \ldots \\
\ldots \ldots \ldots \\
7-6 \\
5^{-2} \\
3^{-2} \\
\ldots \ldots \ldots \\
\ldots \ldots \ldots \\
78-23 \\
\ldots \ldots \ldots \ldots\end{array}$ & 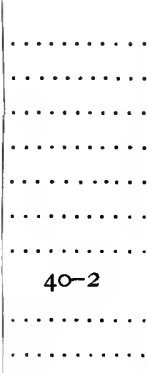 & $\begin{array}{c}\ldots \ldots \ldots \ldots \\
\ldots \ldots \ldots \ldots \\
\ldots \ldots \ldots \ldots \\
\cdots \ldots \ldots \ldots \\
22-17 \\
\ldots \ldots \ldots \ldots\end{array}$ \\
\hline & $\begin{array}{c}428-\mathrm{I} 47 \\
34 \text { per cent. }\end{array}$ & $\left\{\begin{array}{c}6 \mathrm{I}-\mathrm{r} 2 \\
20 \text { per cent. }\end{array}\right.$ & $\begin{array}{c}14 \mathrm{I}-45 \\
32 \text { per cent. }\end{array}$ & $\begin{array}{c}40-2 \\
5 \text { per.cent }\end{array}$ & $\mid \begin{array}{c}22-17 \\
77 \text { per cent. }\end{array}$ \\
\hline
\end{tabular}




\section{CHAPTER VII}

\section{PROPHYLAXIS OF MALARIA}

Prophylactic measures may be directed against the destruction of the malaria parasites within the body of man, the destruction of the mosquitoes which are capable of transmitting the parasites, and the prevention of mosquitoes gaining access to man. The parasite may be opposed either in man or in the mosquito. The mosquito may be combated either in its aquatic or in its aerial stage. Prophylaxis may be conducted by a community or by an individual, may be public or private, offensive or defensive.

As is well known, malaria is now almost or entirely absent from regions in which it was formerly very prevalent, and in other places is rapidly diminishing. In the regions in mind the change was independent of designed efforts for the eradication of the disease; in fact, it occurred in most instances before the discovery of either the malaria parasite or of the rôle of the mosquito in the dissemination of the disease, and was an unexpected result of the progress of civilization.

With the tediously attained and in many cases incomplete results of this unconscious prophylaxis are in decided contrast the consequences of well-organized and vigorous sanitary measures directed toward the prevention of malaria. Many instances could be adduced where within a comparatively short space of time highly malarial localities have been almost completely freed from the disease, but a few examples will suffice.

One of the most successful campaigns against malaria was that at Ismailia, a town of about 8,000 inhabitants near the middle point of the Suez Canal. The town was founded in I 862 , and was celebrated for its salubrity until 1877 , when malaria was introduced and spread rapidly; in I 886 nearly all the inhabitants were attacked. In I 901 the president of the Suez Company, learning something of the results of modern prophylactic methods, dispatched Pressat, a member of the 


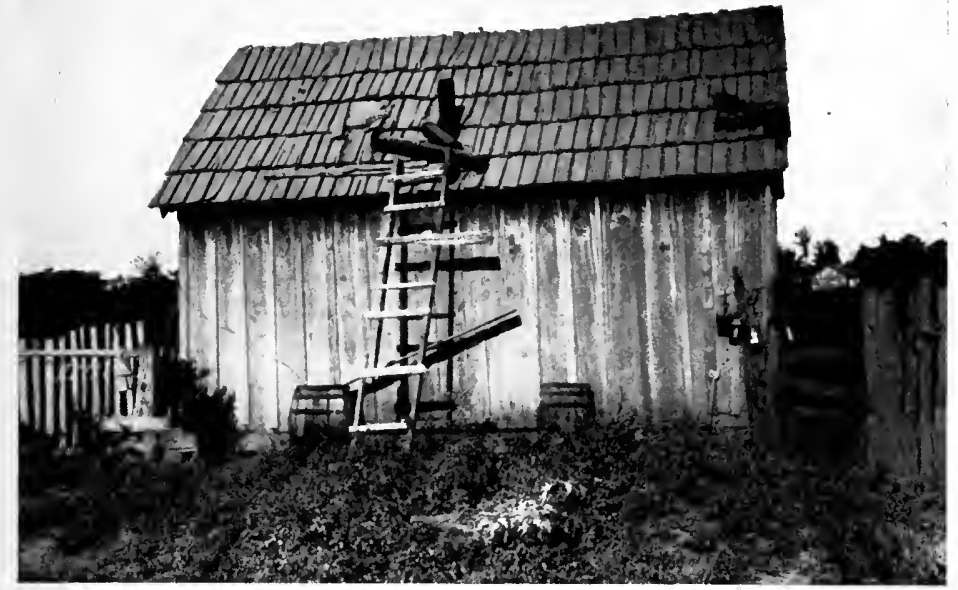

Fig. 45.-The barrels and one of the buckets contained many larva.

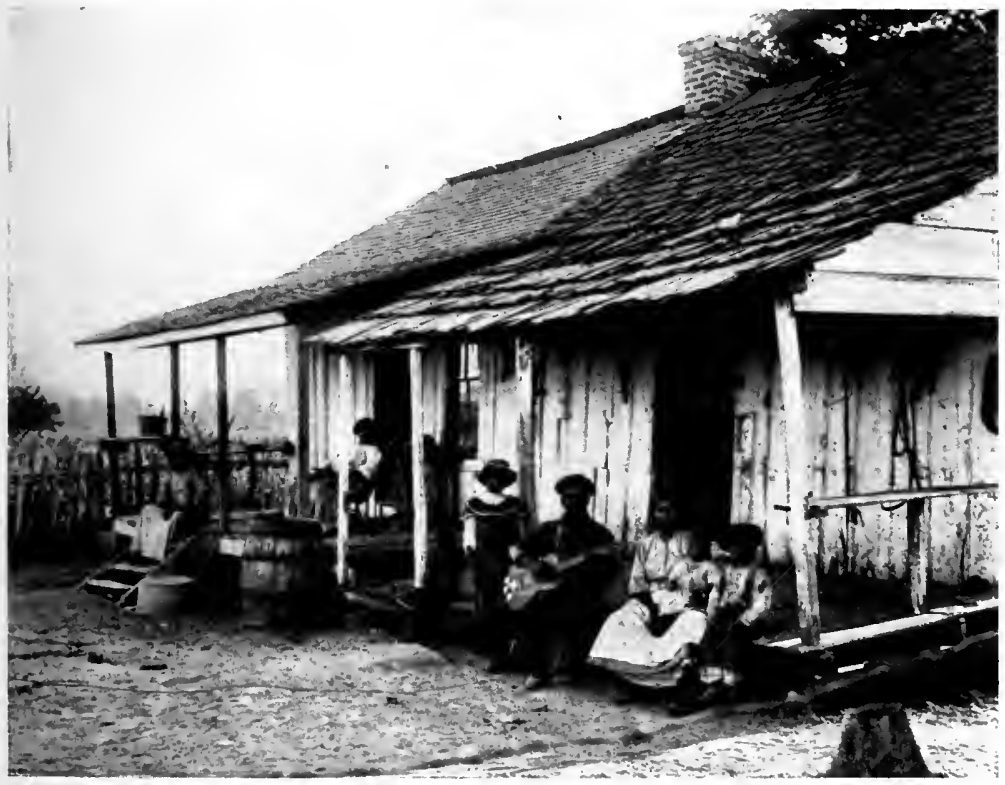

Fig. 46.-Anopheles larvæ in the barrel. A fatal case of comatose malaria occurred here a few weeks before the picture was taken. 
medical staff, to Italy to study the subject, and invited Ross to inspect the place and advise upon the most suitable manner of conducting the campaign. In September, 1902, Ross arrived in company with MacGregor and with Pressat returning from Italy. An abundance of anophelines were found in the houses of the employees, and the larvæ especially in small, brackish marshes in the sand and in some of the waters of irrigation, but not in the main canal, where they were probably destroyed by fish. It was evident that mosquito reduction was to be the chief end, though old cases of malaria received vigorous treatment. Marshes were filled with sand and the irrigation channels

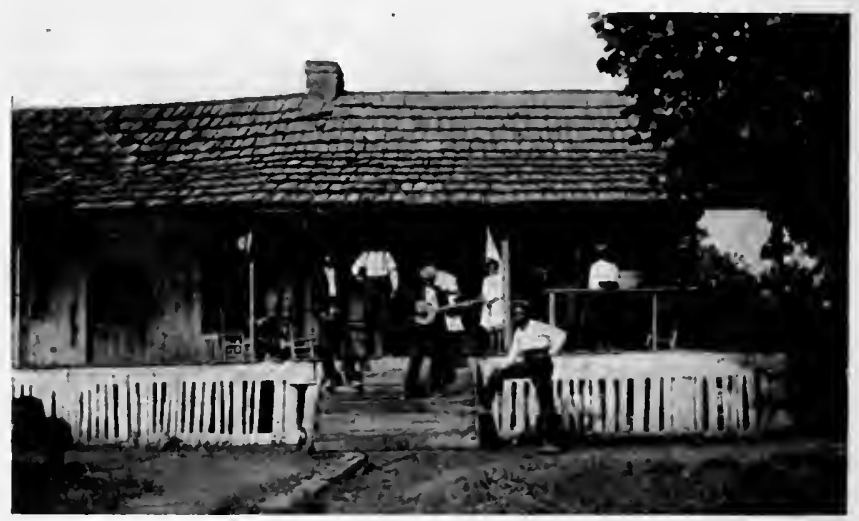

Fig. 47.-Unmindful of the danger lurking in the barrel.

were deepened or treated with oil. This preliminary work was conducted with a brigade of only four men, though many others were employed later for the extensive permanent work. From I 885 until 1902 inclusive the number of cases of malaria at Ismailia had averaged nearly I,800 annually. In 1903 there occurred 214 cases; in 1904, 90 ; and in 1905 only 37 . It is said that it is now possible to sleep with comfort in the place without nets. The cost of the campaign is estimated at an initial expenditure of 6.25 francs and an annual outlay of 2.3 francs per head of population.

The results of the campaign conducted by Travers and Watson at Klang and Port Swettenham, in the Federated Malay States, are hardly less striking. Klang had 3,576 inhabitants 
in r9or. Port Swettenham, five miles away, had a population of about 700. The population of the district surrounding the two towns was about 14,000 . In the latter part of I90 I malaria

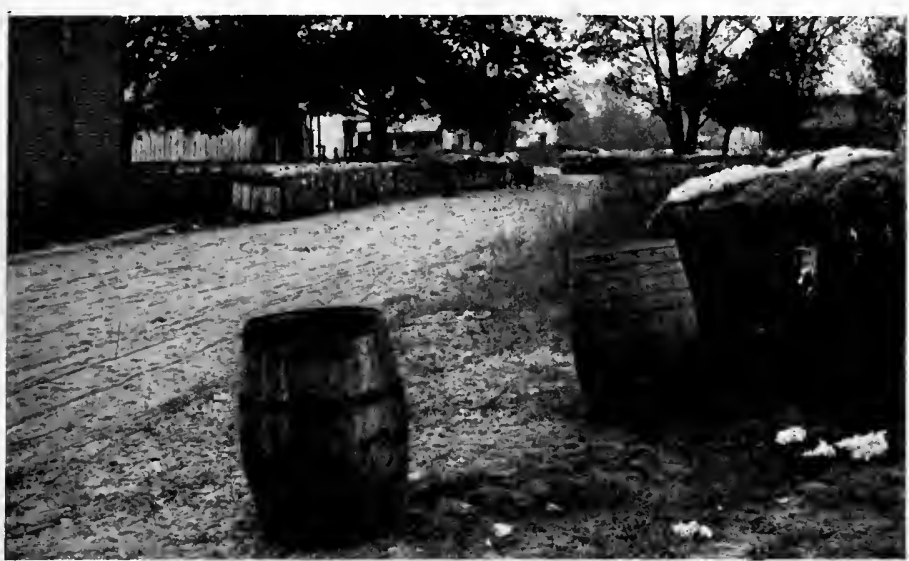

Fig. 48.-Fire barrels containing larvæ.

was so extensively prevalent that probably not more than three houses in Klang escaped infection, and Port Swettenham was being abandoned by the workmen. The antimalarial campaign,

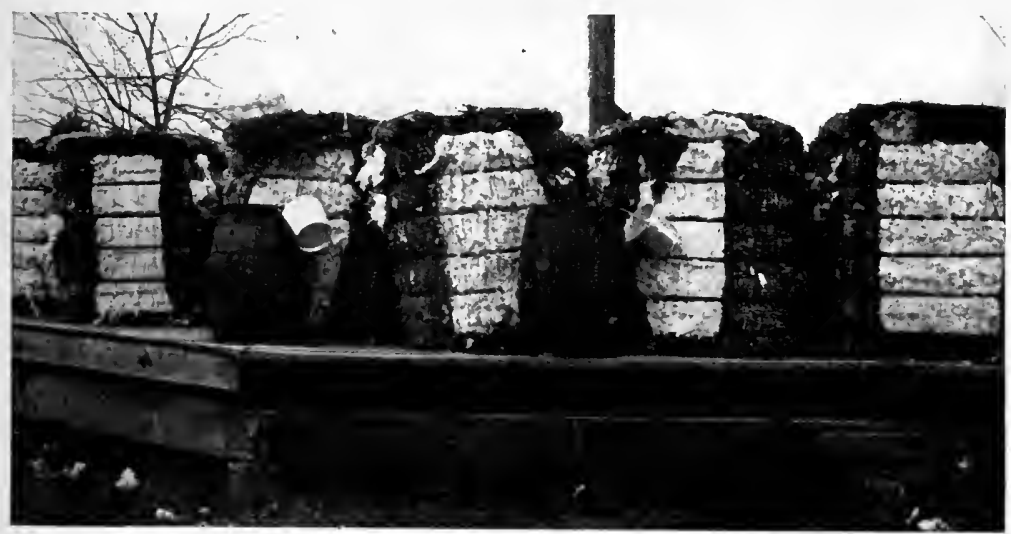

Fig. 49.-Water barrels may prevent the spread of fire, but will hreed mosquitoes unless covered.

which was confined to the towns of Klang and Port Swettenham, began in 1902. Swamps were filled, a contour drain established to intercept incoming water from surrounding springs, and 


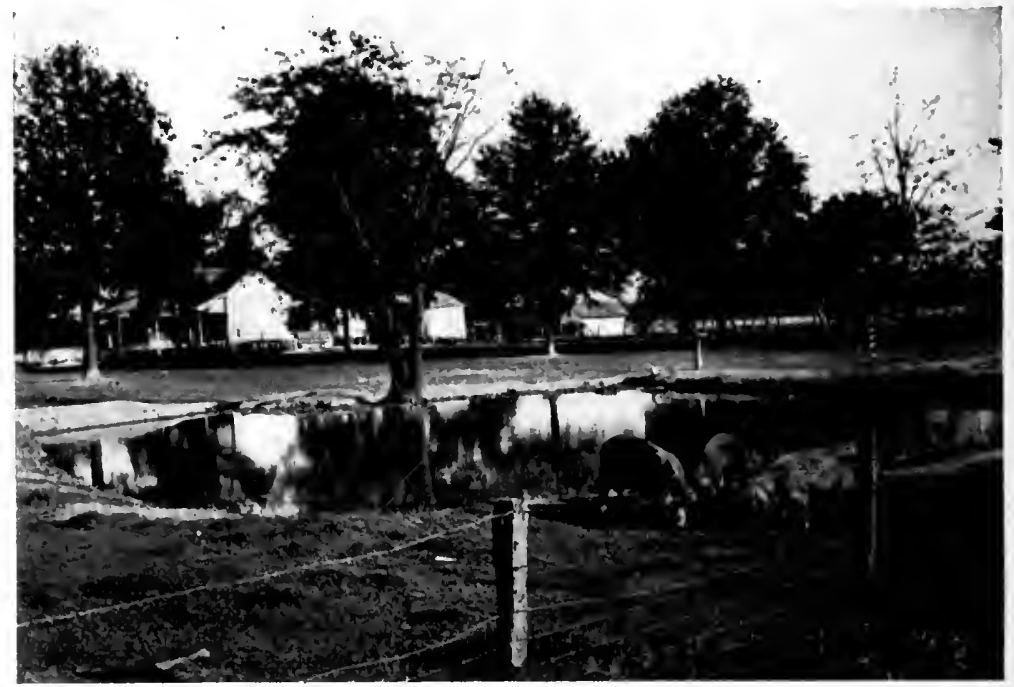

Fig. 50.-Stock pond containing anopheles larvat. Too near the dwelling.

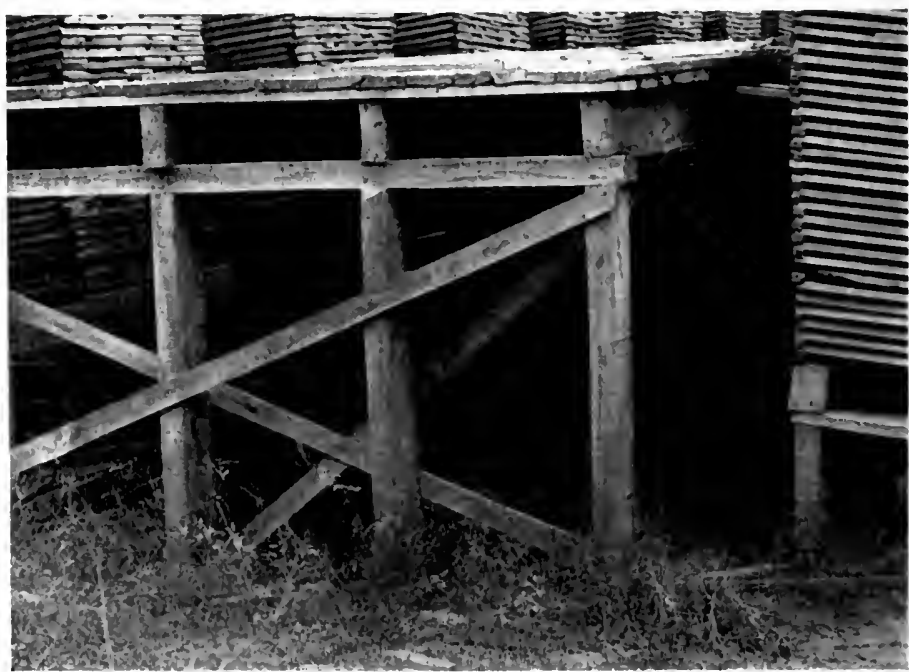

Fig. 5r.-A protected pool in a lumber-yard containing myriads of anopheles larvie. 
forest and mangrove trees were felled. After the epidemic had begun to subside screens were furnished many of the houses and quinine was distributed. The cost of the operations to the end of 1905 consisted of a primary expense of $£_{I 0,100}$ and an annual expense of $£_{410}$.

The following table shows the mortality from malaria within the towns as compared with that of the unprotected district:

\begin{tabular}{l|r|r|r|r|r|r}
\hline & 1900 & 1901 & 1902 & 1903 & 1904 & 1905 \\
\hline Towns............ & 259 & 368 & 59 & 46 & 48 & 45 \\
Districts.......... & 173 & 266 & 227 & 230 & 286 & 351 \\
\hline
\end{tabular}

The most brilliant results in the prophylaxis of malaria were those obtained by Gorgas in Panama, one of the most insalubrious regions upon the face of the earth, having been called during French occupation "the Frenchman's grave." It is a common report that in the railroad between Panama and Colon every cross-tie represents the corpse of a laborer.

The canal zone is 50 miles in length, with Panama and Colon at each end. The average number of employees is 40,000 . The efforts consisted in the destruction of breeding places only within 200 yards of the camps and villages, no attempts being made to deal with those farther off. All the houses were screened and the people were urged to use mosquito bars. Quinine was furnished them and they were advised to take 3 grains daily. The abolition of the breeding pools was regarded as a most important measure. Owing to the heavy rainfall and the luxuriant vegetation the ditches filled rapidly with grass, and it was found much cheaper to concrete them. Subsoiling by means of the tile drain covered with rock and soil was used wherever possible.

The result is that the death rate has been lowered until it does not exceed that of New York City.

\section{MEASURES DIRECTED FOR THE DESTRUCTION OF MOSQUITOES}

Destruction of breeding pools for the anopheles is an effcient preventive measure. It is chiefly through the eradication 
of breeding places that so-called unconscious prophylaxis has accomplished its results. This method has received the chief consideration in the greatest antimalarial campaigns. It is more permanent and possesses the further advantage in many instances of being cheaper in the end.

It is neither necessary nor in every case advisable to remove the surface water from the whole of a malarial country, but only in the regions of inhabitants or where anopheles are known to breed. In the Panama campaign the area of destruction extended only 200 yards from camps and habita-

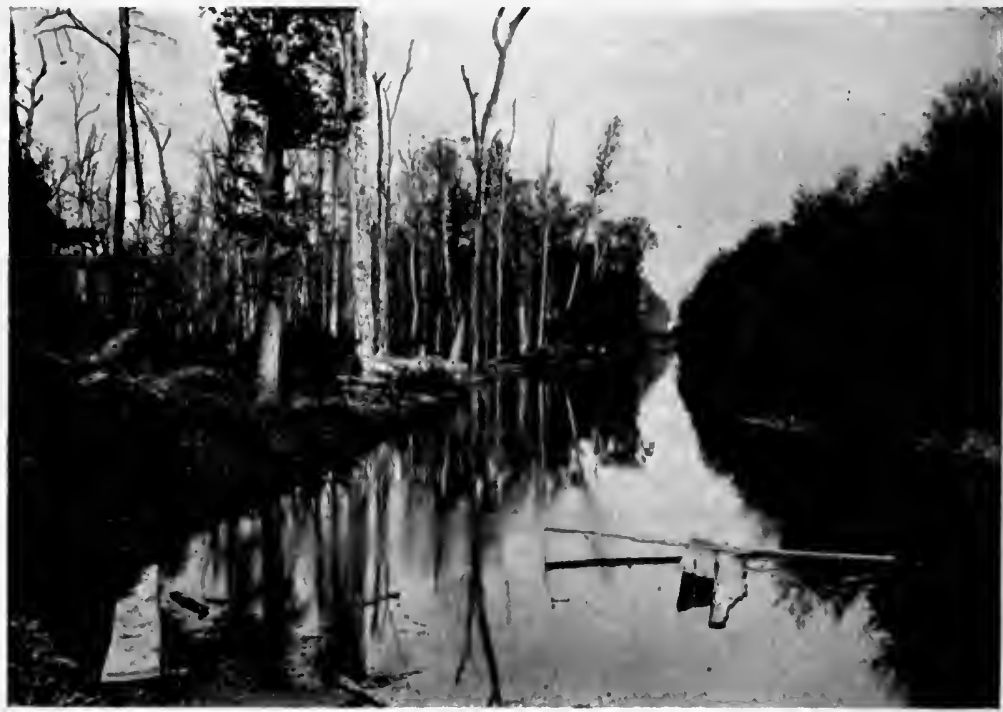

Fig. 52.-A typical "bayou," the headquarters of malaria.

tions. This should probably be the minimum radius, though work at a much greater distance is only a useless expense.

In the area to be protected the land should be cleared of weeds, undergrowth, bushes, and unnecessary trees to promote evaporation and prevent the formation of puddles. Grocery cans, broken bottles, buckets, and old tinware which might retain water should best be buried. Water barrels, tanks, cisterns, and wells should be emptied, filled, or screened. Gutters should be maintained in such a condition that water cannot accumulate. 
The stock pond, so common in the vicinity of habitations in some sections, is a menace to both man and beast and should not be tolerated.

The care of streams and large bodies of water is ordinarily simple, since these rarely threaten sanitation as anopheles breeders. Within the protected area the banks should be cleared of dense weeds and bushes, eddies prevented where possible, and pools along the edges drained into the channel.

In the case of streams that get very low after the rainy season, leaving a chain of pools along the river-bed, these pools

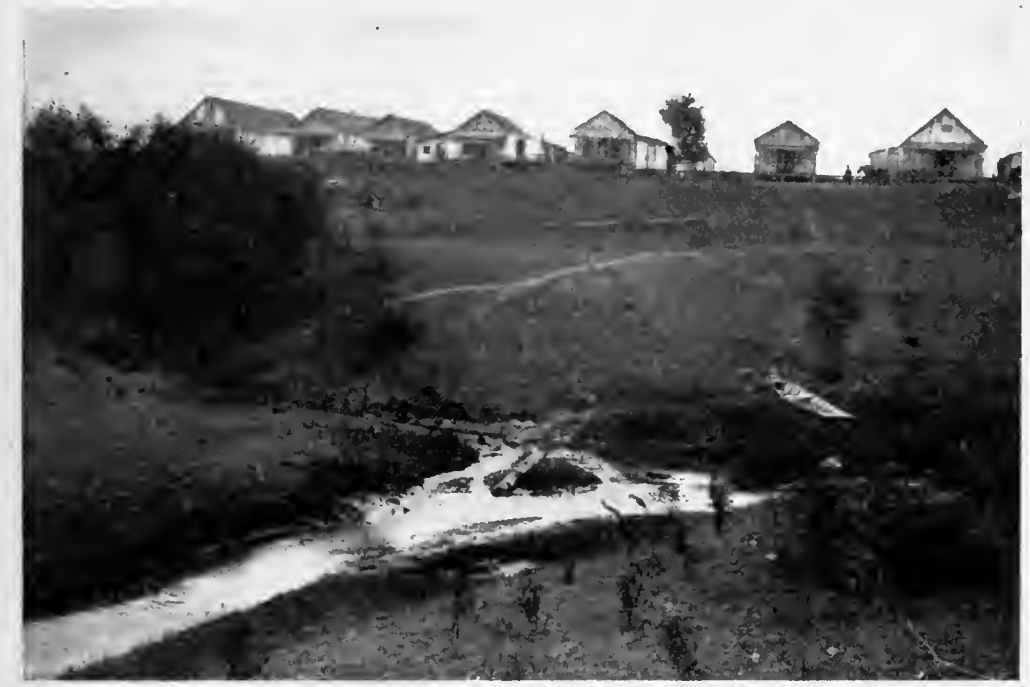

Fig. 53.-An ill-chosen town site along the bayou.

should be drained into each other and an attempt made to reëstablish a flow and to permit of scouring and the access of fish from the larger pools. Where the pools are small much water can be gotten rid of by the use of brooms.

In the case of large bodies of water subject to overflow the problem is more difficult. The primary effect of the submerging of land, while the water is high, is to diminish malaria. The secondary effect, after the waters have receded, is to cause a marked increase. The effect upon malaria of inundations is almost yearly observed in the valleys of the Nile, of the 


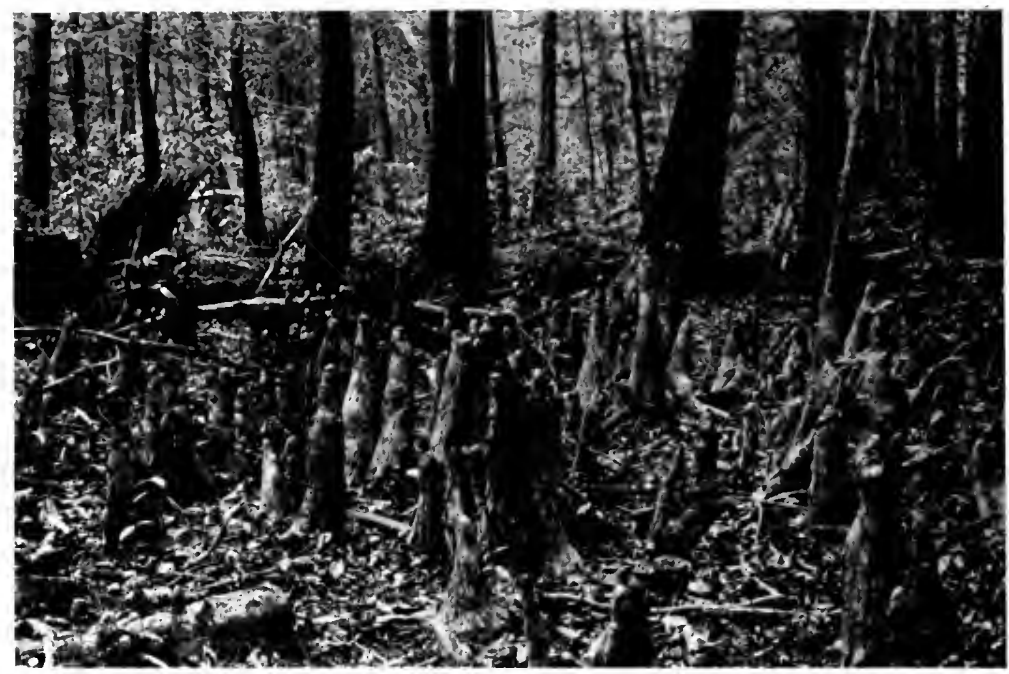

Fig. 54.-Anopheles breed among the cypress knees.

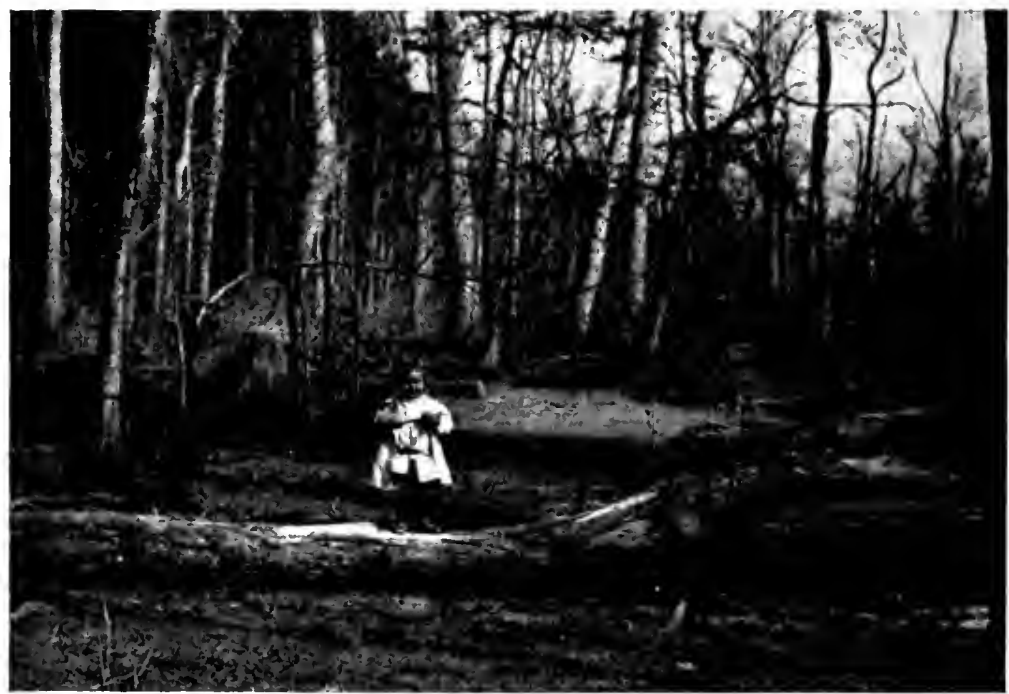

Fig. 55.-Many breeding places are left upon the lowlands after the overflow has recected. 
Mississippi, and of other large streams. Levees, dykes, and other engineering means of large dimensions are the only remedies; these being expensive are rarely employed merely for sanitary purposes.

Marshes and swamps when too extensive to be filled may be effectively drained. The drains should be narrow, of sufficient depth and fall to drain effectively, and may be parallel, crowfoot fashion or otherwise, as best suited to local conditions. If concreted they require less after-treatment

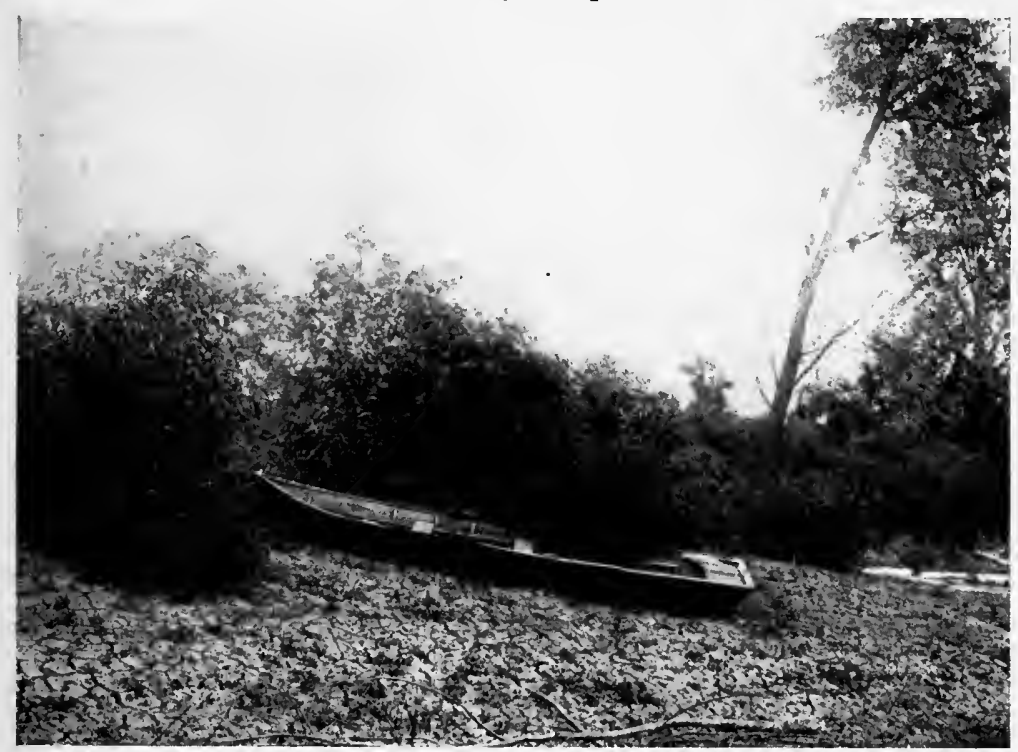

Fig. 56. - A stranded skiff containing a little water and many anopheles wrigglers.

and may be cheaper in the end. If not concreted they should be frequently inspected to prevent caving, deposit, or filling with vegetation. The tile drains are usually very efficient.

Large swamps in the vicinity of streams have been rendered unfit as breeding places by directing the course of the stream through them. The water is thus given a current, and if the stream contains much mud in suspension the bed of the marsh is gradually filled.

- Fresh-water ponds close to the sea have been successfully treated by filling with salt water. Water strong in salt is 
not attractive for breeding purposes, though brackish water may harbor numerous larvæ.

The rendering innocuous of borrow pits along railroad lines is difficult. It is much easier to prevent the stagnation of water during the construction of the road than it is to remedy the defect after completion. Filling and drainage are the best correctives.

The destruction of smaller pools and puddles is usually simple and goes far toward prophylaxis, since it is in such places that anopheline mosquitoes breed by preference. Filling is by far

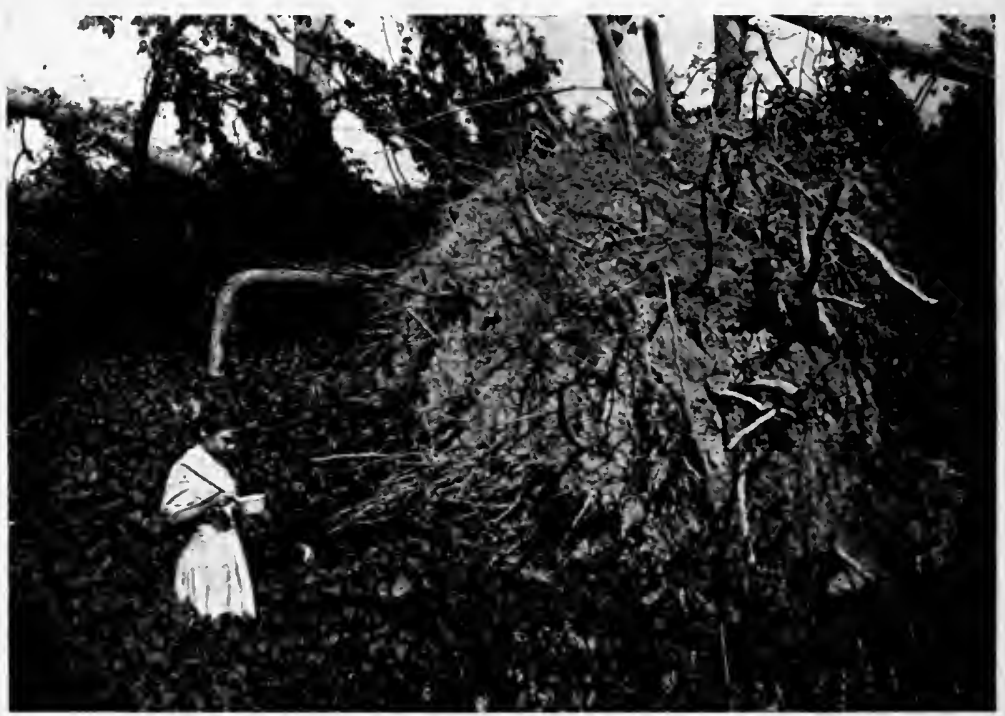

Fig. 57. - The cyclone as a factor in malaria.

the most permanent, hence the cheapest and most desirable method by which to deal with these collections of water. Pools in ditches along the sides of roads, wheel ruts, hoof prints of stock in soft ground, water remaining in natural inequalities in the ground and in excavations for various purposes should be assiduously attended. The work should be conducted by one who is familiar with the rudimentary principles of drainage.

The height of the ground-water is very intimately associated with the prevalence of malaria, since the quantity of surface water depends largely upon the height of the ground- 
water, and the latter, when appearing upon the surface, is a favorite breeding site for malarial mosquitoes. Hence, measures directed toward the lowering of the ground-water are of the highest efficacy in the prophylaxis of malaria. This is evidenced by the results of the "tiling" of land and by the formation of drainage districts for the reclamation of swamp

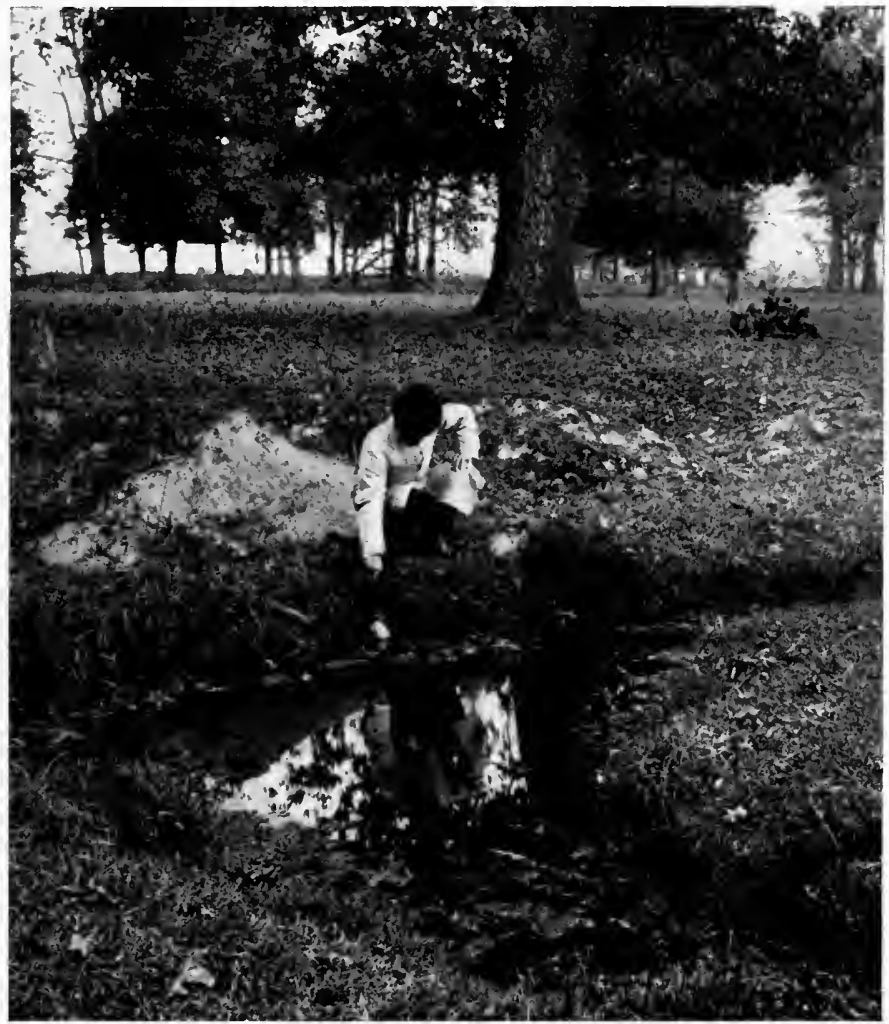

Fig. 58.-This pool, fed from the ice-plant, contained larvæ weeks after most other pools had disappeared.

lands. Such procedures often render unnecessary the expenditure of labor or money for the removal of breeding pools. or other antilarval steps.

In certain regions where the hardpan or impervious stratum is responsible for a high ground-water excellent results have been obtained by boring through this, thereby allowing the 
water to escape into the permeable earth beneath. These are the so-called absorbing wells.

It is well known that rice culture increases the malaria of a region to a great extent. In some of the Oriental countries the crop is a necessity, but in regions not absolutely dependent upon the crop the cultivation of rice must be looked upon as an evil. In fact, some governments have either thrown

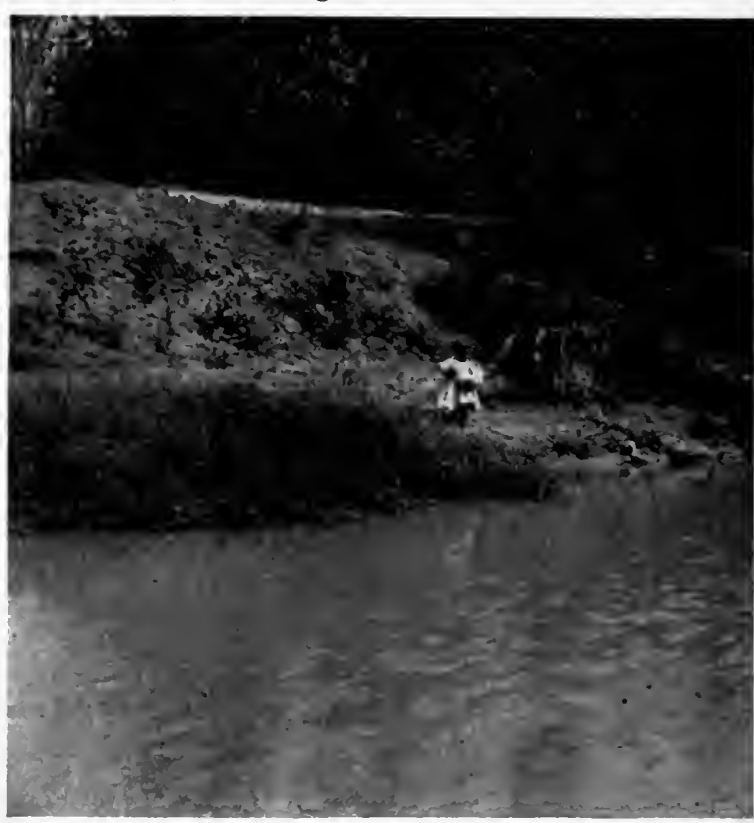

Fig. 59--Hunting for anopheles larvæ along the levée.

certain restrictions around the industry or have altogether prohibited it.

The eucalyptus globulus has attained considerable reputation as a preventive of malaria, probably from a belief that it absorbs moisture from the soil and renders it drier. Recent experiments in Italy have, however, shown that this tree has no effect in decreasing malaria, and that it even affords an excellent shelter for anopheles mosquitoes.

Sunflowers and castor-oil plants, which are reputed to be beneficial in the prophylaxis of malaria, are probably devoid of such virtue. 
There are circumstances under which it is impossible to destroy the breeding pools. Here the use of petroleum is indicated. This oil is also useful in antimalarial campaigns as a temporary measure in part of the work while permanent means are being employed elsewhere.

An oil should be chosen which spreads rapidly and evaporates slowly. The refined illuminating oil evaporates readily, hence is too expensive for work on a large scale. The most suitable is the fuel oil or blast-furnace oil. The oil, forming a film upon the entire surface of the water, chokes the air tubes of

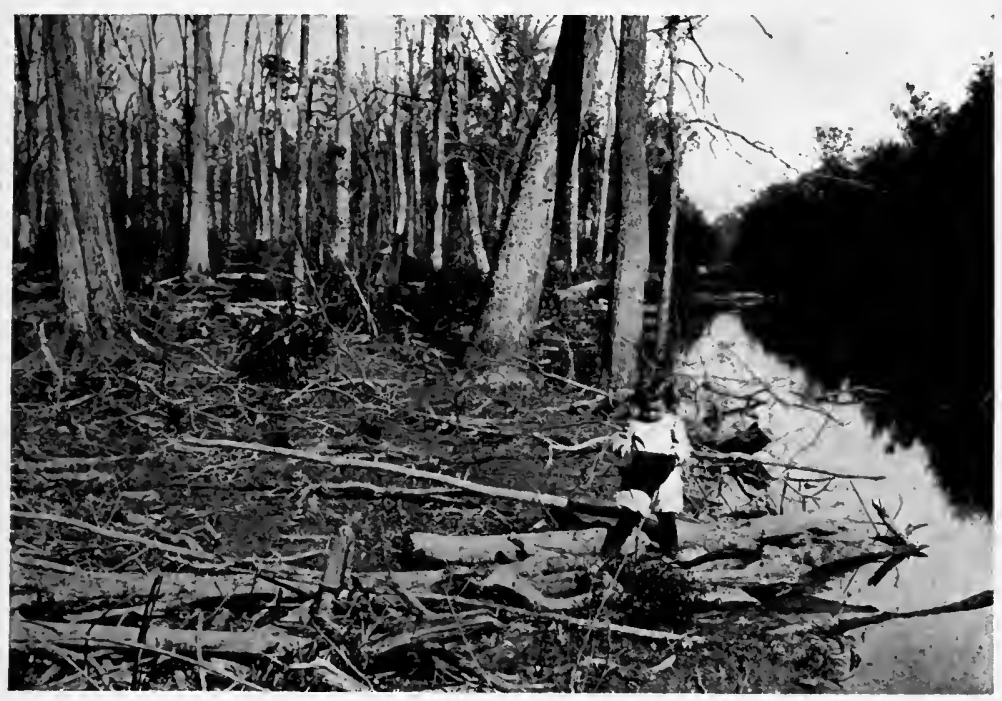

Fig. 6o.-An embryo scientist searching for anopheles larvæ.

the larvæ, which come to the surface to breathe. The pupæ expire even earlier than the larvæ, since they require more air. Furthermore, not a few adult female mosquitoes in the act of oviposition are thereby destroyed.

The pool should be cleared, as far as possible, from weeds and algæ which interfere with the spread of the oil. The oil should be poured from a watering pot, sprayed by means of a force pump, or painted over the surface with saturated cloths tied to the ends of sticks. An automatic oiler may be improvised by placing a barrel of oil a few feet above the water, 
to give the oil the necessary spread, and having a perforation in the bottom of the barrel to drop about twenty times to the minute.

The quantity of oil which has been found amply sufficient is I ounce for each 15 square feet of surface. It has been estimated that a barrel of oil costing only a few dollars is sufficient to cover 96,000 square feet of surface.

Evaporation, rains, and winds prevent permanent results, so that the oiling must be repeated. Intervals of two or three weeks are the proper average, and certain days of the month

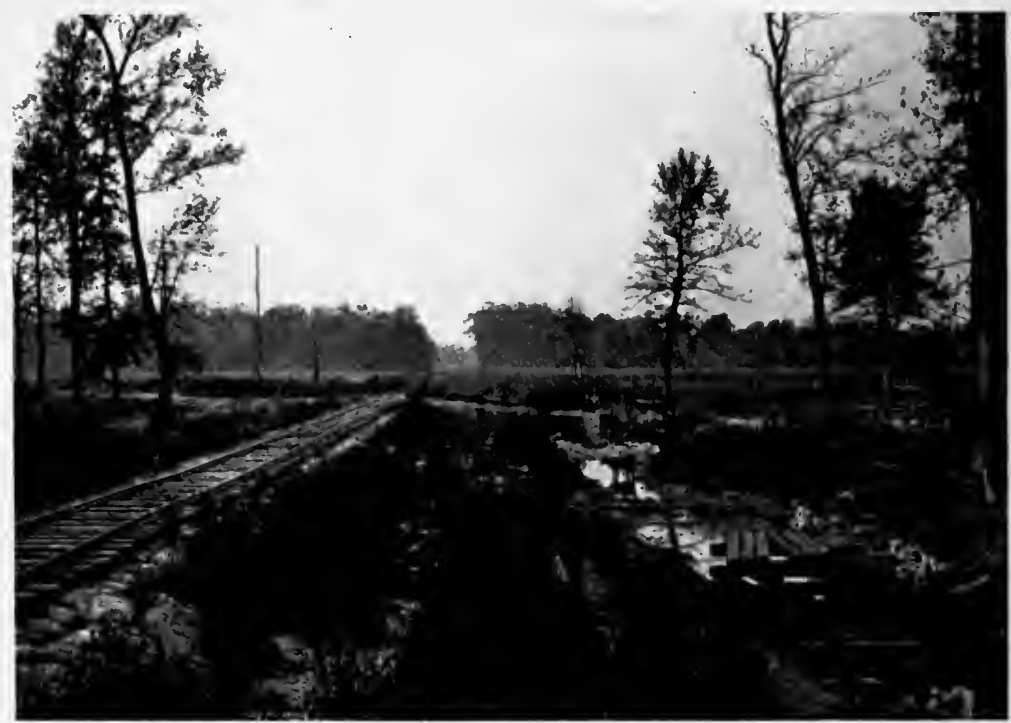

Fig. 61.-Breeding pools in the borrow pits along a railroad.

should be systematically chosen. It is best to begin the oiling in the spring to prevent the first generation.

Nearly every antiseptic and poison has been employed for the destruction of mosquito larvæ. The aniline derivatives are valuable, especially that known as Larvicide, which destroys also fish and other forms of life which may be useful in killing larva. The same objection applies to Phinotas Oil, a cresol combination, and saprol, which are effective larvicides. Formalin, corrosive sublimate, carbolic acid, and lysol are too slow in their effect upon larvæ to be of practical value. 
Permanganate of potash has proved disappointing in all trials made of it.

Where it is not feasible either to drain or oil a breeding pool the introduction of small fish has been practised with success. Certain species of fish prey upon the eggs, larvæ, and pupæ of mosquitoes, and even upon adults when about to emerge from the pupal shell or when in the act of oviposition. The common top minnows (Gambusia and Fundulus) and the sun-fish are excellent for this purpose. The former being very voracious and top feeders are especially adapted for the destruction of

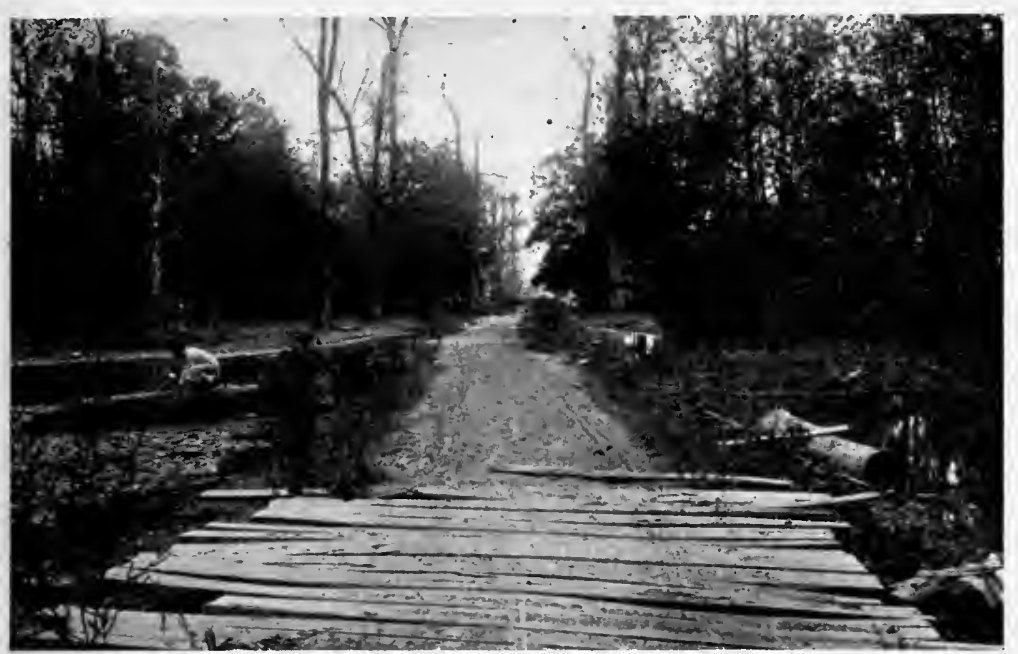

Fig. 62.-Breeding pools along the roadside.

anopheles larvæ. They are fast breeders and resist the drying of pools in a remarkable degree. Sticklebacks, gold-fish, and roach are also larvivorous. It is doubtful whether the common German carp, on account of its feeding habits, is of any use for this purpose. The tadpole is valueless for the destruction of larvæ. Ducks destroy many larvæ. The larvæ of the dragon fly, the water boatman and the hair worm devour mosquito larvæ.

The natural enemies of adult mosquitoes are few and practically insignificant. Dragon flies, night-hawks, whip-poorwills, swallows, bats, and certain species of lizards destroy a 
number and some are killed by parasitic mites and a small suctorial fly.

An ideal prophylaxis destroys the breeding pools or the aquatic stages of mosquitoes, but remedies against the adult insects are sometimes necessary. For this purpose a great variety of substances have been tried. One of the most primitive of measures is the smoldering fire of chips, rags, and feathers, to be seen in summer twilight to the windward of nearly every negro cabin.

The most practical means are the fumes of burning sulphur

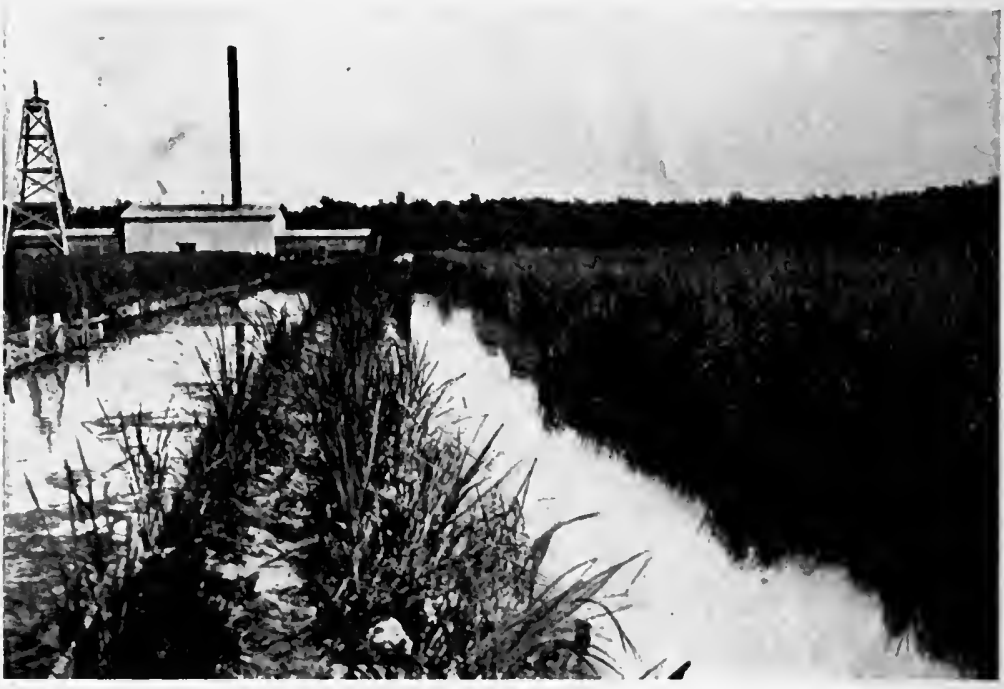

Fig. 63.-Breeding pools on a rice farm.

and of pyrethrum powder. The room to be fumigated should be made as nearly air-tight as possible.

Of sulphur, from 2 to 5 pounds should be used for every $1, \infty 00$ cubic feet of space. Its deleterious effect upon metals and delicate fabrics limits its use somewhat. Sulphur dioxide fumes have been found to be an excellent insecticide.

The room should be kept closed for several hours to insure the complete extermination of the insects.

Pyrethrum powder may be burned in the proportion of a few ounces to a pound for each $1, \infty 00$ cubic feet of space. The 
mosquitoes are suffocated by the fumes and must be swept up and destroyed.

Formaldehyde has been tried and found wanting, but may be effective when very large quantities are rapidly liberated in a tight room with few hiding places for the insects.

The pulverized leaves and stems of the common jimson weed (Datura stramonium), mixed with saltpeter and burned in the proportion of 5 ounces to I,000 cubic feet of space, have been successfully used by the New Jersey Mosquito Commission.

Chlorine gas, generated by adding a few drams of sulphuric acid to an ounce of chloride of lime, is said to be efficient, and burning tobacco leaves is useful.

\section{MEASURES DIRECTED TOWARD THE DESTRUCTION OF PARASITES}

Efforts to destroy the malaria parasites in the human body assumes two modes. The first consists of the radical cure of the malaria-infected individual, the prevention of a relapse, thereby benefiting the individual and annihilating a source of danger to the community. The second mode consists of the administration, to persons not necessarily infected, of a drug which destroys the parasite soon after the latter is introduced into the body, before the incubative stage is completed.

Cases of latent and atypic malaria are of greater importance to prophylaxis, being sources of greater danger to communities than are typic acute cases. The duration of the acute attack is short; the patient is apt to be placed under relatively favorable conditions and to receive quinine; he does not wander and disseminate the disease; and his blood may contain but few sexual forms of the parasite. On the other hand, the subject of latent malaria may harbor parasites for months and, the condition being unrecognized or ignored, he does not take quinine, and is a fountain of infection in diverse places and for prolonged periods.

Koch has maintained that the prompt and permanent cure of 
all acute cases of malaria and the systematic search for, and appropriate treatment of, all latent cases in a region will result in the extermination of the disease from such a locality. This is theoretically possible, but could be practised only in small communities under perfect control. Nevertheless, it is certaintly the duty of physicians to endeavor to effect radical cures of the cases which come under their observation, a duty owed not only to the patient but to the public, and such efforts will go far toward the eradication of the disease.

Quinine Prophylaxis.-Theoretically the administration of quinine to healthy individuals for the prevention of malaria is not an ideal method of prophylaxis, for, strictly speaking, it does not prevent infection, but destroys the parasites in the incubative stage after inoculation into the human body. But no one method satisfies all conditions; each has its advantages and its limitations, and frequently two or more methods must be employed simultaneously.

Quinine prophylaxis is indicated in proportion to the difficulty of pursuing more permanent methods. It is valuable where screens and bars are not available, as in camping, marching, traveling, or where the occupation takes one out at night. When residents of non-malarial countries go into malarial localities, especially in the rural districts, for short spaces of time quinine is a most valuable prophylactic. After infection is known to have occurred quinine is, of course, essential not only as a cure, but as a preventive. It may be employed effectively where it is impossible to destroy mosquitoes or as an adjunct to other measures.

Numerous experiences attest the value of quinine in the prophylaxis of malaria.

The Society for the Study of Malaria in Italy, beginning prophylactic experiments on a small scale in limited areas, have extended their practical efforts until the results are felt throughout the entire country.

Quinine has been the chief reliance of this organization.

The following figures give the malarial mortality in Italy from 1900 to 1910 : 


\begin{tabular}{|c|c|c|c|}
\hline Year & Deaths & Year & Deaths \\
\hline 1900 & ${ }_{15}, 865$. & I906 & 4,871 \\
\hline I9OI & I.3,558 & 1907 & 4,160 \\
\hline 1902 & 9,908 & 1908 & 3,463 \\
\hline $19 \circ 3$ & 8,517 & 1909 & 3,533 \\
\hline 1904 & 8,463 & 1910 & $3,6 \times 9$ \\
\hline 1905 & 7,845 & & \\
\hline
\end{tabular}

Fig. 64 .

Notwithstanding the favorable experiences recorded, there are disadvantages in the employment of quinine as a prophylactic. The obstacles are much greater in its use as a public measure than private.

One objection, varying considerably with individuals, is cinchonism, which may even amount to very unpleasant nervous or gastric disturbances.

To be efficient as a preventive of malaria quinine must be taken in sufficient dose during the entire malarial season. It is difficult to make ignorant people realize the importance of taking treatment during several months to prevent, maybe, merely a chill, and few governments have the authority to force them to do so. No permanent results are to be obtained in this way unless all take the drug throughout the malarial season and all cases of malaria are radically cured.

The expense of public prophylaxis with quinine on a large scale is enormous, in fact, in some instances prohibitory. Money spent for quinine to be given in inadequate doses at irregular intervals is wasted.

The size of the dose and the interval at which the prophylactic is administered are of the utmost importance. Very varying quantities have been employed at different intervals, but the established methods have about settled down to that described below:

The method canonized by Koch consists in giving I gram of quinine every sixth and seventh day, seventh and eighth, eighth and ninth, or ninth and tenth day, according to the danger of infection. This manifestly leaves several intervening days in which there is no quinine in the circulation. In 
localities, therefore, in which estivo-autumnal malaria is prevalent, the shorter interval of administration should be preferred on account of the shorter period of incubation of this form of malaria.

The Koch method, every sixth and seventh day, has been satisfactory in my hands.

Prophylactic quinine should be continued for two or three months after leaving a possible source of infection, even if the disease has not been contracted, and, in the latter event, for yet a longer period.

Combinations in pill form of quinine, iron, and arsenic, known as antimalarial pills, are valuable as tonics and are mildly prophylactic. They do not, however, contain sufficient quinine to be reliable prophylactics, at least in this region. Such pills, Grassi's esanophele pills, were tried in comparison with sulphate and hydrochlorate of quinine by the Italian Antimalaria Society and found to be less efficient. ${ }^{520}$

Arsenic, so long vaunted as an antimalarial, has been thoroughly tried and abandoned.

Tea, coffee, and lemons have very slight preventive virtue, if any at all.

\section{MEASURES TO PREVENT THE ACCESS OF MOSQUITOES}

Exclusion of Mosquitoes.-The prophylactic value of excluding mosquitoes is in proportion to the number of anophelines and the proximity of infected persons.

A properly protected house should have every door and window screened. In some localities it is advisable to cover even the chimneys with wire netting. Doors should be provided with springs to necessitate closure. Where mosquitoes are plentiful and a door is much used, a double door, with an intervening vestibule, after the manner of the Italians is to be preferred. A screened porch permits of sitting in the air in the evening when it would be dangerous to do so otherwise.

The selection of the gauze for screens is of the highest importance. The mesh of the wire netting often used, No. I2, is too large, permitting small mosquitoes to pass. None should be used with fewer meshes than eighteen to the inch. 
In the absence of wire gauze, cotton mosquito netting may be employed, but, being frail, soon becomes torn and useless.

Persons whose occupations keep them out at night in highly malarial places, as watchmen and others, should be protected with veils and with leather gloves having gauntlets.

The mosquito bar is indispensable in malarial countries. Besides being very effective when properly adjusted, it is the most inexpensive of all prophylactic methods.

As with every other method for the prevention of malaria, screens have certain shortcomings. It is evident that if

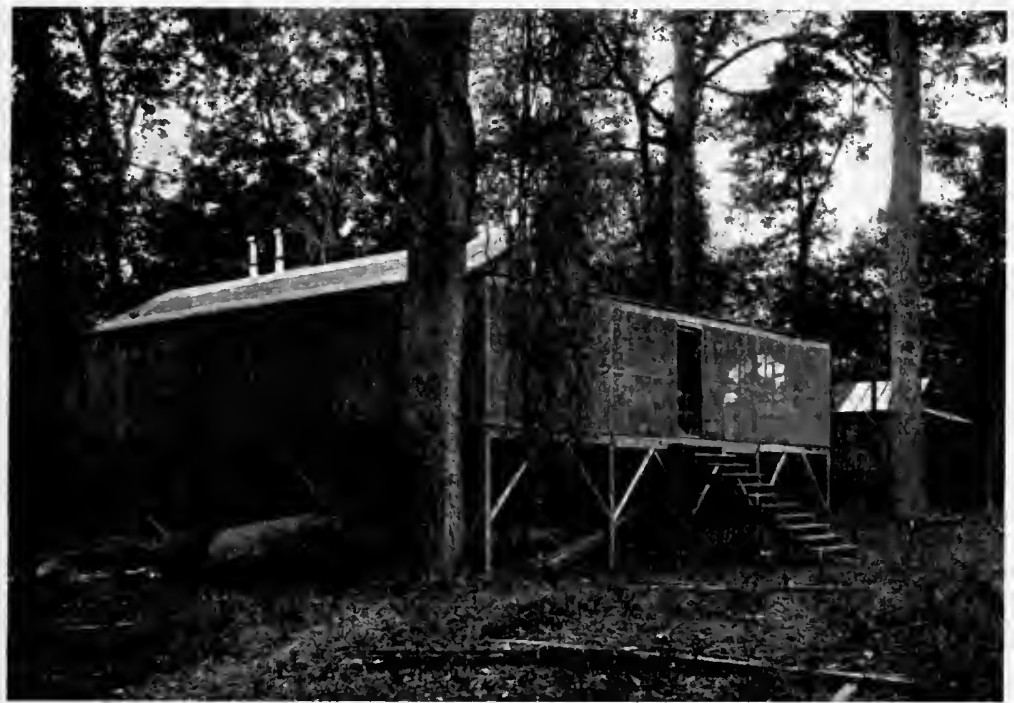

Fig. 65.-A model of house screening.

malaria is to be eradicated by these means from a locality every house should be screened, otherwise only those in the protected houses would be exempt, and only so long as they remain in such houses. It is out of the question in many malarial places to consider the screening of all the houses, both on account of the expense and because of the poor construction of many of them, permitting mosquitoes to enter through crevices and other openings. The fact that screens offer a slight hindrance to the free circulation of air in hot countries is of little moment in the face of the benefits derived from their 
use, and they must be considered as one of the most effective means of private prophylaxis.

Of local applications to drive away mosquitoes many substances have been tried, particularly the essential oils, of which the oils of citronella eucalyptus and lavender are probably the most efficacious. Petroleum, infusion of quassia, naphthaline, powdered sulphur, camphor, garlic, the oils of cloves, tar, pennyroyal, chrysanthemum, and anise have been employed with varying degrees of success.

In India the punka is employed to keep the air in motion, and for this reason is found to be of service in driving away mosquitoes. The electric fan has this effect also, but for obvious reasons should not be employed for this purpose during sleep.

The value of smoke against mosquitoes is well known, though it is not always entirely effective. I recall an occasion while on an island off the Gulf coast of Florida where smoke was of no avail against the terrific onslaught of bloodthirsty mosquitoes, and it became necessary to bury myself up to the neck in the sand and to cover my head with a coat.

Isolation of the malarial patient is as truly indicated as in yellow fever, both diseases being conveyed in the same manner. Mosquitoes must become infected before they can infect man; breaking the vicious circle at this point would extirpate malaria. Isolation is demanded not only for the good of the community, but to prevent reinfection of the patient who should be confined under a well-adjusted bar until a radical cure is effected. It is not to be expected, however, that as much can be accomplished from the isolation of malaria as from isolation of yellow fever. Many cases of malaria entirely escape medical treatment, and a malarial subject may be a source of infection for a year or more, while yellow fever is infectious for only a few days.

Since it has become evident that so great a proportion of the inhabitants, especially the children of tropic countries, harbor malarial parasites in the blood, segregation of the whites from the natives has been proposed and in some instances practised with success. While the question is of some import in this 
country, the negro quarters in most of our towns are fairly well defined from those of the white. Upon the premises the householder should see that his servants' quarters are as thoroughly screened as his own. In the choice of camp sites native houses should be avoided beyond the limit of flight of mosquitoes, if possible.

Great good is being accomplished in the prophylaxis of tuberculosis by education, keeping the main facts in the etiology and prevention constantly before the eyes of the people. So much cannot be expected from malaria on account of the ignorance and carelessness of the class and race of people most scourged, but undoubtedly some good may accrue from this method. The Europeans, at home and in their colonies, have obtained some results in the prophylaxis of malaria by teaching the people the elements of the cause and prevention of the disease.

Lectures, illustrated by stereopticon views, are held publicly. Publications in simple language, in the form of circulars and tracts, and even appropriately illustrated postcards are scattered broadcast. The Italian Society for the Study of Malaria has distributed about two million of these circulars. The principles of prophylaxis are instilled into the minds of the school children, and made attractive and impressed by means of illustrated charts. The lay press has been used to advantage. With such means the formation of an antimalarial league can do much for a community. A little can be accomplished by education, and this little should not be neglected.

To be thorough, malaria prophylaxis should be handled by the Government. Destruction of the breeding places of the mosquitoes, which is by far the most radical method, is, in many instances, too expensive to be done by individuals. The formation of drainage districts, the expenses of which are paid by those benefited, is an effective plan, and so enhances the value of real estate, from both agricultural and sanitary standpoints, that there should be no opposition.

It should be the duty of the authorities of every malarial country to remove the duty from quinine and to maintain a high standard of purity and a low price. 
Private prophylaxis consists of measures having reference to the person and to the premises. Personal prophylaxis is synonymous with proper hygiene. Suitable food, water, and clothing are essential. Regular hours must be kept, and constipation, chilling of the body, and excess of all kinds must be avoided. Prophylactic quinine is not constantly necessary for residents if the premises are in proper condition, but is suitable for strangers and under conditions where mosquitoes cannot be excluded. Persons sleeping upstairs are less liable to infection than those upon the first floor.

Pools are to be filled, drained, or oiled, and vessels emptied. It has been suggested that a tub of water be kept on the place to tempt mosquitoes to breed, and that this be emptied every few days. Stock ponds should be drained, oiled, or stocked with fish. The houses should be thoroughly screened, and where these are not effective, or if infection occurs, bars must be employed. 


\section{CHAPTER VIII}

\section{TREATMENT OF MALARIA}

While quinine is more nearly specific than any other known drug, it has limitations and while the clinical manifestations of malaria usually subside readily after quinine, a radical cure is sometimes difficult. A few grains a day will relieve many cases, though a dram a day will not save some patients. A few of the sequelæ of malaria are but little, if at all, influenced by quinine.

Among the alkaloids of cinchona bark quinine is the one now generally used. The following table shows the alkaloidal strength of the various salts of quinine, as well as their solubility:

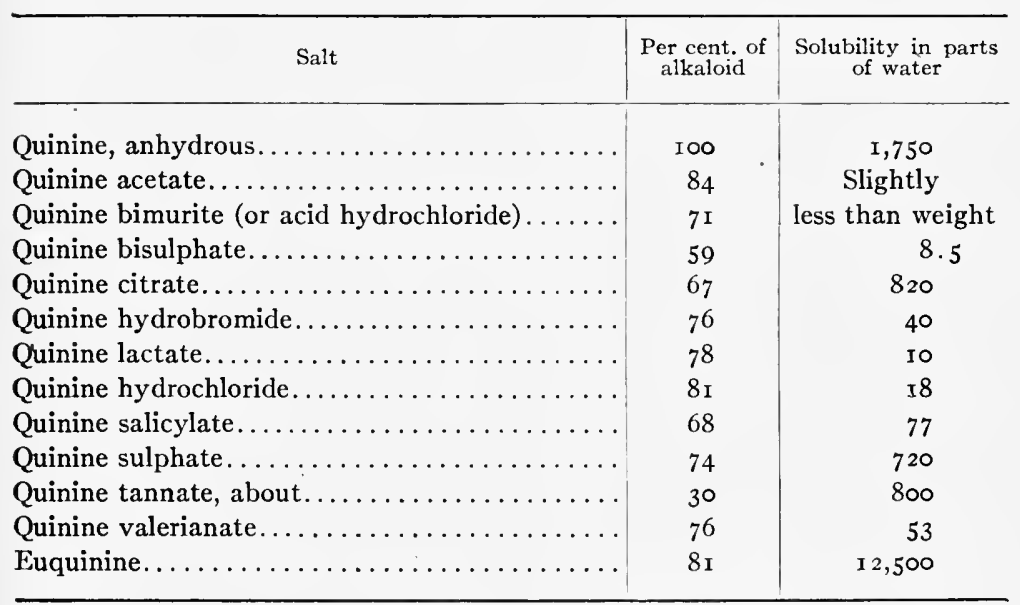

Absorption and Elimination of Quinine.-Most of the salts are readily absorbed from the stomach. It has been shown, however, that the tannate is more largely absorbed from the small intestine. 
The rapidity of absorption varies with the different salts, and is estimated by the length of time required to appear in the urine. The time from the administration of the drug until it begins to appear in the urine is represented as follows:

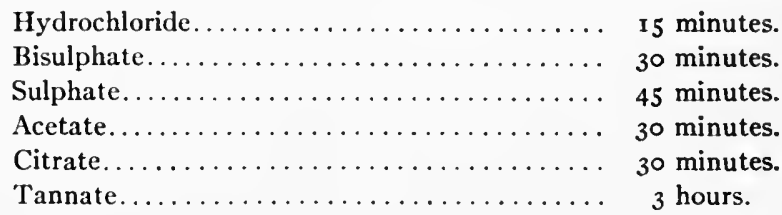

The method of administration of quinine also influences the rapidity with which it is absorbed.

Given by the mouth, a highly soluble salt will begin to appear in the urine within fifteen to thirty minutes, and is eliminated in the greatest quantity within three to twelve hours.

While it is a widely prevalent belief that the soluble salts of quinine are much more rapidly and completely absorbed from the stomach than are the insoluble preparations, experiments show that such is not the case.

Giemsa and Schaumann ${ }^{162}$ observed that the average percentage excreted within the first twenty-four hours after administration of a soluble salt of quinine was 22.9 per cent., while with an insoluble salt it was 24.33 per cent., and they conclude that the salts of quinine, hardly soluble in water, are at least as energetically absorbed from the digestive tract as the soluble ones.

The results of clinic experience with euquinine and the tannate of quinine fully support such a conclusion.

Employed hypodermically the rapidity and thoroughness of absorption depends upon the solubility of the salt and the concentration of the solution. The latter is of the utmost importance, since, no matter how soluble the salt, if given in strong solution it will not be absorbed.

The rate of excretion after injection of solutions of bimuriate of quinine, I gram to Io c.c. of water and I gram to I c.c. of water, is recorded in the following tables, respectively: ${ }^{162}$ 
Total Daily Elimination Estrmation in Per Cent. of Anhydrous QUININE

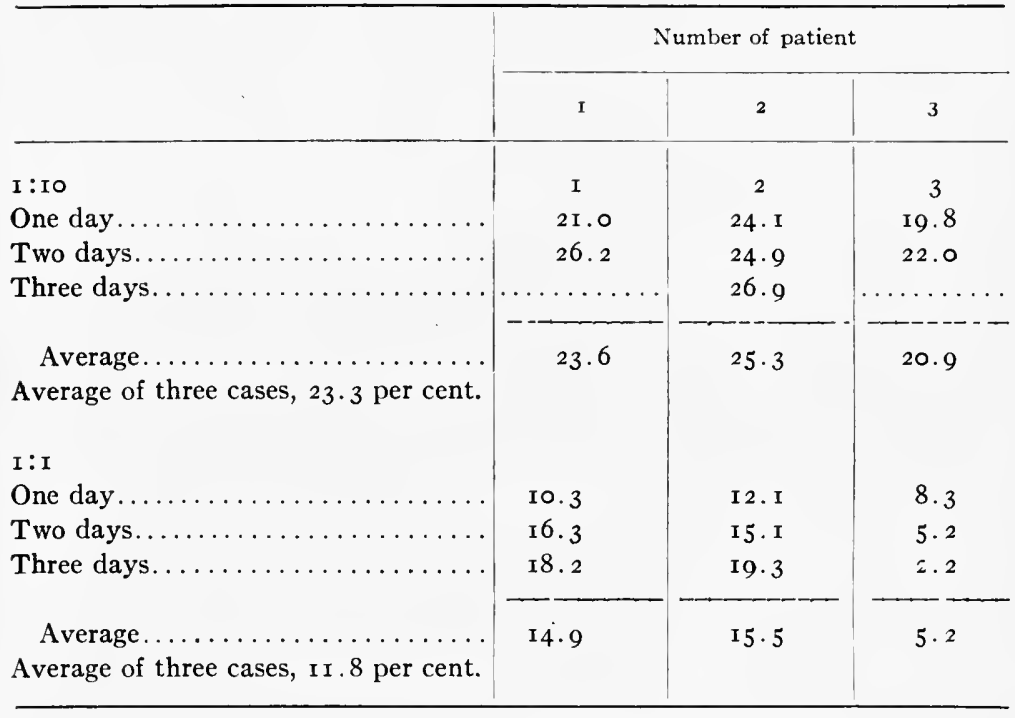

Mariani $^{543}$ found that after the injection of $\mathrm{I}$ gram of the bimuriate of quinine dissolved in Io c.c. of water the maximum excretion occurred between the sixth and the twelfth hours, while after the injection of the same quantity of the salt dissolved in 2 c.c. of water this period occurred between the ninth and the eighteenth hours.

As compared with the oral administration of quinine the hypodermic method has been ascertained to be followed by the absorption of a smaller proportion of the drug. The proportion is, according to Giemsa and Schaumann, $38.5: 17.5$; according to Mariani, $45.63: 31.86$, and according to Schmitz $27.7:$ i 6. ..

After intravenous administration quinine has been detected in the urine in ten minutes.

Injected into the rectum quinine appears in the urine in twenty to twenty-five minutes. On account of the tenesmus which quinine solutions are prone to produce when introduced into the rectum, experiments are not very numerous, but those performed show that the drug, even in a highly soluble form, is much less easily absorbed than when given orally. 
Besides with the urine, quinine is excreted with the feces, the milk, the sweat, the tears, pathologic transudates and exudates, the amniotic fluid, and the first urine of the newborn children of cinchonized mothers.

Action of Quinine upon the Malaria Parasites.-Binz, in r 867 , was the first to assume that the effect of quinine in malaria was due to its action as a protoplasmic poison upon the organisms which he believed to be the cause of the disease. This conclusion was reached from a knowledge of the action of quinine upon infusoria.

In I88 I Laveran found that the parasites were killed by the addition of $I: 10, \infty 00$ solution of quinine, and concluded that "it is because it destroys the parasites that quinine causes the disappearance of the manifestations of paludism."

It is well known that the sexual forms of the malaria parasites are very resistant to quinine, persisting in the blood for weeks and months despite the liberal use of quinine.

While young and half-grown tertian and quartan gametes are sometimes destroyed by quinine, those of the estivoautumnal variety are exceedingly difficult to kill. In fact, it has been maintained that the administration of quinine to patients harboring only the asexual forms favors the development of crescents.

Macrogametes are more resistant to the effects of quinine than are the microgrametocytes. This may possibly be due to the thicker protoplasmic body of the former, and explains the difficulty of interrupting the parthenogenetic cycle, the cycle of chronic or latent malaria.

Binz observed that infusoria were stimulated to increase movement by quinine. The same has been noted with the parasites of malaria.

That small doses of quinine are able to arouse latent malaria is, in my opinion, unquestionable. This can be explained satisfactorily only by assuming that quinine stimulates the parthenogametes into a compensatory reproduction.

It is the uniform result of experience that the stage of the parasite most susceptible to the action of quinine is the merozoite, the spore before it has assumed the protection of the red 
cell. Hence it is desirable to have in the blood as strong a solution of quinine as possible at the time of sporulation, that the young parasites may be born into a toxic medium.

The exact manner in which quinine destroys the parasite of malaria is not certain. Whether it acts as a direct poison to the parasite, or by stimulating phagocytosis, or by increasing the fluorescence of the blood, or by forming indigestible combinations with the blood elements, has not been determined.

Contra-indications to the Use of Quinine.-The mere statement of the patient that he is unable to take quinine should constitute no bar to the use of the specific. The history of the invariable sequence of very severe skin manifestations should perhaps lead the physician to employ one of the substitutes for quinine.

Cardiac depression and dyspnea are decided contra-indications to the administration of the drug.

The treatment of malaria complicating pregnancy is essentially the same as under other conditions. A fear, probably more fancied than real, of the oxytocic properties of quinine is widely prevalent, but of the dilemma, malaria or quinine, the latter is certainly the shorter horn. The pregnant patient runs far less risk of abortion with rational quinine treatment than without. Malaria during pregnancy is notoriously stubborn, and, while the attack should be treated with the smallest doses necessary to effect a prompt cure, systematic and prolonged quinine prophylaxis is necessary to prevent recurrences. If labor pains have begun, opium should be administered with the quinine.

The history of hemoglobinuric fever is no contra-indication to the use of quinine. While the administration of the drug is sometimes the occasion of an outbreak of blackwater fever, the latter is generally due to too little quinine rather than to too much. Nor is it advisable to restrict the size of the dose unnecessarily through fear of an attack, since it has been shown that the amount of quinine is of little importance in the etiology, very small quantities being as prone to occasion the attack as moderate amounts. 
Choice of Preparation.-This is influenced by the age of the patient, the mode of administration, the severity of the attack and other factors.

The sulphate, on account of its cheapness and the ease with which it is obtained, is widely employed. I, however, now rarely employ it, and then only in suspension in syrup of yerba santa for children. It is probable that it gives rise to more gastro-intestinal and nervous disturbances than any other salt of quinine, and it is these manifestations produced, as a rule, by this salt, which cause so many persons to say to the physician that they cannot take quinine.

The bisulphate, the hydrobromide or bromide, and the hydrochloride are useful preparations, being easily dissolved and readily absorbed.

The bimuriate, or acid hydrochloride, or dihydrochloride is the most valuable salt of quinine. Its great solubility adapts it for solution to be given by mouth, by rectum, intramuscularly, or intravenously.

Euquinine, or quinine ethyl carbonate has been thoroughly tried and has given satisfactory results in my hands. Being practically tasteless, it is easily administered, either in powder or suspended in a neutral syrup, to children. An acid with the drug or immediately following develops a bitter taste. The objections to the preparation are its expense and the fact that it is patented.

The tannate of quinine, on account of its small proportion of alkaloid and slight solubility, has been until recently only rarely employed in the therapy of malaria. The Italian Government, in an effort to supply a tasteless salt of quinine for children, has been dispensing tannate of quinine in the form of chocolate confections. A commission of members of the Superior Council of Health, appointed to investigate the results obtained by this method of administration, reported adversely. They concluded that the tannate of quinine is one of the most insoluble preparations of quinine, and that it is weakly and slowly acted upon by the digestive fluids; that the fat of the cocoa retards the action of the digestive fluids upon the quinine and causes it to deteriorate under the influence 
of the air. This report was the occasion of a unanimous remonstrance by numerous physicians who obtained excellent results from the use of the tannate.

My own experience with this salt, together with the reports of the Italian physicians, leads to the following conclusions:

I. The tannate of quinine is almost completely absorbed from the alimentary tract.

2. It is more slowly absorbed and more slowly eliminated than the other salts of quinine, and remains in the system longer.

3. A small quantity only of the salt is acted upon by the gastric juice, but is largely absorbed from the bowel after contact with the bile and pancreatic juice.

4. It is not absorbed when injected into the rectum.

5. It is better tolerated by the stomach, intestines, and nervous system than the sulphate.

6. The clinic results with the tannate of quinine are entirely satisfactory.

7. Being nearly tasteless, it is especially adapted to the treatment of malaria in children.

8. It has a good effect upon diarrhea and dysentery complicating malaria.

9. It is several times less expensive than any other tasteless preparation of quinine.

Methods of Administration of Quinine.-Administration by the Mouth.-In simple cases of malaria, administration of quinine by the mouth is the rule; by other methods the exception. It is probable that ninety-nine-one-hundredths of the quinine consumed is given by the oral route.

There are those, not objecting to the taste of quinine, who will take it in the powdered form; in fact, it is a common method among the Southern negroes to lick it from the palm of the hand. The taste is, however, so repulsive to most persons that, with the exception of the tannate and euquinine, it must be given in some other form.

The same objection applies to giving the drug in solution, though this is unquestionably the most reliable form in which 
to give it by the mouth, but for obvious reasons it cannot be so extensively employed in this manner in private practice. The solution is more quickly and completely absorbed. The bimuriate and the bisulphate are the salts most suitable for solution, but if neither of these is at hand the sulphate may be employed by adding a drop of dilute hydrochloric or sulphuric acid for each grain of the quinine.

The most efficient vehicle for disguising the taste of the sulphate of quinine is the syrup of yerba santa. Two grains of quinine to the dram of the syrup is the suitable proportion. Syrup of chocolate, fluidextract of licorice, ginger, coffee, milk, honey, olive oil, and other media have been recommended, but are far from satisfactory. Acid fruit juices and syrups usually enhance the bitter taste.

Where prejudice against quinine makes it necessary to disguise the appearance of the drug, this may be accomplished effectively by adding a small quantity of charcoal, turmeric, or methylene-blue to the bimuriate, bisulphate, of other salt.

Pills and tablets are convenient to administer and not unpleasant to take, but cannot be relied upon. The coating often becomes so hard as to make solution difficult or impossible. I have several times seen quinine given in this form pass from the bowel wholly unaffected. Pills and tablets should not be given when capsules can be obtained. Capsules, when fresh, are easily dissolved. If there is any doubt as to their quality they may be punctured several times in each end with a pin, or may be followed by a few drops of a dilute mineral acid.

In the absence of capsules quinine has been rolled in a little ball with cigarette paper. Absorption is extremely slow and uncertain, and this method should not be resorted to.

The tannate of quinine has been compounded with the chocolate confection for administration to children, and in this form, if reliably made, is readily taken, and in sufficient dose is efficient.

Hypodermic Method.-The most suitable salt of quinine for injection in unquestionably the bimuriate. The tablets of bimuriate of quinine and urea are convenient and insure 
accurate dosage. The 3-grain tablets contain approximately $2 \frac{1}{2}$ grains of the quinine salt.

The advantages of giving quinine by the needle in pernicious malaria are obviously being able to administer it to patients unable to swallow or to retain it, and the certainty and promptness of absorption. Nevertheless, these great benefits are somewhat discounted by the bad results which sometimes appear. Formerly tetanus was to be feared. But there are other consequences which, while not so deadly, are more commonly met. Nodules, necrosis, sloughing, and abscess formation are referred to.

These results, however, should not prevent the use of quinine injection in the treatment of pernicious malaria, as such effects are, in a great measure, preventable. To this end there are three measures of importance: first, asepsis; second, dilute solutions; and third, deep injections.

The first is at the present day probably the least often neglected, as most physicians realize the importance of sterilization, and a spoonful of water and a few matches are sufficient to effect it on the part of the solution and needle, and soap and water are almost omnipresent.

The necessity of employing a dilute solution has been all but ignored. Nearly all writers on the subject lay great stress on the need of asepsis, but with few exceptions never even mention the evils of too concentrated solutions. Strong solutions of quinine injected into the tissues cause a wall of necrosis around the solution, preventing absorption and paralyzing phagocytosis, resulting, even if the solution is sterile, in nodes or ugly chemic sloughs. It is in all probability this chemic irritation of the cells which allows of bacterial infection following the injections of solutions not properly sterilized, and, no matter how sterile the solution, if too concentrated, a nodule or chemic slough is apt to result. Witness the frequency with which unsterilized solutions of morphine and other drugs are given without the slightest bad consequence.

For injection purposes the following formula is most suitable:

Quinine bimuriate................. I gm. (gr. $x v$ )

Water........................ Io c.c. (dr. iiss). 
As much of this as needed may be injected in one or several locations.

The solutions should not be injected hypodermically, but intramuscularly, since in the latter location the injection is more certainly absorbed, is less apt to cause induration and abscess, and is less painful. In some cases of pernicious malaria the superficial circulation is very poor, absorption correspondingly inadequate, and necrosis almost inevitable if the quinine is not deeply injected. In a case of algid malaria in my practice where the quinine was given hypodermically the site of injection began to turn blue within ten minutes and was almost black within two hours.

The initial dose should ordinarily be $x_{5}$ grains. Afterward from 5 to 10 grains should be injected every six to eight hours as long as the symptoms demand it. For children under five years the first dose may be $1 / 2$ grains for each year of age.

Most of the continental writers recommend the Pravaz or Luer syringes, with platin-iridium needles, but the ordinary antitoxin syringe answers as well. A soft-rubber tubing connection between the needle and the nipple of the syringe is advantageous, as it may prevent the breaking of a needle in a struggling patient. One of these syringes, a small sterilizing pan, and alcohol lamp do not occupy much space, and, being almost indispensable in these cases, should be easily accessible during the malaria season to the physician in a malarial locality, who often sees these cases miles from his office, when time is a matter of life and death. The ordinary hypodermic syringe may be used in an emergency, but to use a sufficient quantity of a properly diluted solution a number of injections have to be made.

The best location for injection is in the gluteal region well above the ischial tuberosities, though the interscapular region is of ten chosen.

The technic of intramuscular injections of quinine may be summarized in these precautions: Have the solution freshly made, thorough, dilute, and sterile; render the syringe and the injection site aseptic; insert the needle into muscular tissue, and avoid breaking the needle. 
Intravenous Method.-In $1890 \mathrm{Bacelli}^{544}$ introduced the intravenous administration of quinine in the treatment of pernicious malaria, claiming thereby to have reduced the mortality from $i 7$ to 6 per cent. The following formula was used:

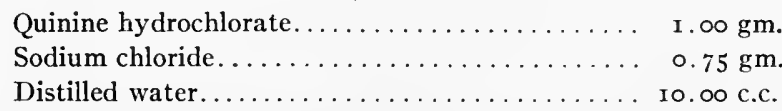

This solution is more highly concentrated than necessary and I would recommend a I per cent. solution. The technic employed is similar to that used in the intravenous injection of salvarsan.

Hypodermoclysis.-Quinine dissolved in normal salt solution given by hypodermoclysis has been recommended. From Io to 30 grains of the bimuriate are dissolved in a pint of normal salt solution, and as much as desired is injected into the loose subcutaneous tissue. This method is probably not adapted to the treatment of algid malaria on account of the deficient superficial circulation.

Rectal Administration.-This method, though uncertain, is of value where quinine cannot be retained by the stomach, and there are objections to the intramuscular injection, especially in children. It may also be used as an adjuvant to the intramuscular or intravenous injection in pernicious cases. A soluble salt should be used, preferably the bimuriate. The water should be about the temperature of the body, and should not exceed a few ounces in quantity. Two or three times as much quinine should be given by the rectum as by the mouth, and the injection should be made high into the bowel. Ten or fifteen drops of tincture of opium should be added to prevent tenesmus and aid retention. Antipyrine has been recommended by some, but should be used with care where there is depression. A cleansing injection should be given first if the patient is conscious.

Epidermic Administration.- - The use of quinine mixed with fats and oils and rubbed into the skin is not to be relied upon, since little, if any, quinine is absorbed by the skin, except in young children. 
Time of Administration and Dose.-With reference to the time when the drug is given, there are three chief modes of giving quinine:

(r) The method of Torti, a single dose before the paroxysm; (2) the method of Sydenham, a single dose in the decline of the paroxysm; and (3) the method of fractional doses.

The first two methods are adapted only to the benign infections.

The efficacy of the method of Torti rests upon the fact that the parasites are most susceptible to the action of quinine immediately after sporulation, while free, before having entered the red cells. It presupposes an accurate knowledge, of the hour at which the next paroxysm will occur, based obviously upon a definite history of repeated paroxysms, a temperature chart, or blood examinations sufficiently accurate to determine not only the type of the organism, but its exact stage. It is evident that in private practice, in the patient seen in the first access, the prediction of the next paroxysm must usually depend upon the results of the examination of the blood, and that this must be repeated if the stage is not recognized at the first examination. Unless this can be done quinine should not be administered in this way, for, even if the type of malaria present is known, there are two conditions which may render the single dose futile: first, anticipation of the paroxysm; second, a multiple infection. Even where the blood is carefully examined, it may happen in double infections, that only one group can be detected in the peripheral blood.

By this method, also known as the Roman method, the quinine is given in a single dose of about 15 grains from four to six hours before the next succeeding paroxysm. This paroxysm is not prevented; in fact, it may be entirely unmodified; but such a dose, properly timed, usually secures apyrexia subsequently for several days.

In double tertian infections a single dose given in this way may change the quotidian paroxysms into tertian, and if repeated, in multiple tertian and quartan infections, constitutes a sort of fractional sterilization of the blood.

The method of Sydenham, the English method, consists of ·a 
single dose, averaging 15 grains, given in the sweating stage of the decline of the paroxysm. This dose usually prevents succeeding paroxysms; if one should occur it is usually abortive.

This method requires less knowledge of the exact nature of the infection and of the stage of development than the former method; hence it may be more effectively applied by the busy practitioner. What experience I have had with it has been satisfactory.

The third method, that of small doses at frequent intervals, has numerous advantages over the one-dose methods.

I. Quinine given in this way is better borne by the digestive and nervous systems.

2. The loss of one dose by vomiting or failure of absorption is not of so much importance.

3. The method is adapted to tertian, quartan, or estivoautumnal infections; this is important, for sometimes these cannot be differentiated clinically.

4. It is adapted especially to estivo-autumnal infections where sporulation is not so nearly synchronous.

5. The time of administration is not dependent on parasitic findings or definite stages, both of which may be obscure where the patient has previously taken quinine.

6. An experience in many hundreds of cases has proved its value.

I give quinine in this way almost exclusively. The average dose is a grain an hour, given usually 2 grains every two hours, 3 grains every three hours, or 4 grains every four hours, day and night. It is especially important that the drug be given uring the night, since thus only may the blood be charged during the day, when sporulation usually occurs.

Cinchonism is no guide to the quantity to be given; it is not the patient toward which the quinine is directed, but the parasite.

The specific should not be discontinued as soon as the temperature is normal, but should be kept up for at least two days longer in the quantity employed during the fever. Thereafter about $\mathrm{I}_{5}$ grains on two successive days of each week should be given for at least two or three months to prevent 
relapse, even though the patient leave the malarial locality. A few days' treatment with quinine no more cures malaria than does a few weeks' rubbing with mercury cure syphilis.

Hygienic and Symptomatic Treatment.-Rest is important in the treatment of malaria not only during the stage of active symptoms, but during convalescence. Exercise may counteract the benefits of quinine; it is not uncommon to see cases yield after confinement to bed which had previously resisted quinine. A relapse may be provoked by a too early resumption of duty. Rest is especially important in the treatment of estivo-autumnal infections.

Buttermilk is one of the most acceptable and easily retained articles of diet. Sweet milk, meat broths, vegetable soups, fruit juices with egg albumin, soft boiled eggs, and toast are usually allowable. Where there is much gastric disturbance food had better be withheld temporarily.

The room and bed should be screened; in this way only can other members of the household be satisfactorily protected. The room should be thoroughly ventilated and the patient protected from draughts.

It is customary to begin the medical treatment with a purge. Calomel is the drug most easily administered and retained. The drug need not exceed 5 or 6 grains, and should be followed by a saline. The quinine should not be delayed for the action of the purgative. Calomel has been frightfully abused in most malarial countries. It was formerly the universal practice to give the drug until the gums were "touched" and the teeth irreparably damaged. It was more the abuse of calomel than of any other drug that led Oliver Wendell Holmes to declare that, excepting a few drugs, "if the whole materia medica, as now used, could be sunk to the bottom of the sea it would be all the better for mankind and all the worse for the fishes."

During the cold stage blankets, hot drinks, and the external applications to the head, tepid sponging, and cold rectal injections may be used. The coal-tar antipyretics are not often indicated. Cold drinks may be given.

For the headache cold applications, codeine, and acetanilid, or chloral and bromide of soda are useful, and if the pain 
demands it, morphine need not be withheld. If nervousness is marked the monobromated camphor should be administered with the quinine in capsules, or the bromide of soda, in solution, with each dose of the specific.

For vomiting, if intense and not relieved by the application of a mustard plaster to the epigastrium, morphine should be employed subcutaneously.

Chronic Malaria.-In the treatment of chronic malaria two parasitic cycles have to be combated, the schizogonic and the parthenogenetic. The treatment of the asexual cycle of parasitic evolution in chronic malaria is that of acute malaria.

The tendency to relapse at multiples of approximately seven days has long been recognized, these periods being known as the septenary periods. It is now known that these relapses depend upon the sporulation of the parthenogametes, a cycle difficult to interrupt except during the stage of free spores.

After successfully meeting the active symptoms by quinine, administered as above outlined, the prevention of a relapse is to be accomplished by giving $x_{5}$ grains of quinine every sixth and seventh days for a period of not less than three months. The administration of a valuable salt of quinine in this manner has rarely failed, in my experience, to cure the most obstinate case of chronic malaria. The quinine is usually given in 3grain doses every three hours until five are taken.

The hygienic treatment is even of greater importance in the management of chronic malaria than in acute. Many secondary factors may arouse latent malaria, and these, which have been mentioned in the section on Etiology, should be assiduously avoided. A change of climate is advisable.

Cachexia.-Nothing is more discouraging to the physician than the treatment of cachectics in whom the poor hygienic conditions cannot be corrected, which is not rarely the case. The two chief principles involved in the treatment of cachexia are, first, the prevention of active outbreaks of malaria, and, second, the improvement of the general condition of the patient by appropriate hygiene.

Quinine is most effectively given upon two successive days 
in each week as described. This alone, however, will rarely effect a cure except in the mildest cases.

Where it is practicable a complete change of climate should be advised. Without this very little can be accomplished for cases of severe degree. A wholesome, nutritious, and digestible diet should be prescribed. The digestion is often impaired and stomachic tonics may be indicated. Exposure to inclement weather must be avoided on account of the dangers of pneumonia. Occupations which subject the cachectic to violent exertion or to bodily harm should be interdicted for fear of rupture of the spleen. Regular hours must be kept and constipation overcome.

Of drugs other than quinine, arsenic has the best reputation. It should be given in rather large doses of the arsenious acid or Fowler's or Donovan's solutions. Atoxyl has recently been introduced into the treatment of malarial cachexia. It is employed hypodermically, 1/3 grain being a moderate dose. My limited experience with this method has been rather favorable than otherwise. The possibility of amaurosis as a toxic result of atoxyl should be borne in mind. Cacodylate of soda has given me good results.

Iron is nearly always indicated; the organic preparations of iron and manganese are usually well borne by the stomach.

Counter-irritation over the splenic area may aid in the reduction of the enlarged spleen. The best agent is the ointment of the red iodide of mercury. A piece the size of a pea or larger should be thoroughly rubbed in, the splenic region being bared to the sun's rays or to the heat of a fire. This should be repeated daily until the skin becomes so irritated as to make friction painful, when it should be discontinued, to be resumed again when the condition of the skin will permit. Iodine, turpentine, mustard, firing with the actual cautery, and other counter-irritants have been recommended.

While the $\mathrm{X}$-rays have a destructive effect upon certain protozoa, they do not appear to have such action upon the parasites of malaria within the circulation.

Splenectomy may be performed in very anemic patients with large, painful spleens, especially if freely movable, in whom a 
change of climate is impossible and therapeutic measures have failed. I had the opportunity of treating an obstinate case of estivo-autumnal malaria in an adult female who had had, several years previously, the spleen removed on account of malarial cachexia. Hemoglobin percentage was only slightly affected by the attack, and convalescence was rapid.

Treatment of Malaria in Children.-In the treatment of malaria in children it is my practice to administer the quinine at short intervals, every two or three hours.

While children bear quinine in relatively larger doses than adults, the size of the dose should be regulated by the severity of the attack and the age of the patient. In average cases children from one-half to two years of age may be given from $1 / 2$ to I grain of quinine every three hours; from three to five years, from $I$ to 2 grains; and from six to ten years, from 2 to $2 \frac{1}{2}$ grains. These quantities may be increased in severe attacks.

The drug is ordinarily given by the mouth. Where capsules cannot be used, recourse must be had to a tasteless preparation or to a disguising vehicle. Euquinine and the tannate of quinine are the best of the tasteless preparations. The former must be given in slightly larger doses, the latter up to double the doses indicated above. The most efficient liquid for disguising the taste of the sulphate of quinine is the syrup of yerba santa, at least I dram of which should be given for each 2 grains of the quinine. In cases with pernicious symptoms the drug should, of course, be injected intramuscularly. Rectal administration of a solution or suppository may be employed to supplement other modes. The buttocks should be pressed together for half an hour after insertion to aid retention.

Calomel, mercury with chalk, and castor oil are efficient purgatives in the treatment of malaria in children.

Treatment of Complications.-When malaria is complicated with other diseases each should receive appropriate treatment, the malaria should be promptly treated as under ordinary circumstances. Only a few complications need special consideration.

For rupture of the spleen immediate laparotomy should be 
performed. With early operation over half recover; without operation the mortality is nearly roo per cent.

Abscess of the spleen is a surgical condition. The choice of operation between splenectomy and splenotomy must be based upon individual conditions.

In the treatment of quinine amaurosis the quinine must, of course, be discontinued. Nitrite of amyl and nitroglycerine, with tonics, are recommended.

Substitutes for Quinine.-In the treatment of malaria there is no drug that can compare in efficacy to the salts of quinine; nevertheless, in rare instances it becomes necessary on account of idiosyncrasy to resort to the use of other remedial agents.

While the newer preparations of quinine, euquinine, saloquinine, aristochin, etc., are purported to be free from the toxic properties of the official salts, I have seen one case in which euquinine caused distressing dyspnea, and another case in which this preparation caused violent urticaria. Euquinine is probably the most valuable of these preparations.

The alkaloids of cinchona, other than quinine, are now but rarely employed, and opinions vary widely as to their merits. Cinchonidine is probably the most useful of these alkaloids. It must be given in doses about twice as large as of quinine. These alkaloids, especially cinchonine and quinidine, are more toxic than quinine, producing nervous and gastric disorder and, in toxic doses, convulsions.

Good results have been obtained with Warburg's tincture. It is claimed that quinine may be given in this form when not tolerated otherwise. The liquid contains, among its many ingredients, about Io grains of quinine to the ounce, and the dose is $1 / 2$ ounce repeated in two to four hours. It is probable that as good effects can be obtained by quinine alone in solution as with this unpalatable and unscientific conglomeration of "quinine concealed in a farrago of inert substances for purposes of mystification." Its composition was for a long time a secret.

Methylene-blue was introduced into the treatment of malaria by Guttmann and Ehrlich ${ }^{545}$ in I89I.

In my experience this is the best substitute for the derivatives 
of Peruvian bark. While it does not compare favorably with quinine, requiring a longer time to effect a cure and failing altogether in not a few instances, it seems to possess some specific action upon the parasites of malaria, and is the most valuable drug where the cinchona preparations are absolutely contraindicated.

Only the purest preparation should be employed for medicinal purposes, otherwise untoward results may follow.

The dose is from $1 / 2$ to 3 grains given every three hours until from $7 \frac{1}{2}$ to $I_{5}$ grains have been given in twenty-four hours. The drug may be continued in this manner for several days.

Untoward symptoms caused by the administration of methylene-blue are headache, nausea, vomiting, diarrhea, strangury, and albuminuria. These effects are less apt to supervene when a pure article is given. The patient should always be forewarned of the blue color imparted to the urine and feces.

After its introduction in 1842 by Boudin arsenic enjoyed, until recently, considerable reputation in the treatment of malaria. The old school of physicians claimed results but little inferior to those with quinine. More recent observations show that if this agent has any value in the treatment of malaria it is in the chronic form. Whether its good effects here are chiefly upon the anemia and as a general tonic or whether it has some action upon the parthenogenetic cycle is not determined. Fowler's solution and the arsenious acid are the preparations usually employed. The dose at the beginning should be small and gradually increased. Upon the appearance of puffiness of the eyelids, colicky pains in the abdomen, and diarrhea the dose should be diminished or its use temporarily discontinued. The arsenious acid may well be combined with iron, quinine, and strychnine in the treatment of chronic malaria.

Two recent preparations of arsenic, arthenal or disodic methylarsenate, and sodium cacodylate have been vaunted as antimalarials. They are rich in arsenic, but less toxic. They are usually given hypodermically in doses of from $3 / 4$ to $1 / 2$ grains daily. I have had good results with the latter preparation, especially in chronic malaria. 
The benefits derived from atoxyl in the treatment of trypanosomiasis led to an investigation of its merits in malaria. Chemically, atoxyl is sodium aminophenyl arsenate, containing about 25 per cent. of arsenic and soluble at $20^{\circ} \mathrm{C}$. in 4.3 parts of water. The usual dose is from $1 / 3$ to $\mathrm{I}$ grain, given every day or every other day, usually hypodermically. It cannot be regarded as a substitute for quinine.

There is no doubt but that salvarsan cures some cases of malaria where all other means fail. Such cases are doubtless those in which "quinine-fast" organisms are present. The drug should be administered by the usual technic in ordinary dosage at weekly intervals.

Opium is not only effective against certain symptoms of malaria, but it has been shown to possess antiperiodic virtue. This is, in all probability, due to the narcotine contained. For this reason opium, in the form of the powder, Dover's powder, laudanum, or paregoric, is more effective than morphine. The immunity of opium smokers to malaria has been remarked upon by a number of tropic physicians.

A large number of other substances have been recommended as substitutes for quinine. The chief of these are phenocoll, eucalyptus, salicin, salicylic acid, sodium hyposulphite, etc. Their antimalarial value, if indeed they possess any, is so slight as to render a detailed consideration not worth the while.

The serum treatment of malaria has not yet reached the stage of practical utility.

Recently Carpenter ${ }^{546}$ claims remarkable success in the treatment of malaria with powdered splenic extract in the dose of 5 grains every two to four hours, preferably in capsules. He states that it is equally as reliable a remedy as quinine.

The Treatment of Pernicious Malaria.-There are certain cases, apparently on the borderline between benign malaria and pernicious malaria of the cerebral type, which may cause hesitation as to the mode of administration. In these cases, usually in children, the patient, though stupefied, or even semicomatose, can be aroused and made to swallow and usually retain the medicine. In such cases, if the patient can be watched, the quinine may be given in solution by the mouth. If vomited 
or if the symptoms do not rapidly improve, the drug in dilute solution should be injected intramuscularly. Where the injection mode is chosen it is advisable to supplement this with oral administration of the solution where it can be swallowed, and even the rectum may be employed also.

As previously stated, quinine for intramuscular injection should be in dilute solution; 15 grains to $2 \frac{1}{2}$ drams of water is a suitable proportion. The gluteal region, above the ischial tuberosities, is the best site for injection. In pernicious cases about 15 grains is the quantity to be used at first injection. Subsequent doses may be from 5 to ro grains injected every six or eight hours as needed. The technic of such injections has already been given.

Just as antisyphilitics may cause the gumma to melt rapidly but are powerless to restore the tissue they have destroyed, so quinine has its limitations in the therapeutics of malaria. It should be borne in mind that in its relation to the parasites quinine is a toxin, but not an antitoxin. It is impossible that where the parasites are accumulated to the extent of thrombosis the quinine in solution in the blood does not reach them in toxic quantities, and where perivascular exudation, hemorrhage, and necrosis have resulted from these thrombi the annihilation of the parasites avails nothing. This is corroborated by those cases ending fatally, notwithstanding a rapid disappearance of the parasites, and in which post-mortem these secondary changes are found. All that can be expected of quinine is to destroy the parasites, and this it may fail to accomplish from not being absorbed or not being present in the blood in sufficient quantities or at the time when the parasites are most susceptible to its action or on account of thrombotic occlusions it may not gain access to the parasites causing the symptoms. Quinine is probably a true specific in those cases of pernicious malaria only in which, in the absence of irreparable changes due to toxins or to thrombi, the prompt destruction of the parasites would be attended by an almost simultaneous cessation of symptoms.

Other than the specific treatment there are important symptomatic indications to be met. 
In cases with high temperature and hot dry skin, cooling baths should be used. For heart depression strychnine or digitalis are useful.

In the cerebral types the ice-bag to the head is called for and an active cathartic should be given if possible. Where this cannot be swallowed, a drop of croton oil on the back of the tongue may be tried. If delirium is marked, a solution of chloral and the bromides should be given by the rectum. Where there are convulsions, chloral and bromides by the rectum, morphine hypodermically, or even inhalations of chloroform may be necessary. Bell employed lumbar puncture in a case of malarial coma to relieve the increase in the cerebrospinal fluid which usually exists in these cases, but the result was disappointing.

In algid attacks for the relief of cold surface and dyspnea, especially if choleraic symptoms are present, nothing is so suitable as a combination of morphine and atropine. The heart usually requires stimulation by strychnine and digitalis. Hypodermics of ether may be necessary. If dysenteric symptoms arise they should be treated with opium and bismuth, together with saline irrigations.

If complications appear they should receive appropriate treatment.

During convalescence a tonic of arsenic, strychnine, iron, and quinine is usually indicated. In cases where it is feasible, a change of climate should be ordered until recovery is thoroughly established. 


\section{BLACKWATER FEVER}

\section{CHAPTER IX}

\section{INTRODUCTION}

Blackwater fever is known by many names, some of which are mere localisms. Among the more general synonyms are: malarial hematuria, hemoglobinuric fever, swamp fever, bilious hematuric fever, and melanuric fever.

History.-It is probably unique in historic pathology that a complex of symptoms so striking as hemoglobinuric fever should have such an obscure history. As this obscurity is intimately associated with the etiology and symptomatology of the condition an investigation of some of the factors in its history is not without interest. After a short statement of the history of hemoglobinuric fever we will briefly consider how far it has been influenced by (I) its confusion with bilious remittent fever and yellow fever, (2) the introduction of cinchona bark and its alkaloids into the treatment of malaria, and (3) the advent of Europeans into endemic regions.

In the years from 1850 to 1853 blackwater fever was described by Lebeau, Daullé, and Leroy de Méricourt, physicians of the French navy, who observed it in Madagascar, and especially on the Island of Nossi Bé, off the northwest coast of the former island. They named the condition icteric pernicious fever. In I86I cases observed in the Antilles, Guiana, and Senegal were described by Dutrouleau as hematuric bilious fever. In the early sixties Barthèlemy-Bénôit also described hematuric bilious fever, and in 1874 appeared the monograph of BérengerFéraud on melanuric bilious fever. This writer states that the disease has existed in Goree since 1845 , and in St. Louis, in Senegal, according to the hospital records of that city, at least since 1820 , shortly after the settling of the Europeans there. The first twenty-three settlers at Gabun, in 1843, 
were attacked with blackwater fever and almost all of them died. A great increase in frequency has occurred since 1850 .

Crosse ${ }^{1}$ believes his own case, in 1888 , to be the first on record in the Niger Territories, though he states that the disease was said by old coasters to have existed in the Niger Delta since 1882 . F. Plehn ${ }^{2}$ does not believe the disease to be of recent introduction into West Africa, but attributes its comparatively late recognition to two facts-first, that the susceptible population, who formerly lived as traders on anchored hulks, began to take up their abode on the shore; second, that the disease was formerly confounded with yellow fever. It has been shown in Senegambia since $1885,{ }^{3}$ and in the Dutch East Indies since the Atjeh War, I874-78. ${ }^{4}$ Calmette saw a number of cases in Gabun in $1886-87$, and Fluit, in San Juan del Sur, has seen numerous cases since $1850 .^{3}$ The condition was not described in India until 1855 , and Sambon $^{5}$ regards this as conclusive of its recent introduction, as "it would be absurd to think that it could have escaped the attention of such men as Annesley, Chevers, Carter, Martin, Fayrer, Morehead, and Maclean had they met with it."

Dr. Elliotson, ${ }^{6}$ in 1832 , mentioned a case of ague accompanied by a discharge of bloody urine during the cold stage.

Todd, ${ }^{6}$ in 1849 , asserted that "a state of general cachexia, such as often occurs in scurvy, may bring on hematuria, or such as results from an aguish state brought on by the malaria of marshy districts."

In the United States hemoglobinuric fever was first described by Dr. J. C. Cummings, ${ }^{7}$ of Monroe, Louisiana, in r859. He reported six cases, and refers to numerous cases during the previous season. Faget $^{8}$ treated the disease as early as 1859 , and states that the cases with hematuria and hematemesis had frequently been seen in New Orleans and been mistaken for yellow fever. Inasmuch as Faget considered hematemesis a common symptom of hemoglobinuric fever, it is possible that he himself confounded the two diseases in some instances. In $\times 867$ Dr. T. C. Osborn ${ }^{9}$ of Greensboro, Ala., observed ten cases, five of which ended fatally, some with anuria and uremia. All the patients had been repeatedly 
attacked with malaria. A few months later his son, Dr. J. D. Osborn, ${ }^{10}$ read a paper before the Greensboro Medical Society from which it is evident that the disease was becoming more prevalent, and that the country people were regarding it as yellow fever. Dr. H. C. Ghent ${ }^{11}$ of Port Sullivan, Texas, in 1866 reported hemoglobinuric fever endemic in parts of Texas. In March, I869, Dr. R. F. Michel, of Montgomery, Ala., read a paper before the Medical Association of the State of Alabama in which he spoke of the disease as "a malignant malarial fever, following repeated attacks of intermittent, characterized by intense nausea and vomiting, very rapid and complete jaundiced condition of the surface as well as most of the internal organs of the body, an impacted gallbladder, and hemorrhages from the kidneys. These phenomena presented themselves in an almost uninterrupted link, attended by remissions and exacerbations. It is a fever peculiar to the United States." He recorded the morbid anatomy in one of his fatal cases. In Arkansas hemoglobinuric fever was first recorded by Dr. E. R. Duvall, of Fort Smith, in a paper read before the State Society in $187 \mathrm{I}$. He believed the case he recorded to be the first to occur in the state. This paper is said to be a model of accurate clinic observation. In 1880 Dr. G. B. Malone, in Monroe County, Arkansas, reported I 55 cases met in his practice. The affection was first reported in Georgia by Dr. W. A. Greene, of Americus, in 1872 , and in North Carolina by Dr. Norcom, of Edenton, in 1874 . Norcom asserts that the disease did not, as some claimed, make its first appearance a few years ago, but that it had long been recognized. Dr. McDaniel, ${ }^{6}$ of Camden, Alabama, described hemoglobinuric fever in 1874 , and says, "In calling up my own reminiscences, I am sure that I have occasionally, ever since my boyhood, seen isolated cases of what was considered intense bilious fever with the surfaces and under tissues stained deeply yellow and with the urine deep red. They were nearly all fatal, and were called in older phrase 'bilious congestive,' and in more recent 'pernicious bilious.' I have also, but more rarely, known groups of similar cases associated, say three or four cases occurring on the same premises or in the same 
family, about the same time. All such cases, in addition to the deep so-called bilious color and the red urine, had jactitation, suspirous breathing, inordinate thirst, and vomiting of various shaded and tinted so-called bilious matters. By diligently inquiring I have ascertained that very many old physicians, some of whom have now retired from practice, are satisfied that they have observed similar cases, sometimes singly and sometimes in groups."

The late lamented Dr. A. G. Mabry, in a report of a case of intermitting icterode hematuric fever made to this association in 1870 , says, "It is a mistake to suppose that this is a new form of disease. More than twenty-five years ago I treated, in the vicinity of Selma, cases of intermitting fever presenting in a marked degree all the symptoms characteristic of these cases at the present day."

The acrimonious dispute of the earliest writers on the subject of what constituted the coloring matter of the urine is paralleled only by that occurring later concerning quinine in the treatment. While Daullé and Bérenger-Féraud stoutly maintained that the dark color was due to the presence of bile in the urine, Dutrouleau, Pellarin, Barthèlemy-Bénôit, Antoniades, and Corre ascribed it to blood. It is remarkable that none of the first American writers attributed the color of the urine to bile, but considered it due to blood. Corre (I88I) and Karamitsas (I882) proved that the process was a hemoglobinuria instead of a hematuria.

The credit of first directing attention to the etiologic relation between quinine and hemoglobinuric fever is generally credited to Tomaselli who published his first observations in 1874 , but this is an error. At a meeting of the Greek Medical Society, Nov. 6, 1858 , Veretas ${ }^{12}$ reported that the majority of physicians practising in the marshy regions of Greece had noticed hematuria following the administration of quinine. He adds, "Among these observers my father has a place, having attentively observed this action of the medicament not only in several other persons, but in himself also, unfortunately, as he was for a long time tormented with intermittent fever during his long residence at Vonitsa." Konsola ${ }^{12}$ is said to have 
observed similar cases in 1858 . During this year also, Antoniades published an article on "Hemorrhages and Particularly Hematuria in Intermittent Fever," in which he opposes the theory that quinine is a cause. Other Greek physicians whose observations were published before those of Tomaselli are Papavassilou, Rizopoulos, and Karamitsas.

I. The close relationship between malaria and blackwater fever renders it easily understood why the latter might have been confounded with bilious remittent fever. Moreover, the early pyretologists almost completely ignored the condition of the urine in fevers. Hence, in a clinical scene, preceded by or opened with ordinary malarial paroxysms and characterized by dark urine, between the color of which and the bilious urine of bilious remittent fever there are all degrees, it is slight wonder that the two conditions were confounded.

This probably occurred chiefly in India and to a less extent in certain portions of Africa and America. One is struck, on reading accounts of the Indian fevers, with the description of the intense jaundice of the skin and sclaræ, out of all proportion to this symptom in the bilious remittent fevers of the present day. In fact, some of these descriptions-for instance, Johnson's ${ }^{13}$ of his first case in India-lack only the mention of the characteristic urine, about which the author is altogether silent, to make a fairly complete case of hemoglobinuric fever. Since it is reasonably certain that there was no yellow fever in Minorca during the period of Cleghorn's sojourn in the island, namely from $\mathrm{I} 774$ to $\mathrm{I} 749$, it may reasonably be inferred that he saw cases of blackwater fever.

In 1804 , Alibert, in the third edition of his work, wrote: "The state of the urine in malignant fevers demands great attention." Thus a diminution of that excretion and its assuming a black color are very alarming symptoms.

The fact that the early history of hemoglobinuric fever opens with disputes as to whether the coloring matter of the urine was due to blood or to bile is evidence of the confusion by some observers between hemoglobinuric fever and bilious remittent fever, since formerly bile and malaria were practically synonymous. 
It has been mentioned that the first reliable records of the existence of hemoglobinuric fever were, according to BerengerFeraud, those of the hospital of St. Louis in Senegal, where it is shown to have existed as early as 1820 . It is a singular coincidence that this city afforded in 1778 , the first epidemic of yellow fever occurring in Africa. ${ }^{15}$ Later Plehn' ${ }^{2}$ gave as one of his reasons for believing that hemoglobinuric fever was not a new disease in West Africa, that it had formerly been mistaken for yellow fever. Besides Senegal, two of the other regions where hemoglobinuric fever was first seen, the West Indies and Guiana, were yellow fever foci. In the United States we have the early statement of Dr. J. D. Osborne that the condition was then regarded as yellow fever.

The similarity of the symptoms and the relative immunity of the black race to both diseases render the mistake somewhat excusable. As recently as 1897 Below $^{16}$ maintained the identity of yellow fever and blackwater fever.

2. Cinchona bark was introduced into Europe in 1640 by the Countess del Cinchon, wife of the vice-regent of Peru, in whose honor it has received its name. The efficacy of the bark in malaria was first known to the Indians in the region of Loxa, in the southern portion of Ecuador. The Corregidor of Loxa, hearing of the severe illness of the countess with tertian fever at Lima, in I638, advised her physician, de Vega, to give the bark a trial, which effected a prompt cure, and in those days was regarded as nothing short of miraculous.

When the countess returned to Spain she took a supply of the bark with her. Here it seems first to have been employed chiefly by the Jesuits who introduced it into Rome in 1649 . It was then known as countess' powder or Jesuits' powder. Its use was antagonized by other religious denominations and by the medical profession. Bark was imported into England in I $_{1} 7$ by Sir Robert Talbot, an English quack, who kept the remedy a secret and sold it for one hundred louis d'or per pound. Louis XIV, who was attacked with a rebellious and severe intermittent in the year 1679 , was cured by Talbot with a concentrated vinous tincture of the bark, purchased and 
made public the secret remedy, for which he paid $£_{4} 8, \infty \circ$ and a life annuity of $£_{2, \infty}, 00$.

In India the remedy was employed by Bogue ${ }^{17}$ as early as 1657. In these times in Spanish-America, where the bark was indigenous, extraordinary methods were employed to prevent the nature of the drug becoming recognized. But during the eighteenth century cinchona bark was almost universally known. Lind is said to have employed in Lower Senegal, during $I_{7} 65$, over $I_{4} \circ$ pounds of the bark. In $I_{7} I_{4}$, Ramazzini ${ }^{18}$ wrote that should a fever patient die it was considered a crime not to have employed cinchona. In fact, so widespread was the use of large doses of bark that Calmenero (I647), Casati (I66I), Daval (I684), Ramazzini (I7I4), and others wrote vehemently against the abuse of the drug.

Pelletier and Caventou, in 1820 , succeeded in isolating quinine from the bark.

The institution of cinchona plantations in Java in 1854 and in Ceylon in 1859 caused a drop in the price of quinine, which had formerly sold for its actual weight in gold, to one-twentieth the original price.

Marchiafava and Bignami ${ }^{19}$ seek to explain the seeming late appearance of hemoglobinuric fever by the use of quinine becoming prevalent at the time when the disease was first described. It is probable that this factor has caused an increase in certain localities, but a comparison of the history of the disease with that of the drug shows no very intimate chronologic relations. Further, blackwater fever is on the decrease in some regions where the use of quinine is becoming more general. This is reported to be the case in German East Africa by Meixner, ${ }^{20}$ in Cameroon by Ziemann, ${ }^{20}$ in Togo by A. Plehn, ${ }^{21}$ and by Kohlbrugge ${ }^{4}$ in the Malay Archipelago. The large number of cases occurring without the previous use of quinine should also be considered.

3. A consideration of the importance, in the history of hemoglobinuric fever, of the immigrations of Europeans into regions where the condition is endemic involves the history of the tropics and subtropics. This factor is manifestly an essential in countries where the natives are nearly immune, 
as in parts of Africa. Historic events, which were probably potent in the development of blackwater fever, were the discovery of America, the Portuguese discoveries and settlements on the coast of Africa, the African slave trade and the later efforts to abolish same, the advent to Africa of missionaries and explorers, especially in the early part of the nineteenth century, and the operations of the East India Company.

The accession of Europeans was influential in the history of hemoglobinuric fever in several ways-by the increase of susceptible population, by the importation of quinine, and by the advent of physicians competent to recognize and to describe the disease.

Geographic Distribution.-In North America hemoglobinuric fever is found in the Southern States, especially parts of Texas, Louisiana, Arkansas, Mississippi, Tennessee, Alabama, Georgia, Florida, North Carolina, South Carolina, and Virginia. It is prevalent in Central America, particularly in Honduras, Nicaragua, and Costa Rica. It is found in the Greater Antilles, but appears to be rare in Hayti. In the Lesser Antilles it is more common on the islands of Gaudeloupe and Martinique. Numerous cases have been reported from Panama. In South America hemoglobinuric fever prevails more notably on the north and east coasts, in Venezuela, Guiana, and Brazil, at least as far south as Rio de Janeiro.:

It is rare in Italy, but rather more common in Sicily, Sardinia, and Greece. Otto ${ }^{22}$ has reported an autochthonous case from Krakau. It has appeared in some of the valleys of Spain, and, according to Schoo, was formerly observed in Holland.

The regions in India in which hemoglobinuric fever is endemic are as follows: Between the Ganges and the Himalayas in Behar Province; between the Godavari and the Mahandi Rivers in the Madras Presidency; a region of which Nagpur is the center; certain localities in the region of Bombay; and in Assam and in Upper Burmah. It is found in Asia Minor, Cyprus, and Syria (being common in Palestine), the Malay Peninsula, Siam, Cochin-China, Tonking, and other portions of French Indo-China, and in Southern China. In the East Indies it appears in Sumatra, Java, Celebes, and more commonly 
in New Guinea and the Bismarck Archipelago. It has been reported from Formosa, but is comparatively rare in the Philippines.

Tropical Africa is the home of blackwater fever. Here between the parallels of $15^{\circ} \mathrm{N}$. and $15^{\circ} \mathrm{S}$., it has been one of the deadliest foes to civilization. On the West Coast it occurs from Senegal to Damara Land, especially in Sierra Leone, Gold Coast, Nigeria, Cameroon, and the Congo Region. On the East it prevails from Somali Land to Delago Bay, particularly in British and German East Africa and the Congo Free State, and is met with in the Bahr-el-Ghazal region and in Sudan. In Algeria, Laveran, ${ }^{23}$ during a residence of five years, did not observe a single case and Brault ${ }^{24}$ saw only one. However, Coste ${ }^{25}$ has recently published his observations of 25 cases treated during I904-0.5 in the region of Arzew. It rages in parts of Madagascar and Reunion, and is known in Mauritius and the Comora Islands, notably Mayotte. The mountainous islands of the Gulf of Guinea afford a few cases.

Thus it seems that, while the peculiar geographic distribution of hemoglobinuria is embraced by that of malaria, it is not coextensive with the latter. And here the relation ceases. While all localities in which blackwater fever exists endemically are highly malarial, there are very extensive regions in which the severest forms of tropical malaria are rampant where hemoglobinuric fever is unknown.

Wellman $^{26}$ maintains a close relationship between the geographic distribution of Myzomyia funesta in Angola and that of blackwater fever. Daniels ${ }^{27}$ believes that if the disease is due to one or all of several varieties of mosquitoes which he mentions, $M$. funestus must be one of those implicated. F. Plehn ${ }^{28}$ suggested a possible relation between the geographic range of hemoglobinuric fever and that of certain mosquitoes.

In certain localities the disease seems to be on the increase. Crosse $^{1}$ says that it is increasing in certain parts of West Africa. Manson ${ }^{29}$ refers to the belief of competent observers that it is yearly becoming more common in Africa. Johnson ${ }^{31}$ and F. Plehn assert that it is undoubtedly becoming more 
prevalent on the West Coast of Africa, and A. Plehn ${ }^{30}$ believes that it is increasing in frequency in New Guinea. The inhabitants of the region of Jalpaiguri, in India, are recently said to be alarmed at its increase in that section. ${ }^{32}$

On the other hand, there are places where it is becoming less frequent. The medical report from German East Africa for the year 1903-04 shows a decrease from the preceding year. The report from Duala shows a steady annual decrease from I90I to 1904 inclusive. $^{33}$ Kohlbrugge ${ }^{4}$ declares that it is becoming rarer in the Malay Archipelago. A. Plehn $^{21}$ after mentioning the decrease in certain sections of West Africa, prophesied that in half a century this scourge of Tropical Africa would become, if not a historic reminiscence, at least an insignificant rarity. It is probably becoming less frequent in some of the Southern States judging from my experience in Eastern Arkansas.

Epidemics of hemoglobinuric fever have been described. Mastermann ${ }^{34}$ states that in $\mathrm{I} 893$ there was a regular epidemic of malaria in and around Jaffa, and among the fatal cases were a great many of hemoglobinuria. Says Plehn, ${ }^{30}$ "Not infrequently the disease appears in epidemic form, as was the case several years ago in Goree, Quittah, and Bonny." Sambon ${ }^{5}$ mentions several epidemics, as follows: The disease broke out among the laborers employed in making the canal through the Isthmus of Corinth; it attacked the Chinese laborers on the Congo Railway; and in 1885 , according to Dr. Wenyon, of Fatshan, China, it ravaged, like a plague, the Chinese army on the Tonquin border of Kwangsi. In collective dwellingssuch as barracks, hospitals, schools-it may attack several persons at the same time. In 1885 it broke out in a prison in Castiades, Sardinia, attacking 24 out of 800 convicts. 


\section{CHAPTER $\mathrm{X}$}

\section{ETIOLOGY OF BLACKWATER FEVER}

Among the conditions other than blackwater fever, under which hemoglobinuria can occur, may be mentioned paroxysmal hemoglobinuria, scarlet fever, typhus and typhoid fevers, acute articular rheumatism, leucemia, pneumonia, streptococcus infection, chronic suppurative conditions, after extensive burns or freezing, occasional injuries, rupture of ectopic pregnancy, transfusion of blood, injection of tuberculin, poisoning with phenocoll, guaiacol, pirodin, salipyrin, salicylic acid, antipyrin, sulphonal, the salts of chloric acid, phenol, pyrogallic acid, sulphuric, nitric, and hydrochloric acids, naphthol, analine, chrysarobin, toluylendiamin, glycerine, nitrobenzol, potassium chlorate, phenacetin, arseniuretted hydrogen, methylene-blue, phosphorus, oxalic acid, certain illuminating gases, helvella esculenta, and snake venom. Hemoglobinuria is a common symptom of Texas fever in cattle, and is seen occasionally in sheep, dogs, goats, horses, and mules, following infection with hematozoa resembling the malaria parasite.

Race.-Hemoglobinuric fever is chiefly a disease of the white race. The negro is not absolutely immune, though not a few observers of wide experience have not seen cases in this race. This relative immunity can be explained only by natural selection. It varies markedly in different tribes and members of an unsusceptible tribe may be attacked on moving to a blackwater fever focus. F. Plehn ${ }^{2}$ refers to an extensive outbreak that occurred among the Cameroon negroes, especially those who came from the interior to the coast. According to Dryepondt, ${ }^{23}$ the negroes recruited for the Congo Free State in 1890 to 1892 paid a large tribute to this malady. DeGreny ${ }^{3}$ saw twenty cases in negroes imported from the British Antilles for railroad construction work on the lower Congo. In the medical report from German East Africa for 
the official year, 1903-04, there were listed eight cases in negroes. Corre, ${ }^{3}$ Donny, ${ }^{3}$ the younger Moncorvo, ${ }^{3}$ Hanley, ${ }^{35}$ A. Plehn, ${ }^{36}$ Rudolph Plehn, ${ }^{37}$ Wittrock, ${ }^{33}$ Brunn, ${ }^{33}$ Curry, ${ }^{38}$ Eyles, ${ }^{29}$ Doering, ${ }^{39}$ Reynolds, ${ }^{40}$ Easmon, ${ }^{29}$ Wicke, ${ }^{41}$ Gaertner,${ }^{41}$ Quartey-Papafio, ${ }^{29}$ O'Sullivan-Beare, ${ }^{41}$ Vieth ${ }^{41}$ Goltman and Krauss, ${ }^{42}$ McElroy, ${ }^{43}$ Berenger-Feraud, ${ }^{2}$ Ziemann, ${ }^{23}$ Fisch, ${ }^{2}$ Ollwig, ${ }^{44}$ Greisert, ${ }^{44}$ Perry, ${ }^{55}$ Lovelace, ${ }^{56}$ Dufranc, ${ }^{57}$ Schumacher, ${ }^{58}$ Jungels, ${ }^{59}$ Hoesemann, ${ }^{60}$ (1 3 cases), Lewis ${ }^{45}$ Francez, ${ }^{46}$ Minor, ${ }^{47}$ McKay, ${ }^{48}$ Tyson, ${ }^{49}$ Gorgas, ${ }^{50}$ and Wendland, ${ }^{33}$ have seen cases in negroes. I have seen two cases in mulattoes and two in black negroes. Chinese imported into blackwater fever regions are almost as susceptible as whites. Manson ${ }^{29}$ says that many of the Chinese laborers on the Congo railway died of hemoglobinuric fever. Imported Indians are affected, but according to Daniels, ${ }^{51}$ are only about one-fourth as susceptible as Europeans. As may be inferred from one of the names, "fièvre jaune des creoles," creoles are not infrequently attacked. Masterman ${ }^{34}$ reports that it is common among the Jews of Palestine. Rothschuh ${ }^{3}$ saw cases in mixed breeds and pure Indians in Nicaragua.

Sex.-Males are more often stricken than females, the latter being less often exposed to malarial infection. In the temperate zone the proportion of males to females is about 3 to I. In persons under $I_{5}$ the proportion is more nearly equal. As we approach the equator the difference becomes wider, owing to the relatively small number of susceptible females and children. Daniels ${ }^{27}$ says the proportion of male to female cases in British Central Africa is 15 to $I$. Cardamatia ${ }^{52}$ believed that pregnancy conferred immunity; however, Krauss ${ }^{53}$ has reported a case in a pregnant woman, who made a tedious recovery after abortion. A few years ago ${ }^{54}$ published brief notes of a case occurring in the practice of a colleague. The woman aborted on the third day of the disease and died on the fourth. Cases have often been observed to follow immediately after menstruation.

Age.-In America more than half the cases occur before the age of thirty, though very young children are relatively exempt. In the tropics it is commoner in the third and fourth decades of 
life because most of the susceptible population is within these ages. Daniels ${ }^{27}$ saw a case in a half-caste about five years old; Wendland, ${ }^{33}$ Van der Scheer ${ }^{69}$ and Karamitsas ${ }^{12}$ observed cases in children of four; Lipari ${ }^{70}$ mentions two cases in children of three, Masterman ${ }^{34}$ one in a girl of two, and Oetker ${ }^{71}$ one in a two-year-old child; Fisch ${ }^{72}$ saw cases in children of fourteen months and two and one-half years, and $\mathrm{McElroy}^{73}$ one at twelve months.

Season.-In the tropics, like malaria, it is perennial, occurring without marked seasonal prevalence, though probably commoner in the transition period from the moist to the dry season. In the temperate zone it appears at the height of, or immediately following, the malarial season, the second half of the year showing by far the greater number of cases, especially August, September and October. A few cases are seen in the first six months. In Greece it is during the months of November and December that the majority of cases occur.

Family Predisposition.-Tomaselli ${ }^{74}$ believed in a wellmarked family tendency, having observed cases in several members of the same family. Daniels ${ }^{27}$ refers to three families in which he noticed this predisposition. Three such families are known to me. Cardamatis ${ }^{12}$ relates the case of a family of seven, of which the father, mother, and one daughter were within a few days attacked and died with blackwater fever. The others, removing to Athens, were all subsequently attacked but fortunately recovered. Sutherland ${ }^{75}$ speaks of a family of which all the children, six in number, died with hemoglobinuric fever.

Idiosyncrasy.-An idiosyncrasy in susceptible individuals has long been assumed and by many passively accepted as the sole explanation of the mysteries of pathogenesis. Foust$\operatorname{anos}^{76}$ holds that idiosyncrasy is either congenital or acquired, as the result of debility or bodily changes due to syphilis, malaria, etc. There is not sufficient evidence to show that heredity plays an important part in whatever is meant by this vague term.

Previous Attacks of Hemoglobinuria.-Who has had blackwater fever is prone to recurrences. In the tropics about 
one-fourth of the subjects have more than one attack. Of 304 cases mentioned by Cardamatis, $8 \mathrm{I}$ occurred in persons who had previously had it. Several tropic physicians record repeated attacks in themselves. Thus F. Plehn ${ }^{37}$ had five attacks, Crosse ${ }^{77}$ at least ten severe attacks, and Banks ${ }^{78}$ twelve or thirteen during eighteen years' residence in Congo. One of Koch's ${ }^{79}$ patients had ten attacks in one year, and the Plehns ${ }^{80}$ state that they know persons who have had fifteen or more attacks. I treated a patient in his twelfth attack. There is, therefore, no active immunity; the only immurity except natural being conferred by prolonged residence in an endemic focus.

Length of residence in the home of the disease is an important factor. A curve showing the number of first attacks to each year of residence would rise from the first to the third years and then fall gradually. This is almost constant for observation in the tropics, being less noticeable in temperate regions. The following table of cases seen in the tropics will illustrate:

\begin{tabular}{|c|c|c|c|c|c|c|}
\hline & $\begin{array}{c}\text { Ist } \\
\text { year }\end{array}$ & $\begin{array}{l}\text { 2nd } \\
\text { year }\end{array}$ & $\underset{\text { year }}{\text { 3rd }}$ & $\begin{array}{l}4^{\text {th }} \\
\text { year }\end{array}$ & $\begin{array}{c}\text { 5th } \\
\text { year }\end{array}$ & Later \\
\hline Burot and Legrand, ${ }^{81}$ Ioo cases. & 6 & 22 & 43 & 20 & $\ldots$ & 9 \\
\hline Daniels, ${ }^{27} \times 14$ cases $\ldots \ldots \ldots$ & $2 \mathrm{I}$ & 40 & 27 & 12 & 5 & 9 \\
\hline Berenger-Feraud, ${ }^{82}$ I 85 cases............. & 10 & $4^{2}$ & 79 & 37 & 9 & 8 \\
\hline \multirow[t]{2}{*}{ Vedy ${ }^{83} 54$ cases............................ } & 6 & 8 & 29 & 5 & 2 & 4 \\
\hline & 43 & I 12 & 178 & 74 & 16 & 30 \\
\hline
\end{tabular}

Fifty cases observed by McElroy ${ }^{84}$ in the Mississippi Valley were distributed as follows: Two in the first year of residence, three in the second, six between the second and the fiftb, twenty-three between the fifth and tenth, i I between the tenth and twentieth, and five after twenty years.

Exceptionally are cases seen after only a short period of tropic residence, as Plehn's ${ }^{2}$ case XXXV, after two months in the Cameroon, and one of Brem's ${ }^{85}$ cases after two months on the Isthmus of Panama. Ziemann ${ }^{86}$ mentions two cases beginning six weeks and twenty-seven days, respectively, after 
arrival in a malaria locality, and Oeconomou ${ }^{12}$ observed a case occurring after ten days of residence in a malarial region. The case showing the longest period of residence before onset in which this is specified is that of Howard, ${ }^{87}$ twenty-three years in Central Africa, though in five of McElroy's ${ }^{84}$ cases the length of residence was longer than twenty years.

Altitude.-Hemoglobinuric fever is often considered a disease of the lowlands, though cases are commonly observed at heights of 3,000 feet. The results of Daniel's ${ }^{27}$ observations on the influence of altitude may be stated as follows:

The greater number of recorded cases have occurred in the highlands at or about 3,000 feet above the sea level. There are two reasons for this: First, the number of residents in these highlands is much greater than in the other districts. This correction alone reverses the figures; secondly, many of these cases were visiting the highlands on account of health or for other reasons. Others were passing through the highlands when invaliaed home. Some had recently visited the lowlands. A true correction that would attribute each case to the district in which the disease was acquired is impossible, but taking an arbitrary period of a fortnight as representing a not improbable latent period we should find that the place of residence a fortnight or more previous to the onset would give a very different district distribution to that given by considering the places of onset. Corrected by proportional numbers of susceptible persons in each district and for place of residence two weeks previous to onset, the distribution per ro of population is as follows: 1.04 in the highlands, 7.28 at the lake level (Lake Nyassa, altitude about I,500 feet), and 3.8 in the lower shore regions. According to Laveran ${ }^{23}$ it was necessary, on account of the frequency of blackwater fever, to abandon certain posts in Congo established at heights of 500 to 700 meters.

Change of residence is a not uncommon cause for an outbreak, especially if the difference in altitude or climate is decided. In Africa not only those coming from the mountains to the lowlands, but also those moving from the insalubrious littoral to the refreshing high-lying districts are predisposed. This change seems to be independent of the hardships of travel. 
After Leaving Endemic Region.-Such cases have been observed in England by Bassett-Smith ${ }^{88}$ Hughes, ${ }^{89}$ Sylivan, ${ }^{3}$ Crosse, ${ }^{1}$ Manson, ${ }^{90}$ Daniels, ${ }^{27}$ and Parker; ${ }^{91}$ in Ireland by Mowbray; ${ }^{92}$ in Germany by Schlayer, ${ }^{93}$ A. Plehn, ${ }^{21}$ Kleine, ${ }^{94}$ Koch, ${ }^{95}$ and F. Plehn $;{ }^{80}$ in France by Burot and Legrand, ${ }^{96}$ Le Dantec, ${ }^{97}$ Kelsch and Kiener, ${ }^{98}$ Rouvier,${ }^{98}$ Vincent, ${ }^{3}$ Boison, ${ }^{23}$ Troussaint ${ }^{23}$ and Laveran; ${ }^{23}$ in Belgium by Dryepondt and Vancanpenhout ${ }^{99}$ and Bertrand $;^{100}$ and in Baltimore by Brem. ${ }^{85}$ Many of these cases were not mere relapses; indeed, in the majority in which the number of previous attacks was specifically stated they were first attacks. The onset may occur from a few days to five months or more after leaving the endemic. area. Inclement weather and fatigue seem to be factors in some of these cases. The mortality is low.

Occupation, which requires residence in a malarial locality and which necessitates overturning of the soil, as gardening, farming, ditching, railroad construction, etc., is largely predisposing. Not a few cases occur among timber workers. The disease prevailed extensively among those engaged in the construction of the canal of Corinth. Manson ${ }^{29}$ tells us that many of the Chinese laborers on the Congo railroad died of hemoglobinuric fever, and DeGreny ${ }^{3}$ found many cases in both negroes and whites in the railroad work on the lower Congo. Crosse $^{77}$ says that it is significant that his first three gardeners died of blackwater fever, and that for some considerable time cases occurred only near the plantations, and as the plantations became more numerous the disease spread to the other stations in the territories.

Occasional Causes.- - Of these, exposure to cold and dampness is probably the most efficacious, showing somewhat analogous to paroxysmal hemoglobinuria. Over-exertion precedes some cases. The influence of alcohol has probably been over-estimated. Trauma has a slight etiologic importance. Thus Mould ${ }^{101}$ mentions a case developing after a sprained ankle; $\mathrm{Plehn}^{30}$ one in which a man was wounded in a bush fight and bled considerably. Crosse $\mathrm{e}^{77}$ and Plehn saw cases immediately following confinement. Psychic states, as anger, grief, and fear, exposure to sun, fatigue, excessive venery, syphilis, and 
the mercury cure have been mentioned as occasional causes. Cardamatis $^{12}$ lays stress on the association with rheumatic diathesis, I 2 of his 30 cases being rheumatic. Alexander Haig ${ }^{102}$ believes there is an intimate relation, most probably causative, between an excess of uric acid in the blood and hemoglobinuric fever. He makes the unfounded statement that the ordinary acid sulphate of quinine is about one-fifth xanthin, which is physiologically and pathologically equivalent to uric acid, and herein, he believes, lies its supposed power to produce hemoglobinuria. Johnson ${ }^{31}$ holds that a meat diet predisposes to blackwater fever.

Previous Malaria.-It may be said with almost absolute certainty that previous infection with malaria is essential. In fact, a majority of careful observers make the unqualified assertion. The extreme rarity of cases in which preceding malarial infection is denied almost forces us to the conclusion that it may have been overlooked, as might occur in latent or masked infection. It is, however, not impossible that hemoglobinuria may exceptionally accompany the first outbursts of malaria, as in cases of F. Plehn, ${ }^{2}$ Goltman and Krauss, ${ }^{42}$ and Brem. ${ }^{85}$ In all of the cases of Tomaselli and Koch, the most ardent advocates of the quinine theory, there was history of antecedent malaria. Tomaselli ${ }^{74}$ states in italicized words that the two conditions which favor the hemolytic action of quinine are: I, malarial infection, chronic or sometimes recent; 2, a special idiosyncrasy, often hereditary.

Cardamatis $^{12}$ cites several writers who have seen cases without preceding malaria, and Van der Scheer $^{69}$ is said to have seen such a case.

Pathogenesis.-There are three chief theories as to the nature of hemoglobinuric fever; I, that it is malaria; 2 , that it is quinine poisoning; 3 , that it is a disease sui generis.

I. Against the malarial nature of hemoglobinuric fever may be urged the following objections:

I. The parasites are often absent; when present they are not numerically proportionate to the severity of the attack and usually disappear as the disease progresses; sporulation does not correspond in time with the symptoms; hemoglobin- 
uria may be associated with different forms of the malaria parasite.

2. In malaria very numerous parasites may be present without producing hemoglobinuria.

3. The geographic range does not coincide with that of malaria.

4. Its seasonal prevalence does not correspond with that of malaria.

5. Blackwater fever is not amenable to quinine.

The frequency with which the parasites are found is shown by the following list of examinations by various observers. The first column of figures shows the number of examinations made, the second the number in which the parasites were found:

\begin{tabular}{|c|c|}
\hline$\ldots \ldots \ldots \ldots$ & 20 \\
\hline Bignami and Bastianellii ${ }^{104} \ldots \ldots \ldots \ldots \ldots$ & 2 \\
\hline Vincent $^{3} \ldots \ldots \ldots \ldots \ldots \ldots \ldots \ldots \ldots \ldots$ & 5 \\
\hline Drypondt and Vancanpenhout ${ }^{99} \ldots \ldots \ldots \ldots$ & I \\
\hline$\ldots \ldots \ldots \ldots \ldots \ldots$ & II \\
\hline 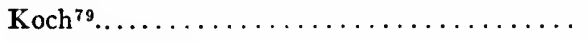 & 16 \\
\hline Hanley $^{35} \ldots \ldots \ldots \ldots \ldots \ldots \ldots \ldots \ldots \ldots \ldots$ & 13 \\
\hline Cardamatis $^{106} \ldots \ldots \ldots \ldots \ldots \ldots \ldots$ & 25 \\
\hline Burns $^{73} \ldots \ldots \ldots \ldots \ldots \ldots \ldots \ldots \ldots \ldots$ & 3 \\
\hline Boison ${ }^{23} \ldots \ldots \ldots \ldots \ldots \ldots \ldots \ldots \ldots$ & 3 \\
\hline$\ldots \ldots \ldots \ldots \ldots \ldots \ldots \ldots \ldots$ & 16 \\
\hline$\ldots \ldots \ldots \ldots \ldots \ldots \ldots \ldots$ & I \\
\hline Crosse $^{107} \ldots \ldots \ldots \ldots \ldots \ldots \ldots \ldots$ & $\mathbf{I}$ \\
\hline Brem $^{85} \ldots \ldots \ldots \ldots \ldots \ldots \ldots \ldots \ldots \ldots \ldots$ & 14 \\
\hline Krauss $^{108} \ldots \ldots \ldots \ldots \ldots \ldots \ldots \ldots \ldots \ldots$ & I I \\
\hline McElroy $^{84} \ldots \ldots \ldots \ldots \ldots \ldots \ldots \ldots \ldots$ & 23 \\
\hline $\operatorname{Thin}^{109} \ldots \ldots \ldots \ldots \ldots \ldots \ldots \ldots \ldots$ & $\mathbf{I}$ \\
\hline Kleine $^{94} \ldots \ldots \ldots \ldots \ldots \ldots \ldots \ldots \ldots \ldots$ & I5 \\
\hline$\ldots \ldots \ldots \ldots \ldots \ldots \ldots \ldots$ & 3 \\
\hline Curry ${ }^{110} .$. & 2 \\
\hline Troussaint $^{23} \ldots \ldots \ldots \ldots \ldots \ldots \ldots \ldots$ & 7 \\
\hline Pezopoules and Cardamatis ${ }^{23} \ldots \ldots \ldots \ldots$ & 7 \\
\hline Ketchen $^{111} \ldots \ldots \ldots \ldots \ldots \ldots \ldots \ldots$ & I \\
\hline Masterman $^{34} \ldots \ldots \ldots \ldots \ldots \ldots \ldots$ & $\mathbf{I}$ \\
\hline Schlayer ${ }^{93} \ldots \ldots \ldots \ldots \ldots \ldots \ldots \ldots \ldots \ldots \ldots \ldots \ldots \ldots$ & I \\
\hline$\ldots \ldots \ldots \ldots \ldots \ldots \ldots$ & 15 \\
\hline Stephens and Christophers ${ }^{27} \ldots \ldots \ldots \ldots \ldots$ & 16 \\
\hline Howard ${ }^{87} \ldots \ldots \ldots \ldots \ldots \ldots \ldots \ldots$ & I \\
\hline Ruge $^{112} .$. & I \\
\hline Goltman and Krauss ${ }^{42} \ldots \ldots \ldots$ & 12 \\
\hline
\end{tabular}




\begin{tabular}{|c|c|c|}
\hline Hartsock ${ }^{113}$ & I & $\circ$ \\
\hline F. Plehn ${ }^{2} \ldots \ldots \ldots \ldots \ldots \ldots \ldots \ldots \ldots \ldots$ & 33 & 22 \\
\hline Broden $^{114} \ldots \ldots \ldots \ldots$ & 20 & 6 \\
\hline $\operatorname{Marchoux}^{115} \ldots \ldots \ldots \ldots \ldots \ldots \ldots \ldots \ldots$ & 9 & I \\
\hline Oeconomou $^{115} \ldots$ & 3 & $\circ$ \\
\hline Cardamatis ${ }^{115}$. & 25 & \\
\hline Le Dantec ${ }^{97}$ & 3 & \\
\hline Bernardo $^{116} \ldots .$. & 20 & 17 \\
\hline Gauducheaun ${ }^{116} \ldots \ldots \ldots \ldots \ldots \ldots \ldots$ & 15 & $\circ$ \\
\hline 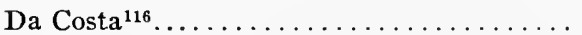 & 20 & I5 \\
\hline $\operatorname{Grattan}^{117} \ldots \ldots \ldots \ldots \ldots \ldots \ldots \ldots$ & II & 4 \\
\hline $\mathrm{Kulz}^{249}$. & I 6 & 3 \\
\hline Kudicke ${ }^{44}$. & 17 & 9 \\
\hline Wellman $^{119} \ldots \ldots \ldots \ldots \ldots \ldots \ldots \ldots \ldots$ & 34 & 3 \\
\hline Lovelace $^{56} \ldots \ldots \ldots \ldots \ldots \ldots \ldots \ldots \ldots \ldots$ & 327 & 145 \\
\hline Woldert $^{61} \ldots \ldots \ldots \ldots \ldots \ldots \ldots \ldots \ldots \ldots \ldots$ & $2 \mathrm{I}$ & 2 \\
\hline Deaderick $^{62} \ldots \ldots \ldots \ldots \ldots \ldots \ldots \ldots \ldots \ldots$ & 34 & 14 \\
\hline & 01 & 331 \\
\hline
\end{tabular}

As stated in the first objection, the parasites when present tend to disappear as the disease progresses. The following figures show the difference in results of examination at different periods. The great frequency with which they are found the day before the attack should be noted:

Stephens and Christophers:120

Day before attack parasites present in 95 per cent. of cases.

Day of attack parasites present in 70 per cent. of cases.

Day after attack parasites present in 20 per cent. of cases. Mannaberg: 82

Day before attack parasites present in 95.6 per cent. cases.

Day of attack parasites present in 63 per cent. of cases.

Day after attack parasites present in 17.1 per cent. of cases.

The reasons for the rapid disappearance of the organisms are, first, that often quinine has been taken before the examination; secondly, that in the terrific hemolysis the weaker cells, including those containing parasites, are usually the first to succumb.

The hemoglobinuria occurring in Texas fever of cattle is cited with some show of reason as an argument for the purely malarial origin of blackwater fever. There are essential differences, however, in the occurrences of blackwater in malaria and Texas fever. First, malaria is followed by blackwater in a 
very small percentage of cases, malaria being common, hemoglobinuria fever much rarer; in Texas fever blackwater is a common symptom, occurring in nearly all severe cases. Second, in blackwater fever in man the number of parasites shows no proportion whatever to the severity of the disease. In Texas fever, on the other hand, as is shown by Smith and Kilbourne ${ }^{483}$ the number of parasites is in direct relation to the severity of the process and increases as a fatal termination approaches. In human malaria the parasites may exist in very large numbers without the development of hemoglobinuria; this is not the case in Texas fever. Bonome found in the icterhemoglobinuria of sheep the same relation between the number and behavior of the hematozoa and the intensity and progress of the attack as obtains with Texas fever.

The form of parasite found in blackwater fever is, in the great majority of instances, the estivo-autumnal. Only exceptionally is hemoglobinuria combined with infections with the benign organisms. The tertian parasite has been observed in cases of Ziemann, ${ }^{86}$ Panse, ${ }^{86}$ Orme, ${ }^{121}$ Pecori, ${ }^{122}$ Carducci, ${ }^{122}$ Van der Horst, ${ }^{1}$ Cardamatis $^{64}$ ( ${ }_{5}$ cases), Deeks and James ${ }^{65}$ (21 cases), Williamson, ${ }^{66}$ Roseo, ${ }^{67}$ Hughes, ${ }^{89} \operatorname{Koch}^{105}$ (5 cases), A. Plehn $^{36}$ (3 cases), Ollwig, ${ }^{33}$ McElroy, ${ }^{84}$ Goltman and Krauss, ${ }^{42}$ Brem ${ }^{85}$ Herrick, ${ }^{85}$ and $\mathrm{Curl}^{85}$ (3 cases). The quartan parasite has occurred in cases of Vincenzi, ${ }^{82}$ Groco ${ }^{82}$ Kleine, ${ }^{94}$ Kudicke, ${ }^{44}$ Christophers and Bentley, ${ }^{63}$ Cardamatis $^{64}$ (5 cases), and Otto. ${ }^{22}$ Thiroux $^{22}$ and Laveran ${ }^{23}$ are said to have found the large form of parasite, but whether tertian or quartan is not stated. The fact that parasites other than estivo-autumnal have been found is no argument against the malarial nature of blackwater fever, since cases of pernicious malaria in which only the large tertian parasites were found have been reported by French, ${ }^{123}$ Ewing ${ }^{124}$ ( 2 cases), Ziemann ${ }^{86}$ and others.

Some writers believe that in addition to the mechanical destruction of the red cells by the parasites the latter give off toxins which have hemolytic powers. The facts, however, that intense hemolysis may occur with very few parasites in the blood, and that the parasites when present do not bear a direct relation to the severity of the disease, but rapidly diminish as the 
disease progresses, speak strongly against the rôle of a parasitic toxin in blackwater fever.

The number of cases in which the parasite is found if the examination is made early constitutes a conclusive evidence of an intimate relationship to malaria. This, however, is not all. The testimony furnished by the parasites is corroborated by the two subsidiary evidences of malaria: first, pigmented leucocytes; secondly, mononuclear leucocytosis. Given, therefore, the presence of the parasites in the first hours of attack, and the almost constant finding of pigmented leucocytes and mononuclear leucocytosis, it is impossible to deny that malaria plays an important rôle in its production.

The peculiarity of the geographic distribution of hemoglobinuric fever is no argument against its malarial nature. While it does not occur in all, even highly, malarial countries, it is not met except in markedly miasmatic regions. Neither does the distribution of quartan fever or some forms of pernicious fever coincide with that of malaria in general; nor is the slight difference of seasonal prevalence of any weight. The different forms of malaria have different seasons of prevalence as "spring tertian," and estivo-autumnal.

Favorable, therefore, to malarial character are:

I. Geographic distribution.

2. Length of residence in endemic region.

3. Previous attacks of malaria.

4. Malarial prophylaxis is prophylactic of blackwater fever.

5. Blood findings; parasites, pigmented leucocytes, mononuclear leucocytosis.

The fact that hemoglobinuric fever does not respond to quinine is one of the strongest evidences that it is not an attack of malaria per se.

My opinion of the relation of malaria to blackwater fever is that the former is essentially and solely the predisposing cause, and that in some cases it may act also as the exciting cause.

II. Tomaselli first published his observations as to the etiologic relation between quinine and blackwater fever in 
1874. More recently Koch has directed attention to it. The widespread controversy that followed the publication of Koch's views was bitter in the extreme; the matter was even aired in the London lay press. The misunderstanding was probably due to two causes: first, ignorance of Koch's utterance at first hand; secondly, the somewhat non-committal manner in which he expresses his idea of the relation to malaria. While he is very emphatic that blackwater fever is not an attack of malaria, he is not clear as to the predisposing rôle of the latter. He does not even assert that quinine is the exciting cause in all cases, but admits that, although he saw no cases of blackwater fever in which quinine could be excluded, he could not go so far as to maintain that every case of blackwater fever is quinine poisoning. There is no doubt but that this acrid dispute was productive of dire results, inasmuch as it brought the specific into discredit not only with the laity, but with many of the profession. Even yet it is necessary in some places on account of a fear of hemoglobinuria to disguise quinine before it can be given.

Tomaselli ${ }^{74}$ was able to collect from the literature only 102 cases of quinine hemoglobinuria.

The objections to the quinine theory are:

I. Hemoglobinuria is restricted in geographic range, and is absent from some highly malarial localities where much quinine is used.

2. Hemoglobinuria does not follow the administration of quinine for maladies other than malaria.

3. In a considerable number of cases the antecedent use of quinine can be eliminated with certainty.

4. The same individual may have an attack following the administration of quinine, and later take it without harmful results.

5. The severity of the attack bears no relation to the size of the dose.

6. One dose of quinine could not cause intermittent hemoglobinuria.

7. The great majority of cases recover even under the continued use of large doses of quinine. 
Objections I, 2, and 6 go to demonstrate that other, and probably more important, factors than quinine are at work even in cases often attributed to it. Objections 4 and 7 are not potent if we assume that only a portion of the erythrocytes are susceptible to the effects of quinine, and that all these are destroyed by the first dose. Objection 5 proves that in cases where an outbreak occurs after quinine it cannot be regarded as mere quinine poisoning. The third is the strongest argument against the theory that all blackwater fevers are cases of quinine poisoning. That quinine is not always the exciting cause is fully attested by the numerous cases in which no quinine had been given, as observed by Boye, ${ }^{83}$ Vedy, ${ }^{83}$ Doering, ${ }^{125}$ Bolden, ${ }^{114}$ Ellenbeck-Hilden, ${ }^{126}$ Legrain, ${ }^{127}$ Grall, ${ }^{128}$ Rossoni, ${ }^{129}$ F. Plehn, ${ }^{27}$ A. Plehn ${ }^{21}$ (22 cases), Marchiafava, ${ }^{105}$ Celli, ${ }^{105}$ Bastianelli, ${ }^{105}$ Beyfuss, ${ }^{105}$ Van der Scheer,${ }^{105}$ Seal, ${ }^{130}$ Powell, ${ }^{105}$ Von Diesing, ${ }^{105}$ Carre, ${ }^{105}$ Schellong, ${ }^{105}$ Laveran, ${ }^{23}$ Quennec, ${ }^{105}$ Navarre, ${ }^{105}$ Reynolds, ${ }^{105}$ Etienne, ${ }^{105}$ Sims, ${ }^{105}$ Donny, ${ }^{105}$ Dryepondt, ${ }^{105}$ Mense,,${ }^{105}$ Rothschuh,${ }^{3}$ Fluit, ${ }^{3}$ R. Plehn, ${ }^{37}$ Dempwolff, ${ }^{37}$ Crosse, ${ }^{107}$ Thin, ${ }^{109}$ Stalkarrt, ${ }^{131}$ Christophers and Bently, ${ }^{63}$ Dufranc, ${ }^{57}$ Deeks and James, ${ }^{65}$ Connor, ${ }^{55}$ Sutton, ${ }^{68}$ Hopkins, ${ }^{132}$ Cargill, ${ }^{107}$ Mould, ${ }^{101}$ Hoffmann, ${ }^{33}$ Daniels, ${ }^{27}$ Rankin, ${ }^{133}$ Cardamatis ${ }^{12}$ (32 cases), Yofe, ${ }^{23}$ Moffatt, ${ }^{134}$ Schlayer, ${ }^{93}$ Curry, ${ }^{110}$ McElroy, ${ }^{135}$ DuBose,${ }^{136}$ Hearsey,,${ }^{137}$ Ziemann, ${ }^{23}$ Brem, ${ }^{85}$ Bignami, ${ }^{105}$ Doering, ${ }^{37}$ and Shropshire ${ }^{138}$ (I 5 per cent. of his cases). I have seen four cases where quinine could be excluded from the etiology.

Hemoglobinuric fever occurring only in malarial subjects and quinine being specific for malaria, it is but a most natural sequence of events that a large number of the cases of hemoglobinuric fever have developed after the administration of quinine. The bare fact that blackwater fever often follows quinine is weak evidence for quinine etiology in the face of the numerous cases in which previous quinine could be absolutely excluded.

When, however, attacks can be produced repeatedly at will by a dose of quinine the question assumes a very different aspect. Such cases are those of Murri, ${ }^{139}$ Hoffman, ${ }^{33}$ Koch, ${ }^{79}$ Manson, ${ }^{83}$ Ketchen, ${ }^{111}$ Hopkins, ${ }^{132}$ Bertrand, ${ }^{100}$ A. Plehn, ${ }^{21}$ I6 
Ollwig, ${ }^{33}$ Marsden, ${ }^{140}$ Daniels, ${ }^{27}$ Kleine, ${ }^{94}$ Tomaselli, ${ }^{74}$ Vincenzi, ${ }^{82}$ and Grocco. ${ }^{82}$

As stated above, there is no relation between the amount of quinine and the intensity of the attack. Ketchen ${ }^{111}$ precipitated an attack, experimentally, with $1 \frac{1}{2}$ grains. This patient stated that $1 / 18$ gram had previously produced blackwater. Karamitsas, ${ }^{23}$ Chomatianos, ${ }^{23}$ Pampoukis, ${ }^{23}$ Kanellis, ${ }^{23}$ Koch,${ }^{79}$ Kleine, ${ }^{94}$ Shropshire, ${ }^{138}$ Moscato, ${ }^{23}$ A. Plehn, ${ }^{21}$ Boxer, ${ }^{141}$ and others report outbreaks elicited by less than $1 / 2$ gram. Panse ${ }^{83}$ believes the usual dose preceding an outbreak to be from $1 / 10$ to I gram. Tomaselli ${ }^{74}$ has observed attacks to follow the administration of doses as small as from $1 / 20$ to $1 / 10 \mathrm{gram}$, and Koch $^{125}$ reports a case after $1 / 10$ gram has been administered. Kudicke ${ }^{44}$ and Marchiafava and Bignami ${ }^{19}$ state the minimum quantity as $1 / 20$ gram, Laveran ${ }^{23}$ and Ziemann ${ }^{86}$ as I centigram, and Ruge ${ }^{41}$ as $\mathrm{I}$ milligram.

Tomaselli74 examined various preparations of quinine to ascertain whether the toxic effect was dependent upon adulteration, and concluded that such was not the case, but that the toxic properties were inherent to quinine itself and to all the preparations containing quinine.

The time intervening between the administration of quinine and the onset of hemoglobinuria is almost uniformly fixed by observers at from one to six hours. With six hours as the maximum interval, the cases really due to quinine would dwindle considerably.

It is believed by some writers that quinine hypodermically does not produce blackwater, even in persons susceptible, when administered orally. This, however, is not the case. Tomaselli $^{74}$ has shown that subcutaneous injections of quinine are followed more promptly by hemoglobinuria than is the oral administration. Kohlbrugge ${ }^{4}$ thinks that only the inorganic salts of quinine are toxic, and states that the tannate, even in the largest doses given to susceptible persons, fails to cause hemoglobinuria. McKay ${ }^{142}$ has recently attempted to show that hemolysis following the administration of the sulphate of quinine is due to the sulphate and not to the quinine. This view, however, is not supported by clinic experience. Further- 
more, the results of experiments upon which McKay based his conclusion could not be verified by Christophers and Bentley. It is probable that neither the mode of administration nor the preparation used, if absorbed, gives any difference in results.

The rôle of quinine in hemoglobinuric fever is probably highly complex. It will be shown that it is of value as a prophylactic when systematically employed; if not thus used, and malarial infection be permitted to occur, it may, in some persons thus predisposed, act as the exciting cause. In the attack itself it is possibly of value in destroying the parasites when these are present, or it may act harmfully in aiding hemolysis.

Even after a careful study it is not easy to define precisely the respective potency of malaria and quinine as etiologic factors. To quote Shropshire, ${ }^{138}$ "To establish the cause of any disease we must apply the agent to the subject, and have, as uniform result, the disease. But if there are two agents suspected as causative which, applied together, produce the disease, but applied separately to the same individual, the one produces it, the other never, we can attribute only to the one a causative place, and to the other an accidental presence. Such is the case before us. Malaria taken as the cause and applied without quinine to an individual of such tendency, hemoglobinuria results in 15 per cent. of the cases before us. Quinine has probably been applied to all the cases before us without the presence of malaria and no hemoglobinuria resulting. Which produces it?"

Favoring malaria as against quinine we have:

r. Antecedent malaria essential.

2. Relative immunity of the negro. Racial immunity to disease well known; racial susceptibility to drugs rare or unknown.

3. Occurs often without the administration of quinine.

We may safely conclude that the predisposing cause is always malaria; the exciting causes are fresh malarial invasion, quinine or other medicaments, exposure, exertion, mental states, etc.

III. The most enthusiastic champions of the view that blackwater fever is neither malaria nor quinine poisoning, but a disease sui generis, are Sambon ${ }^{143}$ and Craig. ${ }^{144}$ Manson $^{145}$ 
formerly advocated this theory. The two reasons for his belief are a similarity to paroxysmal hemoglobinuria and an analogy with Texas fever. Stalkarrt, ${ }^{131}$ Rho, $^{131}$ Vincent, $^{3}$ and others believe that it is a distinc tdisease. While the similarity to paroxysmal hemoglobinuria cannot be denied, the relation to Texas fever, as we have seen, is far from close, and the evidence that it is a disease sui generis is inadequate. Yersin ${ }^{147}$ found bacilli in the casts and epithelium in the urine of two patients, and believed that he had discovered the cause of the disease. Breaudat, ${ }^{148}$ however, showed that these were the Bacillus coli communis.

Collet $^{149}$ has recently, without grounds, however, suggested that there may be a causal relation between the Bacillus Megatherium and blackwater fever.

The theory that green beans and their blossoms were the cause of many cases of hemoglobinuric fever seems to have perished in Greece, Sicily, and Sardinia, where it originated.

It is generally conceded that hemoglobinuric fever consists of a destruction of red blood-cells so widespread that, the liver being powerless to transform the liberated hemoglobin into bile pigment, the greater part is excreted by the kidneys. This conversion into biliary coloring matter is the physiologic fate of free hemoglobin, and indeed its pathologic destiny up to a certain limit which, according to Ponfick's postulate, is the destruction of one-sixth of the entire number of red cellsbeyond which hemoglobinuria ensues. This much seems to be rather unanimously accorded. The nature of the hemolysin is the missing link in the pathogenetic chain.

Several years ago I expressed the belief that the hemolytic agent was neither a specific parasite, a parasitic toxin, nor quinine, and quote the following from my paper: ${ }^{103}$

"The modern study of immunity and cytolysis has thrown a flood of light on hemolysis. It is unnecessary to review in detail the development of our knowledge of hemolysis, but the following facts will be recalled. It has been known for some time that the serum of certain animals has the power of dissolving the blood corpuscles of certain other animals. Bordet showed that this effect may be produced artificially. The 
serum of guinea-pigs naturally has no hemolytic effect on the red cells of the rabbit, but if the rabbit's blood is injected into the guinea-pig and the process repeated the serum of the guinea-pig becomes hemolytic toward the rabbit. It has been shown that the hemolysins are formed by the interaction of two substances: one, the amboceptor or immune body, resisting moderate degrees of heat; the other, called the complement, inactivated by a temperature of about $55^{\circ} \mathrm{C}$. Neither amboceptor nor complement alone is sufficient to dissolve erythrocytes, but for this it is necessary for both to act, the amboceptor sensitizing the cells for the complement. The amboceptor may act alone, but the cells will only be rendered susceptible, not dissolved. The complement has no effect whatever on the red cells except through the immune body. The complement exists in normal serum."

Bignami ${ }^{19}$ states his theory as follows:

I. An alteration in the plasma which is effected, little by little, as a consequence of a specific change in the red blood corpuscles through which a certain number of them come to behave, in respect to the organism, like ,the corpuscles in the blood of another species of animal. 2. The formation, in consequence of this change, of a substance in the plasma which is capable, under certain conditions, of becoming hemolytic.

At the Igro meeting of the American Society of Tropical Medicine I suggested that the hemolysis might be a phenomenon of anaphylaxis.

It is possible that the death of a brood of parasites, either spontaneously or as a result of the administration of quinine, might sensitize the corpuscles, which after an appropriate interval become hemolyzed by a second setting free in the circulation of strange proteid from dead parasites. Proteid anaphylaxis is the only explanation for a number of bacterial diseases and it is known to occur with helminths, for instance ascaris lumbricoides.

It is believed that this hypothesis explains the occurrence of hemoglobinuric fever during and after malarial infection, with or without the administration of quinine; it explains why the malarial attack may precede by months the appearance 
of blackwater; why exposure, exertion, etc., may elicit an attack; why the hemolysis does not always coincide in time with the sporulation of the parasites in the case in which the latter are present; it accounts in a measure for the complex relation with quinine and explains quinine fever, post-malarial secondary fever, and post-hemoglobinuric fever.

Christophers and Bentley, ${ }^{63}$ constituting a committee appointed by the government of India to conduct an inquiry regarding the nature of blackwater fever, have recently published an extensive monograph containing a record of their experiments and the conclusions which they reached as a result of these experiments. They exclude parasitic, osmotic, and chemic actions as causes of hemolysis, and show that the hemolysin is probably derived from auto-immunization against the organism's own red cells-an autolysin.

According to the conditions of its occurrence, hemoglobinuric fever is classified by the Italian school as follows:

r. Malarial hemoglobinuria:

(a) Cases in which the blood contains parasites.

(b) Cases in which no parasites are present.

2. Quinine hemoglobinuria in malarial subjects, occurring:

(a) During the malarial attack.

(b) After the attack (post-hemoglobinuric). 


\section{CHAPTER XI}

\section{PATHOLOGY OF BLACKWATER FEVER}

The pathologic findings vary in proportion to the proximity and intensity of the malarial attack. In addition to the changes characteristic of malaria there are found, in blackwater fever subjects, the results of hemoglobinemia and polycholia chiefly in the kidneys and liver. Occasionally post-mortems do not reveal malarial evidences, as in two cases reported by Curry, ${ }^{110}$ but this is exceptional. The body is usually deeply jaundiced. There may or may not be edema. The muscular system is often icteric.

The spleen is enlarged, often enormously so, and congested. The surface color varies from grayish to reddish brown, almost black. The capsule is thickened and usually strips easily, but may be adherent. The consistence of the organ is often so diminished that it appears like a pulpy sac. The trabeculæ are thickened and fibrous; the pulp is decidedly increased. The Malpighian corpuscles are usually hypertrophied, sometimes giving the appearance of sago spleen. Pigment is usually abundant. It is contained within the cells or lying between them. The cells of the Malpighian bodies show the greatest quantity and largest masses. The large mononuclear cells and giant cells are pigmented. The leucocytes lying external to the walls of the small veins may show more pigment than those scattered here and there throughout the pulp. The color of the pigment varies from yellow to almost black, and may consist of hemosiderin or melanin. The walls of the smaller vessels are thickened, and the lumen may be obliterated. The sinuses may be obliterated with pigmented and other cells. The endothelial cells may be proliferating, and often contain granules of pigment. Parasites and pigmented leucocytes may be present in the spleen when not discoverable in the general circulation. There may be round-cell infiltration around the trabeculæ. 
The liver is enlarged, congested and surcharged with bile. It varies in color from a decided yellow to a dark brown. The capsule is slightly adherent. The surface is usually smooth, but there may be subcapsular nodules from the size of a pinhead to that of a pea, which on section exude a thick, cheesy matter. There is abundant pigmentation, often rod shaped, especially of the endothelial cells, macrophages, and leucocytes. The course of the capillaries may be well marked by the pigment contained in the endothelial cells and that between the wall and the adjacent liver cells. Both the yellow and black pigments are found, the former especially, in the liver cells. Pigmentation is often more pronounced in the center of the lobule. Thrombi of pigmented cells in the capillaries and sublobular veins occur, with cloudy swelling and fatty degeneration of liver cells. These retrogressive processes are in the form of islands. The biliary injection, more intense in the center of the hepatic lobule, may extend to the smallest branches. Regenerative efforts on the part of the liver cells are very much more common than in pernicious malaria(Marchiafava and Bignami). Karyokinetic barrels and monasters predominate. This is interpreted by Bastianelli as evidence of hyperfunction of the liver. Marchiafava and Bastianelli both agree in believing that this multiplication of the hepatic cells is an attempt on the part of the liver to meet the increased demands for work in eliminating the detritus of hemoglobin (Thayer). The gall-bladder is usually distended with bile.

The kidneys are generally congested, weigh more, and are softer than normal. The capsule is loosely attached. On section the cortex is often yellowish; the pyramids may present brownish streaks, more intense toward the apices. In the cortex may be found wedge-shaped hemorrhages with bases toward the capsule and apices pointing toward the medulla. The medullary pyramids may show minute hemorrhages. The glomeruli often escape undamaged; there is rarely any pigmentation of the cells within Bowman's capsule; there may be cloudy swelling and slight epithelial desquamation. The epithelia of the convoluted tubules usually show cloudy swelling, fatty degeneration, or coagulation necrosis. There may be 
pigmentation of the epithelial cells. The lumina are often plugged with hemoglobin casts holding the epithelia in place. The changes in the straight tubules are similar, but casts are more numerous. The epithelium of Henle's loops is better presented, but the lumen is usually choked with casts of hemoglobin and epithelial detritus from the convoluted tubules. Biliary pigment also occurs here. Karyokinesis is sometimes seen in the epithelium of Henle's loops of the convoluted tubules.

The stomach and intestines may be negative. The serous coat may be pale, the mucous membrane congested and bile stained, especially near the opening of the common bile-duct. There may be isolated hemorrhages, excoriations, and pigmentation. The pancreas is normal.

The pleuræ may show punctate hemorrhages and the cavity may contain a quantity of serous fluid. The surface of the lungs may show slaty specks and striæ. The cut surface is very pale, and exudes a very small amount of very pale, frothy, serous fluid. There may be an ashy discoloration in the course of the vessels, hypostatic congestion, and edema.

The pericardium may contain from a very few drams to several ounces of a clear or sanguineous fluid, and may present hemorrhages varying in size from that of a millet seed to that of a cent. The heart is pale and often flabby. The muscular fibers are easily separable; the walls may be very thin. The left ventricle is usually strongly contracted, the right collapsed. Auricles and ventricles may contain coagula or thrombi. Microscopically the fibers stain well and show striations perfectly; there are some areas of slight pigmentation and some of connective-tissue proliferation; the nerve trunks in the transverse section show marked degeneration; empty nerve sheaths are seen, and some connective-tissue proliferation into funiculus (Goltman and Krauss).

The brain is usually pale and unpigmented; the latter ventricles may contain an excess of fluid. The convexity of the pia may show slight cloudiness in the course of the vessels. The puncta vasculosa may be scarcely visible. The bonemarrow shows the usual changes of malaria. Melanin, hemosiderin, and proliferating normoblasts may be found. 


\section{CHAPTER XII}

\section{CLINICAL HISTORY OF BLACKWATER FEVER}

After a severe chill the temperature rises rapidly and a copious discharge of red, almost black, urine is voided. The patient complains of headache and pains in the loins and epigastrium, and is afflicted with nausea and yiolent bilious vomiting. Thirst is torturing and insatiable because of the gastric disturbance. There may be more or less tympanites. The liver and spleen, especially the latter, may be enlarged and tender. In a few hours icterus begins and the patient soon becomes as yellow as a pumpkin. He is very restless and has an anxious expression. If the attack is mild the duration may not be longer than that of an ordinary malarial paroxysm, the vomiting ceases, pain disappears, the urine gradually clears, the temperature falls to normal or a little below, and the patient is comparatively comfortable excepting a degree of weakness. The jaundice usually lasts a day or two longer. In rare cases the duration of the attack is extremely short, the urine voided at a single act only being hemoglobinuric.

In severer cases the temperature may drop, but not to normal; vomiting is incessant; urine continues darkly colored and becomes scantier. Rigors may occur at irregular intervals, followed by a rise of temperature, deepening of the color of the urine, and marked prostration. The urine may become suppressed and death takes place in a few days; or the patient may die suddenly while being raised to use the vessel or to take medicine or nourishment.

The attack may be preceded by one or more malarial paroxysms or may come on suddenly. There may be prodromata consisting of general malaise, aching in the loins and head, nausea, and a slight rise of temperature, though this latter may be imperceptible to the patient. In more than nine-tenths of the cases the onset is with a rigor, usually intense and pro- 
tracted. Sometimes, as in ordinary malaria, the first stage is obscure or wanting, and the attack begins with fever and vomiting. Occasionally the passage of black water precedes the other symptoms, as in one of my cases where the patient had had a mild rigor the day before but felt well enough to ride out on horseback in search of his cow. While five miles from home he was dumbfounded at passing an abundance of almost black urine. He immediately set out to consult me, and had ridden six miles when he was prevailed upon to return home. Death from exhaustion occurred on the fourth day. Rarely the onset is characterized by violent pain in the abdomen. The intensity of the onset is no guide to the severity of the attack.

There are four symptoms which are present in nearly all cases. These are: fever, hemoglobinuria, icterus, and vomiting.

There is nothing characteristic in the temperature. Its usual range is from $101^{\circ}$ to $105^{\circ} \mathrm{F}$. Hyperpyrexia is unusual, though very high temperatures have been recorded. Thus Marsden ${ }^{140}$ noted a case in which the temperature reached Io9 ${ }^{\circ} \mathrm{F}$. Cases in which the temperature is normal or subnormal throughout are not unknown, for example, two reported by Baldwin Seal. ${ }^{130}$ Neither of these patients had taken any quinine. As a rule, in mild cases the temperature reaches the fastigium shortly after onset, from which point it drops progressively to or a little below normal. In other cases it is intermittent, remittent or irregular, and may resemble the curve of septic fever. When rigors occur during the course they are accompanied by a rise in temperature. Periodicity is not a conspicuous feature, nor is the characteristic curve of Marchiafava and Bignami seen. The average duration of the fever is from a few hours to several days. The average in my cases was eighty hours. It usually outlasts the hemoglobinuria, but not in all cases. The height of the temperature in hemoglobinuric fever is possessed of little or no prognostic import.

A rare occurrence is the obstinate tenacity or subsequent rise of the fever after hemoglobinuria has subsided. The duration of this post-hemoglobinuric fever is variable. In two of Brem' $\mathrm{s}^{85}$ cases it was fourteen and eighteen days; in three of 
my cases twelve, nineteen, and twenty-eight days; in one of Bank's ${ }^{78}$ over five weeks; in one of Howard's ${ }^{87}$ six weeks. Outbursts of hemoglobinuria occasionally occur during this fever. In most of the cases the temperature rose higher than during the hemoglobinuric period. It is entirely uninfluenced by quinine and is probably related to the spodogenous fever of Marchiafava and Bignami ${ }^{19}$ or post-malarial secondary fever. The mortality of these cases seems to be very low.

Schellong ${ }^{160}$ observed a peculiar case which showed a postmortem elevation of temperature. The fever began to rise a few minutes after death, and more than an hour later, when last recorded, the temperature was $106.2^{\circ} \mathrm{F}$. The thermometer registered higher in the right axilla than in the left throughout the observation.

Probably in no other condition do we see such rapid and profound transition in the state of the urine. A few hours before the onset the urine is normal; afterward it may show all the characteristics detailed below. In favorable cases the return to normal is remarkable.

The quantity varies within very wide limits. In mild attacks it may not vary from that of health. Often at first there is an increase, a decided decrease at the height of the attack, gradually increasing to normal or above with improvement. In suppression cases there is usually a diminution from the first, resulting in total anuria or the passage of a few ounces daily. Anuria is due to the plugging of the renal tubules and to diminution of blood pressure. Pain resembling that of renal colic may be experienced with anuria. The temperature may remain normal throughout suppression of several days' duration. It occasionally happens that urinary secretion is reëstablished after anuria has persisted, even as long as five days; in such cases, however, most die of complications during convalescence. The outlook is very grave when suppression lasts longer than twenty-four hours. Death usually takes place after three or four days, though $\mathrm{Plehn}^{2}$ reports a fatal case where life was prolonged twelve days after the onset of suppression, and Kudicke ${ }^{44}$ observed two fatal cases in which anuria persisted thirteen days. 
The color, often described as "port wine," varies from a light claret to that of black coffee. The latter color obtains when the urine of a severe attack is examined in a thick layer by reflected light. In a test tube by transmitted light it appears of a lighter color. The froth varies from yellow to reddish; a greenish color is said to be due to the presence of bile. The coloring matter is more often in the form of methemoglobin, though oxyhemoglobin is found. It is probably not present in a true solution, since it is more abundant in the sediment of a centrifugalized urine than in the supernatant fluid, and disappears from the latter first with improvement. The hemoglobinuria may be intermittent or continuous. Stephens and Christophers ${ }^{120}$ observed that blackwater urine, made alkaline with potash and then boiled, produced a purple color, giving the bands of hemochromogen, showing that the urine itself contained reducing bodies. A. Plehn mentions that on boiling the urine and allowing it to stand for some . time a bright purple color appears. On standing an abundant dirty brownish sediment is deposited, the amount varying with the concentration of the fluid. The urine stains linen a dirty red. The reaction is generally slightly acid, but may be neutral or alkaline. The specific gravity varies inversely with the quantity. The average in my cases has been ror 5. Albumin is always present. It is commonly in excess of the hemoglobin and persists for a longer period, though the curves run more or less parallel. Serum albumin, albumose, globulin, and nucleoalbumin are found. Plehn ${ }^{2}$ gives the limits of quantity as $1 / 2^{-2}$ grams per liter, estimated according to Esbach's method. I have very frequently observed twice as much as his maximum limit, and in one of my cases the amount was I4 grams with the Esbach instrument. Some urines on being boiled become almost completely solidified. Bile is, as a rule, absent; it is never present in proportion to the polycholia. It was present in only one of a series of 24 cases reported by me. ${ }^{62}$ It was not found by the Plehns ${ }^{2}$ or by Daniels ${ }^{27}$ in any of their cases. Urobilin is common. Stephens and Christophers ${ }^{120}$ assert that it occasionally appears before the attack, but more constantly after the oxyhemoglobin has disappeared, or together 
with it. In nine cases I examined the urine with negative results in all. Marchoux ${ }^{161}$ maintains that quinine cannot be detected in the urine during the hemoglobinuric period, but appears later. But the observations of Giemsa and Schaumann $^{162}$ do not sustain this opinion. They found the amount of quinine excreted during the attack to be somewhat larger than otherwise, and that the excretion is extended over a longer period of time in a regularly increasing and decreasing curve which is uninfluenced by the hemoglobin content of the urine. This increased excretion of quinine in the urine in hemoglobinuric fever would lead to the inference that the organism is not capable, as it usually is, of protecting itself from the poisonous alkaloid by splitting the molecule. Marchoux ${ }^{161}$ claims that hemoglobinuric fever urine has no hemolytic action on the red blood-cells of normal persons.

On microscopic examination the field appears littered with a brownish amorphous detritus, the products of broken-down red blood corpuscles. Whole red blood-cells are not generally found and rarely in considerable numbers. Casts are abundant, especially the granular; also hyaline and epithelial. These casts are sometimes almost covered with the granular pigment. Renal and vesical epithelium are common, and mucus and crystals of hematoidin may be found. Leucin and tyrosin are rare. Williams, ${ }^{163}$ Mackey, ${ }^{164}$ and Brem $^{85}$ have described different peculiar bodies found in the urine. The nature and significance of these bodies are unknown.

There are often present vesical tenesmus and pain over the bladder. Retention of urine, burning in the urethra, and tenderness over the kidneys are not uncommon symptoms. The urine may be voided drop by drop.

After a few hours jaundice begins to appear and, except in the mildest cases, develops rapidly until the skin and sclera are of a pronounced saffron yellow. It usually outlasts the fever a few days. Itching of the skin is not common. Herpes is relatively infrequent and petechiæ are rare. The occurrence of the latter is said to imply a grave prognosis. Edema or anasarca may be encountered, especially in cases where there is unusual involvement of the kidneys. Sweats may occur with 
the decline of the fever or with collapse. The skin is often dry. Sometimes the perspiration is charged with the pigment. Banks ${ }^{78}$ mentions a peculiar odor emanating from blackwater fever patients. He claims that it enables one, together with the expression, to make a diagnosis before the urine is examined. So far as I know he has not been corroborated, though in suppression cases a uremic odor may be perceptible. Herpes was not observed in any of my cases.

Vomiting is usually one of the earliest symptoms and frequently the most distressing. After the stomach contents are voided the vomit consists of a yellowish or green bile. Occasionally it is a grass green or peculiar bluish green, or it may be very dark, almost black, somewhat resembling the black vomit of yellow fever. The vomiting is independent of the taking of food, and is probably more or less of central origin. In very mild cases vomiting may be very insignificant or absent. Nausea is usually in proportion to the vomiting. The bowels may be constipated or there may be a bilious diarrhea. Occasionally the dejections are thin and watery, of a reddishbrown color, and may closely resemble the urine. This is thought to be due to the extravasation of hemoglobinuric serum into the intestine, and is usually seen in severe cases only. Dysenteric symptoms are infrequent. Hemorrhage from stomach or bowel is rare. Meteolism is not an infrequent symptom. There may be severe colicky pains in the abdomen. Pain is usually present in the epigastrium region or over the liver and spleen. These are usually tender, the spleen often greatly enlarged, the liver less so. The appetite in all but mild cases is completely lost. Thirst is intense and cannot be alleviated for the vomiting. The tongue is anemic and heavily coated. Sordes of the teeth and lips are often seen in extreme cases. The saliva may stain the linen a brownish yellow.

The pulse is rapid, out of proportion to the temperature, at first full and bounding, later small and compressible. A hemic murmur, systolic in time, is sometimes heard over the precordia, not transmitted. Respiration is accelerated. There is often sense of oppression in the chest. Dyspnea may be a 
prominent symptom, due to anemia or to edema of the lungs. There may be, especially after some days of severe illness, slight dullness and diminished respiratory murmur over the dependent portions of the lung, accompanied by slight cough, resulting from hypostatic congestion. Cheyne-Stokes respiration may appear toward the end. Hiccough is present in a large per cent. of fatal cases, and if obstinate is always to be regarded unfavorably. Epistaxis is occasionally seen. The blood pressure is.low.

Anemia increases with intense rapidity, half the red cells sometimes being destroyed in twenty-four hours. The number usually falls to one to two million during the attack. Except for the absolute diminution of the fluid portion of the blood as a consequence of purging and vomiting, the number of red cells per cubic millimeter would appear much smaller than it does. It is occasionally difficult to obtain a drop for examination by the usual method. It appears relatively thinner than normal and the cover-glass may adhere to the oil immersion objective rather than to the slide. On coagulation the serum may appear yellow (cholemia) or reddish (hemoglobinemia), though neither is constant. Macrocytes, microcytes, poikilocytes, shadows, polychromatophiles, and basophiles are found, especially during convalescence. The color index shows nothing characteristic; it may be normal or above, at first falling gradually until convalescence is established. The hemoglobin per cent. usually runs parallel with the red cell count. It is generally reduced to 25 to 50 per cent., sometimes lower, as in a case of Hoffmann ${ }^{33}$ in which the patient recovered notwithstanding a fall to $\mathrm{I} 2$ per cent.

In one of my fatal cases the patient was practically exsanguinated. He had been treated actively with enteroclysis and hypodermoclysis and the fluid obtained by puncture of the lobe of the ear was straw colored. The cover-glass adhered to the lens rather than to the slide. Only a few very pale red cells were to be seen in each field of the microscope. The lowest hemoglobin percentage observed in a case recovering was thirty. It is often asserted that during pyrexia there is a leucocytosis, and the polymorphonuclears are increased often 
to 90 per cent. With falling temperature there is a pronounced large mononuclear increase with leukopenia. However, the average of a number of differential counts made at irregular intervals during the attack shows a marked increase in the large mononuclears, a decided diminution of small mononuclears, and a slight increase of polymorphonuclears. Pigmented leucocytes are common. Christophers and Bentley ${ }^{165}$ have made interesting observations on the phagocytosis of red blood corpuscles in the spleen of a case of blackwater fever. In a differential count of 2,200 spleen cells 1.7 per cent. were large macrophages containing red cells, and I.3 per cent. were small mononuclear cells containing red bloodcells. In both kinds of cells were seen blood corpuscles showing no evident alterations, corpuscles more or less decolorized, and clear vacuoles about the size of red blood-cells. The closest scrutiny of the engulfed cells failed to reveal the presence of parasites or other evidence of parasitic invasion. This extensive phagocytosis of apparently normal cells is of interest from the standpoint of pathogenesis. The platelets are numerous and of large size. The alkalinity of the blood is often diminished. In spite of the destruction of red cells the specific gravity remains relatively high. This is no doubt due to the quantity of material in solution in the serum. During convalescence the specific gravity falls. Stephens and Christophers ${ }^{27}$ give the following as the result of their observation on tonicity: "In blackwater fever there is occasionally a remarkably low tonicity; in other cases it has the normal value or somewhat raised value, as in malaria. The low or normal value in blackwater may be due, as we have previously suggested, to the fact that the weak corpusclesthose of high tonicity - are destroyed; or it may be due to the fact that the tonicity of the corpuscles as a whole is changed after the liberation of hemoglobin." The presence of malarial parasites has been dealt with.

Blackwater fever is not a very painful affection, but the vomiting and thirst make the patient intensely wretched. Besides the abdominal pains there are headache and aching of the back and limbs. He is usually terrified at the appearance 
of the urine. His expression is one of anxiety and apprehension, and a fear of death often seizes him. He is restless and irritable. In children especially there is usually tossing of the head from side to side. Later there is prostration, intense languor, perhaps somnolence. Formication and numbness in the hands and toes are occasional complaints. Delirium, when present, is usually quiet. When suppression ensues the symptoms may be typic of those in uremia, but this is not constant; delirium may be of the low, muttering variety; convulsions are often missing, and the mind may be clear until shortly before death, when coma supervenes. There may be involuntary discharge of urine and feces.

The causes of death are three: Suppression of urine, exhaustion, and cardiac paralysis. In my fatal cases exhaustion has been the commonest mode of exit. Suppression sometimes takes place when the urine is clearing or is already clear. Uremic symptoms do not result from suppression in blackwater fever as frequently as in other conditions. This is probably due to two causes: first, elimination through vomiting and purging is free; secondly, metabolism is diminished as a result of deficient oxygenation. Exhaustion is usually the result of the tremendous destruction of blood-cells, together with inability of the hematopoietic organs to meet the deficiency, or to pyrexia. Occasionally hiccough plays a rôle in exhaustion. The patient may die early with symptoms of shock or may linger several days in a typhoid state. Cardiac paralysis is usually due to thrombosis of the heart. Plehn ${ }^{2}$ regards this as a common cause of death. Goltmann and Krauss $^{42}$ have shown that in some cases of death from syncope there exists a marked cardiac nerve degeneration and empty cylinders.

It would manifestly be of great practical importance if the symptoms of an impending attack of hemoglobinuria could be recognized. This is possible, if at all, only in a very general way. Plehn ${ }^{21}$ says that an onset is to be feared when the patient has lived some six months in a blackwater fever area and has had malaria at short intervals, when this malaria has been treated improperly with insufficient quinine dosage, when 
he looks downcast, and perhaps shows a mild icterus of the sclera and skin. This might, however, forebode a relapse of ordinary malaria. Sometimes, he further states, there occurs a certain depressed condition, a characteristic mental apathy with physical restlessness, phenomena which are hard to describe, but have often been encountered by one who has seen many cases develop. Also the presence of albuminuria should cause suspicion as this tends to be absent in simple tropic fever, even with a temperature of $4 \mathrm{I}^{\circ} \mathrm{C}$. Koch ${ }^{41}$ designates as "blackwater fever candidates" those in whom a few hours after taking quinine the temperature mounts to $38^{\circ} \mathrm{C}$. or more, the urine becomes decidedly darker, and the next morning a mild icterus is evident. Ziemann ${ }^{86}$ has frequently observed in blackwater fever candidates the following blood changes, which, however, are not constant:

I. The more frequent appearance of decided basophile and polychromatophile degeneration of the red cells.

2. The rapid solution of the red cells in a salt solution, in which normal cells do not dissolve.

3. A decided diminution of the coagulability of the blood.

This writer also regards urobilinuria as a valuable prognostic sign.

Relapses are not infrequent and several may occur, often befalling the patient when he is thought to be doing well. It may be difficult to distinguish relapses from recurrences. Convalescence may very properly be regarded as the dividing line, those occurring during convalescence being considered as relapses and those later as recurrences. $\mathrm{Plehn}^{33}$ believes that recurrences are rare unless provoked by quinine. Of eighteen recurrences recorded by $V^{2} y^{83}$ one occurred after less than three months, fourteen from three to six months, two from six to twelve months, and one longer. It is remarkable that nine recurrences happened just three months from the date of the last attack. My experience has been that in persons having more than one attack the attacks are more often separated by intervals of a year or more.

Complications and Sequelæ.-These are singularly few in variety. Nephritis in some degree is an almost constant com- 
plication, and may cause death from suppression during the attack. It may heal in a remarkably short time. As a sequel it is not infrequent and may persist for days or weeks, causing slow and incomplete convalescence or death. The changes in the kidney may be attributed to the irritating effect of hemoglobinuric urine and to the pyrexia.

In sixteen cases I have been able to make examinations of urine at periods varying from a few days to fourteen years after the last attack. In eight cases the examination was negative. The others may be noted as follows:

F. S., white, male, aet. seventeen, one attack, r899; urinalysis, Feb. 27,1907 , showed a trace of albumin, no casts, no symptoms of nephritis.

A. J., white, male, aet. eighteen, two attacks, last one Oct., I904; urinalysis, Sept. 2I, I 906, showed albumin 1/4 gram liter, very numerous cylindroids and hyaline casts, moderate number of granular. Anemia, edema of lids and ankles.

Mrs. H., white, female, aet. twenty-five, one attack fourteen years ago; urinalysis, Sept. 25, I906, showed the presence of albumin and a few granular casts. No symptoms.

M. C., white, female, aet. ten, two attacks, last one Sept. 24, I906; urinalysis, on Oct. 8, I906, showed a moderate amount of albumin, no casts. Anemia, edema of face and ankles, indigestion.

E. C., white, female, aet. seven, five attacks, last one Nov. I 5, I906; urinalysis, Dec. 4, I906, nitric acid test for albumin negative, microscope showed a very few cylindroids and hyaline and granular casts.

R. A., mulatto, male, aet. forty-four, one attack Nov. r 3, 1905; urinalysis, Aug. 22, I906, showed no albumin, moderate number of cylindroids, and a few hylaine casts.

J. P., white, male, aet. thirty-seven, several attacks, last one Sept. 21, I907; urinalysis, July 28, I908, a slight trace of albumin, a few hyaline casts, very few granular.

W. S., male, white, aet. forty-two, one attack, which occurred Feb., I905; urinalysis, June 27, I908, no albumin, a few cylindroids.

The possibilities of the abnormalities of urine and the 
symptoms in the cases being produced by other causes than the hemoglobinuria should be borne in mind.

Anemia and consequent debility and digestive disturbances are not uncommon. Rare complications are: paraplegia, tetanic convulsions, purpura hemorrhagica, dysentery, pneumonia, pancreatitis, abscess of the liver, erysipelas, parotiditis, retinal hemorrhage, pleurisy, and neuralgia. I have seen tonsillitis once as a complication. 


\section{CHAPTER XIII}

\section{DIAGNOSIS OF BLACKWATER FEVER}

This is usually made, and as a rule, correctly before the physician arrives. The history of malaria, the fever, vomiting, jaundice, and blackwater are pathognomonic. Though the parasites are so frequently missed, on examination of the blood there is usually a mononuclear leucocytosis, and pigmented leucocytes may be found.

The diagnosis from paroxysmal hemoglobinuria might prevent difficulties. In this rare condition the attacks usually follow chilling of some portion of the body, and the attack is usually of short duration and seldom fatal. In hemoglobinuric fever there is given a history of several years of residence in an endemic region, repeated attacks of malaria, with often the presence of parasites, pigmented leucocytes, and a mononuclear leucocytosis in the blood.

The conditions which have been most frequently confounded with hemoglobinuric fever are yellow fever and bilious remittent fever.

In localities where yellow fever and blackwater fever prevail their differentiation is not easy. The following are the chief points of difference:

Endemic.

\section{Hemoglobinuric fever}

One attack predisposes.

Occurs usually after several years of residence.

Malarial history always given.

Prodromata common

Icterus intense, early, always present.

Conjunctiva jaundiced.

Hemoglobinuria.

Blood may show malaria parasites, pigmented leucocytes, and mononuclear leucocytosis.
Yellow fever

Epidemic.

One attack confers immunity.

Attacks also newcomers.

May be no history of malaria.

Uncommon.

Icterus usually slight, begins on third or fourth day; may be absent.

Usually congested at first.

Albuminuria or hematuria.

Absent. 
Hemoglobinuric fever,

Bilious vomiting.

Hemorrhages uncommon.

Spleen usually much enlarged.

Increasing pulse.

Albuminuria from beginning.
Yellow fever

Vomit clear or black.

Relatively common.

Enlargement slight.

Pulse retards with stationary or increasing temperature (Faget's sign).

Usually appears from second to fourth day.

A rather striking coincidence is the relative immunity of the negro to both diseases.

Certain cases of bilious remittent fever present points of striking similarity. This is well illustrated by the following case which was presented to me by the messenger and by the family on my arrival as one of "hematuria:"

A. H., white, male, aged thirty-nine, timberman, lived in a malarial country eighteen years. Never had hemoglobinuric fever. He had been having chills at intervals all summer and fall, slight fever, "dumb chills," and slight jaundice for three weeks, no quinine for two months; badly salivated from seven large doses of calomel taken several days ago. Examination Nov. 29, I906, four and a half hours after first passage of "bloody water." Temperature 994/5; pulse, 92; marked jaundice of skin and sclera; has been vomiting; liver region tender; spleen extends to anterior-superior spinous process and to within $1 \frac{1}{2}$ inches of the umbilicus. Blood examination showed two large pigmented, intracorpuscular parasites, hemoglobin 65 per cent. Urine "port wine" color, acid r.or4; nitric acid test for albumin negative, biliary coloring matter abundant, no hemoglobin. Microscopic examination negative. Under quinine treatment the urine cleared in thirty-six hours and the fever left in a few days, going no higher than $101 \frac{1}{2}$. The anemia and enlarged spleen were yet present when I last saw the patient, two weeks after the attack.

The following scheme will help to differentiate hemoglobinuric fever and bilious remittent fever:

Onset sudden.

Hemoglobinuric fever

Jaundice develops rapidly and be- Jaundice develops more slowly and is comes intense.

Parasites frequently absent.

\section{Bilious remittent fever}

Onset slower.

not so intense.

Parasites usually present. 
264 ENDEMIC DISEASES OF THE SOUTHERN STATES

Hemoglobinuric fever

Albuminuria constant.

Urine colored by hemoglobin or its Urine colored by bile. derivatives.

\section{Bilious remittent fever}

Albuminuria not constant.

The differential diagnosis, as attempted by some writers, from "quinine poisoning in malarial subjects" is futile and impossible, as this condition is a mode of hemoglobinuric fever. 


\section{CHAPTER XIV}

\section{PROGNOSIS OF BLACKWATER FEVER}

The prognosis of hemoglobinuric fever is grave, and should be "guarded and Delphic." Probably the most valuable prognostic sign is the quantity of urine; the chemic analysis and microscopic examination are not of great value in prognosis. Anuria, the most dreaded symptom, is to be feared if the daily quantity of urine falls below 200 c.c. If suppression supervenes the outlook is extremely serious and is unfavorable in proportion to early onset. When a patient is tided over a period of suppression, as occasionally happens, he usually dies during convalescence of exhaustion, subsequent nephritis, or embolism. The phenolsulphonephthalein test should be used as the index to kidney efficiency.

Excessive and uncontrollable vomiting is a bad omen, exhausting the sufferer and interfering with nutrition and medication. Diarrhea is probably in many cases, with suppression or a tendency thereto, a life-saving measure, and may be particularly responsible for the relative rarity of uremic symptoms under these circumstances. Singultus is present in a majority of fatal cases, and when obstinate is always unfavorable. Remittent or intermittent temperature is usually favorable. Somnolence, with diminishing amount of urine; coma, especially of early onset; petechiæ, epistaxis or other hemorrhage and algor forebode evil.

Thrombus formation in the heart or large vessels may cause sudden death when the patient is thought to be progressing favorably. Plehn ${ }^{2}$ believes that loud heart murmurs accompanied with weak, irregular pulse denote heart thrombus. This condition is almost certainly fatal, usually in five to eight days.

The larger the share partaken by quinine in the etiology of the individual case the better the prognosis, provided the case is 'not further aggravated by quinine. 
Cases occurring in victims of malarial cachexia or of complications are usually more serious.

The mortality varies unaccountably from year to year, some seasons evincing a series of mild cases, others an appalling mortality. In a certain parish of Louisiana in 1867 many cases are said to have occurred, of which not less than 95 per cent. died. ${ }^{168}$ Fisch, ${ }^{2}$ who placed the mortality on the Gold Coast at 20 per cent., states that until two or three decades previous nearly all who were attacked died. On the other hand, $\mathrm{Banks}^{78}$ makes the well-nigh incredible statement

Treated with Quinine, 2107 Cases, 538 Deaths, 25.5 Per Cent.

\begin{tabular}{|c|c|c|}
\hline & Number of cases & Number of deaths \\
\hline Vieth ${ }^{3} \ldots \ldots \ldots \ldots \ldots \ldots \ldots \ldots \ldots \ldots \ldots$ & 14 & 3 \\
\hline Dryepondt $^{3} \ldots \ldots \ldots \ldots \ldots \ldots \ldots \ldots$ & 28 & $\mathbf{I}$ \\
\hline$\ldots \ldots \ldots \ldots \ldots \ldots \ldots \ldots \ldots$ & 22 & $\ldots \ldots \ldots \ldots$ \\
\hline 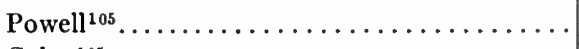 & 9 & 7 \\
\hline Gelpe $^{105} \ldots \ldots \ldots \ldots \ldots \ldots \ldots \ldots$ & 3 & 2 \\
\hline 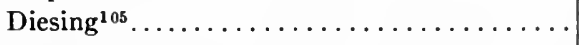 & 2 & 2 \\
\hline $\mathrm{Hagge}^{105} \ldots \ldots \ldots \ldots \ldots \ldots \ldots \ldots \ldots \ldots \ldots \ldots$ & 7 & 2 \\
\hline 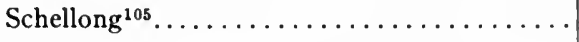 & 7 & 3 \\
\hline Reynolds ${ }^{105} \ldots \ldots \ldots \ldots \ldots \ldots \ldots$ & I & $\mathbf{I}$ \\
\hline Doering $^{39} \ldots \ldots \ldots \ldots \ldots \ldots \ldots$ & 6 & $\ldots \ldots \ldots \ldots$ \\
\hline 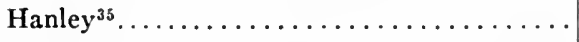 & 13 & 3 \\
\hline 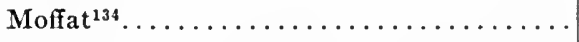 & 9 & 2 \\
\hline $\operatorname{Gorgas}^{170} \ldots \ldots \ldots \ldots \ldots \ldots \ldots \ldots \ldots \ldots \ldots$ & 20 & 3 \\
\hline Steudel $^{82} \ldots \ldots \ldots \ldots \ldots \ldots \ldots \ldots$ & 18 & 3 \\
\hline Malone $^{171} \ldots \ldots \ldots \ldots \ldots \ldots \ldots$ & 120 & 14 \\
\hline Brem $^{85} \ldots \ldots \ldots \ldots \ldots \ldots \ldots \ldots$ & 14 & 2 \\
\hline Coste $^{172} \ldots \ldots \ldots \ldots \ldots \ldots \ldots \ldots$ & 15 & 7 \\
\hline Steggall ${ }^{173} \ldots \ldots \ldots \ldots \ldots \ldots \ldots \ldots$ & 3 & $\ldots \ldots \ldots \ldots \ldots$ \\
\hline Woldert ${ }^{174} \ldots \ldots \ldots \ldots \ldots \ldots \ldots \ldots$ & 5 & $\ldots \ldots \ldots \ldots$ \\
\hline Otto $^{22} \ldots \ldots \ldots \ldots \ldots \ldots \ldots \ldots$ & $\mathbf{I}$ & $\ldots \ldots \ldots \ldots$ \\
\hline Schlayer $^{93} \ldots \ldots \ldots \ldots \ldots \ldots \ldots \ldots$ & $\mathbf{I}$ & $\ldots \ldots \ldots \ldots$ \\
\hline Austin $^{173} \ldots \ldots \ldots \ldots \ldots \ldots \ldots$ & I & $\ldots \ldots \ldots \ldots$ \\
\hline Herrick $^{85} \ldots \ldots \ldots \ldots \ldots \ldots \ldots \ldots$ & 8 & $\ldots \ldots \ldots \ldots$ \\
\hline Curry $^{110} \ldots \ldots \ldots \ldots \ldots \ldots \ldots \ldots$ & $\mathbf{I}$ & $\mathbf{I}$ \\
\hline Burot and Legrand ${ }^{96} \ldots \ldots \ldots \ldots \ldots$ & 3 & $\mathbf{I}$ \\
\hline Cardamatis ${ }^{12} \ldots \ldots \ldots$ & 1,352 & 354 \\
\hline Broden $^{114} \ldots \ldots \ldots \ldots \ldots \ldots \ldots \ldots$ & 12 & 7 \\
\hline Theophanidis $^{176} \ldots \ldots \ldots \ldots \ldots \ldots \ldots$ & 23 & 14 \\
\hline Oeconomou $^{176} \ldots \ldots \ldots \ldots$ & 18 & 5 \\
\hline McDaniel $^{177} \ldots \ldots \ldots \ldots \ldots \ldots \ldots$ & 85 & 35 \\
\hline Berenger-Feraud ${ }^{150} \ldots \ldots \ldots \ldots \ldots \ldots$ & 286 & 66 \\
\hline
\end{tabular}


Treated without Quinine, i 183 Cases, 123 Deaths, 10.4 Per Cent.

\begin{tabular}{|c|c|c|}
\hline & Number of cases & Number of deaths \\
\hline Tomaselli $^{104} \ldots \ldots \ldots \ldots \ldots \ldots \ldots \ldots$ & 30 & 6 \\
\hline Navarre $^{3} \ldots \ldots \ldots \ldots \ldots \ldots \ldots \ldots \ldots$ & 2 & $\ldots \ldots \ldots \ldots$ \\
\hline Henric $^{178} \ldots \ldots \ldots \ldots \ldots \ldots \ldots \ldots \ldots$ & 2 & $\ldots \ldots \ldots \ldots$ \\
\hline Kohlstock $^{179} \ldots \ldots \ldots \ldots \ldots \ldots \ldots \ldots$ & 48 & 8 \\
\hline 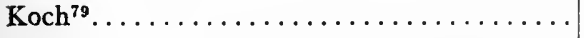 & I6 & 3 \\
\hline Hopkins $^{132} \ldots \ldots \ldots \ldots \ldots \ldots \ldots \ldots$ & 6 & $\mathbf{I}$ \\
\hline Bertrand $^{100} \ldots \ldots \ldots \ldots \ldots \ldots \ldots \ldots \ldots$ & $2 \mathrm{I}$ & 2 \\
\hline Ollwig $^{33} \ldots \ldots \ldots \ldots \ldots \ldots \ldots \ldots \ldots$ & I 5 & $\ldots \ldots \ldots \ldots$ \\
\hline Wittrock $^{33} \ldots \ldots \ldots \ldots \ldots \ldots \ldots \ldots$ & 4 & $\mathbf{I}$ \\
\hline $\operatorname{Ziemann}^{33} \ldots \ldots \ldots \ldots \ldots \ldots \ldots \ldots$ & I 2 & 4 \\
\hline A. Plehn ${ }^{2} \ldots \ldots \ldots \ldots \ldots \ldots \ldots \ldots$ & 53 & 5 \\
\hline Kleine $^{94}$ & I 5 & I \\
\hline Krauss $^{108} \ldots \ldots \ldots \ldots \ldots \ldots \ldots \ldots$ & I 5 & $\cdots \cdots \cdots \cdots \cdots$ \\
\hline McElroy $^{84} \ldots \ldots \ldots \ldots \ldots \ldots \ldots \ldots$ & 25 & 4 \\
\hline Goltman and Krauss ${ }^{42} \ldots \ldots \ldots \ldots \ldots \ldots$ & I 2 & 9 \\
\hline Malone ${ }^{171} \ldots \ldots \ldots \ldots \ldots \ldots \ldots \ldots \ldots \ldots \ldots$ & 35 & $\ldots \ldots \ldots \ldots$ \\
\hline $\operatorname{Coste}^{172} \ldots \ldots \ldots \ldots \ldots \ldots \ldots \ldots \ldots$ & IO & 4 \\
\hline Hearsey $^{137} \ldots \ldots \ldots \ldots \ldots \ldots \ldots \ldots$ & I 5 & 4 \\
\hline $\operatorname{Seal}^{130} \ldots \ldots \ldots \ldots \ldots \ldots \ldots \ldots \ldots$ & 6 & I \\
\hline Ruge $^{112} \ldots \ldots \ldots \ldots \ldots \ldots \ldots \ldots \ldots$ & $\mathbf{I}$ & $\ldots \ldots \ldots \ldots$ \\
\hline Dryepondt and Vancampenhout ${ }^{99} \ldots \ldots \ldots \ldots$ & $\mathbf{I}$ & $\ldots \ldots \ldots \ldots$ \\
\hline Howard ${ }^{87} \ldots \ldots \ldots \ldots \ldots \ldots \ldots \ldots \ldots \ldots \ldots \ldots$ & $\mathbf{I}$ & $\cdots \cdots \cdots \cdots \cdots$ \\
\hline Ketchen $^{111} \ldots \ldots \ldots \ldots \ldots \ldots \ldots \ldots$ & I & $\cdots \cdots \cdots \cdots$ \\
\hline Masterman $^{34} \ldots \ldots \ldots \ldots \ldots \ldots \ldots \ldots$ & $\mathbf{I}$ & $\mathbf{I}$ \\
\hline Herrick $^{85} \ldots \ldots \ldots \ldots \ldots \ldots \ldots \ldots \ldots \ldots \ldots \ldots \ldots$ & $\mathbf{I}$ & I \\
\hline Curry $^{110} \ldots \ldots \ldots \ldots \ldots \ldots \ldots \ldots \ldots \ldots \ldots \ldots \ldots \ldots$ & I & I \\
\hline Cardamatis $^{12} \ldots \ldots \ldots \ldots \ldots \ldots \ldots \ldots$ & 456 & 33 \\
\hline Ensor $^{180} \ldots \ldots \ldots \ldots \ldots \ldots \ldots \ldots \ldots \ldots$ & I I & $\mathbf{I}$ \\
\hline Broden $^{114} \ldots \ldots \ldots \ldots \ldots \ldots \ldots \ldots \ldots \ldots \ldots \ldots \ldots \ldots$ & 25 & 2 \\
\hline Pancot $^{114} \ldots \ldots \ldots \ldots \ldots \ldots \ldots \ldots \ldots \ldots \ldots \ldots \ldots \ldots$ & 7 & $\mathbf{I}$ \\
\hline Theophanidis $^{176} \ldots \ldots \ldots \ldots \ldots \ldots \ldots$ & 9 & $\cdots \ldots \ldots \ldots$ \\
\hline Oeconomou ${ }^{116} \ldots \ldots \ldots \ldots \ldots \ldots \ldots \ldots$ & $3 I$ & 2 \\
\hline McDaniel ${ }^{177} \ldots \ldots \ldots \ldots \ldots \ldots \ldots \ldots \ldots \ldots \ldots \ldots \ldots \ldots$ & 93 & I6 \\
\hline F. Plehn ${ }^{2} \ldots \ldots \ldots \ldots \ldots \ldots \ldots \ldots$ & 25 & I \\
\hline Cardamatis $^{157} \ldots \ldots \ldots \ldots \ldots \ldots \ldots \ldots \ldots \ldots \ldots \ldots$ & I I 5 & 8 \\
\hline Woldert $^{158} \ldots \ldots \ldots \ldots \ldots \ldots \ldots \ldots \ldots$ & 24 & 2 \\
\hline Alexandropoulos ${ }^{159} \ldots \ldots \ldots \ldots \ldots \ldots$ & 38 & $\mathbf{I}$ \\
\hline
\end{tabular}


Treatment Mixed or xot Recorded, 3815 Cases, 779 Deaths, 20.4 Per Cent.

\begin{tabular}{|c|c|c|}
\hline & Number of cases & Number of deaths \\
\hline Kanellis ${ }^{104} \ldots \ldots \ldots \ldots \ldots \ldots \ldots \ldots \ldots \ldots \ldots \ldots \ldots \ldots \ldots$ & 20 & 4 \\
\hline Poole $^{3} \ldots \ldots \ldots \ldots \ldots \ldots \ldots \ldots \ldots \ldots$ & 56 & 15 \\
\hline Rothschuh $^{3} \ldots \ldots \ldots \ldots \ldots \ldots \ldots \ldots \ldots$ & 20 & 18 \\
\hline Guiol $^{2} \ldots \ldots \ldots \ldots \ldots \ldots \ldots \ldots \ldots \ldots$ & 185 & 49 \\
\hline Gouzien $^{132} \ldots \ldots \ldots \ldots \ldots \ldots \ldots \ldots$ & 53 & $\ldots \ldots \ldots$ \\
\hline Meixner $^{33} \ldots \ldots \ldots \ldots \ldots \ldots \ldots \ldots \ldots \ldots$ & 40 & 6 \\
\hline $\mathrm{Hofft}^{33} \ldots \ldots \ldots \ldots \ldots \ldots \ldots \ldots \ldots \ldots$ & 14 & 6 \\
\hline Wendland $^{33} \ldots \ldots \ldots \ldots \ldots \ldots \ldots \ldots \ldots$ & 10 & $\cdots \cdots \cdots \cdots \cdots$ \\
\hline Daniels $^{27} \ldots \ldots \ldots \ldots \ldots \ldots \ldots \ldots \ldots \ldots \ldots \ldots \ldots \ldots \ldots \ldots$ & 184 & $4 \mathrm{I}$ \\
\hline Wellman $^{26} \ldots \ldots \ldots \ldots \ldots \ldots \ldots \ldots$ & 34 & 5 \\
\hline $\operatorname{Ipscher}^{41} \ldots \ldots \ldots \ldots \ldots \ldots \ldots \ldots \ldots$ & 20 & I \\
\hline Kruger $^{41} \ldots \ldots \ldots \ldots \ldots \ldots \ldots \ldots \ldots$ & II & 2 \\
\hline $\operatorname{Simon}^{41} \ldots \ldots \ldots \ldots \ldots \ldots \ldots \ldots \ldots \ldots$ & I 7 & 3 \\
\hline Kerr $\operatorname{Cross}^{181} \ldots \ldots \ldots \ldots \ldots \ldots \ldots \ldots$ & 27 & 9 \\
\hline$\ldots \ldots \ldots \ldots \ldots \ldots \ldots \ldots \ldots$ & 10 & 5 \\
\hline $\mathrm{O}^{\prime} \mathrm{Neill}^{82} \ldots \ldots \ldots \ldots \ldots \ldots \ldots \ldots \ldots \ldots \ldots \ldots \ldots$ & 50 & 2 \\
\hline Burns $^{73} \ldots \ldots \ldots \ldots \ldots \ldots \ldots \ldots \ldots$ & 16 & 6 \\
\hline 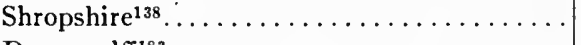 & I 77 & 35 \\
\hline Dempwolff ${ }^{183} \ldots \ldots \ldots \ldots \ldots \ldots \ldots \ldots$ & 17 & 2 \\
\hline 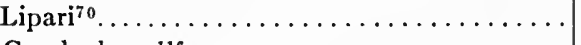 & 19 & 5 \\
\hline Gouducheau $^{116} \ldots \ldots \ldots \ldots \ldots \ldots \ldots \ldots$ & I5 & 4 \\
\hline Cochran ${ }^{184} \ldots \ldots \ldots \ldots \ldots \ldots \ldots \ldots$ & 642 & 158 \\
\hline Kelsch and Kiener ${ }^{98} \ldots \ldots \ldots \ldots \ldots \ldots$ & 109 & 35 \\
\hline Bolton $^{45} \ldots \ldots \ldots \ldots \ldots \ldots \ldots \ldots \ldots \ldots$ & I 75 & 38 \\
\hline 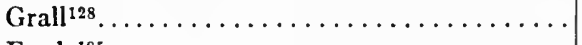 & I I 3 & 13 \\
\hline Forde $^{185} \ldots \ldots \ldots \ldots \ldots \ldots \ldots \ldots \ldots$ & 2 & I \\
\hline Grenet $^{176} \ldots \ldots \ldots \ldots \ldots \ldots \ldots \ldots \ldots \ldots$ & 68 & 8 \\
\hline Rousseau $^{176} \ldots \ldots \ldots \ldots \ldots \ldots \ldots \ldots$ & 22 & 6 \\
\hline Carmouze $^{176} \ldots \ldots \ldots \ldots \ldots \ldots \ldots \ldots$ & 30 & 9 \\
\hline Mericourt $^{176} \ldots \ldots \ldots \ldots \ldots \ldots \ldots \ldots$ & 22 & 3 \\
\hline Koryllos $^{176} \ldots \ldots \ldots \ldots \ldots \ldots \ldots \ldots \ldots$ & 28 & 5 \\
\hline Pampoukis ${ }^{176} \ldots \ldots \ldots \ldots \ldots \ldots \ldots \ldots \ldots \ldots \ldots$ & I 56 & 35 \\
\hline Cardamatis ${ }^{176} \ldots$ & 30 & 6 \\
\hline Parathyris ${ }^{176} \ldots \ldots \ldots \ldots \ldots \ldots \ldots \ldots$ & 23 & 3 \\
\hline Prout $^{186} \ldots \ldots \ldots \ldots \ldots \ldots \ldots \ldots$ & 24 & 8 \\
\hline 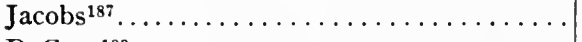 & 147 & 16 \\
\hline DeCruz ${ }^{188} \ldots$ & 13 & 6 \\
\hline DeBlasi $^{189} \ldots \ldots \ldots \ldots \ldots \ldots \ldots \ldots$ & 3 & $\therefore \ldots \ldots \ldots \ldots$ \\
\hline Orme $^{190} \ldots \ldots \ldots \ldots \ldots \ldots \ldots \ldots \ldots$ & 2 & $\cdots \cdots \cdots \cdots \cdots$ \\
\hline Thompstone $^{191} \ldots \ldots \ldots \ldots \ldots \ldots \ldots$ & 27 & 5 \\
\hline Canal Zone Reports ${ }^{151} \ldots \ldots \ldots \ldots \ldots \ldots$ & 423 & 73 \\
\hline Hearsey ${ }^{152} \ldots \ldots \ldots \ldots \ldots \ldots \ldots \ldots$ & I4 & 3 \\
\hline Baker $^{153} \ldots \ldots \ldots \ldots \ldots \ldots \ldots \ldots \ldots$ & 7 & 2 \\
\hline Langley ${ }^{154} \ldots \ldots \ldots \ldots \ldots \ldots \ldots \ldots$ & 27 & 4 \\
\hline Will1ss ${ }^{2} \ldots \ldots \ldots \ldots \ldots \ldots \ldots \ldots \ldots \ldots \ldots \ldots \ldots \ldots \ldots \ldots$ & Ior & 15 \\
\hline Fagan $^{136} \ldots \ldots \ldots \ldots \ldots \ldots \ldots \ldots \ldots \ldots \ldots \ldots \ldots \ldots \ldots \ldots$ & 20 & $\mathbf{I}$ \\
\hline 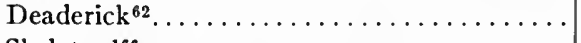 & 34 & 10 \\
\hline Skeleton ${ }^{166} \ldots \ldots \ldots \ldots \ldots \ldots \ldots \ldots \ldots$ & 20 & 2 \\
\hline German Protectorate Reports ${ }^{168} \ldots \ldots \ldots \ldots$ & 538 & 96 \\
\hline
\end{tabular}


that he treated over roo cases in the Congo State without a death.

Pampoukis $^{82}$ gives the mortality of blackwater fever as 6.6 per cent.; Crosse $^{1} 20$ per cent.; Kanellis ${ }^{5} 22.4$ per cent.; Berenger-Feraud $^{82}$ 23.I per cent.; Barthelemy-Benoit ${ }^{82} 25$ per cent.; Bertrand ${ }^{100} 25$ per cent.; Carre ${ }^{2} 27$ per cent.; Manson $^{29} 25$ per cent.; Cassan ${ }^{82}$ 32.I per cent.; Michel ${ }^{169} 33$ to 50 per cent.; Schellong ${ }^{2} 42$ per cent.; Reynolds ${ }^{5} 50$ per cent.; Scott ${ }^{5} 60$ per cent.

The following list of 7105 cases, with 1440 deaths, shows a mortality of 20.2 per cent. It is compiled from various sources. The first column of figures shows the number of cases, the second the number of deaths.

F. Plehn ${ }^{2}$ asserts that mortality is highest in first attacks, but the following table of Daniels ${ }^{27}$ does not bear him out:

Of 136 first attacks........ 31 or 22.7 per cent. were fatal.

Of 33 second attacks....... 8 or 24.0 per cent. were fatal.

Of 15 third or fourth attacks . 2 or 13.3 per cent. were fatal. 


\section{CHAPTER XV}

\section{PROPHYLAXIS OF BLACKWATER FEVER}

A. Plehn has shown that hemoglobinuric fever is preventable to a greater degree even than malaria. In 1897-99 among the officers of Cameroon who used no prophylaxis there occurred in 578 months of residence 287 cases of malaria and $3 \mathrm{I}$ of blackwater fever, or I malaria case for every 2 months and I of blackwater for each 18.5 months. Ten per cent. of the blackwater cases terminated fatally. During the same period among those who used prophylaxis there were in 446 months of residence 90 cases of malaria and 6 of hemoglobinuric fever, or I case of malaria for each 5 months of residence and I of hemoglobinuric fever for each 74 months, none of which were fatal. Thus, while malaria was reduced by half, the morbidity of blackwater fever was lowered to one-fourth. The lowered mortality of these cases is even still more remarkable; similar results were observed by Moffatt. ${ }^{134}$ Even $\mathrm{Koch}^{79}$ believes that through appropriate quinine prophylaxis not only malaria but blackwater fever, in an overwhelming majority of instances, can be exterminated.

The prophylaxis of hemoglobinuric fever consists of the prophylaxis and proper treatment of malaria. There are two chief methods in vogue for the use of quinine as a preventive of hemoglobinuric fever: Plehn's method, $1 / 2$ gram every fifth evening, and Koch's, I gram on two successive days of each week.

The results of A. Plehn, recorded above, were obtained with 1/2-gram prophylaxis, but Ruge ${ }^{41}$ maintains that better consequences follow Koch's method, and gives the following figures: According to the 1903 statistics of Cameroon, there were among those who used quinine regularly $\mathrm{I} 2$ cases of blackwater fever, of which 8 employed the Plehn method; 3 first Plehn's, then Koch's; and only I Koch's method 
regularly. Of 35 cases among irregular users, i 7 employed the $1 / 2$-gram method and only 3 the I-gram method. From these figures it is evident that Koch's method is preferable even when not systematically employed.

It is necessary to persist in prophylaxis not only while in the blackwater fever district, but for several months thereafter. As a majority of the first cases occur from the second to the fourth year of residence, it is evident that greater care should be exerted during this period. 


\section{CHAPTER XVI}

\section{TREATMENT OF BLACKWATER FEVER}

The discussion of the treatment of hemoglobinuric fever has probably been productive of more harsh and prejudiced controversies than has any other question in therapeutics. The bone of contention is quinine.

It is unnecessary to review the discussions or to rehearse the arguments for or against the etiologic relation of quinine to blackwater fever. No valid conclusion can be reached except through results of a large series of cases treated with and without quinine. The collection recorded under Prognosis shows a mortality of 25.5 per cent. in cases treated with quinine, and 10.4 per cent. in cases in which no quinine was used. This number of cases probably eliminates all errors and should be convincing. While the results of the series prove that the mortality is higher under the routine treatment with quinine, they should not be taken to exclude absolutely the use of quinine in some cases of hemoglobinuric fever, for under certain circumstances quinine may be of value. It is difficult-in fact, sometimes impossible - to say whether quinine is indicated or contra-indicated in a certain case.

Mannaberg ${ }^{82}$ gives the following general rules to aid in a decision:

I. When, without quinine preceding, hemoglobinuria occurs and the blood examination shows the presence of malarial infection, quinine is undoubtedly to be exhibited.

2. When the hemoglobinuria occurs after one dose of quinine, while the anamnesis shows that the patient previously took quinine without bad effect, and the parasites are present in the blood, quinine is also to be exhibited. If a paroxysm of hemoglobinuria should follow within a few hours, the repetition of the drug should be made dependent upon whether or not the parasites have in great part disappeared. In the for- 
mer case the quinine may be stopped, at least for a time. But if the blood examinations show that the parasites have increased in number the quinine is to be continued.

3. When anamnesis shows that the patient suffered previously from hemoglobinuria following quinine and the blood examination is negative, quinine is to be absolutely avoided.

4. When the case manifests a severe malarial infection (numerous parasites on examination) and at the same time an assured intolerance to quinine in the shape of hemoglobinuria, the decision is very difficult.

Marchiafava and Bignami ${ }^{19}$ believe that the only guide indicating to the physician whether to give or to withhold quinine ought to be the result of a blood examination.

Bastianelli' ${ }^{29}$ canon is as follows:

I. If a hemoglobinuria occurs during a malarial paroxysm and the parasites are found in the blood, quinine should be given.

2. If parasites are not found in the blood, quinine should not be given.

3. If quinine has already been given before the hemoglobinuria has appeared and no parasites are found, its use should be suspended; but if parasites persist it should be continued.

Thayer ${ }^{192}$ states his rules, modified from Bastianelli, thus:

I. If the attack occurs spontaneously with a malarial paroxysm, the blood showing the presence of parasites, quinine should be freely administered hypodermically or intravenously.

2. If the parasites have disappeared, either as a result of the paroxysm itself or of doses of quinine already given, it may be as well to abstain, at least for a time, from the administration of the drug. It cannot ameliorate the further course of the paroxysm, and the possibility, if it has been already given, that the symptoms may be in part due to quinine may be thought of.

3. If an attack arise in the middle of an ordinary malarial infection, after taking quinine, it is best to abstain, for a time, at any rate, from the further use of the drug. That which has been given may have been enough to control the affection.

4. If, however, in an attack coming on after quinine, the I8 
parasites continue to develop, quinine should be again administered, despite the slight possibility of its injurious action. The dangers from the further development of the parasites are probably the greater.

5. In post-malarial hemoglobinuria quinine is, of course, useless.

The following rules of $V e d y^{83}$ are practical:

I. If living parasites (not merely evidence of their former existence, pigment) are detected twenty-four hours after the beginning of the attack 80 centigrams of a salt of quinine may be injected subcutaneously.

2. If the parasites are not visible do not administer quinine.

3. If in doubt, that is to say if the microscopic examination of the blood cannot be made, do not give quinine.

It may be seen that the authorities quoted lay great stress on the presence of the parasites as a guide to the administration of quinine. I, however, cannot agree with those who hold that quinine should be exhibited in every case where the microscopic examination shows the presence of parasites. It has been shown conclusively that parasites are present in a very large proportion of cases examined early. It has also been shown that in an equally large number of cases the parasites disappear spontaneously. In these cases quinine is, to say the least, superfluous.

In my opinion, the only conditions in which quinine is indicated are: first, where the parasites show no tendency to disappear after forty-eight hours from onset; second, in the infrequent cases of intermittent hemoglobinuria where the outbreak corresponds with parasitic sporulation.

If it is decided to give quinine, it should be injected, in dilute solution, into the muscles as directed for the treatment of pernicious malaria. Given by the mouth it upsets the stomach and may not be absorbed.

Even in cases of mildest onset the patient should be confined to bed from the start, and should be kept quiet, either by persuasion or by sedatives. Sudden death on slight exertion sometimes occurs. The patient should not be transported from one place to another; the Plehn brothers observed 
anuria as a frequent consequence of moving patients from place to place. Chilling of the body, especially when the temperature is low, should be carefully avoided. When vomiting is not a prominent feature, liquid nourishment may be given freely; buttermilk and albumen water are the most suitable. Sweet milk is often ejected as a thick curd, molded ropy by the esophagus in the act of vomiting. Animal broths, barley and oatmeal water, lemonade, and orange juice are allowable. Rectal alimentation is unsatisfactory.

There is no specific. Methylene-blue has proved disappointing. Besides being a renal irritant, it masks the color of the urine, a most serious objection. Salicylic acid probably has no effect further than to upset the stomach and increase the discomfort. With the false idea that a hemorrhage has to be checked, gallic and tannic acids, ergot, and similar drugs are frequently given; these cannot possibly be of any benefit. Carbolic acid and other renal irritants should not be used.

The bowels should move early and often, and calomel possesses advantages over other purgatives; it is more easily retained, is a bland diuretic, and is the best of intestinal antiseptics. Two large doses are usually advised; 3 to 5 grains are, as a rule, sufficient, repeated pro re nata.

Quennec's ${ }^{193}$ chloroform treatment has been successful in some hands. The originator claims for the method three points of value:

I. Controls vomiting.

2. Increases output of urine.

3. Diminishes albuminuria.

He treated more than fifty cases with no mortality. The following is his formula: Chloroform, 6 grams; gum arabic, 8 grams; sweetened water, 250 grams. This amount is used daily, a sip taken every ten minutes. In addition, Quennec used quinine I gram daily subcutaneously, and sulphate of soda and senna by rectum. The excessive administration of chloroform might be harmful, as it is a cardiac depressant, renal irritant, and lowers the blood pressure.

Cardamatis ${ }^{12}$ gives ether in every case of hemoglobinuric fever. In ordinary cases he prescribes a teaspoonful in sweet- 
ened water every three hours, and increases the dose if the urine diminishes in quantity. In cases of suppression he gives as much as a teaspoonful every hour, at the same time injecting hypodermically I c.c. every two or three hours. $\mathrm{He}$ maintains that by this means the pulse is strengthened, precordial anxiety, dyspnea, and vomiting are relieved, and a profuse diuresis is provoked.

I have had no experience with ether in the treatment of blackwater fever, but would consider it too irritating to the kidneys for general use.

Hearsey ${ }^{152}$ used with good results a modification of Sternberg's yellow fever treatment. The original Sternberg formula is: sodium bicarbonate, I 50 grains; mercury perchloride $1 / 3$ grain; water, 2 pints. Sig.: $1 \frac{1}{2}$ ounces every hour. Hearsey gives sodium bicarbonate, Io grains; liquor hydrargyri perchloride, 30 minims, every two or three hours.

A method of treatment recently introduced and extravagantly extolled by its originator is that of Vincent. ${ }^{194}$ This writer maintains that calcium chloride is not only a preventive, but has extraordinary curative powers. During the attack from 4 to 6 grams are given daily by the mouth, or from I to 2 grams dissolved in normal salt solution hypodermically. $\mathrm{He}$ asserts that it acts as an antihemolysin, and that in persons in whom an attack of blackwater fever may be provoked at will, by a dose of quinine, the previous administration of calcium chloride will forestall the outbreak. It is worthy of mention that this drug has been used successfully in paroxysmal hemoglobinuria by Saundby, and in hemophilia by Wright and others.

I have employed calcium in six cases, of which three ended fatally. The series is too small to permit of very definite conclusions as to results of treatment, but it would appear that the results claimed by Vincent were not obtained. The three fatal cases were in persons whose health was probably not more undermined from previous attacks of malaria or other causes than the average patient who is attacked with hemoglobinuric fever. It is worthy of note that the cause of death in these three cases was not syncope nor suppression, but 
exhaustion due directly to hemolysis, the very process which calcium chloride was used to combat. No treatment other than supportive was used which might modify the antihemolytic effects of the calcium chloride.

Hyposulphite of soda, introduced into the treatment of malaria by Polli ${ }^{195}$ in 1867 , has been used extensively in the treatment of hemoglobinuria. Its use is probably not attended with any signal results. O'Sullivan-Beare ${ }^{196}$ used with good results a decoction of the root of cassia beareana, a native plant. Gouzien employed an infusion of the leaves of cassia occidentalis.

Teas made from the leaves of folia combreti alti and of aphloia theaeformis are also highly recommended. ${ }^{23}$

The fever does not usually run sufficiently high to call for treatment. The coal-tar preparations should be assiduously avoided. Cold baths may be productive of harm by increasing the blood destruction, but the hyperpyrexial cases sponging with tepid water may be resorted to.

Vomiting, if not intense, is often benefited by a mustard plaster on the epigastrium. The fly-blister formerly used should be abandoned. Draughts of hot water or carbonated water sometimes assist in relieving this troublesome symptom. Cracked ice may be tried. Morphine hypodermically should be given unhesitatingly when other measures fail. Any evil effects are more than outweighed by its enabling the stomach to retain liquids.

An important measure toward the prevention and relief of nausea and vomiting is to maintain the recumbent position. Medicine, water, and nourishment should be taken through a drinking tube or the ordinary invalid's cup, and the bed pan or urinal should be used when evacuating the bowels or bladder.

It is imperative to allay the restlessness often present in these cases. For this purpose chloral and bromide of soda by rectum, morphine hypodermically, or sulphonal or small doses of chloroform by mouth are useful.

Probably the most important indication in the treatment is the prevention of suppression. Medicinal diuretics usually do harm. One, turpentine, widely used in some sections, should be condemned in the strongest terms. It is one of the most 
violent renal irritants, and in some persons small doses may cause suppression or hematuria. Water is the best diuretic, and as much should be given by mouth as can be retained. Lewis, ${ }^{45}$ of North Carolina, was the first to recommend the use of normal salt solution by hypodermoclysis and by the rectum in the treatment of hemoglobinuric fever, though Laveran ${ }^{23}$ attributes the priority to Gouzien. The latter recommends the daily injection of 100 to 300 grams of a $9 / 10$ per cent. solution, in conjunction with the rectal injection of 200 grams four to six times in twenty-four hours. The use of salt solution is the very best means of combating and treating anuria. It is probably better to use a hypertonic solution. In mild cases where the urine is free the rectal use is usually sufficient, but in cases where suppression threatens or is imminent the solution should be given subcutaneously or intravenously and in larger quantities and of tener than advised by Gouzien. Mild counter-irritation over the region of the kidneys may be tried.

Werner ${ }^{197}$ in 1902 , suggested nephrotomy for anuria. Such an operation has been recorded in only three instances. Zie$\operatorname{mann}^{86}$ mentions a case in a young female patient in whom suppression had existed two days. The capsule of the right kidney was split and peeled off to the hilum and nephrotomy performed through the convexity of the organ. The operation was well borne, and subsequently 200 c.c. of cloudy, albuminous urine was voided from the bladder. During the following days complete suppression recurred, and the patient died.

In Kruger's ${ }^{198}$ case decortication of one kidney was done five days after the onset of anuria, and, although the secretion of urine was profusely reestablished, the patient died of progressive weakness.

$\mathrm{Kul}^{199}$ reports a case in a man during his second attack. Three and a half days after the onset of anuria nephrotomy upon one kidney was performed through Simon's incision. Vomiting, which was formerly uncontrollable, ceased immediately. Three hours after the operation 30 c.c. of blood were voided from the bladder. In eight hours the dressing was saturated with bloody icteric urine, which necessitated changing 
the dressing every three hours. Twenty-four hours after the operation the patient died. Though a microscopic examination of the kidney could not be made, upon gross inspection the nephrotomized kidney appeared much more nearly normal than the other.

Supportive measures are essential. Alcohol in all its forms is inadmissible. Strychnine is useful, and should be given hypodermically when circumstances permit. Digitalis has proved serviceable in my hands. Doering ${ }^{39}$ had good effects from strophanthus. The aromatic spirits of ammonia and hypodermic injections of ether have been recommended. Transfusion of blood has been used, it is said, with excellent results. The elder Plehn $^{30}$ says that he had four attacks, in which Kohlstock treated him with inhalations of oxygen, and that nothing else did him so much good. Unfortunately this method of treatment is not often possible in private practice.

The after-treatment should have a care for the diet, which should be non-nitrogenous and consist largely of liquids at first. A tonic of organic iron is indicated, and digestive disorders when present should receive appropriate treatment.

A question of practical importance is, how soon after the attack to begin the administration of quinine. A dose given too early might possibly, in some persons, precipitate hemolysis. On the other hand, delay may permit an outbreak of malaria accompanied by hemoglobinuria. Upon the ground that most of the sensitive cells have succumbed during the attack and that the newly formed cells are probably more susceptible than those that have withstood the attack, I am of the opinion that quinine should be begun, carefully at first, a short time after the attack has subsided and before blood regeneration is fairly established. One grain of quinine three times daily, increased gradually every other day, is a safe procedure. If the temperature rises or the urine becomes distinctly darker no further attempt to increase the dose should be made.

In the present state of our knowledge it is probably utopian to discuss the treatment of hemoglobinuric fever by antihemolytic sera, but such has been successfully accomplished by Widal and Rostaine in paroxysmal hemoglobinuria. 



\section{PELLAGRA}

\section{CHAPTER XVII}

\section{INTRODUCTION}

Pellagra is an endemic disease, the cause of which is at the present time unknown, is usually of slow progress, and characterized by more or less seasonal periodicity, and by lesions of the skin, alimentary tract and nervous system, terminating in recovery, cachexia, insanity or death.

The list of names by which the disease is known is a long one, the chief of which are Alpine scurvy, dermotagra, Asturian leprosy, Asturian rose, disease of the Landes, Italian leprosy, maidismus, corn bread disease, sun disease, elephantiasis italica, psychoneurosis maidica, mal de rosa, mal del sole, mal de misere, etc.

History.-Our history of pellagra begins in Spain. It was first observed in the province of Oviedo by Gaspar Casal who in 735 wrote a treatise in which he described it, which, however, was not printed until i 762 . He called the disease mal de rosa. In 1787 Townsend in his description of his travels through Spain refers to his observation of the disease in Oviedo and definitely states that the subjects ate little meat and largely of corn and other vegetable foods. Corn is said to have been introduced into Spain between 1680 and $\mathrm{I} 700$. Oviedo remains to this day a hot bed of the disease.

Pellagra was, according to Terzagli, known in Italy even earlier than $\mathrm{I} 730$, isolated cases having been observed in the neighborhood of Sesto Calende. The disease appears to have spread rapidly appearing simultaneously in the districts of Milan, Brescia, Bergono, and Lodi and soon after in the vicinity of Como, Cremona, Mantna and Paira, toward the end of the century extending almost entirely throughout Lombardy. The name Pellagra was given the malady by Prapolli in I $77 \mathrm{I}$, and 
probably means "rough skin." As the disease invaded new areas in Italy the number of cases in the earlier foci increased rapidly. In 1784 a pellagra hospital was established in Legnano under the supervision of the elder Strambio. Marzari in 1810 is said to have been the first to infer an etiologic relationship

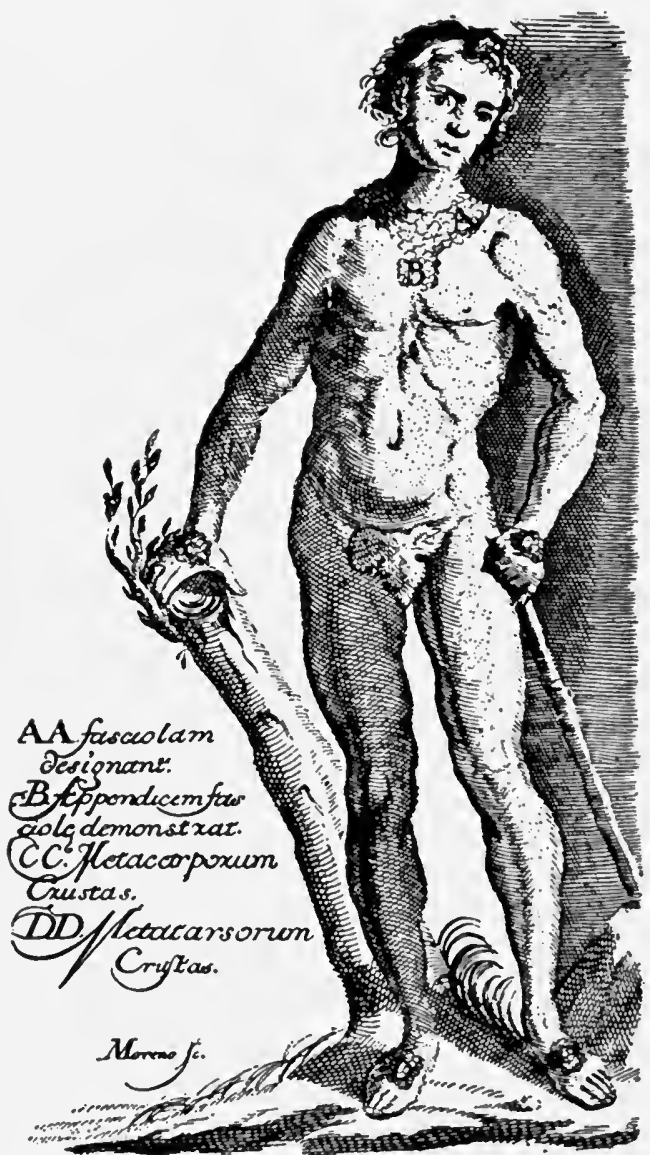

Fig. 66.-Casal's illustration of the cutaneous lesions in pellagra.

between the consumption of maize and pellagra. On account of the early invasion of Italy, of the extensive prevalence of the disease in that country and of the attention paid to the scourge by scientists and by the government, Italy may well be regarded as the home of the disease. 
Our first knowledge of pellagra in France originates with Hameau who, in 1829 , published his observations of cases since I 818 in the vicinity of Teste-de-Buche and in the plain of Arcachon and in the coast region of the Giroude. For the last quarter of a century there has been little if any pellagra in France.

Pellagra was first described in Roumania by Caillat in 1854 , who states that the disease was unknown there prior to 1846 . In Corpi isolated cases were observed in 1839 , but only since I 856 has the malady assumed an endemic character. Nicholas and Hambon reported cases in Austria near Vienna in 1794 and in 1846 an epidemic occurred in Roumania. It is stated that pellagra was observed in Great Britain in $\mathbf{1} 860$.

In Africa pellagra was first described by Prumer in Egypt in 1847. The disease was practically ignored until 1893 when Sandwith found it to be prevalent in patients he was treating for anchylostomiasis.

In the United States the definite history of pellagra goes back to I 864 when a case was reported by Dr. John Gray of Utica, New York, and another by Dr. Tyler of Somersville, Massachusetts. Both patients were insane. Shewell of Brooklyn reported a case of pellagra in $\mathrm{I} 883$ in an Italian sailor. Bemis of New Orleans, in 1889 , left a written diagnosis of a case in a white female in the Charity Hospital.

Babcock ${ }^{280}$ has adduced very strong evidence in favor of the existence of pellagra in the South Carolina State Hospital from its opening in 1828 . A case history which he reproduces in full from the records of the institution is certainly very convincing. This case was admitted in 1834 .

It is believed that the terrific mortality among the prisoners in the Confederate prison at Andersonville, Georgia, was due in part to pellagra. The medical records of the Civil War, however, do not conclusively support this theory.

Harris, ${ }^{281}$ Atlanta, in r902, reported a case of ankylostomiasis in an individual presenting all the typical symptoms of pellagra. The patient was a native of Georgia and had always resided there. It is worth noting that both Sandwith and Harris discovered the disease in the investigation of uncinariasis. 
The report of Harris does not seem to have excited any interest and it was not until 1907 that the disease was next re-

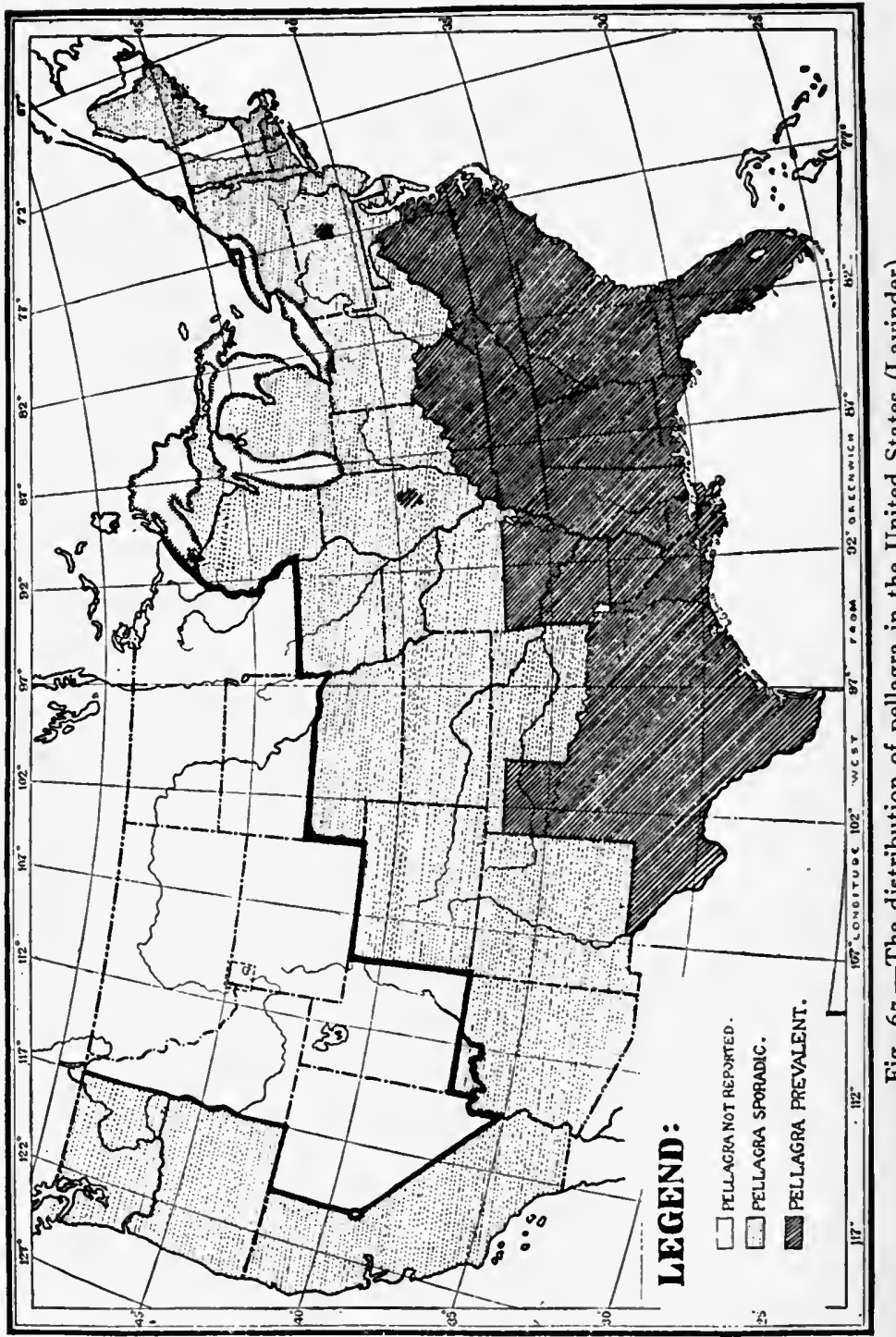

ported, this time by Searcy ${ }^{282}$ as an epıdemic at the asylum in Mt. Vernon, Alabama. Reports of cases then followed 
rapidly. Merrill ${ }^{283}$ reported a case in Texas in 1907 ; Babcock and Watson ${ }^{284}$ in South Carolina in 1908; Bellamy ${ }^{285}$ in North Carolina in 1908; $\mathrm{Card}^{286}$ in Mississippi in I908; Williamson ${ }^{287}$ in Arkansas in 1909; Hewit ${ }^{288}$ in Virginia in 1909; Pollock ${ }^{289}$ in Illinois in 1909 and King ${ }^{290}$ in Tennessee in r 909 . Thayer ${ }^{291}$ is said to have observed cases in Maryland in 1905 and 1909.

Geographic Distribution.-The distribution of pellagra is peculiar. In Europe it is found in Italy, Roumania, Austria, Hungary, France, Northern Portugal, Northern Spain, Bessarabia, Poland, Kherson, the Island of Corfu, Bosnia, Herzegovina, Servia, Bulgaria, Turkey, Greece, England, Scotland, Ireland, Wales and the Shetlands.

In Asia it has been found in Asia Minor, Persia, Straits Settlements and in North Behar, India.

In Africa it is encountered in Lower Egypt, less in Upper Egypt, in the coastal regions of the Red Sea, in Algeria, Tunis, Nyassa and Rhodesia, South Africa and Robben Island.

Pellagra has been found in New Caledonia, Hawaii, Cuba, Porto Rico, Barbadoes and Jamaica.

In South America it has been reported from Brazil, Argentina and the Columbian Republic.

Numerous cases are recorded in the Canal Zone and the disease is found in Mexico.

In the United States there is no other disease known to be so widely disseminated so soon after its recognition, pellagra being found in thirty-nine states including the District of Columbia. The most intense foci of infection are in Virginia, North Carolina, South Carolina, Georgia, Florida, Kentucky, Tennessee, Alabama, Mississippi, Arkansas, Louisiana, and Texas. The disease is frequent in part of Illinois; pellagra is found sporadically in Maine, Vermont, Massachusetts, Connecticut, Rhode Island, New York, New Jersey, Pennsylvania, Maryland, District of Columbia, West Virginia, Kansas, Wisconsin, Oklahoma, Ohio, Indiana, Iowa, Missouri, Michigan, Minnesota, Colorado, New Mexico, Arizona, Washington, Oregon and California.

Prevalence.-As pellagra is not a reportable disease except in a small portion of the country in which it is endemic, accu- 
rate statistics of its prevalence are not available. In I9I2 Niles $^{305}$ estimated that there were between 6,000 and 10,000 cases in the United. States. Roberts ${ }^{300}$ believed that there had been at least 30,000 cases in the United States between the years 1907 and 1912 .

Basing our opinion from personal observation on the increasing prevalence of the disease in Arkansas and upon the published reports from other localities we believe that there are at least 25,000 cases of pellagra in the United States at this time (August I, I915). 
CHAPTER XVIII

\section{ETIOLOGY OF PELLAGRA}

Season.-Pellagra is subject to decided seasonal variations. In the United States the months of most frequent occurrence are from March to July, particularly April, May and June.

The following table is compiled from cases reported in the United States and shows the occurrence of the disease by months.

\begin{tabular}{|c|c|c|c|c|c|}
\hline & Grim $^{292}$ & Walker ${ }^{293}$ & Tucker 294 & Mizell 291 & Total \\
\hline January.......... & 2 & 2 & 2 & 2 & 8 \\
\hline February..... & I I & I & 2 & 2 & I6 \\
\hline March..... & I8 & 2 & IO & I 7 & 47 \\
\hline April....... & 27 & 2 & 9 & 24 & 62 \\
\hline 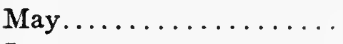 & 37 & 3 & 7 & 39 & 86 \\
\hline June................ & 36 & 3 & 9 & 40 & 88 \\
\hline July............... & 35 & I & $\circ$ & $3 \mathrm{I}$ & 57 \\
\hline August............... & 14 & 4 & 4 & 8 & 30 \\
\hline September......... & 44 & 5 & $\circ$ & 4 & 13 \\
\hline October.............. & 5 & Io & $\mathbf{I}$ & 2 & 18 \\
\hline November............ & 2 & 5 & I & $\circ$ & 8 \\
\hline December............. & $\circ$ & 4 & $\circ$ & I & 5 \\
\hline
\end{tabular}

Most of the cases seen by one of us in eastern Arkansas came for consultation for the first time in May and June, while of ninety-one cases seen by one of us in the Arkansas State Hospital for Nervous Diseases, the admissions for pellagra by months were as follows:
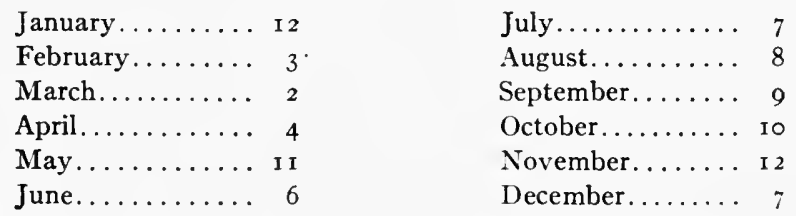

By an analysis of I 5 cases the Thompson-McFadden Pellagra Commission ${ }^{295}$ have shown that there is no particularly marked 
tendency for the seasonal recurrences to reappear during the same month year after year. Relapses may occur during the fall months of the same year in which the onset appears.

The onset appears earlier in countries lying farther south. In Florida more cases originate in the early spring months than in states farther north. In Italy the majority of first cases are said to occur from the middle of March to the middle of May and the disease appears somewhat earlier in the central provinces than in the northern ones. Sandwith ${ }^{205}$ states that in examining 300 patients in Egypt two-thirds of them stated that their skin lesions were first seen during the months of January and February.

Rainfall.--The Thomson-McFadden Commission ${ }^{295}$ observed that if during the spring precipitation is high, the temperature low, and the number of rainy days excessive the appearance of acute symptoms, more particularly those involving the brain, is delayed.

Altitude and Topography.-In the United States pellagra is found in mountainous regions, as in southeastern Kentucky and northwestern Georgia, in rolling upland regions and in lowland and swampy regions. In the eastern hemisphere also pellagra occurs from the Tyrolean Alps to the delta of the Nile. In Europe the disease is especially prevalent about the lower slopes and foothills of mountainous regions.

Sambon ${ }^{296}$ has emphasized the frequency with which pellagra cases are found on the narrower valleys of hilly and wooded country trenched by swift-running streams. His observations were conducted in northern and central Italy. In Kentucky, Georgia and South Carolina, Grimm ${ }^{292}$ classified 323 cases with reference to the distance from water courses with the following results:

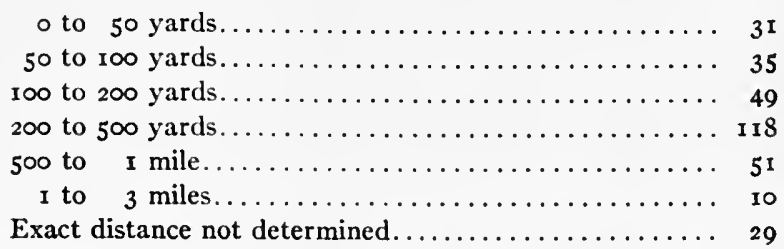


The Tennessee Pellagra Commission ${ }^{299}$ record their observations with reference to proximity to creeks as follows:

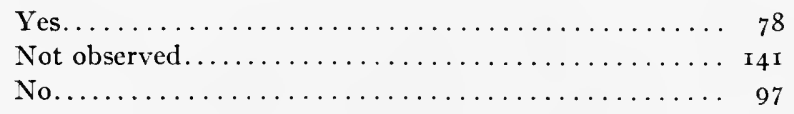

Wood's ${ }^{297}$ experience in North Carolina was similar to that of Sambon in Italy.

On the other hand, the Thomson-McFadden Commission ${ }^{298}$ investigated conditions on a large island off the coast of South Carolina, the distinguishing feature of which was the absence of streams. Pellagra was found to be endemic here. Investigations were also undertaken in the Panhandle of Texas where the rainfall is very slight and running streams remarkably scarce. Pellagra cases were found originating here.

The most that can be said regarding the relation of altitude and topography to pellagra is that it seems to be restricted to certain areas which vary widely in these characteristics.

Race.-Generally speaking pellagra is commoner in the white race than in the negro as shown in the table below:

\begin{tabular}{|c|c|c|}
\hline & White & Colored \\
\hline Lavinder ${ }^{291}$. & 9,88 I & 4,048 \\
\hline Roheren ${ }^{291}$. & 25 & 6 \\
\hline Roberts $^{300}$. & 36 & 24 \\
\hline Albright ${ }^{299} .$. & 298 & I8 \\
\hline Randolph and Green ${ }^{286}$. & 5 & 6 \\
\hline Siler, Garrison and MIcNeal ${ }^{298}$. & 680 & 100 \\
\hline Tucker ${ }^{294} \ldots \ldots \ldots \ldots \ldots$ & 46 & 9 \\
\hline Grimm $^{292} \ldots \ldots \ldots \ldots \ldots \ldots \ldots \ldots \ldots \ldots$ & 1,223 & 203 \\
\hline 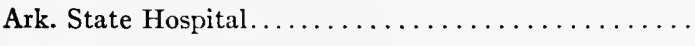 & 74 & I 7 \\
\hline Total. & I 2,266 & $4,43 \mathrm{I}$ \\
\hline
\end{tabular}

Siler, Garrison and $\mathrm{McNeal}^{298}$ believe that this ratio is not necessarily indicative of a relative racial resistance to pellagra in negroes but rather as the end-results of the influence of several factors, prominent among which are the poor hygienic conditions under which the people live in villages as compared with the relative isolation of the bulk of the colored population on farms. 
There are undoubtedly areas in which the disease is far commoner in negroes than in whites. This was the experience of one of us in the lowlands of eastern Arkansas where many cases were seen in negroes and only few in whites. It is true that relatively few white persons live in the rural districts of that particular locality.

As recently shown by Wolff $^{301}$ pellagra is an exceedingly rare disease among Jews.

Sex.-Foreign observers have failed to note any significant influence of sex on the prevalence of pellagra. In the United States, however, and particularly in the South, the disease is known to occur with a notable preponderance among females. Sixty-five per cent. of Niles $^{305}$ cases and 8I per cent. of Lowery's ${ }^{306}$ were in females. At least two-thirds of the eastern Arkansas cases seen by one of us and 73 per cent. of the cases in the Arkansas State Hospital were in females.

A compilation of cases from numerous sources shows that females are affected about two and a half times more frequently than males:

\begin{tabular}{|c|c|c|c|}
\hline & & Males & Females \\
\hline Clark $^{302}$. & Canal Zone...... & 12 & 25 \\
\hline Harrington ${ }^{291}$. & Rhode Island...... & 5 & 27 \\
\hline Mizell $^{291} \ldots$ & Maryland.... & 15 & I6 \\
\hline Siler and Nichols ${ }^{286}$. & Illinois $\ldots \ldots \ldots \ldots$ & 44 & 56 \\
\hline Thomson-McFadden Commission & South Carolina..... & 212 & 528 \\
\hline Beall $^{303} \ldots$ & Texas............ & 36 & 53 \\
\hline Tucker 294. & Virginia.......... & 16 & 39 \\
\hline Grimm $^{292}$. & $\begin{array}{c}\text { Kentucky, } \\
\text { Carolina.............. }\end{array}$ & 420 & 1,006 \\
\hline Lavender. . & United States........ & 4,982 & I 2,689 \\
\hline Thomson-McFadden Commission & South Carolina..... & 71 & 211 \\
\hline Albright ${ }^{299} \ldots \ldots \ldots \ldots \ldots \ldots$ & Tennessee........... & 102 & 214 \\
\hline Roberts $^{300}$. & United States....... & 91 & I 55 \\
\hline \multirow[t]{2}{*}{ Thompson............... } & Arkansas............ & 24 & 67 \\
\hline & & $6,05 \mathrm{I}$ & 15,265 \\
\hline
\end{tabular}

The sex distribution of the cases investigated by the ThomsonMcFadden Commission is interesting. $\mathbf{I}$. The rate of prevalence among children under to years of age and among adults 
aged 45 years and older is practically equal in the two sexes. 2. The rate of prevalence drops among males between the ages of I9 and 45 years, whereas for females there is a remarkable excess of prevalence between these ages. 3. In both males and females there is a striking fall in prevalence between the ages of 10 and 20 years.

Age.-No period of life is free from the attack of this disease. It has been found in infants at the breast and in the centenarian. The youngest pellagrin of which we can find a report is one mentioned by Haase. ${ }^{307}$ which was but 4 weeks old. $\mathrm{He}$ also speaks of two others respectively 6 and 8 weeks of age. The age of the oldest pellagrin on record is IO2 years, that being the age of a pellagrous woman reported by Roberts. ${ }^{300}$

These extremes of age, however, are rare. The youngest pellagrin we have seen was a child of 2 , while the oldest was 69. The following table shows the ages of the 9r cases seen by one of us in the Arkansas State Hospital for Nervous Diseases:

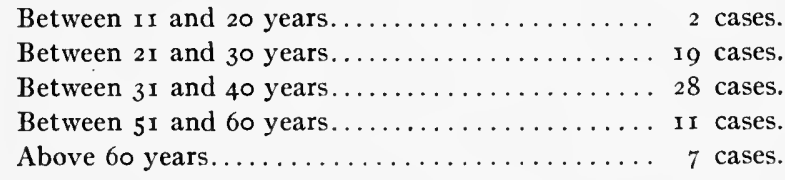

Lavinder ${ }^{304}$ found 532 cases in children under 5 years of age, 2,192 cases between the ages of 5 and 20 years, 9,404 between 20 and 40 , and 5,309 cases in individuals over 40 years old.

Merck's ${ }^{300}$ table of ages shows that the greatest number of cases occur between the ages of 40 and 50 years, and the smallest number of cases under 5 years.

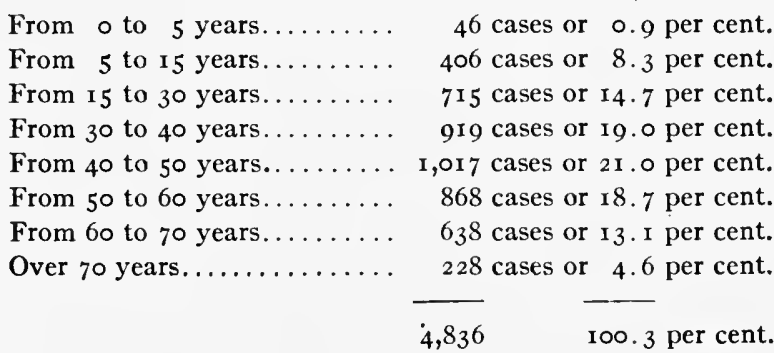


Heredity.-The rôle of heredity in pellagra has been a moot question for many years, and is one that in all probability will not be settled until the etiologic factor of the disease is discovered. Some investigators contend that pellagra is truly hereditary, that it may be transmitted from parent to child, but there is little or no evidence to prove that such is the case. No instance of pellagra has been reported in the new-born and as stated above the youngest case on record was in a child of four weeks of age.

On the other hand, the majority of investigators contend that pellagra is not hereditary in the sense that syphilis is, but that pellagra in the parents predisposes the children to the disease.

Civil Condition.-While it would seem from our present knowledge of pellagra that the civil condition of an individual could have little or nothing to do with him contracting the disease, it will be seen from the accompanying table that pellagra is much more prevalent among the married than those of any other civil condition.

\begin{tabular}{|c|c|c|c|c|c|c|c|}
\hline & Grim ${ }^{292}$ & Tucker 294 & Roberts $^{300}$ & Albright 299 & \begin{tabular}{c|} 
Thomson \\
and \\
McFadden
\end{tabular} & $\begin{array}{c}\text { Arkansas } \\
\text { State } \\
\text { Hospital }\end{array}$ & Total \\
\hline Married. & 208 & 37 & 42 & 204 & 150 & 53 & 698 \\
\hline Single... & 83 & 14 & I 2 & 92 & 25 & 20 & 246 \\
\hline Widowed... & 32 & 4 & 5 & 20 & 9 & I 8 & 88 \\
\hline Divorced... & $\circ$ & $\circ$ & I & $\circ$ & $\circ$ & $\circ$ & I \\
\hline - & 323 & 55 & 60 & 316 & I 84 & 91 & 1,033 \\
\hline
\end{tabular}

Occupation.-It has long been considered in Italy that pellagra is found almost exclusively among farmers or field laborers, and that "peasant life, poverty and polenta" are the three p's responsible for this disease. While it is undoubtedly true that the majority of the pellagrins of Italy are of the farmer class, this does not seem to be the case in America. According to the Thompson-McFadden Pellagra Commission, ${ }^{295}$ the most significant fact concerning occupation brought out by their investigation is the prevalence of the disease among housewives. They state, however, that this means little more than that 
pellagra is most prevalent among women of the age at which they are usually employed in the house.

Social Condition.-As stated above, poverty has long been considered as one of the prime causes of pellagra, and the studies of numerous investigators show that while pellagra may attack those of affluent circumstances it is much more prevalent among the poor.

Thus the Thompson-McFadden Pellagra Commission ${ }^{295}$ report as follows:

Cases living in squalor................ 2

Cases living in poverty..................... 28

Cases living where only the necessities of life were obtained 200

Cases living in comfort................ 4 I

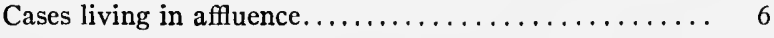

Lavinder, ${ }^{304}$ in a study of 16,960 cases of pellagra, found $8,49 \mathrm{I}$ were poor, 6,970 were of moderate means, and only I,499 were well-to-do:

Hygiene.-That the hygienic surroundings of an individual may play an important rôle in the etiology of pellagra seems at least possible.

Grimm ${ }^{292}$ found that of 296 cases I I lived under good sanitary conditions in the home, 36 under fair conditions, while 249 cases came from homes where the sanitary conditions were bad.

Marie $^{308}$ states, however, that lack of cleanliness in the home is not a cause, as many peasants keep their houses neat.

Siler, Garrison and $\mathrm{McNeal}^{298}$ consider inefficient methods of the disposal of human excreta a material factor of the epidemiology of pellagra in those sections where the disease is endemic. In Spartanburg County, S. C., they found pellagra endemic in all of the mill villages, and in all they found unscreened surface or pail privies. In two mill villages of other counties where every house contained a water-carriage flush closet they failed to find a single case of pellagra which had certainly originated in these villages. In the city of Spartanburg these investigators observed that the active foci of the disease were found in the portions of the city where unscreened pail and surface privies were used. They found that 230 cases 
of the $24 \mathrm{I}$ observed in the city came from homes where the above-named privies were used, and several of the remaining eleven cases, while using the water-carriage system themselves, lived in communities where unscreened surface privies were common, and some of them used by pellagrins.

Rural and Urban Dwellers.-Aside from the tilling of the soil it has been alleged that the mere fact of dwelling in rural districts predisposes to pellagra. Practically all writers on Italian pellagra are agreed upon this point, and Roberts ${ }^{300}$ writing of conditions in general, including America, states that this is a disease of rural districts, that "pellagra stops at the city gates."

This statement is not in accord with the findings of most observers in the United States. The Thompson-McFadden Pellagra Commission ${ }^{295}$ found the disease much more prevalent in the congested districts of the mill villages than in either the urban or rural districts. This coincides with the findings of Grimm, ${ }^{292}$ who observed more pellagra among dwellers of towns than among city or country residents.

Of the 17,763 cases reported by Lavinder ${ }^{304} 5 x^{2} \mathrm{I} 2$ were city dwellers, 784 lived in towns, while 11,767 were inhabitants of rural districts.

In Arkansas, however, the inhabitants of even the largest cities have easy access to the country and most of such make visits of more or less extent to rural districts. This statement is true also of a great deal of the pellagrous area of this country, and we have never seen a city pellagrin who has not made visits to the country.

Other Predisposing Causes.-Among the other factors which seem to be predisposing causes of pellagra the incidence or previous occurrence of other diseases is important. However, the occurrence of disease in childhood does not seem to predispose the adult to pellagra.

Alcoholism appears to play an important rôle in predisposing the individual to this disease, while many pellagrins are found to have indulged in venereal excesses. Pregnancy and frequent child bearing may account for the development of the disease in some cases. One case in this connection which has 
been under treatment by one of us for some time is of more than ordinary interest as it followed pregnancy and puerperal convulsions.

Malaria and hook-worm are frequent concomitant diseases with pellagra, the former having been seen by us in several cases.

It seems to us that the factor to be considered in this connection is not that the previous or concomitant disease per se predisposes the individual to pellagra, but that by lowering his vitality and power of resistance it renders him more prone to contract it.

Theories of Etiology.-There are two theories as to the etiology of pellagra: (I) that it is due to some dietetic error; and (2) that it is an infectious disease, caused by some as yet unknown parasite.

It might be well to mention here, not because it has any scientific bearing upon the subject of the etiology of pellagra, but for historic interest, that there are certain French pellagrographers who contend that pellagra is not a disease entity, but that it is a symptom-complex which may develop in any cachectic state. Outside of a small group of writers this theory has received scant attention.

Dietetic Dyscrasia.-From the earliest description of pellagra by Casal down to the present time Indian corn or maize has been incriminated in connection with the etiology of the disease. However, Casal and his contemporaries, some of whom in all probability independently thought of corn as the cause of pellagra, did not formulate their views on the subject and only in a vague indefinite sort of way laid the malady at the door of maize. Pellagra was a new disease - at least it had only newly been observed - and Indian corn was a new product, so it was rather natural that the physician of the times should connect the two.

It was not until I810, however, that any definite theory as to the manner in which corn caused pellagra was formulated. At this time Marzari propounded his theory that the disease was due to a lack of certain nutritive elements in the corn, and almost immediately there sprang up two schools of thought: 
the zeists (from $Z a M a y s$ ), who supported the corn theory, and the anti-zeists, who discredited it. However, it was not long before the zeists became divided among themselves and many new theories were formulated as to the exact manner in which corn caused this disease.

The following list comprises the principal theories as to the manner in which corn acts as an etiologic factor in the production of pellagra:

I. Lack of nutritive elements.

2. Toxicity of normal corn itself.

3. Toxicity of corn due to germination process.

4. Toxicity of corn due to products elaborated by the action of certain microörganisms.

5. Toxicity of corn due to products produced in the alimentary tract after ingestion.

6. Toxicity of corn due to poisonous substances in the grain which act only after being sensitized by the rays of the sun.

7. Action of certain parasites of corn ingested with that grain.

The first and second of these theories have been thoroughly disproven by the many careful analyses of corn which show that this grain is rich in nutritive value, containing a large percentage of fat and nitrogenous substances, and is readily assimilated by the body. Further, it has been shown that pellagra is often found in well-nourished persons, thus proving that it is not a disease due to lack of nutritive elements; and finally, there are many districts in which corn has been very extensively used as an article of diet for long periods of time in which pellagra has never been found.

The third theory, that pellagra is due to a toxin elaborated during the process of the germination of the corn, has also been shown to be untenable by the above-mentioned facts, and even the most ardent zeists have abandoned these theories.

The fourth theory, the so-called toxico-chemical theory, that pellagra is due to toxins produced in corn by the action of certain microörganisms, has probably had the widest vogue of any theory as to the etiology of this disease. It was to the 
elaboration and promulgation of this theory that the great Lombroso gave the last twenty-five years of his life.

A great many microörganisms, moulds and bacteria have been described by investigators in this field, but even Lombroso, himself, was unable to incriminate any one of them.

The most frequent parasite of spoiled corn is the Penicillium glaucum or common blue mould. This organism penetrates into the interior of the corn and has been thought by many to produce the specific toxin of pellagra.

Other microörganisms, which have been described and defined as the cause of the toxin of pellagra, are Sporisorium maidis, Eurotium Herbariorum, Aspergillus glaucus, Oidium lactis maidis, misentericus vulgaris, bacterium thermo, etc.

Numerous chemical studies of spoiled corn have been made and several substances have been isolated, the chief of which, according to Marie, ${ }^{308}$ are the so-called red oil, pellagrosine, and a resinous substance.

With these substances and with the spoiled corn itself Lombroso conducted many experiments in animals, especially in dogs and chickens, and considered that he had produced a true pellagra in them.

So firmly convinced were the Italian people that Lombroso was correct in his contentions that they instituted the most elaborate measures calculated to prevent the use of spoiled corn as food and thus act as a prophylaxis to the disease.

De Giaxa ${ }^{309}$ considered that pellagra was due to a toxic substance elaborated in the intestinal tract by the action of the colon bacillus on ingested corn. He considered that the properties of the organisms were changed by growth with the corn, and alleged to have produced a toxic substance from the growth of this bacillus on a corn culture media. Another auto-intoxication theory, advanced by Neusser, ${ }^{310}$ was that a toxin was produced in the alimentary tract from a so-called "receptive mother substance" which was the product of the action of the Bacillus Maydis on corn.

The so-called photodynamic theory of the etiology of pellagra was advanced by Ravbitschek ${ }^{311}$ and others. They maintained that all corn contains a certain toxic substance which, after 
ingestion of the corn, is taken up by the blood stream where it remains inactive until it becomes sensitized by the action of the sun's rays on the exposed portion of the body.

Next to the toxico-chemical theory so strongly defended by Lombroso and his followers the idea that pellagra is due to a specific microörganism, either mould or bacteria ingested with corn, has probably been the most popular.

The organisms which have been described and incriminated in this respect are legion and only a few of them will be mentioned. The ordinary blue mould, Penicillium glaucum, has probably been accused in this respect by more investigators than any of the other parasites of corn.

$\mathrm{Cini}^{312}$ contended that pellagra was an aspergillosis due to two moulds, Aspergillus fumigatus and Aspergillus flaverscens.

Tizzoni $^{313}$ described a specific bacterium which he termed Streptobacillus pellagre, which he isolated from spoiled corn and from the blood, spinal fluid, and tissues of pellagrins.

This organism usually appears as a short bacillus, grows both in short and long chains, stains readily with the ordinary aniline dyes and often shows polar staining. It is nor-spore forming, and obtains its optimum growth on human or rabbit blood agar at $37^{\circ} \mathrm{C}$.

The main arguments advanced by the zeists for their beliefs as to the etiology. of pellagra are based upon the following assumptions:

I. Pellagra first developed in Europe almost immediately subsequent to the introduction of Indian corn from America.

2. Pellagra appeared everywhere that corn was cultivated, and the number of cases increased as the new grain became more popular as an article of diet.

3. Pellagra is found only in localities where corn is cultivated or imported and only in individuals who consume it as food.

4. And finally, since the institution of the prophylactic measures of the Italian government which have reduced the consumption of spoiled corn, the number of cases of pellagra has diminished.

The anti-zeists offer the following arguments against the maize theory: 
I. That pellagra first appeared in Europe soon after the introduction of corn from America is without foundation in fact.

2. That many districts exist, where corn has been cultivated and used as an article of diet for generations, in which pellagra is unknown.

3. That pellagra is often found in individuals who have seldom or never partaken of corn as food.

4. That the peculiar topographic distribution of pellagra even in the worst endemic centers is untenable with the corn theory.

5. That the erythema and other characteristics of the disease may recur each spring for several years in patients removed from the endemic centers and who have been on a corn-free diet.

6. That all prophylactic measures based on the corn theory have failed to prevent the disease.

Other dietetic factors than corn have from time to time been proposed as the cause of pellagra. Thus Strombio, as far back as 1784 , considered bad food as an important cause. Others thought the lack of salts in the diet was responsible, while by some the abstinence from wine by those who were used to it was considered the cause of the disease.

In I9II Mizell ${ }^{314}$ proposed the theory that pellagra was due to the use of cotton-seed oil; this theory is untenable because of the fact that cotton-seed oil is not used as an article of diet by the vast majority of European pellagrins, if at all, and that it is used very extensively in this country in localities where pellagra is unknown. We are very much in accord with Lavinder, ${ }^{315}$ who states that the publication of such speculations founded upon personal opinion, especially when they add to the fears of the laity, are strongly to be deprecated.

Goldberger $^{316}$ in I9I4 asserted that pellagra is a disease essentially of dietary origin and that it is caused in some way by the absence from the diet of essential vitomines or perhaps, as is suggested by the work of Meyer and Voegtlin, by the presence in the vegetable food of such poison as soluble aluminum salts in excessive amounts. 
Goldberger cites as evidence of the correctness of his theory the fact that of 996 patients admitted to the Georgia State Sanatorium during the year I9I0, excluding those who died, were discharged or had pellagra upon admission, there were remaining in the institution after one year 418 patients and of this number 32 or 7.65 per cent. developed the disease. $\mathrm{He}$ continues that none of the 293 employees of the Sanatorium who came into contact with the pellagrins developed pellagra, while if the disease had developed in the employees at the same rate as in the patients 22 of them should have been afflicted.

Further studies at the Jackson orphanage showed that 68 or 32 per cent. of the 2 I I inmates had pellagra. To Goldberger the remarkable fact to be observed is that practically all of the cases developed in children between the ages of 6 and 12 years, that only 2 cases developed in the group of 25 children under 6 years of age and only $I$ case in the group of 66 children over 12 years of age.

Goldberger found that in both the institutions cited the groups which were exempt from pellagra subsisted on a better diet than those who developed the disease, that the diet of the pellagrin was noticeable for the marked lack of meat or otker animal protein and that of the vegetable food, such as corn and sirup, were in excess and legumes relatively inconspicuous. $\mathrm{He}$ therefore concluded that this one-sided diet is the cause of pellagra.

That the disease develops in certain groups of individuals outside of institutions is due mainly to economic conditions such that the more expensive foods, meat, milk, eggs, legumes, etc., are not procurable among the poorer classes.

That it develops in certain well-to-do individuals is accounted for by Goldberger by more or less well-recognizable eccentricities of taste, which causes them to partake of a one-sided diet.

He further states that such eccentricities of taste are found in the insane, some of whom will not eat at all. This, Goldberger thinks, accounts for the development of pellagra among residents of insane asylums where the diet is otherwise satisfactory. 
Goldberger (547) has recently conducted a feeding experiment upon eleven convicts on the Mississippi State Penitentiary farm. These men were fed a proteid-free diet from April I9, I9I 5 to October 3I, I9I5. Of the eleven subjects not less than six are claimed to have developed symptoms, including a typical dermatitis, justifying a diagnosis of pellagra. The nervous and gastro-intestinal symptoms are said to have been mild but distinct. The skin lesions were first recognized on the scrotum. Later there appeared lesions on the backs of the hands in two cases and on the back of the neck in one case.

This experiment while suggestive would have proved more convincing had it been conducted in a pellagra-free territory.

The theory that water may be responsible for pellagra is an old one, and many attempts have been made to connect the etiology of pellagra with the source of the water supply of the individual. Numerous investigators have collected statistics of this nature, and many theories as to the manner in which water could cause the disease have been advanced.

Quite novel among these theories, however, is the contention that pellagra is due to the presence of certain chemical substances in drinking water.

Alessandrini and Scala ${ }^{317}$ propounded the idea that the etiological factor of pellagra is to be found in the silica in the colloidal state contained in certain potable waters. In support of their view they cite a large amount of experimental data, the work being conducted on guinea-pigs, dogs, rabbits and monkeys. They injected the animals with and caused them to drink artificial colloidal solutions and gelatinous suspensions of silica and natural potable waters of various pellagrous districts. From the results of their experiments they conclude that they have produced in animals a chronic intoxication which very closely simulates, even in the details, pellagra as seen in human beings. They claim to have produced typical clinical symptoms as well as typical pathologic findings.

Infectivity.- - The second great etiologic theory of pellagra, that it is an infectious disease, has, especially of recent years, received the enthusiastic support of many investigators. There are four well-known theories concerning the infectivity of 
pellagra: first, that it is a bacterial disease; second, that it is a parasitic disease transmitted by a blood-sucking insect; third, that it is a form of amebiasis; fourth, that it is a parasitic disease due to a water-borne nematode worm.

Contagion.-One of the first questions asked when the infectious theory is considered is concerning the contagiousness of pellagra. Can non-pellagrins associate with pellagrins without fear of contracting the disease? This is one of the most important questions which confronts us and one which cannot be answered with certainty. Most of the early observers considered pellagra as non-contagious, although by some it was considered to be spread by direct contact.

Marie, ${ }^{308}$ who holds so religiously to the corn theory, naturally does not believe in its infectivity.

Sambon $^{318}$ says pellagra is not contagious and tells of having an Italian pellagra girl as nurse for his children, and this intimate contact failed to convey the disease from one person to another.

Other facts, which seem to refute the idea of contagion are: first, pellagra is usually confined within certain narrow centers while there is free intercourse between the inhabitants of these centers and those of neighboring communities; second, pellagra is very rarely, if ever, found in physicians, nurses or attendants who come into almost daily contact with pellagrins; third, pellagra is never transmitted from a pellagrous wet nurse to a child, or from a pellagrous child to a healthy mother.

Krauss $^{319}$ mentions two cases which bear out the latter contention: one, a pellagrous infant of twelve months of age which had contracted the disease at the age of six months and had had no nourishment except the milk of a healthy mother; the other, a mother with a virulent pellagra, had nursed an infant for four weeks, the child remaining in perfect health.

The case mentioned above under other predisposing causes which followed pregnancy and puerperal convulsions is of interest also in this connection. Five months after conception the mother who has active symptoms of pellagra is nursing the child which is perfectly normal.

Family Tendency.-The opinion of most observers has been 
that family tendency is of little or no consequence in the etiology of pellagra, and that by far the vast majority of pellagrins are members of families of which they are the only member affected. Thus, according to Roberts, ${ }^{300}$ Alessandri found only five families with more than one pellagrin. Lavinder ${ }^{291}$ found in nearly 16,000 cases more than one case to the family 933 times and more than two cases 235 times. Grimm ${ }^{292}$ gives the following table covering this point:
(a) Families having one case
(b) Families having two cases
(c) Families having three cases
(d) Families having four cases
264
23
6
I

The findings of the Thompson-McFadden Pellagra Commission, ${ }^{295}$ however, are slightly at variance with those quoted above. Of $3^{1} 6$ cases of pellagra they found 160 or 50.6 per cent. occurred only one case in a family, 84 or 26.6 per cent. two cases in a family, 42 or 13.3 per cent. three cases in a family, 20 or 6 per cent. four cases in a family, and ro or 3.2 per cent. five cases in a family.

One case which we have seen was the only surviving member of a family of four, the father, mother and sister having died from three months to seven years previously of pellagra.

Immunity.-When the various facts concerning the contagion and family incidence of pellagra are considered, the question of immunity naturally arises.

Sambon $^{291}$ is inclined to the opinion that a certain kind of immunity is acquired by residents of pellagrous districts. This he thinks is especially apparent in children, as pellagra is more prevalent in very young children than in older ones.

Roberts $^{300}$ contends that there is probably no immunity either natural or acquired to pellagra, but that there is a variation in susceptibility.

Bacterial Disease.-The theory that pellagra is a disease caused by bacteria other than those of corn has had many enthusiastic supporters, and many attempts to isolate the infecting organism both microscopically and culturally have been made.

Bravetta $^{291}$ after exhaustive cultural studies with the blood 
and spinal fluids of pellagrins reached the conclusion that these are sterile.

MacNeal and Hamilton ${ }^{291}$ isolated approximately 100 strains of bacteria from the intestines of pellagrins and subjected them to agglutination tests with the blood serum of pellagrins. All but two of these strains reacted negatively. These two strains, however, showed a very definite agglutination with the sera of pellagrins in the acute state and less agglutination with the sera of pellagrins taken during the interval between attacks. The possible significance of these facts was much lessened, according to the authors, by the fact that agglutination occurred, sometimes as definite as with pellagrins, with the sera of insane non-pellagrous patients and normal individuals.

Early in 1915 Page $^{320}$ announced the isolation of a bacillus from the feces of pellagrins which he insists "must be the cause of the disease." According to this author the organism is from 4 to ro microns long with the ends more sharpened than those of the colon group. It is a spore bearer and is found in many forms. Just before the formation of a spore an area of cloudiness or bright refraction appears and the bacillus generally elongates. The spore grown, the membrane of the bacterium bursts and the young organism emerges through the opening. At first the young bacillus is very active and assumes a spiral or cork-screw shape. It gradually grows in length and the motility decreases. The organism is aerobic and Gram negative. Sterile feces, according to Page, is the best culture medium, and the bacilli grow well at a temperature of $80^{\circ}$ to $90^{\circ}$.

Page states that a cat fed on food inoculated with pure cultures developed mild diarrhea and nervousness, and that a man accidentally infected with the organism developed pellagra.

Page says that he has found the organism many times in sixty-four cases of pellagra and that he has been unable to find it in normal individuals and in those suffering from other diseases.

Sanders $^{321}$ describes a similar organism which he isolated 
from the spleen of a pellagrin at necropsy and grew on a special culture media containing corn meal. He considers it the same organism as that described by Page.

Intermediate Host.-The theory that pellagra is an insectborne disease had its origin with Sambon, ${ }^{322}$ who in 1905 stated before the British Medical Association that such was his belief. He later incriminated the Simulium reptans as the offending insect and backed his theory by extensive epidemiologic studies.

Sambon gives as his reason for this belief the following:

I. Pellagra is a parasitic disease because:

(a) The characteristic eruption and other symptoms of the disease may recur each spring for a number of years, notwithstanding the removal of the patient from the endemic districts and the strict elimination of maize from his diet. This peculiar periodicity of symptoms can be explained only by the agency of a parasitic organism presenting definite alternating periods of latency and activity. Analogous periodicities are met with in other parasitic diseases-as, for example, in tertian fever, in which the periods of activity of the parasite (Plasmodium vivax) recur each summer in correlation with the activity period of its anophelic definitive host. No toxic substance could account for it.

(b) It presents the peculiarities of distribution and seasonal incidence found in all parasitic diseases.

(c) Its symptoms, course, duration, and morbid lesions are analogous to those of other parasitic diseases.

2. It is an insect-borne disease because:

(a) It is not directly contagious.

(b) Neither food nor drinking water accounts for its peculiar epidemiology.

(c) It is limited to certain rural districts only, towns and villages almost invariably escaping.

(d) It presents a definite and peculiar seasonal incidenceviz., spring and autumn.

(e) It is practically restricted to only one class of peopleviz., the field laborer, owing to greater exposure to infection.

3. It is conveyed by a Simulium, because: 
(a) Simulium, so far as we know, appears to effect the same topographic conditions as pellagra.

(b) In its imago stage it seems to present the same seasonal incidence.

(c) It is found in rural districts and, as a rule, does not enter towns, villages, or houses.

(d) It explains most admirably the peculiar limitation of the disease to agricultural laborers, a limitation which nothing else can explain in a satisfactory manner.

(e) It has a wide geographic distribution which seems to cover that of pellagra, although certainly exceeding it, in the same way that the distribution area of the Anopheline exceeds that of malaria, and the range of Stegomyia calopus that of yellow fever.

(f) It is known to cause severe epizootics in Europe and America.

(g) Other similarly minute blood-sucking diptera, such as Phlebotomus papatassi and Dilophus feverilis, are strongly suspected of being propagators of human diseases.

While Sambon does not state definitely the nature of the hypothetical infecting organism transmitted by the sand-fly, he is inclined to regard it protozoal in nature.

Since the formulation of Sambon's theory many investigators have written pro and con concerning it and much valuable information has been obtained.

Babes, ${ }^{291}$ who holds to the corn theory, considers that the fact of being unable to find any relation between the Simulium and corn is sufficient reason for discarding Sambon's theory.

Roberts ${ }^{291}$ discards the theory of Sambon, that the etiologic factor of pellagra is borne by the sand-fly, and incriminates the mosquito. He bases his opinion on alleged analogies of pellagra and the mosquito. He does not accuse any one species of this insect, but states that it will probably prove to be a ruralbreeding, house-living, day-biting mosquito. Jennings and King ${ }^{295}$ after a most exhaustive study of the insects of Spartanburg, S. C., including ticks, lice, bedbugs, cockroaches, horseflies, fleas, mosquitoes, buffalo gnats (Simulium), house-flies, and stable flies (Stomoxys), reach the following conclusion: 
Horseflies have nothing and cockroaches little to support them as the intermediate host.

On account of their scarcity and the nature of their biting habits ticks and fleas may be excluded. It is even doubtful if the existence of an animal reservoir of infection would cause these insects to become prominent.

The sex and age incidence and the rural nature of the disease cannot be accounted for by lice and bedbugs. Further, lice are rather scarce. Mosquitoes are eliminated on account of their comparative rarity, the night-biting habit of the local species, which would not account for the sex incidence, and the lack of coincidence between their distribution and that of pellagra.

House-flies (Musca domestica) are considered from the point of view that pellagra may be an intestinal infection, the organisms being passed with the feces and infecting others through contaminated food.

Buffalo gnat (Simulium) could hardly have been considered had it not been for Sambon's theory. In Spartanburg County they seldom attack man, and when they do it is only locally and their attacks are largely confined to field workers. Further, they are present in this locality only in comparatively moderate abundance.

In the stable fly (Stomoxys calcitrans) is found certain prominent peculiarities which, according to Jennings and King, lead them to consider it a possible intermediate host of pellagra.

The range of the stable fly covers and exceeds that of pellagra. While the season of the greatest numbers of the stable fly is somewhat later than the season of greatest prevalence of pellagra, it however appears earlier in the spring than do most recurrences and new cases of the disease, and when these do occur the stable fly is found in great numbers. This insect is exceedingly plentiful and is found most in rural districts, thus corresponding to the rural distribution of pellagra.

On account of the great number of stable flies it seems an efficient transmitter of disease.

The stable fly is intimately associated with man whom it bites often and persistently. It is a day-biting insect, which explains the sex and age incidence. The life of the stable fly 
seems long enough for the development of a possible infecting organism.

This insect is easily and of ten transported over long distances, which may account for the development of sporadic cases of pellagra.

Amebiasis.-In I909 Siler and Nichols ${ }^{286}$ called attention to the large number of pellagrins in whom amebæ were found.

Jelks $^{328}$ reported having found amaba histolytica in the mucopurulent material taken from the rectum of a pellagrin.

Long ${ }^{329}$ proposed the hypothesis that pellagra is a disease due to the injury of the intestinal mucosa caused by amebas, and in support of this theory reported finding the ameba in the stools of fifty out of fifty-two pellagrins, and that the ulceration and damage to the intestine was shown by the presence of blood, mucus and pus in the feces.

Nematode Worm.-To Alessandrini ${ }^{329}$ is due the theory that pellagra is due to a water-borne nematode worm which he found constantly in shallow wells and slowly flowing streams of pellagrous regions. These were found only rarely or were entirely absent from the potable waters of non-pellagrous districts. Later Alessandrini was forced to abandon this theory and while he continued to incriminate potable waters, he adopted the colloidal silica theory mentioned above.

Inoculation Experiments.-As far back as I 780 Gherardini attempted the inoculation of healthy individuals with pellagrous material, such as ichorous matter from the skin lesions, the blood and saliva, but with negative results.

Since that time numerous attempts have been made to reproduce pellagra in the lower animals.

In I9Io Anderson and Goldberger ${ }^{323}$ attempted to infect the Rhesus monkey with the blood and spinal fluid of pellagrins. Similar experiments were conducted by Lavinder ${ }^{323}$ and by Singer, MacNeal and Rooks, ${ }^{324}$ all with negative results.

In the summer of I $_{913}$, however, came the announcement of Harris $^{325}$ of New Orleans that the experimental production of pellagra in the monkey had been accomplished. The tissues from different parts of the body, central nervous system, portions of skin lesions and alimentary tract, were removed at 
necropsy soon after death from a typical case of pellagra. After mixing with an equal amount of normal saline solution and ground in a mortar they were allowed to stand in the ice chest over night. They were then coarse filtered and the juice passed through a Berkefeld filter, Letter $N$. The filtrate was injected subcutaneously, intravenously and intracranially in

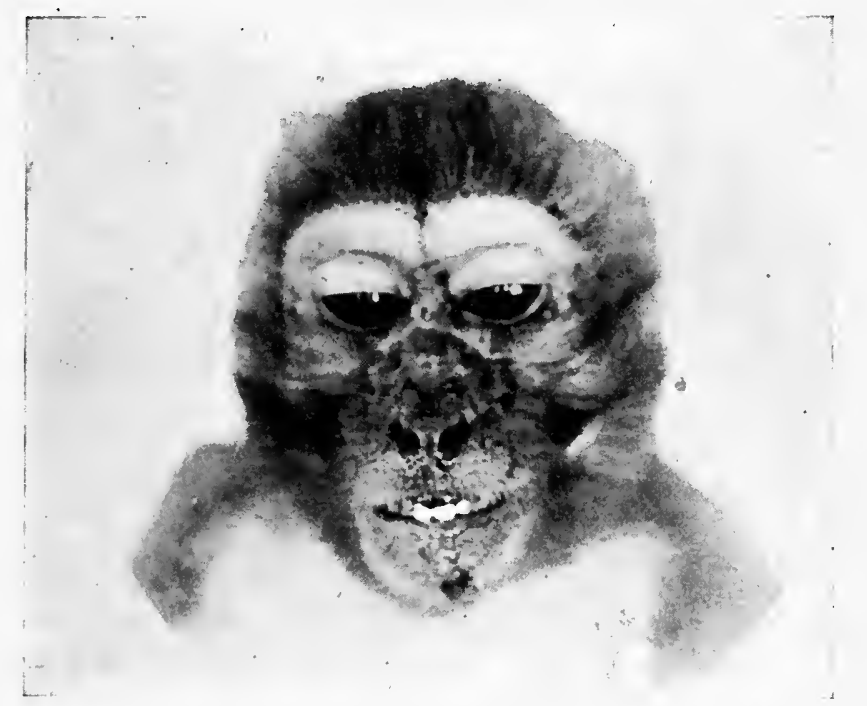

Fig. 68.-Monkey 2, showing lesions of face. (Courtesy of Dr. W. H. Harris.)

large quantities into monkeys (Macacus rhesus). The first of these animals remained apparently normal for many months when he developed irregular dark patches on the hands, forearms, face, back and sides of the body. He gradually became emaciated and weak and finally died "with all the signs of pellagra."

The second monkey after a period of about three months developed similar lesions to monkey $\mathrm{I}$, and at the time of publication was still living, though growing progressively weaker and thinner.

Stimulated by this work of Harris, Lavinder, Francis, Grimm and Lorenz ${ }^{326}$ attacked the problem anew and by Iо3 experiments with 77 rhesus monkeys, 2 Java monkeys and 3 female baboons administered pellagrous material from every conceiv- 
able source and in every conceivable manner. The pellagrous material consisted of tissue extracts, pericardial and spinal fluids from necropsies, and blood, urine, feces and spinal fluid collected during life.

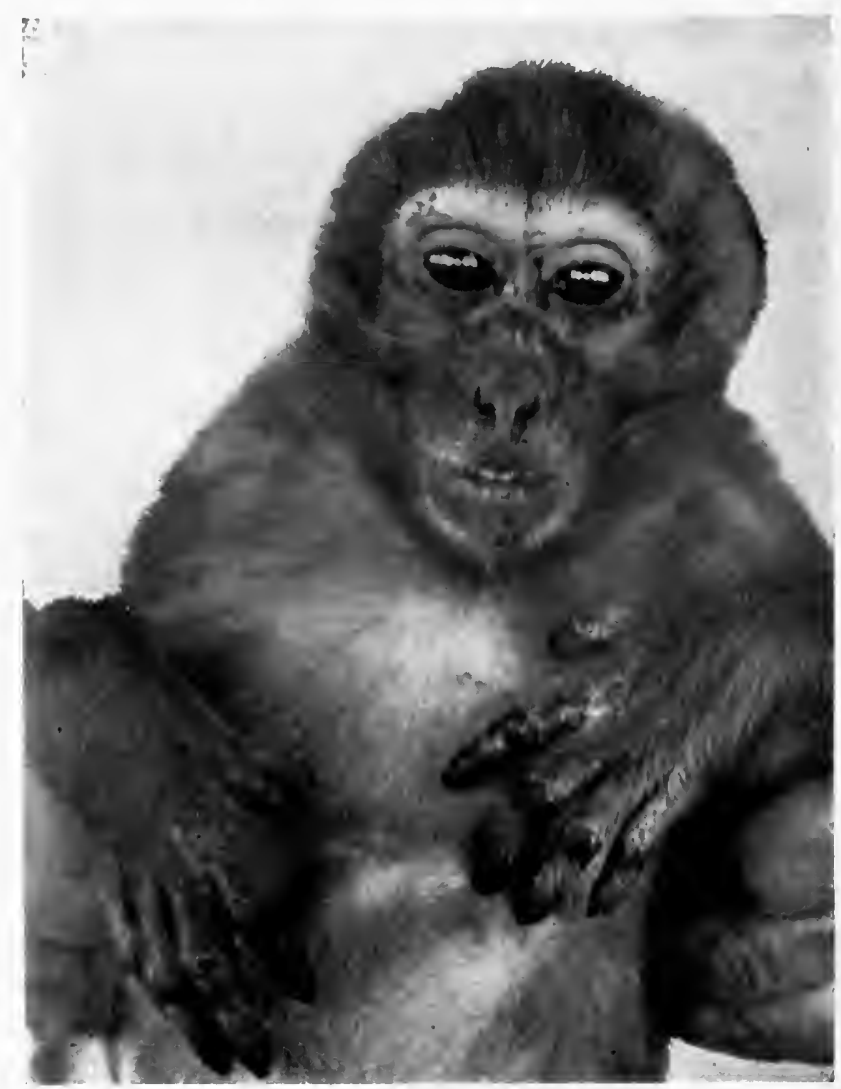

Fig. 69.-Monkey 2, showing lesions of hands and face. (Courtesy of Dr. W. H. Harris.)

The inoculations were made intravenously, intraperitoneally, intraspinally and material was fed by stomach tube.

Eight of the animals died after varying periods following inoculation. Four of them showed plainly death was not due to pellagra. The cause of death was not determined in the other four.

The remaining monkeys with one exception had up to the time 
of the publication of the report shown no indications suggesting pellagra.

The following is the history of the exception:

M. Rhesus, 98, was injected intraspinally with 6 c.c. of spinal fluid, April 14, r914, and again inoculated, May 2, intraspinally with 4 c.c. of spinal fluid drawn from another pellagrin. The first change in this monkey was noted May 4, I9I4, at which time the right forearm appeared slightly swollen and looked as though some of the hair was falling out. The following day the left forearm showed a similar condition. Later both forearms became entirely denuded of hair, the skin became roughened and scaly with red cracks in which appeared a slight serous exudate. Over both wrists the superficial skin seemed to be denuded and the condition gave the appearance of superficial ulceration. On the posterior surface of each hand a similar condition was noticed; the knuckles of the fingers were swollen and reddish and presented cracks and broken skin. May 9 the skin was dry and more scaly. The bowel movements were occasionally loose. Later thick crusts on the skin came away, leaving a pale and slightly scaly surface. The monkey is now again in his usual condition.

These investigators admit that the interpretation of the above manifestations is not clear and state that they may be accidental or that they may indicate pellagra. If they do indicate pellagra they were brought about either by a living organism or by an unorganized toxic element.

Pathogenesis.- The subject of the pathogenesis of pellagra as with every other disease is, of course, intimately associated with the etiologic factor, and until this is determined any statements concerning this feature of the disease must perforce be little more than conjectures.

The sun has long been accused of exerting some influence over pellagra and has even been considered the sole etiological factor. Sandwith ${ }^{327}$ says the sun is certainly responsible for some of the skin eruption.

As stated above those who hold to the photodynamic theory consider that the sun's rays acting on the exposed surfaces of the body sensitize a hypothetical toxin of ingested corn. 
It has been suggested that the actinic rays of the sun are responsible for the erythema and others consider the direct heat of the sun the cause. It is a fact that those pellagrins who are more exposed to the sun's rays usually develop the skin eruption before the onset of the other manifestations and those who are well protected from the sun usually develop the gastro-intestinal and other symptoms before the erythema. Nevertheless it is not the sun which causes the erythema but the pellagra. Allessandrini and Scala ${ }^{317}$ who proposed the theory of silica in drinking water contend that there is more or less affinity for mineral salts exhibited by colloidal silica and they conclude from their experiments on dogs that "without doubt silica in the animal organism acts by accumulating mineral substances and produces in consequence a destruction of tissues." They trace the chemical changes and relations which the silica undergoes and further conclude. "Therefore it seems to us without doubt that the silica fixes the mineral salts on the proteins of the tissues with a continuous incessant action quite similar to the action of an enzyme or diastase."

They finally arrive at the following conclusion, that "pellagra is a malady caused by the forced retention of mineral salts which in turn produces a liberation of acids in excess of the needs of the particular organism; or in other words, pellagra is nothing more than a mineral acidosis with all of its consequences."

Gosio and Anatonini ${ }^{291}$ contend that pellagra is a form of anaphylaxis, that the organism becomes sensitized by the toxins produced by the parasitic moulds of Indian corn, and that repeated ingestion of these toxins brings on the phenomena.

Long $^{328}$ who proposed the ameba-intoxication theory of the disease assumed that the skin lesions are caused by pressure on the nerves at their exit from the spinal canal due to a deposit in the foramina, and to degeneration of the nerves themselves as a result of toxemia.

Jelks $^{330}$ thinks that pellagra is not a morbid entity but a series of symptoms-complex, due to the absorption of certain toxins or toxin-developing bacteria carried into the blood stream or to the central nervous system by ameba which he claims are almost constantly found in these cases. 


\section{CHAPTER XIX}

\section{PATHOLOGY OF PELLAGRA}

The pathology of pellagra presents no characteristic features which are absolutely constant, but certain morbid changes usually take place which serve as distinguishing factors. For example, there is usually anemia and more or less emaciation, but we have seen pellagra in well-nourished individuals. Again the eruption is a very constant manifestation but "pellagra sine pellagra" is not unknown.

The pathologic findings described are usually those of the skin, gastro-intestinal tract and the central nervous system, although other organs and tissues are frequently involved.

Skin.-The skin lesions are more or less characteristic. Among foreign writers, and to some extent among those of America also, it is customary to divide the skin manifestations into three stages, viz.: first, the stage of congestion or erythema; second, the stage of thickening, pigmentation and added scaliness; and third, the stage of atrophy. To us this seems an artificial classification and that the skin manifestations of pellagra do not present stages, so much as degrees of the lesions.

The lesions of the skin in pellagra usually start as mild erythema scarcely distinguishable from the erythema of sunburn. They may, however, as Merk has shown, start as discreet maculæ which last for a few days to a few weeks. The pellagra erythema is of a vivid red color and perhaps slightly darker in shade than erythema of sunburn. After a variable period of time a hyperkeratosis may take place and the lesions are covered with scales and shed-off epithelium. As a result of this process the tissues are swollen due to the increase of blood and serum to the derma, causing the skin to have a wrinkled appearance. In some instances there is a pigmentation following the desquamation while in others the skin is of lighter hue than before. 
Frequently, instead of a hyperkeratosis, there may be superimposed upon the erythema vesicular or bullous lesions. These may be quite large in size and are always monolocular. In a few days they gradually dry, leaving a thickened crusted condition of the skin. This class of lesions is not infrequently the seat of secondary pyogenic infection. Often the edema is sufficient to produce marked fissures.

It not infrequently occurs that the bulla is broken by mechanical action and the surface of the lesion appears raw and bleeding. After this class of lesion has cleared, the skin is somewhat thinner than formerly and there is no pigmentation.

The microscopic picture of the skin in pellagra is one very similar to erythema multiforme. It is evidently due to disturbances of the nervous system and the peripheral circulatory apparatus; in other words, it is an angioneurosis.

The greatest pathologic change is seen in the superficial part of the corium, the most marked infiltration being found in the stratum papillare.

With low power magnification the stratum corneum is seen to be more or less thickened while the stratum granulosum and the rete Malpighii are usually not involved. The upper portion of the corium usually shows a considerable inflammatory process while the subcutaneous tissue is more or less edematous. With the higher magnifications the hypertrophy of the corneum is seen to be quite marked. Usually more or less parakeratosis is demonstrable by the finding of nucleated cells in the upper layer of the corneum, and pigment granules are seen scattered through it. The only pathologic finding in the rete Malpighii is a slight infiltration of cells. The most marked cellular infiltration is seen in the pars papullaris, especially around the blood-vessels. Collagen, showing edematous change and elastin are seen. The other layers of the corium show little or no pathologic change.

Gastro-intestinal Tract.-Here again, as with the skin lesions, the pathologic findings, while usually quite marked, have no absolutely characteristic features.

The tongue is usually swollen and more or less denuded and nearly always presents a bright red appearance. Along the 
edges and on the undersurface ulcerated areas may be seen and sometimes yellowish sloughs which bleed easily are found. The lips and cheeks also, in the worst cases show similar ulceration and sloughing.

The stomach often shows a chronic gastritis. The mucosa is frequently found pale, while there is more or less atrophy of the muscular coat.

The intestines usually show a similar condition. There may be either anemia or hyperemia. Ulcers may be found along the entire tract but are more frequently seen in the ileum and jejunum. There is usually a thickening of Peyer's patches and the mesenteric lymph glands are often markedly enlarged.

The histopathology of the gastro-intestinal tract in pellagra presents nothing absolutely characteristic. Sections of the tongue show a similar picture to sections of the skin. There is a desquamation of the epithelium and some infiltration of the tunica propria.

Sections of the stomach wall show more or less superficial necrosis with destruction and exfoliation of the glandular epithelium. The gland tubules may show cystic dilatation with infiltration of connective tissue between them. The bloodvessels of the submucosa are usually dilated, while the muscular coats are more or less atrophied. As a rule the condition is less marked in the cardiac end than in the pyloric end.

The microscopic picture of the intestines shows chronic inflammation with atrophy and disappearance of some of the columnar cells. There is sometimes entire destruction of the epithelium, only a narrow line of necrotic tissue marking its location.

Central Nervous System.- The most exhaustive study of the pathologic anatomy of the central nervous system which has been made in this country is that of Singer and Pollock. ${ }^{331}$

These investigators found that the dura mater is usually thickened, streaked with increased formation of connective tissue and sometimes adherent to the pia mater. The piaarachnoid is found to be cloudy, opaque, edematous and thickened. This is most marked over the convexity of the brain, and at times the membranes are separated from the 
brain by the collection of fluid. Occasionally instead of being separated from the brain by fluid the pia-arachnoid is adherent to the cortex. In the meninges of the spinal cord are seen similar pathologic findings to those found in the cerebrum. Small bony plaques are sometimes noted. No abnormalities are found in the basal ganglia, cerebellum, pons and medulla as a rule.

I. Pia Mater.-Microscopically the pia always shows rather marked change consisting of a thickening of the connective tissue with a proliferation of the fibroblasts. Occasionally the glia of the cortex invades the pia, especially in the neighborhood of the sulci. The most marked changes are found in the blood-vessels. An exudate, consisting of cells which are swollen and undergoing fatty degeneration, and fibrinoid pigment granules and lymphocytes, is often seen. This exudate is scanty compared with that found in cerebral syphilis, paresis, etc. These changes in the pia are most pronounced over the convexity of the brain and very rarely are severe. Similar findings are observed in the pia mater of the spinal cord.

2. Blood-vessels. - Singer and Pollock describe the pathologic changes which are found in the blood-vessels under two headings, acute and chronic, and state that the former may be related to pellagra, while the latter certainly are not.

The larger arterioles show the most evidence of chronic change. All the coats of the vessel are thickened while there is some proliferation of the cells, which are swollen and frequently are found to contain pigment granules. Some endothelial cells of the intima also are swollen and often contain granules due to degeneration. The vessels are usually surrounded by numerous fatty and fibrinoid granules, and sometimes basophilic granules are seen. Granule cells (Abraümzellen) containing fatty and fibrinoid material are very often noted. In the smallest vessels also is seen to some extent the chronic thickening and occasionally splitting of the intima. Neither hyaline change of the vessel wall nor occlusion of the lumen is noted.

The smallest vessels present the more acute changes. The intima is thickened by proliferation of the endothelial cells 
which are found either swollen or shrunken. Fatty degeneration is frequently seen and often the intima is split, although few, if any, cells are found in the spaces thus formed. Proliferation, with swelling and distortion, of the muscle cells is seen. These are elongated, poor in chromatin and contain pigment of various kinds. Thickening of the adventitia with proliferating and degenerating cells is noted. Singer and Pollock state that in some cases these changes are very slight.

These authors tell of a whole set of specimens from one case in which were found in the adventitial cells a number of metachromatic basophile bodies presenting various shapes; "rosettes curved and elongated masses with small projecting buds or lobules, etc." They further state that these bodies resemble very closely the bodies described by Borrel in carcinoma, and, slightly, Councilman's variola bodies. They stained a purplish red with thionin, similar in color to the nucleus of the specimens but as these authors were unable to demonstrate them again with an apparently identical technic they conclude that if they were not degenerative products, they were cell nuclei.

Singer and Pollock state that in all cases there is more or less perivascular infiltration. This consists of vessel cells, pigmented and degenerating lymphocytes, fatty and fibrinoid pigment granules, glia cells and granule cells. No basophile cells are noted, and no example of etat crible or blood cyst was seen. These authors state that there is nothing in the picture to indicate a local invasion of the nerve tissue with microörganisms.

3. Neuroglia.-(a) Fibers. Increase of the glia fibers is noted in the outermost layer of the cortex, especially prominent in and about the sulci and around the blood-vessels. Numerous amyloid bodies are observed among these fibers. The glia fibers sometimes are found in the pia mater and cause it to adhere to the cortex. This proliferation of the glia fibers is also noted in the spinal cord, especially marked around the periphery and the central canal. Singer and Pollock state that while this increase of the glia fibers is sometimes quite extensive it is never found as great as usually found in senile dementia. 
(b) Cells. In regard to the cells it is stated that while several types of glia nuclei and cell bodies with thionin staining are found no definite conclusions can be drawn from them, since the normal limits of these cells are not well known. A large number of pyknotic distorted cells with long fibers, "star-shaped lanceolate, biscuit-shaped, and semilunar" nuclei are seen in the superficial layers of the cortex. The protoplasm of the cells takes stains poorly, while many bluish-red or pink-staining granules are observed. The deeper layers of the cortex present glia cells containing a small dark nucleus rich in chromatin, and a small amount of poorly staining protoplasm. Here also are found cells with a pale nucleus often swollen and distorted and filling almost the entire cell, containing a few fine chromatin granules and sometimes one or two pink-staining nucleolus-like bodies. Occasionally a cell with a similar nucleus but with deeply staining protoplasm is seen. Two types of cells are seen in the white matter, one containing a small dark nucleus, while the other contains a large pale one. In the bodies of the glia cells of the cortex is noted marked fatty degeneration and pigment material staining blue, green and reddish with thionin.

Satellitosis is said by Singer and Pollock to be constantly present in a varying degree depending on the amount of chronic change in the ganglion cells. The satellites are sometimes found directly around a ganglion cell, even forming a fence around it leaving a space between them and the cell. Satellitosis is most marked about the cells undergoing degeneration and also the shadow cells. It is not present about the cells showing axonal reaction.

The astrocytes are said always to be increased in number, but especially so in the deeper layers of the cortex and the white matter. This condition, however, is not so marked as in paresis or senile dementia.

When stained by Alzheimer's Methods IV and V the cell processes are usually thickened and show darkly staining streaks of wavy outline, although they may be small and thin and are frequently broken. The cell bodies vary in size. The large ones are more homogeneous. The protoplasm often contains 
granules corresponding to the Alzheimer methyl-blue granules which probably is a degenerative condition. Variously shaped nuclei containing granules are seen.

Some of the astrocytes are observed near but parallel to the blood-vessels; others encircle them, but seem to bear no relation to them.

In some cases cystic degeneration, not fatty, is seen. The significance of this condition is in doubt, but, according to Singer and Pollock, it suggests a degenerative condition. Rarely giant astrocytes are found. These investigators state that while the increase in the astrocytes is less than found in senile dementia they take issue with Kozowski in his assertion that this increase is the exception rather than the rule. They further state that the significance of the increase in astrocytes is undetermined. According to Alzheimer, Wever and Schroeder it would seem to indicate the presence of an active process, while Orlow and others consider them more numerous in chronic conditions.

Alzheimer considers of great importance the finding of the ameboid transformation of astrocytes and small glia cells, which are found by him mainly in recent and active diseases of the brain, such as infection deliria, alcoholic delirium, dementia præcox in the acute stages, paresis, cerebral syphilis, etc., and never in old standing softening, old hemorrhages, old dementia præcox, etc.

Singer and Pollock state that these ameboid glia cells are found in pellagra, often clinging to a vessel by means of the insertion process, but they draw no conclusions from such findings.

4. Nerve Cells.-The most marked changes in the cells of the cortex are found in the large pyramidal cells, which consist of a decrease in the number of cells, changes in their relative positions, and an increase in the glia elements.

The changes in the ganglion cells are most marked and are considered by Singer and Pollock of great importance. These changes they divide into two classes: (I) the indirect or axonal types of reaction, and (2) the direct types.

I. In the indirect or axonal reactions two grades of intensity 
are seen, the first or typical reaction in which the cell is swollen and rounded, the nucleus displaced to the periphery, distorted, staining more or less uniformly with a pale color and containing a well-preserved nucleolus rich in chromatin; and the second or central chromatolysis, in which the cells are not swollen, the nucleus is well reserved with a darkly staining nucleolus, and showing a marked disappearance of the Nissl granules from the central portion of the cell.

These changes are most marked in recent cases, that is, in those dying during or soon after an acute attack.

Singer and Pollock state that the cells showing axonal reaction are, except in very severe degrees, capable of recovery and that the findings are probably due to the condition which causes the acute pellagrous attack.

The widespread axonal chromatolysis involving especially the Betz cells and those of the column of Clark has been observed by all workers in pellagra with modern methods of staining. Singer and Pollock state, however, that this picture is identical to the one described by Meyer as central neuritis, and to one seen by them in a case of alcoholism in which there was nothing to suggest a history of pellagra. They therefore conclude that pellagra is not recognizable post-mortem, except by finding typical skin lesions, although this is contrary to the opinion of Kozowski. Singer and Pollock further state that these changes can be interpreted in only one way, namely, that they are reactions to "some harmful agent circulating in the blood, acting on the axis-cylinder processes of the neurons at some point in their course." Further, it seems clear to these investigators that various ultimate causes may account for the reaction while it is "conceivable that the actual excitant of the reaction is the same in all and a product of body metabolism under morbid conditions."

2. Direct cell reaction is due to direct injury to the cell and according to Singer and Pollock six types are observed as follows:

Type 1.-Cell body shrunken and distorted with crenated edges but in some cases normal in size, more or lessuniformly staining, pale in color. Nucleus rich in chromatin. 
Type 2.- Similar cell bodies with pale nucleus which may be of the same color or lighter than that of the cell body which may be reticulated. Within the nucleus or nucleolus are granules staining a bluish green with thionin. In some of these the nucleus is lost, with or without the nucleolus.

Type 3.-Shrunken, uniformly dark-staining cell body and dark nucleus sometimes filling almost the whole cell.

Type 4.-Simple chromatolysis. Body normal or a little larger. Nissl granules are scattered diffusely throughout, rarefied and pale or present only as a fine dust. Nucleus approximately normal in staining and position. Nucleolus dark.

Type 5.-Shadow cells, a more extreme degree of the same type as the last. Nucleus absent. The outline of the cell indistinct.

Type 6.-Vacuolated cells. Nucleus present. No NissI bodies. The cell body contains vacuoles.

The first four of these types of reaction are found constantly in all regions of the cortex, while Types 5 and 6 are found in the majority of cases. These changes, especially Type 3, are found in the cells of the gray matter of the cord, principally in the posterior horns.

That these reactions are due to intoxication of the cells is shown by the satellitosis which is here present.

The following changes in the neurofibrils were found by Singer and Pollock:

(a) Agglutination.

(b) Fragmentation.

(c) Loss of febrils (in small cells only with one exception).

(d) A peculiar encircling of the nucleus and pigment masses with agglutinated fibrils.

Pigment is found in all types of cell change as well as in normal staining cells in all cases, and is especially marked in the Betz, large pyramidal and other axonal reacting cells. The pigment is of various kinds, black staining with osmic acid, red with scharlach, and blue with nilblue sulphate.

5. Nerve Fibers. - Scattered degeneration of radial fibers of the cortex is shown by the Marchi method of staining. This is not seen in the superadiary or tangential fibers. 
Degenerated fibers are seen in all regions of the spinal cord, the posterior columns being most involved. In the crossed pyramidal tracts the reaction is not more marked than in the other portions of the cord, in fact is sometimes practically absent here.

Some degenerated fibers are seen in the anterior and posterior roots, and occasionally the number is quite large.

6. Amyloid Bodies and Pigment Granules.-All cases of pellagra show an excess of amyloid bodies, beneath the epindyma, along the blood-vessels, in the thickened glia of the most superficial layers of the cortex, the periphery of the spinal cord and around the central canal. The origin of these bodies is not certain, but they are found in chronic degenerative conditions such as the senium.

The occurrence of pigment granules of fatty and fibrinoid nature in the tissues and perivascular lymph spaces is seen in all cases.

By their most exhaustive study of the pathology of the central nervous system Singer and Pollock reach the following conclusions:

I. The acute pellagra attack is accompanied by evidence of both acute and more chronic intoxication.

2. In common with other intoxicative conditions, the acute pellagra attack gives rise to a "central neuritis" reaction.

3. None of the changes are characteristic of. this particular form of intoxication.

4. There is no evidence of a local infection of the nervous system with microörganisms.

5. From our cases it is impossible to determine whether the more chronic changes found in the absence of a recent pellagrous exacerbation belong to the pellagra picture.

6. There is no evidence to show that chronic vascular changes are essential to the picture of pellagra.

Lungs.-No definite lesions of the lungs due to pellagra per $s e$ are found. This disease may be found complicated with many pathologic conditions of the lungs, such as pleurisy, pneumonia, edema, hyperemia and emphysema. It has been stated that pellagra is rarely complicated by phthisis. 
Heart.-While this organ frequently shows changes from the normal such as hypertrophy, atrophy, hydropericardium and myocardial softening, none of these lesions can be ascribed to the pellagra itself. Microscopically brown atrophy and fatty degeneration are often seen.

Spleen.-The spleen of most pellagrins is atrophied, although it may be found enlarged in some instances.

Pancreas.-The pancreas is usually normal, but may be atrophied.

Kidneys.-These organs may appear normal, but usually show some pathology, being nearly always diminished in size. Chronic interstitial nephritis is often seen, while fatty degeneration of the epithelium of the tubules is not uncommon.

Adrenals. - The adrenals are said always to be normal.

Liver.-Atrophy of the liver is very frequent, it of ten being decreased in size to half the normal. Hypertrophy may occur, while fatty infiltration is sometimes seen.

Bones.-The most marked changes in the bones are seen in the ribs which are very frequently found to be fragile. Other bones are at times also fragile.

The bones of the calvarium may be thickened although sometimes they are thinner than normal.

Muscles.-The muscular system is usually atrophied although it may be normal and even well developed. Fatty degeneration has been noted. 


\section{CHAPTER XX}

\section{CLINICAL HISTORY OF PELLAGRA}

As may readily be inferred a disease showing so varied and marked pathology as pellagra will have a symptomatology as varied and marked. This fact led to Lomborso's famous epigram, "there is no disease; only the diseased." Nevertheless pellagra is a definite morbid entity and may be differentiated from other diseases.

The clinical history of pellagra may be divided into those symptoms referable to the skin, those of the gastro-intestinal tract and those referable to the central nervous system. To these may be added certain general signs and symptoms. Most pellagrographers divide the clinical history of pellagra into stages, as follows: The prodromal stage, during which there is little deviation from the normal but a general malaise and vague undescribable symptoms; the first stage, during which the gastro-intestinal and skin symptoms are manifest; the second stage, which is marked by the nervous and mental manifestations; and the third or terminal stage, in which cachexia is seen.

While this artificial and arbitrary classification of symptoms is in some respects convenient for ease of description, pellagra does not by any means always follow such a regular course, nor can the stages definitely be separated by a sharp line of demarcation. Further, no definite period of time can be named as covering the course of the disease.

Pellagra is essentially a chronic disease and as stated above is marked by more or less seasonal periodicity, exacerbations alternating with periods of remission. The average pellagrin will give a history of having "felt bad" for a varying time previous to the active outbreak of the disease. The first actual symptoms noted are usually those of the gastro-intestinal tract, red and coated tongue, dyspepsia with flatulence and distention, occasionally abdominal pain, and diarrhea. The 
latter symptom may be replaced by constipation. Vertigo and headache are frequent and the deep tendon reflexes may be exaggerated.

The skin manifestations usually occur early and as a rule select the exposed surfaces of the body. They may be the first symptoms noted.

Sometimes at a very early date there are psychic disturbances, usually of a mild character, although as a rule they occur later in the course of the disease. The first outbreak may be mild in

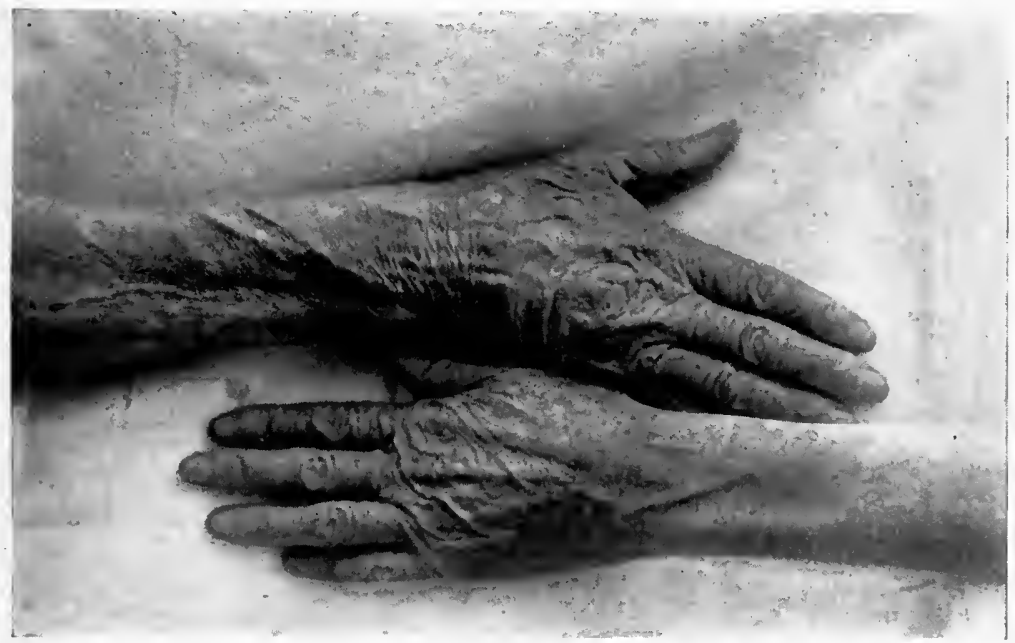

Fig. 70.-Showing wrinkled condition of skin following eruption.

nature and pass almost unnoticed, to recur at a variable time, usually the following spring in an exaggerated form or the first outbreak may be severe in type.

The skin eruption will be more marked and the gastro-intestinal symptoms more severe, while the nervous and mental manifestations will take on a more serious aspect.

The disturbances of motility are usually marked, such as muscular weakness, and even paralysis.

The psychic manifestations are most variable, ranging from the deepest depression to great excitability.

There may again be periods of remission followed by exacerbations, each one usually becoming more severe until finally 
there is a most distressing picture of cachexia, dementia and paralysis and the chapter is closed with death.

Incubation Period.-It seems paradoxical to attempt to assign an incubation period to a disease about the etiology of which so little is known, and it is a fact that the majority of writers who have so assigned an incubation period have done so on the assumption that the cause of the disease is known.

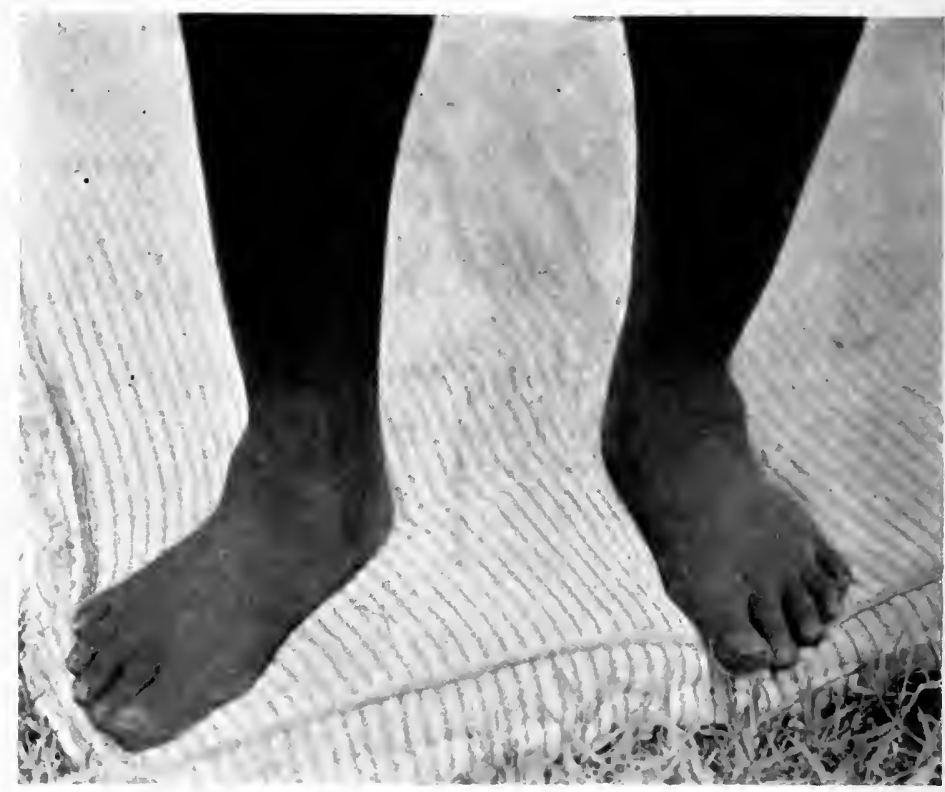

Fig. 71.-Slight dermatitis of feet and ankles of negress.

Thus Sandwith, ${ }^{327}$ who so religiously holds to the spoiledcorn theory of the etiology of pellagra, considers the incubation period to be from nine to twelve months in length. $\mathrm{He}$ bases his opinion on the fact that corn is harvested in Egypt in November and December, while in the majority of patients the disease begins in January. From these facts he assumes that the corn could not have become sufficiently spoiled in this time to cause the disease; and he therefore incriminates the crop of the previous season.

Merk, ${ }^{322}$ who also believes in the corn theory, states that the incubation period is from seven to nine months. 
Sambon, ${ }^{322}$ the author of the Simulium theory, is of the opinion that the incubation period is short, and of course bases his opinion upon the assumption that the bite of the insect is necessary to the development of the disease. He states that he has seen pellagra in infants of five months of age who have not been taken to the fields until two or three weeks previous to the appearance of the eruption; he therefore fixes the period of incubation at about two weeks.

While the above-mentioned pellagragraphers are undoubtedly

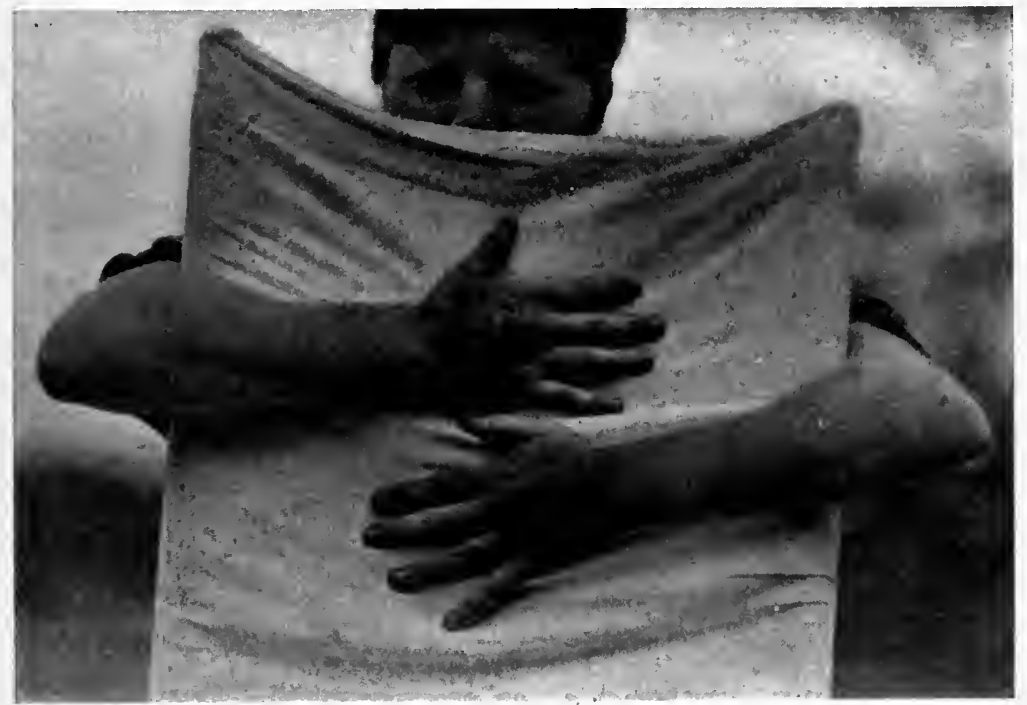

Fig. 72.-Typic eruption on backs of hands of girl of sixteen.

convinced of the correctness of their views, it seems to us that all discussion as to the incubation period of pellagra is irrelevant until the etiologic factor of the disease has been scientifically demonstrated.

Skin Symptoms.-While as stated above the symptoms of the gastro-intestinal tract are usually the first to be observed, it of ten occurs that the skin eruption develops first. The most frequent time of the first occurrence of the skin lesions is in the spring, but they may develop first in the fall. Recurrences in the fall following a spring eruption may occur.

These manifestations are usually spoken of as an erythema, 
328 ENDEMIC DISEASES OF THE SOUTHERN STATES

but, as Fox $^{322}$ has pointed out, the erythema with which the lesions usually begin is of minor importance owing to the

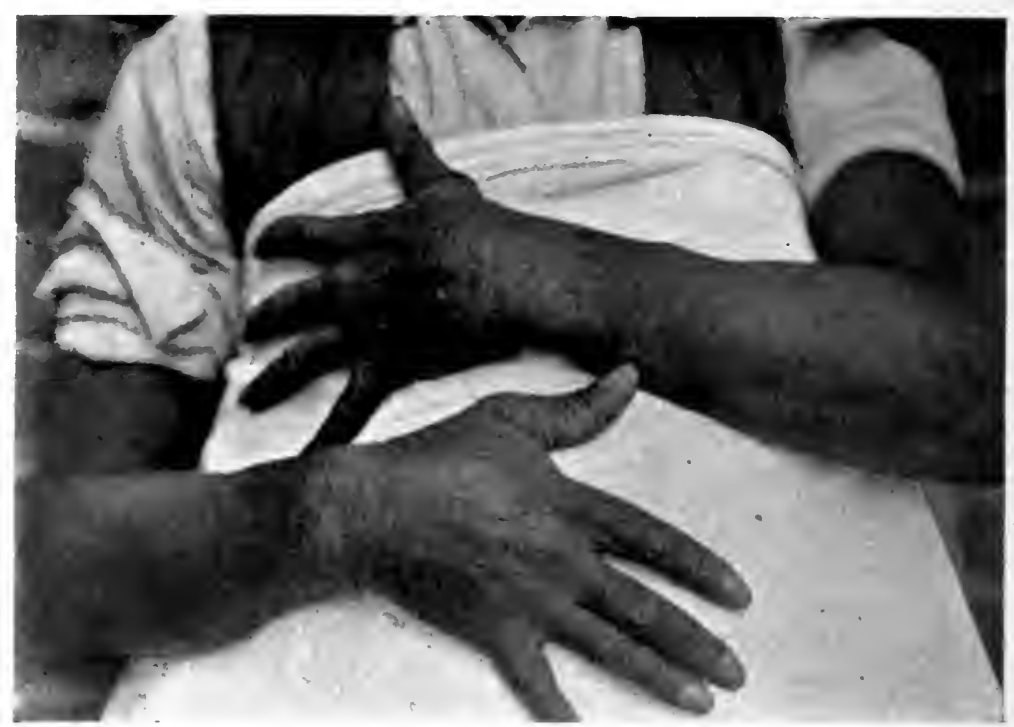

Fig. 73.-Dry dermatitis on backs of hands of negro.

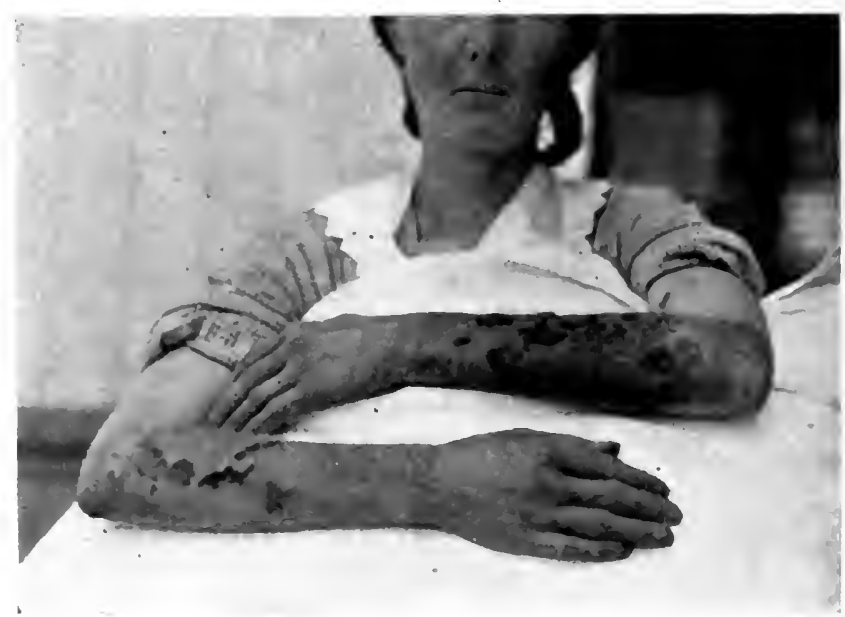

Fig. 74.-Extensive and severe eruption on arms.

comparatively short time it lasts, and dermatitis would be a more appropriate term. 


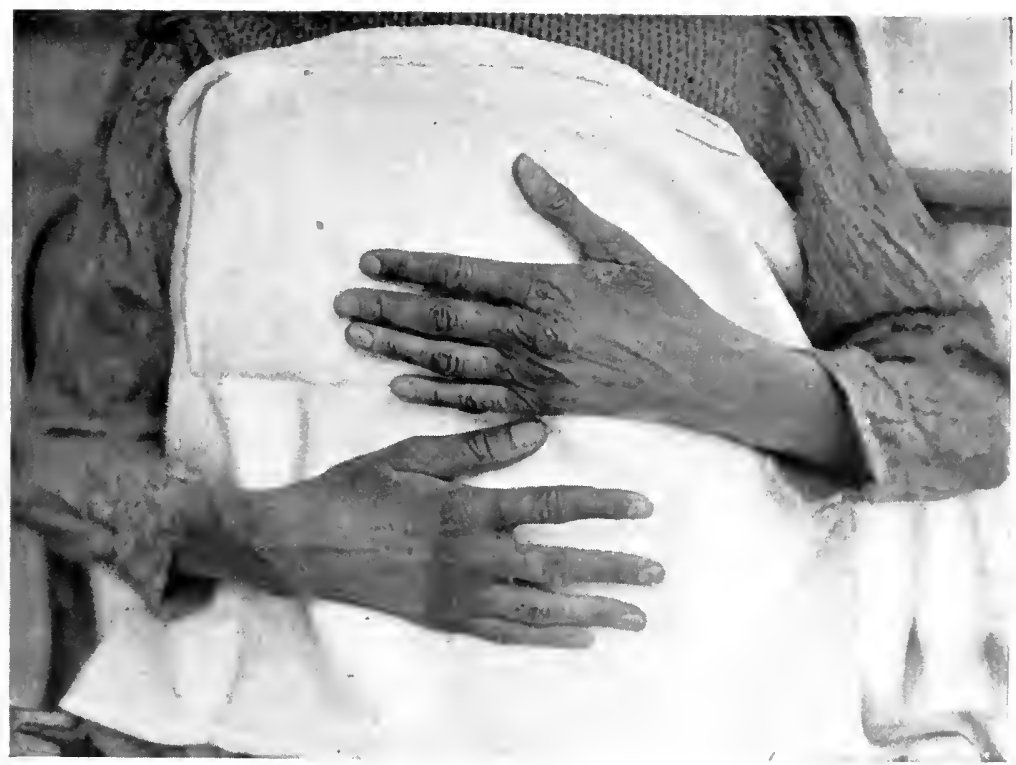

Fig. 75--Rather unusual eruption affecting but small portion of backs of hands.

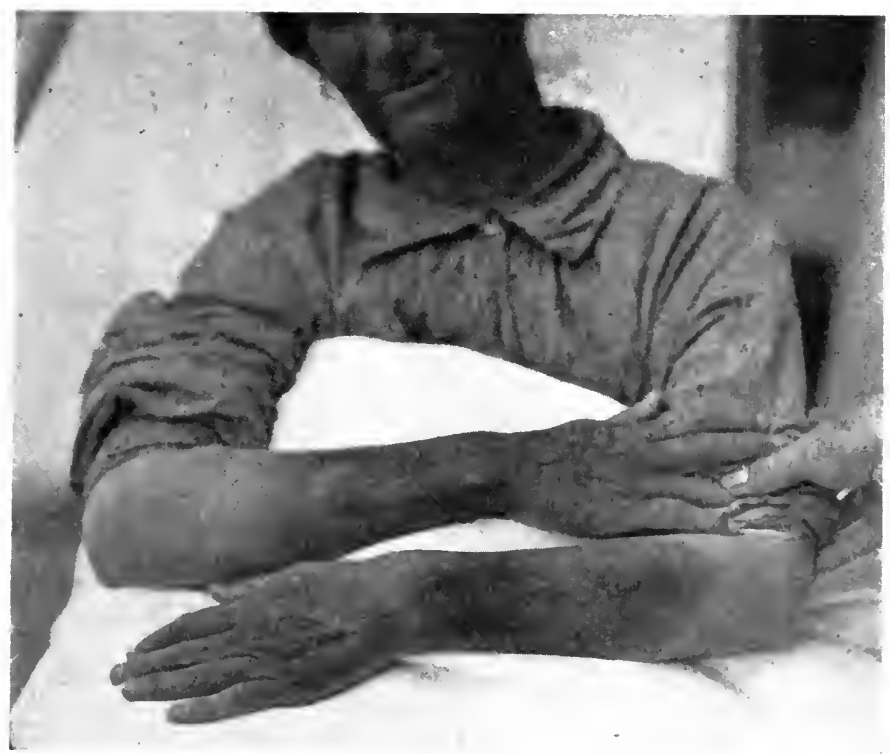

Fig. 76.-Typic eruption on backs of hands and arms. 
The most frequent location of the skin lesions of pellagra is the backs of the hands. The palms are rarely affected and the last two phalanges of the fingers sometimes are not involved, while the nails are never attacked. The eruption may extend to the wrist and for some distance up the extensor surface of the forearm. Occasionally the lesion may completely encircle

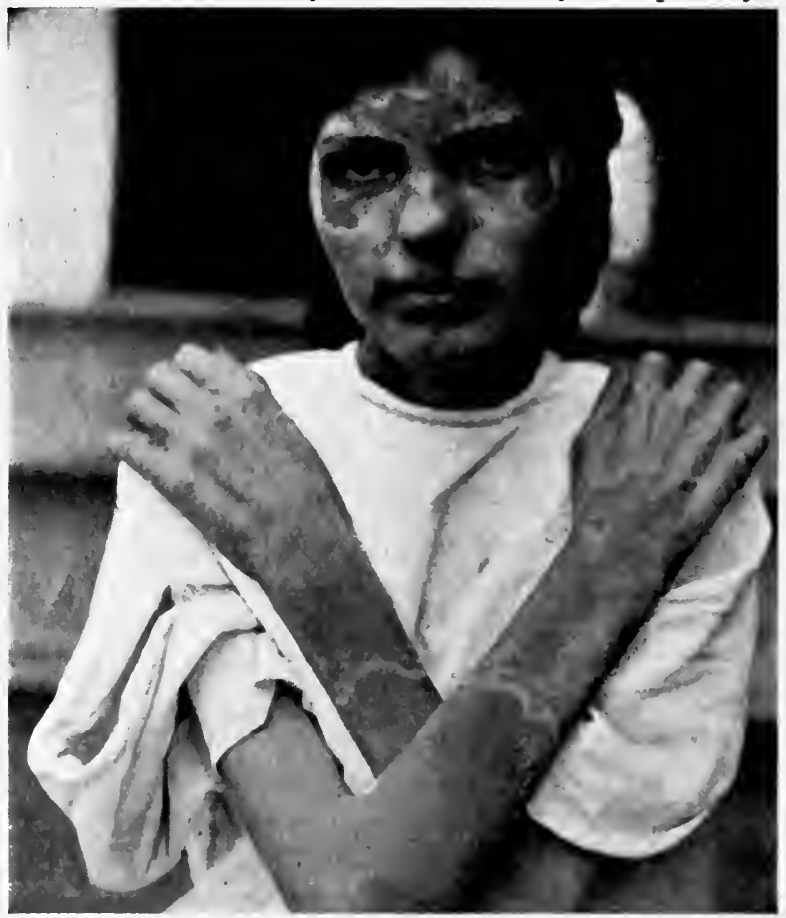

Fig. 77.-Fxtensive eruption of face and backs of hands. Note white band parallel to border of dermatitis.

the forearm. The line of demarcation between the normal and diseased portion of the skin is usually quite pronounced and the so-called "pellagrous glove" is not infrequently seen.

A condition which we have observed in one case and have not seen described elsewhere, is a distinct narrow white line in the dermatitis about a half inch from the line of demarcation between the diseased and sound skin. This condition is shown in Fig. 77.

Probably the most characteristic feature of the skin mani- 
festations of pellagra is the symmetry. This condition is most constant and may be considered almost pathognomonic, although the lesions on both sides of the body need not develop simultaneously.

The lesions seem to prefer the exposed surfaces of the body

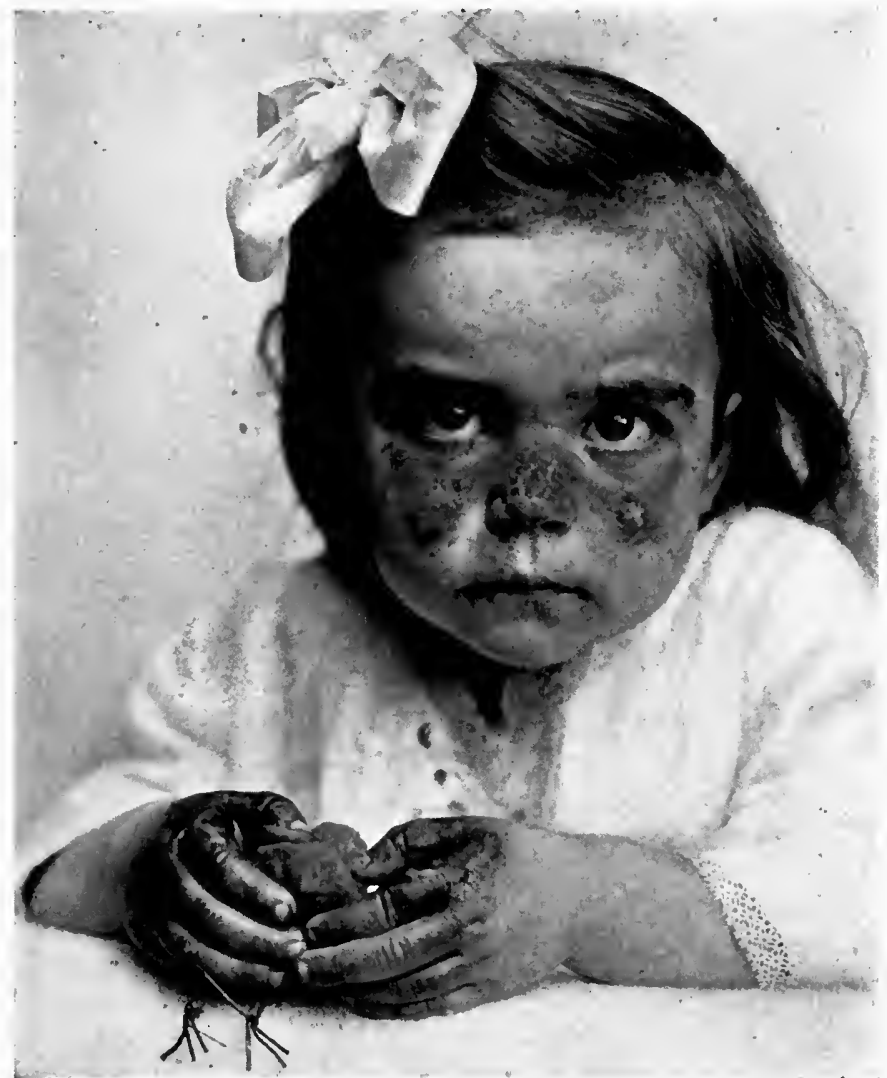

Fig. 78.- "Butterfly" eruption on face of child two years old.

and are found very frequently on the face, back of the neck, chest and dorsal surfaces of the feet. The attacking of the exposed surfaces is by no means constant, as the lesions are frequently found on the covered portions of the body.

The upper arms, elbows, knees and thighs are often the seat of the dermatitis. Sometimes the external genitalia, the vulva 
in women and the scrotum in men, are involved and the eruption has been noted on the buttocks extending from one cheek to the other, while complete involvement of the integument of the body is occasionally observed.

It is rare to see lesions on other parts of the body when the hands are not involved.

The lesions on the backs of the hands may cover the entire surface or they may occur in spots 4 or $5 \mathrm{~cm}$. in diameter with

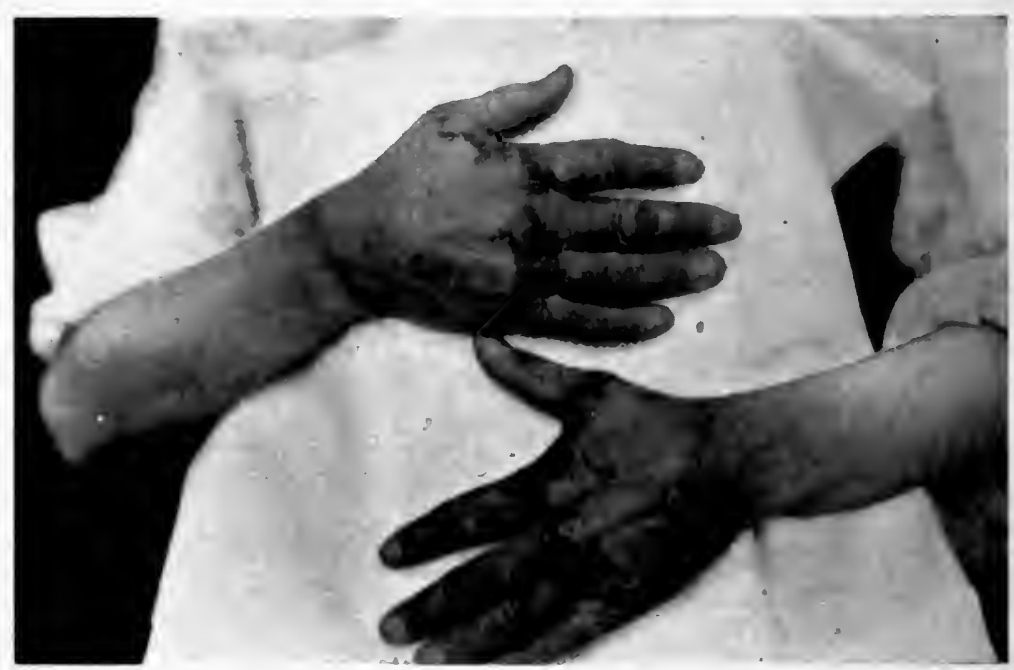

Fig. 79.-Desquamation of skin of backs of hands.

areas of normal skin between. The spots may become confluent or they may remain separate.

On the face the lesions may also occur in spots, the most frequent location being the alæ of the nose, after which in the order of frequency the lesions occur on the forehead, cheeks and chin. The lips usually escape as do the ears and eyelids. The face may be the seat of a diffuse eruption known as the pellagrous mask.

The lesions of the neck are very variable in character. The back of the neck alone may be involved or there may be a complete circle of the eruption corresponding to the exposed portion, such as in women accustomed to low-necked clothing or in men who leave the shirt unfastened at the neck. The 
so-called "Casal collar" is described by Merk as beginning on the back of the neck a little below the margin of the hair and

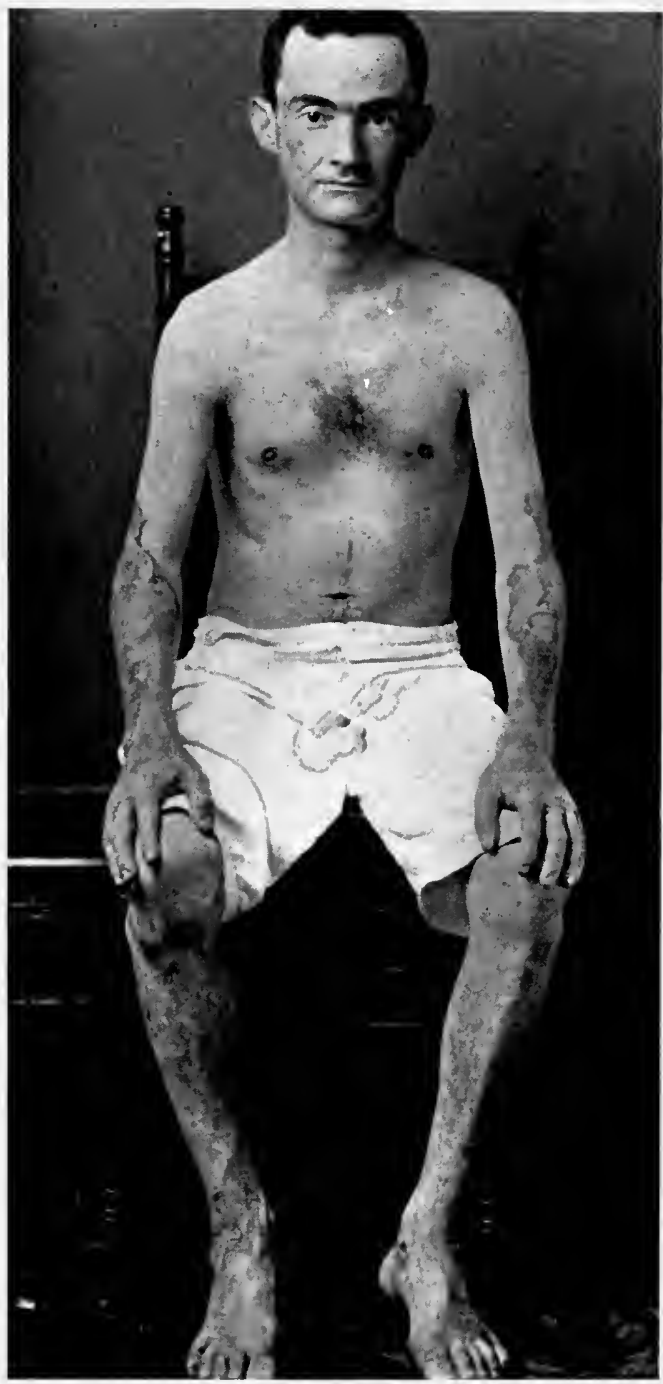

Fig. 80.-Extensive dermatitis of arms and feet. (Dr. T. E. Sanders.)

extending around the neck parallel to the lower jaw and several finger-breadths from it. The two lines meet on a level with the larynx above. The lower border is said to begin a little be- 
low the vertebra prominens, pass around the root of the neck on either side and unite over the upper part of the manubrium. The pellagrous eruption of the feet is of rather frequent oc-

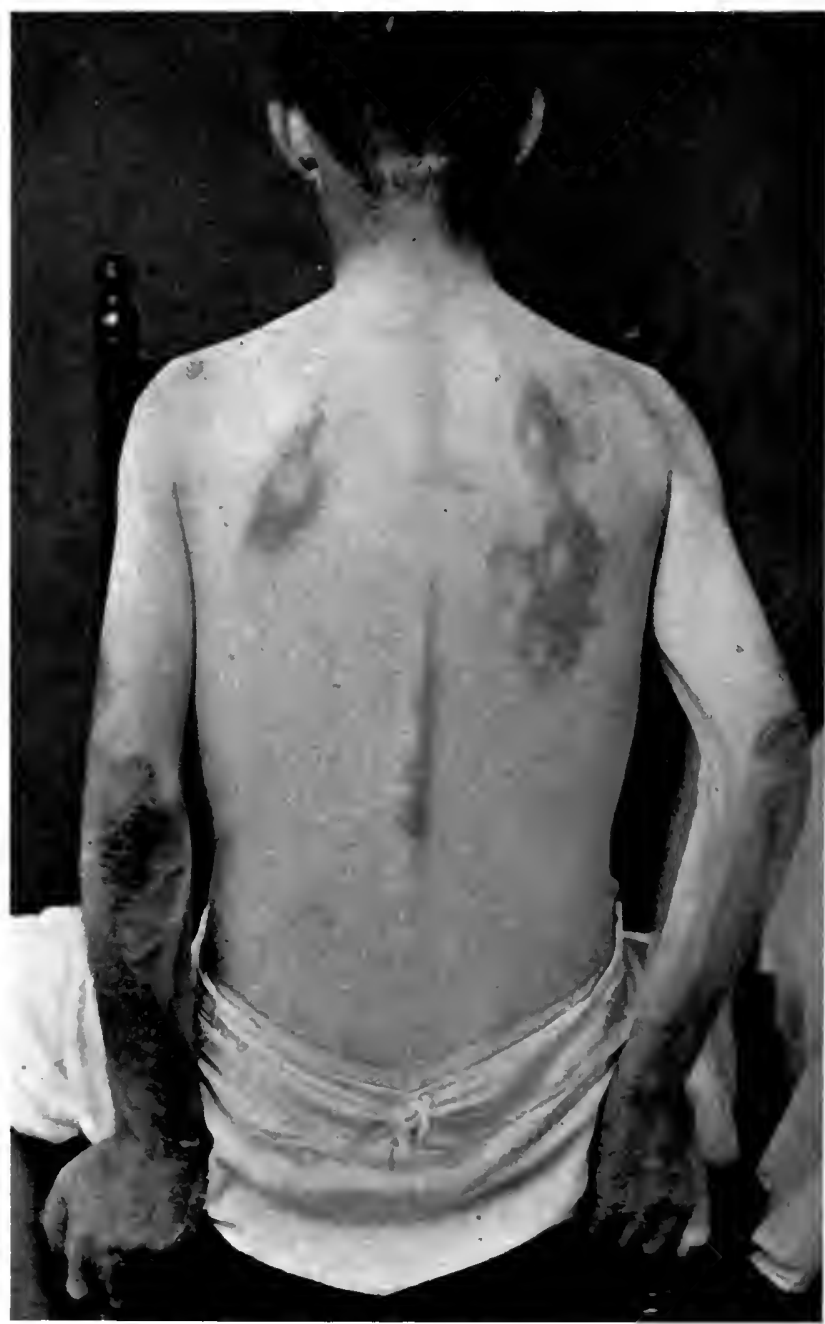

Fig. 81.-Back of same patient as shown in Fig. 80.

currence. The dorsal surfaces alone are usually involved, although rarely the soles of the feet are affected. The line of demarcation is usually in the region of the malleolus, although the lesion may pass on up the ankle. While the toes are 
generally not involved there are exceptions, the great toe being most frequently the seat of the eruption.

While the pellagrous dermatitis of the feet is most frequent in those who do not wear shoes and stockings, it is often noted in those who do and even in those who are bedridden. There is nothing especially characteristic about the skin lesions of the

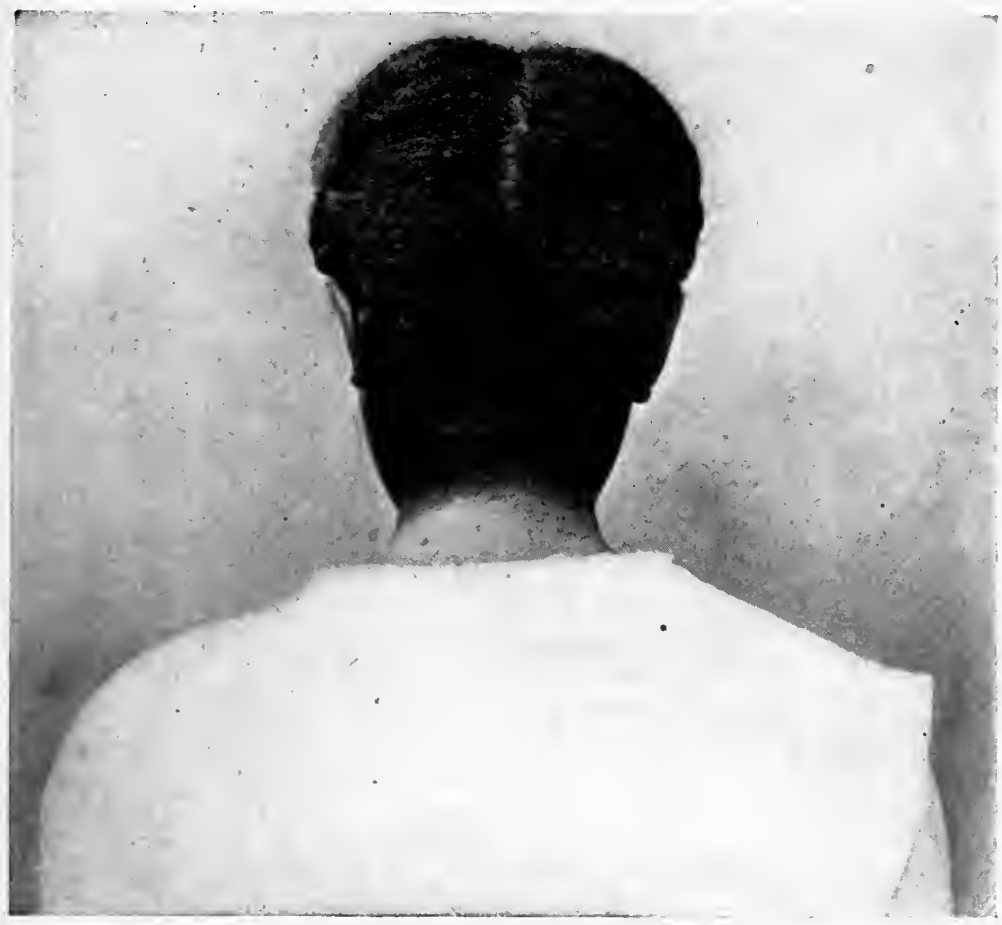

Fig. 82.-Casal's collar in negress.

upper arms, elbows, knees and thighs except the symmetry. It is of importance not to confuse the simple roughening of the skin over the knees and elbows with the pellagrous eruption.

Lesions of the external genitalia are not of rare occurrence and should not be overlooked. Dermatitis in the anal region, especially in patients with diarrhea, is sometimes observed.

It is customary to divide the skin eruptions into "wet" and "dry" lesions depending on whether or not bullæ are formed.

As stated above, the usual beginning of the skin manifesta- 
tions very markedly resembles a severe sunburn and often is indistinguishable from it.

It is usually, however, darker in color without the usual pinkish hue of the erythema of sunburn. In dark-skinned individuals, especially the negro, the color of the eruption may take a grayish hue, while the normal pigment of the part deepens

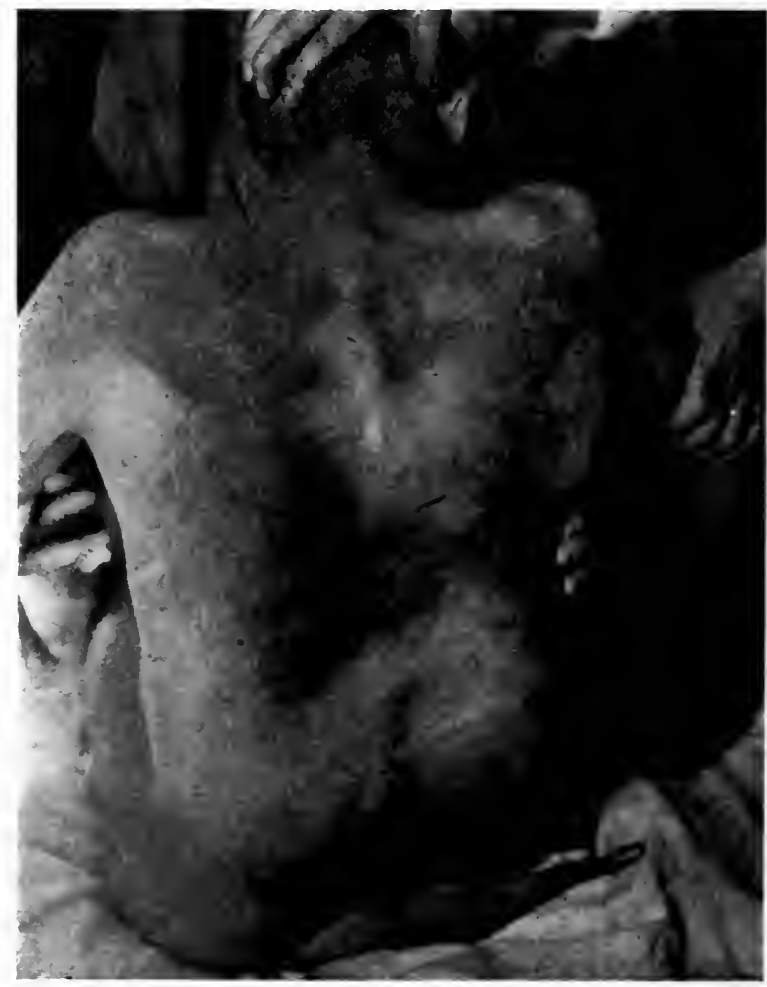

Fig. 83.-Extensive dermatitis on back.

in color. The skin of the affected area is generally slightly puffed and a slight burning sensation is complained of, while there is some pruritis. At first the color disappears on pressure but returns when the pressure is removed. Later this phenomenon is not observed.

The color of the eruption gradually deepens and when at its height is a reddish brown or chocolate shade, this depending upon the color scheme of the individual. The first eruptive 
symptoms may disappear in two or three weeks with exfoliation of the epidermis usually in light scales, although exfoliation may occur in large flakes. The area is left pigmented according to the severity of the lesion.

As stated above, the pellagrous eruption may begin not as an erythema but with discrete maculae which may persist for two to three weeks and then disappear.

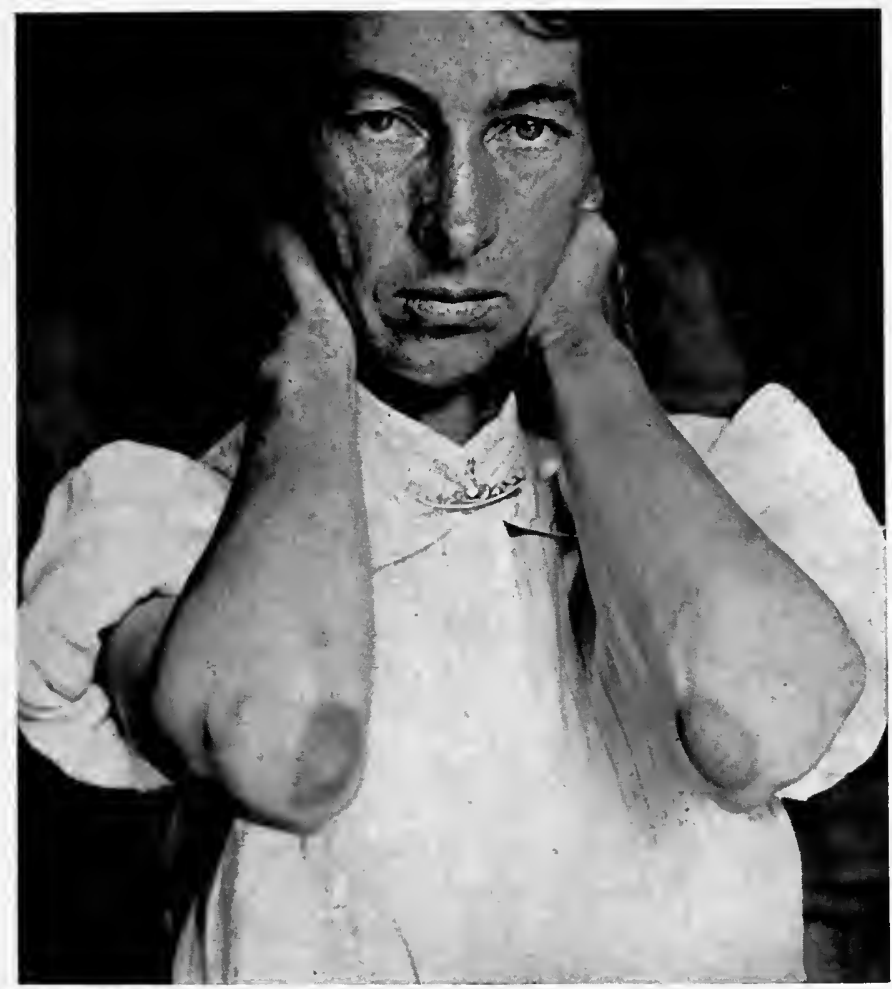

Fig. 84.-Typic eruption on elbows.

The so-called "wet" pellagrous eruption, as distinguished from the "dry" form just described, begins with the typical erythema, upon which in a short time are superimposed vesicular or bullous lesions of greater or less extent. These lesions are always monolocular, and the contents which in the beginning is sterile fills the space without, leaving the covering epidermis in a flaccid condition. 
The bullæ are not found in all regions where the dry dermatitis is found, but are located chiefly on the backs of the hands, the neck and occasionally on the feet and legs.

Secondary infection with pyogenic organisms is not an infrequent occurrence, the bleb being converted into an abscess. Following this there is edema of the underlying skin and that adjacent, with more or less inflammatory reaction.

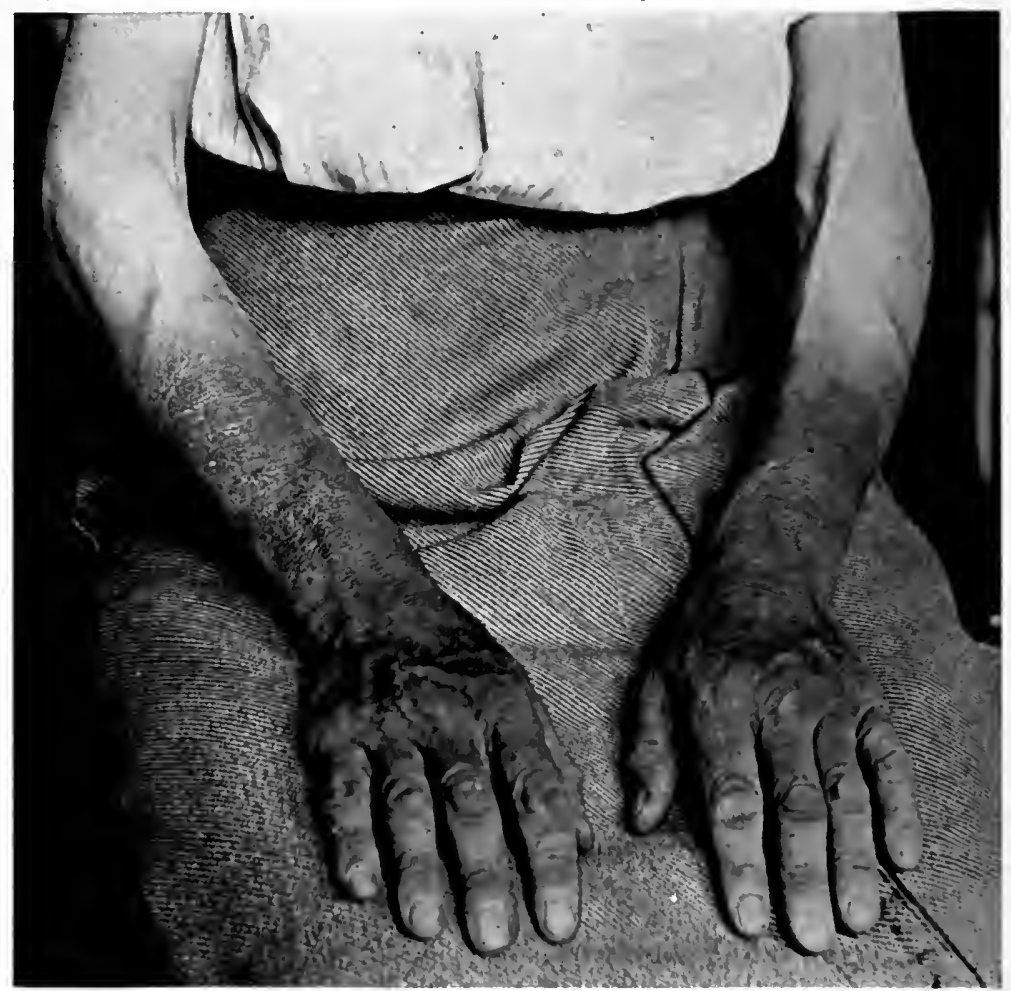

Fig. 85.-Typic dry eruption on hands and forearms.

The bullæ may be ruptured by some mechanical means and the lesions become granulating wounds, or they may gradually dry up, the covering being converted into a fine crust of a yellowish-green color beneath which the epidermis gradually becomes regenerated.

Skin lesions developing from the original erythema in which fissures are developed have been noted. These at first are 
confined to the corium, but later invade the deeper layers. This type of lesion may heal, leaving little or no trace if infection does not occur. In the event of the latter a granulating wound develops, which is followed by cicatrization. Not infrequently, when this class of lesion develops, blebs also are seen.

Desquamation without erythema is sometimes seen, but this is certainly rare.

Pustular lesions have been reported, especially on the face. The pustules vary from the size of a millet seed to that of a pea and are said to occur either without the erythema or complicating it. We have never seen a case of pustular pellagra except one in which the bullous lesions had become infected.

Pellagra hemorrhagica is another rare form of the skin lesions. It occurs as small hemorrhagic areas beneath the skin and is seen on the backs of the hands, arms, face and neck.

Pellagra sine pellagra, the disease without the skin manifestations, has been described. This condition will be discussed more fully later.

The eruption of pellagra begins rather suddenly, reaches its maximum intensity in a variable time from several days to a few weeks, and retrogresses gradually, requiring from a few weeks to several months to disappear. After the first attack the skin remains pigmented for some time. Following attacks leave the skin much thickened, roughened, hard and pigmented. The color is a peculiar bronze or yellowish green. After several attacks the skin may become atrophied and lose its elasticity almost entirely. White spots are also of ten seen.

Gastro-intestinal Symptoms.-The symptoms referable to the gastro-intestinal tract are usually, as stated above, the first to appear and undoubtedly they are the most important. Not only are there many symptoms present due to the actual demonstrable organic changes, but usually there are also many functional symptoms observed.

Stomatitis of varying degree with glossitis is one of the most constant symptoms of pellagra. Often this condition is so mild as to pass unrecognized by the patient, and is detected only after a careful and painstaking examination by the physician. 
The tongue is usually denuded of epithelium and is always red, especially along the edges. It may be thick and beefy or it may present a pointed appearance. Along the edges there may be ulcers.

The outer borders of the lips are dry, and in severe cases present a more or less cyanotic appearance. The inner borders of the lips in severe cases are tender, raw and swollen. Black spots may be noted.

The stomatitis and glossitis may be so severe that the tongue cannot be protruded and eating and swallowing may be accomplished only with the greatest difficulty, while taking into the mouth of even weak acids is accompanied with considerable burning. Most pellagrins complain of a "bad taste" in the mouth. The breath is usually very foul and saliva may flow so freely that the patient cannot speak. The teeth are not always affected, but pyorrhea is not uncommon. There is sometimes a bilateral, non-suppurating, painless parotitis. The other salivary glands are usually normal in size.

There is a sensation of heat in the mouth, throat and stomach; in fact, these symptoms may precede any noticeable pathologic change. This sensation of heat is undoubtedly due, in part at least, to the pathologic changes, but may also be caused by a neurosis.

The appetite is usually diminished, although some patients are loud in their clamor for food. Some suffer from thirst, while others will only drink when forced to.

The principle gastric symptoms are those of gastritis, distention, belching, gastralgia, nausea and rarely vomiting except in advanced cases.

It not infrequently occurs that a patient consults a physician for more or less vague indefinite gastric symptoms and is treated for the same for some time, when the typical stomatitis or skin eruption develops and the true nature of the condition is revealed.

The distention and belching are often most severe causing the patient great discomfort.

Gastralgia varies from the vague indefinite "hunger pains" to most severe epigastric pains and bear no relation to the in- 
take of food. These are often mistaken for the gastric crises of tabes dorsalis, especially in the later course of the disease when the nervous system is involved. There is usually more or less epigastric tenderness.

The gastralgia is usually intermittent even during the acute attack, but often persists after the dermatitis and other symptoms have disappeared, in fact, it may last in a more or less severe form until another acute attack occurs.

The nausea of pellagra is generally not marked. It may occur early in the disease, but more frequently as a later manifestation. Sandwith ${ }^{327}$ states that vomiting does not occur. Niles $^{305}$ says that in 200 cases vomiting was seen less than twenty times. Other observers find it a rather frequent accompaniment to the final stages of the disease. We have recently had a case in which vomiting was an early and obstinate symptom.

Roberts $^{300}$ is of the opinion that the burning pains are referred, having a common origin with the burning sensations of the hands and feet, that they do not arise from a gastric condition, "but are rather due to cord involvements and impulses reflected through the sympathetic ganglia."

With these views we cannot agree. In the first place these symptoms occur in the vast majority of cases long before any demonstrable nervous symptoms exist; and in the second place, the pathologic anatomy of the stomach in pellagra is usually sufficient to account for the gastric symptoms.

One of the most frequent symptoms, and also one of the most difficult to control is diarrhea. However, instead of diarrhea there may be constipation, and not infrequently the two symptoms alternate one with the other.

Various investigators report that from 85 to roo per cent. of their cases show diarrhea at one time or another. The time of the appearance of the diarrhea is somewhat variable. Very often it is the first symptom to be noted; however, it is not at all unusual for the three symptoms, diarrhea, stomatitis, and dermatitis to appear at nearly the same time.

The development of the diarrhea is nearly always gradual, and its disappearance with the recession of the disease is also gradual. 
In the beginning the diarrhea is usually of a spasmodic character and is accompanied with more or less pain and griping. The stool is dysenteric and muco-sanguinolent in character.

It is hard for the patient to control the sphincter and often his clothing may become soiled. In the mild cases the diarrhea as described gradually improves with the other symptoms. In the severe cases it becomes worse. The stools assume a more watery appearance, and are of more frequent occurrence. Neither rest nor food influence the condition, there being as many bowel movements at night as during the day. There may be distention and paralysis of the intestines.

Opium even in large doses has little or no effect, and the condition persists until a remission occurs or death ensues. In the severe cases the stool may be almost watery in consistence, of a light gray color, often tinged with blood, and is usually very irritating to the parts.

The odor of the pellagrous stool is most characteristic, some writers even going so far as to state that a diagnosis may be made upon this feature alone.

Diarrhea is frequently the only symptom to appear in the fall after an acute pellagrous attack the previous spring, and this symptom is a rather good index to the severity of the disease; the less severe the diarrhea the more mild the case and vice versa. Often the cases which show constipation run a mild course.

Should the patient become convalescent for a long time his appetite is variable, indigestion is usually brought on by slight indiscretions in diet, the flatulence and eructions are rather frequent.

Nervous Symptoms.-The nervous symptoms of pellagra as usually described are most varied, and by most pellagragraphers great importance is attached to them.

Thus Sandwith ${ }^{327}$ states that in the acute stage there is usually pain in the back, which may be so severe as to compel the patient to walk with body arched. The pain is elicited on both sides of the vertebral column by pressure over the spinal nerves. These symptoms generally disappear if the patient remains in the hospital for a month. 
The lower tendon reflexes are found disturbed in the majority of cases. According to Sandwith 46 per cent. show marked exaggeration, 29 per cent. slightly increased, 9 per cent. diminished, I4 per cent. absent and 2 per cent. normal.

While these reflexes are usually on both sides they may be asymmetrical. Ankle clonus is occasionally noted. Tremor of the fingers and of the tongue are often observed rather early in the disease, while in the later stage tremors are usually most marked.

There is no typical "gait" in pellagra; nevertheless there may be a spastic gait. A positive Romberg is often noted. Vertigo is a frequent symptom, and usually occurs when the patient is standing, but may develop when the patient is in bed.

Singer ${ }^{333}$ has pointed out that there are none of these symptoms but may be observed in other severe intoxicative conditions such as tuberculosis. He further states that "faulty nervous organization seems for some reason to be associated with a predisposition to pellagra."

Singer gives in detail the history of the only case which came under his observation in which there were evidences of chronic structural change in the nervous system. In this case there were noted occasional brief attacks of loss of power in the lower extremities following an attack of pellagra. These attacks lasted from fifteen to twenty minutes followed by apparently complete recovery. Following a second attack of pellagra there was atrophy of the small muscles of the hands and spastic paraplegia of gradual development.

In spite of these manifestations Singer is of the opinion that the pellagra merely acted as a precipitating force and was not the cause per se of the nervous symptoms.

Mental Symptoms.-For a long time it has been recognized that pellagra is very frequently accompanied by mental disorders, and of later years some investigators have even gone so far as to consider the involvement of the central nervous system (both nervous and mental symptoms) as the principle evidence of this disease. Others ascribe to it the cause of mental derangement and describe a pellagrous insanity. 
This insanity according to most writers takes various forms; thus Marie $\mathrm{e}^{308}$ states that the most frequent form of psychosis in pellagra is mental confusion, with depressive states and with delirium, while Sorbets, according to Babcock, ${ }^{291}$ says, "For alienists, hallucinations are the chief characteristics, the pathognomonic phenomenon of pellagrous insanity."

Green as quoted by Roberts ${ }^{300}$ states that at the present time it is impossible to recognize a pellagrous insanity as an entity, and temporarily it is best to classify the mental condition as the "psychosis accompanying pellagra" and to subdivide the groups into types according to the symptoms. He therefore describes the following types: infective exhaustive, manic depressive, dementia præcox, general paralysis, senile, involutional melancholia and unclassified.

Gregor $^{334}$ has distinguished between the abnormal mental pictures directly attributable to pellagra, that is, caused by change in the brain due to the presence of the pellagra toxin and those which are secondary.

Singer ${ }^{33}$ has proposed the following classification of the types of mental disorder associated with pellagra.

Group I. Disorders directly due to the pellagra toxin (or toxins).

I. Symptomatic depressions.

2. Delirious pictures.

Group II. Disorders based on peculiarities in the personal make-up, the attack of insanity being precipitated by pellagra.

I. Manic depressive disorders.

2. Hysteria.

3. Psychasthenia.

4. Dementia præcox.

5. Paranoic developments.

Group III. Disorders due to definite brain changes with pellagra merely as a complication.

I. Arteriosclerosis dementia.

2. Senile dementia.

3. Presenile psychoses.

4. General paralysis of the insane.

Singer considers the relation of pellagra to mental disturb- 
ance the same as that of other general diseases, and that while similar pictures as observed in pellagra are encountered in typhoid fever, yet a separate grouping is not made.

There may be cases in which the mental condition, such as delirium, is due to the action of toxins of the disease on the brain, or there may be a picture of dementia præcox or manicdepressive insanity which runs a similar course to those cases in which there is no infection. If pellagra develops in a dementia præcox predisposed individual it will be the "last straw" to bring on the symptoms of the mental disease. Another view of the matter is taken by Singer, which is that the relation of the functional psychoses (including dementia præcox) and pellagra is the reverse of the usually accepted theory. That instead of the pellagra being the cause of the psychosis, it may be that the psychosis or "the poor adaptability and peculiarity of make-up," predisposes the individual to pellagra. It is certainly a fact that pellagra very frequently develops among the chronic insane.

Singer admits that "the relation of pellagra to the psychoses due to more definite brain disease or degeneration such as the presenile, senile and arteriosclerotic dementias, is a somewhat different question. He states that there is no doubt of the occurrence of pellagra with these conditions, and that they may predispose the body to pellagra or that the pellagra might favor the early development of involution change or even provoke arterial degeneration.

While admitting the occurrence of pellagra with paresis, Singer cannot agree that there is a type of mental disturbance in pellagra comparable with the general paralysis of the insane. The picture which is generally so described is that of general intoxication with central and possibly peripheral neuritis, and much more resembles the severe toxic forms of the infective fevers, such as typhoid, than it resembles paresis.

In describing the symptomatic depressions which occur in pellagra, Singer states that they are by far the most frequent if cases outside hospitals for the insane are included. There is a marked depression, although there need be no objective signs of fatigue. The most striking characteristic of this class of 
cases is that the mental symptoms are severe or mild as the other symptoms progress or recede. There is more or less hopeless sadness, thinking is difficult and general lowering of tone exists while apprehension without definite fear is often observed. This may be expressed by the patient by words or by restlessness.

The delirium sometimes found in pellagra, Singer says, in the vast majority of cases, is the result of intoxication of the brain causing changes in the functional activity involving the highest cerebral levels. All degrees are met with in the mild cases; there are short periods of clouding of consciousness, while in the intervals the mind may be practically clear, yet with depression manifest. There is nothing to distinguish these conditions from those found in other intoxications, such as in typhoid.

In pellagra, as with other conditions, these manifestations probably depend upon two factors, viz., the virulence of the toxin, and the ability of the individual to resist the poison. The relative frequency of delirium in pellagra may be due to the extreme virulence of the pellagra toxin or that the individuals who contract pellagra are of poor nervous organization.

The mental pictures of pellagra which are due to the personality of the individual, manic-depressive, dementia præcox, etc., are in no way distinguishable from those conditions in which pellagra is not present. There may be some delirium due to the intoxication which will make the diagnosis of the type more difficult, but this delirium will run a course parallel to the other symptoms of the pellagra.

The frequency of mental disorders in pellagra is the subject of some differences in opinion. Wood ${ }^{297}$ states that the "probable outcome of the ordinary types of pellagra will be insanity."

Grimm $^{292}$ found 7.5 per cent. of $\mathrm{I}, 436$ cases were insane.

Roberts $^{300}$ states that it has been estimated that ro per cent. of the pellagrins in Italy are insane, and considers that figure much too high for this country; in fact that 5 per cent. would be nearer.

In regard to the various "types" of mental change found in pellagrins, Green's ${ }^{300}$ table is as follows: 


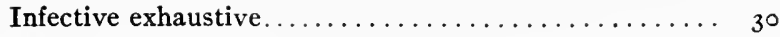

Manic-depressive..................... 9

Dementia præcox..................... 9

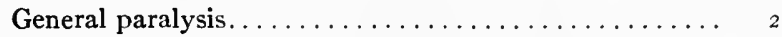

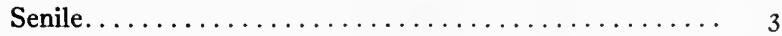

Involution melancholia . . . . . . . . . . . . . . 2

Unclassified..................... 5

Total............................ 60

Of the cases reported by Singer ${ }^{333}$ the following table shows the number and percentage of types:

Types of Mental Disorders in a Series of Pellagrins

\begin{tabular}{|c|c|c|c|c|c|c|c|c|}
\hline \multirow[b]{2}{*}{ ' } & \multicolumn{2}{|c|}{$\begin{array}{l}\text { Thirty-four } \\
\text { unselected } \\
\text { cases seen by } \\
\text { the author }\end{array}$} & \multicolumn{2}{|c|}{$\begin{array}{l}\text { One hundred } \\
\text { and thirty } \\
\text { unselected } \\
\text { cases seen by } \\
\text { commission }\end{array}$} & \multicolumn{2}{|c|}{$\begin{array}{l}\text { Total } \\
\text { unselected } \\
\text { cases }\end{array}$} & \multicolumn{2}{|c|}{$\begin{array}{c}\text { Eighteen } \\
\text { hospital cases } \\
\text { selected for } \\
\text { severity }\end{array}$} \\
\hline & $\begin{array}{l}\text { No. of } \\
\text { cases }\end{array}$ & $\begin{array}{l}\text { Per } \\
\text { cent. }\end{array}$ & $\begin{array}{c}\text { No. of } \\
\text { cases }\end{array}$ & $\begin{array}{l}\text { Per } \\
\text { cent. }\end{array}$ & $\begin{array}{l}\text { No. of } \\
\text { cases }\end{array}$ & $\begin{array}{l}\text { Per } \\
\text { cent. }\end{array}$ & $\begin{array}{l}\text { No. of } \\
\text { cases }\end{array}$ & $\begin{array}{l}\text { Per } \\
\text { cent. }\end{array}$ \\
\hline Group I: & & & & & & & & \\
\hline Symptomatic depression... & 9 & 26.5 & $4 I$ & 3 I. 5 & 50 & 30.5 & IO & $55 \cdot 5$ \\
\hline $\begin{array}{l}\text { Delirious pictures } . . . \ldots \ldots \\
\text { Group II: }\end{array}$ & 2 & 5.9 & Io & $7 \cdot 7$ & I2 & $7 \cdot 3$ & 2 & II. I \\
\hline Manic-depressive disorders & $\circ$ & 0.0 & 0 & 0.0 & 0 & 0.0 & $\mathbf{I}$ & $5 \cdot 5$ \\
\hline Hysterical disorders....... & I & 3.0 & $\circ$ & 0.0 & I & 0.6 & $\circ$ & 0.0 \\
\hline $\begin{array}{l}\text { Dementia præcox disorders } \\
\text { Group III: }\end{array}$ & $\circ$ & 0.0 & I & 0.8 & I & 0.6 & I(?) & $5 \cdot 5$ \\
\hline Arteriosclerotic dementia .. & $\circ$ & 0.0 & $\circ$ & 0.0 & ० & 0.0 & $\mathbf{I}$ & $5 \cdot 5$ \\
\hline Presenile psychoses....... & I & 3.0 & $\circ$ & 0.0 & I & 0.6 & ० & 0.0 \\
\hline Senile dementia. & $\circ$ & 0.0 & $\circ$ & 0.0 & $\circ$ & 0.0 & I & $5 \cdot 5$ \\
\hline
\end{tabular}

The following table shows the mental condition of the ninetyone cases seen by one of us at the Arkansas State Hospital for Nervous Diseases:

Intoxication psychosis ................ 55

Dementia præcox........................

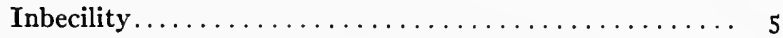

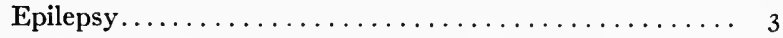

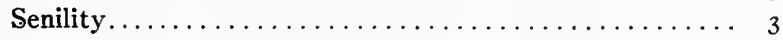

Manic-depressive.................... 2

Paranoia...................... I

Arteriosclerosis......................

Paretic .........................

Total...................... 
Sperial Senses.-Eye.-While the eyes of pellagrins are rarely normal there are, with possibly one exception, no pathognomonic findings in the eyes in this condition. This exception is one reported by Whaley, ${ }^{286}$ who states that as his number of cases is small (thirty-five) he wound not like to be too sanguine about its being pathognomonic. He further states that Lombroso found it. The symptom as described by Whaley "appears as if the retinæ were thickened and gives the fundus reflex a peculiar indistinct yellowish color, and is not so pronounced as the senile reflex."

The pellagrous eruption may appear on the eyelids and ptosis is not rare. Ectropion has been observed and conjunctivitis is a rather frequent occurrence. The ocular muscles and visual powers fatigue rapidly so that pellagrins are rarely able to perform work which requires close inspection for any length of time.

Cataracts are not infrequently observed.

Diplopia, hemianopia, and photophobia, especially the latter, are very common. Marie ${ }^{308}$ states that he has seen patients who remained for years with eyes closed owing to the fear of light. Bilateral and monolateral mydriasis is seen. If the condition is confined to one eye the right is the one usually affected. The pupillary reflexes, both to light and accommodation, are frequently sluggish.

In very advanced cases are seen ulcers of the cornea, retinitis, choroiditis, retinochroiditis and inflammation of the optic nerve.

Sclerosis of the retinal vessels and dilatation of the retinal veins are frequently noted.

All these symptoms, as would be expected, appear more exagerated in the severe cases and less so in the mild ones.

Marie $^{308}$ gives the following table of the ocular findings of Ottolenghi, Manfredi and Flarer:

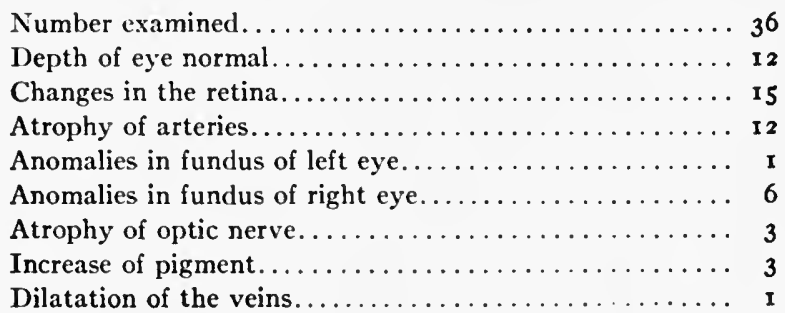


The other special senses show little or no derangements, and certainly nothing which may be regarded as pathognomonic.

General Symptoms.-Cachexia, varying from slight loss of weight to great emaciation, is observed. The cachexia varies with the severity of the disease and especially with the severity of the diarrhea. In the first attack, which may be mild, there may be little or no loss of weight, while in subsequent attacks the cachexia often advances very rapidly.

The temperature in uncomplicated pellagra is usually normal. Marie $^{388}$ quotes the observations of Alpato-Novello on Io० pellagrins. He states that 22,274 observations were made, the maximum temperature being $41.5^{\circ} \mathrm{C}$. and the minimum $35^{\circ} \mathrm{C}$; 2,059 were above normal and $5,25^{\mathrm{I}}$ below normal. Marie further states, that when fever does occur it is very irregular, rarely continuous and often remittent, almost always with an evening rise.

The pulse rate in pellagra is usually increased, and its rate depends upon the severity of the disease. During the acute attacks it may run as high as 100 or more, gradually dropping to normal as a remission occurs. In the final stages it often runs as high as I 30 or more, which is considered a grave sign.

In spite of its rapidity the pulse is usually regular. It is, however, soft and compressible and as the disease progresses, decreases in volume.

The blood pressure, as would be expected, is usually low, except in pellagrins with arteriosclerosis. Those cases which are seen in the terminal stages with marked cachexia show a very low blood pressure; one case in particular observed by one of us had a blood pressure of only $85 \mathrm{~mm}$.

As stated in the chapter on pathology, the heart frequently shows hypertrophy, atrophy, hydropericardium and myocarditis. Endocarditis with incompetent valves is rare, although it may be noted as a complication.

The lungs show no characteristic symptoms, though as stated above pellagra may be complicated by various pathologic conditions in the lungs, which will display typical symptoms.

Blood.-Most investigators have found that there is usually a deficiency in the blood stream in pellagra. Not only is the 
total volume of the blood diminished, but the red blood corpuscles and hemoglobin are below normal.

The anemia is usually said to be secondary in type, that is, the decrease in the hemoglobin is greater than the decrease in the erythrocytes.

Most investigators find either a normal or slightly increased leucocyte count.

Lavinder ${ }^{286}$ in a review of the literature of the hematology of pellagra gives the findings of the following investigators: Carletti reviews the work of Lombroso, Capezzuli, Sepilli, Agostini and D'Ancona, and Randi, and noted that all found similar results, viz., reduction in both the red blood-cells and the hemoglobin which is fairly constant. Sepilli was the only one who reported the leucocyte count, which he found to be normal. In his own work Carletti found a constant slight reduction in the erythrocytes, a variable number of whites (never a leucocytosis), constant reduction in the hemoglobin (65-75 per cent.) with a low color index. He found rather a large number of small red cells and the varieties of leucocytes in about the normal proportions with a possible increase in the large mononuclears; eosinophiles were rarely found.

Galesesco and Slatinéano, according to Lavinder, found in - thirty-one cases a constant diminution in the number of red cells (three to four million) without qualitative changes; hemoglobin (von Fleischl) from 70 to $9 \circ$ per cent.; slight leucocytosis (nine to ten thousand) of which the polynuclears constituted 55 to 78 per cent.; lymphocytes $\mathrm{I} 7$ to 33 per cent.; large mononuclears ro to 22 per cent.; eosinophiles 2 to 4 per cent.

These investigators conclude that there is a large mononuclear increase from which no deduction can be drawn as to etiology.

In a study of thirty-four cases, Frateni using the Thoma-Zeiss counting apparatus and the von Fleischl hemoglobinometer, found a rather constant reduction in the number of red cells (three to four and one-half million) and in the hemoglobin (55 to 92 per cent.) leucocyte counts from $7,4 \mathrm{I} 2$ to $\mathrm{II}, 4 \mathrm{I} 8$. Differential counts showed polynuclears 55 to 76 per cent., large lymphocytes 2 to 5 per cent., small lymphocytes 18 to 39 per cent., eosinophiles 2 to 9 per cent. 
There was also a rather constant finding of macrocytes, microcytes and poikilocytes. This investigator states also that he found nothing resembling a protozoal parasite.

Lavinder continues, that Manson states that Sambon and Terni in Italy, and Grigoresou and Galasesco in Roumania, have noticed a relative increase in the large mononuclear leucocytes.

Fiorini and Gavini in non-alienated pellagrins found no leucocytosis, but a typical mononuclear increase and a decided eosinophilia.

Masini, Lavinder states, "in a study of the eosinophile cell in the blood of pellagrins concludes that, contrary to what is found in many acute infectious diseases, there is produced in the pellagrous intoxication a conspicuous and decided eosinophilia, which occurs in cycles corresponding with the increase or diminution of the pellagrogenous toxines; that is, the more toxemia the greater the eosinophilia. He suggests that this constant eosinophilia may prove at times a valuable aid in the early differential diagnosis."

Peserico found the following percentages of the varieties of leucocytes: polynuclears, 53.7 to 64.4 ; lymphocytes, 26.1 to 37.4 ; large mononuclears and transitionals, 1.2 to 7.7 .

These results, Lavinder says, in many ways seem decidedly discordant, but continues, that it may be concluded that a usually mild secondary anemia is very frequent; such qualitative changes in the red cells as are found are only such as would be expected. The only factor concerning the differential leucocyte count upon which there is an agreement, is that there seems to be a majority opinion of a definite relative large mononuclear increase. Lavinder's own work consisted of twenty-four examinations. He used a Thoma-Zeiss counting chamber with Turck's ruling; in counting the red cells the four corner unit squares (twenty-five small squares each) were counted in two preparations, and if the results were discordant, a third preparation was counted; in counting the leucocytes the whole ruled space was counted in two preparations, and again if results were discordant, a third preparation was counted. A new Dare hemoglobinometer which gave very uniform results in normal individuals was used for estimating the hemoglobin. 
Lavinder states that most of the cases were of an advanced type showing nervous and mental symptoms, and nearly all in negro women. The same time of day (morning) was chosen for making nearly all the counts. Many had been taking arsenic in some form with full dosage either at the time of the examination or a very short while previously.

Lavinder's results were given in a table which shows that the red cell count ranged from $2,826,640$ to $5,520,000$, with an average of $4,474,000$; the lowest hemoglobin reading was 38 per cent. (two cases), the highest 95 per cent. and the average 68 per cent., while the leucocytes varied in number from 4,000 to 17,000 , with an average of 9,040 . The average color index was .77 .

Lavinder does not tabulate his differential counts which were not made in all cases, but states that there was an increase in the large mononuclears and no eosinophilia except in cases complicated with round worms or hook-worms.

Hillman ${ }^{335}$ in thirty-two examinations of twelve pellagrins found the average number of erythrocytes to be $4,758,000$, the average hemoglobin 83 per cent., with the following percentages for the different varieties:

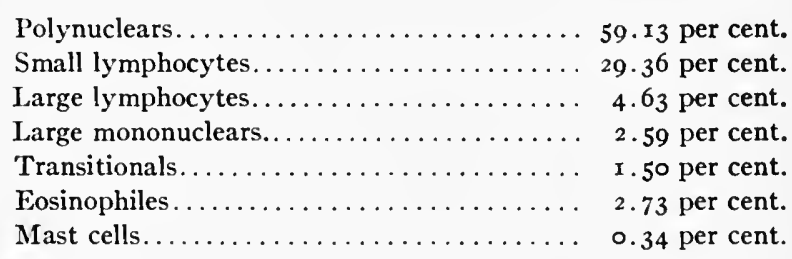

Hillman in connection with Schule ${ }^{336}$ later published the results of further investigations, which corroborated the findings described above.

Of the ninety-one cases seen by one of us at the Arkansas State Hospital for Nervous Diseases complete blood counts were made on forty-eight. The table on page 353 shows the results.

The average number of erythrocytes was $3,800,000$, the average hemoglobin being 79 per cent. This gives a rather high average color index.

The average number of leucocytes was 8,500 with an average 


\begin{tabular}{|c|c|c|c|c|c|c|c|c|c|c|c|c|}
\hline $\begin{array}{l}\dot{0} \\
Z \\
\dot{U} \\
\tilde{U}\end{array}$ & $\begin{array}{l}\stackrel{x}{0} \\
\ddot{\mho}\end{array}$ & $\underset{x}{x}$ & 䓌 & 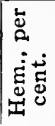 & $\underset{\oplus}{\vec{\Xi}}$ & 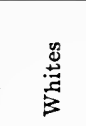 & 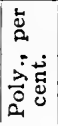 & $\begin{array}{c}\text { 总 } \\
\text { 号 }\end{array}$ & 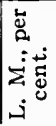 & 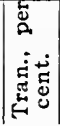 & 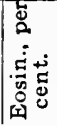 & 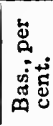 \\
\hline 7,216 & $\mathrm{~F}$ & 50 & $3,220,000$ & 71 & I. I & 6,400 & 68 & 29.0 & 2 & I & o & o \\
\hline 7,193 & F & 48 & $3,460,000$ & 75 & I .O & 6,200 & 39 & 59.0 & 2 & o & o & o \\
\hline 6,019 & F & 40 & $3,950,000$ & 90 & I . . 3 & 6,400 & 56 & 39.0 & 4 & o & I & o \\
\hline 6,578 & F & 37 & $4,390,000$ & 90 & I . I & 5,860 & $7 I$ & 2 I.O & 5 & 3 & 0 & $o$ \\
\hline 6,222 & $\mathbf{F}$ & $4 I$ & $2,000,000$ & 60 & I. 5 & 4,700 & $7 \mathrm{I}$ & 26.0 & 2 & o & I & \\
\hline 5,982 & $\mathbf{F}$ & 30 & $4,180,000$ & 85 & I.O & 9,500 & 71 & 25.0 & I & o & I & $\mathrm{o}$ \\
\hline 7,090 & $\mathbf{F}$ & 45 & $3,920,000$ & 80 & I.O & I 8,400 & 80 & 20.0 & o & o & o & o \\
\hline $5,52 \mathrm{I}$ & F & 22 & $4,020,000$ & 80 & 1.o & 10,500 & 79 & 19.0 & $\mathbf{I}$ & o & o & 0 \\
\hline 7,119 & F & 32 & $4,640,000$ & 90 & 0.9 & 15,400 & 79 & 17.0 & 3 & o & I & o \\
\hline 6,968 & $\mathrm{~F}$ & 52 & $3,360,000$ & 73 & 1.0 & 4,400 & 59 & 40.0 & o & I & o & o \\
\hline 6,432 & $\mathrm{~F}$ & I 4 & $4,870,000$ & 90 & 0.9 & 8,000 & 65 & 33.0 & I & I & o & o \\
\hline 6,977 & $\mathrm{~F}$ & 45 & $3,320,000$ & 73 & I. I & 5,500 & 85 & I 4.0 & o & o & I & o \\
\hline 5.906 & $\mathbf{M}$ & 60 & $2,880,000$ & 65 & I. I & I I,600 & 79 & I9.0 & 2 & o & o & o \\
\hline 6,797 & M & 45 & $5,590,000$ & 95 & 0.9 & 9,200 & 60 & 37.0 & 2 & I & 0 & 0 \\
\hline 4,893 & $M$ & 44 & $4,500,000$ & 78 & 0.8 & 22,200 & 82 & I 5.0 & 3 & o & 0 & o \\
\hline 6,880 & $\mathrm{M}$ & 35 & $4,240,000$ & 80 & 0.9 & 7,700 & 75 & 21.0 & 2 & o & I & I \\
\hline 6,174 & $\mathrm{M}$ & 45 & $3,710,000$ & 75 & I.O & 12,200 & 75 & I 7.5 & 6 & o & I & o \\
\hline 5,970 & $\mathrm{M}$ & 35 & $5,050,000$ & 95 & 0.9 & 6,200 & 71 & 22.0 & 4 & 2 & I & o \\
\hline 7,167 & M & 37 & $3,650,000$ & 70 & I.O & 6,200 & 83 & I 2.0 & 2 & o & 2 & $\mathbf{I}$ \\
\hline 6,231 & $\mathbf{M}$ & 30 & $4,400,000$ & 70 & 0.7 & 4,100 & 70 & 26.5 & 2 & I & 5 & o \\
\hline 6,846 & $\mathbf{M}$ & 22 & $4,430,000$ & 82 & I.O & 7,600 & 65 & 27.0 & 2 & 4 & 2 & o \\
\hline 6,0 I 2 & M & 25 & $2,880,000$ & 56 & I. O & 6,600 & 60 & 37.0 & 2 & o & I & 0 \\
\hline 7,292 & $\mathbf{F}$ & 45 & $3,680,000$ & 75 & I. $O$ & 9,600 & 66 & 30.0 & 4 & o & o & 0 \\
\hline 7.301 & $F$ & 34 & $3,510,000$ & 78 & I . I & I I ,OOO & 85 & I 3.0 & o & I & I & 0 \\
\hline 7.319 & $\mathrm{~F}$ & 39 & $3,410,000$ & 75 & I. I & 9,200 & 77 & 20.0 & 2 & 0 & $\mathbf{I}$ & 0 \\
\hline 5,276 & $\mathrm{~F}$ & 27 & $2,760,000$ & $6 I$ & I. I & I I,OOO & 45 & I 9.0 & 4 & I & I & o \\
\hline 7,248 & $\mathrm{~F}$ & 36 & 3, I 20,000 & 69 & I . I & 6,400 & $7 \mathrm{I}$ & 27.0 & 2 & o & 0 & o \\
\hline 7,243 & $\mathrm{~F}$ & 49 & 4,2 I0,000 & 85 & I.O & 4,600 & 76 & 20.0 & 4 & o & o & o \\
\hline 7,247 & $\mathrm{~F}$ & 33 & $3,880,000$ & 82 & I.O & 7,400 & 75 & 25.0 & o & o & o & o \\
\hline 7,276 & M & 40 & $4,400,000$ & 80 & 0.9 & 10,200 & 79 & 19.0 & 2 & o & o & 0 \\
\hline 7,148 & $\mathrm{~F}$ & 29 & 4, I 30,000 & 85 & I. $O$ & 8,200 & 84 & 16.0 & o & o & o & o \\
\hline 5,653 & $\mathrm{~F}$ & 53 & $4,040,000$ & 80 & I.O & 7,600 & 72 & 25.0 & 2 & o & I & o \\
\hline 5,967 & $\mathrm{~F}$ & 57 & 3.960,0́00 & 75 & 0.9 & 6,200 & 71 & 24.0 & 3 & 2 & o & o \\
\hline 7,164 & $F$ & 30 & $4,350,000$ & 90 & I.O & I I , 200 & 82 & 17.0 & I & o & o & o \\
\hline 6,4 I 8 & $\mathrm{~F}$ & 35 & $3,670,000$ & 65 & 0.9 & 4,400 & 70 & 24.0 & 3 & 2 & I & o \\
\hline $6,70 \mathrm{I}$ & $F$ & 40 & $4,740,000$ & 90 & 0.9 & 5,600 & 68 & 28.0 & 3 & I & o & o \\
\hline 6,992 & $\mathrm{~F}$ & 34 & $3,180,000$ & 65 & I .O & 5,200 & 57 & 43.0 & 0 & o & $o$ & o \\
\hline $6,69 \mathrm{I}$ & $F$ & 30 & $4,830,000$ & 90 & 0.9 & I 8,200 & 79 & 17.0 & 3 & I & o & o \\
\hline 6,755 & $F$ & 34 & $4,370,000$ & 80 & 0.9 & 5,600 & $5 \mathrm{I}$ & 38.0 & 7 & 4 & o & o \\
\hline 6,380 & $\mathrm{~F}$ & 28 & $4,760,000$ & 80 & 0.8 & 5,480 & 70 & 25.0 & 3 & 2 & o & o \\
\hline 6,720 & $\mathrm{~F}$ & 69 & $3,550,000$ & 80 & 0.9 & 9,000 & 79 & 14.0 & 5 & 2 & o & o \\
\hline 7,036 & F & 66 & $3,040,000$ & 64 & 0.9 & 9,000 & 67 & 33.0 & 0 & o & o & o \\
\hline 6,334 & $\mathrm{~F}$ & 43 & $4,190,000$ & 90 & I .O & 7,000 & 76 & I 8.0 & 5 & $\mathrm{o}$ & I & o \\
\hline 7,130 & F & 45 & $4,230,000$ & 85 & 0.9 & 10,000 & 79 & 16.0 & 3 & I & o & o \\
\hline 7,135 & $\mathrm{~F}$ & 45 & $3,940,000$ & 82 & 0.9 & 9,400 & 69 & 26.0 & 4 & 0 & I & $o$ \\
\hline 7,323 & $\mathrm{~F}$ & 29 & $4,670,000$ & 88 & 0.9 & 10,800 & 80 & 20.0 & 0 & O & o & 0 \\
\hline 5,004 & $\mathrm{~F}$ & 53 & $3,660,000$ & 68 & 0.9 & 9,600 & 78 & 28.0 & 3 & 0 & $\mathbf{I}$ & 0 \\
\hline 6,390 & $\mathrm{~F}$ & 28 & $3,580,000$ & 70 & 0.9 & 12,200 & 75 & 19.5 & 5 & 5 & o & 0 \\
\hline
\end{tabular}


of 72 per cent. polymorphonuclears and 24.5 per cent. lymphocytes. Eosinophiles were found in only twenty cases, the highest percentage being 2 and that in only one case.

Numerous investigators have made blood cultures from pellagrins, using every conceivable medium and under both aerobic and anaerobic conditions, but with uniformly negative results.

While no definite data are to be found concerning the coagulability of the blood of pellagrins, in a rather extensive experience with such blood in making various tests and examinations we have never noticed any abnormalities in the time of coagulation.

Wassermann Test.-Several investigators have applied the Wassermann complement fixation test to the blood of pellagrins.

Bass ${ }^{339}$ using an alcoholic lecithin antigen, reported six positive reactions in pellagra. Later ${ }^{340}$ he reported on ten more cases. Four of the sixteen cases were ruled out on account of malaria, syphilis or autopsy blood. Of the remaining cases eight were positive, seven of them being mild or chronic cases and one a severe case, while four were negative cases.

Fox, ${ }^{286}$ using the Noguchi modification of the Wassermann, secured eight weakly or moderately positive and twenty-two negative reactions with thirty cases. Carletti, using the original Wassermann technic, tested the sera of twenty pellagrins, securing all negative results. With Bass' lecithin antigen he secured one positive, the others all being negative. One of us using the technic described by him $^{337}$ performed the Wassermann reaction on thirty-five pellagrins with uniformly negative results.

In attempting to develop a complement fixation test for pellagra one of us prepared an antigen by macerating portions of the brain, spinal cord, skin (the area affected with the dermatitis) and intestinal mucosa washed thoroughly, and extracting with alcohol.

The technic employed was that described by one of us, ${ }^{337}$ while the amount of antigen used was an amount a little less than would slightly inhibit hemolysis with the pooled sera of several known normal individuals.

The sera of ten pellagrins with active lesions were examined with absolutely negative results. 
Nitzesco ${ }^{338}$ claimed to have been able to diagnose pellagra with a dialization test, using Abderhalden's ninhydrin technic with a maize albumin or zein. The reaction was particularly pronounced with eight cases with predominating gastro-intestinal symptoms, and negative with fifty-six non-pellagrins and two pellagrins who had been in the hospital for over two years and had eaten no maize during this time and had had no symptoms except slight mental confusion at times. Another case with merely nervous manifestations was shown by the serologic test to be suffering from an otherwise latent pellagra and later developed other characteristic symptoms.

Nitzesco's work has not been confirmed and in the light of the present evidence against the corn theory of the etiology of pellagra must be taken with the greatest reserve.

Urine.-The urine of pellagrins shows nothing characteristic, although certain abnormalities very frequently occur. Marie $\mathrm{e}^{308}$ states that in roo pellagra patients the 24 -hour quantity showed an average of 900 c.c. with a minimum of 500 c.c. and a maximum of $\mathrm{I}, 900$ c.c. He also found marked variation in the reaction as follows: 76 times slightly acid; I4 times neutral; and Io times alkaline. The specific gravity ranged from $I, 005$ to $I, 025$. Albumin was found but twice in $\mathrm{I} r \mathrm{O}$ urines.

Most investigators find indican a very frequent constituent of pellagrous urine. Thus Watson ${ }^{308}$ found indican present $\mathrm{I} 75$ times in 180 urines. Meyers and Fine ${ }^{291}$ found indican in large quantities in all of the 14 cases, while the Illinois Pellagra Commission ${ }^{356}$ found a very marked reaction in all cases.

Of the ninety-one cases seen by one of us at the Arkansas State Hospital for Nervous Diseases, urinalyses were made for thirty-nine.

The table on page 356 shows the results.

It will be seen that the specific gravity varied from $\mathrm{I}, \infty \mathrm{O}_{3}$ to $\mathrm{I}, 030$ with an average of $\mathrm{I}, \mathrm{OI} 8$. The reaction was acid in all but two cases, it being neutral in them. Alkaline urine was not observed. Indican was found eleven times usually only in traces, although some cases showed a fairly strong reaction.

Albumin was found five times, hyaline and granular casts 
twice-once in a urine containing albumin and once in one free from that protein. Sugar was not found in any of the cases.

\begin{tabular}{|c|c|c|c|c|c|c|}
\hline Case No. & Sp. gr. & Reaction & Indican & Albumin & Sugar & Casts \\
\hline I & I,o1 6 & Acid & Trace & Negative & Negative & Negative \\
\hline 2 & I,OI 6 & Acid & Trace & Negative & Negative & Negative \\
\hline 3 & 1,026 & Acid & Negative & Positive & Negative & Negative \\
\hline 4 & 1,003 & Acid & Negative & Negative & Negative & Negative \\
\hline 5 & 1,022 & Acid & Trace & Positive & Negative & Negative \\
\hline 6 & 1,022 & Acid & Positive & Negative & Negative & Negative \\
\hline 7 & $\mathrm{I}, 024$ & Acid & Negative & Positive & Negative & Negative \\
\hline 8 & 1,014 & Acid & Negative & Negative & Negative & Negative \\
\hline 9 & $\mathrm{I}, 028$ & Acid & Negative & Negative & Negative & Negative \\
\hline IO & 1,020 & Acid & Trace & Negative & Negative & Negative \\
\hline II & 1,022 & Acid & Negative & Negative & Negative & Negative \\
\hline 12 & I,OI8 & Acid & Negative & Positive & Negative & Negative \\
\hline 13 & 1,024 & Acid & Negative & Negative & Negative & $\mathrm{H}$ and $\mathrm{G}$ \\
\hline 14 & 1,010 & Neutral & Negative & Negative & Negative & Negative \\
\hline 15 & I,OIO & Acid & Negative & Negative & Negative & Negative \\
\hline 16 & $x, 030$ & Acid & Positive & Negative & Negative & $H$ and $G$ \\
\hline$I_{7}$ & $I, 020$ & Acid & Negative & Negative & Negative & Negative \\
\hline I 8 & 1,016 & Acid & Negative & Negative & Negative & Negative \\
\hline 19 & 1,020 & Acid & Negative & Negative & Negative & Negative \\
\hline 20 & 1,026 & Neutral & Negative & Negative & Negative & Negative \\
\hline $2 \mathrm{I}$ & I,OI 2 & Acid & Negative & Negative & Negative & Negative \\
\hline 22 & $\mathrm{I}, 026$ & Acid & Negative & Negative & Negative & Negative \\
\hline$=3$ & 1,020 & Acid & Negative & Negative & Negative & Negative \\
\hline 24 & 1,022 & Acid & Trace & Negative & Negative & Negative \\
\hline 25 & $\mathrm{I}, 022$ & Acid & Negative & Negative & Negative & Negative \\
\hline 26 & 1, oI 8 & Acid & Negative & Negative & Negative & Negative \\
\hline 27 & 1,015 & Acid & Negative & Negative & Negative & Negative \\
\hline 28 & 1,020 & Acid & Positive & Negative & Negative & Negative \\
\hline 29 & 1,004 & Acid & Negative & Negative & Negative & Negative \\
\hline 30 & 1,020 & Acid & Positive & Negative & Negative & Negative \\
\hline $3^{I}$ & 1,020 & Acid & Negative & Positive & Negative & Negative \\
\hline 32 & 1,028 & Acid & Negative & Negative & Negative & Negative \\
\hline 33 & $\mathrm{I}, 026$ & Acid & Trace & Negative & Negative & Negative \\
\hline 34 & I,OI 6 & Acid & Negative & Negative & Negative & Negative \\
\hline 35 & I,oro & Acid & Positive & Negative & Negative & Negative \\
\hline 36 & 1,010 & Acid & Negative & Negative & Negative & Negative \\
\hline 37 & 1,028 & Acid & Negative & Negative & Negative & Negative \\
\hline 38 & $1, \infty 08$ & Acid & Negative & Negative & Negative & Negative \\
\hline 39 & I,OI 8 & Acid & Negative & Negative & Negative & Negative \\
\hline
\end{tabular}

Gastric Contents. - The gastric contents following a test meal have been analyzed by numerous investigators. Lom- 
broso, Filippi and Roncoroni, according to Marie, ${ }^{308}$ made four tests each of the gastric contents of two pellagrins. The test meal consisted of a porringer of soup, 85 grams of meat, 200 grams of bread and roo grams of wine. The length of time the meal was allowed to remain in the stomach is not stated.

The following table shows the results in detail:

\begin{tabular}{|c|c|c|}
\hline Analyses & Case I & Case 2 \\
\hline Reaction of gastric juice. & $\begin{array}{l}\text { Once neutral; three times } \\
\text { slightly acid. }\end{array}$ & Acid. \\
\hline $\begin{array}{l}\text { Hydrochloric acid (Guns- } \\
\text { berg reaction). }\end{array}$ & Always negative. & Always negative. \\
\hline $\begin{array}{l}\text { Lactic acid (Uffelmann } \\
\text { reaction). }\end{array}$ & $\begin{array}{l}\text { Present three times out of } \\
\text { four. }\end{array}$ & Always appreciable. \\
\hline Percentage of acidity.... & Average 60 per cent. & Average 50 per cent. \\
\hline $\begin{array}{l}\text { Peptone (Biuret reac- } \\
\text { tion). }\end{array}$ & Present. & Present. \\
\hline Digestion of starches. & $\begin{array}{l}\text { Twice complete; once } \\
\text { arrested in the second } \\
\text { stage. }\end{array}$ & $\begin{array}{l}\text { Three times complete; } \\
\text { once arrested in the } \\
\text { first stage. }\end{array}$ \\
\hline
\end{tabular}

The following table is part of one quoted by Roberts ${ }^{300}$ from the work of $\mathrm{J}$. Clarence Johnson:

\begin{tabular}{cl} 
Case No. & \multicolumn{1}{c}{ Test meal } \\
$\mathbf{x}$ & $\mathrm{HCl}-\mathrm{R}-$ \\
2 & $\mathrm{HCl}-\mathrm{R}+$ \\
3 & $\mathrm{HCl}-\mathrm{R}-$ \\
4 & $\mathrm{HCl}-\mathrm{R}+$ \\
5 & $\mathrm{HCl}-\mathrm{R}+$ \\
6 & $\mathrm{G}+\mathrm{F} 36 \mathrm{~T} 66$ \\
7 & $\mathrm{G}+\mathrm{F} 38 \mathrm{~T} 74$ \\
8 & $\mathrm{HCl}-\mathrm{R}-$ \\
9 & $\mathrm{HCl}-\mathrm{R}+$ \\
ro & $\mathrm{G}+\mathrm{F} 32 \mathrm{~T} 5$
\end{tabular}

Case No.
II
I 2
I 3
I 4
I 5
I6
I7
I 8
I9
20

Test meal $\mathrm{HCl}-\mathrm{R}+$ $\mathrm{HCl}-\mathrm{R}+$ $\mathrm{HCl}-\mathrm{R}-$ $\mathrm{G}+\mathrm{F} 30 \mathrm{~T} 38$ $\mathrm{G}+\mathrm{F} 24 \mathrm{~T} 42$ $\mathrm{G}-\mathrm{F} \mathrm{I}_{8}-\mathrm{T} 38$ $\mathrm{HCl}-\mathrm{R}+$ $\mathrm{HCl}-\mathrm{R}-$ $\mathrm{HCl}-\mathrm{R}-$ $\mathrm{HCl}-\mathrm{R}-$

$\mathrm{HCl}=$ hydrochloric acid $; \mathrm{R}=$ renin $; \mathrm{F}=$ free (presumably free $\mathrm{HCl}, \mathrm{L}$. T.) ; $\mathrm{T}=$ total acid; $\mathrm{G}=$ Gunzburg's test.

It is not stated what test meal was used by this investigator nor the length of time it remained in the stomach.

Nisbet ${ }^{286}$ made fifteen analyses on ten cases but he also failed to give data concerning the test meal and the period of digestion. The following table is compiled from his findings: 


\begin{tabular}{|c|c|c|c|c|}
\hline Case No. & T. A. & Free $\mathrm{HCl}$ & Ferments & Mucus \\
\hline I. & 30 & ○ & $\mathrm{XXX}$ & Excess \\
\hline 2. . . . & 28 & 4 & Diminution & Excess \\
\hline 3. . & 80 & 35 & Normal & Normal \\
\hline 3. Second analysis....... & 40 & Io & $\mathrm{XXX}$ & Normal \\
\hline 3. Third analysis......... & 28 & 4 & $\mathrm{XXX}$ & Normal \\
\hline 4. $\cdots$ & 33 & 8 & Diminution & Excess \\
\hline 4. Second analysis.. & 24 & o & $\mathrm{XXX}$ & Excess \\
\hline $5 . \ldots \ldots \ldots \ldots \ldots \ldots$ & I8 & 4 & Normal & Normal \\
\hline $6 . \ldots \ldots \ldots$ & $5^{6}$ & 24 & Normal & Excess \\
\hline $7 . \ldots \ldots \ldots \ldots \ldots \ldots$ & 76 & 39 & $\mathrm{XXX}$ & Normal \\
\hline 7. Second analysis........ & 42 & 20 & $\mathrm{XXX}$ & Normal \\
\hline 7. Third analysis......... & 22 & 8 & $\mathbf{X X X}$ & Excess \\
\hline $8 . \ldots \ldots \ldots \ldots \ldots \ldots$ & 40 & 19 & Normal & Normal \\
\hline $9 . \ldots \ldots \ldots \ldots \ldots$ & 50 & 20 & $\mathrm{XXX}$ & Normal \\
\hline ro. $\ldots$. & $\circ$ & $\circ$ & $\mathrm{XXX}$ & Excess \\
\hline
\end{tabular}

It will be seen from the above that the most constant findings in the gastric contents in pellagrins is a diminution in the total acidity and the free hydrochloric acid. This is no more than would be expected from the pathologic findings and cannot be considered at all pathognomonic.

Feces.-The feces of pellagrins vary greatly in consistency and volume. The consistency varies with the degree of diarrhea or constipation, from watery stools to hard stools, and according to Myers and Fine ${ }^{291}$ the daily elimination varies from 60 to 700 grams of moist feces, yielding 20-40 grams of dry excrement, the moisture varying between 75 and 95 per cent. There is usually an increase-in the undigested food particles, especially muscle fibers, starch granules and fats.

The fact that the feces of pellagrins very frequently contain amebas, which are considered by some of etiologic significance, has been mentioned in the chapter on etiology. Other animal intestinal parasites such as uncinaria, strongyloides, trichiuris, ascaris, etc., have been found but are without doubt merely incidental and bear no relation to the etiology of the disease.

The fecal flora of pellagrins has been the subject of study by numerous investigators who have hoped to discover the etiological factor.

$\mathrm{McNeal}^{295}$ found that there were present unusual quan- 
tities of certain types of bacteria which are normally found in the intestinal tract, such as B. bifidus, B. welchii and micrococci, as well as a large number of organisms not found in normal feces. McNeal states that none of these changes appear constant. During the acute attack when diarrhea is present the Gram-positive cocci are nearly always abnormally numerous and Gram-negative bacilli less numerous than normal. McNeal continues that these changes are also observed in the subacute cases and might persist to a slight degree after recovery from the skin lesions. They are nearly constant, but are such as might be expected as a natural result of digestive derangement. This investigator further states that there was no indication that a substitution of normal bacteria by abnormal ones occurred.

Spinal Fluid.-The spinal fluid of pellagrins has been the subject of investigation by several workers.

$\mathrm{Marie}^{308}$ states that the spinal fluid is found to be negative.

Boveri, ${ }^{342}$ on the other hand, found an increase in albumin and a slight pleocytosis in the spinal fluid of pellagrins.

This is somewhat in accord with the findings of Hindmann ${ }^{291}$ who in I9I 2 reported the results of the examination of the spinal fluids of over ninety insane pellagrins. He found an average cell count of 16.69 cells per cubic millimeter, with 64 as the maximum where no complication existed. Seventy-four, or over 87 per cent., of eighty-five specimens showed increase in globulin by Noguchi's butyric acid test.

Later Hindmann ${ }^{343}$ revised his conclusions to a certain extent, stating that some of the cases in which a pleocytosis and increase in globulin exist show positive evidence of syphilis, although "many cases have shown a decided increase in globulin and slight increase in cells, and invariably gave negative Wassermann reactions."

The Illinois Pellagra Commission ${ }^{344}$ found the spinal fluid of pellagrins without increase in the cellular elements and globulin and sterile.

Lorenz, ${ }^{316}$ who has made the most detailed study of the spinal fluid in pellagrins yet reported, after examining ${ }_{5} 53$ fluids from ro6 cases of pellagra reached the following conclusions: 
I. A lymphocytosis of the cerebrospinal fluid does not occur in uncomplicated pellagra.

2. Globulin excess is only occasionally observed.

3. Lange's colloidal gold chloride test is uniformly negative in pellagra.

4. The Wassermann is negative with a few exceptions. In this investigation the exceptions were moribund cases which gave weakly positive reactions with blood serum.

5. The spinal-fluid findings would seem inconsistent with a conception that pellagra is an infectious disease of the central nervous system.

In a limited number of cases the findings of one of us have been in accord with those of Lorenz. In six cases upon whom lumbar puncture was performed at the Arkansas State Hospital for Nervous Diseases the spinal fluid showed no pleocytosis nor increase in globulin and the Wassermann was negative.

Types of Pellagra.-While pellagra is without doubt a morbid entity showing many characteristic symptoms and lesions, it very frequently assumes different forms, certain symptoms predominating, so that various types of the disease have been described. One of the most interesting conditions seen in pellagra is that described as typhoid, the so-called typhus pellagrosus. This is a most unfortunate term, for, while pellagra is occasionally complicated by typhoid, there is nothing in the condition usually termed typhoid pellagra to warrant this name.

This condition is, however, characterized by great prostration. It may begin either as an acute outbreak developing suddenly, or as an incidence to the chronic course of pellagra, or finally it may mark the fatal ending of the disease.

Fever is usually present to a more or less marked degree, the temperature ranging from $99^{\circ}$ to $105^{\circ} \mathrm{F}$. The fever is continuous, without morning or evening variation. The pulse is rapid, rarely falling below I Io, and may run as high as I30-r40. This height is out of proportion to the temperature, which is the reverse of the condition in typhoid fever. The pulse is small and often irregular.

The gastro-intestinal symptoms are accentuated, the sto- 
matitis and gastroenteritis being present to a marked degree. Diarrhea of a persistent type is seen, the stools being as a rule of watery consistency.

The mental symptoms are nearly always aggravated. There may be delirium or there may be stupor.

The nervous phenomena are most marked. Tremors of the hands, tetanic contractions, convulsions, and opisthotonos are seen. There may be incontinence of the urine and feces.

The course of the attack is usually short-from one to two weeks - and nearly always ends fatally, often with a terminal bronchitis.

Recovery from the attack may, however, occur, but there is always a recurrence.

Pellagra sine pellagra, mention of which has been made above, is a condition in which, as the term implies, other symptoms of pellagra than the skin lesions alone are present. Whether or not pellagra can exist without the skin manifestations being present at some time during the course of the disease is a moot point. Lombroso as well as Strambio recognized this condition, the former considering that it appeared only in an hereditary form.

It seems to us that if pellagra sine pellagra does exist it is an extremely rare condition, and that in any given case the skin eruption has either already occurred without recognition and has faded or it will occur. Most cases exhibiting other symptoms of pellagra will, upon a careful anamnesis, give the history of some sort of skin manifestation attributable to pellagra, perhaps a severe sunburn or a roughening of the skin of the elbow.

Other types of pellagra have been described, such as cerebral, spinal, tabetic, spastic, gastric, atrophic, cutaneous, enteric, etc., depending upon the symptoms which are predominant.

Duration.-The duration of pellagra is most variable. The severe acute attacks that sometimes occur. may end fatally in from one to two weeks or the disease, which is, as has been reiterated, a chronic one, may last with recurrences and periods of remission for five, ten, fifteen years or longer. Sambon ${ }^{322}$ states he has seen pellagrins who declared they had had the disease for twenty, thirty or more years. He tells of cases 
eighty years of age who claimed to have been pellagrous since childhood.

Albright $^{345}$ gives the following concerning the duration of the disease:

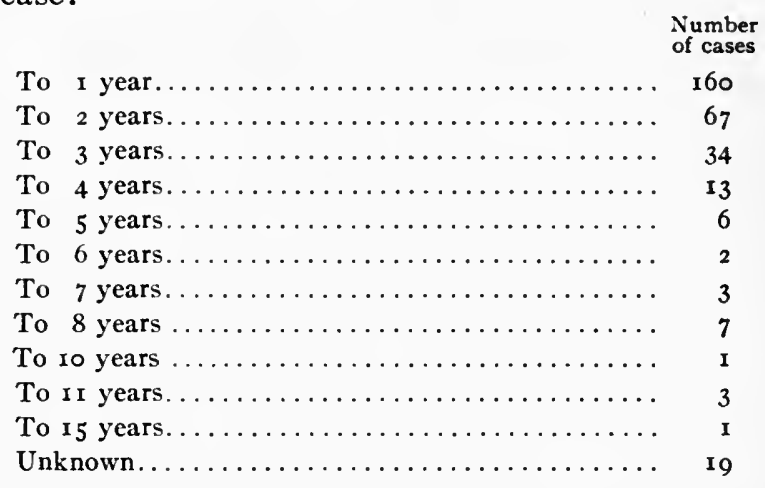

Of course the duration of pellagra will depend upon the severity of the symptoms.

As stated, the acute attacks sometimes terminate in death in as short a time as one week, or they may last as long as three months, a remission following and the disease recurring again the following year.

The number of recurrences will, as can readily be seen, depend upon their severity, the first, second, third or later attack proving fatal, or the attacks may be mild, extend over a period of years, and the patient succumb to some intercurrent disease.

Complications.-As may readily be imagined, a disease so chronic in its nature as pellagra will very frequently be complicated by other diseases. This is certainly true, and there is scarcely an ill to which human flesh is heir which may not be found in conjunction with pellagra.

As has been mentioned above, pellagra has been found very frequently complicated by ankylostomiasis and amebæ by various investigators, and according to Sambon ${ }^{346}$ the infection with hook-worm disease accounts in a large measure for the anemia found in pellagrins. Other intestinal parasites found more or less frequently complicating pellagra are Ascaris lumbricoides, Trichiuris trichiura, oxyuris vermicularis, Cercomonas hominis and Hymenolepis nana. 
Sandwith $^{327}$ says that in Italy pellagra is often complicated by alcoholism, syphilis and malaria, but that in Egypt alcohol plays a small part. He finds it, however, frequently associated with ankylostomiasis, bilharziosis, and favus of the scalp.

Alcoholism is certainly a frequent complication in this country, and Marie ${ }^{308}$ considers that it may be difficult to determine which of the symptoms are due to pellagra and which to alcohol.

Tuberculosis is a rather frequent complication of pellagra and may be found affecting either the lungs or other portions of the body, especially the intestines.

Sambon ${ }^{346}$ states that in Italy pellagra is frequently found in conjunction with scurvy.

That pellagrins are also not infrequently syphilitics is undoubted. This is borne out by the statements of many observers-Marie, ${ }^{308}$ Sandwith ${ }^{327}$ and others.

It has been pointed out above that pellagra not infrequently develops in the insane, even in individuals who have been patients in insane hospitals for many years. It may therefore be said to complicate any of the psychoses. (See page 359.)

Many pellagrous women are sufferers from gynecologic complications, dysmenorrhea, amenorrhea, menorrhagia, leucorrhea, etc., and in male pellagrins a urethritis is not uncommon.

According to Lavinder ${ }^{348}$ certain investigators, especially in Italy, consider that chronic alcoholism may produce in its victims a condition much resembling pellagra, even including skin manifestations similar to those of this disease. This condition comes under the classification of pseudopellagra according to the Italians, but Lavinder considers it a true pellagra.

Roberts $^{300}$ gives the following table showing the complications seen by him:

Number of patients.....................

Pulmonary tuberculosis $\ldots \ldots \ldots \ldots \ldots \ldots \ldots \ldots$ ro

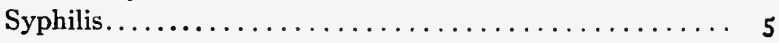

Valvular heart disease.................... Iо

Arteriosclerosis........................ 6

Chronic nephritis.................... I

Enlarged thyroid $\ldots \ldots \ldots \ldots \ldots \ldots \ldots \ldots \ldots \ldots \ldots \ldots \ldots$

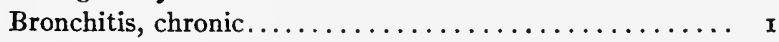

Drug habit.................... I 
Albright $^{330}$ in an analysis of 298 cases gives the following table of complications:

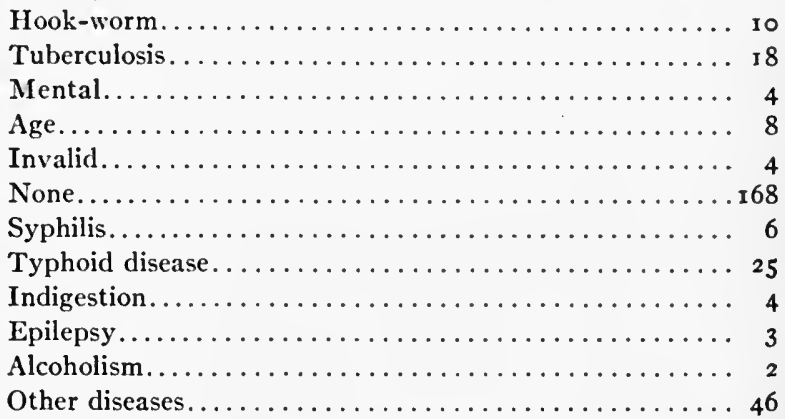

Pregnancy and Pellagra.-Mention of a possible relation of pregnancy in pellagra as a predisposing cause of the malady has been made above.

Saunders ${ }^{286}$ states that I7 per cent. of pregnant pellagrous women are liable to abort, to give birth to still-born infants, and at delivery to post-partum hemorrhage. This author further states that gestation and lactation, especially when frequent, predispose to pellagra, and that parturition is often an exciting cause for the outbreak of the dermatitis.

Recurrences.- - In what has gone before we have made use of the term recurrences in describing the repeated outbreaks of the pellagrous symptoms, but it is not an absolutely proven fact that these repeated outbreaks are recurrences and it is recognized that they may be reinfections.

It seems to us, however, that the well-known fact of certain individuals suffering from repeated attacks of pellagrous symptoms year after year and the comparative rarity of those suffering from one attack and remaining well is almost proof of the theory of recurrent attacks. However, we admit that this question cannot be settled with finality until the etiologic factor is known, or at least until some means of determining when a pellagrin is cured other than the absence of pellagrous symptoms is developed.

Pseudopellagra.-This term was first applied by Roussel to a certain symptom-complex which, while resembling pellagra to a certain extent, was to his mind not related 
to that disease. Pseudopellagra has received scant attention in this country, and such attention as it has received is more or less in the nature of ridicule of the idea. How can there be a pseudopellagra when the essential nature of the disease is unknown? Of course most of those who employ this term believe in the corn theory of the etiology of pellagra and therefore make the disease conform to the supposed etiology. That is, if a symptom-complex resembling pellagra develops in an individual who does not use, nor has not used, maize, it is termed pseudopellagra.

Of course inasmuch as the etiologic factor of pellagra is unknown, we admit it is possible that there are other conditions which resemble this malady but are distinct from it. Thus, if the disease is due to a parasite, there may be similar parasites which cause similar clinical pictures but when the cause is known will be readily differentiated. A similar condition is found in typhoid and paratyphoid infections, which show great similarity-and which are caused by very similar bacteria but which may be differentiated by cultural procedures.

The following case observed by one of us at the Arkansas State Hospital for Nervous Diseases is illustrative:

Case No. I.-J. S. U., white man, aged twenty-two years, single, college student.

Family History.-His father is said to have shown signs of mental derangement for the past ten years, being of a sad disposition and continually wanting to "trade." $\mathrm{He}$ was brought to the Arkansas State Hospital for Nervous Diseases in October, $\mathrm{x} g \mathrm{Ir}$, where he remained for six months when he was discharged. Eight months later he again developed acute mental symptoms and when upon his way to the above hospital escaped and has not been heard of since. The patient's grandmother also was said to have been insane.

Previous History.-No history of previous illness was obtained, the patient stating that he had been healthy all his life.

Present Illness.-The patient states that an eruption on the backs of the hands has appeared each spring for the past six years, seeming to grow a little worse each time. Attacks were accompanied by diarrhea and sore mouth. He says that 
the duration of the present attack has been longer than any other.

Examination July 25, I913.-The patient is not emaciated, the muscles being well formed and firm. The general nutrition is good. The chest is full and well formed, expansion is thoracic-abdominal in character. The lungs are negative to auscultation, palpation and percussion. The heart is normally situated and there are no murmurs audible. His pulse is of good volume, medium tension and regular. The rate is 96 per minute, reclining posture.

The systolic blood pressure is r ro taken in the right arm, patient in reclining posture. The abdomen is negative and the spleen and liver are not palpable. The tongue is moist and only slightly coated and the teeth are in fair condition. The skin is dark over the entire body and is free from eruption, except beginning at the elbow on each arm and extending down over the backs of the hands and forearms there is a dry condition and the superficial layer of the epidermis is exfoliating in places.

'Blood Examination.-Erythrocytes 2,870,000, hemoglobin $5^{8}$ per cent., leucocytes $\mathrm{I} 7,000$.

A diagnosis of pellagra was made, but on account of lack of sufficient evidence no diagnosis of a psychosis was made and the patient was paroled August 2, I9I3. For a few weeks his condition was favorable, but he soon developed symptoms of restlessness and insomnia and attempted suicide, and was therefore readmitted to the hospital November 27, I9I3. At this time his cutaneous manifestations were most marked, and he soon developed diarrhea and fever.

A Widal test was negative as was a Wassermann upon his blood. The spinal fluid showed a negative Wassermann, three lymphocytes per cubic millimeter and no increase in globulin. He was placed upon sodium cacodylate I grain three times a day, but steadily grew worse.

On December I 2, I913, he received 0.3 gram neosalvarsan intravenously. On December 15 this treatment was repeated. December 16 his temperature became subnormal and he gradually passed into coma, dying Dec. I7, I9I3. 
Case No. II.-H. M., white man, aged thirty-nine years, no occupation.

Family History.-Four members of the immediate family have been addicted to the use of morphine. No cases of pellagra have occurred in the family.

Previous Illness.-He has had very little sickness, none of a serious nature. About twenty years ago he began the use of morphine and is now taking 25 to 30 grains daily. He has abused alcohol for a number of years and has been drinking regularly for the past three months. He smokes about a sack of tobacco daily as cigarettes. The regular weight is about 200, now weighs I60.

Present Illness.-About four weeks ago the legs began to ache, then got weak and numb and began to tingle. They pain considerably now. He feels very weak from the knees down but is strong elsewhere. The eruption began on the backs of the hands three weeks ago. Four or five weeks ago the mouth became sore and is still inflamed. He has no appetite and is drinking a pint of whiskey daily. The bowels are fairly regular.

Examination May 6, I9I4.-The temperature is $994 / 5$, the pulse Iro, and the blood pressure 195 . The radials are not palpable. The apex beat is normally located and there are no adventitious sounds, excepting an accentuation of the aortic sound. The lungs are negative. The tongue is coated in the center and red and sore looking along the sides; the gums are sore and there are ulcer patches on the cheeks. The liver is palpable half way between the costal margin and the umbilicus, the upper border being normally located. The knee jerk is absent, the pupils normal, and the Romberg sign is present. The eruption on the backs of the hands is symmetrical, extending from the wrists to the knuckles, being more intense toward the radial sides. There is a suppurating area on the right hand near the base of the index-finger. The urine has a specific gravity of $\mathrm{I}, 02 \mathrm{O}$, acid reaction, contains no sugar but an abundance of albumin and some bile; microscopically are seen numerous cylindroids and a few hyaline casts; the hemoglobin is 90 per cent. and a stained blood film is negative. 
The patient was started on small daily injections of iron cacodylate, but on account of its effect on the urine this had to be discontinued after a few injections, the Hot Springs baths being continued daily. On May 25 a diarrhea began which lasted about a week. By June 2 the hands were nearly healed, Beck's bismuth paste having been used. The mental condition was normal except when the whiskey was diminished a mild delirium supervened. The patient continued to drink and use morphine until in December, I9I4, he was committed to an institution for the cure of those habits. After his release in March, 1915, he remained free from the morphine habit but resumed the abuse of whiskey.

The only symptom which reappeared in the spring of 1915 was the symmetrical lesion on the backs of the hands, and at the present writing the patient is free from any symptoms of pellagra. 


\section{DIAGNOSIS OF PELLAGRA}

Owing to the fact that the etiology of pellagra is unknown there is no laboratory procedure, such as the examination of the blood for a parasite, as in malaria, or the feces for ova, as in hook-worm infection, which will aid in the diagnosis. So it is upon the symptomatology past and present that the physician must rely.

The diagnosis of this disease when the classical symptomsdermatitis, stomatitis, diarrhea and nervous disturbances-are found presents little or no difficulty, but it is in those cases in which some of the above-mentioned symptoms are absent or which are complicated by other diseases that try the acumen of the most experienced physician.

One of the most important factors in arriving at a diagnosis in pellagra is the anamnesis, which should be most thorough. If no eruption or evidence of past eruption is present, most diligent inquiry should be made concerning a severe sunburn or burning sensations of the hands, feet and face. A thorough investigation should be made into a history of sore mouth, coated tongue, indigestion and diarrhea if these symptoms are not present, and finally a thorough interrogation concerning abnormal nervous and mental states, especially depression and excitability, should be made.

Skin Lesions.-As stated above the most frequent location of the skin lesions of pellagra is the backs of the hands, and their most typical feature is their bilateral occurrence, and finally, that the line of demarcation is most clear cut between the diseased and sound skin; and while the erythema does occur on any part of the body surface, the diagnosis of pellagra would be unjustified in the absence of the symmetrical lesions on the exposed surfaces of the body, unless other symptoms are most marked. 
Menage $^{347}$ considers the shriveling and furfuraceous exfoliation of the horny layers of the epidermis described above as absolutely characteristic of pellagra, whether accompanied or not by the whole symptom-complex.

The most common condition which must be differentiated from the pellagrous eruption is the erythema of sunburn. The two conditions are strikingly similar, the pellagrous lesion, however, usually being darker in shade than the sunburn. This is especially noticeable in blonde individuals. The sunburn may be bilateral, on the backs of the hands, and the line of demarcation most distinct. If such is the case, in the absence of other symptoms of pellagra a diagnosis cannot be made at once, and the future course of the erythema must be noted. A sunburn, if not severe, will usually fade in the course of a few days leaving nothing more than a slight pigmentation, while the color of a pellagrous eruption will deepen to a reddishbrown or chocolate shade, and either disappear in two or three weeks with exfoliation of the epidermis or go on to the bullous state. A severe sunburn may result in a dermatitis with vesicle formation, and present an impossible problem for differential diagnosis from pellagra in the absence of other symptoms.

Eczema, especially of the vesicular type, might be mistaken for pellagra, but is differentiated by the persistence of the vesicles and by the distinct line of demarcation in the latter disease.

Erythema multiforme often presents an appearance similar to the pellagrous eruption and is sometimes most difficult of differentiation. Here again the symmetry, the line of demarcation and the symptoms of pellagra not referable to the skin will serve as distinguishing features.

Pellagra may be differentiated from pityriasis rubra pilaris by the absence of itching (usually), the configuration and the associated symptoms.

Not infrequently pellagra may be mistaken for vitiligo, especially if seen during exfoliation. The history of the condition will usually clear up the diagnosis, as vitiligo is as a rule of insidious growth and does not begin with an erythema.

The common roughening of the skin of the backs of the hands frequently seen in men and occasionally in women who are 
continually exposed to the weather has been mistaken for the pellagrous eruption. This error is especially likely to occur if the patient is suffering from stomatitis or diarrhea. The absence of a marked line of demarcation in these cases should serve as a distinguishing feature.

Acrodynia is mentioned by most pellagrographers as a disease which may be mistaken for pellagra. This disease, which is of obscure origin, begins with anorexia, nausea, vomiting and diarrhea, followed by swelling of the face, hands and feet, and injection of the conjunctivæ. Soon symptoms of the nervous system develop, such as prickling and burning sensations. In the beginning there is marked hyperesthesia of the extremities which is followed by anesthesia. Severe pains in the extremities are characteristic. The skin manifestations make their appearance early in the form of erythematous spots, first on the hands and feet, especially on the palms and soles, and later spread to the arms and legs and even to the trunk. The portions of the skin affected desquamate, are thickened and brownish and may even take on a black pigmentation.

It usually runs a favorable course of one or two month ; , but may be fatal in debilitated individuals or in those of advanced years.

Acrodynia should not present much difficulty of differentiation from pellagra owing to the main seat of the eruption (palms and soles) which is rare in the latter disease, the original marked hyperesthesia followed by anesthesia, and the usual short course and favorable outcome.

Pellagra has undoubtedly often been mistaken for syphilis, especially before pellagra was recognized in this country.

The characteristic location of the skin lesions, the absence of history or evidence of a chancre or enlarged glands, and finally the negative Wassermann test (certainly in the vast majority of cases) will serve to differentiate the two conditions.

Scurvy and leprosy have been mistaken for pellagra and vice versa but these conditions should offer little or no difficulty in differentiating when a careful history and examination are made.

Gastro-intestinal Symptoms.-There are very few conditions other than pellagra in which the classic gastro-intestinal symptoms are observed. 
In sprue is found a gastro-intestinal syndrome which in many respects very closely resembles that of pellagra. There is more or less stomatitis, gastric indigestion with distention and diarrhea. In a well-marked case of sprue it is impossible to differentiate the stomatitis from that of pellagra except by observing the other symptoms. The diarrhea is also of a similar nature to that of pellagra, except that it may be the stools are more copious and of a so-called yeasty consistence and the movements occur more frequently in the morning, the patient being undisturbed during the remainder of the day. This is usually not the case in pellagra, where the diarrhea is as marked during the afternoon and night as in the morning.

Of course, where a characteristic skin eruption exists the differential diagnosis will be easy, but if no such eruption is observed and no history of such can be obtained, in short, if a true pellagra sine pellagra exists, the diagnosis may be most difficult.

One distinguishing feature which may be of value is the leucocyte count which in sprue is nearly always low $(6,000$ 2,800 , Castellani and Chalmers), while in pellagra it is usually increased. Finally, if a patient with pellagra be instructed to sit in the sun for a couple of hours with the backs of the hands exposed the characteristic eruption will usually develop.

Dysentery, both amebic and bacillary, may be mistaken for pellagra, and in the absence of skin manifestations in the latter may present some difficulty in differentiation. The dysenteries usually present little or no stomatitis, and as a rule are accompanied by more or less rise of temperature.

Of course the finding of the ameba in the stools, while not proof positive that the patient is not suffering from pellagra, in the absence of skin manifestations and typical mouth findings, should be strong presumptive evidence that the disease is dysentery without pellagra complication. The same may be said of the cultural isolation of the dysentery bacilli.

It is possible that the characteristic odor of the pellagrous stool might serve as a differentiating feature, but too much stress should not be laid upon this.

In these conditions, again, the diagnosis may be cleared up by placing the patient in the sun. 
Nervous Symptoms.-Although nervous symptoms develop in a large percentage of cases of pellagra, there are none which are typical and which in the absence of other findings would justify a diagnosis. Further, they are usually found in the later course of the disease when other symptoms will be manifest or at least a history of such other symptoms may be obtained.

Thus we find disturbances of the lower tendon reflexes, ankle clonus, tremors, pain, vertigo, etc., in many other conditions than pellagra.

Mental Symptoms.-From what has been written in the chapter on clinical history it is evident that there is no typical mental picture in pellagra, that we may have symptomatic depressions or delirious pictures due to the pellagrous toxin or toxins, in short, intoxication psychoses which are in no way characteristic of pellagra but may be brought about by other intoxications. Further that certain definite psychoses, manicdepressive disorders, dementia præcox, paranoid developments, etc., may occur in certain predisposed individuals with the pellagra acting as an exciting cause but in no way differing from these conditions in non-pellagrins. And finally certain mental disorders due to definite brain changes, arteriosclerosis dementia, senile dementia, presenile psychoses and paresis, may be present with pellagra merely as a complication. It is therefore impossible to make a diagnosis of pellagra on the mental symptoms alone, and even in the presence of symptomatic depression or delirium with the absence of other pellagrous symptoms the examiner must look for another cause than pellagra.

It should here be reiterated that the diagnosis of pellagra on one symptom alone usually cannot be made, that the whole history and clinical findings must be considered and a diagnosis made only when the various symptoms can be reconciled to the symptom-complex of pellagra. It must also be remembered that pellagra is very frequently complicated by other diseases, both those that resemble it in some respects and those that do not, which condition will render the diagnosis more difficult. 


\section{CHAPTER XXII}

\section{PROGNOSIS OF PELLAGRA}

Spontaneous Recovery.-That a disease so protean in its manifestations and varying so in the degree of its severity may undergo spontaneous cure is not beyond the range of possibility, although we are unable to find any evidence of its occurrence in this disease. In fact, on the contrary, Lavinder and Bab$\operatorname{cock}^{308}$ state that untreated pellagra "advances inexorably until finally with marked involvement of the central nervous system little can be hoped for from treatment."

That pellagra may vary in its severity in different localities and the prognosis be more favorable in one district than in another is undoubted. It has for some time been considered by most American observers that the disease in this country runs a more fatal course than in Italy, although there is a tendency at the present time among American pellagrographers to consider that the severity of the disease here is gradually decreasing.

There seems to be some difference in the prognosis of pellagra in various races; thus Grimm ${ }^{292}$ graphically shows the death rate from pellagra in the negro to be 44.5 per cent. against 27 per cent. in the white race. This seems to us to be more on account of the usual differences in sanitation between the two races than to a racial difference, for, as will be pointed out later, in those living under insanitary conditions the prognosis is worse than in those living under good sanitary conditions, and as the negro as a rule lives in poorer sanitary surroundings than the white we would expect a worse prognosis in the former.

The prognosis of pellagra in children is as a rule good, as the disease usually runs a milder course. On the other hand, in elderly people the prognosis is bad, the severity of the disease depending to a greater or less extent upon the debilities of the patient incident to old age. 
It has been pointed out that women are more frequently affected by pellagra than men and the death rate is higher also. According to Grimm ${ }^{292}$ the death rate for white females is 28 per cent. and white males 26 per cent., while in the negro race there is more difference, the death rate for females being $5^{\text {I }}$ per cent. against $3^{8}$ per cent. for males.

Occupation. - The only consideration in the prognosis in regard to occupation of the individual is found in the amount of work he has done. Those who have led lives which have debilitated them to a more or less extent are more susceptible to the ravages of this disease.

As stated above, the prognosis in pellagra is less favorable in those living under insanitary surroundings, and as this condition is found to a marked degree associated with poverty it would be expected that in those of poor circumstances the prognosis would be worse than in those better off. Of course it may be contended that this is due to a large extent to the fact that those of affluent circumstances can better afford the services of physicians and can receive better nursing than those of poorer classes. While this undoubtedly is true to a certain extent, it would seem that the surroundings of the individual with regard to sanitation have more bearing upon the subject.

It has been pointed out that the majority of cases of pellagra develop in the spring of the year, and it is also a fact that the advent of cold weather has a favorable effect upon this disease; therefore it would seem, although we have no figures to substantiate this statement, that cases developing in the fall would have a better prognosis than those developing in the spring.

That the occurrence of previous diseases in an individual may have a bearing upon the prognosis of pellagra can be easily imagined. It has been pointed out above that pellagra not infrequently follows such diseases as typhoid fever, malaria, etc., and it would seem that individuals contracting pellagra following diseases of a debilitating character would give a less favorable prognosis than those in whom the disease developed without previous illness. 
The knowledge of the habits of an individual is of more or less importance in making a prognosis. Alcoholics and those who have indulged in venereal excesses are of necessity less resistant to any form of disease than those who have lived temperate lives, and therefore in the former class of individuals the prognosis is more grave than in the latter.

It is natural to consider that the complication of pellagra by other diseases would tend to make the prognosis more grave. This is indeed the case, and we find that the chances for recovery in pellagra are less when this disease is associated with other maladies.

The associated infection of intestinal parasites, such as hookworm and amebæ, with pellagra undoubtedly renders the prognosis more grave, partially, at least, owing to infecting organisms attacking the intestines which are the seat of so much of the pathology of pellagra.

Niles $^{305}$ states that pellagra when found in hard drinkers almost invariably runs a speedy and fatal course. This undoubtedly is generally true, but one case seen by one of us is an exception. This patient, a man of 39 years of age, developed the symptoms of pellagra in the spring of $19 \mathrm{r}_{4}$ with the characteristic findings-stomatitis, diarrhea and dermatitis on the backs of the hands. He was at that time consuming a quart of whiskey per day and also taking 25 to 30 grains of morphine in the twenty-four hours. Under treatment the symptoms and lesions of pellagra cleared up with only a slight recurrence of the dermatitis in the spring of $\mathrm{I} \mathrm{I}_{5}$, which lasted but a short time and disappeared. When last seen he was still consuming the above-mentioned amount of whiskey and had no evidence of pellagra.

The complication of pellagra by tuberculosis, whether of the lungs or of other portions of the body, is nearly always rapidly fatal. The development of nearly any of the infectious fevers, such as typhoid, in pellagrins nearly always makes the prognosis extremely grave.

Most observers state that when pellagrins show nervous and mental symptoms the prognosis is rendered less hopeful. This undoubtedly is true of those psychoses due to the pellagra 
toxin or toxins, the symptomatic depressions and delirious pictures, and also no doubt of those psychoses due to changes in the brain-arteriosclerotic dementia, senile dementia, paresis, etc.- and perhaps also of idiots and imbeciles in whom pellagra develops, but we do not believe pellagra associated with dementia præcox or paranoia, for example, need prove any more fatal than pellagra in normally mental individuals. There is one exception to this statement, however, and that is the more frequent suicidal intent of insane individuals than those of normal mentality.

The prognosis of pellagra in pregnant women is more grave than in non-pregnant women. This is due, partially, at least, to the liability of pregnant pellagrins to abort as pointed out above.

The gravity of the skin lesions seems to have little or no effect on the prognosis, those with the most severe skin manifestations often making complete recoveries. This is more especially true of the "dry" lesions, as the "wet" type usually are associated with more serious general symptoms. The appearance of so-called typhoid pellagra is to be considered as of most grave import, as most cases go on to a fatal termination. The development of fever in the course of the disease presents a grave condition and should be looked upon with concern.

Continued and severe diarrhea is one of the gravest symptoms as it usually leads to marked emaciation. The appearance of nervous symptoms such as tremors, absence of knee jerks, ankle clonus, etc., is of grave import and, according to Wood, ${ }^{297}$ the appearance of a coarse clonic contraction of the muscles of the forearms is the most positive indication of the approaching end.

As in nearly all other diseases the prognosis depends considerably upon the promptness of the diagnosis. The earlier the diagnosis the earlier treatment may be instituted, and certainly more treated cases recover than untreated ones.

It must be considered, however, especially in our present state of knowledge of pellagra, that any outbreak of this 
disease, no matter how mild, is not to be considered lightly, and that the most diligent methods of treatment are sometimes of no avail.

Mortality.-As pointed out, the death rate from pellagra in this country is higher than in Italy, although according to some observers it is decreásing.

Lavinder ${ }^{354}$ quoted Lombroso as stating that in 1883 there were treated in 866 Italian civil hospitals 6,025 pellagrins of whom 923 died, while in 1884 there were 6,944 cases treated in 993 hospitals, 790 dying, making in a large number of cases a death rate of about 13 per cent.

Wollenberg ${ }^{255}$ reports a total of 55,029 cases of pellagra in Italy in 1905 with a total mortality of 2,359 , which is a little over 4 per cent.

Sandwith ${ }^{327}$ states that of his cases at Kasr el Ainy 18 were cured, 72 relieved, 6 unrelieved and 4 died, making a mortality of 4 per cent.

Statistics in this country are based largely upon insane hospital cases in which the mortality is very high in spite of the fact that in these institutions better facilities for treatment are usually found than outside them. This high death rate of hospital cases is no doubt due to the fact that pellagra cases do not as a rule enter hospitals until they have reached the less hopeful stages of the disease.

According to the Illinois Pellagra Commission, ${ }^{356}$ in 258 cases at the Peoria institution there was a death rate due to the pellagra per se of 49.6 per cent.

Searcy ${ }^{357}$ reported 88 cases occurring in the Mount Vernon Insane Hospital in 1906 , of which 57 or 64 per cent. died.

Harrington ${ }^{291}$ states that of 32 cases occurring in the State Hospital for the Insane of Rhode Island I 2 died, giving a mortality of 37 per cent.

Of the 9I cases seen by one of us at the Arkansas State Hospital for Nervous Diseases, 57 or 63 per cent. died.

The Thompson-McFadden Pellagra Commission ${ }^{295}$ gives the following table of cases and deaths, as far as could be determined, occurring in Spartanburg County from 1894 to I 9 r 2 inclusive: 


\begin{tabular}{|c|c|c|c|c|}
\hline Year & Number of deaths & & Number of cases & Per cent. of deaths \\
\hline 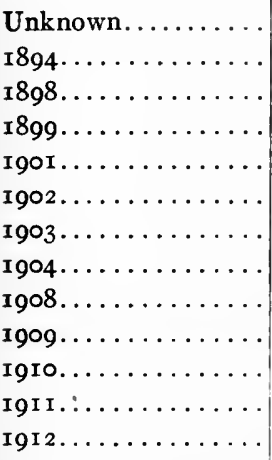 & $\left.\begin{array}{r}8 \\
I \\
I \\
I \\
2 \\
2 \\
1 \\
I \\
2 \\
14 \\
32 \\
54 \\
47\end{array}\right\}$ & 25 & $\begin{array}{l}\text { I I } 5 \\
285 \\
376\end{array}$ & $\begin{array}{l}28 \\
\text { I } 9 \\
\text { I } 2\end{array}$ \\
\hline
\end{tabular}

This shows that the number of cases is increasing but the percentage of deaths is decreasing.

Grimm $^{292}$ of a total of $\mathrm{I}, 426$ cases gives a death rate of 30.2 per cent., while Lavinder ${ }^{358}$ reports $19,9{ }^{1} 5$ cases with 8,085 deaths, or a mortality of 40.59 per cent.

Beall ${ }^{359}$ states that the prevalence of pellagra in the United States is decreasing, although he estimated the death rate to be over 25 per cent. 


\section{CHAPTER XXIII}

\section{PROPHYLAXIS OF PELLAGRA}

The methods of prophylaxis proposed for pellagra are many and are based to a large extent upon the theories of etiology as upheld by those proposing them. In Italy, where the spoiled maize theory is almost universally accepted, the most thorough and extensive prophylactic measures have been instituted with this theory as a basis. These measures are directed against the sale or use of spoiled corn.

According to Sambon ${ }^{360}$ these measures are:

I. The inspection of maize, the seizure of unsound grain and its products.

2. The exchange of deteriorated maize for good maize.

3. The providing of drying apparatus for damp maize.

4. The providing of suitable ovens in rural districts for the proper baking of maize bread.

5. The abolition of late varieties of maize which do not ripen properly.

6. The compulsory notification of all cases of pellagra.

7. The obligation, in all affected districts, upon the municipal authorities to supply free meals to all their pellagrins twice every year for periods of not less than forty days.

8. The institution of special retreats, "pellagrosari," for the housing, feeding and treatment of the more advanced cases.

9. The dispensation of free salt to all pellagrins and their families.

This author continues with statistics and conclusions of Professor G. Sanarelli, Under-Secretary of State for Agriculture, of Italy who claimed that beneficial results were seen almost immediately after the application of the law of 1902, stating that the pellagrins within the kingdom were reduced to barely 55,000 in 1905 , showing a reduction of over 17,000 in the last six years. Further figures by Sanarelli show that the victims 
of pellagra in Italy in the three years $1897-99$ were 10,284; in $1900-02$ they fell to 9,219 ; in $1903-05$ they declined to 7,267 ; while in the three years I906-08 the number was only 4,649 .

More comforting to Sanarelli was the fact that in 1907 $4,95^{\circ}$ new cases were notified, while in 1908 only 2,846 were reported, and that before the application of the present law the yearly mortality from pellagra reached or exceeded 3,000 , while immediately after 1902 the number of deaths only just exceeded 2,000 , in 1907 were 1,635 , and in 1908 were only about 1,000 .

The conclusion reached is that these measures have reduced the number of pellagrins by 50 per cent., and that if the law of I902 "had only been fully and vigorously enforced in all the forty-four provinces of the kingdom more or less severely affected by the fatal disease, at this hour (rgog) the whole of Italy would be entirely free."

Sambon then attempts to prove that instead of the law of 1902 bringing about the results claimed by Sanarelli, it "failed to produce any results at all." He states that the decline in the number of cases and deaths had already begun in a very striking manner some years before the law was enacted, and further that the law of 1902 did not come into effect until three years later. Then Sambon quotes the remarks of Sanarelli made upon another occasion in which he directly contradicts himself, after giving statistics, by remarking: "A legitimate doubt, therefore, arises as to whether it be right to ascribe the gradual decline of pellagra throughout the kingdom to the measures contained in the law of $1902 . "$

Following this Sambon goes into the details of the prophylactic propaganda of the Italians and proves, to his own mind at least, that they have failed entirely to prevent pellagra.

While the bulk of evidence is against the theory that corn is responsible in any way for the spread of pellagra the evidence is negative, and certainly no other theory has been proven correct. And further since spoiled maize is unquestionably not suited for human consumption, whether or not it has anything to do with pellagra, an effort should be made to have only sound corn used for food.

While Sambon has advanced and defended the theory of the 
transmission of pellagra by the Simulium, he has not, as far as we are aware, proposed any definite measures of prophylaxis.

Wood, ${ }^{297}$ who considers this theory the most interesting one at this time, states that as the buffalo fly is a field pest its control would be most difficult if not impossible. However, should the Simulium finally be proven guilty of being the intermediary host of pellagra, such measures as screening, destruction of larvæ, etc., could be instituted. The spread of the knowledge of the connection of this insect with pellagra would in itself make those who come into contact with it wary of its bite and certainly lessen the number of cases.

Should the stable fly (stomoxys calcitrans) prove to be the intermediary host, as suggested by Jennings and King, ${ }^{295}$ the prophylaxis of pellagra would become comparatively simple, first by screening and second by the destruction of the larvæ and eggs of this insect in manure.

The contagiousness of pellagra has been discussed in the chapter on etiology, and as no evidence has been produced to show that this disease is contagious the quarantine or isolation of pellagrins would be unwarranted at this time. However, owing to the possibility of pellagra being infectious, we consider that such close contact as kissing of pellagrins should be avoided, and further, owing to the rather strong evidence that if pellagra is an infectious disease the primary location of the infecting organism is in the gastro-intestinal tract, we consider the effective disposal of the excreta of pellagrins of importance. Also we consider the destruction of the scales and flakes of epidermis which come from the skin lesions during the desquamating stage as not out of place. At least such proceedings can work but little hardship upon either the patient or his attendants and possible sources of the spread of the disease will be removed.

The removal of secondary or alleged predisposing causes of pellagra in endemic districts would certainly not be out of place. One of the most important of these, as pointed out above, is alcoholism, which, even if it is not a predisposing cause of pellagra, is a bane upon civilization and should be destroyed.

Other predisposing causes mentioned are other previous and 
concomitant diseases, such as malaria, typhoid, hook-worm, etc.; so measures instituted against these diseases might in a measure, at least, react against pellagra.

The theory of Allesandrini and Scala that pellagra is due to certain silica compounds contained in drinking water, while brilliant in conception, lacks confirmation. If, however, it should be proven correct, the prophylaxis of the disease would be most simple, namely, the substitution of a water free from these substances.

Goldberger, ${ }^{261}$ whose deficiency diet theory has been discussed in the chapter on etiology, states that "the prevention and eradication of pellagra will depend essentially upon the substitution of a mixed, well-balanced, varied diet for the restricted, one-sided diet that the individual will be found to have consumed prior to the development of symptoms."

He further states that the amount of corn or other starchy foods should be reduced. The most important change in the diet, however, is an increased consumption of fresh (lean) meat, milk, eggs and legumes ("beans and peas, fresh or dried but not canned"). This should be carried out especially in the late fall, winter and early spring months.

The poor, especially the poor of the South, should be encouraged to cultivate beans and peas and preserve them by drying for winter consumption.

Goldberger states that at orphanage "B. H." there were seventy-five cases of pellagra in the spring of 1913 , following apparently milder outbreaks in I9I I and I9I2. A modification of the diet along the lines mentioned was instituted and not a case was known to have developed in the spring and summer of I9I4 among a total of 234 children of the orphanage. He further states that there is reason to believe this experience is not unique.

In the insane hospitals care must be taken to see that not only is a well-balanced, mixed and varied diet furnished to the patients, but that they actually eat it.

Whether or not Goldberger's theory is proven true, we consider his tenets as to diet sound, and especially in the absence of proof of any other etiologic theory they should be carried out. 
The public health aspect of pellagra assumes the proportions of a colossus. The disease should be made reportable where it is not so, and in every state where it is endemic appropriations should be made for the carrying on of study of it; not only an attempt to ascertain the etiology, but a study of the epidemiology, symptomatology, prophylaxis and treatment should be made.

The laity should be informed of what we already know of the disease, both by pamphlets and lectures, and should be encouraged in instituting such prophylactic measures as are outlined above. 


\section{CHAPTER XXIV}

\section{TREATMENT OF PELLAGRA}

The methods of treatment of pellagra are as varied as are the theories of its etiology, and as may readily be imagined many of them are of no avail.

The methods of treatment may be discussed under three headings, namely: hygienic treatment, specific treatment (so called), and symptomatic treatment.

Hygienic Treatment.-The first requisite in the treatment of pellagra is that the patient be placed under sanitary surroundings. If he is ambulatory he should take a certain amount of exercise daily, but should avoid the direct sunlight except in certain nervous and mental conditions where no skin lesions have been manifest for several years, in which sunlight seems beneficial. In all acute cases and those with fever the patient should be put to bed, in which case, or if he is already confined to bed, his room should be well ventilated but with the light subdued. It goes without saying that bed patients should receive the best of nursing and their beds kept clean and fresh. Change of climate has been noted by many observers to be very beneficial to pellagrins, the change from a warm climate to a cold one being most recommended, although it would seem that a change of surroundings, per se, is helpful. In this connection Bass $^{362}$ has recommended an artificial refrigerated ward, which he states could be equipped for $\$ 3,000$ and maintained at an expense of \$I per day per patient in addition to the regular expense.

Hydrotherapy of various forms has been recommended in pellagra. Sufficient bathing for cleanliness is of course to be carried out in all cases.

Other hydrotherapy must be governed by the condition of the patient. In children and in elderly individuals warm baths should be employed. In weak individuals the treatment should 
be begun with a warm bath of short duration and the temperature reduced and the time prolonged with the improvement of the patient. Massage during the bath is often beneficial. The use of salt baths or rubs is often recommended, from which children derive the most benefit. The patient should be placed in a tub of warm water and after the body is wet all over should stand up in the tub and the attendant, after wetting the hands, takes a handful of salt (any good fine cooking salt will do) and thoroughly, but not roughly, rubs the entire body with the salt for fifteen to twenty minutes, after which the patient lies down in the water, the salt is washed off and if the patient is sufficiently robust a cold douche is given, after which he is put to bed for a time. A shower bath is perhaps more satisfactory than a tub for this purpose. Medicated baths of various kinds, such as arsenic and sulphur, are sometimes used, but are of doubtful value.

Baths in water with radio-activity, such as at the Hot Springs of Arkansas and Virginia, have proven beneficial in certain cases. These baths should of course be controlled by the condition of the patient, and should be given in conjunction with other methods of treatment.

Whether or not we accept the teachings of Goldberger concerning the etiology of pellagra, the diet he recommends as treatment is far from undesirable. It has long been recognized that, inasmuch as pellagra is usually found in the undernourished, one of the first and most essential procedures in the treatment is to prescribe good, wholesome, and nourishing food.

The diet recommended by Goldberger ${ }^{361}$ is as follows:

Milk.-Fresh milk, alone or in alternation with buttermilk, should be given freely. An adult should be urged to take not less than a pint and a half to two pints in twenty-four hours.

Eggs.-Fresh eggs should be allowed freely. In addition to the milk and meat, an adult should take not less than four eggs a day. In certain of the severer forms it may be necessary to give the eggs in the form of albumin water.

Meat.-The meat should be fresh lean meat. Whether all fresh meats are equally valuable in treatment we do not know; future studies will have to determine this. Our experience has 
been with beef alone. This may be served as scraped beef, as a roast, or as steak. Where mastication is painful, meat juice may be given instead. An adult should be urged to take at least a half pound of lean meat a day in addition to the milk, eggs, and legumes. It may be necessary in some instances to work up gradually to the point where these quantities can be taken.

Legumes.-We have been much impressed with the favorable results following the use of beans and peas alone. The beans and peas should be fresh or dried, not canned. A palatable pea or bean soup should be prepared and may be given freely. In addition to or in alternation with the soup the beans or peas should be served and eaten in any one of the other well-known forms.

To this diet of Goldberger we feel that little can be added. We wish to say, however, that in view of the absence of proof as to the etiology of pellagra and out of deference to the zeist theory, although we do not subscribe to it, we are accustomed to advise our pellagra patients to abstain from eating corn in any form. This can work but little hardship and adds an element of safety until the exact etiology is learned.

Specific Treatment.-The so-called specific treatment of pellagra consists of the empirical administration of various medical substances upon the theory that they are specifics for the hypothetical microörganism of the disease; the practice of transfusion of blood and the administration of blood serum from cured pellagrins upon the theory that "antibodies" against the hypothetical microörganism are being introduced; and finally, the practice of auto-serum therapy.

Lavinder and Babcock ${ }^{308}$ quote Holland as having written in 1817 : "In short, it appears certain that mere medicine has done very little for the relief of pellagra; and Strambio frankly confesses that he never saw a case distinctly cured by the remedies that were employed."

These writers then state that it was Lombroso who first employed arsenic in the treatment of pellagra. The idea of the great Italian was not, however, that arsenic is a true specific for the disease, that it did not cure all cases, but that it was a 
valuable remedy acting as a sort of antidote for the toxins of spoiled maize. Lombroso's method of administration of arsenic, according to Lavinder and Babcock, was in the form of Fowler's solution in dosage of $5,10,15,20$, and 30 drops, or as pure arsenous acid (arsenic trioxide) dissolved in slightly alcoholized water, in doses of $1 / 40$ to $1 / 20$ milligram, increasing according to tolerance to $0.001,0.002$, or 0.003 gram and very rarely even o.or gram. The drug is discontinued for a few days from time to time.

Lombroso cautions against certain untoward effects of the arsenic and mentions certain types of the disease which are benefited by the treatment and certain types which are not. Symptomatic and dietetic treatment are also recommended by the Italians.

Other methods of administering arsenic in pellagra than by mouth have been employed by many investigators. Thus Babes, according to Wood, ${ }^{291}$ was the first to use atoxyl in the treatment of this disease. The latter writer has had a wide experience in the use of this drug, recommending its use in dosage of 5 to 7 grains every fourth day in the beginning and later increasing the length of the interval.

The untoward effects sometimes seen, such as chills, fainting, headache, blindness from retrobulbar neuritis, colic, loss of appetite, albuminuria, etc., have not been observed by Wood, although expected and looked for. He attributes this to the careful preparation of the solution of the drug without the use of heat which it is claimed will decompose it. Wood reported very favorably upon the use of atoxyl, but stated that he was instituting the use of soamin because it is said to be less toxic.

Soamin has been used very extensively by many investigators with more or less satisfactory results. Martin ${ }^{363}$ reported its use in six cases of pronounced pellagra and five of suspected pellagra. In the latter the suspicious symptoms were promptly relieved by a few injections of soamin, and of the six pronounced cases all but one, who died within a week after the institution of treatment, made apparent recoveries. Martin injected this drug in 3 - to 5-grain doses on alternate days, giving 100 grains as a maximum for one course of treatment. 
Probably no other drug has ever received the attention that Ehrlich's salvarsan or "606" has, and it is natural to suppose that a drug which contains arsenic and is said to be less toxic than any other so far produced should, in view of the results obtained with other arsenical preparations, be given a trial in pellagra.

As far as we are able to determine, Nice, McLester and Torrence $^{364}$ were the first to report on the use of salvarsan in the treatment of pellagra. These workers reported apparent recoveries in three cases.

Cole and Winthrop ${ }^{365}$ collected the reports of twenty-one cases of different investigators with only $33^{1 / 3}$ per cent. improvement lasting seven or more days.

King and Crowell ${ }^{366}$ reported nineteen cases in which as a rule three doses of salvarsan were administerea ten days apart with apparent recoveries in all but one case.

Cranston $^{367}$ administered salvarsan to eleven pellagrins in I9II with the following rather discouraging results: Two left the hospital clinically cured; one was considered well until nausea developed a few days before the report was made and was therefore considered suspicious; three relapsed and were treated a second time; one was unimproved and four died.

Probably the most enthusiastic supporter of salvarsan in the treatment of pellagra is Martin ${ }^{368}$ who considers this drug a true specific for the hypothetical microörganism of the disease. This investigator has used salvarsan in a great many cases (he does not state how many) and contends that one may confidently expect to cure over 80 per cent. of cases by the use of soamin or salvarsan or both.

Martin's method of procedure is to administer salvarsan intravenously in doses of 0.1 gram per 20 pounds of weight, at weekly or ten-day intervals until no so-called endotoxin reaction occurs. He claims perfect cures in favorable cases (he states he is unable to say why favorable) after three or four doses, but considers that six, eight or ten doses will usually be required. Martin states that occasionally salvarsan seems to cause a recrudescence of the disease, and that several days following the first or second dose the mouth becomes red, diar- 
rhea begins and new skin lesions occur. This, Martin thinks, is an indication that a specific drug has been used, though in insufficient dosage.

In a comparatively small experience with salvarsan in the treatment of pellagra we are forced to the conclusion that it does not act as a specific. One case treated by one of us in the spring of IgII is very much to the point.

This patient, a man of forty-nine years, had suffered with diarrhea for two summers, accompanied by a breaking out on the backs of the hands. When examined there was a characteristic eruption on the backs of the hands extending about 4 inches up the forearm. Marked diarrhea was present, there being an average of fifteen bowel movements per day. Three doses 0.6 gram of salvarsan were administered intravenously ten days apart with absolutely no improvement. The patient grew steadily worse and died three weeks after the administration of the last dose of salvarsan.

We are of the opinion that arsenic in any form, which exerts no untoward effect, is to be recommended in pellagra. This we do not consider as acting so much in the nature of a specific as in the nature of a tonic and alterant, although we are not prepared to say that it does not have specific action. We have found that sodium cacodylate has given the best results in our hands. This is administered intramuscularly, usually in 3grain doses daily. The dosage may, however, be increased, we having given as high as 9 grains daily without any untoward effects.

A number of investigators have reported favorable results from the use of hexamethylamine. Other drugs, sodium chloride, gelsemium, potassium permanganate, thyroid extract, calcium sulphide, castor oil, nitric acid, opium, etc., have had their advocates, but do not seem to offer much.

Dyer $^{373}$ advocates very strongly the use of quinine hydrobromate and considers it as much of a specific as any drug so far proposed. It is given in doses of 2 to 5 grains three times a day in mild cases, and as much as Io grains every two to three hours in severe cases. Dyer states that he has given as much as ro grains every three hours night and day for four to five 
days and that he has never lost a case of pellagra treated entirely and throughout by himself.

Recently Page ${ }^{320}$ has advocated the use of ichthyol, and states that one or two 5-grain pills three or four times a day for three weeks seems to cure the average case.

We have had experience with this drug used in this manner in but one case, but have been informed that at the Arkansas State Hospital for Nervous Diseases, it was used in several cases with apparently no beneficial results.

According to Cole and Winthrop ${ }^{369}$ Antonini and Marianni secured curative results in pellagra by the injection of blood serum from cured pellagrins.

Cole $^{370}$ early in I 909 published the results of blood transfusion from a cured pellagrin into a case which was considered absolutely hopeless, but which completely recovered.

In I910 Cole with Winthrop ${ }^{369}$ reported six recoveries in eleven cases. These investigators considered that if the blood serum of cured pellagrins contained specific antibodies the whole blood would also contain them, as well as help replenish the impoverished blood stream of the patient. They point out four dangers of transfusion, aside from the technic difficulties, as follows: the transmission of disease, hemolysis, agglutination and acute dilatation of the heart.

Later these workers ${ }^{311}$ reported twenty cases of pellagra in whom transfusion was performed with twelve recoveries. At this time, however, they stated that it seemed to make no difference whether the donor was a cured pellagrin or any other healthy individual.

According to Castellani and Chalmers ${ }^{372}$ a most unique method of treatment has been devised by Nicolaidi. This consists of horse-serum together with all the organic and mineral salts of the blood, in a solution saturated with carbon dioxide gas, which is then rendered radioactive. The preparation is administered by injections until twenty or twenty-five are given. Neither dosage nor the method of injection is stated. Remarkable improvement is reported by Nicolaidi and according to Castellani and Chalmers his results are supported by several eminent authorities. 
Palmer and Secor ${ }^{375}$ after a short review of the literature of auto-serotherapy in other diseases report the treatment of seven cases of pellagra by this method with most gratifying results. Two cases, according to these authors are well, having passed a spring and fall season without recrudescence of the disease. Two others are apparently well but as yet have not passed the critical seasons, while the other three showed marked improvement but have been lost track of.

The technic employed is to apply a piece of cantharides plaster $1 \frac{1}{2}$ inches square, smeared with olive oil, to the chest at bedtime. In the morning a blister will be raised. Without entirely removing the plaster a hypodermic needle is thrust into the blister from an upper corner, I c.c. of serum withdrawn and injected into the arm. No visible reaction should occur.

Palmer and Secor state that their therapy is based upon the theory that the beneficial effects derived from the old-time blister in pneumonia were not entirely due to the counterirritation but that antigens were produced in the serum and reabsorbed, thus stimulating the production of antibodies.

We have seen this method tried in one case with apparently good results, although this patient was at the same time under treatment with sodium cacodylate. We consider this treatment rational and as it is very simple and devoid of much pain and discomfort to the patient, should be given a thorough trial.

Instead of cantharides plaster, however, we recommend the application of cantharides collodion.

Symptomatic Treatment.-The skin lesions are treated according to their severity, and it is best to err on the side of too little treatment rather than too much. In the beginning when there is nothing but an erythema a dressing of boric acid solution is quite sufficient. Later when the eruption has started to desquamate zinc oxide ointment should be applied. The main requisite in this condition to our minds is to exclude the light, for which purpose zinc oxide has been found by one of us best suited. By coating photographic plates with various substances, zinc oxide, ich thyol, bismuth subnitrate and belladonna ointment, exposing them to the light for several minutes, removing the coating and developing, those covered with the 
zinc oxide showed almost absolute exclusion of the light while the other substances permitted more or less light to penetrate. For the so-called "wet" lesions ordinary surgical treatment should be employed. Moist dressings of bichloride (I to $5, \infty 00$ ) or I per cent. p cric acid are good. If there is secondary infection with pyogenic organisms the parts should have hydrogen peroxide applied in dilute solution once daily and aristol or some similar powder dusted over them.

Of the gastro-intestinal symptoms the anorexia and nausea are usually relieved by nux vomica, condurango or some other bitter. Leroy ${ }^{374}$ has recommended a mixture of cerium oxalate, chloretone and bismuth subnitrate in troublesome vomiting.

For the stomatitis if ulceration exists daily application of silver nitrate solution (4 per cent.) will be of benefit. An astringent mouth wash such as the following should be used:

R. Potassii chloratis............ 3 ii

Tinct. myrrhæ. . . . . . . . . . f $\mathfrak{y}^{\mathrm{s} s}$

Mellis depurati...............f f 5 ss

Aquæ camphoræ............. q.s. ad f 5 viij

Sig.-Shake; use as mouth wash, t.i.d.

If salivation exists atropin $1 / 200$ grain every four hours until checked, may be used.

For the gastric symptoms when there is a deficiency of hydrochloric acid, this drug may be administered ro- 5 drops of the official dilute t.i.d. after meals. Pepsin also may be given.

Many drugs have been advocated for the diarrhea, bismuth in some form being most frequently used. Ergot, albumin tannate, and salol are also extensively employed.

Dyer ${ }^{373}$ states that the quinine hydrobromate he recommends as a specific will control the diarrhea usually within the first five days. Tablets of B. bulgaricus have been used by one of us for the control of the diarrhea but without very flattering results.

When all other means fail opium in some form may be given.

Where constipation is present instead of diarrhea it may best be controlled by castor oil. Phenolphthalein or sodium phosphate may also be employed.

Of the nervous symptoms insomnia will usually be overcome 
by the administration of chloretone in doses of 3 to 5 grains. Trional, veronal or sulphonal may be used. For the pains and burning sensation phenacetin, aspirin or some other salicylate may be administered.

The various mental symptoms can be treated satisfactorily only in a hospital for the insane. The depressed conditions improve with the general condition. For the delirium small doses of deodorized tincture of opium is best.

The other mental conditions associated with pellagra but not directly due to the toxins must be treated as if the pellagra were not present.

An important part of the treatment of pellagra is that which is carried out between attacks. The patient should be under observation and the general condition should be made as good as possible, with careful diet, exercise, tonics, etc. A careful record of the weight should be kept as the season approaches for the attack, the development of slight symptoms should be noted and treated at once. If possible a change to a colder climate is highly to be recommended.

It goes without saying that concomitant diseases should be recognized and treated. Thus in the advent of malaria, quinine should begiven, hook-worm should be treated with salol, and cases with amebiasis should receive emetine. All major surgical procedures, except those absolutely necessary, should be postponed until the symptoms of pellagra subside. 


\section{AMEBIC DYSENTERY}

\section{CHAPTER XXV}

\section{INTRODUCTION}

Amebic dysentery is an infectious disease, caused by a specific protozoal microörganism, the Endamœba histolytica, characterized clinically by abdominal pain, diarrhea and tenesmus, anatomically by ulceration of the colon, sigmoid and rectum, with a tendency to recurrences and chronicity and to the formation of hepatic abscess.

Amebic dysentery is also known as amebic enteritis, amebic colitis, amebiasis, tropical dysentery, amöbenruhr, dysenterie amibienne and dysenterie à amibes.

History.-The history of amebic dysentery may well be said to begin with $\mathrm{Lambl}^{376}$ who in 1859 discovered a minute, motile, unicellular organism in the intestinal mucus removed at necropsy of a child dead of diarrhea. Lambl described the organism as being roughly spherical in shape when at rest, of $0.009 \mathrm{~mm}$. by $0.016 \mathrm{~mm}$. in size, but assuming an elongated shape when in motion. Motility was attained by throwing out club-shaped pseudopodia which were of the same substance as the body. Lambl observed vacuoles in the body of the protozoon and occasionally very minute ones in the nucleus. The peculiar vibration of the granules in the protoplasm was noted. Both classes of movement were stated by this investigator to be very marked at first but to cease gradually within a few hours.

While Lambl did not definitely incriminate the organism he described as the cause of dysentery he did state that its importance should not be underrated.

The importance of Lambl's work was unrecognized and in I870 Lewis and Cunningham ${ }^{377}$ described quite fully certain amebæ found in the feces of cholera patients but ascribed to them no pathologic significance. 
In 1875 Loesch $^{378}$ published an account of the findings of ameba in the feces of a patient with dysentery. This patient had been suffering intermittently with the disease for two years and finally succumbed. Just before death the organisms were absent from the feces but were found at necropsy in great numbers in the intestinal contents and in ulcers of the colon and sigmoid. Loesch also did not definitely incriminate the amebæ as the cause of the disease, considering that they possibly merely aggravated the condition. Nevertheless he injected rectally four dogs with some mucus containing amebæ, producing in one instance a dysentery, recovered the amebæ from the feces and found ulceration in the lower colon.

Grassi ${ }^{397}$ also found amebæ in the feces of dysenteric patients as well as in normalindividuals. $\mathrm{He}$, therefore, considered them as non-pathogenic. This worker described a cystic stage of the amebæ which occurred more frequently when the organisms were forced to live under unfavorable circumstances.

Following the work of Grassi numerous investigators reported the finding of amebæ in the feces of both normal persons and those suffering from dysentery (Leuckart, ${ }^{380}$ Sonsino, ${ }^{381}$ Perroncito $^{382}$ ).

$\mathrm{Koch}^{383}$ in $\mathrm{r} 88_{3}$, while studying cholera in Egypt described amebæ as occurring in sections of the intestinal walls. While Koch considered these as of possible pathologic significance owing to his other investigations he did not pursue the work further at that time.

However, stimulated by the work of Koch, Kartulis ${ }^{384}$ undertook extensive investigations of this important subject and added greatly to the knowledge concerning it. This worker found the amebr in many cases of dysentery both during life and at autopsy and failed to find them in cases of typhus, typhoid, tuberculosis and Bilharzia disease. He therefore rightly concluded that the amebæ were the cause of the disease. $\mathrm{He}$ attempted to cultivate the organism and to reproduce the disease in guinea-pigs but without success. He, however, showed the relationship of hepatic abscess to the amebæ.

The findings of Kartulis were very quickly confirmed by several investigators. 
Hlava $^{385}$ in Prague found the organism in sixty cases of dysentery and was able to produce the disease in cats and dogs by injection into the rectum of feces containing amebæ.

The first worker in America to report the finding of amebæ in dysentery was Osler ${ }^{386}$ who in 1890 discovered these organisms in the feces and pus from an hepatic abscess of a patient who had returned from Panama.

In a short time Musser ${ }^{387}$ reported four cases and Stengel ${ }^{388}$ three from Philadelphia, while Dock ${ }^{389}$ found amebæ in twelve cases of acute and chronic dysentery in Galveston, which were the first reported from the South.

In I891 Councilman and Lafleur ${ }^{390}$ published a most complete description of the disease with a critical study of the literature. These authors concluded that a disease entity with definite pathologic findings was produced by the amebæ and proposed the name "Amœba dysenteriæ" for the organism. They, however, considered that other amebæ, perhaps nonpathologic might be found.

The work of Councilman and Lafleur created a stir both among zoologists and physicians and an extensive literature quickly sprang up, some investigators accepting the work and others rejecting it.

Maggiora $^{391}$ in 1893 after reviewing the evidence at hand reached the conclusion that the ameba coli could not be considered as the cause of any kind of dysentery.

Gasser ${ }^{392}$ injected garden mould into cats producing a colitis and recovered amebæ from their feces apparently identical with those of Loesch and Kartulis.

However, Quincke and Roos ${ }^{393}$ confirmed the work of Councilman and Lafleur and concluded that there were three varieties of amebæ, one being very virulent and causing a marked dysentery, another being less virulent and causing only a mild dysentery, while the third was non-pathogenic. Their conclusions were partially based upon experimental evidence, their first organisms producing marked symptoms when injected into the rectum of cats while the other organisms so injected produced no symptoms.

Kruse and Pasquale, ${ }^{394}$ working in Egypt, also confirmed and 
extended the work of Councilman and Lafleur, finding a pathogenic and non-pathogenic type of ameba in the intestine. They also produced dysentery in cats by the injection of pus from an hepatic abscess bacteriologically sterile, but containing amebæ, into the rectum.

During the next ten years numerous investigators contributed to the study of the intestinal amebæ of man, but it was left for the zoologist, Schaudinn, whose name will be immortal as the discoverer of the specific organism of syphilis, to differentiate scientifically the pathogenic and the non-pathogenic species. The former he termed Entameba histolytica and showed it to be the definite cause of amebic dysentery, while the latter was designated Entamaba coli. However, it would seem that the term Endamœba is the more correct as Leidy ${ }^{432}$ as far back as I879 established the genus Endamœba for parasitic amebæ. Schaudinn's untimely end was probably brought about as a result of his investigations into the amebæ as he allowed himself to become infected by swallowing the developmental cysts of the organisms and suffered with intermittent attacks of dysentery until the time of his death in 1906 following suppuration around the sigmoid.

Schaudinn's work was confirmed by many writers, among them Craig, ${ }^{395}$ Sandby and Miller, ${ }^{396}$ Kartulis $^{397}$ and others.

In 1906 Viereck ${ }^{398}$ described an organism that he considered a third species and termed it Endameba tetragena. This he thought the cause of a certain form of dysentery, but by most investigators Viereck's ameba is considered identical with the endameba histolytica.

Numerous other workers have described amebæ which they considered as distinct species, but their findings have not been confirmed and are considered by most authorities as based upon insufficient evidence and that they will eventually be shown to belong either to the species of Endamaba histolytica or Endamaba coli.

Geographic Distribution.-The distribution of amebic dysentery is widespread although as with most infectious diseases there are certain endemic centers in which it is found more prevalent than in others. 
In Europe the disease is less prevalent than in some of the other continents. It is endemic, however, in parts of Italy, Malta, Sicily and in the Balkans. It has been observed more or less frequently in Austria, Hungary, Germany and Russia. In I9OI Jagers ${ }^{399}$ reported an epidemic among the German troops of East Prussia. In England ${ }^{396}$ and France sporadic cases have been reported from time to time, some of them in individuals who have never been out of their native countries. We are unable to find the reports of any cases occurring in the Scandinavian countries.

Many hot beds of amebic dysentery exist in Asia. Thus in southern China, India and Siam it is found most prevalent, but in northern and central China and Japan, while dysentery is found, the bacillary type is more frequent.

In the East Indies and the Philippines are found extensive endemic centers.

Africa is found to contain many endemic centers of amebic dysentery. In Egypt along the lowlands of the Nile it is probably found more frequently than in any other country of the wor.d, although in the upper portion of the country it is rare. It is found in the Mediterranean countries, Morocco, Tunis and Algiers, while in South and Central Africa it is more or less rare. In Australia and Polynesia it is endemic, and is of frequent occurrence.

In South America, amebic dysentery is found quite frequently in Chili, Brazil, and Venezuela, but less often in the southern countries.

Central America, Panama and Mexico contain many endemic centers.

In the United States amebic dysentery is endemic in most of the Southern States while sporadic cases have been observed from New England to the Western Coast.

Economic Importance.-It would be hard to estimate the economic importance of a disease so prevalent as amebic dysentery. According to Walker ${ }^{400}$ this disease, with the possible exception of malaria, is the most widespread of all the endemic tropical diseases. Walker quotes Gauducheau as stating that nearly half of the deaths of Europeans at Tonkin, 
400

ENDEMIC DISEASES OF THE SOUTHERN STATES

Indo-China, are caused by amebic dysentery and Walker thinks where the disease is not modified by sanitation about the same position of morbidity and mortality statistics is held by it in other tropical countries. 


\section{CHAPTER XXVI}

\section{ETIOLOGY OF AMEBIC DYSENTERY}

Season.-There seems to be some evidence that the incidence of amebic dysentery varies with the season. Thus, according to most observers, in the Philippines the disease is most prevalent from June to September. In Egypt it is said to occur most frequently in the late summer and autumn, while in this country by far the most cases are observed in the warm months, that is, from May to September.

Inundation.--Strong ${ }^{401}$ states that following the great flood in Manila in 1904 amebic dysentery became almost epidemic, while Brown ${ }^{402}$ states that numerous observers in Egypt have pointed out the relation of the frequency of the disease to the annual overflow of the Nile. Following the overflow of the Mississippi River in I9I 2 one of us had medical charge of 3,000 flood refuges, a large percentage of them being accommodated in tents and amebic dysentery was very prevalent.

Altitude.-Many observers have noted the apparant effect of altitude on amebic dysentery, it being largely confined to the low lying countries, along the seashore and the valleys of great rivers and rarely encountered in the uplands.

Race.-Strong ${ }^{401}$ states that while the natives of the Philippine Islands, owing to their mode of life, are more exposed to infection with amebic dysentery they are not nearly so frequently affected as Americans or Europeans; that while the ratio of white to native patients at the Government Civil Hospital has been as 2.5 to $\mathrm{I}$, that of amebic dysentery has been as 9 to r. In direct contradiction to this statement Walker ${ }^{400}$ concludes that there is no definite evidence of racial immunity of the native Filipino to this disease. In the South there seems to be no difference as to susceptibility between the black and white races, and while amebic dysentery is probably more frequent in the negro than the white this is undoubtedly due to more frequent exposure. 
Sex.-That males are much more frequently affected with amebic dysentery than females is the observation of all investigators. That this is due to the more active life led by the former and not to any greater susceptibility is also conceded by all. According to Strong ${ }^{401}$ at the Government Civil Hospital of $40 \mathrm{I}$ cases the ratio of males to females was 4.I to I, while of 200 personal cases he states only 23 were females.

Age.-Musgrave ${ }^{405}$ is of the opinion that children present a partial natural immunity to infection with Amœba histolytica. He states that not only are children more frequently exposed and less frequently infected, but when infection does occur it is usually less severe than in adults. This investigator also considers that the aged are also partially immune, but states that when attacked the aged are usually more severely affected.

DeBuys ${ }^{404}$ does not agree that children possess any natural immunity, but considers the less frequent occurrence of the disease in children to be due to their less frequent exposure. $\mathrm{He}$ is of the opinion that perhaps owing to the usual mild character of the infection in children it is often overlooked.

The youngest case observed by Musgrave ${ }^{403}$ was six months, while other observers have reported cases in children as young as ten months.

Occupation.-The occupation of an individual seems to play a certain rôle in the development of amebic dysentery, those whose occupation leads them into the open being affected more frequently than those who remain within doors the greater part of the time. This seems to be especially true of field laborers.

Other Predisposing Causes. - It would seem that such factors as eating of indigestible foods, chilling of the body, over-indulgence in alcohol, etc., may act as predisposing causes of amebic dysentery.

Also the existence of hemorrhoids, malaria, typhoid fever and other diseases may serve to render the individual more susceptible to infection with Amœba histolytica.

Immunity.-As stated above there is some difference of opinion as to the relative immunity of children and different 
races. We are inclined to agree with Musgrave that children probably are less susceptible to infection, but do not consider that there is any racial immunity.

Epidemics.-While amebic dysentery does not occur in widespread epidemics, such as have been known in many other infectious diseases, numerous small epidemics have been observed in non-endemic centers. Such an epidemic was reported by Allen $^{405}$ in which a number of persons were infected, supposedly from water from a well used in common with a man suffering from amebic dysentery.

Parasite.-Amebic dysentery is now recognized to be a morbid entity caused by a specific microörganism, the Endamœba histolytica.

The biologic position of the organism of amebic dysentery is of importance for a thorough understanding of its life history and relation to disease.

The Endameeba histolytica is of the protozoa, a subkingdom of the animal world.

The protozoa are divided into four orders: the Infusoria, the Flagelata, the Sporozoa and the Rhizopoda. It is to the latter order, which is the lowest of the protozoa, that the organism under discussion belongs.

The Rhizopoda ( $\dot{\rho} i \zeta a$, root $+\pi$ oús $(\pi \circ \delta)$ foot) consists of single cells which move by the extrusion of so-called pseudopodia or parts of the cell substance and maintain their existence by enclosing food in a similar manner. Of a very extensive list of suborders into which the Rhizopoda are divided only one, the Amoba, contains organisms which are parasitic to man.

The order of Amœba is composed of three genera: Chlamydophrys, Leydenia and Amœba. Schuadinn divided the genus $A m \propto b a$ into two subgenera: Amøba and Endamaba, and finally as stated above, the endameba into Endameba coli, a nonpathogenic intestinal parasite, and Endamoba histolytica the causative agent in amebic dysentery.

For purposes of biologic study as well as of diagnosis it is well to compare Endameba histolytica with Endameba coli.

Endamøeba Histolytica.-The average diameter of Endamaba 
histolytica in the vegetative stage is from 25 to 40 microns, thus averaging larger than Endameba coli. Some specimens measure as much as 70 microns in diameter. In color the ectoplasm is glassy, while the endoplasm is light gray, or when blood is present in the feces it may have a slight greenish tint.

At rest the organism is roughly spheric or oval. In locomotion it becomes irregular or fantastic, dependent upon the extrusion of pseudopodia.

In young amebæ it may be difficult to distinguish the endoplasm from the ectoplasm, but in large organisms, especially in motion the differentiation is easy. The endoplasm comprises about two-thirds of the protoplasm and is coarsely granular, more so than that of E. coli. The ectoplasm comprising onethird of the protoplasm is hyaline and lightly refractive. In fresh specimens it is frequently very difficult to distinguish the nucleus. It is situated to one side of the center of the endoplasm. The average diameter is 5 microns. In shape it is circular or when located at the periphery or at the junction of the endoplasm and ectoplasm may be slightly flattened. The nucleus contains less chromatin than that of E. coli. The great variations in stained specimens in the appearance of the nucleus of this parasite were largely responsible for the former division of the species into E. histolytica type of nucleus. The nuclear membrane is delicate, the chromatin granules are few and lie upon the inner side of the nuclear membrane, the karyosome is very small and appears as a dot of chromatin near the center of the nucleus, and there is no centriole. In the so-called tetragena type of nucleus the nuclear membrane is thicker and better defined, the chromatin is more abundant and lies upon the inner side of the membrane or between the membrane and the karyosome. The latter is comparatively large and appears as irregular or circular masses of chromatin, and a well-marked centriole is often present.

The endoplasm always contains one or more non-contractile vacuoles. There may be as many as ten or more. When numerous they vary in size and are apt to be small. When single they are frequently large. It is thought to be dissolved hemoglobin within the vacuoles that give the endoplasm a 
PLATE III

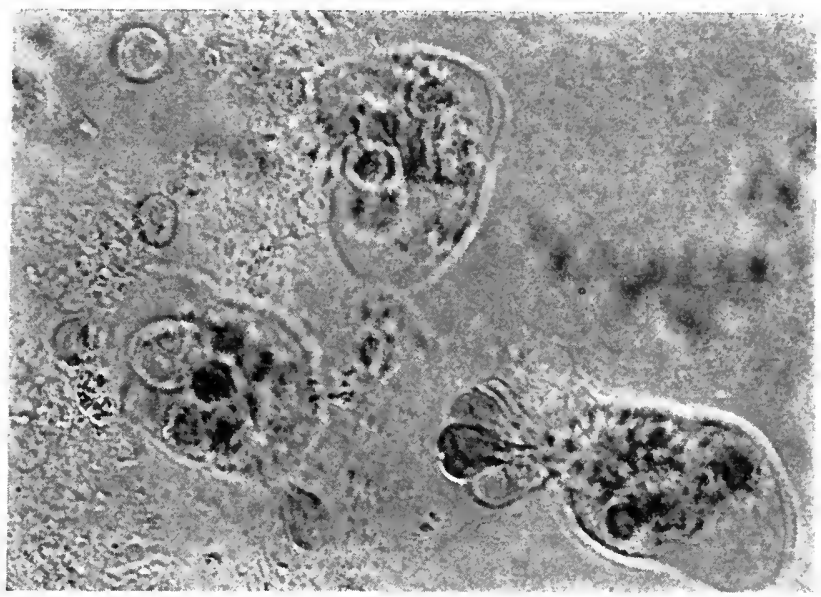

I

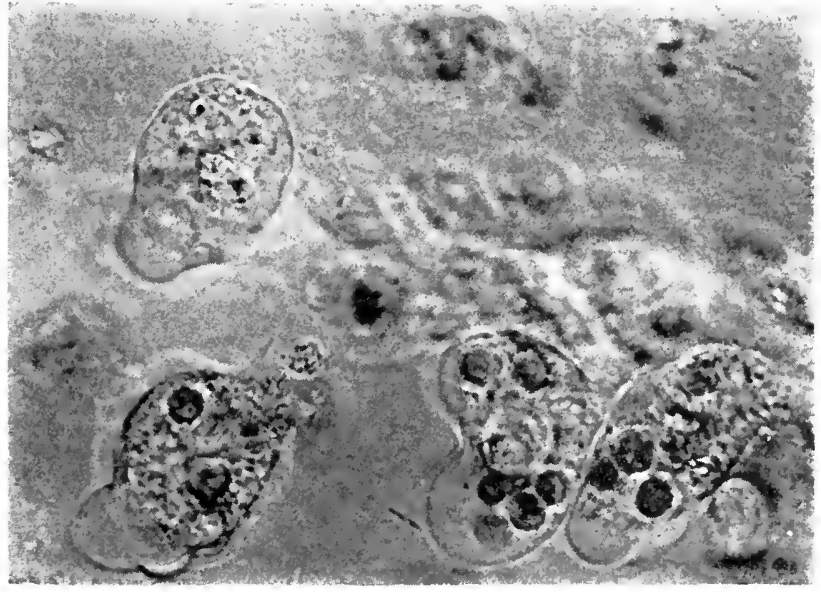

Endamœba histolytica. I. Living organisms. Note absence of nucleus. All three of the parasites contain red blood-corpuscles. 2. Living organisms. Note nucleus in upper organism. The three lower specimens contain red blood-corpuscles. (Bulletin No. I, Medical Department, U. S. Army.) 

greenish tint. When the parasite is at rest the vacuoles are spheric in shape, but in motion they may become elongated.

Endameba histolytica is much more actively motile than in Endamaba coli. Locomotion takes place by the protrusion of pseudopodia. The clear granular endoplasm follows, pouring in until it appears filled. The pseudopodia are larger and more distinct than in the non-pathogenic parasite. Motion without locomotion consists of the extrusion of pseudopodia and the ebb and flow of endoplasm.

Endameba histolytica is actively phagocytic. Within the endoplasm are engulfed bacteria, crystals and amorphous granules, and in contra-distinction to $E$. coli this parasite normally envelops red blood-cells. As a rule from two to six are present, but the parasite may appear markedly distended by the number of erythrocytes which it contains.

In the cystic stage this ameba measures from to to 20 microns in diameter and is spheric in shape. The outer contour of the organism is particularly distinct in stained specimens but in fresh specimens may appear double. The chromatin is arranged in large spindle-shaped masses. The cystoplasm appears uniform, phagocyted bodies having been extruded before encystation begins. The nucleus elongates, becomes constricted and then divides. Each of these two nuclei then divides, forming four daughter nuclei. The four-nucleated cyst is characteristic of E. histolytica. In fully developed cysts the chromatin is not infrequently found to have disappeared.

In the vegetative stage the only known method of reproduction is by simple division. In this process the nucleus elongates, becomes constricted and divides, to be followed by a corresponding process in the cytoplasm, resulting in the formation of two organisms.

Degenerative forms of $E$. histolytica are not infrequently observed. They may appear entirely filled with vacuoles, the nucleus may appear almost entirely filled by the karyosome, or the nucleus may be broken and scattered through the cytoplasm.

The staining reactions for $E$. histolytica are similar to those of E. coli. 
Endamoba Coli.-The limits of size of Endamoba coli may be put at from 6 to 50 microns in diameter. The average, however, is from $\mathrm{I}_{5}$ to 25 microns. While the largest Endamaba coli is larger than the average Endamaba histolytica, the average size of the former is smaller than the average of the latter. A differentiation should not be made, therefore, upon size alone but, taken with other morphologic data, is an aid.

At rest this ameba is spheric in shape. In motion it varies in form with the protrusion of the pseudopodia.

The color of Endameba coli is uniformly dull grayish yellow. This color is independent of the composition of the feces, whether bloody or otherwise.

It is ordinarily impossible to differentiate between the endoplasm and the ectoplasm of $E$. coli, almost the entire protoplasm being endoplasm, and this is a valuable point in the determination between the two parasitic species in man. The endoplasm appears as finely granular in structure.

In $E$. coli, unlike $E$. histolytica, a well-defined nucleus may nearly always be determined. Its position is usually to one side of the center. In size it is from 5 to 8 microns in diameter. The nucleus is commonly spheric, sometimes oval, in shape and contains numerous refractive granules and shreds of chromatin and one or more small nucleoli.

Vacuoles are not commonly present in this ameba and when found are single. In common with the other parasitic species in man this vacuole is never contractile. It is usually indistinct and of small size.

Endameba coli is only slightly motile as compared with E. histolytica. Motion in the resting position may be detected by the flow of the granular protoplasm and the protrusion of minute pseudopodia. Locomotion is performed by means of small rounded pseudopodia. It is very slow compared to that of $E$. histolytica and ceases altogether after the specimen has stood at room temperature for half an hour.

This ameba frequently engulfs bacteria and crystals, but very rarely red blood-cells. Even when blood is found in the feces it is rare to find the corpuscles phagocyted by this ameba 
and then only one or two, whereas with $E$. histolytica several corpuscles are frquently found in a single organism.

Cysts of $E$. coli are about a third smaller than the vegetative forms. They possess a limiting wall of double outline and are motionless. The protoplasm is hyaline and contains nothing but the nucleus, bacteria and crystals having been extruded before the formation of the cyst wall.

Reproduction takes place in the vegetative stage by two methods: by simple division, resulting in two daughter amebæ; and by schizogony, resulting in eight daughter amebæ.

In reproduction by simple division the nucleus elongates, its membrane thins and the chromatin collects at each pole. Next a constriction appears near the center, narrowing in hourglass form until complete separation occurs and two nuclei are formed. While this is taking place a corresponding constriction and division of the protoplasm occurs and two amebæ are the result. Schizogonic reproduction is less common than that by simple division. In schizogony the nucleus becomes swollen and the quantity of its chromatin is greatly increased. The chromatin next collects in eight hemispherical masses within the nucleus. The nuclear membrane then disappears and the eight chromatin bodies are free in the protoplasm of the ameba. The chromatin masses become surrounded with nuclear membrane, thus forming daughter nuclei arranged with more or less regularity throughout the cytoplasm of the parasite. The cytoplasm then divides in proportion to the number of daughter nuclei and new organisms are formed.

During reproduction within the cyst, the nucleus elongates, becomes constricted and finally divides much after the manner described for simple division. The further process of autogamy is obscure, but after division from two to eight nuclei are observed within the cyst, eight being the normal number for this ameba and less than eight denoting that the organism is still in the act of reproduction. More than eight nuclei, sometimes as many as sixteen, are occasionally observed. The daughter nuclei are spheric in shape and have a distinct nuclear membrane.

Degeneration forms are sometimes observed. These are 
characterized by poorly defined cystic membranes, coarse granulation of the cytoplasm, shreds or lumps of chromatin, and distortion or disappearance of the nuclei. Vacuoles may almost entirely replace the endoplasm.

In the vegetative stage stained with hematoxylin there is no distinction between endoplasm and ectoplasm, while the nucleus takes a dark purple color. With Wright's or Giemsa's stain the ectoplasm stains light blue and appears structureless, while the endoplasm is darker blue and appears granular. On account of its chromatin the nucleus takes a bright red or crimson. The nucleolus stains dark violet.

Cultivation of Organisms.-Numerous investigators have attempted the artificial cultivation of the infective organism of amebic dysentery, and many reports of its accomplishment have been published.

Thus Musgrave and Clegg ${ }^{406}$ considered that they had been successful in obtaining growth of Amaba histolytica on a medium of bouillon and agar, I per cent. alkaline to phenolphthalein, plus a trace of peptone. They were unable, however, to obtain the organism in pure culture, but upon the addition of some bacterial form, S. cholere asiatica being best suited, they obtained good development at room temperature.

Lesage $^{407}$ considered that he had obtained cultures of $E$. histolytica but that $E$. coli could not be grown artificially.

Whitmore ${ }^{408} \mathrm{Craig}^{409}$ and others after thoroughly investigating the matter have reached the conclusion that the organisms which have so far been cultivated are not the parasitic amebæ, but are free-living species which have gained entrance either in the cystic stage with the food and passed through the intestines or as contaminations of the culture media.

Inoculation Experiments.-One of the first to attempt to transmit amebic dysentery to the lower animals was Loesch. ${ }^{378}$ Since his time numerous investigators have performed inoculation experiments on various animals and in man, both by feeding and by rectal injections, and in one instance ${ }^{400}$ intravenously, of feces containing amebæ and of pus from liver abscess.

In the classic experiments of Schaudinn ${ }^{410}$ he fed kittens both 
with feces containing $E$. coli and those containing $E$. histolytica, and showed conclusively that the former was harmless while the latter produced typical amebic dysentery. He further showed by an ingenious experiment that $E$. histolytica is infectious only when spores are present.

Craig ${ }^{411}$ found that amebic dysentery developed in 50 per cent. of young kittens injected per rectum with feces containing $E$. histolytica, and in 65 per cent. of those fed with that material. This investigator considers that the negative results obtained were due to the feces containing the organisms only in the vegetative state and without spores.

In I9I3 Walker and Sellards ${ }^{412}$ published the results of most exhaustive feeding experiments on human beings. The work was carried on with long-term prisoners at the Bilibid prison, all of whom were volunteers, fully understanding the nature of the experiments and having signed an agreement to the conditions.

The experiments were most carefully controlled by previous fecal examinations for parasites, by controlling the food and water supply, etc.

The first series of experiments consisted of 20 feedings of cultures of 13 strains and 8 species of amebæ to ro different men. It was found that the amebæ could be cultivated from the feces on Musgrave and Clegg's medium for a few days following the feeding, but never later. The amebæ could not be recognized microscopically by examination of the feces. No dysentery developed. These investigators therefore concluded that cultivable amebæ will not live as parasites in the intestinal tract of man, are not pathogenic, and when obtained in cultures from stools are derived from cultural contamination or from encysted amebæ which have been ingested with water and food and passed unchanged through the intestinal tract.

The second series of experiments consisted of 20 feedings of 5 strains of Endamœba coli given to 20 different men. Of these 20 men 17 became parasitized at the first feeding, while 3 did not: In the I7 the Endamoba coli was found microscopically in every case, while cultures on Musgrave and Clegg's medium were invariably negative. None of the men developed dys- 
entery. From these experiments Walker and Sellards concluded that the Endam $\propto b$ a coli is a strict parasite, non-cultivable on Musgrave and Clegg's medium and non-pathologic.

The third series of experiments consisted of 20 feedings with Endamaba histolytica. Of the 20 men 17 became parasitized after the first feeding, I after 3 feedings, while the other 2 were reserved as controls. Of the 18 men who became parasitized 4 developed endamebic dysentery. The organisms were recovered by microscopic examination in all who became parasitized, but cultures on Musgrave and Clegg's medium were invariably negative.

Encysted "Endameba tetragena" was obtained exclusively in the stools of men who ingested Endameba histolytica only. Motile Endamaba histolytica were observed exclusively from men who ingested "tetragena" cysts only. From the above experiments these authors. conclude that Endamoba histolytica is also a strict parasite and cannot be cultivated on Musgrave and Clegg's medium; that Endameba tetragena and Endameba histolytica are identical; that Endameba histolytica is the essential etiologic factor in endemic tropical dysentery.

Sellards and Baetjer ${ }^{415}$ in I9I4 introduced the procedure of direct inoculation into the cecum for the experimental production of amebic dysentery in cats. Their method was to perform a laparotomy under general anesthesia and inject the material with a sterile needle directly into the cecum. By this method they were able to produce dysentery in all of ten animals with the use of eight strains, two of which were from distinctly atypical cases. These authors consider that their method of inoculation is of value for the propagation of a strain of amebæ through a series of animals for a period of at least several months; that it may be used to determine the etiology of some obscure diarrheas; and that it is of value for the study of the morphology of some atypical amebr of low virulence.

Mode of Infection.-As the life history of the Endamaba histolytica outside the human body is unknown, it is rather difficult to determine the source of infection. All authors are agreed that practically the only method of infection in amebic dysentery is by the ingestion of the organisms. It is a somewhat 
moot point, however, whether or not drinking water is the sole source of infection. It is well within the range of possibility that the infective organism may be present on various kinds of uncooked food such as lettuce, cress, etc.

Brown ${ }^{402}$ states that a case developed following the use of cold water enemas for constipation. While such cases are obviously possible they must be exceedingly rare.

Pathogenesis.-The question as to the rôle of the amebre found in the intestinal canal of man in causing dysentery has been a greatly mooted one and many views have been expressed. Cassagrandi and Barbagallo ${ }^{414}$ considered that amebx were not only harmless but that they actually combated disease, while Musgrave and Clegg ${ }^{406}$ consider that all amebæ are, or may become, pathogenic. Other views are that the amebx are infectious only in the presence of bacteria, that they may prepare the soil, as it were, for the action of certain bacteria, or vice versa.

The view is becoming more and more prevalent that the Endamaba histolytica is the true and only cause of the disease; however, this can be proven definitely only after the successful cultivation of the organism free from contamination, the reproduction of the disease in animals, and the recovery in pure culture of the amebæ. Even the successful accomplishment of this experiment may be open to the objection that the bacteria always found in the intestinal canal of animals may in some manner be partially responsible for the production of the disease.

This objection might be overcome after the successful cultivation of the organism has been accomplished by removing animals at term by Cesarean section, keeping them in a sterile chamber supplied with sterile air and fed on sterile food until old enough for experimental purposes, and then injecting with pure cultures of the organisms.

It is generally conceded that the usual portal of entry of the amebæ into the tissues is by way of the glands of Lieberkühn. This is shown to be the case by examination of the intestine early in the course of infection, when the organisms may often be found lying free in the lumen of the glands and the 
epithelial lining remaining intact. Later they are found to have thrust themselves between the lining cells which to a large extent degenerate as a result. The organisms soon penetrate the basement membrane and spread out on either side of it. Here they develop and push on into the submucosa and feed upon the tissue cells, red blood-cells and perhaps the leucocytes. In fresh tissues which are cut with a warm knife, movements of organisms in the tissue can be watched for several hours.

The amebre may enter the radicles of the portal vein and be carried to the liver where they may cause hepatic abscess. 


\section{CHAPTER XXVII}

\section{PATHOLOGY OF AMEBIC DYSENTERY}

The morbid anatomy of amebic dysentery is confined mainly to the large intestine, the sigmoid and rectum, though occasionally the lower end of the ileum may be involved by extension through the ileocecal valve, and the appendix has been found affected. There are also certain complications which may arise, causing pathologic processes in other locations than the bowels.

The pathologic anatomy as observed in the large intestine in amebic dysentery presents many features which are characteristic of this disease. This is especially true of the chronic protracted cases, but as may readily be imagined the condition will depend largely upon the severity of the infection, and in very acute cases when death results early, the lesions may not present typical findings.

In the latter type of cases the colon shows evidence of marked inflammation, the mucous membrane being hyperemic and inflamed with but little change in the submucosa. It is swollen, of a dark bluish or purple color, and presents many extravasations from congested vessels. Ulceration is infrequent, though small superficial areas of necrosis are noted. Diphtheritic-appearing membranes are often seen, composed of the excessive mucous secretion and portions of the mucosa which have separated from the intestinal wall. The whole picture presents a marked resemblance to that observed in acute bacillary dysentery.

This, however, is not the case in the more chronic type of amebic dysentery. In such cases of moderate severity the peritoneum is found more or less injected, contains some fluid, but otherwise presents little that is abnormal. The large intestine is bright in color, smooth and shows patches of congestion. The mucosa is seen to possess an excess of secretion 
and presents some points of hyperemia and ecchymosis. The enamebæ usually first enter the crypts of Lieberkühn and penetrate the epithelial lining by boring between the cells with their pseudopodia and after passing through the basement membrane enter the submucosa. Here they multiply rapidly and are reinforced by others from the lumen of the intestine. By their presence in the submucosa, areas of congestion are formed; the mucosa is covered by a more or less copious secretion of viscid mucus and in severe cases with a diphtheroid membrane. It is, however, in the submucosa that the principal changes take place. There are evidences of acute inflammation and proliferation of the fibers of the connective tissue. Small tumors of adenoid tissue are formed which may be seen on the surface of the mucosa. Later these suppurate in the center and present one of the characteristic lesions of the disease. In the early stage, also, are seen small erosions and areas of necrosis. They are in the beginning superficial and separate, but they gradually extend, deepen and are joined together and cover rather large areas of the surface. These erosions later constitute the typical ulcers of the disease. Between them the mucosa appears normal except for the congested vessels.

Two types of ulceration are seen: First, the typical undermined amebic ulcers which in the early stages, as has been pointed out, are seen as small erosions on the mucosa. As the process continues, a pocket is formed in the submucosa which is extended in all directions parallel with the surface. The base of the ulcer rests upon the circular muscular coat with overhanging edges of mucosa. The size of the ulcer varies from I $\mathrm{mm}$. in diameter to 8 or $10 \mathrm{~cm}$. As may be expected, the submucosa becomes markedly thickened and edematous, and usually the muscular and serous coats are somewhat involved in a like manner. It is not unusual in severe cases for two or more ulcers to coalesce either in the submucosa alone, forming tunnels under the mucosa, or by sloughing away of the mucosa itself. In very severe cases the muscular coat may be involved, necrosis or even perforation taking place and the peritoneum or omentum forming the 
base of the ulcer. The ulcer may perforate into the subperitoneum where it may remain circumscribed, may burrow widely, or may cause general peritonitis.

The second type of ulcer seen in amebic dysentery is the socalled type of Harris. These ulcers are rarer than the typical or undermined ulcer but are not infrequently seen, especially in early and rather acute cases. The Harris ulcers are in the beginning confined mainly to the mucosa, and for this reason there is some doubt as to their exact connection with amebic dysentery. They may extend into the submucosa, but rarely penetrate it and never extend into the muscular coat. The edges of the ulcer are abrupt, thickened and congested. They are sometimes described as of punched-out appearance. The base of the ulcer is clean, edematous and of a grayish color.

Histopathology.-The mucosa between the ulcers generally presents little abnormality upon microscopic examination. Near the ulcers the mucosa may be hypertrophied, with some mucoid degeneration and occasionally cyst formation. Often the cells lining the glands are seen to have separated from the basement membrane and amebæ may be found among these cells.

In the beginning the most noticeable condition is a marked congestion, and often capillary hemorrhages are seen beneath the mucosa. The submucosa may also show congestion with thickening due to edema. There is an infiltration of lymphoid cells into the interglandular tissue.

Later in the disease a slight superficial necrosis is seen and the glands surrounding the lesions show hypertrophy and mucoid degeneration. There is more infiltration and more congestion. Within the interglandular connective tissue amebæ are seen in the blood-vessels and lymph spaces. As the process advances the submucosa is seen to be more affected. Congestion and edema are more pronounced and amebæ are seen to be more plentiful. Sometimes there is an infiltration of polymorphonuclear leucocytes which seems to be accompanied by activity of bacteria. The ulcer contents in uncomplicated cases are composed of a granular base with degenerated cells, amebæ, bacteria and erythrocytes. 
Amebic ulcers show a marked tendency to heal. After the necrotic area has sloughed, red granulations are seen in the base of the ulcer. These granulations are unstable and break down easily, and as long as amebre are present no permanent repair takes place. In small ulcers complete repair may occur, but in large ones scar tissue is formed followed by more or less marked contraction.

Complications. - The most important complication of amebic dysentery is hepatic abscess. According to Brown ${ }^{402}$ there are four routes by which the amebæ may gain access to the liver as follows: (I) Directly from the lumen of the intestinal canal; (2) transperitoneally from the intestinal wall; (3) by the portal vessels; (4) by the general circulation.

Infection of the liver by way of the portal circulation is by far the most frequent route.

According to Craig ${ }^{415}$ Roux in 639 collected cases of hepatic abscess found the location to be as follows: 435 , or 70.8 per cent., in the right lobe; 85 , or 13.3 per cent., in the left lobe; and 2 , or 0.3 per cent., in the lobus Spigelli.

It was formerly considered that hepatic abscess of amebic origin are usually single but of recent years it has been shown that this is usually not the rule and that multiple abscesses are observed at least as frequently as in 50 per cent. of cases.

The size of liver abscesses varies greatly. They may be so small as to be microscopic or they may attain a size so large as to fill almost completely the abdomen.

The first change noted in beginning hepatic abscess is the formation of one or more small irregularly spheric nodules which appear like commencing coagulation necrosis and to some degree resemble the lesions observed in the intestinal tract. The nodules are solid and upon being cut appear of a cheesy consistency, the lobular markings being obliterated. Later the patches may coalesce and begin the breakdown. The process may be stopped at this point and occasionally found as described. As a rule the breaking down continues and liquefaction takes place, followed by the formation of a cavity which is filled with viscous fluid. This fluid is usually dark red in color 
and of gelatinous consistence. It may, however, be of a greenish color owing to the mixture with bile. As the process advances the contents become more liquid and necrotic tissue may be found floating in the mass. There is usually no lining membrane of the cavity especially when of large size but occasionally the small abscesses may have clearly defined smooth walls. It may be that the vascular structures are not destroyed and are seen crossing the cavity. Microscopically the contents of liver abscess are seen to be made up of disorganized liver cells, red blood corpuscles and granular matter. Fat globules, cholesterin, Charcot-Leyden crystals and hemotoidin are recognized. Pus cells are usually not present. The amebæ are found if the abscess is not old. They are, however, found in the walls of practically all abscesses. The-liver cells show hyaline and granular destruction. If the abscess is contaminated by pyogenic bacteria the tissues will show many leucocytes.

Other complications of the liver that are frequently noted are fatty degeneration and cirrhosis.

The spleen is frequently the seat of cirrhosis; infarcts are occasionally seen, while splenic abscess has been reported. The route of infection is probably usually the blood stream although the infection may extend from the splenic flexure of the colon.

Chronic gastritis is not a rare complication of amebic dysentery and is usually of a rather severe character.

Chronic enteritis is often seen, while, as pointed out above, acute peritonitis may occur and amebic appendicitis is not uncommon.

The kidneys are very often the seat of nephritis, usually of a chronic character.

In the heart, valvular disease is rarely seen, while edema of the pericardium is occasionally observed. Arteriosclerosis is sometimes found.

The lungs are quite frequently found to be the seat of bronchopneumonia. This is in all probability mainly due to the fact that the patient remains in a recumbent position for so long a time. This complication is almost always fatal. Lobar pneumonia is occasionally seen and usually ends with death. 
4 I 8 ENDEMIC DISEASES OF THE SOUTHERN STATES

Empyema may occur, due to the perforation into the pleural cavity from an hepatic abscess.

Brain abscess is a rather rare complication of amebic dysentery and usually follows hepatic abscess. The abscesses are usually small and may even be microscopic in size. The amebæ are readily demonstrable in the pus and the walls of the cavity, and bacteria of various kinds are usually present. 


\section{CHAPTER XXVIII}

\section{CLINICAL HISTORY OF AMEBIC DYSENTERY}

The clinical course of amebic dysentery varies greatly. It may be ushered in with a chill, nausea and vomiting, and pass on with acute griping pain and diarrhea followed by prostration, exhaustion, collapse, cardiac failure and death. Or after such an acute attack either with or without treatment the system may overcome the infection and after four or five days improvement begin. Complete recovery is rather rare and a tendency to relapse and chronicity may continue.

On the other hand, the onset of the disease may be insidious and persist for years with only mild disturbances, such as irregularity of the bowels, occasional abdominal discomfort, and gastric derangement. This type of the disease may develop acute symptoms at any time and terminate in death or recovery.

The period of time elapsing from the ingestion of E. histolytica until they could be found in the stools microscopically in the experimental cases of Walker and Sellards ${ }^{412}$ varied from one to forty-four days with an average of nine days. Of the four cases which developed dysentery the incubation periods until the advent of symptoms were respectively, twenty, fifty-seven, eighty-seven and ninety-five days. These authors consider that it is at least probable that the number of organisms ingested accounts for the varying incubation periods.

In the experiments of Sellards and Baetjer ${ }^{412}$ on cats the incubation period of animals injected from acute and chronic amebic dysentery varied from six to ten days, while in the animals injected with atypical strains the incubation period was one month.

In the acute form of the disease there may be some prodromal symptoms such as malaise and headache. After three to five 
days marked nausea, which vomiting does not altogether relieve, is usually noted. A distinct rigor may be the first marked symptom or the nausea and vomiting may be accompanied by chilly sensations. In a short time diarrhea with severe griping pain develops. The pain is at first of an intermittent acute character most marked in the umbilical regions; later this is less acute but continuous and is located in the region of the sigmoid.

At first the stools are copious and watery but later are scanty and composed mainly of mucus, blood and cast-off fragments of intestinal epithelium: The movements become more frequent, sometimes as many as fifty a day, and urgent, and are attended with burning and tenesmus. Following defecation there is no relief and even though the rectum be almost entirely empty the desire to defecate remains and violent efforts are made to do so. Not only is rectal tenesmus present but very frequently vesical tenesmus is one of the most distressing symptoms. In the malignant cases there is a continued increase in the severity of the symptoms. The pain and loss of sleep often cause mental symptoms, either depressions or delirium, to develop. There may be severe hemorrhages of the bowels. Exhaustion soon appears and in a few hours death results from cardiac failure and collapse.

If the system is able to throw off the infection, or sometimes under proper treatment the acute stage is followed in four or five days by beginning recovery. The pain becomes less, the bowel movements less frequent, but contain more fecal matter. Complete recovery is unusual and the disease passes into either the chronic or the intermittent form. In the former the stools vary from 2 or 3 to Io or 15 per day with more or less pain. As the disease progresses there is loss of weight, anorexia, indigestion and flatulence. Extreme emaciation may result with great exhaustion. Death may follow from exhaustion or intercurrent infection. In the intermittent type there are periods of diarrhea, more marked in the mornings, consisting of two to four semifluid stools without much mucus and little pain. The diarrhea lasts for one to six or eight days and is followed by a period of constipation. These alternate periods of diarrhea 
and constipation may last for many years and the patient die of intercurrent disease or an acute exacerbation may lead to a fatal outcome.

That mild cases of infection with Endameeba histolytica, in which there are no dysenteric symptoms, do occur has been pointed out by many observers. Thus Musgrave ${ }^{416}$ reports a series of fifty cases in which characteristic amebic lesions were present at autopsy but in which dysentery symptoms were absent. Such symptoms as abdominal "aching," more pronounced at night and early in the morning, flatulence and constipation are noted. The constipation is usually resistant to the ordinary doses of the usual cathartics, or their actions may be unusually severe and prolonged. Loss of weight is often noticed but this may not result, and the patient may even gain in flesh. Anorexia is often first noticed at breakfast time and is frequently accompanied by nausea and accumulation of mucus in the mouth and throat during the night. One of the most frequently noted symptoms is excessive perspiration, especially of the palms of the hands and the soles of the feet. Often, as Musgrave points out, the whole chain of symptoms, dullness, headache, loss of memory, weakness, desire for sleep, etc., is noted in these mild infections. The disease often begins insidiously and passes into a slightly more accentuated form which may persist for months or years. This type of infection is similar to the chronic type following an acute outbreak and may develop at any time an acute attack.

Physical Signs.-By physical examination in amebic dysentery little or nothing distinctive may be learned. During acute attacks there is more or less tenderness over the large intestine, especially over the sigmoid. The liver is usually enlarged and in some cases of hepatic abscess enormously so. At first the abdomen is more or less distended with gas but later is flat and the abdominal muscles are flaccid.

Temperature.-The temperature in amebic dysentery varies from normal or subnormal in the mild cases to $104^{\circ}$ to $105^{\circ} \mathrm{F}$. in the severe attacks of acute onset. Just before death in the acute cases the temperature may drop to subnormal. In uncomplicated cases even of moderate severity there is usually 
no rise in temperature, but in cases of secondary infection the temperature may be high. In the acute attacks, which occur during the course of a chronic case, even if of marked severity, the temperature is usually not so high as in the attacks of acute onset.

Pulse and Respiration.-The pulse and respiration as a rule follow the variation in temperature. In the mild cases and those of chronic character the pulse may be normal, while in moderately severe cases the pulse changes from 80 to roo. In the acute cases the pulse rate may run as high as I Io to I 20 , and in those of fatal termination I 20 to 140 and more have been noted. The respirations as a rule increase with the pulse, except as collapse occurs in which they are shallow and infrequent. The blood pressure in amebic dysentery depends upon the general condition of the patient; where there is emaciation and cachexia the blood pressure is low, while in mild and even in some chronic cases it may be normal. In cases complicated by other conditions such as nephritis and arteriosclerosis the blood pressure may be high. As a rule there are no symptoms referable to the heart, though pathologic changes in this organ may occur.

The blood stream in amebic dysentery shows nothing which is typical. In those cases of acute onset there is nothing abnormal seen, the erythrocytes show no alterations in shape or numbers, and the hemoglobin is of normal percentage. There may be a slight leucocytosis.

In the more chronic cases there is a secondary anemia depending in degree upon the severity and length of the infection. The red blood-cells are decreased in numbers to as low as 1,500 ,$\infty$ or less, while poikilocytes, normoblasts and megaloblasts may be found. The hemoglobin is diminished in amount in a proportion greater than the reduction of the erythrocytes. It may be as low as 20 to 30 per cent. There is usually a moderate leucocytosis in the later stages of the disease depending to a large extent upon the amount of secondary infection. The . relative numbers of the various types of leucocytes are usually normal. There is rarely an eosinophilia in cases uncomplicated by other intestinal parasites, although as pointed out 
by Amberg $^{418}$ in children this condition is very frequently encountered.

All symptoms observed which are referable to the lungs occur as complications.

The gastro-intestinal symptoms are most marked in amebic dysentery. Thus nausea and vomiting are early symptoms in acute attacks, and as pointed out above in those mild infections without dysenteric symptoms the only symptoms may be those referable to the stomach. The appetite varies with the severity of the condition. In mild and chronic cases it may be normal while in the more severe cases there may be complete anorexia.

As pointed out above, the intestinal symptoms may vary all the way from constipation to the most marked diarrhea of the dysenteric type. It is not at all to be inferred that the pathologic findings are to be interpreted in the light of the clinical evidence for it not infrequently occurs that cases showing marked dysenteric lesions come to autopsy without having shown dysenteric symptoms.

Macroscopically the stools of amebic dysentery show nothing characteristic of this disease. They vary in consistence from solid and semi-solid in the mild and chronic conditions to watery in the acute. When formed stools are passed there may or may not be a coating of mucus. The fluid stools show the most marked variations. There may be little present but blood, mucus and intestinal débris, or there may be noted particles of undigested food. Again they often assume a watery consistence with little mucus. When ulceration is present the stools may contain blood clots and portions of intestinal mucosa. The odor of the amebic feces is usually very offensive and according to Musgrave ${ }^{417}$ "all but characteristic." This author further states that the odor is so nearly characteristic that diagnostic importance may be attached to it.

The nature of the blood as passed in the feces is of importance both as an indication of the severity of the pathologic process and the location of the lesions. If no blood is present macroscopically, it is an indication that ulceration probably has not taken place. This is not invariable as in very acute cases 
perforation may take place before ulceration. If the blood is of dark brown or black color, it is an indication that the cecum is the chief seat of the infection. If the blood is fresh, bright red and clotted, it may be assumed that the lower end of the colon is most affected, while if the blood is intimately mixed with mucus and fecal matter and the stools are of a dark red or reddish-brown color, it is probable that the bleeding is taking place above the sigmoid flexure.

Microscopically the appearance of the feces in amebic dysentery varies with the stage of the disease and the severity of the infection. Red blood-cells are practically always found and more or less epithelium. There are varying quantities of food particles, epithelioid and pus cells, bacteria and the distinguishing feature, Endamaba histolytica. It must be remembered that Endamaba coli are also frequently present as well as the ova of certain other intestinal parasites.

The urine in amebic dysentery shows nothing characteristic. The amount is usually greatly diminished during the acute attacks, but may be normal during the intervening periods. The specific gravity corresponds to the amount secreted and the reaction is usually acid. Retention may occur in the severe cases when the reaction may become alkaline. Albuminuria is seen only when the disease is complicated by nephritis. Albumoses have been noted and may be indicative of hepatic abscess. Indican is usually present in considerable quantity, depending on the severity of the pathologic processes. It has been noted that the chlorides are diminished or absent during acute attacks, especially when the diarrhea is severe and the stools watery. Microscopically may be found hyaline and granular casts, if nephritis is present, and epithelial cells and various crystals.

Complications.-As stated in the chapter on pathology the most important complication of amebic dysentery is hepatic abscess. This may occur early in the disease but is usually a later complication.

Cases have been reported in which liver abscess preceded other manifestations of amebic infection but this is undoubtedly rare. In cases of acute onset hepatic abscess may be found 
within a week of the onset of dysenteric symptoms, but as a rule is not noted till about the sixth week. In chronic cases, however, this complication may not develop for months or even years after the initial attacks. In one case reported by Brown ${ }^{402}$ the patient developed hepatic abscess nine years after the last attack of dysentery.

According to Manson ${ }^{419}$ Europeans are more liable to develop hepatic abscess than natives of tropical countries (India) although more dysentery is seen in the natives. He further states that while European women contract amebic dysentery as frequently as European men they rarely develop hepatic abscess and children hardly ever. One of us has operated on half a dozen cases of amebic abscess in adult negroes, all but one being males. The onset is usually insidious and may last for a considerable length of time without any symptoms. Symptoms of perforation may be the first indication. Pain usually develops some time in the course of the disease and is usually dull and aching but may be sharp. The pain may be over the hypochondrium or epigastrium, or, as is very frequently the case, it may be reflex and observed in the region of the right scapula. If pain is absent, it may sometimes be elicited by pressure over the liver. A rise in temperature is usual but not constant. The morning temperature may be nearly normal and in the evening a rise to $100^{\circ}$ to $101^{\circ} \mathrm{F}$. be noted. On the other hand, a temperature of $103^{\circ}$ to $104^{\circ} \mathrm{F}$. is not infrequent. The temperature is usually intermittent but it may be continuous for a long time with slight morning remissions. Chills and excessive perspiration may occur. The pulse usually corresponds to the temperature and may run as high as 140 or more.

Marked jaundice is rare, although the conjunctivæ are frequently tinged with yellow. Vomiting is not usual and anorexia is the rule. The tongue is usually coated.

Physical examination will reveal an enlarged liver and if the abscess is located anteriorly and of very large size fluctuation may be noted. If it is located under the vault of the diaphragm, there will be bulging upward and an area of dullness will be noted in the thorax. The intercostal spaces may be obliterated and protrude. 
The blood in hepatic abscess usually shows a leucocytosis, although this condition is by no means constant. The total number of leucocytes may run as high as 30,000 or 40,000 mainly due to an increase in the polymorphonuclears.

If the patient is not operated upon and lives long enough, spontaneous rupture may occur. This most frequently is into the right lung, less often into the peritoneum and pleural sac, and rarely into the colon, the stomach, small intestine, bile ducts, vena cava, pericardium, kidney, or through the skin in the lumbar or right hypochondriac region.

Manson ${ }^{419}$ gives the following table of $5^{6} 3$ cases of hepatic abscess collected by Rendu in which rupture occurred in 159:

Rupture occurred into the pericardium in I case, 0.13 per cent.

Rupture occurred into the pleura in $3 \mathrm{r}$ cases, 5.5 per cent.

Rupture occurred into the lung in 59 cases, I0.5 per cent.

Rupture occurred into the peritoneum in 39 cases, 6.9 per cent.

Rupture occurred into the colon in 6 cases, I per cent.

Rupture occurred into the stomach and duodenum in 8 cases, 1.4 per cent.

Rupture occurred into the bile duct in 4 cases, 0.7 per cent.

Rupture occurred into the vena cava in 3 cases, 0.5 per cent.

Rupture occurred into the kidney in 2 cases, 0.3 per cent.

Rupture occurred into the lumbo-iliac region in 6 cases, $r$ per cent.

One of us has recently treated a case in which rupture of an abscess occurred into the right lung. Ten months later, though pus was being expectorated freely, the abscess pointed just to the right of the second lumbar vertebra where it was evacuated through an incision.

Peritonitis may occur as a result of rupture of an hepatic abscess into the peritoneum or following perforation of the intestine. This condition usually proves fatal.

Other Diseases.-Amebic dysentery is very frequently complicated by other diseases, such as malaria, pellagra, hook-worm and other intestinal parasites, which complications will cause variations in the symptomatology. We have seen pyorrhœa alveolaris complicating amebic abscess of the liver. 


\section{CHAPTER XXIX}

\section{DIAGNOSIS OF AMEBIC DYSENTERY}

The absolute diagnosis of amebic dysentery upon clinical findings alone is usually impossible, although certain symptoms are very suggestive. A disease of acute onset with nausea and vomiting and griping diarrhea, especially in endemic centers of amebic dysentery, must be looked upon with suspicion. Again, a disease of insidious onset with chronic diarrhea, emaciation, etc., should be considered at least possibly amebic dysentery. It is, however, upon the microscopic examination of the feces or pus from a hepatic abscess for the Endamoba histolytica that the final diagnosis must rest.

In collecting feces for examination for the infecting organism most writers enjoin the giving of a purgative beforehand. However, as Walker ${ }^{412}$ has pointed out, if a purgative is given, the Endamœba histolytica, if present, will be found in the preencysted state when it most resembles Endamœba coli, and that if the stools are formed, the organisms are usually found in the encysted state when they may most certainly be differentiated from Endamaba coli. The examination of dysenteric or diarrheal stools should be made as soon as possible after passage, as entamebæ in these stools are motile but quickly lose their motility. If the surrounding temperature is much below that of the body it is well to collect the stools in a glass jar of some description and place this in a vessel of water at a temperature of about $100^{\circ} \mathrm{F}$.

This is not necessary with formed stools as the entamebæare in the encysted state and preserve their characteristics for several days. If the fluid stools are to be examined for the organisms in the fresh state, a platinum loop full of the material is placed upon a microscope slide and covered with a coverglass. Gentle pressure should be applied to spread the material in a thin layer. If particles of mucous or bloody material are 
present, preparations of them also should be made. Naturally it is more easy to recognize the endamebæ when they are still motile but after some experience they may readily be recognized after motility ceases if disintegration has not commenced.

A rapid survey with a low-power objective should be made and all suspicious-looking objects examined with the oil immersion. When motility is present even the inexperienced should have little or no difficulty in recognizing the organisms. If, however, motility has ceased, amebæ may be distinguished from other bodies found in the feces by their size which, even when smallest, is larger than pus cells and other protozoa except Balantidium coli, by their refractiveness which is greater than pus cells or epithelial cells, and by their distinctness of outline. The nucleus of the ameba is also characteristic in that it is usually ring shaped and relatively small. If the developmental characteristics of the entamebæ are to be studied. it is well to employ a warm stage.

In examining the solid stools for the organisms a small portion of stool is rubbed up on a slide with a drop of water or salt solution and covered with a cover-glass as described above. In these stools the amebæ are found in the encysted state, when they are round or slightly oval, more refractive than when in the vegetative state, and the cyst wall is usually distinctly seen. The cysts will be observed to contain from two to eight nuclei depending upon the species of entameba and the stage of development.

While for diagnostic purposes it is usually best to examine the feces for the amebæ in the fresh state, it is sometimes desirable for preparing permanent specimens and for bringing out some of the details of the nuclei to stain them.

A simple method which is sometimes employed is to add a few drops of a weak aqueous solution of acid fuchsin or methylene-blue which will stain epithelial cells and débris, leaving the endameba almost free from stain.

The vapor of osmic acid applied to an air-dried film of feces for twenty minutes, followed by washing in water, is another simple and satisfactory method of staining. 
Staining by one of the Romanowsky modifications is largely used and gives very satisfactory results. The following method, originated by Darling and described by Deeks ${ }^{420}$ has given very good results in our hands.

A small portion of material is smeared on a slide by covering with another slide and drawing the two apart lengthwise, and dried in the air.

The slide is then fixed for five minutes with undiluted Hasting's stain, after which distilled water is added until no more will stay on the slide. The staining is continued for one hour. More water and stain may be added from time to time to prevent drying, or it may be covered with a small bell jar. When the staining is completed the slide is rinsed in tap water and dried, following which it is placed in any good Giemsa solution. The one used by us is prepared by mixing 5 c.c. each of 0.1 per cent. aqueous solution of yellowish eosin and Azure II and diluting with 40 c.c. of distilled water.

The slide is stained in this solution over night, after which it is rinsed in tap water, dried and differentiated by dipping a few times in 60 per cent. alcohol containing I per cent. aqueous solution of ammonia.

Methods of dry fixation, however, are not as satisfactory, owing to the distortion of the nucleus, as certain wet fixation methods. For this purpose we have found Mallory's chloride of iron-hematoxylin stain as modified by James and described by Craig ${ }^{421}$ most satisfactory.

I. Thin smears of material are immersed without drying into Schaudinn's sublimate-alcohol solution and fixed for two to five minutes. (Schaudinn's solution was originally composed of one part of absolute alcohol and two parts of saturated aqueous solution of bichloride of mercury. It has been modified to contain one part absolute alcohol to two parts of normal salt solution saturated with bichloride, plus 2.5 per cent. glacial acetic acid.)

2. After fixing, the smears are hardened in 80 per cent. alcohol for ten minutes.

3. Smears are washed in 50 per cent. alcohol and then in distilled water. 
4. Smears are flooded with a mordant which consists of freshly prepared so per cent. aqueous solution of chloride of iron.

5. After pouring off the mordant the smears are flooded with freshly prepared solution of hematoxylin made by adding crystals of hematoxylin to 250 c.c. of warm distilled water, until a deep port wine color is produced: The stain is added to the slides until the hematoxylin is precipitated, which is shown by the smear appearing through the solution. The staining is continued for five to twenty minutes.

6. Following the staining the smears are washed in at least ten changes of distilled water.

7. They are then decolorized with freshly prepared 0.5 per cent. solution of chloride of iron in distilled water.

8. Smears are washed in tap water over night.

9. They are next dehydrated in 95 per cent. followed by absolute alcohol.

Io. Cleared in xylol.

II. Mounted in neutral balsam.

The cytoplasm of the endamebæ stains a grayish blue, the nuclear membrane and chromatin a deep black, while the structure of the nucleus is beautifully brought out.

While it is necessary to differentiate amebæ from certain other cells and artefacts which appear in the feces, the main point in the diagnosis in amebic dysentery is the differentiation of Endamœba histolytica from Endamœeba coli.

As far as we are aware no one has reported the finding of Endamøba buccalis in the feces, but it seems at least possible that in severe cases of Rigg's disease these organisms might be observed in the feces and perhaps mistaken for Endamœba histolytica.

The following table shows the main differentiating features between Endamœba histolytica and Endamœba coli: 


\begin{tabular}{|c|c|c|}
\hline & E. coli & E. histolytica \\
\hline Size......... & $\begin{array}{l}\text { Variable, average } 15 \text { to } 25 \mathrm{mi}- \\
\text { crons in diameter, in vegeta- } \\
\text { tive stage. }\end{array}$ & $\begin{array}{l}\text { Variable, average } 25 \text { to } 40 \mathrm{mi} \text { - } \\
\text { crons in diameter. }\end{array}$ \\
\hline Shape. . & Spheric at rest. & Roughly spheric or oval. \\
\hline Color.... & Dull greenish yellow. & $\begin{array}{l}\text { Ectoplasm hyaline, endoplasm } \\
\text { light gray or greenish tinted. }\end{array}$ \\
\hline Structure. & $\begin{array}{l}\text { Ordinarily impossible to differ- } \\
\text { entiate between endoplasm } \\
\text { and ectoplasm. Protoplasm } \\
\text { finely granular. }\end{array}$ & $\begin{array}{l}\text { Differentiation of endoplasm } \\
\text { and ectoplasm easy. Endo- } \\
\text { plasm coarsely granular. }\end{array}$ \\
\hline Nucleus. & $\begin{array}{l}\text { Well defined, to one side of } \\
\text { center, } 5^{-8} \text { microns in diame- } \\
\text { ter, one or more nucleoli vis- } \\
\text { ible. }\end{array}$ & $\begin{array}{l}\text { Poorly defined, average diame- } \\
\text { ter } 5 \text { microns. }\end{array}$ \\
\hline Vacuoles. . & $\begin{array}{l}\text { Generally absent, generally al- } \\
\text { ways single, usually small and } \\
\text { indistinct. }\end{array}$ & $\begin{array}{l}\text { Always present, may be nu- } \\
\text { merous, distinct. }\end{array}$ \\
\hline Motility....... & $\begin{array}{l}\text { Sluggish, pseudopodia rounded } \\
\text { and very small. }\end{array}$ & $\begin{array}{l}\text { Active, pseudopodia rounded } \\
\text { or finger-like, larger and more } \\
\text { distinct. }\end{array}$ \\
\hline Phagocytosis .. & $\begin{array}{l}\text { Bacteria and crystals, rarely } \\
\text { blood-cells. }\end{array}$ & $\begin{array}{l}\text { In addition to bacteria and } \\
\text { crystals, red blood-cells often } \\
\text { present. }\end{array}$ \\
\hline Cysts. . & $\begin{array}{l}\text { A third smaller than vegeta- } \\
\text { tive forms, limiting wall of } \\
\text { double outline, protoplasm } \\
\text { hyaline, no phagocytosis, } \\
\text { eight nuclei. }\end{array}$ & Four nuclei. \\
\hline Reproduction.. & $\begin{array}{l}\text { In vegetative stage by binary } \\
\text { fission and by sporulation, in } \\
\text { cystic stage by formation of } \\
\text { slight daughter cells. }\end{array}$ & $\begin{array}{l}\text { In vegetative stage by binary } \\
\text { fission, in cystic stage by pro- } \\
\text { duction of four daughter cells. }\end{array}$ \\
\hline
\end{tabular}

In staining the amebæ in tissues the following method of Mallory ${ }^{422}$ gives very satisfactory results:

I. Harden in alcohol.

2. Stain sections in a saturated aqueous solution of thionin three to five minutes.

3. Differentiate in a 2 per cent. aqueous solution of oxalic acid for one-half to one minute.

4. Wash in water.

5. Dehydrate in absolute alcohol.

6. Mount in xylol balsam. 
By this method the nuclei of the amebr, the granules of the amebre and the granules of the mastzellen are stained a brownish red; the nuclei of the mastzellen and of all other cells are stained blue.

Differential Diagnosis.-There are a few conditions which closely resemble amebic dysentery and must be differentiated from it. The most important of these is bacillary dysentery, which, while its tendency is much more to assume an acute form than amebic dysentery, is usually without microscopic examination impossible of differentiation from the latter.

In amebic dysentery the stools will almost always be found to contain Endameba histolytica upon microscopic examination. If this organism is not found it might be well to attempt animal inoculation as suggested by Sellards and Baetjer. ${ }^{413}$ In bacillary dysentery the $B$. dysenteria can usually be recovered from the feces by cultural methods, and in all but very mild cases the blood serum of patients suffering with bacillary dysentery will agglutinate either the Shiga bacillus or the Flexner bacillus after seven or eight days.

Hemorrhagic typhoid may be mistaken for amebic dysentery, especially in the tropics where it usually runs an atypical course. In the absence of amebæ in the stools a positive blood culture would clear up the diagnosis. Later a Widal test would be of service.

Not infrequently malaria may simulate amebic dysentery, but an examination of the blood should make it impossible to confuse the two conditions, even in the absence of amebæ in the stools.

Tuberculosis of the intestines is sometimes mistaken for chronic amebic dysentery and when amebæ cannot be found in the feces may be difficult of differentiation. A diagnostic point is that in tuberculosis of the intestine tenderness is usually more marked in the right iliac fossa, while in amebic dysentery it is usually more marked over the sigmoid. The various tuberculin tests may be of value, while the finding of the tubercle bacillus in the feces will be conclusive.

Malignant involvement of the sigmoid and rectum may be mistaken for amebic dysentery, and especially in old people the 
rectum should be examined, when little difficulty should be encountered in arriving at a correct diagnosis.

The invasion of the alimentary tract by other intestinal parasites, such as hook-worm, may cause symptoms simulating amebic dysentery, but microscopic examination of the feces should clear up the diagnosis.

The diarrhea of pellagra has been mistaken for amebic dysentery, but here again even in the absence of the typical eruption of pellagra the diagnosis should be easy upon examination of the feces.

It must be remembered that it is not only possible for amebic dysentery to be complicated by any of the above-mentioned conditions, but that such complications as malaria, pellagra and hook-worm are by no means rare.

The physician should therefore be upon his guard to recognize such complications by careful examinations not only of the feces but also of the blood.

Therapeutic Test.-Since the introduction of emetine into the therapy of amebic dysentery, the injection of this drug may be of importance in clearing up the diagnosis of obscure cases. If the symptoms improve under such treatment it would be strong presumptive evidence that the disease is amebic dysentery. If they do not improve the reverse would be the case.

Hepatic Abscess.-This condition following amebic dysentery may usually be diagnosed by increase in size of the liver, by pain, which if not present can be elicited by pressure, by the temperature and leucocytosis. Or, as a last resort, aspiration with a needle may be performed. Of course the finding of pus by aspiration does not prove the abscess to be of amebic origin and a search for the organisms should be made. This may be done by similar methods to those described above for feces.

The endamebæ are found in the aspirated pus of hepatic abscess in only about 50 per cent. of cases, but can be found in all cases by scraping the wall of the abscess. 


\section{CHAPTER XXX}

\section{PROGNOSIS OF AMEBIC DYSENTERY}

Death in amebic dysentery may occur from the gravity of the intestinal lesions, from exhaustion in long-protracted cases, from severe complications, such as hepatic abscess, peritonitis due to perforation, etc., from severe intestinal hemorrhage, from terminal infection, or from intercurrent disease.

The severity of the intestinal lesions and hepatic abscess are the most frequent causes of death.

Musgrave ${ }^{416}$ gives the following as the causes of death of fifty fatal cases of intestinal amebiasis without diarrhea:

Three from peritonitis following perforation of the appendixtwo of these produced by amebic ulceration, the other by an unknown cause, not amebic.

Four from liver abscess-one perforating in to the right pleura, one in to the abdominal cavity, and two without perforation.

One from acute pericarditis.

Eight from pulmonary tuberculosis, and in three of these abdominal tuberculosis was also present.

Two from chronic estivo-autumnal fever.

Five from perforation of amebic ulcers in the large intestinefour times in the cecum and ascending colon, and once in the transverse colon.

Seven from acute beriberi.

Twenty from lobar pneumonia.

Mortality. - The mortality in amebic dysentery varies with the severity of the infection, the time of beginning treatment, the resistance of the patient, and the method of treatment.

Duncan $^{423}$ states that among sixty cases in the Niger Protectorate, of those treated with ipecac the mortality was 32 per cent., while of those treated with magnesium sulphate the mortality was only 2.9 per cent. In the Malay States, of 337 cases the mortality with ipecac treatment was 3 I.I per cent., 
with saline treatment 23.6 per cent., while with boric acid treatment the mortality was i 8 per cent. In the Nigri-Sembilin Hospital the mortality sank from 34.2 per cent. to I 7.88 per cent. after the introduction of the boric acid treatment.

Of 100 cases treated by Musgrave ${ }^{417}$ in private practice, including all types and stages of the disease, ninety-six permanently recovered without leaving the Philippines, one died and three returned to the United States.

Prognosis.- - In making a prognosis of amebic dysentery the fact of the great tendency of this disease to recur must be kept in mind.

In children, as a rule, the prognosis is good, while in the aged it is bad. It has been stated that alcoholics are less liable to infection. This we do not believe to be the case as the chronic gastritis so frequently observed in alcoholics predisposes to the disease, and certainly the prognosis in this class of individuals is not good.

Good general physical condition and previous good health make for a favorable prognosis.

According to Musgrave ${ }^{417}$ the location of the lesions is important as a prognostic indication. Thus the higher up the lesions the less favorable the prognosis.

The outlook in cases with acute onset is always grave, while if the onset is gradual and the condition recognized early the prognosis is good.

Complications will, of course, render the prognosis less favorable; thus in hepatic abscess the chance for recovery is lessened, depending upon the size of the abscess.

Strong ${ }^{424}$ states that in twenty-seven cases reported by Futcher there were nineteen deaths. Of these cases seventeen were operated upon with only five recoveries. In his own cases Strong reports twelve cases of abscess with three recoveries after operation. One of us has operated on six cases with no death. 


\section{CHAPTER XXXI \\ PROPHYLAXIS OF AMEBIC DYSENTERY}

Since the Endamoba histolytica is primarily an inhabitant of the intestinal canal and is passed with the feces of the vast majority of patients suffering from amebic dysentery, obviously one of the most important measures of prophylaxis is the disinfection of the stools in all cases. This is best done by the use of chloride of lime. Bichloride of mercury, $1-1, \infty 00$, or ro per cent. phenol is very satisfactory.

The so-called "carriers" are a source of danger. It is these individuals, in whom the organisms exist in the encysted state without any active manifestations of the disease, that should as far as possible be sought out and treated. It is obviously impossible to make examination of the feces of all persons to detect carriers, but with certain units of population, such as in eleemosynary institutions, hospitals, prisons, etc., it is not only possible, but very desirable to make such examinations. It goes without saying that the excreta of such individuals should be carefully disinfected.

While the life history of the Endameba histolytica outside of the human body is unknown, inasmuch as certain animals, especially the cat, are known to be capable of artificial infection, it is well within the range of possibility that they may become infected without the intervention of artificial means. It therefore seems to us that cats as household pets should be eradicated from endemic centers of the disease.

As stated above, all authors are agreed that practically the only source of infection is by the ingestion of the infective organisms, and in all probability the main source of these is drinking water. It therefore becomes an essential to the prevention of the disease that nothing but water free from the amebæ be used for drinking purposes. Obviously in endemic centers of amebic dysentery it is impossible to secure a raw 
water that is absolutely above suspicion. It is therefore advisable to boil all water before use, and to store it in as nearly a sterile condition as possible. It is well also to prepare only a sufficient quantity for use for a short time at once, to avoid possible contamination during storage. Of course in endemic centers the use of distilled water or bottled and carbonated waters is to be recommended where possible. However, care should be exercised that they come from reliable sources.

The eating of raw foods such as lettuce, cress, etc., is not to be recommended.

The use of raw milk is also to be looked upon as a possible source of danger in endemic centers, and unless the most rigid sanitary inspection is exercised over the source of the milk supply, this should be boiled before use.

Alcoholics are perhaps more susceptible to the disease than other individuals, therefore the use of alcohol as a beverage should be interdicted.

It goes without saying that the general health of individuals, especially those residing in endemic centers of amebic dysentery, should be kept as nearly perfect as possible and that all measures of prophylaxis of other infectious diseases should be used.

And, finally, it is well to remember the case cited above in which infection followed the use of cold water enemas and guard against such a possibility. 


\section{CHAPTER XXXII}

\section{TREATMENT OF AMEBIC DYSENTERY}

The treatment of amebic dysentery consists of hygienic measures, general and symptomatic treatment, surgical procedures, and specific treatment. To these may be added the surgical and specific treatment of hepatic abscess.

Hygienic Treatment.-In the acute attack of amebic dysentery the patient should be placed in bed and should not be permitted to get up to urinate or go to stool, using the urinal and bed pan. The room should be well ventilated, but chilling draughts of air which may increase congestion of the internal viscera should be avoided. In the chronic course of the disease it is not necessary for the patient to remain in bed, in fact is not desirable. A certain amount of exercise, depending upon the general condition of the patient, should be taken, although over-exertion and fatigue should be avoided. Care should be taken to avoid exposure to cold and dampness, and the clothing should be sufficient to keep the body warm at all times.

The diet is of the utmost importance in treating amebic dysentery. During the early acute stage it should consist of nothing but albumin water. Later, when the symptoms have shown some improvement, chicken or beef broth may be given. Milk is usually contra-indicated owing to its tendency to form bulky residue. As improvement continues soup and eggs may be eaten, while minced chicken, fish, etc., can usually be allowed within a few days. The diet in chronic amebic dysentery should be sufficient to restore the body weight, yet it should not be excessive and should contain as little residue as possible. A diet composed largely of meat is very desirable. There is little residue in such a diet and it furnishes a large amount of nourishment; further, meat stimulates a flow of the gastric and intestinal juices.

In prescribing a meat diet care must be taken to change frequently the kind of meat used, as well as the method of cooking 
it. Eggs, tea, small amounts of toast and butter are permissible, as well as fresh stewed fruits and small quantities of fresh cooked vegetables.

General and Symptomatic Treatment.-In the acute stages of the disease when griping is intense and rest is imperative the use of morphine is to be recommended. This drug should be pushed to the physiologic limit if needed. Enemas of laudanum may be given. As the symptoms improve Dover's powders may be substituted. Nausea and vomiting when troublesome may usually be controlled by the alkaline carbonated waters. Pepsin, hydrochloric acid and pancreatin may be employed for the dyspepsia seen in the chronic stages.

If abdominal pain is severe it may usually be relieved by turpentine stupes, hot fomentations or the ice-bag. It may be necessary to administer opium in some form for the pain.

Bismuth was formerly given quite extensively for the diarrhea, but its use is not to be recommended.

In the beginning of treatment, whether in the acute or chronic stage, the administration of a dose of castor oil is usually advisable. This clears the bowel, and in a measure, at least, assists in removing the infecting organisms. The use of any purgative, however, is contra-indicated where the diarrhea is severe and the stools thin and bloody.

The number of drugs which have been recommended in amebic dysentery is legion, and only the most important of them can be mentioned.

Copper sulphate and opium have been recommended in chronic dysentery. Oil of turpentine has also been used very extensively. Salol, benzosol, benzoyl-acetyl-peroxide, salophen and many other similar preparations have been recommended and have had more or less extensive use.

The use of rectal injections of various drugs for their amebacidal action has been widely recommended. After a preliminary douche of warm water to cleanse the lower bowel the injection of the drug at a temperature of about $96^{\circ} \mathrm{F}$. is made.

Solutions of quinine have been extensively employed for this purpose since the time of Loesch. This drug is usually used in a I to I,500 dilution and about I liter injected. Argy- 
rol has been highly recommended in I per cent. solution, while thymol, potassium permanganate, silver nitrate, protargol, boric and tannic acids, creosote, etc., have had more or less vogue.

Surgical Treatment.-Several surgical procedures have been advocated in the treatment of amebic dysentery. For the most part, however, they have been recommended only in the most severe cases and those which have not yielded to other methods of treatment. According to Herrick, ${ }^{425}$ White, in 1895 , was the first to suggest surgical treatment in this disease. This worker recommended making an artificial anus on the right side for the purpose of giving the colon rest and to administer local treatment in severe cases of ulcerative colitis. Later other writers advocated cecostomy and appendicostomy with lavage of the colon with various antiseptics, such as argyrol, silver nitrate, potassium permanganate, etc.

In certain extreme cases of amebic dysentery where the structure of the colon has been very extensively destroyed and the patient almost moribund, the excision of the entire colon has been performed.

Specific Treatment.-While, as mentioned above, certain drugs such as quinine, salol, etc., have been injected rectally for the purpose of destroying the infecting organisms, they are not considered true specifics. Ipecac, however, has been employed as a specific for dysentery for over two centuries. This drug was first known in Europe in ${ }^{6} 6_{72}$, having been brought from Brazil where it was used by the natives as an emetic. ${ }^{462}$ John Helvetius, grandfather of the famous author of that name, used it so successfully in the treatment of the Dauphin that he was given a large sum of money and public honors by Louis $\mathrm{XIV}$ upon the condition that he make the nature of his treatment public. While in Europe the use of ipecac fell into disrepute, it was employed with considerable success in India, and in 1858 Docker, an English army surgeon, brought it again into prominence by advising its use in comparatively large doses. It has since that time been used with varying success in nearly all countries where amebic dysentery is known. The usual method of procedure in administering ipecac is to instruct the patient to abstain from food for three or four hours, and about 
twenty minutes after giving a dose of ten to twenty drops of laudanum to give 20 to 60 grains of the powdered ipecac root, usually in capsules. After this the patient is instructed to lie on his back in bed, absolutely quietly for at least four hours. Should nausea and vomiting of the drug be caused within an hour he should receive another dose as soon as the nausea ceases.

The main objection to the ipecac treatment of amebic dysentery has been that in doses sufficiently large to exert any specific action nausea and vomiting are almost sure to occur. Even the pills coated with substances insoluble in the stomach do not entirely overcome this effect.

The real specific treatment of amebic dysentery may be said to have begun with the introduction of emetine for this purpose.

This drug is one of the alkaloids of ipecac discovered in 1867 by Pelletier and has the formula $\mathrm{C}_{15} \mathrm{H}_{22} \mathrm{NO}_{2}$. It is a white amorphous powder with a melting point of $60^{\circ} \mathrm{C}$. With the halogens and with nitric acid it forms crystalline salts. It is readily soluble in chloroform, ether, benzol or alcohol. Its solution in the latter gives no coloration with ferric chloride. Emetine is insoluble in solutions of caustic or carbonated alkalies, but is soluble in acetic acid in which latter it effects no substitution. The most frequently employed salt of emetine is the hydrochloride $\left(\mathrm{C}_{15} \mathrm{H}_{22} \mathrm{NO}_{2} \mathrm{Cl}_{2} \mathrm{H}_{2} \mathrm{O}\right)$.

The first use of emetine in amebic dysentery is accredited to Walsh, ${ }^{427}$ who in $189 \mathrm{r}$ employed it in combination with Mayer's reagent. He reported 34 cases diagnosed clinically, treated in this manner, of which 32 were clinically cured in an average of 4.9 days, one was unimproved and one died. It was not until twenty years later that any scientific work was done to determine the action of emetine on the amebæ. In I9I I Vedder, ${ }^{428}$ working with cultures of the free living amebæ, showed that ipecac in dilutions of $\mathrm{I}$ to 10,000 to I to 50,000 was amebecidal, and deematized ipecac in dilution of $\mathrm{r}$ to 5,000 was not amebecidal, while emetine in dilution of $I$ to 100,000 killed the amebæ.

To Rogers, ${ }^{429}$ however, is due the credit of applying emetine, clinically to amebic dysentery. This worker first found that solutions of emetine hydrochloride in normal saline in dilutions 
of I to I0,000 immediately killed the active pathogenic amebæ (E. histolytica) present in mucus, and that in dilutions of $\mathbf{I}$ to I 00,000 they were rendered inactive and were apparently killed. After these experiments Rogers tried the drug hypodermically in cases of amebic disease and reported most favorably upon it. In twenty-four cases treated in this manner twenty were cured, two moribund patients died within three days after admission to the hospital, and two died from other diseases following the cure of the dysentery. The average time in the hospital was 7.2 days, while the average time until the stools became normal was 2.35 days.

Following the brilliant results of Rogers many investigators have used emetine in the treatment of amebic dysentery, with such generally favorable results that there can be no doubt that this drug should rank as a specific with quinine in malaria and mercury and salvarsan in syphilis.

The dosage employed by Rogers was $1 / 3$ to I grain of the hydrochloride dissolved in about I c.c. of salt solution administered hypodermically. This amount has been increased by various workers up to $5^{-6}$ grains. Baermann and Heinemann ${ }^{430}$ recommend the subcutaneous or intravenous injection of one or two doses of $2 \frac{1}{3}$ to 3 grains, followed in the next eight or ten days by four or five subcutaneous injections of $1 \frac{1}{2-2}$ grains given at intervals of two or three days according to the results of examination. According to these authors this after-treatment should be repeated at intervals of three or four weeks and the stools should be examined carefully for amebæ at frequent intervals for several months.

We consider that for ordinary cases such doses are too large and prefer to give not more than I grain daily, best in two injections, one in the morning and one at night. This seems as effective as the larger doses and much less irritating.

In administering emetine intravenously the dose, not to exceed 0.25 gram (3.8 gr.) per 60 kilograms ( $3_{2} 2$ pounds) of body weight, should be given in about roo c.c. of normal salt solution. This, to our minds, is the method of choice in acute cases. Emetine may be given by mouth, but is neither as effective nor as pleasant to take, as nausea and vomiting are 
much more liable to occur. And further, the drug is more or less irritating to the intestinal mucosa.

Nausea and vomiting are rare following the subcutaneous and intravenous injection of emetine except in very large doses (5-6 grains).

Depending upon the size of the dose there is some local reaction at the point of injection when the subcutaneous route is used, and a general urticaria has been noted.

Hepatic Abscess.- If the hepatic abscess be small, it will probably usually yield to the general emetine treatment alone. Rogers ${ }^{429}$ early reported the aspiration of liver abscess and the injection of the cavity with I grain of emetine dissolved in I ounce of normal salt solution. This with small and medium sized abscesses is usually sufficient, but with large abscesses a radical operation may be necessary, although Cantlie ${ }^{431}$ considers "cutting" operations, "overheroic" and that better results are obtained by the use of a large trocar and canula. A recent case treated by one of us was promptly cured after evacuation of the pus and daily injections of $1 / 3$ grain of emetine.

The operations most frequently employed are the abdominal and transthoracic. The former is employed when the abscess is in the lower portions of the liver and the latter when it is found in the superior portions or extends into the thorax.

The abdominal operation is performed by placing the patient on his back or if a lateral incision is necessary on his left side. A sand bag is placed under him to raise the liver up as close to the abdominal wall as possible. An incision 8 to $10 \mathrm{~cm}$. in length over the most prominent part of the swelling is made through the skin and superficial fascia and is deepened to the peritoneum. If adhesions exist between the abscess wall and the peritoneum, walling off the abscess from the peritoneal cavity, the abscess may be opened at once. If, however, no adhesions are present, a series of sutures are made between the capsule and some of the liver substances and the parietal peritoneum and part of the rectus muscle. The peritoneum is walled off with a thick layer of gauze, after which an incision is made into the abscess cavity and the pus allowed to escape. Some authorities advocate the use of a cautery, but this is usually unnecessary. After the 
evacuation of the pus the abscess cavity may be washed out with normal salt solution followed by 2 or 3 grains of emetine dissolved in several ounces of salt solution. A large drainage tube is now inserted and anchored with two stitches to the skin.

In performing the transthoracic operation an incision 8 to to $\mathrm{cm}$. in length is made along the rib under which the abscess seems to be. This incision should separate skin, fascia, muscle and periosteum. The latter is separated from the bone with a periosteal elevator for about $8 \mathrm{~cm}$. and the rib cut at either end of the incision with bone forceps or a saw. The same care must be taken not to contaminate the pleura as was advocated for protection of the peritoneum. The remainder of the operation is performed in a similar manner to the abdominal operation.

Other complications, such as severe hemorrhage, a peritonitis, and intercurrent disease must be met and dealt with as if the amebic infection did not exist. 


\section{HOOK-WORM DISEASE}

\section{CHAPTER XXXIII}

\section{INTRODUCTION}

Uncinariasis, ankylostomiasis, tropical chlorosis, tunnel workers' anemia, brickmakers' anemia, mountain cachexia, miners' anemia, dirt-eating disease, etc.

History.-Uncinariasis is a parasitic infestation with certain species of hookworm and is characterized by the discharge of ova and worms in the feces, progressive anemia, and digestive and nervous phenomena.

There is to be found in the Ebers papyrus, written by the Egyptians about $155^{\circ}$ B. C., a reference, thought by Joachim and Sandwith, to relate to hook-worm infection. The authenticity of this reference must of necessity remain doubtful owing to the incompleteness of the clinic picture.

The earliest undoubted accounts of this disease are those of Piso, in Brazil, in I648, Pere Labot of Guadeloupe in his travel narratives in $\mathrm{I} 74^{2}$, and Bryon Edwards in $\mathrm{I} 793$, in his history of the British Colonies in the West Indies. The latter who was a planter in Jamaica for many years attributed a large proportion of the deaths among the negroes to this disease. Following these were numerous reports by English, French and Danish practitioners in the West Indian Islands and in Guiana.

The etiology of the condition was completely dark until the paper of Dubini in 1843 . From 1838 this observer at several autopsies had discovered these little worms to which he attributed the production of chronic diarrhea.

Castaglioni found the parasite in Milan in I844. Pruner in Egypt mentioned, in 1846 , that he had found it in cachectic, scrofulous and dropsical adults, but he failed to note any etiologic relationship. 
Bilharz discovered independently the worms in Egypt in I $85^{2}$, and it was with him that Griesinger established the fact that the hook-worm caused the disease which he declared affected one-fourth of the population of Egypt.

Wucherer in Brazil in 1866 called attention to the prevalence of the disease in that country and succeeded in arousing some interest.

In Italy Paletti and Maliverria, in $\mathrm{I} 877$, suggested that brickmakers' anemia was due to hook-worm infection and in the same year recognized in the feces the typical ova of the parasite, and attributed grave results to the blood-sucking propensities of the parasite.

The epidemic occurring in 1879 among the workers of the St. Gothard tunnel marks an epoch in the prophylaxis of the disease. Hundreds of these laborers sickened and many died; no cause was known, and gases, faulty ventilation and lack of sunshine were advanced. Colormiatti found $I, 500$ uncinarix in the intestines of one of the dead laborers. As a result of the investigation which followed the true cause was determined, and to Perroncito and Bozzolo is given the credit for the campaign against the epidemic.

Profiting by the lessons of this epidemic, investigations were then made into the anemia of mines and brickyards of Europe with the uniform result of finding the hook-worm responsible.

For more than roo years anemia has been recognized as the scourge of Porto Rico. On August 8, 1899, a hurricane swept a part of the island. Ashford, of the Army Medical Corps, was ordered to establish a provisional hospital for the care of some of the sick natives who were thronging the streets. Blood examinations of these natives showed an eosinophilia which led to an examination of the feces disclosing the ova of the parasites responsible for the trouble. On November 24, I899, Ashford wired the chief surgeon of his findings. This culminated in the appointment of the Porto Rico Anemia Commission, which has contributed much toward the eradication of the infection from the island and toward the scientific study of the disease.

In the United States the earliest reference which seems to allude to hook-worm infection is that of Pitt, in 1808 , writing of 
the Roanoke Valley in North Carolina. Chalbert, in Louisiana, in 1832 , Cotting, in Georgia, in 1836 , Le Conte, in Georgia, in I845, Little, in Florida, in 1845 , and Duncan, in Louisiana, in I850, refer to what was probably uncinariasis.

Blickhahn, of St. Louis, in 1893 , is apparently the first in this country to observe and recognize as such, a case of hook-worm infection. His patient was a German brickmaker who had been in America but seventeen months, and it is probable that the infection was imported.

In I893, Allen J. Smith, of Galveston, Texas, found ova of hook-worm in a specimen of feces but was unable to locate the patient. Cases were reported from Buffalo, N. Y., by Mohlau in I897; from Richmond, Va., by Gray in I901; from New Orleans, by Tebault in I899; from Galveston, Texas, by Smith in I901; from Washington, D. C., by Clayton in I $90 \mathrm{I}$; from St. Louis, by Dyer in I90I; from Georgia, by Claude Smith in I902; and by Harris in 1902 .

The credit, however, for the discovery that hook-worm is endemic in the Southern States belongs to Stiles. Upon the evidence of specimens from Washington, D. C., from Porto Rico, sent by Ashford, from Cuba, sent by Guiteras, and from Galveston, sent by Smith he stated positively in a paper published September 25, 1902, that there existed in the United States an endemic uncinariasis, which had been generally overlooked. He was thereupon commissioned by the Public Health and Marine Hospital Service to investigate, and after a preliminary survey in Virginia, North Carolina and South Carolina he reported, October 22, 1902, that the disease was resulting in loss of wages, loss in productiveness of the farms, loss in the school attendance of the children, etc. $\mathrm{He}$ announced also that the parasite was a new species which he termed uncinaria americana.

Stiles' observations were abundantly confirmed 'from many sections and great interest was aroused, the matter being. discussed pro and con in the lay press. The concern culminated in the organization on Oct. 26, 1909, of the Rockefeller Sanitary Commission, financed by John D. Rockefeller, for a campaign against the endemic in the South. 
Geographic Distribution.-Uncinariasis does not occur north of latitude $5^{2}$, and north of $47^{\circ}$, it is found only in the hospitable warmth of mines. Thus it is a disease of tropic and subtropic countries, but there are countries with endemic foci in more northerly climes.

In Germany the Westphalia mining districts are heavily infested. The disease prevails also in localities in Rhenish Prussia. Cases have been reported from other parts of Prussia. In Austria and Hungary the disease has been known to be prevalent for many years, particularly among miners, but among other classes as well. From Limburg in Holland several cases have been recorded. The Belgian mines are hot beds of infection where public measures have been directed against the suppression of the disease. In France, Auzin, Vallenciennes, Commentry, Lyons and St. Etienne are centers of infection. Uncinariasis is said to occur in the Spanish mines and in Servia and Bulgaria. In Italy, Sicily, and Sardinia the disease prevails extensively. In the Dolcoath coal mine in Cornwall, England, nearly 20 per cent. of the miners have been found infested.

In Asia a number of the Indian Provinces, particularly Bengal, Madras, and Travancore are scourged. It has been stated that in Ceylon the ravages of the hook-worm exceed those of cholera. Siam, Indo-China, Korea, and portions of China are intense foci. In Japan the mountainous regions of the island of Kiou-Siou are said to be intensely infested. The disease prevails in the Malay States and is found in the Province of Bagdad.

The continent of Africa, especially the coast regions are foci of hook-worm infection. Parts of Egypt are heavily scourged. Abyssinia, German East Africa, Nyassaland, British Central Africa and Orange Free State show a high infection index. British South Africa, parts of Cape Colony, and Madagascar are infested. In the north, besides Egypt, Tunis and Algeria habor the disease. On the west coast it prevails from the Senegal River to the Congo River.

In Australia the infection is known upon the west coast particularly in Queensland. Other islands where the disease 
is known to be endemic are the Leeward Islands, Comore Islands, Mauritius, New Guinea, Bcrneo, Java, Sumatra, Fiji, Sandwich Islands, West Indies, Samoa, Formosa, Philippines and Guam.

In South America the disease has been studied particularly in Brazil. Paraguay and northern Argentine are endemic foci. Other South American States where the infection prevails are Bolivia, Peru, Ecuador, Columbia, Venezuela and Guyana.

Central American States from which cases are reported are Panama, Costa Rica, Nicaragua, Salvador, Guatemala, and British Honduras.

Cases have been found in southern Mexico and in Lower California.

In the United States the disease is endemic in all the states south of the Ohio and Potomac Rivers, and in Arkansas, Louisiana and Texas. Autochthonous cases have been reported from Oklahoma, Nevada, California and Missouri and it is probable on clinical grounds that the disease exists in Maryland.

To illustrate the extent of infection in some of the Southern States the following is quoted from the I9r2 Report of the Surgeon General of the U. S. Public Health Service:

"Hook-worm infection has been demonstrated in 93 of the roo counties in Virginia; in 99 of the roo counties in North Carolina; in 140 of the 146 counties of Georgia; in every county in South Carolina; in 66 of the 67 counties in Alabama; in 77 of the 79 counties in Mississippi; in 27 of the 59 parishes of Louisiana; in 57 of the 75 counties in Arkansas; in 95 of the 96 counties in Tennessee; in 22 of the II9 counties in Kentucky. The foregoing statistics include the reports up to December $3 \mathrm{I}$, I $9 \mathrm{II}$, as reported by the state boards of health in question. Of the 884 counties in these ten states, infection has been demonstrated by the state boards of health in 719; the remaining I 56 counties had not been surveyed when these statistics were summarized."

The comparative degree of infection at all ages in eleven states is shown in the following table from the r913 Report of the Rockefeller Sanitary Commission: 


\begin{tabular}{|c|c|c|c|}
\hline & Examined & Infested & Per cent. \\
\hline 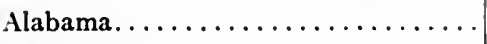 & 25,821 & I 1,204 & 43 \\
\hline 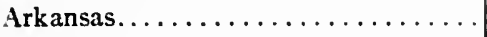 & 17,169 & 4,151 & 24 \\
\hline 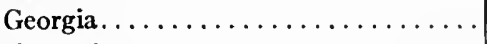 & 31,251 & 19,034 & 60 \\
\hline Kentucky $\ldots \ldots \ldots \ldots \ldots \ldots \ldots$ & 64,485 & 22,862 & 35 \\
\hline 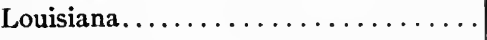 & 35,472 & 17,533 & 49 \\
\hline Mississippi $\ldots \ldots \ldots \ldots \ldots \ldots \ldots \ldots$ & I 10,007 & 42,722 & 38 \\
\hline 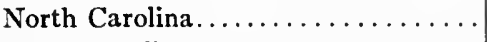 & 247,870 & 77,625 & $3 I$ \\
\hline South Carolina............. & 47,692 & 16,386 & 34 \\
\hline 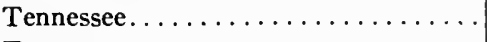 & 32,432 & 10,369 & $3 I$ \\
\hline Texas............... & 38,913 & I 3,447 & 34 \\
\hline Virginia ................... & 49,622 & I 2,888 & 25 \\
\hline Total. . & 700,734 & 248,221 & 35 \\
\hline
\end{tabular}

The new-world hook-worm, Necator americanus, besides being the parasite of the disease in the United States, has been found in Porto Rico, Cuba, Guam, Panama, Brazil, Philippine Islands, Australia, Ceylon, Mysore, Burmah, Assam, Cochin China, China, Egypt, Rhodesia, Gold Coast, Uganda, and South Africa. Imported cases have been recorded in Italy and Spain. 


\section{CHAPTER XXXIV}

\section{ETIOLOGY OF HOOK-WORM DISEASE}

Climate.-As has been stated hook-worm disease is one of tropic and subtropic climates with occasional endemic foci in temperate climates, these latter being chiefly in mines. It has been determined that temperatures from $78.5^{\circ}$ to $95^{\circ} \mathrm{F}$. are best adapted to the hatching of ova and that while they still matured below $7 \mathrm{I}^{\circ} \mathrm{F}$. their numbers were few and many perished. Direct exposure to sunlight inhibits the development of the ova. Altitudes from the sea level to 3,000 feet are had by endemic areas.

Season.--In cold weather larval life and activity are diminished or abolished and the hook-worm larva is no exception. Freezing prevents ova from hatching but they may develop after gentle thawing out. Infection in temperate climates is commoner in the summer months.

Moisture.-Moisture is necessary for the development of both ova and larvæ. Complete desiccation destroys both ova and larvæ. The ova will not develop in water but Lambinet ${ }^{201}$ has shown they will then enter a resistant state and hatch later under favorable circumstances. Bruns ${ }^{\prime 202}$ experiments show that (I) in undiluted feces the larvæ appeared in four days but in ten days all were dead; (2) the best cultures were obtained from a dilution of from $\mathrm{r}$ : ro to $\mathrm{I}: \mathrm{IOO}$; (3) dilutions of from I : I,, 00 to $I: 2,000$ killed the larvæ and prevented the ova from developing after six days.

Soil.--The character of the soil exerts an important influence on the distribution of the disease. Stiles ${ }^{203}$ has shown that in the South the infection prevails chiefly upon sandy soils. My experience in eastern Arkansas was that alluvial soil did not afford a favorable environment and this conclusion is further strengthened by the work of the Rockefeller Sanitary Commission. The Porto Rico Anemia Commission ${ }^{202}$ found that 
sandy regions were not those which contributed the majority of their cases on that island.

Race.-It was shown by Zinn and Jacoby ${ }^{72}$ that the negro possesses a relative immunity to the effects of hook-worm infection. It is probable, however, that negroes in the Southern States are more frequently infested than whites. Hence the carrier problem is, as in malaria, a weighty one. No immunity other than racial is known.

Sex.--Stiles ${ }^{204}$ believes the infection to be commoner in females than in males in the South. In Egypt Sandwith ${ }^{205}$ found only I per cent. of his cases in females. Of 89,219 cases examined by the Porto Rico Anemia Commission 52.10 per cent. were males and 47.90 per cent. were females. ${ }^{206}$

Age.-In the Southern States the disease is commoner in the young, 58.5 per cent. of Stiles' ${ }^{\prime 204}$ cases being in children under sixteen years old. Two years experience of the Porto Rico Commission $^{207}$ gave the following age distribution:

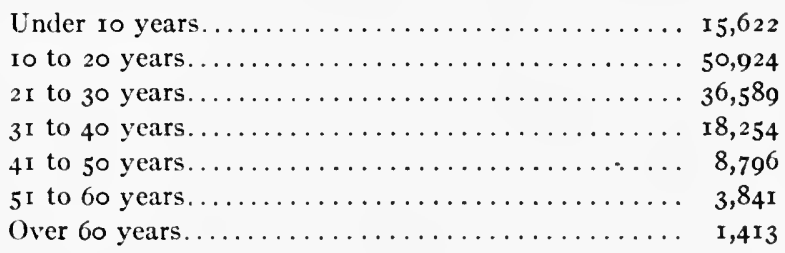

Of 18,865 cases found by this Commission ${ }^{208}$ in 1905 there were 240 under five years of age. Sandwith's ${ }^{205}$ youngest patient was four years old, Giles' ${ }^{\prime 203}$ four years old, and Stiles reports one in a child of three.

Occupation.-Those occupations which necessitate intimate relations with the soil predispose to hook-worm infection. Miners and brickmakers are the classes chiefly infested in Europe. In the South the agriculturists are especially predisposed, particularly those who go into the fields barefoot or poorly shod. Dock and Bass ${ }^{209}$ mention cases occurring in charcoal burners and turpentine dippers. The cotton mill operatives through the South are heavily infested, but this is probably due more largely to the insanitary condition of their homes than to the occupation. 
Social Condition.-While no class is exempt from infection those who use concrete sidewalks and modern sewerage are far less frequently infested than those who walk the fields and defecate in the fence corners. Uncinariasis is chiefly a disease of poverty, ignorance and bad hygiene. Surface closets and soil pollution are largely responsible for the dissemination of the disease, hence its over-whelming prevalence in the rural districts.

Mode of Infection.- There are two routes by which the parasites gain access to the host, through the mouth and through the skin.

There is no doubt that the former method is more common than is supposed by some authorities. Infection through unclean vegetables, fruits and drinking water, and soiled hands undoubtedly occurs. A considerable proportion of hook-worm patients in this country deny having had ground itch, and while some of these may have been unaware of an existing dermatitis, it cannot be denied that many of them are correct.

In $\mathrm{x} 898$ while working in his laboratory in Cairo Looss inadvertently allowed a drop of water containing more than $\mathrm{I}, \infty 00$ hook-worm larvæ to fall between the fingers. This was immediately followed by burning and redness. To be sure that the symptoms were due to the fluid he permitted another drop to fall upon another part of his hand and this was followed by the same results. Examining the fluid remaining upon his hand he found that most of the larvæ had disappeared leaving behind a few sluggish ones and numerous empty sheaths. In due time ova were found in the feces and anemia and debility ensued necessitating prolonged treatment with thymol.

Looss' next experiment was upon the leg of a boy, one hour before the leg was amputated. After thorough cleansing a drop of fluid containing larvæ was placed on the skin. After amputation the skin was removed, hardened and sectioned. The larvæ were found in the skin having penetrated through the hair follicles.

Another experiment was made upon a volunteer nurse who was infected through the forearm. Ova were found in the 
feces seventy-one days later. Looss succeeded in infecting puppies in a similar manner.

These experiments with ankylostoma duodenale were confirmed by Schaudinn in Germany, Herman in Belgium, and others.

The same mode of infection was proven for Uncinaria americana by Claude Smith of Atlanta. A mixture of soil and feces containing larvæ was bound upon the wrist of a volunteer. In five minutes a sharp stinging sensation was complained of. During the night and the next day the itching was severe, and the area was covered with vesicles. These crusted and desquamated and by the eighth day had disappeared. By the middle of the sixth week ova were found in the feces.

After piercing the skin through the hair follicles the larvæ gain entrance to the circulation and are carried through the heart to the lungs. Here they leave the blood-vessels, entering the air cells, traverse the bronchi to the larynx, and down the esophagus through the stomach to the intestine. From the entrance into the skin until ova appear in the feces averages from five to ten weeks.

The dermatitis produced by the entrance of the larvæ into the skin is known as "ground itch." Its commonest location is upon the feet and ankles, oftenest between the toes, but may occur upon any part of the body exposed to infection. Turner ${ }^{210}$ states that in the South African mines the miners sitting on damp wood contracted the sores on their buttocks.

The Parasite.-While Looss succeeded in infecting dogs with human hook-worms both through the skin and through the mouth, this parasite is not known in nature to infest any host but man. Uncinariasis is known to occur in many domestic and other animals but their parasites are not communicable to man.

The new-world hook-worm, Uncinaria americana, or Necator americanus, has a cylindrical body, narrowed anteriorly and of a light reddish or grayish color. The male measures from 7 to $9 \mathrm{~mm}$. in length, and 0.3 to $0.35 \mathrm{~mm}$. in diameter; the female 9 to I I mm. in length by 0.4 to $0.45 \mathrm{~mm}$. in diameter. The buccal capsule is small, presents an irregular border and is pro- 
vided with a ventral pair of prominent semilunar plates, there being dorsally a pair of slightly developed chitinous plates. The dorsal tooth projects prominently into the buccal cavity. The four buccal lancets are situated deeply. The tail of the
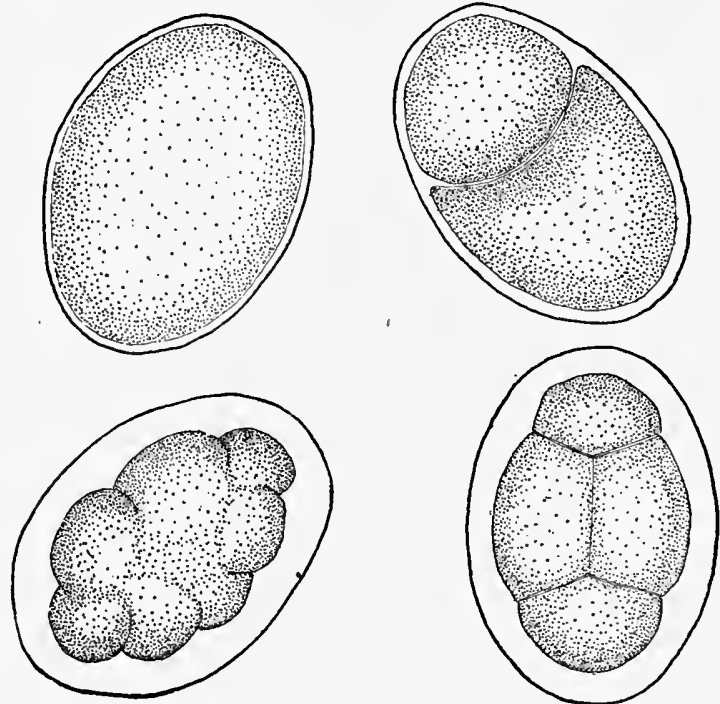

Fig. 86.-Ova of uncinaria americana, enlarged.

male flares into an umbrella-like caudal bursa, composed of dorsal and ventral lobes and prominent lateral lobes supported by rays like the ribs of an umbrella. Two barbed spicules proceed from the bottom of the caudal bursa and the genital organ lies at the bottom. The tail of the female is conical. The vulva is situated slightly anterior to the middle of the body. The eggs are symmetrically oval and vary from 64 to 76 microns in length by 36 to 40 microns in

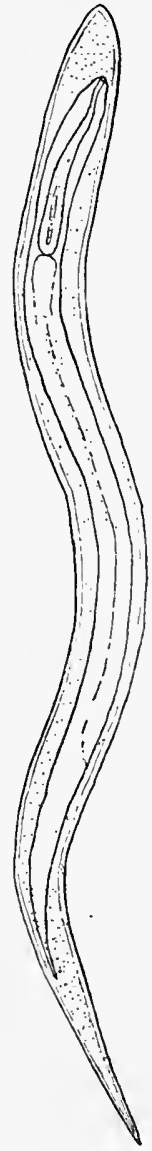

Fig. $87,-$ Hookworm larva, infective stage, enlarged. breadth. They are laid in a state of segmentation, the number of segments being most commonly four.

The old-world hook-worm is longer and broader, the mouth is heavily armed bearing two pairs of ventral teeth, curved like 


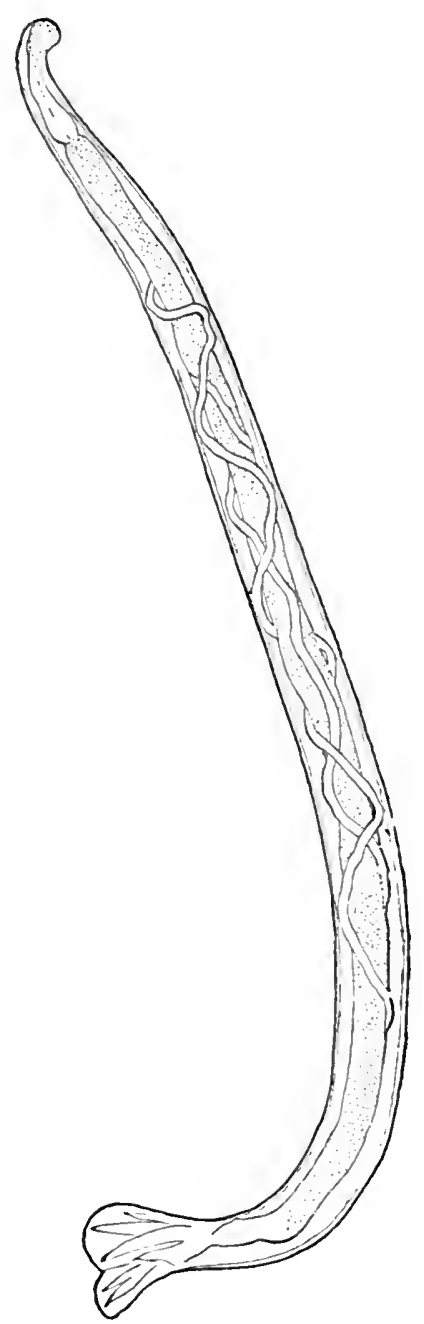

Fig. 88.-Uncinaria americana, male, enlarged.

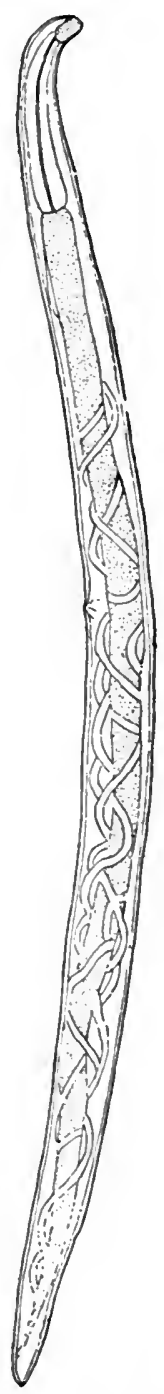

Fig. 89.-Uncinaria americana, female, enlarged.

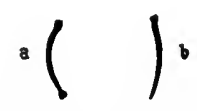

Fig. 90.-Showing natural size of hook-worms. $a$, Male; $b$, female. 
hooks, and one pair of dorsal teeth, the buccal capsule is larger, and the ova are smaller.

The ova never hatch in the intestine, but pass out with the feces. The ova hatch under proper conditions in from one to three days or less, into rhabditiform larvæ, measuring about $0.2 \mathrm{~mm}$. in length. In a few days the larvæ attain a length of 0.5 to $0.6 \mathrm{~mm}$. and moult. On the fifth to the ninth day a second moult or ecdysis occurs and the larvæ are in the infective stage or stage of encystation, and measure $0.65 \mathrm{~mm}$. long by 0.025 to $0.027 \mathrm{~mm}$. broad. The larvæ may live for months in this stage but cannot reach maturity except in the intestine of man. They arrive at maturity from four to six weeks after infection. The third stage in the evolution of the larva marks the beginning of its parasitic existence. Its sojourn in the stomach is said not to exceed fifteen hours. During the fourth stage the mouth parts are further elaborated, a provisional buccal capsule appearing, and the sexes are differentiated. In the fifth or adult stage all the organs become maturely developed.

These parasites infest the small intestine, chiefly the duodenum and jejunum, but the ileum may also harbor them.

The number of parasites varies greatly and is not necessarily in ratio to the severity of the disease. In 40 cases of the Porto Rico Commission ${ }^{208}$ in which the worms expelled were counted, they varied from 96 to 4,395 . Of a series observed by Leichtenstern ${ }^{211}$ in 9 cases the parasites were less than IoO, in 14 cases between 100 and 500 , in 3 cases over 500, numbering respectively 99I, I,066, and 2,763. Turner ${ }^{212}$ states that in his experience in South Africa it was exceptional to collect 50 specimens from one body. Of 58 cases reported from North Carolina by Stiles ${ }^{213}$ the greatest number of worms was 2,277, the smallest number, I, the average 135 .

With reference to the proportion of sexes of the parasites existing in given cases, Bilharz found the proportion of males as I to 3 ; Leichtenstern as ro to 24 ; Lutz as 2 to 3 ; Schulthess as I to $6 ;^{211}$ Sandwith ${ }^{205}$ reports that in about 50 cases the proportion of males to females was 56 to 44 . Stiles ${ }^{213}$ observed I02 cases from which 13,080 parasites were obtained; 46 per 
cent. of the specimens were males and 53 per cent. females. Of the 102 cases, 37 presented an excess of males, 9 presented an equal number of males and females, and 56 presented an excess of females.

Pathogenesis.-A satisfactory explanation of the proximate cause of the symptoms of uncinariasis is yet lacking. There are three factors which may be concerned in the pathogenesis: (I) The abstraction of blood by the parasite and hemorrhage subsequent thereto; (2) mechanical injury to the intestinal mucosa followed by infection; (3) the production of a toxin.

I. The structure of the mouth parts, the habit of the worms clinging to the mucosa, a portion of which it partially swallows, and the finding of blood in the alimentary canals of the parasites renders it certain that the hook-worm is a blood sucker. It is furthermore possible that the worm injects a substance at the site of feeding to prevent the coagulation of blood to cause it to flow more easily as the leech and mosquito are supposed to do. This is rendered probable by the finding of visible and occult blood in the feces, by the appearance of the mucosa, and by experiments of Loeb and Smith. ${ }^{209}$ It seems hardly possible that the amount of blood merely consumed by the parasites in certain cases of light infection could account for the symptoms present so that subsequent bleeding or some other factor must be at work in these cases.

Looss claims that the mucous membrane itself is the food of the hook-worm and that the abstraction of blood is only accidental.

2. The mechanical wounding of the intestine and ensuing infections may possibly play a rôle in the pathogenesis of hookworm disease but nothing definite is known.

3. The belief that a toxin is produced by the parasite and is responsible for the symptoms is widespread. The reasons for this theory are:

(a) The blood changes, eosinophilia, almost or quite normal red cell count with low hemoglobin percentage.

(b) Nervous symptoms out of proportion to the anemia and which may improve before the anemia has been compensated. 
(c) The relative immunity evinced by certain individuals and races.

(d) Analogy to other helminth infestations.

(e) The appearance of symptoms before the parasites have reached the suctorial phase.

(f) Symptoms in some cases out of proportion to the number of worms.

(g) Deposits in liver and kidney cells giving reaction of hematoidin.

(h) Results of experiments.

The question of the existence of a toxin, however, is far from settled. A toxin is said to have been demonstrated by the experiments of Bohland ${ }^{261}$, Lussano, ${ }^{261}$ Micheli, ${ }^{202}$ Crisapilli, ${ }^{202}$ de Renzi, ${ }^{202}$ Preti, ${ }^{262}$ Tenholt and Peiper, ${ }^{261}$ Arslan, ${ }^{202}$ Battistine, ${ }^{202}$ Gabbi and Vadala, ${ }^{209}$ Goldman, ${ }^{209}$ Muller, ${ }^{263}$ Rieder, ${ }^{263}$ Gappert, ${ }^{263}$ Ehrlich, ${ }^{263}$ Leichtenstern ${ }^{263}$ and Herman, ${ }^{263}$ while Loeb and Smith, ${ }^{209}$ and Whipple, ${ }^{264}$ on the basis of their experiments deny the existence of a toxin. 


\section{CHAPTER XXXV}

\section{PATHOLOGY OF HOOK-WORM DISEASE}

Even in severe cases the body usually shows no emaciation. The skin is pallid and anemic. The subcutaneous fat is well preserved and of a light yellow color. Edema is commonly present, ascites is shown in greater or less degree, hydrothorax and hydropericardium are often found and the condition may amount to general anasarca. The muscles appear anemic and may be atrophied or normal in development.

The stomach is often dilated and sometimes the seat of gastritis. Hemorrhagic erosions of the mucosa have been observed. Hook-worms are occasionally found in this organ either free or attached.

Important changes are shown in the intestine. The duodenum, jejunum, and part of the ileum are in a catarrhal condition, thickly coated with mucus in which the worms are embedded. This part of the bowel may be studded with erosions produced by the bites of the parasites. Sometimes these may be visible to the naked eye and sometimes they may show through the serosa. There are usually more erosions than parasites. Free blood is rarely found in the bowel but the mucus may be blood stained. The majority of the parasites appear in the upper and middle third of the jejunum. Interstitial inflammation, infiltration of the solitary follicles and Peyer's patches, and atrophy of the valvulæ conniventes are microscopic findings in many cases.

The liver is commonly of normal size. In color it is very pale being of a light brownish-yellow color. On section it is soft, greasy and friable. These changes depend upon fatty degeneration which is present in practically every case. The outer third of the lobule is chiefly affected where the entire lobule is not involved. Yellow pigment and dilated capillaries have been found in a few cases. Amyloid degeneration is a rare phenomenon. 
The pancreas is anemic but further changes are not constantly found.

The spleen may be slightly enlarged but is more often normal in size or atrophied. In the Porto Rico Commission's ${ }^{206}$ autopsies microscopic sections revealed a great reduction of lymphoid elements and a decrease in the protoplasm of the cells. The size of the Malpighian corpuscles was greatly reduced, the cells were scanty and widely separated, and the central artery showed hyaline degeneration. The increase of connective tissue was only relative and apparent. Pigmentation is rare. Changes in the hemolymph glands, particularly those about the bifurcation of the abdominal aorta, have been described. These are larger than normal, of a dull reddish hue and on section show mitosis and phagocytosis of red cells.

The marrow of the long bones presents changes similar to those of pernicious anemia, being reddish and soft and in addition showing groups of eosinophiles and an abundance of myeloplaxes.

Cardiac hypertrophy is a common post-mortem finding. Flabby musculature and fatty degeneration predispose to valvular incompetency. An increase in the pericardial fat is not infrequent. Arteriosclerosis is often found.

In the lungs extreme pallor and edema are almost constant changes. Passive congestion is a finding in many cases. Pleural effusion is present in the majority of cases.

Practically all cases show chronic parenchymatous or diffuse nephritis. The kidneys are usually slightly enlarged. The convoluted tubules show the most marked changes consisting of fatty degeneration and desquamation of epithelium. Yellow pigment is a rare finding.

The brain usually shows an intense anemia and effusion into the ventricles of a clear, pale yellow fluid. 


\section{CHAPTER XXXVI}

\section{CLINICAL HISTORY OF HOOK-WORM DISEASE}

Classifications of clinical forms of hook-worm disease are purely arbitrary and rest solely on differences of degree. Lutz ${ }^{263}$ has proposed the following rather complicated schedule:

I. Stage of exclusively local symptoms.

(a) Acute form.

(b) Chronic form. In the two forms the symptoms are similar and characterized by disorders of digestion; no pallor nor acceleration of pulse.

II. Stage of anemia.

(a) Acute form. I. First degree: the vessels of the conjunctiva are visible, the nails and the lips are pale, the pulse is frequent, no anemic murmur. 2. Second degree: conjunctiva colorless, nails and lips pale, pulse always accelerated, anemic murmur.

(b) Chronic form. Anemia more or less intense, cardiac hypertrophy and dilatation, valvular insufficiency rare, pulse rapid.

III. Dropsical stage.

(a) Acute form. Profound anemia, rapid pulse, anemic murmur, edema and serous effusion.

(b) Chronic form. Symptoms of organic dissolution, arrhythmic heart, myocarditis, asystole, cyanosis, anasarca, marasmus, death.

Ashford and King's ${ }^{202}$ division into slight, moderate, and marked cases, and Stiles' ${ }^{\prime 203}$ into light, medium, and severe are much simpler and more satisfactory.

Light cases are those in which the feces contain a few ova, but symptoms, if present, are slight. Anemia is not particularly noticeable, but fatigue is out of proportion to exertion and there may be slight uneasiness in the epigastrium. 
Moderate cases are those in which there are definite anemia and digestive disorders with their resulting symptoms.

In severe cases symptoms of the first two forms are intensely exaggerated, the heart weakens, and edema and ascites supervene.

A not altogether fanciful classification-of the stages of hookworm disease is into primary, secondary and tertiary, after the stages of syphilis. The primary stage is the stage of the initial

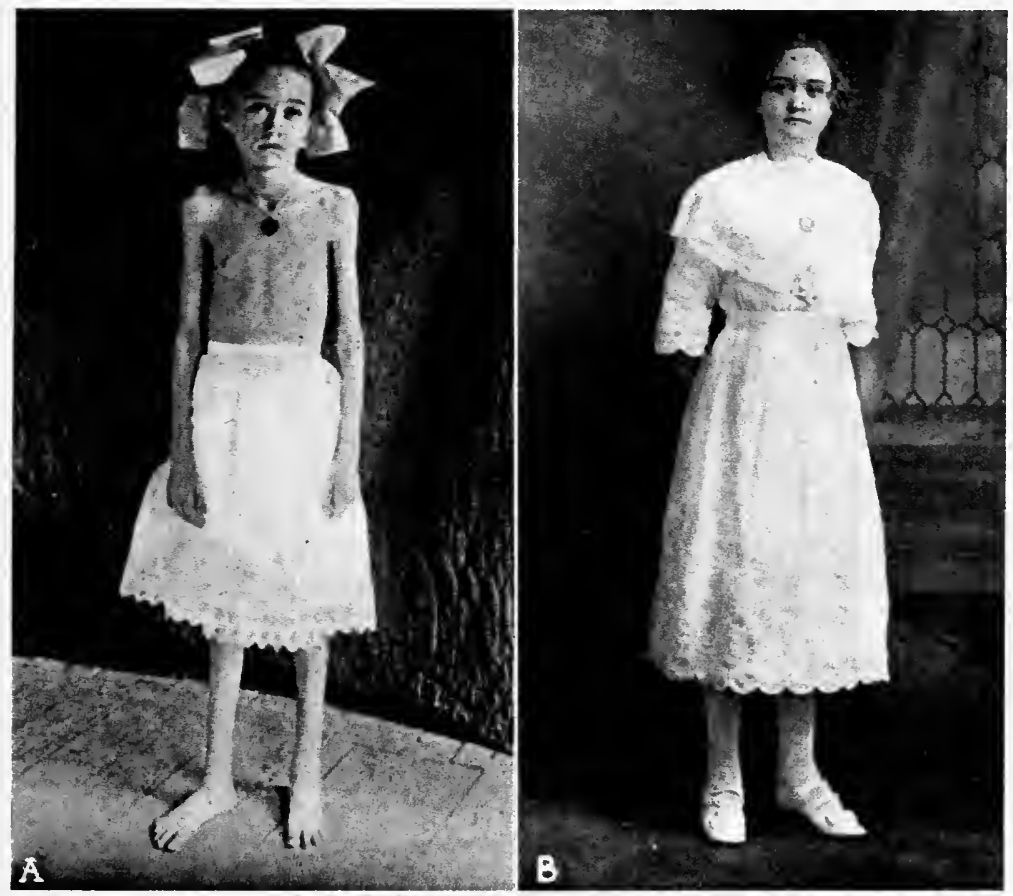

Fig. 9r.-A. Della Carder, Grant County, Arkansas, aged sixteen, practically an invalid from childhood, treated for malaria and tuberculosis, found heavily infected with hook-worms, treated. B. Della Carder as she is today. (Rockefeller Sanitary Commission.)

lesion or port of entry of the organisms into the host-ground itch. The period of incubation of this stage is from a few hours to a few days. This is followed by a secondary period of incubation, as in syphilis, during which time the parasites are immature and unable to produce marked symptoms. The secondary stage begins after the parasites have reached the alimentary 
canal, have matured, and have begun to produce ova in the feces, anemia and digestive disorders. As tertiary symptoms may be designated evidences of organic changes in other organs resulting in heart weakness and anasarca.

General Description.-It is ordinarily from five to ten weeks from the time the larvæ enter the skin until ova appear in the feces.

The symptoms are essentially those of anemia and impaired digestion. A slight uneasiness in the epigastrium is often the first symptom, or the patient may complain of indigestion. Pressure often increases the uneasy sensation or causes actual pain, while it may be relieved by food. The appetite is not radically affected early but is apt to be capricious. Gastralgic pains may be complained of. Meteorism appears in many cases and abdominal tenderness is not infrequent. The bowels are constipated or regular, occasionally loose. In mild cases nausea and vomiting are not early symptoms. A certain degree of pallor, as evidenced by bloodless conjunctivae and pale mucous membranes, is observed in all but mild cases. The skin is dry and inactive. Headache is a common complaint and dizziness is frequent. The patient is subject to palpitation and shortness of breath on slight exertion and this, with muscular weakness, gives him an aversion to labor. In chronic cases the mentality is substandard, initiative and ambition are lacking, and the victim is insensitive to the stimuli of the daily routine of life. Hence it is that the hook-worm has been called the "germ of laziness." In severe cases the pulse is accelerated and weak, the blood pressure is low, and the anemia reaches a low degree. An anemic murmur is often heard over the precordia and the heart may be dilated. There are pains in the thorax and joints. The appetite becomes perverted and the patient eats earth, chalk or soot. Sometimes blood is passed from the bowels. The face becomes puffy, the ankles swell and fluid may collect in the serous cavities.

Hook-worm disease in childhood, if neglected, results in retarded mental and physical development. The children are over-age in school. Puberty is delayed.

Sandwith ${ }^{205}$ found that 100 infested men weighed $\mathrm{r} 7.5$ pounds 
less than normal. Dock and Bass ${ }^{209}$ report that fifty-six grown men affected with hook-worm disease averaged $81 / 4$ pounds lighter and $2 \frac{1}{3}$ inches shorter than normal.

The duration of the disease in untreated cases is governed by two factors, the opportunity for reinfection and the life of the worm. It is evident that there is no limit to the number of reinfections nor the duration of cumulative infections in the absence of prophylaxis. As to the life of the parasite it is certain that this may be as long as twelve or fifteen years.

The cause of death is most commonly exhaustion. Intercurrent affections play a prominent rôle. Fatty degenerations are frequent as secondary factors. Exodus may occur from intestinal perforation.

Temperature.-This is normal, subnormal or elevated. I have noted nothing characteristic in its occurrence or course. In Porto Rico, fever at the onset of the disease is said to be a fairly constant symptom. ${ }^{202}$ In Ceylon, Castellani ${ }^{265}$ observed fever in about 25 per cent. of the cases of medium gravity and in 50 to 75 per cent. of those of a serious nature. Sandwith ${ }^{205}$ in Cairo found that one-third of his patients had a normal temperature during their stay in the hospital and that twothirds had a distinctly subnormal range. In cases having fever it rarely exceeds $102^{\circ}$.

Skin.-Ground itch, known also as dew poison, toe itch and water itch, is usually the first evidence of the disease. It occurs oftenest between the toes and on the sides and tops of the feet, but may appear upon the hands, buttocks, or indeed upon any portion of the skin. Its commonest site is explained by the habit of going barefoot, and squeezing mud between the toes. Its first appearance is of small macules or papules which soon proceed to vesiculation and pustulation. Scratching and mechanical irritation follow, producing crusts and scabs. It is highly probable that bacterial infection occurs both with the entry of the larvæ and later when the lesions are scratched. The exudate from the ruptured lesions is sticky and causes the stockings or bandages to adhere tightly. The average duration of ground itch is one or two weeks, but deeply ulcerated lesions, particularly between the toes, sometimes take weeks in healing. 
The pallor of the skin varies from a fish-belly white through yellowish to tan. True icterus is rare. It should be borne in mind that it is impossible to determine the degree of anemia by the appearance of the skin.

Edema is present in many moderate and most severe cases. In the absence of heart and kidney lesions its frequency is in direct ratio to the degree of anemia. It is commonest in the lower extremities, then in the face. The entire body may be water-logged in severe cases. Of the serous cavities the peritoneal is of tenest involved. Injuries of the skin usually heal slowly and may result in ugly ulcers.

Diminished activity of the skin, indicated by lack of perspiration, is a common manifestation. Atrophy of the skin results from chronic infections.

Petechiæ are occasionally observed in those of low vitality. Urticaria is an infrequent symptom. Pruritus is not uncommon.

Digestive System.-A vague uncomfortableness in the epigastrium is rarely absent except in the mildest cases. Sensations of weight and distention after eating are the rule. Gnawing pain is frequently complained of and gastralgia is sometimes present. Not infrequently the epigastric region is tender to pressure, a sign of significant diagnostic import.

The tongue is furred, enlarged and shows the imprints of the teeth. Not infrequently a purplish smear is observed on each side of the tongue as if a pencil had been sucked. This appeared in nearly 5 per cent. of a series of cases reported from Porto Rico. ${ }^{207}$ These phenomena are attributed to engorgement of the veins. Pharyngitis may occur early. Catarrhal stomatitis is occasionally observed.

The appetite is very variable. It is said to be frequently greatly increased in the beginning, but in cases seen in practice it is usually capricious or diminished. In severe cases it is nearly always diminished.

One of the most striking features of the disease is geophagy or dirt-eating. This runs the scale from the desire for coarse food to actual dirt-eating. It was formerly thought to be the cause of the anemia. Among the many articles which may be 
eaten are coffee, chalk, ashes, soot, brittle wood, paper, earth, mortar, clothing, sand, etc. I have known subjects to walk I 2 miles and back to obtain a'small box of a certain kind of earth. I am convinced, however, that this is not pathognomonic of uncinariasis and that it is a habit which is widely prevalent among the negro women of the South.

Flatulence and heart-burn are common complaints. Nausea is infrequent in mild cases but common in severe infections. Vomiting may occur in severe cases. Hematemesis is occasionally reported.

Yoshida ${ }^{266}$ states the results of his observations upon. IOI subjects as to the acidity of the gastric juice as follows:

I. The content of free hydrochloric acid in the gastric juice in uncinariasis is usually normal, then follows hypochlorhydria, then hyperchlorhydria.

2. The greater the intensity of the anemia the lower the content of free hydrochloric acid.

3. The appetite is usually in the most intimate relation to the content of free hydrochloric acid.

The bowels are usually constipated or irregular, often normal, occasionally loose or even dysenteric. Blood is not often observed macroscopically in the feces. Of over 22,000 specimens of feces examined by the Porto Rico Commission, ${ }^{207}$ only six contained blood and five blood and mucus from a nakedeye inspection. Microscopically blood is occasionally noted. Charcot-Leyden crystals are not infrequently found.

The chief characteristic of the feces in these cases is of course the presence of hook-worm ova. These vary greatly in numbers and may be present in every field, or several slides may have to be examined before finding a single specimen. Leichtenstern ${ }^{72}$ states that it is not unusual for a single stool to contain four million ova. The number of ova in the feces is a guide to the number of parasites in only a very general way, if at all.

Adult parasites are very exceptionally found in the feces except after the administration of a proper anthelmintic.

Circulatory System.- In mild cases the only symptoms from the circulatory system are palpitation and acceleration of the pulse on exertion. Pain in the precordial region is common in 
the more severe cases. This pain may be lancinating or may be dull. In the mildest cases the pulse rate may not depart from the normal except after exertion, but in moderate and severe cases it is always accelerated. It is usually soft and compressible, even dicrotic, and later may become small and irregular. The blood pressure is low. In mild cases the apex beat is normal in location and force. Later it is apt to be displaced downward and to the left, and diffuse and weak. Pulsation in the epigastrium is often observed. Acute dilatation and cyanosis have been observed. An anemic murmur is the rule when the hemoglobin per cent. is markedly reduced. Insufficiency from dilatation also may cause characteristic murmurs. Dilatation is not uncommon in chronic infections and hypertrophy may result. Pericardial effusion often occurs in the cases with anasarca. Exaggerated pulsations in the neck and throbbing in the supraclavicular spaces may be detected at a distance. The superficial abdominal veins are often distended. Uncinariasis in childhood predisposes to organic heart lesions in later years and probably also to arteriosclerosis.

Upon the blood is spent most of the force of the infestation. In mild cases there may be no reduction of red cells. Dock and Bass $^{209}$ examined forty university students who were hook-worm carriers and found the average number of red cells to be 5,246,322. However, there is usually a reduction of these cells. The average count is around three millions, though it may run in severe cases below one million.

The most frequent qualitative changes in the erythrocytes are polychromatophilia, poikilocytosis, and the appearance of microcytes and macrocytes. Nucleated red cells are not rarely present. Notwithstanding these changes occur, they are not as prevalent as in primary anemia.

The hemoglobin falls more rapidly and to a lower degree than the red cells so that the color index is nearly always below normal. It is occasionally noted that the color index is high, even as high as $\mathrm{I} .35$ having been observed. In the mildest infections there may be little or no reduction. The average in this country is perhaps between 40 and 60 per cent. The Porto Rico Commission report one case in which it was as low as 9 per cent., and 
they record it that persons with as low as i6 to 20 per cent. can and do keep at their accustomed labor. ${ }^{208}$

The white cells range from 5,000 to ro,000 per cubic millimeter. Leucopenia is common in cases of long standing and leucocytosis may usually be accounted for by complications. The increase in the eosinophiles is the only characteristic change in the leucocyte formula. This is the rule in mild and moderate cases, but eosinophilia is usually lacking in severe and fatal cases. Ehrlich and Leichtenstern ${ }^{263}$ report a case where these cells were 72 per cent. of the total leucocytes. The highest count of Dock and Bass ${ }^{209}$ was 34 per cent., mine was 26 per cent. Fifteen per cent. is not uncommon for moderate cases. Regarding the variation of the eosinophiles the Porto Rico Commission draw the following conclusions:

I. Very severe chronic cases with poor resisting power and exhausted blood-making organs have little or no eosinophilia.

2. A rise in eosinophiles is generally found in cases which progress favorably and should influence the prognosis.

3. If very severe cases presenting little or no eosinophilia fall in their eosinophile percentage without improving in their general condition, the prognosis for such a case is less favorable.

Under appropriate treatment the eosinophiles rise to a varying degree, then gradually fall to normal.

Extreme blood changes are much less frequently found in the negro race than in the white.

Respiratory System.-Symptoms on the part of the respiratory system are few. Dyspnea is very common and usually in proportion to the anemia. Hydrothorax often occurs in anasarca cases and edema of the lung may supervene. Bronchitis is mentioned by some observers as an early symptom and attributed to the passage of the larvæ through the air cells and bronchi, but I have had no experience with this symptom. So far as I am aware larvæ have not been found in the sputa.

Urinary System. - In cases of edema or anasarca attended with grave anemia it is often difficult to determine how much of the former are attributable to the anemia and how much to the kidneys. That nephritis does play a rôle is credible from both clinic and urinary findings. 
In the early stages the quantity of urine is normal; later it becomes diminished. The Porto Rico Commission ${ }^{207}$ determined that one-fourth of cases of all grades have albuminuria. Casts are not very frequent and when present are in relatively small numbers. The kinds of casts found are in the order of frequency hyaline, granular, fatty and epithelial. Urobilin and indican are common findings. Biliary coloring matters are not common.

Generative System.-Puberty is delayed in both sexes. The male organs are apt to remain undeveloped. Impotence is very common. In the Porto Rico ${ }^{207}$ cases 58.5 per cent. of the males were completely impotent, and 9.7 per cent. were partially so. In Sandwith's ${ }^{205}$ series 63 per cent. were completely impotent and 13 per cent. were partially so.

The age at which menstruation begins is often postponed several years. Irregularity of the menses is very common. Total suppression of the menses is not uncommon in moderate cases and is the rule in severe ones. Sterility is frequent. Stiles $^{267}$ showed that of thirty-four pregnancies in hook-worm subjects 8 . I per cent. terminated in miscarriage. Still-births are said to cause havoc among the women of Porto Rico. Lactation may be impaired or suppressed in severe cases.

Muscular System.-The muscles are weak and lack tone. Fatigue on exertion is due not only to dyspnea but to muscular weakness. To this weakness is largely attributable the reputation for laziness which the subjects of uncinariasis have. Pains in the joints and bones, especially in the sternum, are very common.

Nervous System.--Headache is one of the commonest symptoms and many patients complain of vertigo. Neuralgia is sometimes very annoying. Tingling and formication are the commonest paresthesias present in these cases, and many of the subjects complain of over-sensitiveness to cold. The patellar reflex is diminished in many cases and abolished in some severe infections. After the infection has persisted some time, neurasthenia not infrequently develops and outbursts of hysteria and hystero-epilepsy may supervene. Drowsiness is almost constant except in mild cases but insomnia is very com- 
monly met with and night terrors are not rare. Edema of the brain sometimes occurs in cases with a tendency to anasarca.

The expression is usually dull and stupid. Lack of ambition and intellectual laziness are psychic characteristics. Children are retarded and over age in school. There is difficulty in concentration and delay in answering questions which must ofttimes be repeated. Apathy exists to a marked degree. Defective memory is common. Melancholia and other psychoses may develop. Green ${ }^{268}$ believes that hook-worm infection is in many cases the direct or indirect cause in the production of a distinct psychiatric entity, the principal symptoms being psychic retardation, irritability, depression, lackadaisicalism and blunting of the higher sensibilities.

The moral perversions dependent on uncinariasis are among the most interesting of the aspects of the disease. Macdonald's ${ }^{269}$ observations show that the infection is responsible for disobedience, cunning, lying, stealing, forgery, and sexual perversions, which are cured by thymol.

Special Senses.-Tinnitus aurium is a frequent manifestation. The conjunctiva of the lids shows anemia. The pupil is readily dilatable. Stiles ${ }^{203}$ describes a symptom which was present more constantly than almost any other: "If the patient is directed to stare intently into the observer's eyes there will be noticed a symptom which is difficult to describe, but which I have found more constant than almost any other noticed, namely: after a moment, the length of time apparently varying slightly according to the degree of the disease, the pupils dilate and the patient's eyes assume a dull, blank, almost stupid, fishlike or cadaveric stare, very similar to that of extreme alcoholic intoxication." This sign is not thought by other observers to be pathognomonic of hook-worm disease. Blurred vision and specks before the eyes are frequent complaints. Cataract was common and corneal ulceration occasional among the Porto Rico cases. Examination of the fundus shows the retina pale, occasional hemorrhages, and pulsation and convolutions of veins. Other eye manifestations which have been described are nystagmus, diplopia, amblyopia, neuritis, asthenopia, restriction of the visual field, and chemosis of the bulbo-palpe- 
bral fold. From observations on the eye changes, especially cataract, in hook-worm infections in the South, Calhoun ${ }^{270}$ believes that the former are due directly to the disease, while Jervey ${ }^{271}$ concludes that these lesions are only indirectly dependent upon the infection, and that they are in no sense sufficiently distinctive or characteristic to be of any diagnostic value.

Latent Infections.-There are many cases of hook-worm infestation which cannot be classified as either mild, moderate, or severe, inasmuch as symptoms are entirely absent. Such a host is known as a "carrier." From a public-health standpoint these cases are the most important of all since they do not seek treatment for their parasites, hence are difficult to locate. When such a person is a soil polluter he may spread the infection broadcast without being suspected.

Complications and Sequelæ.-It is, of course, possible that almost any disease may be found associated with uncinariasis. ln the majority of instances this association is merely a coincidence. The Porto Rico Commission ${ }^{208}$ record 657 complications in $\mathrm{I} 7,354$ cases. The most common were diarrhea, dysentery, malaria and tuberculosis. Other than intercurrent diseases hook-worm infection may bear an etiologic relation to gastritis, dilatation of the stomach, enteritis, and dysentery. As sequellæ disturbances dependent on fatty degeneration of the heart, kidneys and liver may be mentioned, and nervous disorders have been attributed as sequelæ. Pernicious anemia and leukemia are supposed to bear a direct relation to hook-worm infection in some cases. In the Southern States infestations with other intestinal parasites is extremely common in hook-worm disease. The commonest worms associated with hook-worms are ascaris lumbricoides, trichuris trichiura, and hymenolepis nana. 


\section{CHAPTER XXXVII}

\section{DIAGNOSIS OF HOOK-WORM DISEASE}

While it is as easy in hook-worm disease to examine the feces and obtain the signs of certainty as it is to examine the patient for the signs of probability, it is as a rule true that the disease must first be suspected before any examinations are made. The symptoms which should cause any one to suspect hook-worm disease are especially anemia, weakness, digestive disorders and a history of ground itch. Many cases come to the physician to be treated for heart disease and some for dropsy.

The following conditions should invariably lead to an examination of the feces: anemia not due to malaria or other obvious cause, a history of ground itch, fatigue out of proportion to exertion, and the presence of eosinophilia. In many instances the diagnostic clue is obtained from the blood examination. However, it should be borne in mind that the absence of eosinophilia does not exclude uncinariasis nor does even the presence of malaria parasites.

Too much stress should not be laid upon geophagy as a diagnostic sign particularly in the female negro. In an investigation which I made upon this point in eastern Arkansas the result was that of the female negroes who came to me for troubles other than hook-worm disease, about 80 per cent. confessed to having been dirt or soot eaters.

Occupation, social position and sanitation of surroundings may be taken into consideration in the probable diagnosis but it is to be remembered that only the detection of the ova in the feces is decisive.

When requesting a specimen of feces the physician should explain to the patient that only a small quantity is necessary and should furnish a container, otherwise he is apt to be brought an entire stool in a large jar or a portion of liquid stool in a match box. Small wide-mouthed bottles such as those for vaseline and quinine are suitable. 
The best slides for this purpose are said to be the 2 by 3 -inch slides but I have always used the I by 3 -inch. A drop or two of water should be placed in the center of the slide and a quantity of feces the size of a match head thoroughly mixed to the proper consistency and spread well over the slide except for a narrow margin along each edge. A tooth pick serves the purpose of mixing and spreading. A cover-glass should not be used. A I-inch eyepiece and $2 / 3$-inch objective are best adapted to this work. A mechanical stage is a great convenience but not an essential. A moderate degree of illumination is most appropriate.

While this method of examination will certainly detect moderate or severe infections it is well known that even the most careful scrutiny of a single slide will fail to detect ova in many cases of light infection. Stiles ${ }^{220}$ holds that a negative opinion cannot be given until at least ten such slides have been examined, consuming from thirty minutes to one hour, and Dock and Bass ${ }^{209}$ have shown that in some cases twenty-five slides may have to be examined before detecting ova. The time necessary for this would, of course, preclude such examinations by the general practitioner, and as the importance of detecting these mild infections is of the greatest degree from a public-health standpoint, it becomes necessary to use some special method of concentrating the eggs for examination before pronouncing the patient free from infection.

Pepper's method is to mix the feces thoroughly with water and then centrifugalize. The supernatant fluid is poured off and fresh water added, mixed well and again centrifugalized. This process is repeated half a dozen times and in this way the bacteria, free coloring matter, light vegetable and other matter are eliminated and only the ova and heavy particles remain in the bottom of the tube whence they may easily be removed by means of a pipette. Pepper further showed that ova of the hook-worm thus placed on a slide after being allowed a few moments in which to settle would adhere to the glass when the water was drained off and would even withstand the slide being rinsed in water. Additional drops may be placed on the slide, the fluid drained off and if this process is frequently repeated the glass becomes studded with ova. 
Bass' method is a most valuable one. It is as follows: It is known that the specific gravity of the hookworm egg is between $\mathrm{r}, 050$ and $\mathrm{r}, \mathrm{roO}$. A quantity of the feces is diluted with about ten parts of water and strained through gauze to eliminate coarse matter. This filtrate is centrifugalized and the fluid poured off. This is repeated several times to wash thoroughly. A fluid of heavy specific gravity is then added and again centrifugalized, the ova remaining in the top of the liquid. A ninetenths saturated solution of sodium chloride, or a solution of calcium chloride having a specific gravity of $\mathrm{r}, 25^{\circ}$ may be used for this. A small amount of the top fluid is then poured off into a centrifuge tube, water added and when centrifuged the ova go to the bottom. Dock and Bass ${ }^{209}$ state that without the adoption of this method, of ro4 cases of intestinal parasite infection, 47 per cent. were found by the usual technic and 53 per cent. were missed until the centrifuge technic was employed. It is of course in the mild infestations that it is of such great service.

For making permanent specimens of ova I have sedimented them in 2 per cent. formalin, and after making a cell of damar varnish by means of the turntable, filled the cell with the preservative containing the ova, applied a circular cover-glass while the varnish was still soft and ringed again with damar.

Reference to the chapter on other intestinal parasites will assist in differentiating between hook-worm ova and those of other helminths. Besides these the beginner may confound vegetable cells and other food remnants with hook-worm ova. A striking characteristic of the ova of most intestinal parasites is their uniformity and when once recognized, or even when good plates are studied there should be no difficulty in their determination.

Attempts to estimate the number of parasites from the number of ova detected on examination have been made. It is estimated that a vigorous female will lay between two and six thousand eggs in twenty-four hours. Leichtenstern's ${ }^{207}$ method

- of making this estimate is to divide the number of ova found in I gram of feces by 47 . Lutz's ${ }^{207}$ method is to make a 25 per cent. suspension of the feces in water and by a specially 
ruled counting chamber to estimate the number of ova in the entire stool. Grassi and Parona ${ }^{207}$ estimate that $I_{50}$ to 200 ova per centigram of feces indicate $I, 000$ uncinariæ, three-fourths females and one-fourth males. The Porto Rico Commission, ${ }^{207}$ however, maintain that no more shifting ground can be assumed than that one can judge of the number of uncinariæ inhabiting an intestine save in a general way.

Stiles ${ }^{203}$ proposed as a rough diagnostic method the blottingpaper test, which he claims is useful in the absence of a microscope examination. An ounce or more of the fresh stool is placed on a piece of white blotting paper; after being allowed to stand from twenty minutes to an hour the stool is removed and the color of the stain is examined. A reddish-brown stain is suggestive of uncinariasis. This test has not found wide favor.

Herman $^{263}$ endeavored to apply the method of serodiagnosis to uncinariasis but the result of his experiments was completely negative.

The therapeutic test is occasionally advocated as a diagnostic measure. This consists of administering a dose of anthelmintic and examining the stool for worms. Inasmuch as it is now readily possible in most Southern States to have feces examined free of charge there is rarely any justification for the therapeutic test.

There are several diseases from which uncinariasis may have to be diagnosticated.

In malaria there can often be elicited a history of typical paroxysms, splenic enlargement may be detected, and herpes is sometimes present. The blood examination may show the presence of malaria parasites, a mononuclear leucocytosis, a relatively high color index, and the absence of eosinophilia. Malaria is usually amenable to quinine. The examination of the feces should settle the diagnosis.

In pernicious anemia the color index is high, the blood contains megaloblasts as well as normoblasts and poikilocytes. Eosinophilia is not present. The skin may show icterus or even pigmentation. The fecal examination is negative.

In leukemia the blood examination on one hand shows an 
enormous increase in the leucocytes with the advent of abnormal cells together with splenic enlargement, and on the other hand the examination of the feces should render the diagnosis certain.

In chlorosis the anemia and the sometimes perverted appetite may cause temporary uncertainty. The blood examination and the color of the skin together with the microscopic examination of the feces should clear all doubt.

In many cases of uncinariasis heart disease is suspected. Careful examination of the heart, however, with the examination of the feces will usually settle the diagnosis.

Bright's disease, tuberculosis, syphilis and enteritis may be differentiated from hook-worm disease by the history, physical examination and the microscopic investigation of the feces. 


\section{CHAPTER XXXVIII}

\section{PROGNOSIS OF HOOK-WORM DISEASE}

While the majority of cases of uncinariasis tend to recover spontaneously it is known that the worms may live for years and that reinfection is extremely common.

Under favorable conditions the prognosis of hook-worm disease is good because the disease is usually chronic, affording time for diagnosis and treatment, because reinfection is preventable, and because we possess a specific for its treatment.

The prognosis depends largely on the severity of the disease, the presence or absence of complications, and on the age of the patient.

In the presence of organic changes resulting directly from the infection and in the event of intercurrent diseases the outlook is less favorable. The prognosis is more favorable in the young because the blood-making organs are commonly more active and complications in vital organs are less likely to supervene.

No case of hook-worm infection should be treated lightly on account of the depletion of vitality due directly to the disease and of the predisposition to and aggravation of other diseases.

Pregnancy, miscarriage and labor are unfavorable complications.

Even after all worms are expelled some cases will die from lack of recuperative power.

Contra-indications to specific treatment render the prognosis very grave.

According to the Porto Rico Commission ${ }^{202}$ good resistance to the toxin of uncinaria is expressed by eosinophilia and they conclude:

I. Very severe chronic cases with poor resisting power and exhausted blood-making organs have little or no eosinophilia.

2. A rise of eosinophiles is of good prognostic import.

3. If very severe cases, presenting little or no eosinophilia, 
fall in their eosinophile count, the prognosis is not generally good.

The cause of death is most commonly from exhaustion, diarrhea, intercurrent affections, fatty changes in the heart, kidneys or liver, or complications of pregnancy and labor. Intestinal perforation has been observed as a cause of death.

It is very difficult to determine the true mortality of uncinariasis for unquestionably many cases never come under the observation of any physician. Furthermore it is doubtful if, as in the Porto Rico Commission figures below, all cases can be traced to termination by cure or death. The following figures show a mortality of twenty-three hundredths of I per cent.:

Porto Rico Commission ${ }^{202} \ldots \ldots \ldots 287,568$ cases, 473 deaths.

Canal Zone Reports ${ }^{151} \ldots \ldots \ldots \ldots . \quad 445$ cases, 3 deaths.

German Protectorate Reports ${ }^{167} \ldots$ I, 158 cases, 200 deaths.

Total................ 289,171 cases, 676 deaths.

Of Sandwith's ${ }^{205}$ hospital cases 89.5 per cent. were cured or greatly relieved, 2.5 per cent. were unrelieved, and 8 per cent. died. 


\section{CHAPTER XXXIX}

\section{PROPHYLAXIS OF HOOK-WORM DISEASE}

Hook-worm disease is preventable. Caused as it is by a parasite having also an extracorporeal phase of development and for which there exist specific remedies it may be attacked in both phases by several methods.

The following table of deaths from anemia demonstrates the results obtained through the campaign of the Porto Rico Commission ${ }^{207}$ :

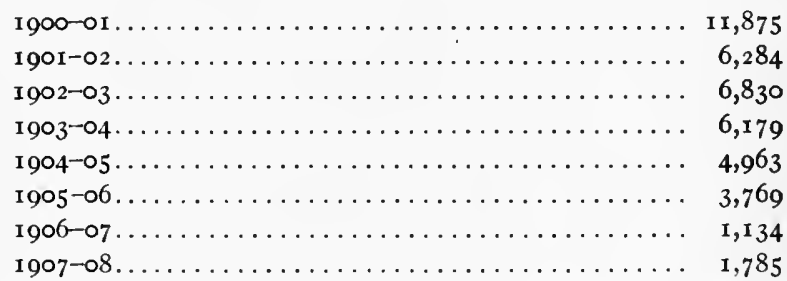

The methods to be employed against hook-worm disease fall naturally into three classes: (I) The sterilization of carriers; (2) the prevention of soil pollution; and (3) the prevention of access of larvæ, by mechanical means or by destruction. For success in any method education in the methods of causation and prevention is a sine qua non. There are many who will follow if intelligently led, though there will remain a few who must be forced even after they have been informed and the policy of any campaign should be education for the willing and legislation for the unwilling.

I. If every person who has hook-worms could be treated to a radical cure the disease would be exterminated. Most of those seeking medical advice are so cured but there are very many who have the disease who do not take treatment, and there are many more who are infested but have no symptoms which lead them to suspect it. These carriers are a greater source of danger than actual cases of the disease for the latter sooner or

- later are compelled to take treatment, while the former rarely 
do. It is impossible to force hook-worm patients to accept competent treatment, and with the carrier there is the double difficulty of first locating him and then sterilizing him. It is, of course, the duty of every physician treating a hook-worm patient to explain to him the importance of complete cure. It is, furthermore, the duty of physicians to seek out carriers and to educate their clientele in hook-worm etiology and prophylaxis.

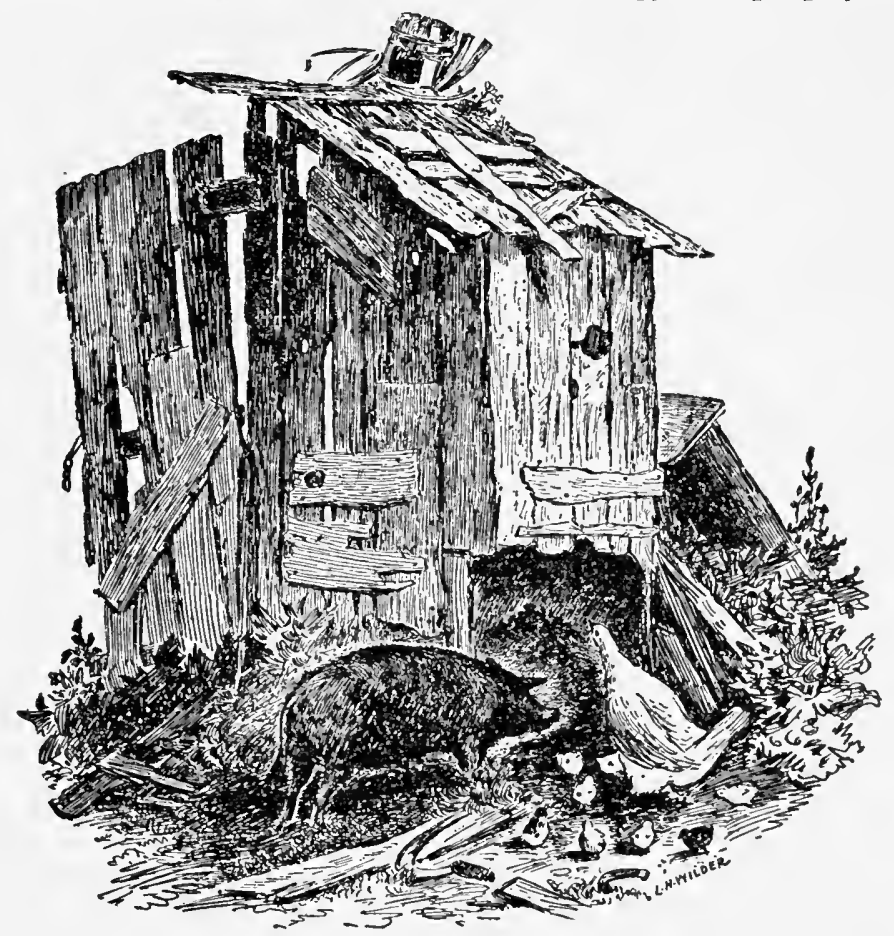

Fig. 92.-An insanitary privy, found too frequently on our farms. Notice how the animals are spreading soil pollution. (Public Health Reports.)

If each physician, expecially in the rural districts, would constitute himself health officer among his patients the hook-worm problem would solve itself and the collateral benefits ensuing, the prevention of typhoid fever and other diseases, would be almost incredible.

As to guides in the search of carriers, residence in an endemic area, other cases in the family or neighborhood, and a history of ground itch, must be regarded in those lacking symptoms. 
In industrial concerns it would be advantageous if employees were periodically inspected for hook-worm infection as is done in some European mines.

2. Since it is largely through infected earth that uncinariasis is propagated it is evident that any scheme of prevention which ignores the significance of soil pollution will work in vain.

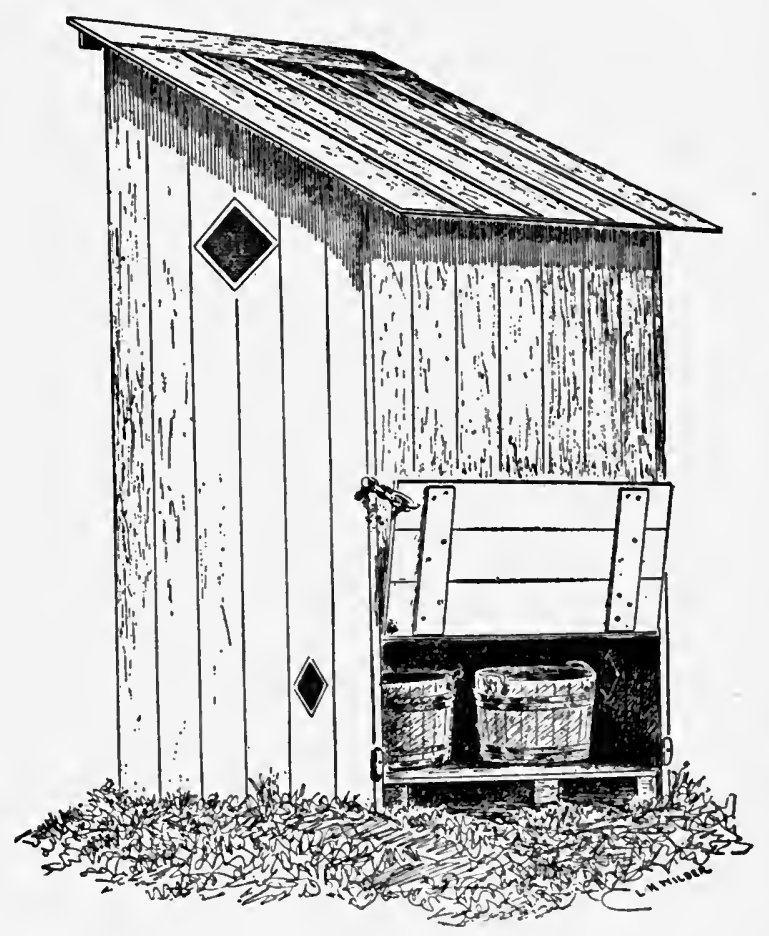

Fig. 93.- Sanitary privy designed to prevent soil pollution. Galvanized pails may be used instead of tubs. (Public Health Reports.)

Throughout the rural districts of the South soil pollution is the rule rather than the exception. In relatively thickly populated communities and along main roads there is usually an excuse for a privy, but in more remote districts there is often none, and the observance of the hygienic rules against soil pollution does not equal the instinct of the cat or the hygienic knowledge of the time of Moses.

Only a very small percentage of such privies as exist prevent 
to any degree whatever soil pollution. They are surface closets, open at the back with nothing to prevent the access of flies and domestic animals.

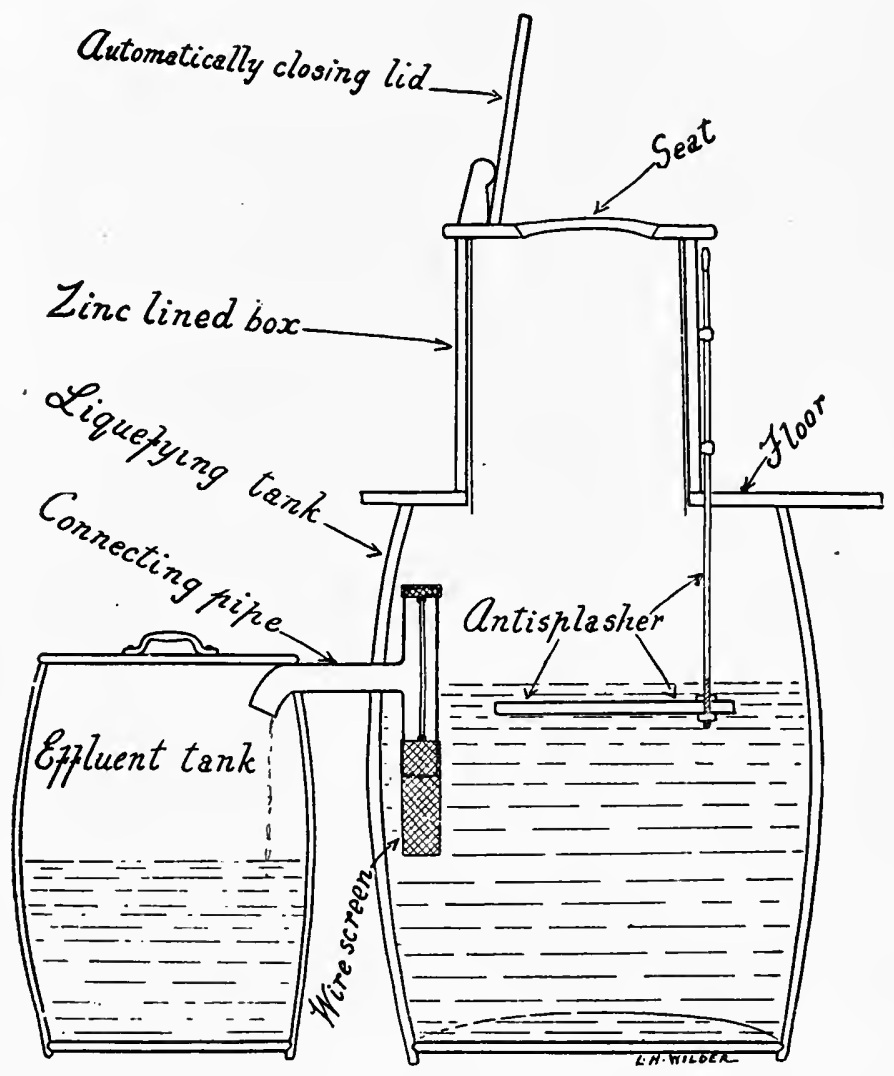

Fig. 94.-Mechanism of the Lumsden-Roberts-Stiles privy. (Public Health Reports.)

There are five requirements for a sanitary privy:

I. The feces must not lie upon the ground.

2. Domestic animals must be excluded.

3. Washing rains must be guarded against.

4. Flies must be excluded.

5. The receptacle must be easily accessible and capable of being cleaned.

A simple and at the same time efficient privy is illustrated in 
Fig. 93. A small quantity of earth or sand in the bottom of the receptacle facilitates cleaning.

Several methods of disposing of night soil have been proposed; heat, chemicals, burial, fermentation, etc. While burial is perhaps not the ideal method, it is reasonably safe if properly done and by far the most practical of all. The excreta should be buried at least 18 inches deep and at least roo yards distant, and down hill from any source of water supply. They should never be thrown into any stream or spread upon the surface for fertilizer unless absolutely sterilized.

The Lumsden-Roberts-Stiles ${ }^{272}$ privy illustrated in Fig. 94 is ideal for rural homes. The device consists of the following parts:

I. A water-tight barrel serving as liquefier.

2. A covered water-tight barrel, can or other container to receive the effluent.

3. A connecting pipe about $2 \frac{1}{2}$ inches in diameter, about $\mathrm{I} 2$ inches long, and provided with an open " $T$ " at one end, both openings of the " $\mathrm{T}$ " being covered by wire screens.

4. A tight box, preferably zinc lined, which fits tightly on the top of the liquefying barrel; this is provided with an opening on the top for the seat, which has an automatically closing lid.

5. An anti-splashing device consisting of a small board placed horizontally under the seat and $\mathrm{I}$ inch below the level of the transverse connecting pipe; it is held in place by a rod, which passes through eyes or rings fastened to the box, and by which the board is raised and lowered. The liquefying tank is filled with water up to the point where it begins to trickle into the effluent tank.

A thin film of oil should be poured upon the surface of the liquid in each barrel.

The devisers claim that the following requirements are met:

x. It solves the fly and mosquito problems so far as the privy is concerned.

2. It liquefies fecal matter and reduces its volume so that it may be safely disposed of more easily and cheaply than night soil.

3. It reduces odor. 
4. It reduces the labor of cleaning the privy and makes this work less disagreeable.

5. It is of simple and inexpensive construction.

The location of the privy is important. When inaccessible or even when too public it is not used. It should be placed within easy reach of the house. Proximity encourages its use and care.

3. Chemical and physical means of destroying ova and larvæ are not practical, and in the European mines where they have been so thoroughly tried they have been abandoned in favor of the restriction of soil pollution and of the protection of the feet.

Since it is known that the skin route of infection is the usual one, and that ground itch is commonest on the feet, the wearing of shoes in infected areas has been shown productive of good results. This means, of course, the annihilation of the barefoot boy immortalized by Whittier and not a few artists, but serves to translate the whistled tunes, red lips and sunshiny face from the realm of poetry to reality.

Ashford ${ }^{273}$ mentions the case of a planter in Porto Rico, employing a large number of laborers, who provided them all with shoes at his own expense and declared that he never made a better investment, since the increase in efficiency repaid him many fold. As this writer states, the providing of shoes for the poor should receive the attention of educationalists and philanthropists.

In this connection Manson ${ }^{274}$ cites an interesting incident:

"A planter from Trinidad, West Indies, told me some time ago that he was at one time seriously inconvenienced by coolie itch among his field hands. He remarked that the attack of coolie itch was often followed by profound anemia, and he also remarked that the anemia occurred only or principally among the coolies who either passed through or who worked in certain fields. He argued that in these fields were certain germs that, coming into contact with the legs and feet of the coolies, produced the dermatitis and, on subsequently entering the body, the specific anemia. He knew nothing about the ankylostoma; the observation was made long before Looss' discovery. The planter had some knowledge of bacteriology and he, like many 
others, thought that the germ in question was a bacterium, and that by some antiseptic and protective procedure he could either kill the germ or prevent its access to the skin. In casting about for a means to effect this he bethought him of a practice he had seen in operation in a certain part of Germany during one of the annual pilgrimages he made to that country in search of health. In that particular part of Germany geese are raised in large numbers. The goose market is a long way from many of the goose farms, so that the birds, when ready for sale, have to be delivered for many miles over the hard roads. To enable their feet to stand the journey the farmer provides each of his birds with a pair of close-fitting socks and sandals. This he fits on in this wise: He fills a shallow trough with tar and through this trough he drives the geese onto a piece of ground covered with fine sand. The tar sticks to their feet and the sand to the tar; the birds are shod for the road. Thus provided they perform the long journey without injury. Acting on this hint the planter made his coolies, on their way to their work in the morning, dip their feet and legs in a bucket of Barbadoes tar and then walk across a layer of sawdust or sand. The result was excellent. Coolie itch and coolie anemia almost disappeared from the plantation."

Children should constantly be drilled to keep the fingers out of their mouths. It is impossible to keep their hands clean, and diseases other than hook-worm are frequently conveyed in this manner. Those who till the soil or handle the products of the soil should be taught to wash the hands frequently.

In many European mines shower baths have been installed for the miners.

Bathing in common tubs by those exposed to hook-worm infection is conducive to the spread of the disease.

After all is said and done in the prophylaxis of uncinariasis the results will not be proportionate to the efforts until a popular demand for sanitation is created throughout the rural districts. This must be accomplished through a campaign of education beginning with the children. The Rockefeller Sanitary Commission has, in a few years, come nearer this end than all other combined agencies throughout the past. 


\section{CHAPTER XI}

\section{TREATMENT OF HOOK-WORM DISEASE}

Hook-worm disease is a curable affection, there being one or more specifics for its relief. It is true that patients die of hookworm infection even under treatment, but if appropriate treatment is begun reasonably early and persisted in practically all these cases recover promptly.

The most efficient remedy for uncinariasis is thymol, first used for this disease by Bozzolo ${ }^{275}$ in 1879 . Thymol consists of large colorless, translucent, hexagonal crystals, with an aromatic odor and pungent taste. It is obtained from the volatile oils of thymus vulgaris, thymus monarda and carum ajowan. It is soluble in I,100 parts of water, and in less than one part of alcohol, ether or chloroform, it is readily soluble in glacial acetic acid, carbon disulphide and in fixed and volatile oils. It liquefies when triturated with equal quantities of camphor, menthol or chloral. When liquefied by fusion it is lighter than water.

Thymol has poisonous properties, but in proper dosage administered with due precautions, it is a very safe drug. It is neither uncertain nor unreliable, and so far as I am aware especiai idiosyncrasies toward it are not common. It is a powerful antiseptic, said to be ten times less toxic than carbolic acid; indeed it is not unlike the latter in some of its systemic effects as well as in chemical nature.

The commonest symptoms following thymol administration are nausea, depression, burning in the epigastric region and vertigo. It is eliminated principally by the lungs and kidneys, and the urine may become very dark and increased in quantity. These symptoms are usually aggravated by getting out of bed. Everything possible should be done to prevent the absorption of the drug, as it is not the patient that is to be treated but the worms, and the more of the medicine absorbed by the host the less his parasites receive. When absorbed, nausea and vom487 
iting, diarrhea and tremors may occur. The temperature is frequently lowered, the pulse may be rapid and weak and dyspnea and cyanosis may develop. Profuse sweating and collapse have been observed, and convulsions and delirium may ensue. The drug is said to irritate the kidneys in some cases. The heart symptoms are due to the effect of the thymol on the heart muscle and to the lowered blood pressure.

Stiles and Boatwright ${ }^{276}$ have recently studied the subjective effects in 464 administrations of thymol to 243 patients. The results of their observations are recorded in the following tabulation:

\begin{tabular}{|c|c|c|}
\hline & Number & $\begin{array}{l}\text { Per cent. } \\
\text { of total }\end{array}$ \\
\hline Total treatments....... & 464 & 100.0 \\
\hline 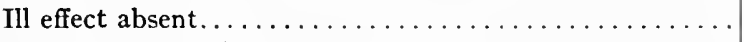 & 259 & 55.8 \\
\hline Ill effect present (as follows)............... & 205 & $44 \cdot 1$ \\
\hline Sickness at the stomach $\ldots \ldots \ldots \ldots \ldots \ldots \ldots$ & 66 & 14.2 \\
\hline 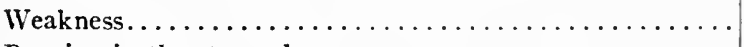 & 62 & $13 \cdot 3$ \\
\hline Burning in the stomach $\ldots \ldots \ldots \ldots \ldots \ldots \ldots \ldots$ & 45 & $9 \cdot i$ \\
\hline Dizziness (including also " giddiness," "drunk" "staggery" & 44 & 9.4 \\
\hline 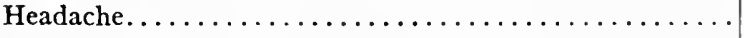 & 14 & 3.0 \\
\hline 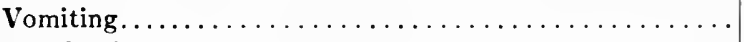 & I 3 & 2.8 \\
\hline Burning in throat $\ldots \ldots \ldots \ldots \ldots \ldots \ldots \ldots \ldots \ldots \ldots \ldots$ & 8 & 1.7 \\
\hline 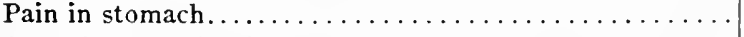 & 7 & 1.5 \\
\hline Drowsiness or sleepiness............... & 5 & I. I \\
\hline Sick after discharge from treatment $\ldots \ldots \ldots \ldots \ldots \ldots$ & 3 & 0.64 \\
\hline Chill apparently not due to treatment. . . . . . . . . . & $\mathbf{I}$ & 0.21 \\
\hline 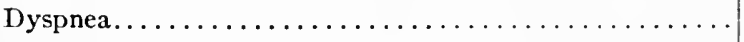 & I & $0.2 \mathrm{I}$ \\
\hline Irregular heart following thymol $\ldots \ldots \ldots \ldots \ldots$ & I & $0.2 \mathrm{I}$ \\
\hline Fainted................. & I & $0.2 \mathrm{I}$ \\
\hline 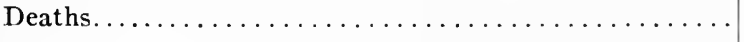 & $\mathbf{I}$ & $0.2 \mathrm{I}$ \\
\hline
\end{tabular}

Stiles and Leonard ${ }^{278}$ state that they know of at least eleven deaths in this country, due, so far as it has been possible to determine, either to following thymol with castor oil instead of with salts, or to carelessness in respect to following out the usually adopted procedure.

Bozzolo, ${ }^{277}$ Thornhill ${ }^{29}$ and others report cases of fatal poisoning by thymol.

Thymol poisoning should be treated systematically. Mor- 
phine and atropin are usually indicated, and digitalis, ergot or strychnine may be needed. Hot coffee should be avoided as it is a solvent of the drug. External warmth is not to be used.

Thymol is contra-indicated in great debility, advanced old age, organic heart disease, nephritis, gastritis, dysentery, and pregnancy. In some such cases, however, it may be deemed safer to administer the drug than to withhold it. The Porto Rico Commission sometimes treated pregnant women with thymol to save life, and on several occasions nursing mothers were given the drug without bad effect to either mother or child.

The American school is inclined to regard with disfavor the large doses of thymol, even up to 120 grains, advocated by the Europeans. In gauging the dose of thymol the apparent age, real weight and degree of debility should be the determining factors rather than the real age. There are few diseases in which the real and apparent ages have such a wide degree of variation and the latter must be taken into consideration. Subjects with contra-indications to thymol should, if treated, be given small doses and repeated; they should be "fractionally sterilized." The following scale of dosage is recommended by Ashford and King, and widely used in the Southern States:

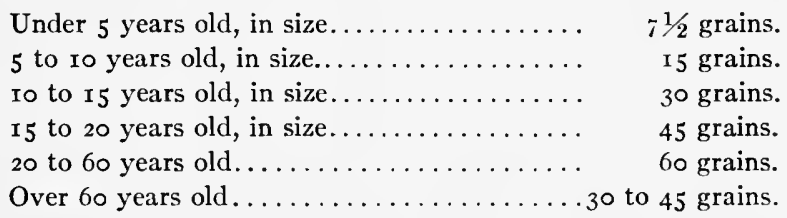

The parasites lie in the small intestine, many of them with head and neck imbedded in the mucosa. They are covered with the normal mucus of the gut, together with that produced by mechanical irritation. In addition the food contents of the bowel envelops the worms. It can be seen that under these circumstances a dose of anthelmintic might kill none and sicken and dislodge only a few parasites. Hence it becomesimperative to prepare the intestine for the medicine. The patient should eat sparingly the day before treatment and take only liquids for the evening meal. At bedtime a purge should be administered. Sulphate of soda and the sulphate of magnesia are the most 
suitable; the former especially seems to liquefy and remove the protecting mucus. On the day of treatment the patient should remain in bed and should not be allowed any nourishment until through with the treatment. Especially should no milk, butter, other oils or fat, alcohol, turpentine, ether or chloroform be permitted, as these are solvents of thymol. The thymol should be thoroughly triturated with an equal quantity of sugar of milk to prevent packing. It may be given in capsules or in cachets. To children who cannot swallow these it is usually given suspended in syrup or mucilage of acacia. The dose selected is ordinarily divided into two parts and administered two hours apart, say at 8 a.m. and at roa.m. At noon another dose of the saline is given and in an hour the patient may be allowed nourishment, excluding rigidly the solvents of the drug. The directions to the patient should be given in the minutest detail, and should be reduced to writing. The patient should be instructed to save the stool for inspection. He should not be obliged to walk to a privy or water closet, but should use a bed pan or vessel at the edge of the bed. As Stiles suggests, Sunday is a convenient day for treating working people and school children.

Although thymol is a specific for hook-worm disease, this does not mean that cures are always accomplished by a single dose, a therapia sterilisans magna. On the other hand, while at least three-fourths of the parasites are expelled at the first treatment, usually several repetitions are necessary to effect a radical cure. In many cases a single dose expels so many worms that the patient is greatly improved clinically, but is not sterilized and remains a carrier to infect others. Sandwith ${ }^{205}$ found that an average of 2.6 treatments were necessary to cure hospital cases. His observations on 184 cases are as follows:

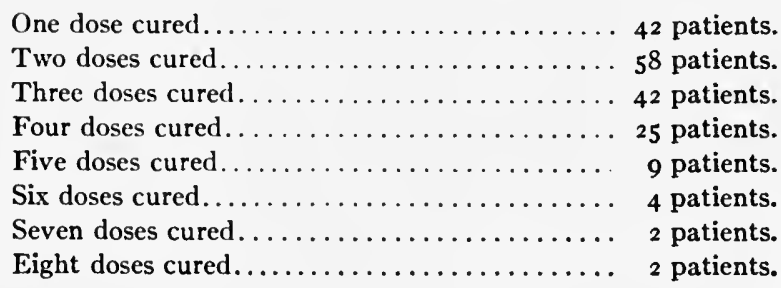


Ashford, King and Igaravidez ${ }^{202}$ recorded in 3,630 patients the number of treatments necessary to effect a complete cure, averaging 2.04 doses per patient:

$\begin{array}{cccc}\text { Number of doses } & \text { Number of patients } & \text { Number of doses } & \text { Number of patients } \\ \text { I } & \text { I, 5 } 8 & 7 & \text { I9 } \\ 2 & \text { I, I } 66 & 8 & 6 \\ 3 & 5 \text { I } 8 & 9 & 3 \\ 4 & 247 & \text { Io } & \text { I } \\ 5 & \text { IO4 } & \text { II } & \text { I } \\ 6 & 47 & & \end{array}$

While most authorities recommend at least three doses for all patients as routine, I am of the opinion, inasmuch as many patients are radically cured by one dose, that repetitions after the first dose be based solely upon microscopic evidence of more parasites in the intestines. These examinations should be made at weekly intervals and should be repeated at least three times, because a dose of thymol which may not destroy all worms may suspend ovulation for a week or two. Weekly intervals are the most suitable intervals also for the repetition of treatment should it be necessary.

To recover the parasites from the stools I have found useful a large flat baking pan, known in the South as the "biscuit pan." This should be painted black and in one end about an inch from - the bottom should be punctured a row of small holes so that the diluted feces may be retained or released by tilting without agitating. Straining through gauze is also a very satisfactory method.

After treatment patients usually begin to show unmistakable evidence of improvement in from three to five days. Improvement depends largely upon the intensity of the infection and the resistance of the patient, considered under Prognosis.

In certain cases thymol does not give the good results anticipated. In some of these it is because the alimentary canal has not been properly prepared and the drug does not gain access to all the parasites. In other cases the thymol becomes lumped in the alimentary canal and passes through like a marble. There remains a small group of cases in which the only explanation seems to be that the worms are of a thymol-resistant 
strain. Analogies are found in other parasitic diseases, notably the quinine-fast parasites in certain resistant cases of malaria.

Of other drugs for the specific treatment of uncinariasis beta-naphthol introduced by Bentley ${ }^{279}$ in 1904 is the most serviceable. Bentley used it in several thousand cases with excellent results and prefers it to thymol. It is used in half the dose of thymol and is very much cheaper. It is less depressing than thymol but more irritating to the kidneys, a serious drawback in view of the frequency of albiminuria in the disease. The treatment preparatory to beta-naphthol is the same as that for thymol. The Porto Rico Commission ${ }^{207}$ made a comparative study of the value of thymol and of beta-naphthol as shown by the percentage of the total number of parasites expelled by successive doses of each drug. Their observations may be summarized as follows:

\begin{tabular}{|c|c|c|}
\hline Doses & Thymol & Beta-naphthol \\
\hline 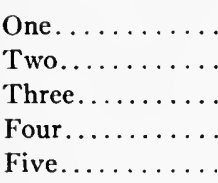 & $\begin{array}{l}76.84+ \\
92.39+ \\
97.56+ \\
99.07+ \\
99.80+\end{array}$ & $\begin{array}{l}72.24+ \\
88.13+ \\
93.68+ \\
96.49+ \\
98.75+\end{array}$ \\
\hline
\end{tabular}

This shows that thymol is somewhat more efficient than betanaphthol in the treatment of uncinariasis. Even a slight degree of difference is important in the prevention of carriers as a result of lack of complete cure.

Male fern has been used largely and successfully in Europe for the treatment of infection with the old-world hook-worm, but seems to be relatively inert against the new-world species.

Herman's mixture, consisting of chloroform 3 grams, oil of eucalyptus 2 grams, and castor oil 40 grams, to be given in two divided doses an hour apart, has been used successfully and has the advantage for research purposes of expelling the worms alive. It is said to be a nauseous dose and will hardly supplant thymol in popularity.

Oil of chenopodium has recently come into repute as a remedy for hook-worm disease. Liquid diet and a saline should be used 
preparatory to the drug. The next morning three doses of the oil are given at two hourly intervals. The following individual doses have been recommended:

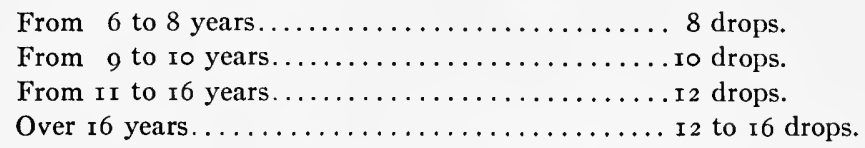

It is not disagreeable to take, is not accompanied by unpleasant symptoms and is said to be very effective.

The number of other drugs advocated for the specific treatment is almost legion, but none possess any particular virtues.

Archibald has recently suggested an autogenous vaccine prepared from bacteria of the colon group on the theory that the intoxication is largely due to the absorption of the toxins of these germs.

Uncomplicated ground itch is not often seen by the physician, but secondary infection may cause the patient to seek medical aid. Pustules should be opened, crusts removed and the parts soaked in a hot antiseptic solution and then dressed with wet antiseptic dressings.

The treatment of uncinaria infection before ova have appeared in the stools is futile inasmuch as the encysted larvæ are very resistant to treatment.

The majority of patients should receive aid to the bloodmaking organs to restore the lost blood. Proper hygiene, air, sunshine and nutritious food are of prime importance. Iron is usually indicated, and the preferable forms are Blaud's pills and the peptonate of iron and manganese. 



\section{CHAPTER XII}

\section{OTHER INTESTINAL PARASITES}

It is probable that the pathogenic importance of intestinal parasites other than hook-worm and the dysenteric ameba has been as frequently under-as over-estimated. There is a marked tendency on the part of the laity to magnify the importance of these worms, and all the symptoms in the category of disease have been attributed to them. On the other hand, most physicians who have not paid particular attention to this branch of the pathology of our Southern States are apt to. minimize their significance and regard them as innocent commensals of their host.

The numerous cases of mechanical injury caused by intestinal parasites, as in obstruction and appendicitis, as well as the many instances in which toxins have provoked trouble, forces on us the realization that they are potential factors for evil.

Every child infested with intestinal worms is more or less handicapped and most adult hosts are at least less efficient. Something of the bearing these parasites have on the predisposition to, and aggravation of other diseases, may be gathered from the fact that after quarantining the prisoners in Bilibid prison who were entertaining intestinal worms the death rate fell from 75 per $\mathrm{r}, 000$ to 9 per $\mathrm{I}, 000 .{ }^{256}$

\begin{tabular}{|c|c|c|}
\hline & Stiles and Garrison & Canal Zone \\
\hline Hook-worm. . . & 6,219 & $15,13 i$ \\
\hline Ascaris lumbricoides............ & 3,792 & 6,359 \\
\hline Trichuris trichiura..... & 2,846 & 13,879 \\
\hline Hymenolepis nana.. & 19 & 9 \\
\hline Oxyuris vermicularis. & $1,5 \circ 3$ & 26 \\
\hline Tænia saginata............. & 95 & 53 \\
\hline Strongyloides intestinalis $\ldots \ldots \ldots \ldots \ldots \ldots \ldots$ & 62 & $3, \mathbf{1} 49$ \\
\hline Tænia solium $\ldots \ldots \ldots \ldots \ldots \ldots \ldots \ldots$ & 39 & $\cdots \cdot$ \\
\hline Balantidium coli. . & ..... & 97 \\
\hline Dibothriocephalus latus................. & $25^{\circ}$ & I \\
\hline
\end{tabular}


The different helminths occur with great variation in frequency in different localities. In the foregoing tabulation the first column is the result of a compilation by Stiles and Garrison ${ }^{214}$ of results by observers in many parts of the world. The second column shows the finding of the laboratories of the Canal Zone as recorded in the reports from 1907 to 1913 .

Since the inauguration of the work of the Rockefeller Sanitary Commission the relative frequency of the various parasites of the Southern States is determinable, and the magnitude of their figures probably eliminates any large degree of error. Below are given the results of the work of this commission as well as of other investigators.

\begin{tabular}{|c|c|c|c|c|c|}
\hline & $\begin{array}{l}\text { Stiles and } \\
\text { Garrison } \\
\quad(214)\end{array}$ & $\begin{array}{l}\text { Kohl- } \\
\text { heim } \\
(257)\end{array}$ & $\begin{array}{c}\text { Rockefeller } \\
\text { Sanitary Com., } \\
\text { I9I I-I } 913\end{array}$ & $\begin{array}{c}\text { Rockefeller } \\
\text { Sanitary Com., } \\
\text { 2nd qtr. I9I4 } \\
(258)\end{array}$ & Total \\
\hline Hook-worm......... & $3^{6}$ & 269 & 29,743 & 25,873 & $55,94 I$ \\
\hline Ascaris lumbricoides... . & 17 & $2 \mathrm{I}$ & 18,820 & 8,330 & 27,188 \\
\hline Trichuris trichiura..... & 266 & $\ldots \ldots$ & 5,757 & 2,424 & 8,447 \\
\hline Hymenolepis nana.... & 12 & $1_{7}$ & I, 879 & 806 & 2,714 \\
\hline Oxyuris vermicularis... & 45 & 15 & 177 & $5^{81}$ & $8 \mathrm{r} 8$ \\
\hline Trenia saginata....... & 2 & $\ldots \ldots$ & 102 & 23 & 125 \\
\hline $\begin{array}{l}\text { Strongyloides intesti- } \\
\text { nalis . . . . . . . . }\end{array}$ & 8 & 5 & 53 & 23 & 89 \\
\hline Balantidium coli...... & 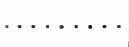 & $\ldots \ldots$ & 3 & $\ldots \ldots$ & 3 \\
\hline Total... & 386 & 327 & $5^{6,543}$ & 31,588 & 95,325 \\
\hline
\end{tabular}

From the beginning of the work of the commission in Arkansas to June 30 , I9I4, the following results were obtained: $:^{259}$

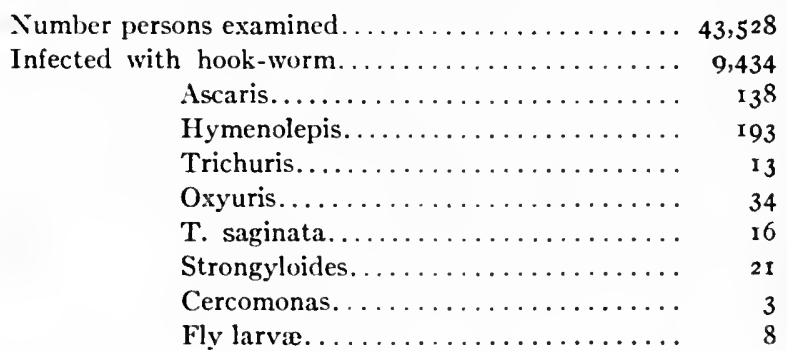

Mixed infections are common. The combinations do not seem to depend on any particular affinities or elements of sym- 
biosis but are in direct ratio to the frequency of single infections by the particular species concerned.

Negroes are much more susceptible to infection than are the whites. Indians are said to be infrequently the hosts of helminths. ${ }^{260}$

Females are more frequently infested than males.

Children entertain most varieties of intestinal worms much more frequently than adults.

While the inhabitants of cities are not immune to the invasion of parasites, they are much less frequently infested than residents of rural districts.

The diagnosis of intestinal helminthiasis rests solely on the discovery in the feces of worms or their segments or ova.

The determination of the various ova is among the simplest of microscopic procedures and with a little experience should suffice to enable one to make an accurate diagnosis. A small bit of fecal matter the size of a match head should be rubbed on a glass slide with sufficient water to spread it well and examined with a $2 / 3$-inch objective.

\section{T ENIA SAGINATA}

The beef tapeworm, the fat tapeworm, T. mediocanellata, T. inermis.

While the tapeworms have been known from the earliest times,

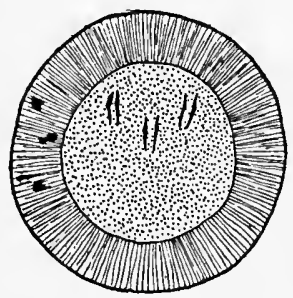

Fig. 95.-Ovum of tenia saginata, enlarged.

it was Kuchenmeister, in $185^{2}$, who first distinguished between the unarmed Tania saginata and the armed Tania solium.

The geographic distribution of the parasite comprises all continents and in most localities is the commonest of the larger cestodes infesting man. In Abyssinia infestation is said to be 
well-nigh universal owing to the prevailing practice of eating uncooked beef. In the United States Tania saginata is far commoner than Tania solium but rarer than the dwarf tapeworm. Of 56,543 cases of intestinal parasites recorded by the Rockefeller Sanitary Commission there were 102 of Tania saginata.

It is stated that this tapeworm is found in Jews far oftener than is the pork tapeworm. The negro is not immune.

In my experience males are more of ten infested than females. Stiles and Garrison ${ }^{214}$ found infestation in females more than twice as prevalent as in males. Of Mosler and Peiper's ${ }^{211}$ cases there were eighty-eight men and twenty-four women.

The age of most hosts is from twenty to forty-five years,

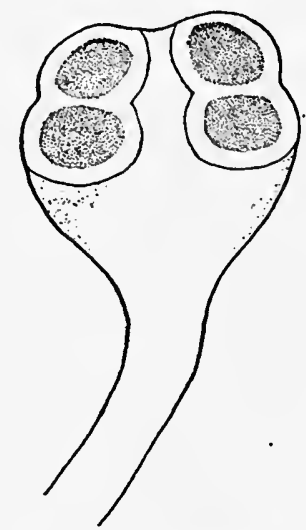

Fig. 96.-Scolex of tenia saginata, enlarged.

though no age can be said to be exempt. My youngest patient was nine years old and he had then harbored the worm for several years. Schloss ${ }^{215}$ reports five cases in children from six to twelve years of age.

The beef tapeworm in the adult state is found only in man. Cattle serve as intermediate hosts for the larvæ which are imbedded in the tongue and other muscles.

The source of infection in man is the eating of raw or insufficiently cooked "measly" beef, that is, beef infected with the cysticercus bovis, or larvæ of Tania saginata.

The beef tapeworm is usually from $\mathrm{x} 2$ to 30 feet in length, but specimens up to 36 and even 74 meters long are recorded. 
The proglottides average from $\mathrm{I}, 000$ to $\mathrm{I}, 300$ in number. The head is subcubical and from $x .5$ to $2 \mathrm{~mm}$. in diameter. There are no hooklets. The suckers are circular and about $0.8 \mathrm{~mm}$. in diameter. The rostellum is rudimentary being replaced by a sucker-like organ. The neck is long and narrower than the head. The proglottides gradually increase in size, when sexually mature measuring 4 to $6 \mathrm{~mm}$. long by 8 to ro $\mathrm{mm}$. broad. Gravid segments measure $x 2$ to $20 \mathrm{~mm}$. long by 4 to 7 broad. The uterus has from 20 to 35 dichotomous branches on each side of and shorter than the median trunk.

The eggs are subglobular and if the shell remains intact one or two filaments are found. The six-hooked embryo is surrounded
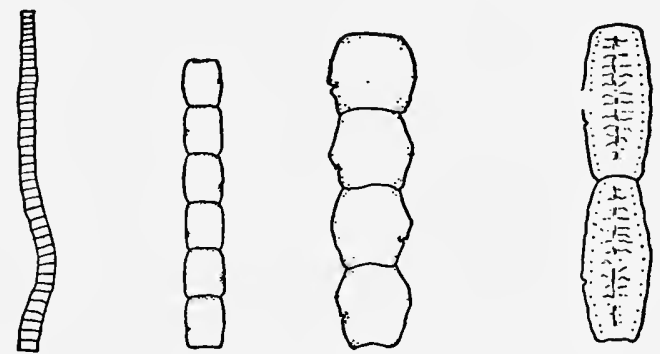

Fig. 97.- Segments of tenia saginata, natural size.

by a radially striated capsule. The ova are transparent or bile stained and measure 30 to 40 microns by 20 to 30 microns.

Abnormalities in the morphology of this parasite are not rare and monstrosities of scolex and proglottides are reported.

Symptoms.-The majority of persons infested with the beef tapeworm do not complain of symptoms, at least until after they discover the presence of the parasites. When symptoms are present there is nothing characteristic about them. The old idea that tapeworm patients have enormous appetites is in a measure erroneous. The appetite is capricious in many cases and anorexia or bulimia occur in a few. Dyspeptic symptoms, increased flow of saliva, belching and nausea are complained of in some cases. Colicky pains in the epigastrium or hypogastrium are sometimes felt. Constipation may be present and diarrhea is an occasional symptom. Nervousness may be marked, especially in young patients. Poor and disturbed sleep are not 
infrequent. Vertigo, tinnitus, palpitation, anal and nasal pruritus, and malaise sometimes appear. Syncope, convulsions and visual disturbances are rare symptoms. Some patients complain of being conscious of the movement of the worm. Segments may be passed in defecation or may escape spontaneously and may be found in the clothing or bedding or about the room.

The blood examination usually shows an increase in the eosinophiles and the feces may contain Charcot-Leyden crystals.

It is not known how long the beef tapeworm may live in the intestines but since certain of the cestodes may live as long as thirty-five years it is probable that tænia saginata may survive a number of years. From the time of swallowing the cysticercus until segments appear in the stools is about sixty days. The average daily growth of the parasite is said to be $7 \mathrm{~cm}$.

Infection with a single parasite is the rule but as many as fiftynine are recorded in one patient. ${ }^{216}$

The diagnosis can be made only by the passage of segments or the finding of ova in the feces.

The differentiation between tænia saginata and tænia solium may be made by examination of the scolex or of mature proglottides. If the scolex is armed with hooks the specimen is tænia solium. Proglottides should be examined pressed between glass slides. In the beef tapeworm the lateral uterine branches number from twenty to thirty-five, while in the pork tapeworm they are usually less than fifteen.

The prognosis is favorable but the beef tapeworm, of all the cestodes, being the most resistant to anthelmintics, several treatments may be necessary before the scolex is removed.

The prophylactic measures consist of the prevention of soil pollution, and efficient system of meat inspection, cold storage for three weeks, or thorough cooking of beef.

In the treatment of tapeworm infection the preparatory treatment is of scarcely less importance than the specific medication. Directions to the patient should be minutely detailed and reduced to writing.

The patient should subsist upon liquids only during the entire 
day preceding the administration of the specific. At bedtime half an ounce of magnesium sulphate is given. The next morning no breakfast is allowed and the anthelmintic is given on an empty stomach.

The best remedy against the beef tapeworm is a fresh preparation of the oleoresin of male-fern. An average dose is I dram. In children from five to ten years old I have given from twenty to thirty drops. It may conveniently be administered in several large capsules. The parasite is not killed by anthelmintics, but merely stupefied and it must be removed by a purgative. Two hours after giving the male-fern is the proper interval and the sulphate or citrate of magnesia are suitable. Castor oil should not be used for this purpose.

Pomegranate is an efficient tæniafuge in many cases. It should be used in the form of the fluid extract, dose half a dram. Pelletierin is a mixture of the alkaloids of pomegranate. An average dose is ro grains. This as well as all other anthelmintics should be preceded and followed by brisk purgatives.

Pumpkin seed is a safe and fairly efficient remedy. One or 2 ounces of the ground seeds should be given in sugar and water. Cusso, kamala and turpentine are rarely used at the present day.

As the worm is about to be passed the patient should be seated on a vessel containing warm water over which gauze or coarse cloth is suspended to receive the parasite. Care should be taken to avoid breaking the worm and no traction should be made. If the bowels cease to move before expulsion is complete an enema of warm water should be given.

Search should be made for the head. On account of its small size, however, failure to find it does not necessarily indicate that it has not been passed. In the latter event proglottides will reappear in the feces within three months.

\section{T AENIA SOLIUM}

The pork tapeworm, the armed tapeworm.

While Tania solium is almost cosmopolitan and in certain localities the commonest cestode, it is far rarer in the South than either the beef tapeworm or the dwarf tapeworm. There are 
no cases of this parasite recorded in the reports of the Rockefeller Sanitary Commission.

In the adult stage this parasite inhabits the small intestine of man only. In the cysticercal or larval stage the common host is the domestic swine, but it is known also in the wild boar, man,

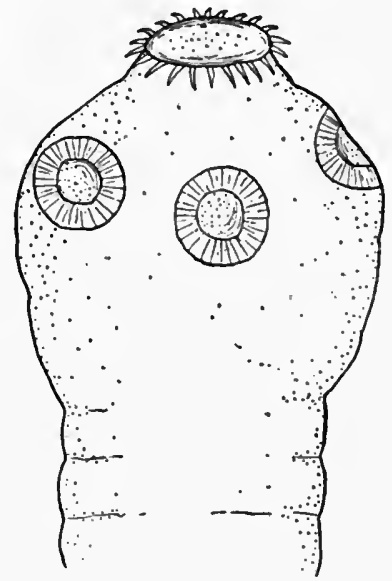

Fig. 98.-Scolex of tenia solium, enlarged.

certain species of monkeys, bears, the dog, rat, sheep, cat and deer.

The mode of infection in man is eating pork infested with the larvæ of the pork tapeworm.

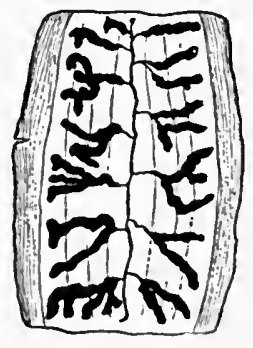

$a$

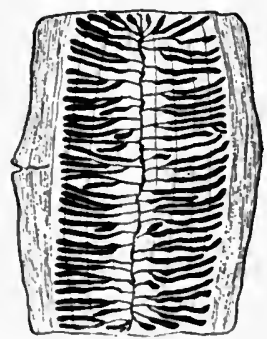

$b$

Fig. 99.-Ripe proglottides of $a$, tenia solium and $b$, tenia saginata.

Tania solium does not attain the length of saginata, averaging from 6 to ro feet and consisting of 800 or 900 segments. The scolex is subglobular, 0.6 to $\mathrm{I} \mathrm{mm}$. in diameter. The short, 
thick rostellum is armed with a double wreath of hooks, 22 to 32 in number, of tenest 26 or 28 . The 4 circular suckers are 0.4 to $0.5 \mathrm{~mm}$. in diameter. The neck is slender. The proglottides increase gradually in size, mature ones measuring to to I $2 \mathrm{~mm}$. in length by 5 to $6 \mathrm{~mm}$. in breadth. The uterus is composed of a median stem with 7 to ro lateral branches on each side. The eggs are globular, 30 to 36 microns in diameter, the shell radially striated, and the oncosphere contains 9 hooks.

The symptoms of infestation with the pork tapeworm do not differ from those of the beef tapeworm. The anatomic differentiation has been made under Tania saginata.

The length of life of the pork tapeworm is not definitely known, but it may infest man for fifteen years or more.

Multiple infections are much more common with this worm than with the beef variety. Mosler and Pfiefer ${ }^{211}$ recount infections with I8, 2I, 2I, 33, and 4I parasites.

The prophylactic measures against the armed tapeworm are identical with those against Tania saginata excepting that in the former the meat concerned is pork.

On account of the danger of somatic teniasis in case of infection with the pork tapeworm, treatment should be instituted immediately. It is possible for the patient to infect himself with cysticercus by swallowing the eggs of his own parasite or by eggs gaining access to the stomach by reverse peristalsis. This does not occur in the case of the beef tapeworm.

The treatment is the same as laid down for Tania saginata.

\section{HYMENOLEPIS NANA}

The Dwarf Tapeworm, Tænia murina, Tænia nana, Tænia ægyptica, Diplacanthus nanus, Hymenolepis murina

The dwarf tapeworm was found in man for the first time by Bilharz in Cairo, Egypt, in $185 \mathrm{I}$. He recovered a "countless number" of them at a post-mortem on a boy who had died of meningitis. Besides in Egypt, this parasite has been found in England, Italy, Servia, Russia, Germany, Siam, Japan, North and South America.

Until r 909 only thirty-three cases had been reported in the United States, seven of these having been reported by me. These cases may be tabulated as follows: 


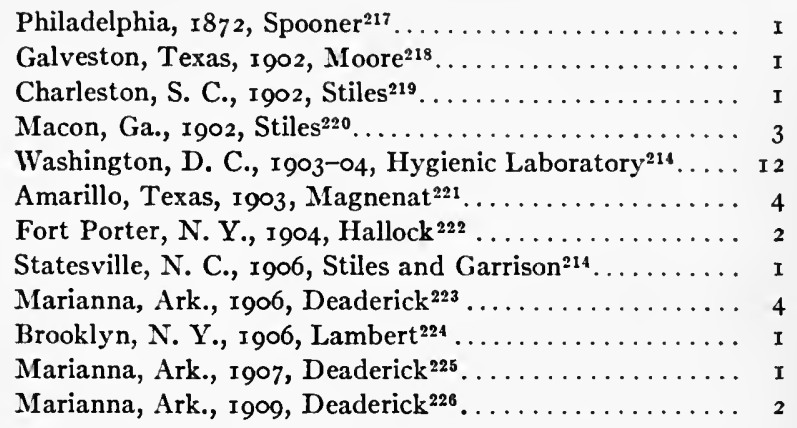

Within the past four years the dwarf tapeworm has been shown to be the commonest cestode in the South. During the three years, I9II-I9I3 of 56,543 infections found by the Rockefeller Sanitary Commission I, 879, or 3.3 per cent., were Hymenolepis nana. It has been found in all Southern States surveyed by this Commission. Schloss ${ }^{230}$ reports twenty cases in 280 consecutive examinations in New York.

Negroes are affected as well as whites. Males are affected more frequently than females.

Children from five to ten years of age are most susceptible to infestation, but no age seems exempt, cases being recorded past fifty years of age.

The only intermediate hosts yet determined are the rat and probably man; the definitive hosts are man and a certain species of rats and mice.

Direct infection from man to man seems possible. In support of this are the two cases of Stiles ${ }^{220}$ in an orphan asylum, members of the staff of the Hygienic Laboratory ${ }^{214}$ finding five cases in the same ward of an insane asylum, ${ }^{1}$ Magnenat' ${ }^{221}$ four cases in the same family, and $\mathrm{my}^{226}$ two cases in the same household. In all of Schloss' ${ }^{227}$ cases, with one exception, more than one child in each family was infected. Venuti ${ }^{17}$ found twentythree cases among 2I4 boys examined in an institution in Catania, while no cases could be found among roo boys of the general population.

The usual mode of infection seems to be the ingestion of food soiled by the excrement of infested rats and mice.

The length of my specimens of Hymenolepis nana has been 
from 8 to $18 \mathrm{~mm}$.; the breadth of mature proglottides from 0.33 to $0.58 \mathrm{~mm}$; and the number of segments approximately from 100 to 200 . In a specimen measuring $15 \mathrm{~mm}$. in length there were 185 segments. The head is subglobular, elongated, possessed of a retractile rostellum surrounded by a single row of hooklets, numbering from twenty-two to thirty, and has four suckers. The average length of the head with rostellum protruded was $0.34 \mathrm{~mm}$., with rostellum retracted $0.27 \mathrm{~mm}$., and the width of the head $0.27 \mathrm{~mm}$. The diameter of the suckers averaged 86 microns. The hooklets averaged I4 microns in length. The neck is slender and unsegmented. In a specimen measuring $15 \mathrm{~mm}$. the distance from the tip of the rostellum to the first segment was I.I $8 \mathrm{~mm}$.; the breadth of the neck $0.14 \mathrm{~mm}$. The segments of the strobila gradually increase in breadth toward the posterior extremity, the last fifteen to forty proglottides being usually stuffed with ova. Occasionally a sterile segment is seen between two fertile ones. The genital pores are single, near the anterior border of the segment, and usually on the left side. Each oviparous segment may contain from 80 to 80 ova. The mature ova are round or slightly oval, averaging in my cases: outer membrane 50 by 42 microns, inner membrane 32 by 30 microns. Between the outer membrane and the inner one which encloses the membrane is a hyaline substance which surrounds the latter like a wide halo. Within the embryo can be distinguished six hooklets lying parallel or radially. A few very delicate filaments may commonly be detected arising from opposite poles of the inner membrane and permeating the hyaline substance.

I have examined the ova in my last three cases with reference to Foster's ${ }^{228}$ interesting observations on the movements of the embryo within the ovum, but have not been able to verify them.

The symptoms are similar to those of infection with the beef tapeworm, strikingly exaggerated in children. In one of my cases abdominal pain, diarrhea and vomiting were prominent; in another nausea, vomiting, dizziness, headache and dyspnea; in a third vomiting, abdominal pains, headache, dyspnea, dizzi- 


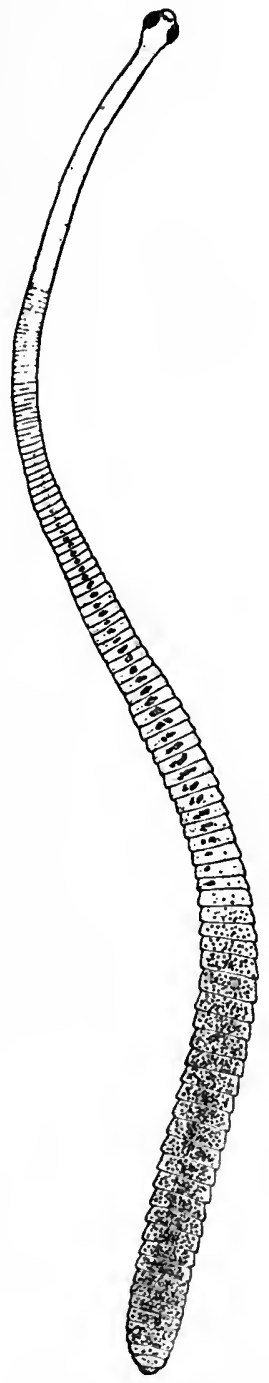

liig. rat.-Hymenolepis nana, enlarged.
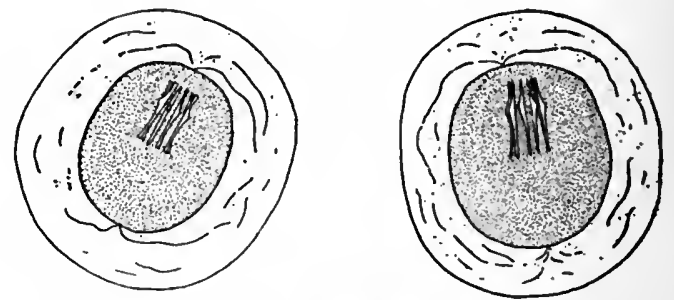

Fig. roo.-Ova of hymenolepsis nana, enlarged

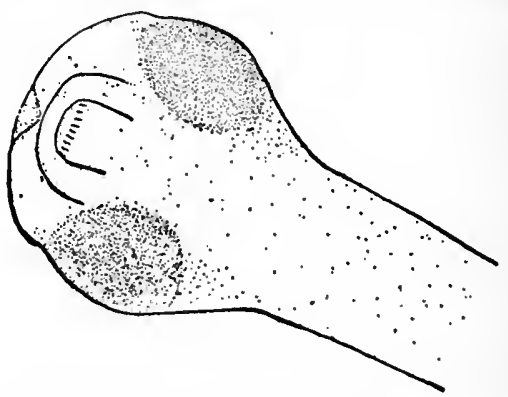

Fig. 102.-Scolex of hymenolepsis nana, enlarged.
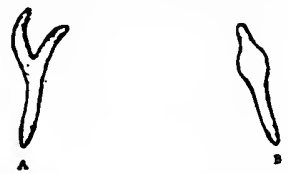

Fig. 103.-Hooklets from hymenolepis nana, enlarged: $a$, lateral aspect; $b$, view from edge.

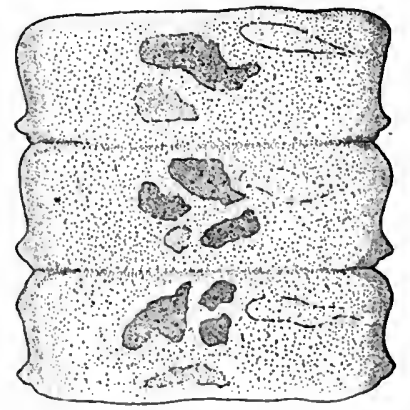

Fig. 104.-Proglottides of hymenolepis nana, enlarged. 
ness and diarrhea. A boy aged eleven developed edema of the hands, feet and legs up to the knees, of the eyelids and upper lip. A young girl had convulsions every time she had a slight fever; this was promptly stopped by removal of a large number of dwarf tapeworms.

Many cases are symptomless.

The appetite may be increased, decreased or otherwise perverted. Abdominal pain is frequent, so are nausea and

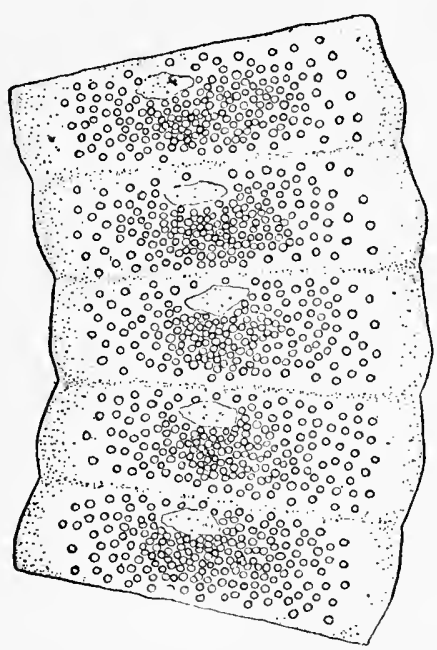

Fig. 105.-Hymenolepis nana, proglottides containing ova.

vomiting. Gnawing and crawling sensations are occasionally complained of. The bowels may be loose, regular or constipated.

Nervousness and disturbed sleep are not infrequent manifestations. In children choreic movements and convulsions have been recorded.

A slight degree of anemia is not uncommon. In seven of my cases the eosinophiles varied between 7.8 and 26 per cent. Of fourteen cases of Schloss ${ }^{227}$ seven showed eosinophilia ranging from 6.5 to 22 per cent., while in the remaining seven cases these cells were normal.

I have not found Charcot crystals in the feces of any of my cases, but in mounted specimens of the parasite, have frequently 
noticed the formation of these crystals at points where the worm was lacerated or contused.

Multiple infestation is the rule, as many as several thousand occasionally being present in the same individual.

Mixed infestations are common, the combination with hookworm being the most frequent. One of my cases showed in addition to Hymenolepis nana, Ascaris lumbricoides and Trichomonas intestinalis.

The infection may persist for several years if untreated.

The diagnosis depends solely upon the detection of the ova or parasites in the feces. The ova are likely to be confounded with no others excepting the rare parasite Hymenolepis diminuta. The latter may be distinguished by the somewhat larger size, the presence of two other membranes with delicate radial striations between, and by the absence of filaments in the hyaline substance.

Preventive measures consist of treating promptly and radically infested persons, guarding against pollution of food or hands with feces of infested persons and the protection of food stuffs from rats and mice.

Treatment.-The most efficient vermifuge in these cases is male-fern. It should be given as for the treatment of the beef tapeworm. While the worms are readily expelled by this.agent, an analysis of the cases in which the results of treatment by malefern were verified by subsequent microscopic examination of the feces shows that more than one treatment is usually necessary. There are twenty-two cases in which the results were thus followed up. Of these there are only five cases in which no ova were found after one treatment with male-fern; in six cases two treatments were necessary; in five cases three doses were needed; in one case four or five treatments were administered; in four cases after one dose the ova were still present but no further treatment is recorded; in one case after several treatments the ova persisted in the feces; and in two of the cases in which three doses were given the ova were yet found after the last dose. The drug should, of course, be given only after thorough purgation with salines. 
HYMENOLEPIS DIMINUTA

Tænia diminuta, Tænia leptocephala, Hymenolepis flavopunctata, Tænia flavopunctata, Tænia flavomaculata, Tænia minima, Tænia varesina

In 1858 Weinland first described a case of Hymenolepis diminuta occurring in man. The parasites were recovered in $185^{2}$ from a healthy infant nineteen months old. Two fragments labelled as coming from man were found in the museum at Alfort, France, by Raillet before I8Io, and were identified as Hymenolepis diminuta by both Raillet and Zschokke.

The following is a synopsis of cases which have thus far been reported in man: $:^{29}$

Alfort, France, by Raillet, before $1810 \ldots \ldots \ldots$ I case.

Boston, r842, Weinland................. I case.

Philadelphia, 1884 , Leidy................. I case.

Varese, Italy, I884, Parona................ I case.

Catania, Sicily, I887-8, Grassi............... I case.

San Paulo, Brazil, 1893, Lutz................ I case.

Pisa, Italy, 1895 , Sonsino............... I case.

Rio Janeiro, Brazil, r896, Magalhaes ........... I case.

Centuripe, Sicily, rgoo, Previtera............. 2 cases.

Philadelphia, I900, Packard............... I case.

Marianna, Ark., I906, Deaderick ${ }^{223} \ldots \ldots \ldots \ldots \ldots$ I case.

Hanley Falls, Minn., I906, Nickerson ${ }^{231} \ldots \ldots \ldots \ldots$ I case.

Of the above thirteen cases four were in males, five in females, and in four the sex is not recorded.

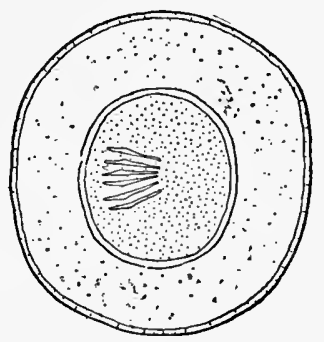

Fig. ro6.-Ovum of hymenolepis diminuta, enlarged.

The ages were 16 months, I9 months, 20 months, 2, 2, 3, 8, II, I2 and 40 years, the ages of four patients not being recorded. According to Ransom ${ }^{229}$ the number of worms present in each individual varied from one to four. In my case there were three 
or four specimens, while in Nickerson's ${ }^{231}$ there were about twenty-five.

No marked symptoms have been recorded in any of the cases. Increased appetite, nausea, and pain in the epigastrium were the complaints in my case. Slight "peevishness" was noted by the mother of the child in Nickerson's case. In my case the eosinophiles were 7 per cent.

This parasite in the adult stage inhabits the intestine of certain species of rats and mice. It is probably accidental in

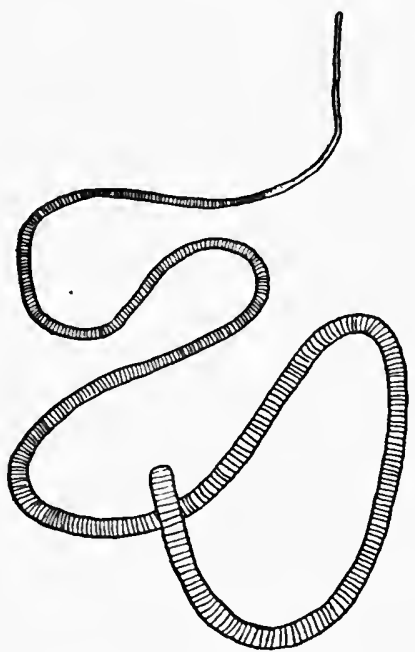

Fig. 107.-Hymenolepis diminuta, natural size.

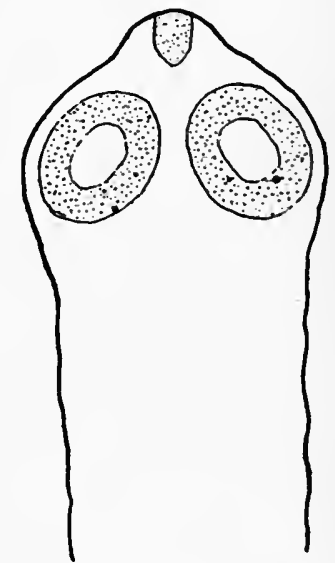

Fig. 108.-Scolex of hymenolepis diminuta, enlarged.

man. The larva occurs in the meal moth, the earwig and certain species of beetles. Recent experiments of Nickerson ${ }^{232}$ implicate certain myriapods, Fontaria and Julus, as intermediate hosts in America.

Hymenolepis diminuta is from 20 to $60 \mathrm{~cm}$. in length and up to 3.5 to $4 \mathrm{~mm}$. broad. The segments number from 600 to $\mathrm{r} 300$. The head is club shaped, 0.2 to $0.5 \mathrm{~mm}$. in diameter, and has a rudimentary unarmed rostellum. There are four suckers. The ova resemble those of Hymenolepis nana. The average of ten measurements of ova in my case gave: outer membrane 35 by 40 microns. Some were slightly bile stained, others perfectly 
clear. The outer membrane appears thickened or doubled and may be radially striated. No filaments are found in the hyaline substance as in the ova of the dwarf tapeworm.

The diagnosis is made by the discovery of segments of the worm or its ova in the feces.

Prophylaxis of infection with this parasite consists of guarding against contamination of food by the meal moth, rats, mice, and other hosts.

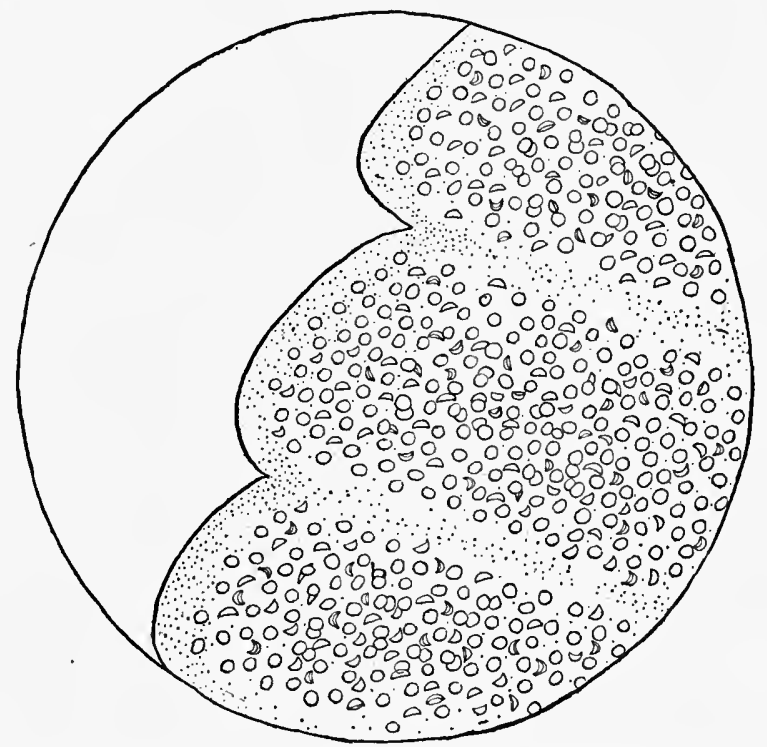

Fig. rog.-Hymenolepis diminuta, proglottides containing ova, enlarged.

Treatment.-Any anthelmintic or merely a cathartic is usually successful in expelling the parasite. In the thirteen cases santonin was used twice, male-fern three times, calomel once, the worm was expelled without medicine once, and the anthelmintic used is not recorded in six cases.

\section{STRONGYLOIDES INTESTINALIS}

Anguillula intestinalis, Anguillula stercoralis, Rhabdonema strongyloides, Rhabdonema intestinalis, Leptodera stercoralis, Leptodera intestinalis

In 1876 Normand treated a number of French soldiers returned to Toulon from Cochin China suffering severely from diarrhea. A large number of small nematodes in the feces were 
identified as strongyloides intestinalis. Both Normand and Bavay, who made a careful study of these parasites, regarded them as the causative factors of Cochin China diarrhea.

The geographic distribution at large seems strikingly in con-

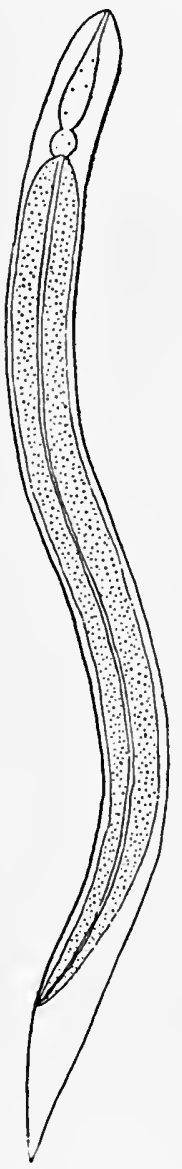

Fig. r ro.-Larva of strongyloides intestinalis, enlarged. formity with that of hook-worm. The reports of the Rockefeller Sanitary Commission for the years I9II-I9I3 record cases in Mississippi, North Carolina, Tennessee, Alabama and Georgia. Cases were observed in Baltimore by Strong ${ }^{233}$ and Thayer, ${ }^{234}$ and in Tennessee by Brush. ${ }^{235}$ I observed a case in eastern Arkansas, and the State Director of Sanitation in this state $^{236}$ informs me that his field men have found twenty-one cases in this state to June 30, I914. Simon ${ }^{237}$ records it in Louisiana.

Besides man the only other host for this parasite appears to be the monkey.

The modes of infection by Strongyloides intestinalis are two: by the mouth with infected food or water, and by the skin after the manner of hook-worm infection. It is maintained by Fulleborn and Torgau ${ }^{238}$ that even those larvæ which are swallowed must pass through the stomach wall into the vessels and by way of the right heart, lungs, trachea and esophagus back to the stomach and intestines.

The complicated life cycle of this parasite is, according to Stiles, ${ }^{216}$ as follows:

I. (a) The parasitic adults, inhabiting the intestine, are parthenogenetic females measuring 2.2 to $3 \mathrm{~mm}$. long by 34 to 70 microns broad. The eggs are deposited in the intestinal lumen of the host or in galleries in the intestinal mucosa made by the females, and develop into

(b) Rhabditiform embryos 200 to 240 microns long by $\mathrm{I} 2$ microns broad which may grow to 450 to 600 microns long by 16 to 20 microns in diameter by the time they are discharged with the feces. These embryos develop within two or three days into 
II. (c) Free-living diœcious adults. The males measure $0.7 \mathrm{~mm}$. long; the tail is curved ventrally to form a hook; the spicules are curved and 38 microns long. The females measure I $\mathrm{mm}$. long; the vulva is situated slightly posterior to the equator of the body. Each female develops thirty to forty eggs which may or may not segment in the uterus; these eggs develop forming the

(d) Free-living rhabditiform embryos which measure 220 microns long; when they attain a length of $55^{\circ}$ microns they moult and become

(e) Filariform embryos. This is the infecting stage which enters man by the mouth or through the skin, reaches the duodenum and upper part of the jejunum and develops directly into

(a) The parthenogenetic females.

Instead of the cycle $a-b-c-d-e-a$ an abridged cycle $a-b-e-a$ may occur.

Strongyloides intestinalis may be cultivated by adding sterile water to the feces and placing in the sunlight.

The parthenogenetic females are found only in the upper part of the small intestine.

The pathogenic equation of this worm has long been debated and is still unsolved. Earlier writers on the subject were confident of an etiologic association with diarrhea. Of recent years the tendency is increasingly to belittle this relation. In many cases in which the parasite is associated with diarrhea, enamebæ and other organisms have been found. It is not infrequently associated with hook-worm. It is probable that its pathologic status is not important, though in some cases it appears to excite a catarrhal enteritis.

The diagnosis is possible only through the discovery of the rhabditiform embryo in the feces. Occasionally after free purgation strings of eggs may be found.

Treatment.-No known anthelmintic is of any particular value in the treatment of infection with this parasite. Thymol is most frequently recommended, but the results are far from satisfactory. 


\section{ASCARIS LUMBRICOIDES}

\section{Round Worm, Eel Worm, Lumbricoid Worm}

This worm is practically cosmopolitan. The only country on the globe in which it is not endemic is said to be Iceland. It is second only in frequency to the hook-worm in the South, onethird of all infection found by the Rockefeller Sanitary Commission for the years I9II-I9I3 being the round worm.

The negro race is more often infested than the white. In some sections of the South it is a common custom for the little negroes to be "wormed" (treated for round worms) regularly every year.

While seasons are not known to influence the frequency of their occurrence, in my experience they are more evident in the summer and fall. The fact that this is the malarial season is probably responsible for this, since febrile conditions cause the wandering and expulsion of the parasites.

Females seem more prone to infestation than males. Children are more frequently the hosts of these worms than are adults. Miller ${ }^{27}$ reports an interesting case in a child of three weeks. After the separation of the cord ulceration of the umbilicus occurred leading to perforation of the intestine, and from the resulting fistula a round worm $18 \mathrm{~cm}$. long was discharged. Stiles and Garrison ${ }^{214}$ report a case in a patient over eighty years old. These writers have collected 534 cases in which the age was given and which are distributed as follows:

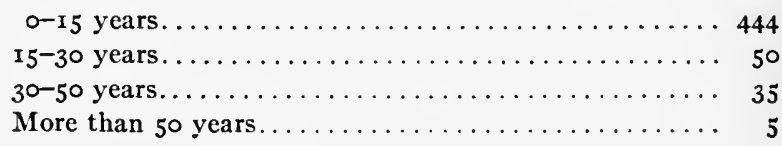

Ascaris lumbricoides is known to occur in man only.

An intermediate host is not concerned in the dissemination of the infection. After passing out with the feces the ova in the course of a month or more develop embryos within. When these ova are swallowed the contained embryo develops directly into the adult stage. Freezing, if not too prolonged, and drying of the ova at ordinary temperatures do not destroy the embryo, and they may be blown about as dust. Infection 


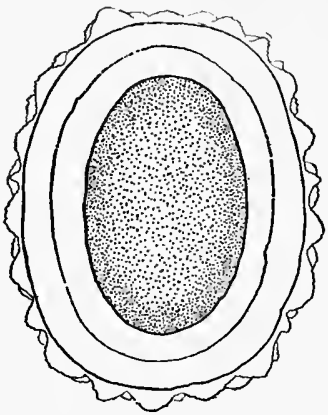

Fig. II - Ovum of ascaris lumbricoides, enlarged.

through water, vegetables or soiled hands are the usual methods. Stiles ${ }^{4}$ believes that flies may carry the ova in their intestines and thus spread the infection.

The worms are reddish or grayish yellow in the fresh condition. The oval cavity is surrounded by three papillæ, one dorsal and two ventral. The body is round and tapers toward both extremities. The male is $I_{5}$ to $25 \mathrm{~cm}$. in length and about $3 \mathrm{~mm}$. in diameter. The posterior end is flexed ventrally. The females are 20 to 40 $\mathrm{cm}$. long and about $0.5 \mathrm{~cm}$. in diameter. The posterior extremity is straight. The vulva is anterior to the middle of the body.

The ova are elliptical, 50 to 70 microns in length by 40 to 50 microns broad. The external covering is thickly set with processes and usually bile stained. The ova are deposited before segmentation.

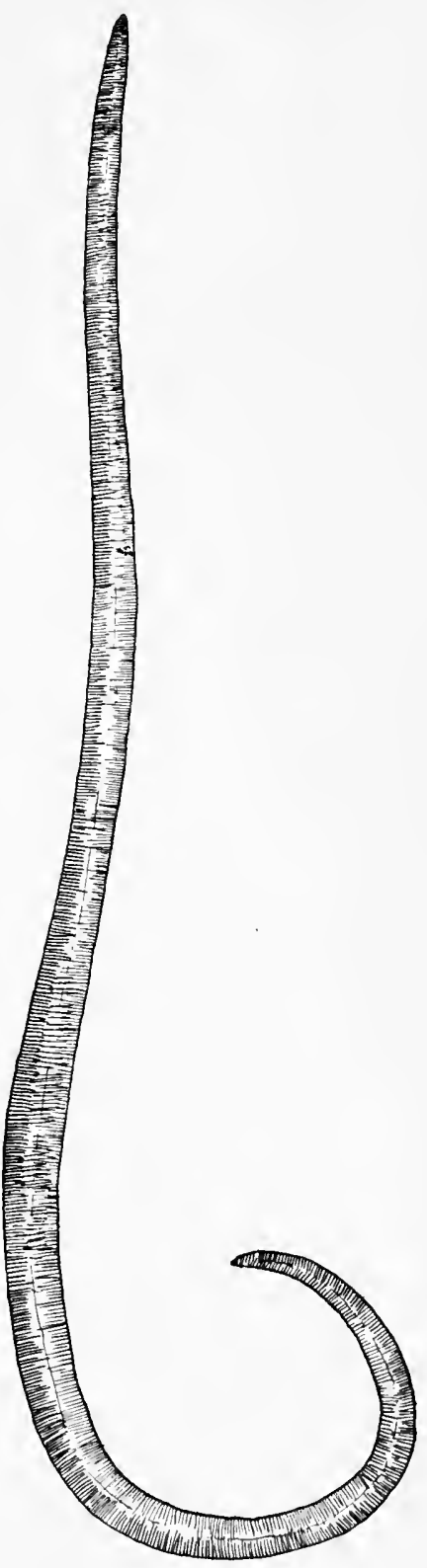

Fig. I $12 .-$ Ascaris lumbricoides, female, natural size 
It is probable that the pathogenic power of ascaris lumbricoides depends, besides mechanical disturbances, upon a toxin. Goldschmidt ${ }^{240}$ records the symptoms experienced by himself and others while dissecting fresh ascaris. The first dissection often caused no symptoms, but a repetition was followed by tenderness, irritation of the respiratory tract, sneezing, coryza, conjunctivitis and typical asthmatic attacks recurring periodically, strikingly resembling hay fever. These suggest anaphylactic phenomena. By the intraperitoneal injection of an aqueous extract of Ascaris lumbricoides Herrick ${ }^{241}$ produced a notable eosinophilia after previous sensitization, and shows that the substance causing such an eosinophile increase is a protein. Flury ${ }^{241}$ has shown that the tissues and excreta of these worms contain numerous compounds capable of inducing local hyperemia, inflammation and necrosis.

Multiple infections are the rule, from two to six being the usual number. However, large numbers may be present. I have felt masses of these worms through the abdominal wall. Raines ${ }^{242}$ describes the unique case of a child of twenty months who discharged 834 worms in one day, 634 the next, and within a short period 1,992 in all. Fauconneau-Dupresne ${ }^{211}$ observed the case of a twelve-year-old boy who passed 5,126 of these worms in less than three months.

Many cases present no symptoms. On the other hand, the symptoms may be alarming. The commonest manifestations are fretfulness, restless sleep, dreams, itching of the nose and anus, gritting the teeth, meteorism, nausea and vomiting. There may be perversion of hearing, taste, sight and smell, vertigo, hysteria and convulsions. Other digestive symptoms are dyspepsia, griping pains in the abdomen, irregular bowels, diarrhea, constipation, increased, deficient or perverted appetite, malnutrition and jaundice. I have seen severe dysenteric symptoms subside after riddance of round worms. Anemia is present in some cases, and if symptoms are present the blood shows eosinophilia.

At times infestation with eel worms assumes dangerous significance. This is especially true when the parasites are in such numbers as to cause intestinal obstruction, or when they pene- 
trate communicating passages or cause perforation. Febrile conditions cause the parasites to become active, and it is often under such circumstances that these accidents occur. The worms may escape spontaneously from the anus, mouth or nose, or may be vomited. Perforation into the thoracic or abdominal cavity may result, or the parasite may er.ter the Eustachian tube or cause suffocation by entering the trachea. It is stated that there are recorded about ninety cases in which round worms have entered the bile ducts, nine cases into the pancreatic duct, and over twenty cases into the urinary passages. ${ }^{14}$ Over eighty cases are recorded in which the worms have escaped through the body wall at various points.

The following unique case occurred in my practice: A colored boy, aged nine years, shot himself while playing with a $4 \mathrm{I}$-caliber Derringer. I saw him several hours after the accident occurred. The ball entered the abdomen in the median line immediately above the symphysis and could be felt beneath the skin a little to the left of the anus. There was some abdominal rigidity; he had vomited once and his bowels had moved twice with blood in both actions. He had urinated once and the urine contained one or two small clots of blood. The temperature was 102, pulse I 20 , otherwise good. The insanitary surroundings and lack of facilities and the belief that the course of the bullet was extraperitoneal led me to treat the case expectantly. His condition did not get any worse nor did he pass more blood from the bowel or bladder. A week or ten days after the accident he began to have difficulty in starting the flow of urine, which would sometimes be interrupted suddenly after having been started-typical "stammering urination." This difficulty continued until the fifteenth day after the accident, when a large living lumbricoid worm made its exit from the bladder through the urethra. The boy made an uneventful recovery.

The diagnosis is easily made by the detection of the ova in the feces or the expulsion of the worms.

The longevity of Ascaris lumbricoides is not known. Eggs may be found in the feces about a month after infection.

Prophylaxis consists of abstaining from contaminated food and water, infection by soiled hands, and exclusion of flies. 
Treatment.-The cure of this infection is easy, santonin being practically a specific. The dose for a child under one year is $1 / 3$ to $1 / 2$ grain; for a child one to five years of age, I or 2 grains; for older children, 2 or 3 grains. It is well to combine calomel with the vermifuge, and preliminary diet and purge should be prescribed as for teniasis. The treatment is usually repeated once or twice at forty-eight-hour intervals. The feces should be examined periodically to ascertain whether the cure has been radical.

\section{OXYURIS VERMICULARIS}

\section{Ascaris vermicularis, Fusaria vermicularis, Pin Worm, Seat Worm,} Thread Worm, Maw Worm

These worms are practically cosmopolitan. Even in Iceland, where the round worm is not endemic, oxyuris is enormously frequent. In the Southern States they do not seem to be as common as they were in former years. The Rockefeller Sanitary Commission has found them in each of the states surveyed, and it is probable that they occur with greater relative frequency than their figures indicate, since statistics based on ova findings under-estimate pin worms as their ova are not as numerous in cases of infection as is the case with many other parasites.

More cases are observed in spring and summer than in other seasons.

Males are less frequently infested than females.

Infections are found from early childhood to old age. Heller ${ }^{243}$ observed a case in a child of five weeks. A number of cases compiled by Stiles and Garrison ${ }^{214}$ may be arranged according to age as follows:

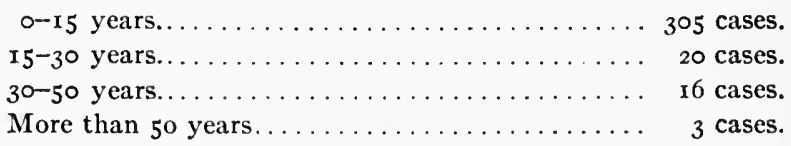

Oxyuris vermicularis is not known to occur in any other animal.

No intermediate host is concerned in the transmission of this parasite from one person to another. The ova gain access to the alimentary canal of man through infected hands, food, or drink. Reinfection of an infected individual is common after 


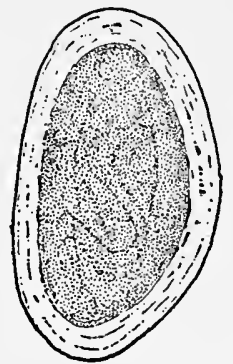

Fig .II3.-Ovum of oxyuris vermicularis, enlarged.

scratching the anus and putting the hands to the mouth or nose. It is possible that flies may carry the ova.

The worm is white in color. The female is about $\mathrm{I} \mathrm{cm}$. in length and $0.6 \mathrm{~mm}$. in diameter, and has a long sharp-pointed tail. The vulva is in the anterior half of the body, the anus in the posterior half. The male is 3 to $5 \mathrm{~mm}$. in length, the body curved ventrally. The males die early and are not often seen in the feces. For a long time it was not known. The eggs are oval, flattened on one side, and average 50 by 20 microns. The sexes are usually present in equal numbers. The worms may be from a very few to so many that the mucous membrane of the gut looks like fur.

Symptoms are usually due to the nocturnal wanderings of the worms.

These consist chiefly of irritation of the anus, disturbed sleep, gritting the teeth, and itching of the nose. Pain in the lower part of the abdomen is common and there may be meteorism, nausea, Fig. 114.-Oxdis vermicudiarrhea or constipation. The appetite may be laris, adult fedeficient, increased or irregular. Genital irrita- male, enlarged. tion leading to masturbation is observed in some cases, and the worms may enter into the vagina. Enuresis is occasionally caused by pin worms. Loss of weight, headache, anemia, convulsions and chorea may exist. 
Eosinophilia above 5 per cent. existed in seventeen of Schloss' ${ }^{222}$ twenty-two cases.

Numerous cases are recorded in which the appendix has been invaded by pin worms with resulting appendicitis; indeed it was at one time erroneously supposed that the appendix was the normal habitat of these parasites.

A diagnosis of this infection cannot be made on symptoms alone and usually rests on the discovery of adult females discharged from the rectum. The ova may be found in the feces in the majority of cases, but a negative result does not exclude infection.

Since reinfection is common it is impossible to determine the length of life of the individual worms. Infection may persist for a number of years, however. The males are short lived.

Prophylaxis.-Since infection is carried from the anus to the mouth by the fingers, the hands should be washed after defecation and the nails kept trimmed and clean. Food should be guarded against contamination and flies should be excluded.

In outlining the treatment it should be considered that the normal habitat of oxyuris is the cecum, and only impregnated females descend to the rectum to ovulate; hence the treatment should be continued for several weeks. Internal treatment should at the outset be combined with irrigations, and santonin, given as recommended for ascaris, is the most useful drug. Quassia is an effective irrigation. It should be used as an infusion using two tablespoonfuls of the chips to a pint of hot water. No more should be injected than can be retained and it should be given with the buttocks elevated, or in the kneechest position. In the cases where this has failed me I have usually had success on obtaining a fresh supply of quassia. Strong salt water used in the same manner is often effective. For the anal pruritus weak mercurial ointment or zinc oxide ointment should be applied locally at night. Reinfection should be guarded against and the treatment persisted in until the cure is radical. 


\section{TRICHURIS TRICHIURA}

Trichocephalus dispar, Trichocephalus trichuris, Trichocephalus hominis, Ascaris trichiura, Whip-worm

The whip-worm is said to be cosmopolitan. It ranks third in frequency among the infections found by the Rockefeller Sanitary Commission. I failed to find ova of this parasite in about 500 examinations in the Mississippi River "bottom" in eastern Arkansas.

Infections are commoner among negroes than among whites. The frequency of this parasite is sometimes regarded as an index to the sanitary condition of a community.

Females are more frequently infested than males.

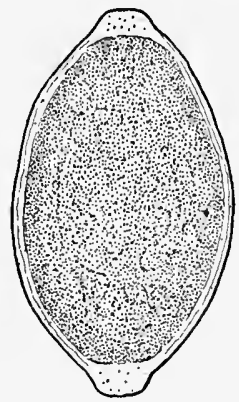

Fig. I I 5.-Ovum of trichuris trichiura, enlarged.

Infections have been found from the age of less than three months until old age. Stiles and Garrison ${ }^{214}$ collected from the literature a number of cases, which fall according to age in the following limits:

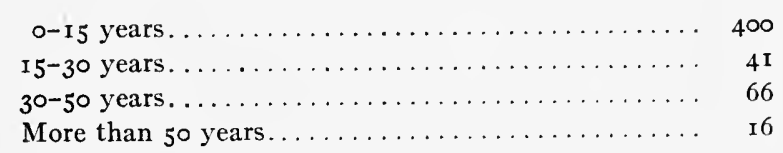

Besides in man the whip-worm is found in certain monkeys and lemurs.

No intermediate host is necessary in the propagation of infection, the partially developed ova being swallowed with contaminated water or food.

The whip-worm has a thick club-shaped body which tapers radially into the long thread-like neck. The male is $40-45$ 
$\mathrm{mm}$. in length, the female $45^{-50} \mathrm{~mm}$., about three-fifths being comprised by the anterior portion of the body. The anus is terminal and the vulva is situated near the commencement of the posterior portion of the body. This part of the body in the male is spirally rolled up. The ova are very characteristic.

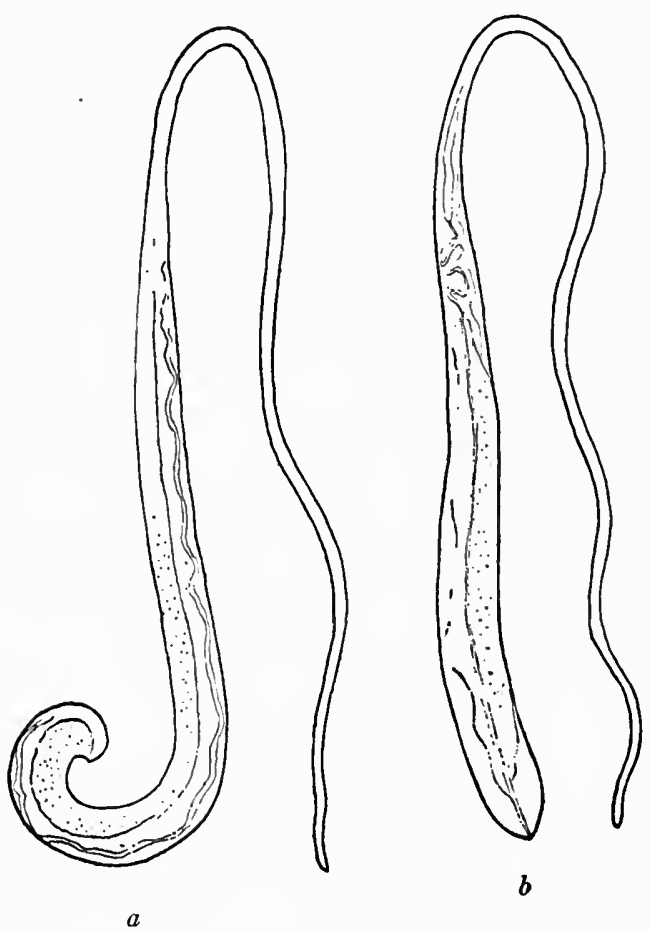

Fig. I16.-Trichuris trichiura. $a$, Male; $b$, female, enlarged.

They are barrel-shaped, possessed of a thick brownish shell and perforated at the poles. They measure about 50 microns in length by about 23 microns in breadth.

There are usually about a dozen worms present, but thousands have been found at autopsy. The cecum is the normal habitat.

This parasite can no longer, as formerly, be regarded as a harmless commensal of man. Various theories have been adduced to explain its pathogenicity, such as that the worm is 
a blood sucker, that it produces a toxin or hemolysin, or that it causes mechanical injury, but none of these is satisfactory. It is known that the whip-worm transfixes the mucous membrane, embedding beneath it the anterior portion of the body and producing hyperemic or even eroded areas, but these are not sufficiently extensive to be pathogenic.

This worm has been accused of a part in the infection with typhoid fever but the evidence is not convincing.

While many cases present no symptoms there may be anemia, severe nervous symptoms and gastro-intestinal disturbances, shortness of breath on slight exertion, dyspeptic manifestations, vomiting, diarrhea, nervousness, insomnia, and even convulsions and coma are symptoms. There is rarely any eosinophilia in these cases, contrary to the case in most helminthic infections. There are a number of fatal cases on record. Metchnikoff ${ }^{244}$ emphasized the importance of these parasites in the production of appendicitis.

Accurate diagnosis can be made only by examination of the feces.

It is not known how long infection with whip-worms may persist.

Prophylaxis.-The prevention of soil pollution and care against contamination of food are the requisite prophylactic measures.

Treatment.-It is proverbially difficult to rid the bowel of these unwelcome guests. Many anthelmintics bring away part but none is known to be specific. Thymol perhaps has the best reputation. High enemas of solutions of benzene have recently been used with apparent success and this treatment deserves further trial.

\section{BALANTIDIUM COLI}

\section{Paramœcium coli, Holophyra coli}

Infections with Balantidium coli have been found in Russia, Sweeden, Finland, Germany, Italy, North and South America, Cuba, Africa, Sunda Isles, Cochin China and the Philippines. In the United States, Bel and Couret ${ }^{245}$ reported a case in Louisiana, Gray ${ }^{246}$ three cases in Arkansas, I observed one 
case in eastern Arkansas, Sistrunk ${ }^{247}$ one in Minnesota, and the Rockefeller Sanitary Commission ${ }^{248}$ two cases in North Carolina and one in Mississippi. Altogether less than 50 cases have been recorded in man.

Males are twice as frequently infested as females.

Adults are far more frequently subject to this infestation, but two cases having occurred in children.

Association with pigs or the preparation of sausage predispose to infection.

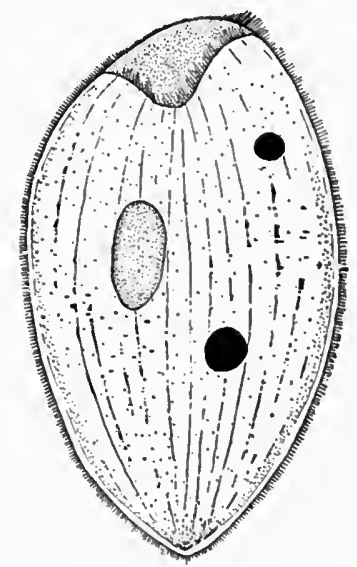

Fig. I 7 7.-Balantidium coli, enlarged.

Four cases have been reported in the same household. ${ }^{249}$

Besides man, the domestic pig and certain species of monkeys serve as hosts for balantidium.

It is possible to acquire this infection through food or water infected with the feces of pigs or through eating infected hog meat.

The body of this infusorium is nearly oval, one side being usually somewhat longer than the other. The length is from 0.06 to $0.1 \mathrm{~mm}$. and the breadth from 0.05 to $0.07 \mathrm{~mm}$. The anterior end shows the short, broad, funnel-shaped peristome. The anus is terminal at the posterior end. There is a distinct ectosarc thickly set with cilia. The endosarc shows ordinarily two contractile vacuoles and phagocyted particles. The nucleus is commonly bean-shaped. Reproduction occurs through binary fission and budding conjugation is known in 
this parasite, and encystation is known to occur. The organisms are highly motile. Bel and Couret ${ }^{245}$ were unable to cultivate them.

The habitat of this parasite in man is the large intestine. The organisms invade the coats and vessels of the gut, producing ulcers, hyperemic or a hemorrhagic condition of the intervening mucosa, and swelling of the mesenteric glands.

Nearly all infested patients suffer with diarrhea or dysentery. The feces are liquid and contain undigested food, mucus or blood. Tormina and tenesmus are frequent symptoms, and in persistent cases there may be anemia, loss of weight and edema. Eosinophilia is present in many cases.

The diagnosis is impossible without the detection of the characteristic organisms in the feces.

The duration of the infection may be as long as fifteen years. The mortality in II I cases collected by Strong ${ }^{249}$ was 30 per cent.

Prophylactic efforts should be directed against infected pigs.

Treatment.-The list of drugs recommended for the treatment of the condition indicates that there is no specific. Enemas of quinine solutions are perhaps more highly recommended than other measures. Enemas of tannic acid, silver nitrate. iodine, acetic acid, salicylic acid, and boric acid have been used. Internal treatment has so far been unavailing. Emetine should be given a trial.

\section{MYIASIS INTESTINALIS}

In 1902 Schlesinger ${ }^{250}$ was able to find in the literature some Ioo cases of intestinal myiasis. Since then a number of such cases have been recorded. I have observed two cases in Arakansas. ${ }^{251}$

Most of the cases are reported in adults, but children of one year or under may be affected.

As is well known, the larval stage of some diptera is necessarily passed in the alimentary canals of mammals and other animals, for instance, the bot-fly of cattle, but fly larvæ must be regarded as accidental parasites of man. 
The following species may give rise to intestinal myiasis in this climate:

Musca domestica, the common house-fly, deposits its eggs on horse manure, occasionally on that of the cow, or even in human feces, rarely on decaying animal or vegetable matter.

Musca vomitoria, the blue bottle meat fly, deposits its ova by preference upon decaying animal matter.

Anthomyia canicularis, the little house-fly, deposits ova upon decaying vegetable matter.

Chrysomyia macellaria, the screw-worm fly, deposits ova in wounds or on decaying flesh.

Sarcophaga carnaria, the green flesh fly, larvæ placed on decomposing animal matter, possibly also on human feces.

Piophila casei, the cheese fly, deposits its ova upon human feces.

Eristalis tenax, the drone fly, the larvæ are found on decaying animal and vegetable matter, in soft mud and in foul water.

These creatures may gain entrance to the body either as ova or as larvæ. Probably the most common mode of infection is through eating food containing ova or larvæ, though the anus may be the port of entry. Wirsing ${ }^{252}$ believes that infection occurred through the anus in the case of a breast nursing infant taking an air bath in an open window. In the case reported by Finlayson ${ }^{253}$ due to Anthomyia canicularis, it appears that the ova were not swallowed but were deposited at the anus while the patient was defecating and were taken up within the bowel after defecation. Nicholson ${ }^{254}$ reports three cases of infection with larvæ of Musca domestica in which it seems probable that infection was by way of the anus. Prolapse, hemorrhoids and external lesions predispose to such infections. It is possible that infestation may occur through the swallowing of pregnant viviparous flies, as sarcophaga.

Among the species of this order of insects whose larvæ occur in man as pseudoparasites there are two modes of reproduction, the oviparous and the viviparous. The former is the more frequent among the species in question, but the latter is usual among the sarcopagids especially with Musca domestica; each female lays about I 20 eggs; the duration of the egg stage is 
about eight hours, of the larval stage about five days, and the pupa stage the same. These figures vary with the climate and season.

The symptoms produced by these loathsome creatures are abdominal pains, nausea, diarrhea, dysentery, nervousness, abdominal tenderness, crawling sensations in the bowels, migrain, neuralgia and even hysterical symptoms and convulsive seizures.

The longevity of intestinal maggots is not known, but infection may persist for years. One case is recorded of twelve years' standing. ${ }^{255}$

The broad diagnosis of intestinal myiasis is easy to any one familiar with the ordinary parasites, but a specific determination should not be attempted by any one but an expert. In all these cases it is well to breed out the maggots to mature insects as determination is much easier in the adult state. A little earth in a pot covered by gauze is all that is necessary for this experiment.

Prophylaxis consists of prevention of access of flies to food and avoidance of defecation in the open, especially by those predisposed by anal or rectal disease.

The treatment which is usually successful is castor oil, alone, or given with santonin. The oil should be given on an empty stomach, one dose for three successive days. In one of my cases, an infestation with Chrysomyia macellaria, I used an enema of a pint of cotton-seed oil with a satisfactory result. 


\section{REFERENCES}

${ }^{1}$ Crosse, Blackwater Fever, London, I899.

${ }^{2}$ Plehn, Die Kamerum Küste, Berlin, r898.

${ }^{3}$ Mense, Arch. f. Sch. u. Trop. Hyg., iii, 4.

${ }^{4}$ Kohlbrugge, Arch. f. Sch. u. Trop. Hyg., iii, 2.

${ }^{5}$ Sambon, Practitioner, London, Mar., 1901.

${ }^{6}$ Sternberg, Malaria and Malarial Diseases, New York, 1884.

${ }^{7}$ Cummings, New Orleans Med. News and Hosp. Gaz., vi, 8I I.

${ }^{8}$ Faget, New Orleans Med. Jour., Oct., 1868.

${ }^{9}$ Osborne, Ibid., r868, 644 .

${ }^{10}$ Osborne, Ibid., xxii, 61 .

${ }^{11}$ Ghent, Richmond and Louisville Med. Jour., v, 27 r.

${ }^{12}$ Cardamatis, LaFièvre Bilieuse Hemoglobinurique, Paris, 1902.

${ }^{13}$ Johnson, Influence of Tropical Climates, etc., New York, 1826.

14 Cleghorn, Diseases of Minorca, London, 1762 .

${ }^{15}$ Hirsch, Handbook of Geog. and Hist. Path., London, $188_{3}$, i.

${ }^{16}$ Below, Berl. klin. Wchnschr., Nov. 15, 1897 .

${ }^{17}$ Rogers, Fevers in the Tropics, London, I908.

${ }^{18}$ Ramazzini, Sur l'Abus du Quinquina, Paris Reprint, 1905.

19 Marchiafava and Bignami, Malaria, New York, I900.

${ }^{20}$ Medizinal Berichte über die Deutschen Schutgebiete, 1903-04.

${ }^{21}$ A. Plehn, Arch. f. Sch. u. Trop. Hyg., vii, 12.

22 Otto, Deutsch. med. Wchnschr., I902, No. 4.

${ }^{23}$ Laveran, Traité du Paludisme, Paris, 1907.

${ }^{24}$ Brault, Janus, Nov. 15, 1903.

${ }^{25}$ Coste, Presse Med., Sept. I 2, I906.

${ }^{26}$ Wellman, Jour. Am. Med. Assn., xlv, 1736.

${ }^{27}$ Reports to the Malaria Committee, 5 th ser., London, I9or.

${ }^{28}$ Plehn, Deutsch. med. Wchnschr., r 90 r, p. 838 .

${ }^{29}$ Manson, Tropical Diseases, London, 1903.

${ }^{30}$ Plehn, Deutsch. med. Wchnschr., 1895, 25-27.

${ }^{31}$ Johnson, Jour. Trop. Med., Dec. 15, Ig00.

${ }^{22}$ Jour. Am. Med. Assn., xlviii, I 95.

${ }^{33}$ Medizinal Berichte über die Deutschen Schutzgebiete, 1904-05, I905-06.

${ }^{34}$ Masterman, Brit. Med. Jour., Feb. Io, 1906.

${ }^{35}$ Hanley, Jour. Trop. Med., I899, p. 85.

${ }^{36}$ Plehn, Die Malaria der afrikanischen Neger Bevölkerung, Jena, I902.

${ }^{37}$ Plehn, Arch. f. Sch. u. Trop. Hyg., iii, 6.

${ }^{38}$ Jour. Am. Med. Assn., xxxviii, I1 1 O.

${ }^{39}$ Doerring, Deutsch. med. Wchnschr., Nov. 14, 1895 .

${ }^{40}$ Reynold, Jour. Trop. Med., Jan., I 899.

41 Ruge, Einführung in das Studium der Malariakrankheiten, Jena, r9o6.

42 Goltman and Krauss, Memphis Lancet, Dec., 1898 .

43 Personal communication. 
${ }^{44}$ Medizinal Berichte über die Deutschen Schutzgebiete, I904-05.

${ }^{45}$ Lewis, N. C. Med. Jour., Mar. 5, I899.

${ }^{46}$ Francez, New Orleans Med. and Surg. Jour., July, 1902.

${ }^{47}$ Minor, Med. News, London, Nov. 24, 1883.

$48 \mathrm{McKay}, \mathrm{Am}$. Pract. and News, June I, 1902.

${ }^{49}$ Tyson, Med. News, May I 2, 1883.

so Gorgas, Ann. Rep. Dept. Sanitat., Isthmian Canal Commis., I907-08.

51 Daniels, Laboratory Studies in Tropical Medicine, Phila., 1903.

52 Cardamatis, Grèce Med., Apr., I900.

${ }^{53}$ Krauss, Memphis Med. Month., Apr., 1902.

54 Deaderick, Memphis Med. Month., Aug., 1907.

${ }^{55}$ Proc. Canal Zone Med. Assn., Apr., I909-Mar., I9ı.

${ }^{56}$ Lovelace, Arch. Intern. Med., June, 1913.

${ }^{67}$ Dufranc, Le Caducee, Sept. 18, 1909.

${ }^{58}$ Schumacher, Arch. f. Sch. u. Trop. Hyg., xv, 672.

${ }^{59}$ Jungels, Ibid., xv, $36 \mathrm{r}$.

${ }_{60}$ Medizinal Berichte über die Deutschen Schutzgebiete, r906-07.

61 Woldert, New York Med. Jour., Sept. 28, r 912.

62 Deaderick, Malaria, Leipzig, 1910, ii, i95.

${ }^{63}$ Christophers and Bentley, Blackwater Fever, Simla, r 908.

64 Cardamatis, Centralbl. f. Bakteriol., lxi, 378 .

${ }^{65}$ Deeks and James, Report on Hemoglobinuric Fever in the Canal Zone, Mount Hope, IgII.

${ }^{66}$ Williamson, Jour. Trop. Med., 1909, No. 15.

${ }^{67}$ Atti della Societa per gli Studi della Malaria, x, Rome, 1909.

${ }^{68}$ Sutton, U. S. Nav. Med. Bull., July, igr I.

${ }^{69}$ Mense, Arch. f. Sch. u. Trop. Hyg., June, I899.

${ }^{70}$ Lipari, Il Morgagni, Sept., 1899.

71 Medizinal Berichte über die Deutschen Schutzgebiete, 1905-06.

${ }^{72}$ Scheube, Die Krankheiten der warmen Länder, Jena, I903.

${ }^{73}$ Burns, Jour. Am. Med. Assn., Nov. I7, 1900.

74 Tomaselli, La Intossicazione Chinica, Catania, 1897.

${ }^{75}$ Sutherland, Memphis Med. Month., July, I905.

${ }^{76}$ Foustanos, Grèce Med., Apr., 1900.

${ }^{77}$ Crosse, Brit. Med. Jour., Oct. 8, I898.

${ }^{78}$ Banks, Jour. Trop. Med., Dec. 15, I900.

${ }^{79}$ Arb. a. d. k. Gsndhtsamte., xiv, 2.

${ }^{80}$ Plehn, Tropenhygiene, Jena, rgo6.

${ }^{81}$ Burot and Legrand, Maladie du Soldat aux Pays Chauds, Paris, I897.

${ }^{62}$ Mannaberg, Malarial Diseases, Phila., I905.

${ }^{83}$ Vèdy, La Fièvre Bilieuse Hemoglobinurique, Brussels, 1907.

${ }^{84}$ McElroy, Memphis Med. Month., May-June, 1905.

${ }^{85}$ Brem, Jour. Am. Med. Assn., Dec. 8-15, 1906.

${ }^{86}$ Ziemann, Mense's Handbuch der Tropenkrankheiten, Leipsic, 1906.

${ }^{87}$ Howard, Jour. Trop. Med., Mar. I, I907.

${ }^{88}$ Bassett-Smith, Jour. Trop. Med., I907, x, 69

89 .Hughes, Jour. Trop. Med., June, 1899.

${ }^{90}$ Manson, Brit. Med. Jour., May I6, 1903.

${ }^{91}$ Parker, Brit. Med. Jour., Sept. 9, I899. 
92 Mowbray, Lancet, London, Aug. 26, I905.

${ }^{93}$ Schlayer, Deutsch. med. Wchnschr., July Io, rgo2.

${ }^{94}$ Kleine, Brit. Med. Jour., Sept. I4, Igor.

${ }^{25}$ Koch, Jour. Trop. Med., July I5, I899.

${ }^{96}$ Burot and Legrand, Thérapeutique du Paludisme, Paris, 1897.

${ }^{97}$ LeDantec, Pathologie Exotique, Paris, I905.

${ }^{98}$ Kelsch and Kiener, Maladies des Pays Chauds, Paris, r889.

${ }^{99}$ Dryepondt and Vancampenhout, Jour. de med. de Bruxelles, r899, 9.

100 Bertrand, Am. Soc. Med.-Chi. d'Anvers, Nov.-Dec., 1899.

101 Mould, Brit. Med. Jour., Sept. 9, 1899.

102 Haig, Lancet, London, Apr. 2, I898.

${ }^{103}$ Deaderick, Jour. Am. Med. Assn., June I, 1907.

104 Mense, Arch. f. Sch. u. Trop. Hyg., iii, 2.

105 Plehn, Ibid., iii, 4.

${ }^{106}$ Cardamatis, Grèce Med., Apr., I900.

${ }^{107}$ Crosse, Lancet, London, Jan. 6, I900.

108 Krauss, Memphis Med. Month., Apr., I902.

109 Thin, Brit. Med. Jour., Sept. 1, 1900.

${ }^{110}$ Curry, Jour. Am. Med. Assn., xxxviii, Ir 30.

111 Ketchen, Brit. Med. Jour., Nov. Io, I906.

${ }^{112}$ Ruge, Deutsch. med. Wchnschr., July Iо, 1902.

${ }_{113}$ Hartsock, New York Med. Jour., Sept. 13, 1902.

${ }^{114}$ Broden, Trav. du Lab. Med. de Leopoldville, Brussels, Ig06.

${ }^{115}$ Cardamatis, Progrès. Med., Nos. 37-40, 1902.

116 Virchow's Jahresbericht, i, I907.

117 Grattan, Jour. Roy. Army Med. Corps, Lond., 1907, ix, 237.

${ }^{118} \mathrm{Kül}$, Arch. f. Sch. u. Trop. Hyg., xii, 242.

119 Atti della Societa Per gli Studi della Malaria, Rome, 1907.

${ }^{120}$ Stephens and Christophers, Practical Study of Malaria, London, 1904.

${ }^{121}$ Orme, Jour. Trop. Med., Feb. I, Ig08.

122 Koch, Deutsch. med. Wchnschr., Feb. I, I900.

${ }^{123}$ French, New York Med. Jour., May 23, 1896.

${ }_{124}$ Ewing, Jour. Exper. Med., Feb. 5, I902.

${ }^{125}$ Koch, Arch. f. Sch. u. Trop. Hyg., June, I899.

${ }^{126}$ Ellenbeck-Hilden, Beobachtungen über Malaria, Berlin, 1905 .

${ }^{127}$ Legrain, Introd. a l'Étude des Fièvres des Pays Chauds, Paris, I899.

${ }^{128}$ Grall, Pathologie Exotique, Paris, I900.

${ }^{129}$ Rossoni, Il Morgagni, Jan., I899.

${ }^{130}$ Seal, Jour. Trop. Med., Feb. 15, 1899.

${ }^{131}$ Stalkarrt, Brit. Med. Jour., Sept. 9, 1899.

132 Hopkins, Dublin Jour. Med. Sc., June, I903.

${ }^{133}$ Rankin, Brit. Med. Jour., Sept. I, I900.

${ }^{134}$ Moffatt, Brit. Med. Jour., Jan. 25, 1902.

${ }^{133}$ McElroy, Jour. Am. Med. Assn., xli, 605.

136 Dubose, Ibid., Mar. I r, 1899.

${ }^{137}$ Hearsey, Brit. Med. Jour., Jan. 26, I901.

${ }^{138}$ Shropshire, Jour. Am. Med. Assn., xli, 600 .

${ }^{139}$ Murri, Deutsch. med. Wchnschr., Feb. 20-27, I896.

${ }^{140}$ Marsden, Brit. Med. Jour., Sept. I, I900. 
141 Boxer, Brit. Med. Jour., May 7, 1904.

142 McKay, Glasgow Med. Jour., Mar., I908.

${ }^{143}$ Sambon, Brit. Med. Jour., Sept. 24, 1908.

144 Craig, The Malarial Fevers, New York, I 909.

145 Transactions of the Epidemological Society, i 892-93.

146 Deaderick, Practical Study of Malaria, Phila., ı

${ }^{147}$ Yersin, Compte Rend. Soc. Biol., Paris, I895, ii, 447.

148 Breaudat, Arch. Méd. Nav., I896, 457.

${ }^{149}$ Collett, Lancet, London, Dec. 28 , 1904.

${ }^{150}$ Sternberg, Malaria and Malarial Diseases, New York, I 884.

${ }^{151}$ Reports of Dept. of Sanitat., Isthmian Canal Commission, 1907-I913.

152 Hearsey, Jour. Trop. Med., Sept. I, 1909.

163 Baker, Ibid.

${ }^{154}$ Langley, Ibid., Apr. I, I909.

${ }^{165}$ Will, Ibid., May I 5, I909.

${ }^{156}$ Fagan, Ibid., Nov. I, I909.

${ }^{167}$ Cardamatis, Bull. Soc. de Path. Exotique, iv, 303.

158 Woldert, Texas State Jour. Med., Dec., 1913.

${ }^{169}$ Alexandropoulos, Progrès. Med., I909, p. 17.

${ }^{160}$ Schellong, Die Malariakrankheiten, Berlin, 1890.

${ }^{161}$ Marchous, Caducèe, Aug. 20, 1904.

162 Giemsa and Schaumann, Studien über Chinin, Leipsic, 1907.

${ }^{163}$ Williams, Jour. Trop. Med., Dec. I 5, I900.

${ }^{164}$ Mackie, Lancet, London, Dec. 6, I898.

${ }^{165}$ Christophers and Bentley, Indian Med. Gaz., Mar., 1908.

${ }^{166}$ Skelton, Jour. Roy. Army Med. Corps, June, 1908.

${ }_{167}$ Medizinal Berichte über die Deutschen Schutzgebiete, 1903-I908.

${ }^{168}$ Jenkins, Trans. Ark. Med. Soc., I904, 203.

169 Michel, New Orleans Jour. Med., 1869.

${ }^{170}$ Gorgas, Jour. Am. Med. Assn., xlvi, 1417.

${ }^{171}$ Malone, Trans. Ark. Med. Soc., r880, 74.

172 Coste, Presse Méd., Sept. I2, I906.

${ }^{173}$ Steggall, Med. Rec., New York, lvi, 259.

${ }^{174}$ Woldert, New York Med. Jour., Feb. 23, I895.

${ }^{176}$ Austin, Brit. Med. Jour., Feb. Io, I900.

${ }^{178}$ Cardamatis, Fièvre Bilieuse Hémoglobinurique, Syra, I9or.

177 McDaniel, Med. News, Nov. 24, 1883.

${ }^{178}$ Henry, Arch. d. Méd. Nav., May, 1898.

${ }^{179}$ Kohlstock, Deutsch. med. Wchnschr., Nov. I4, I895.

${ }^{180}$ Ensor, Arch. f. Schf. u. Trop. Hyg., xii, 108.

181 Prentice, Brit. Med. Jour., Sept. 24, I898.

182 Osborne, New Orleans Med. and Surg. Jour., xxii, 6r.

${ }^{183}$ Dempwolff, Arch. f. Sch. u. Trop. Hyg., June, I 899.

184 Hare, Therap. Gaz., July I5, 1892.

${ }^{186}$ Forde, Jour. Trop. Med., Feb. I, 1908.

186 Prout, Brit. Med. Jour., Nov. 9, 1907.

187 Jacobs, New York Med. Jour., Oct. 12, 1907.

188 DeCruz, Indian Med. Gaz., Nov., 1907.

${ }^{189}$ DeBlasi, Gazz. d. Osp., Apr. 26, rgo3. 
${ }^{190}$ Orme, Jour. Trop. Med., xi, 38.

191 Thompstone, Ibid., xi, r4.

192 Thayer, Lectures on the Malarial Fevers, New York, I90I.

${ }^{193}$ Quennec, Arch. f. Sch. u. Trop. Hyg., iii, 2.

194 Vincent, Compte Rend. Soc. Biol., Dec. $15,1905$.

${ }^{195}$ Polli, Brit. Med. Jour., Nov. 16, I867.

196 Beare, Lancet, London, Feb. I, 1902.

197 Werner, Deutsch. med. Wchnschr., 1902, 42.

${ }_{198}$ Werner, Die Nieren beim Schwarzwasserfieber, Leipsic, 1907.

${ }^{199} \mathrm{Külz}$, Arch. f. Sch. u. Trop. Hyg., xi, 508.

${ }^{200}$ Jour. Am. Med. Assn., June 24, 1905.

${ }^{201}$ Lambinet, Bull. de l'Acad. Roy. de. Belg., July 25, 1903.

${ }^{202}$ Report of the Porto Rico Anemia Commission, San Juan, IgO4.

${ }^{203}$ Stiles, Prevalence and Geographic Distribution of Hook-worm Disease in the U. S., Wash., I 903 .

${ }^{204}$ Stiles, Hook-worm Disease, Public Health Bull. No. 32, Wash., rgro.

${ }^{205}$ Sandwith, Medical Diseases of Egypt, London, r905.

${ }^{206}$ Report on Anemia in Porto Rico, San Juan, 1907.

${ }^{207}$ Ashford and Igaravidez, Uncinariasis in Porto Rico, Sen. Doc. 808, Wash., Igri.

${ }^{203}$ Preliminary Report, Porto Rico Anemia Commission, San Juan, I905.

${ }^{209}$ Dock and Bass, Hook-worm Disease, St. Louis, 1910.

${ }^{210}$ Turner, Brit. Med. Jour., Oct. 31, 1908.

${ }^{211}$ Mosler and Peiper, Thierische Parasiten, Vienna, 1894.

212 Turner, Jour. Trop. Med., Feb. I 5, 1910.

${ }^{213}$ Stiles, Public Health Report, Jan. 3, I913.

${ }^{214}$ Stiles and Garrison, A Statistical Study of the Prevalence of Intestinal Worms in Man, Bull. No. 28, Hyg. Lab., U. S. Pub. Health and Mar. Hosp. Serv., Wash., I906.

${ }^{215}$ Schloss, Am. Jour. Med. Sc., May, r9ro.

${ }^{216}$ Stiles, Osler's Modern Medicine, vol. i, Phila., 1907.

${ }^{217}$ Spooner, Am. Jour. Med. Sc., lxv, p. I36.

${ }^{218}$ Moore, Med. News, Feb. 6, I904.

${ }^{219}$ Stiles, Illustrated Key of the Cestode Parasites of Man, Bull. No. 25, Hyg. Lab., U. S. Pub. Health and Mar. Hosp. Serv., Wash., 1906.

${ }^{220}$ Stiles, Prevalence and Geographic Distribution of Hook-worm Disease in the U. S., Bull. No. 1o, Hyg. Lab., U. S. Pub. Health and Mar. Hosp. Serv., Wash., I903.

${ }^{221}$ Cited by Stiles, N. Y. Med. Jour., Nov. 7, 1903.

${ }^{222}$ Hallock, Jour. Am. Med. Assn., xlii, 89 r.

${ }^{223}$ Deaderick, Jour. Am. Med. Assn., xlvii, 2087.

${ }^{224}$ Lambert, Med. Rec., lxx, 928.

${ }^{225}$ Deaderick, Jour. Trop. Med., Mar. I, 1907.

${ }_{226}$ Deaderick, Internat. Clin., iv, 19.

${ }^{227}$ Schloss, Arch. Pediat., Feb., r 910.

${ }^{228}$ Foster, Jour. Am. Med. Assn., Sept. I, 1906.

${ }^{229}$ Ransom, An Account of the Tapeworms of the Genus Hymenolepis Parasitic in Man, Bull. No. 18, Hyg. Lab., U. S. Pub. Health and Mar. Hosp. Serv., Wash., I904. 
${ }^{230}$ Sihloss, Am. Jour. Med. Sc., Mar., 19 ro.

${ }^{231}$ Nickerson, Unpublished mss.

${ }^{232}$ Nickerson, Science, Feb., I9I I.

${ }^{233}$ Strong, Johns Hopkins Hospital Reports, I 9or, x, 9r.

${ }^{234}$ Thayer, Jour. Exp. Med., Nov. 29, I90I.

${ }^{235}$ Brush, South. Med. Jour., Oct., I908.

${ }^{236}$ Personal communication, Dr. C. W. Garrison.

${ }^{237}$ Simon, Jour. Am. Med. Assn., Nov. 6, I909.

${ }^{238}$ Fülleborn and Torgau, Arch. f. Sch. u. Trop. Hyg., xv, 569.

${ }^{239}$ Miller, Jahrb. f. Kinderh., xxxvi, 39.

${ }^{240}$ Goldschmidt, München. med. Wchnschr., lvii, r99ז.

${ }^{241}$ Editorial, Jour. Am. Med. Assn., lviii, I 377.

${ }^{242}$ Raines, Memphis Med. Month., Mar., I 904.

${ }^{243} \mathrm{Ziemssen}$, Cyclopedia of the Practice of Medicine, vol. vii.

${ }^{244}$ Metchnikoff, Bull. de l'Acad. de Méd., Paris, I90I, pp. 30I-309.

${ }^{245} \mathrm{Bel}$ and Couret, Jour. Infect. Dis., Oct., rgro.

${ }^{246}$ Grey, St. Louis Med. Rev., Apr. 27, 1907.

${ }^{247}$ Sistrunk, Jour. Am. Med. Assn., lvii, I 507.

${ }^{248}$ Reports of the Rockefeller Sanitary Commission, I9I I, I9I 2 and I9I 3.

${ }^{249}$ Strong, Bureau of Government Laboratories, Bull. No. 26.

${ }^{250}$ Schlesinger, Wien. klin. Wchnschr., Jan. 2, 1902.

251 Deaderick, New Orleans Med. and Surg. Jour., Oct., I908, and one unpublished case.

252 Wirsung, Ztschr. f. Klin. Med., lx, I-2.

${ }^{253}$ Finlayson, Glasgow Med. Jour., Mar., I 889 .

${ }^{254}$ Nicholson, Jour. Am. Med. Assn., liv, I687.

${ }^{255}$ McCampbell and Corper, Jour. Am. Med. Assn., liii, 1 160.

${ }^{256}$ Stitt, Am. Jour. Trop. Dis., i, I69.

${ }^{257}$ Kohlheim, Jour. Am. Med. Assn., lvi, 1503.

${ }^{258}$ Personal communication, Dr. Ferrell.

${ }^{259}$ Personal communication, Dr. Garrison.

${ }^{260}$ Hrdlicka, Physiological and Medical Observations among the Indians of the S. W. United States and Northern Mexico, Wash., I908.

${ }^{261}$ Ashford and King, Am. Med., Sept. 5-1 2, 1903.

${ }^{262}$ Preti, München. med. Wchnschr., lv, 436.

${ }^{263}$ Calmette and Breton, L'Ankylostomiase, Paris, I905.

${ }^{264}$ Whipple, Jour. Exper. Med., Mar., I909.

${ }^{265}$ Castellani, Jour. Trop. Med., Sept. I, I 9 Iо.

${ }^{266}$ Yoshida, Arch. f. Sch. u. Trop. Hyg., xii, 696.

${ }^{267}$ Stiles, South. Med. Jour., Apr., I912.

${ }^{268}$ Greene, South. Med. Jour., Apr., I9I I.

${ }^{269}$ MacDonald, Jour. Trop. Med., Jan. I 5, I 908.

${ }^{270}$ Calhoun, Jour. Am. Med. Assn., lix, ro75.

${ }^{271}$ Jervey, Jour. Am. Med. Assn., lxiii, I 5 I.

${ }^{272}$ Lumsden, Roberts and Stiles, Pub. Health Reports, Nov. II, I9IO.

${ }^{273}$ Ashford, Trans. XVth International Congress on Hygiene and Demography, Wash., I9I2.

${ }^{274}$ Manson, Lectures on Tropical Diseases, Chicago, 1905.

${ }^{275}$ Bozzolo, Gior. Internaz. del. Sc. Med., 1879. 
276 Stiles and Boatwright, Public Health Reports, July I8, I913.

277 Bozzolo, Jour. Am. Med. Assn., lviii, 1745.

${ }^{278}$ Stiles and Leonard, Public Health Reports, Jan. I7, I913.

${ }^{279}$ Bentley, Indian Med. Gaz., xxix, No. 4.

${ }^{280}$ Babcock, Am. Jour. Insan., July, I9I 2.

281 Harris, Am. Med., July I9, 1902.

282 Searcy, Jour. Am. Med. Assn., xlix, 37.

${ }^{283}$ Merrill, Jour. Am. Med. Assn., xlix, 940.

284 Babcock, Jour. S. C. Med. Assn., Feb., I908.

${ }^{285}$ Bellamy, Jour. Am. Med. Assn., li, 397.

${ }^{286}$ Trans. Nat. Conference on Pellagra, rgog.

287 Williamson, Jour. Am. Med. Assn., liii, 717.

${ }^{288}$ Hewitt, Jour. Am. Med. Assn., liii, 1085.

${ }^{289}$ Pollock, Jour. Am. Med. Assn., liii, 1087.

290 Albright, South. Med. Jour., Mar., 1912.

${ }^{291}$ Trans. Nat. Conference on Pellagra, Igr 2.

${ }^{292}$ Grimm, Public Health Reports, Mar. 7-14, 1913.

293 Walker, Jour. Am. Med. Assn., July 3, rgog.

294 Tucker, Jour. Am. Med. Assn., Jan. 28, Igrir.

${ }^{295}$ First Prog. Rept., Thompson-McFadden Pell. Com., 1913.

${ }^{296}$ Sambon, Jour. Trop. Med., xiii, $271-319$

297 Wood, A Treatise on Pellagra, New York, IgI 2.

${ }^{298}$ Siler, Garrison and MacNeal, Jour. Am. Med. Assn., Sept. 26, 1914.

${ }^{299}$ Albright, South. Med. Jour., Mar., I9I 2.

${ }^{300}$ Roberts, Pellagra, St. Louis, Igr 2.

301 Wolff, South. Med. Jour., Mar., Igr 2.

${ }^{302}$ Clark, Am. Jour. Trop. Dis., Dec., I914.

${ }^{303}$ Beall, Jour. Am. Med. Assn., Nov. I8, I9ri.

${ }^{304}$ Lavinder, Public Health Reports, July 25, 19 I3.

${ }^{305}$ Niles, Pellagra, Phila., I9I 2.

${ }^{306}$ Lowery, Med. Rec., New York, Aug. 29, 1914.

${ }^{307}$ Haase, Jour. Am. Med. Assn., July 6, I9 2.

${ }^{308}$ Marie, Pellagra, Columbia, S. C., rgro.

${ }^{309} \mathrm{De}$ Giaxa, Annali d'Igiene Sper., Roma, 1892 and 1903.

${ }^{310}$ Neusser, Wien. med. Presse, 1887.

${ }^{311}$ Public Health Reports, Feb. 24, I9ri.

${ }^{312}$ Cini, Rivista Sper. di Freniat., etc., 1902.

${ }^{313}$ Tizzoni, Estrat to del Boletino del Minist. di Agricolt., Indust. e. Comm., Rome, r 909 .

${ }^{314}$ Mizell, The Journal Record of Medicine of Atlanta, I9ir.

${ }^{315}$ Lavinder, Pellagra, A Précis, Public Health Bulletin, 48.

${ }^{316}$ Goldberger, Public Health Reports, Sept. II, I9I4.

${ }^{317}$ Alessandrini and Scala, Ed. Jour. Am. Med. Assn., Sept. 5, 1914.

${ }^{318}$ Sambon, Cin. Lancet-Clinic, Oct. 25, r913.

${ }^{319}$ Krause, South. Med. Jour., March, I91 2.

${ }^{320}$ Page, Ibid., Feb., r915.

${ }^{321}$ Sanders, Ibid., March, rgr 5.

${ }^{322}$ Sambon, Jour. Trop. Med. and Hygiene, Oct. 15, 1910.

${ }^{323}$ Anderson and Goldberger, Public Health Reports, June 30, IgrI. 
${ }^{324}$ Singer, MacNeal and Rooks, Report of Pellagra Commission, State of Illinois.

${ }^{325}$ Harris, Jour. Am. Med. Assn., June 21, I9I3.

${ }^{326}$ Lavinder, Francis, Grimm and Lorenz, Ibid., Sept. 26, i9i4.

${ }^{327}$ Sandwith, The Medical Diseases of Egypt, London, 1905.

${ }^{328}$ Jelks, Jour. Am. Med. Assn., Apr. 30, 19ro.

${ }^{329}$ Alessandrini, Policlinico, May i 5, June 26, 19 го.

${ }^{330}$ Jelks, South. Med. Jour., March, I9 2.

${ }^{331}$ Singer and Pollock, Arch. Int. Med., June, 1913.

${ }^{332}$ Fox, Medical Record, Feb. 5, I 9 ro.

${ }^{333}$ Singer, Second Progress Report Thompson-McFadden Pellagra Commission.

${ }^{334}$ Gregor, Jahrb. f. Psychiat. u. Neurol., 1907, xxviii. Trans. by Alleman,

Alienist and Neurologist, I9I I, xxxii, No. 4.

${ }^{335}$ Hillman, Am. Jour. Med. Sci., Apr., 1913.

${ }^{336}$ Second Progress Report, Thompson-McFadden Pellagra Commission.

${ }^{337}$ Thompson, Arch. Int. Med., May, x9r3, p. 5 I 2.

${ }^{338}$ Deutsch. med. Wchnschr., xl, No. 32.

${ }^{339}$ Bass, Jour. Am. Med. Assn., Oct. 9, 1909.

${ }^{340}$ Bass, Ibid., Nov. I3, I909.

${ }^{341}$ Carletti, Gazz. d. Osp., June 8, igr I.

${ }^{342}$ Boveri, La Presse Méd., Paris, April ro, 1912.

${ }^{343}$ Seventieth Ann. Report, Ga. State Sanitarium, I9I3.

${ }^{344}$ Arch. Int. Med., Aug. and Sept., I 121.

${ }^{345}$ Albright, South. Med. Jour., Mar., I9I 2.

${ }^{346}$ Sambon, Jour. Trop. Med. and Hygiene, Oct. I, I9ro.

${ }^{347}$ Menage, South. Med. Jour., Mar., I9I 2.

${ }^{848}$ Lavinder, Ibid.

${ }^{349}$ Bass, Jour. Am. Med. Assn., I9I I, lvii, 1534.

${ }^{850}$ Bass and Johns, Am. Jour. Trop. Dis., i, 546, Feb., I914.

${ }^{851}$ Lavinder, Jour. Am. Med. Assn., lx, 42, I9I 3.

${ }^{352}$ Ziemann, Centralbl. f. Bakteriol., lxvii, 482, I9 3 .

${ }^{353}$ Thompson and Thompson, Ann. Trop. Med. and Parasit., vii, I 53.

${ }^{354}$ Lavinder, Pub. Health Rep., Sept. I0, I909.

${ }^{355}$ Wollenberg, Ibid., July 23, I909.

${ }^{356}$ Arch. Int. Med., Aug. and Sept., r 9 I 2.

${ }^{357}$ Searcy, Jour. Am. Med. Assn., July 6, 1907.

${ }^{358}$ Lavinder, Pub. Health Rep., July 25, 1913.

${ }^{359}$ Beall, Proc. XVIIth International Cong. of Med., London, 1913.

${ }^{360}$ Sambon, Jour. Trop. Med. and Hygiene, Sept. I 5, 19 Io.

${ }^{361}$ Goldberger, Public Health Reports, Oct. 23, 1914.

${ }^{362}$ Bass, South. Med. Jour., Aug., I9I I.

${ }^{363}$ Martin, Memphis Med. Monthly, Dec., rgro.

364 Nice, McLester and Torrence, Jour. Am. Med. Assn., Mar. 25, I9 I r.

${ }^{365}$ Cole and Winthrop, Ibid., June 17 , I9I r.

${ }^{366} \mathrm{King}$ and Crowell, Ibid., Nov. I8, I91 r.

${ }^{367}$ Cranston, Ibid., May 18, I91 2.

${ }^{368}$ Martin, Pan-American Surg. and Med. Jour., June, I9I4.

${ }^{369}$ Cole and Winthrop, Jour. Am. Med. Assn., Apr. 23, I910.

${ }^{370}$ Cole, Ibid., Feb. 20, r909.

${ }^{371}$ Cole and Winthrop, Ibid., Feb. 25, r9Ir. 


\section{ENDEMIC DISEASES OF THE SOUTHERN STATES}

${ }^{372}$ Castellani and Chalmers, Manual of Tropical Medicine, New York, I913.

${ }^{373}$ Dyer, Texas State Jour. of Med., July, 1914.

${ }^{374}$ Leroy, South. Med. Jour., Mar., 1912.

${ }^{375}$ Palmer and Secor, Jour. Am. Med. Assn., May 8, 1915.

${ }^{376}$ Lambl, Beobachtungen und Studien der path. Anatomie u. Histologie Prag., I860.

${ }^{377}$ Lewis and Cunningham, Ann. Rep. Sanit. Commiss. Govt. of India, Calcutta, i 870 .

${ }^{378}$ Loesch, Arch. f. Path. Anat., 1875.

${ }^{379}$ Grassi, Gaz. med. Ital., Lombardia, I879, p. 445; Ibid., I882, xxiv, p. 135.

${ }^{380}$ Leuckart, Die Parasiten des Menchen, Leipzig, I 879.

${ }^{381}$ Sonsino, Davidson's Hygiene and Diseases of Warm Countries, Edinb., 1893.

382 Perroncito, I. Parassiti dell Uomo e degli Animali Utili, Milan, 1882.

${ }^{383}$ Koch. Arb. a. d. k. Gesundh., I 883.

${ }^{384}$ Kartulis, Arch. f. Path. Anat., I885; Ibid., 1886; Ibid., 1889; Centralbl. für Bakt., I89I; Ztschr. f. Hyg., I89I; Centralbl. f. Bakt., 1904. In Kolle u. Wassermann's Handbuch der Pathogenen Microorganismen, Jena, 1907.

${ }^{385}$ Hlava, Centralb. f. Bakt., I88\%.

${ }^{386}$ Osler, Bull. Johns Hopkins Hosp., 1890.

${ }^{387}$ Musser, Univ. Med. Mag., I89o.

${ }^{388}$ Stengel, Med. News, I 890.

${ }^{389}$ Dock, Texas Med. Jour., 1891 .

${ }^{390}$ Councilman and Lafleur, Johns Hopkins Hospital Reports, 1891.

${ }^{391}$ Maggiora, Centralbl. f. Bakt., I 893 .

${ }^{392}$ Gasser, Arch. de méd. expér. et d'anat. path., I 895.

${ }^{393}$ Quincke and Roos, Berl. klin. Wchnschr., 1893.

${ }^{394}$ Kruse and Pasquale, Ztschr. f. Hyg., 1893.

${ }^{395}$ Craig, Am. Med., I905.

${ }^{396}$ Saundby and Miller, Brit. Med. Jour., I 909.

${ }^{397}$ Kartullis, in Kolle u. Wassermann's Handbuch der Pathologenen Microorganismen, Jena, 1907.

${ }^{398}$ Viereck, Arch. f. Sch.-u.-Trop.-Hyg., 1907.

${ }^{399}$ Jagers, Berl. klin. Wchnschr., I90I.

${ }^{400}$ Walker, Philippine Journal of Science, 1913.

${ }^{401}$ Strong, in Osler's Modern Medicine, Philadelphia and New York, 1907.

102 Brown, Amebic Dysentery, New York, I9II.

${ }^{403}$ Musgrave, Jour. Am. Med. Assn., Sept. 16, 1905.

${ }^{404}$ De Buys, Jour. Am. Med. Assn., Nov. 21, I9I4.

${ }^{405}$ Allen, Jour. Am. Med. Assn., Nov. 6, I909.

${ }^{406}$ Musgrave and Clegg, Reports of the Biological Laboratories, Manila, I904.

${ }^{407}$ Lesage, Ann. de l'Inst. Pasteur, I905.

${ }^{408}$ Whitmore, Am. Jour. Trop. Dis. and Prev. Med., Sept., I913.

${ }^{409}$ Craig, Jour. Med. Research, April, J9I 2.

${ }^{110}$ Schaudinn, Arb. a. d. k. Gsndhtsamte., 1903.

${ }^{111}$ Craig, Jour. Infec. Dis., June 4, 1908.

112 Walker and Sellards, The Philippine Jour. Sc., Aug., I9r3.

${ }^{413}$ Sellards and Baetjer, Bull. Johns Hopkins Hosp., Nov., I914.

${ }^{414}$ Cassagrandi and Barbagello, Bull. Acad. Gioenia, Sci. Nat., Catania, 1895. 
${ }^{415}$ Craig, Am. Jour. Med. Sci., July, 1904.

${ }^{418}$ Musgrave, Philippine Journal of Science, July, I9ı.

${ }^{417}$ Musgrave, Jour. Am. Med. Assn., Sept. i6, I905.

${ }^{418}$ Amberg, Bull. Johns Hopkins Hosp., I9or.

${ }^{419}$ Manson, Tropical Diseases, New York, Igog.

${ }^{420}$ Deeks, Ann. Trop. Med. and Parasit., July, I9r4.

${ }^{421}$ Craig, South. Med. Jour., June, I913.

${ }^{422}$ Mallory and Wright, Pathologic Technic, Philadelphia and London, r 908.

${ }^{423}$ Duncan, Brit. Med. Jour., I902.

${ }^{424}$ Strong, in Osler's Modern Medicine, vol. i, Philadelphia and New York, 1907.

${ }^{425}$ Herrick, Medical Record, Nov. 13, I909.

${ }^{426}$ U. S. Dispensatory, 1907.

${ }^{427}$ Walsh, Indian Med. Gaz., Sept., I891.

${ }^{428}$ Vedder, Military Surgeon, 1900, Bull. Manila Med. Soc., March, I9I I.

${ }^{429}$ Rogers, Brit. Med. Jour., June 22, I912, Indian Med. Gaz., Nov., 1912. Therapeutic Gazette, Dec. 15, I9I 2.

${ }^{430}$ Merck's Annual, i914.

${ }^{431}$ Cantlie, Internat. Cli., vol. iv.

${ }^{432}$ Leidy, The Fresh Water Rhizopods of North America, Govt. Printing Office, Washington.

${ }^{433}$ Sutherland and Mitra, Indian Jour. Med. Research, Apr., r91 5.

${ }^{434}$ Nott, New Orleans Med. and Surg. Jour., iv, ${ }_{56} 6_{3}, 1848$.

${ }^{435}$ King, Popular Science Monthly, Sept., I883.

${ }^{436}$ Moore, Jour. Trop. Med., Mar. 15, 1902.

${ }^{437}$ Curry, Boston Med. and Surg. Jour., Nov. 23 , I899.

${ }^{438}$ Charity Hospital Reports, New Orleans, I906, I907.

${ }^{439}$ Thayer and Hewetson, The Malarial Fevers of Baltimore, Baltimore, I 895.

440 Kendall, Jour. Am. Med. Assn., xlvi, I 270.

${ }^{441}$ Gray and Low, Brit. Med. Jour., Jan. 25, I902.

${ }^{442}$ Gorgas, Ann. Rept. Dept. Sanitat., Isthmian Canal Commis., 1907-08

${ }^{443}$ Koch, Deutsch. med. Wchnschr., Feb. 2, I899.

${ }^{444}$ Koch, Ibid., Sept. 14, I 899.

${ }^{445}$ Cardamatis and Diamessis, Grecè Méd., Nov. I-1 5, 1906.

${ }^{446}$ Mollow, Malaria, i, 75.

${ }^{447}$ Atti della Soc. per gli Studi della malaria, Rome, I90I-08.

${ }^{448}$ Wright, The Malarial Fevers of British Malaya, London, Igo2.

${ }^{449}$ Craig, Jour. Trop. Med., June I 5, I904.

${ }^{450}$ Hope, Ibid.

151 Williamson, Brit. Med. Jour., Sept. I4, rgor.

${ }^{452}$ Koch, Deutsch. med. Wchnschr., Apr. 26, rgoo.

${ }_{453}$ Chamberlain, Jour. Am. Med. Assn., xlvi, 304.

454 Buchanan, Mal. Fever and Mal. Parasites in India, Calcutta, 1903.

${ }^{455}$ Thiroux et d'Antreville, Le Paludisme au Sénégal, Paris, 1908.

${ }^{456}$ Sternberg, Malaria and Malarial Diseases, New York, 1884.

${ }^{457}$ Smith, Brit. Med. Jour., Dec. I7, 1898 .

${ }^{458}$ Celli, La Malaria Secondo le nuove Richerche, Rome.

459 Davidson, Hygiene and Diseases of Warm Climates, Edinb., I893.

${ }^{460}$ Sims and Warwick, Jour. Am. Med. Assn., li, gr6.

${ }^{461}$ Atti della Soc. per gli Studi della Malaria, Rome, 1906. 
462 Ross, Brit. Med. Jour., Sept. 14, 1901.

${ }^{463}$ Report to the Malaria Committee, 6th ${ }^{\circ}$ Series, London, 1902.

${ }^{464}$ Russell, Malaria and Injuries of the Spleen, Calcutta, 1880.

${ }^{465}$ Felkin, Edinburgh Med. Jour., June, 1889.

${ }^{486}$ Watson, Practice of Physic, Phila., I 854.

${ }^{467}$ Thayer, Lectures on the Malarial Fevers, New York, 1901.

${ }^{468}$ Pezopoulos and Cardamatis, Arch. de Méd. des Enfants, Jan., I907.

${ }^{469}$ Winslow, Boston Med. and Surg. Jour., May 27, 1897.

${ }^{470}$ Peters, Bull. Johns Hopkins Hosp., June, 1902.

471 Moffat, Brit. Med. Jour., May 4, 1907.

472 Crespin, Précis du Paludisme, Paris, 1905.

${ }^{473}$ Hitte, Thèse de Montpelier, I902.

${ }^{474}$ Holt, Diseases of Infancy and Childhood, New York, Igo8.

475 Economous, Bull. de la Soc. d'Obstet., $x, 70,1907$.

476 Bel, Jour. Am. Med. Assn., li, 1993.

477 Jeffries, Med. Rec., New York, lvii, 654.

478 Daniels, Brit. Med. Jour., Jan. 26, I gor.

479 Thompson Yates Laboratory Reports, v, I.

${ }^{430}$ Galli-Vallerio et De Jongh, Manuel pour la Lutte, etc., Paris, I 906.

481 Banks, Philippine Jour. Sc., Dec., 1907.

${ }^{482}$ Austin, Practitioner, London, Mar., rgor.

${ }^{483}$ Smith and Kilbourne, Texas or Southern Cattle Fever, Washington, $x 893$.

484 Giles, The Gnats or Mosquitoes, London, I902.

${ }^{485}$ Pressat, Le Paludisme et les Moustiques, Paris, 1905.

${ }^{486}$ Eysell, Arch. f. Sch. u. Trop. Hyg., xi, 6.

${ }^{487}$ Woldert, Jour. Am. Med. Assn., l, r 249.

488 Mitchel, Mosquito Life, New York, I907.

${ }^{489}$ Stephens, Ann. Trop. Med. and Parasit., i, Apr., r914.

${ }^{490}$ Rowley-Lawson, Jour. Exper. Med., xix, No. 6, 19r4.

${ }^{491}$ Craig, Estivo-autumnal Malaria, New York, r 901.

492 Ewing, Jour. Exper. Med., Mar. 25, I 901.

${ }^{493}$ Mannaberg, Die Malaria Parasiten, Vienna, I 893.

494 Schaudinn, Arb. a. d. k. Gsndtsamte, I9, 2.

${ }^{495}$ Maurer, Centralbl. f. Bakteriol., Nov. 5, 1902.

496 Blüml and Metz, Arch. f. Sch. u. Trop. Hyg., xii, 249.

${ }^{497}$ Craig, Internat. Clin., I 7 th ser., iii.

${ }^{498}$ Ford, Jour. Am. Med. Assn., xlviii, 133.

${ }^{499}$ Rosenau et al, Exp. Stud. in Yellow Fever and Malaria, Wash., I905.

${ }^{600}$ Brown, Jour. Exper. Med., xv., No. 6, I91 2.

${ }^{501}$ Ewing, Am. Jour. Med. Sc., Oct., I gor.

${ }^{602}$ Craig and Nichols, Studies of Syphilis, Wash., 1913.

${ }^{503}$ Boehm, Malaria, vol. i, p. rgr.

${ }^{504}$ Thayer, Am. Jour. Med. Sc., Nov., Dec., 1898.

${ }^{505}$ Anders, Jour. Am. Med. Assn., June 15, 1895.

${ }^{506}$ Atkinson, Am. Jour. Med. Sc., July, 1894.

${ }^{507}$ Wurtz and Thiroux, Diag. et Sém. des Malad. Trop., Paris, 1905.

${ }^{508}$ Cohen, Am. Jour. Med. Sc., cxxxvi, 344 .

${ }^{509}$ Rist and Boudet, Pres. Med., Dec. 4, 1907.

$\$ 10$ Berl. klin. Wchnschr., Aug. 24, 1885 . 
511 Goth, Zeitschr. f. Geb. u. Gynäk., vii, I, I88I.

612 Bonfils, Paludisme et Puerperalité, Paris, I 885.

${ }^{513}$ Williams, A Text-book of Obstetrics, New York, 1903.

514 Winfield, New York Med. Jour., Aug. 2, 1902.

515 Anders, Phila. Hosp. Repts., iv, 1895.

${ }^{516}$ Loeffler, Deutsch. med. Wchnschr., r9or, No. 42.

${ }^{617}$ Henson, Malaria, St. Louis, I9r3.

618 Fornario, Deutsch. med. Wchnschr., Jan. 22, 1903.

619 McElroy, Memphis Med. Month., Nov., I902.

${ }^{620}$ Atti della Soc. per gli Studi della Malaria, Rome, 1902.

621 Plehn, Weiteres über Malaria, etc., Jena, I9or.

${ }^{522}$ Delaney, Brit. Med. Jour., Mar. 28, I903.

${ }^{623}$ Cardamatis, Bull. Soc. Méd. de Gand., Feb., r9or.

${ }^{624}$ Ross, Lancet, London, Nov. 17 , I906.

${ }^{525}$ Ross, Ibid., Sept. 28, I907.

${ }^{625}$ Med. and Surg. History of the War of the Rebellion, iii, Med. Vol.

${ }^{627}$ Hagen, Arch. f. Sch. u. Trop. Hyg., iv, iii.

${ }^{528}$ Jour. Trop. Med., vol. xi.

${ }^{629} \mathrm{Haw}$, Jour. Trop. Med., Oct. 16, I899.

${ }^{630}$ Laveran, Bull. Acad. Méd., lxix, 32.

${ }^{631}$ Erni, Arch. f. Sch. u. Trop. Hyg., June, 1899.

${ }^{532}$ Annual Reports, U. S. P. H. and M. H. S., 1905-07.

${ }^{533}$ Medizinal Berichte über die Deutsch. Schutzgeb 1903-06.

634 Wood, Practical Medicine, Phila., r847.

${ }^{635}$ Atti della Soc. per gli Studi della Malaria, Rome, rgor.

${ }^{636}$ Ibid., 1904 .

${ }^{637}$ Carda matis, Bull. Soc. Méd. Gand., Nov., I900.

${ }^{638}$ Billet, Rev. de Méd., Dec., I902.

639 Maurel, Maladies Paludeennes a la Guyana, Paris, 1883.

${ }^{540}$ Charity Hospital Reports, New Orleans, r906-07.

541 Neer, Jour. Am. Med. Assn., 1, r 89o.

542 Atti della Soc. per gli Studi della Malaria, Rome, Igo8.

${ }^{543}$ Ibid., I904.

${ }^{544}$ Bacelli, Gaz. degli Osp., Feb., I890.

${ }^{545}$ Guttmann and Ehrlich, Berl. klin. Wchnschr., r89r, 39.

${ }^{646}$ Carpenter, Med. Rec., New York, lxx, I65.

547 Goldberger, Public Health Reports, Nov. I2, I9I5. 



\section{INDEX}

Abdominal forms of malaria, 116 Abortion and malaria, I3 I

Abscess of liver, 424, 433, 443 and malaria, I6I

Acute malaria, 97

Age and amebic dysentery, 402 and blackwater fever, 230 and hookworm disease, $45^{2}$ and malaria, 36, 137

and pellagra, 29I

Algid malaria, I I6, I65

Altitude and amebic dysentery, $40 \mathrm{I}$ and blackwater fever, 233

and malaria, 28

and pellagra, 288

Ameba in pellagra, 308, 312

Amebic dysentery, clinical history, 419

diagnosis, 427

etiology, 4 OI

geographic distribution, 398

history, 395

pathology, 4I 3

prognosis, 434

prophylaxis, 436

treatment, 438

Anaphylaxis and blackwater fever, 245

Anemia in malaria, 85

Ankylostomiasis. See Hookworm Disease.

Anopheles crucians, $6 \mathbf{I}$ maculipennis, 60 mosquitoes, 50

Ardent fever, I 5

Ascaris lumbricoides, 514

Autoserotherapy in pellagra, 392

BACTERIA in pellagra, 303

Balantidium coli, .523

Banti's disease and malaria, 164

Bass' method in hookworm disease,
Beta-naphthol, 492

Bilious pernicious malaria, I 18

Biology oi malaria parasites, 66

Blackwater fever, a disease sui generis, 243

and malaria, 235

clinical history, $25^{\circ}$

diagnosis, 262

etiology, 229

geographic distribution, 226

history, 2 I 9

pathology, 247

prognosis, 265

prophylaxis, $27^{\circ}$

treatment, 272

Blood in amebic dysentery, 422

in blackwater fever, 256

in hookworm disease, 468

in malaria, 106, 125, 142, 157

in pellagra, 349

Bone-marrow in malaria, 93,96

Bowels in amebic dysentery, 423 in malaria, 92, 96, 109, 125

in pellagra, 372

Brain in amebic dysentery, 418 in blackwater fever, 249

in malaria, 93

Breeding places of mosquitoes, 50

Cachexia in pellagra, 349

malarial, 126

treatment of malarial, 2 I I

Cancer and malaria, I37

Carriers in hookworm disease, 472 in malaria, 189

Cause of death in blackwater fever, $5^{8}$

Cerebellum and malaria, I 4

Cerebrospinal malaria, 112

Change of residence and blackwater fever, 233

and malaria, 39

Children and malaria, 137 
Choleraic pernicious malaria, 117

Chronic malaria, 119.

$$
\text { treatment, } 2 \text { I I }
$$

Circulatory system and malaria, 123

Civil condition and pellagra, 292

Civilization and malaria, 4o

Climate and hookworm disease, $45^{\mathrm{I}}$

Clinical history of amebic dysentery, 419

of blackwater fever, $25^{\circ}$

of hookworm disease, 462

of malaria, 97

of pellagra, 324

Comatose malaria, 112, 164

Complications of amebic dysentery, 424

of blackwater tever, 259

of hookworm disease, 472

of malaria, 123

of pellagra, 362

Congenital malaria, 42

Contagion of pellagra, 302

Convulsions and malaria, II 5

Corn in pellagra, 295

Cultivation of amebæ, 408 of malaria parasites, 80

DiABETES and malaria, 136

Diagnosis of amebic dysentery, 427 of blackwater fever, 262

of hookworm disease, 473

of malaria, I 40

of pellagra, 369

of pernicious malaria, 164

Diaphoretic pernicious malaria, I I 7

Diarrhea in pellagra, 341

Diet in pellagra, 295, 386

Differential diagnosis of malaria, I6I

Digestive system in hookworm disease, 466

Dirt eating in hookworm disease, 466

Duration of hookworm disease, 465 of pellagra, 36I

Dwarf tapeworm, 503

Dysenteric malaria, I 18

Dysentery and malaria, 125

EAR in malaria, 134

Emetine in dysentery, 44I
Endamœba coli, 406, 43I

hystolytica, 403, 427, 43I

Endemic index and malaria, 37

Endocarditis and malaria, 162

Epidemics of amebic dysentery, 403 of malaria, 4 I

Estivo-autumnal fever, 102 parasites, 72

Etiology of amebic dysentery, 401 of blackwater fever, 229 of hookworm disease, 45I of malaria, 25

of pellagra, 287,295

of pernicious malaria, 85

Experimental blackwater fever, 24I

Exposure and malaria, 40

Eye in hookworm disease, $47 \mathrm{I}$

in malaria, 133

in pellagra, 348

FAMILY predisposition to blackwater fever, 231

tendency to pellagra, 302

Feces in amebic dysentery, 423

in hookworm disease, 467,473

in pellagra, 358

Flagella of malaria parasites, 70

Gametes, 7o, 73

Gangrene and malaria, 135

Gastro-intestinal tract, 313

Generative system in hookworm disease, $47^{\circ}$

Genito-urinary organs and malaria, 130

Geographic distribution of amebic dysentery, 398

of blackwater fever, 226

of hookworm disease, 448,466

of malaria, 21

of pellagra, 285

Goldberger's theory, 299

Ground itch in hookworm disease, 465

Heart in blackwater fever, 249

in hookworm disease, 467

in malaria, $93,96,123$

in pellagra, 323 
Hemoglobinuria, 253

Hepatic abscess in amebic dysentery, 424

Heredity and pellagra, 292

Hibernation of mosquitoes, 64

History of amebic dysentery, 395

of blackwater fever, 219

of hookworm disease, 445

of malaria, 17

of pellagra, 28I

Hookworm disease, clinical history, 462

diagnosis, 473

etiology, 45I

geographic distribution, 448

history, 445

pathology, 460

prognosis, 478

prophylaxis, 480

treatment, 487

Hymenolepis diminuta, 509

nana, 503

Hygiene and pellagra, 293

Hygienic treatment of malaria, 210

IDIOSYNCRASY and blackwater fever, 231

Immunity and amebic dysentery, 402

and malaria, 32

and pellagra, 303

Incubation in malaria, 97

in pellagra, 326

Individual predisposition in malaria, 88

Infectivity in pellagra, $30 \mathrm{r}$

Influenza and malaria, 1 36, I 63

Inoculation experiments in amebic dysentery, 408

of malaria, 45

of pellagra, 308

Insanity in pellagra, 343

Intermediate host in pellagra, 305

Intestinal parasites, 495

and malaria, 125

and pellagra, 362

Intestine in amebic dysentery, 4r 3

in blackwater fever, 249

in hookworm disease, 460
Intestine in pellagra, 315

Inundations and amebic dysentery, 401

and malaria, 30

Ipecac in dysentery, 440

JAUNDICE in blackwater fever, 254

Kidneys in blackwater fever, 248 , 259

in malaria, $92,95, \times 30$

in pellagra, 323

LARVA, anopheles, 54

Latent malaria, 84, I 19

Length of residence and blackwater fever, 232

and malaria, 39

Leucocytes in malaria, I08

Leukemia and malaria, I64

Liver abscess in amebic dysentery, 424

in blackwater fever, 248

in malaria, 91, 94

in pellagra, 323

Lumbricoid worm, 5I4

Lungs in blackwater fever, 249

in malaria, 92, 96

in pellagra, 322

Localizations of parasites in malaria, 87

Macrogametes, 7 I, 75

Maggots, 525

Malaria, cachexia in, 126

clinical history, 97

chronic, II 9

diagnosis, 140

etiology, 25

geographic distribution, 2 I

history, I7

pathologic anatomy, 90

pernicious, II I

prognosis, 166

prophylaxis, $\mathbf{1} 72$

treatment, 197

Malarial mosquitoes, 46, 50, 59

paroxysms, 97

Malignant tertian fever, I03 
Masked malaria, $\mathbf{2} 3$

Mental symptoms in pellagra, 343 , 373

Methylene blue in malaria, $2 I_{4}$

Microgametocytes, $7 \mathrm{r}, 75$

Mode of infection in amebic dysentery, 410

in hookworm disease, 453

in malaria, 42

Moisture and hookworm disease, $45 \mathrm{I}$

Mortality of amebic dysentery, 434 of blackwater fever, 266

of hookworm disease, 474

of malaria, 168

of pellagra, 378

Mosquitoes and malaria, 46 destruction of, 177

Muscular system in hookworm disease, 470

Myiasis intestinalis, $\mathbf{5 2 5}$

NeCATOR americanus, 454

Negro and malaria, 138

Nephritis in blackwater fever, 259 in malaria, 130

Nervous system in hookworm disease, 470

in malaria, $111,112,132$

in pellagra, 342,373

Number of parasites in malaria, 86

Occupation and amebic dysentery, 402

and blackwater fever, 334

and hookworm disease, 452

and malaria, 39, 375

and pellagra, 292

Ova of hookworm, 457

of mosquitoes, $5 \mathrm{I}$

Oxyuris vermicularis, 518

Parasites, frequency, in malaria, I 5 I

of amebic dysentery, 403

of blackwater fever, 236

of hookworm disease, $45^{\circ}$

of malaria, 64,74

of pellagra, 298, 303

Paralysis in malaria, 114,132
Paroxysms in malaria, 97

Parthenogenesis, 77

Pathogenesis of amebic dysentery, 4 I I

of blackwater fever, 235

of hookworm disease, $45^{8}$

of malaria, 82

of pellagra, $3 \mathbf{I I}$

Pathology of amebic dysentery, 413

of blackwater fever, 247

of hookworm disease, 460

of malaria, 90

of pellagra, $3^{1} 3$

Pellagra, clinical history, 324

diagnosis, 369

etiology, 287

geographic distribution, 285

history, 28r

pathology, 3I 3

prognosis, 374

prophylaxis, 380

sine pellagra, $36 \mathrm{I}$

treatment, 385

Periodicity in malaria, 140

Pernicious malaria, II I

diagnosis, 164

etiology, 85

prognosis, 169

treatment, 216

Pin worm, 518

Plasmodium tenue, 65

Pneumonia and malaria, I 16, 125

Pork tapeworm, 501

Prevention of blackwater fever, 270 of hookworm disease, 480

of malaria, 172

Pregnancy and pellagra, 364

Previous attacks of blackwater fever, $23 \mathrm{I}$

malaria and blackwater fever, 235

Prognosis of amebic dysentery, 434

of blackwater fever, 265

of hookworm disease, 478

of malaria, 166

of pellagra, 374

Prophylaxis of amebic dysentery, 436

of blackwater fever, 270

of hookworm disease, 480 
Prophylaxis of malaria, 172

of pellagra, 380

Pseudopellagra, 364

Puerperal septicemia and malaria, 162

Pupa of anopheles, $5^{8}$

Quartan fever, IOI parasites, $7 \mathbf{I}$

Quinine, absorption and elimination, I97

action on parasites, 200

and blackwater fever, 224, 239

choice of preparation, 202

contra-indications, $20 \mathrm{I}$

dose,, 208

in blackwater fever, 272

in malaria, 197

methods of administration, 203

prevention of malaria, 190

substitutes, 214

Quotidian estivo-autumnal, I05

RACE and amebic dysentery, $40 \mathrm{I}$ and blackwater fever, 229 and hookworm disease, $45^{2}$ and malaria, 32, 138 and pellagra, 289

Rainfall and malaria, 26 and pellagra, 288

Recurrences in pellagra, $36+$

Relapse in malaria, 84, I 19

Relative frequency of malaria, 24

Respiratory organs in hookworm disease, 469

in malaria, 109,124

Round worms, 514

Salvarsan in malaria, 216

Schizogonic cycle, 67

Season and amebic dysentery, $40 \mathrm{I}$ and blackwater fever, 231 and hookworm disease, $45 \mathrm{I}$ and malaria, 26 and pellagra, 287

Sepsis and malaria, I62

Sequels of blackwater fever, 259 of hookworm disease, 472 of malaria, 123
Sex and amebic dysentery, 402 and blackwater fever, 230 and hookworm discase, 452 and malaria, 36 and pellagra, 290

Skin and malaria, I I I , I 34 and pellagra, 313, 327, 369

Smallpox and malaria, I 37

Social condition and hookworm disease, 453

and malaria, 40

and pellagra, 293

Soil and hookworm disease, $45 \mathrm{I}$ and malaria, 27

Sources of error in malaria, 149

Spinal fluid in pellagra, 359

Spleen in blackwater fever, 247 in malaria, 91 , 94, 125

in pellagra, 323

rupture, 129

Spontaneous recovery in malaria, I 66

in pellagra, 374

Sporogonic cycle, 76

Staining ameba, 428

Stains for malaria, 147

Stomach in blackwater fever, 249

in malaria, 92, 96, 109, 125

in pellagra, $315,34^{\circ}, 356,37 \mathrm{I}$

Stomatitis in pellagra, 339

Strongyloides intestinalis, 5 I I

Surgery and blackwater fever, 278 and malaria, 139

Symptomatic treatment of malaria, 210

Symptoms of amebic dysentery, 419 of blackwater fever, 250

of hookworm disease, 462

of malaria, 97, 105

of pellagra, 324

Syphilis and malaria, I 37, I63

TæenIA saginata, 497

solium, 501

Tapeworm, 497, 501, 503, 509

Technic of blood examinations, $1+2$

Temperature in amebic dysentery, 42 I

in malaria, 105 


\section{Tertian fever, 99} parasites, 69

Therapeutic test in hookworm disease, 476

in malaria, 159

Thoracic forms of malaria, I 16

Thymol, 487

Topography and malaria, 28 and pellagra, 288

Toxin in malaria, 82

Transfusion and pellagra, $39 \mathrm{I}$

Trauma and malaria, 139

Treatment of amebic dysentery, 438 of blackwater fever, 272 of hookworm disease, 487 of malaria, 197 of pellagra, 385

Trees and malaria, 30

Trichocephalus dispar, 52 I

Trichuris trichura, $5^{2} \mathrm{I}$

Typhoid fever and malaria, 135, 163

Typhoid pellagra, 360

pernicious malaria, II 5
Typhomalarial fever, 135

Tuberculosis and malaria, I36, 163

UNCINARIA americana, 454

Uncinariasis. See Hookworm disease.

Urine in blackwater fever, 252 in hookworm disease, 470 in malaria, 109

in pellagra, 355

Vegetation and malaria, 30

WASSERMANN reaction in malaria, 108

in pellagra, 354

Whipworm, 521

Wind and malaria, 31

YeLLow fever and blackwater fever, 262 and malaria, 163 

,

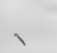

(5)

I
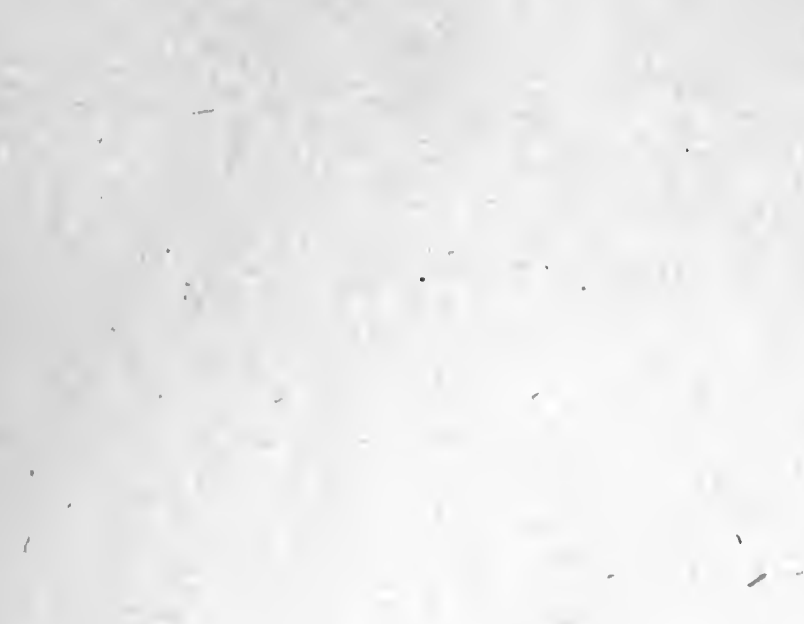

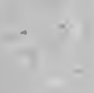

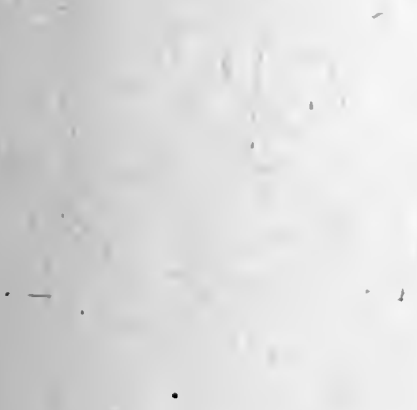

,

$x^{1}+2 x+10$ 



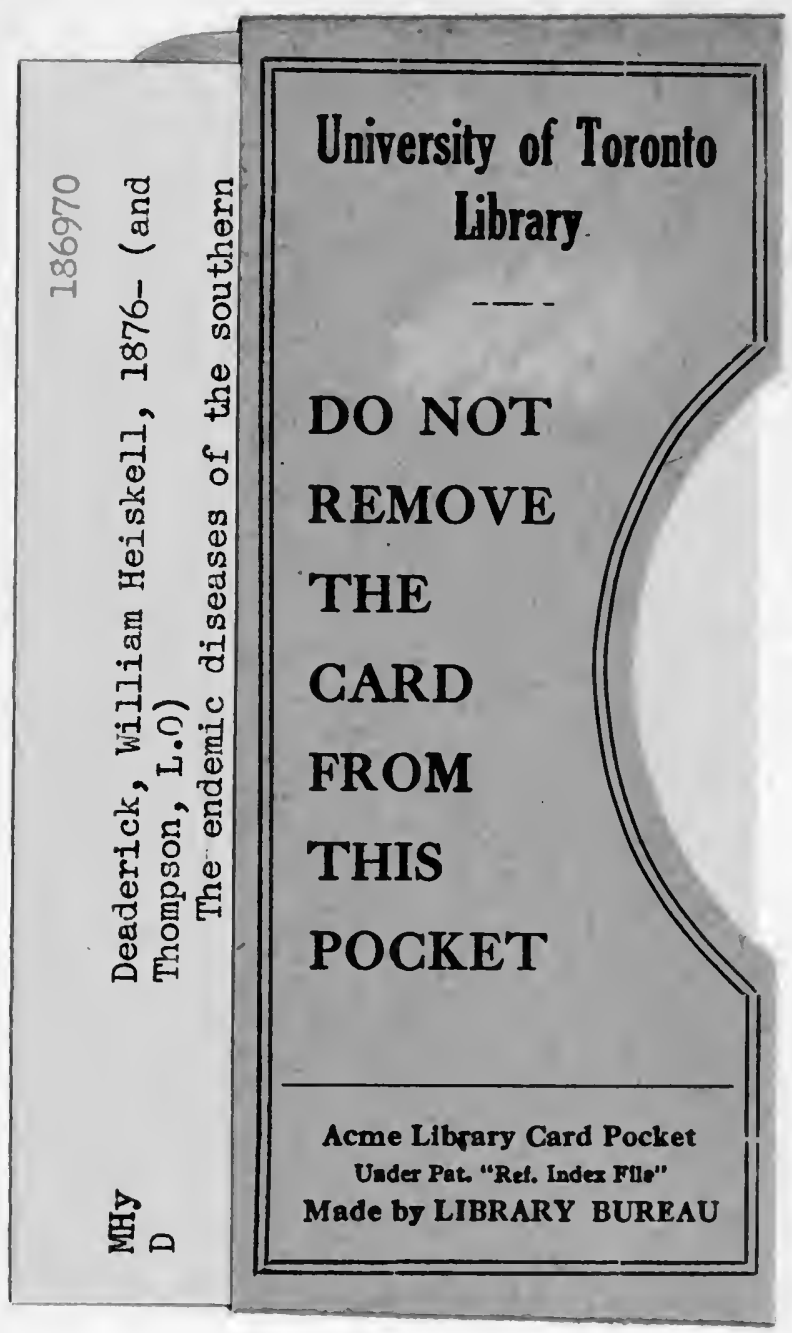


